\title{
Two-Phase Synthesis of Taxol ${ }^{\circledR}$
}

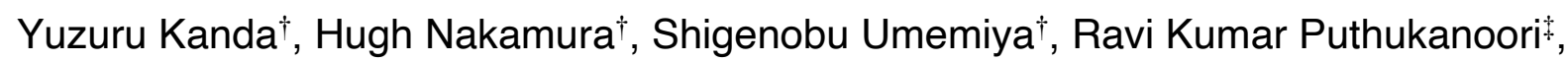
Ravi Kumar Puthukanoori , Venkata Ramana Murthy Appala $\$$, Gopi Krishna Gaddamanugu ${ }^{\ddagger}$, Bheema Rao Paraselli§, Phil S. Baran ${ }^{\dagger *}$

† Department of Chemistry, Scripps Research, 10550 North Torrey Pines Road, La Jolla, CA 92037, United States.

Chemveda Life Sciences, Pvt. Ltd., Plot No. B - 11/1, IDA Uppal, Hyderabad, Telangana 500039, India.

$\S$ Chemveda Life Sciences, Inc., 9920 Pacific Heights Blvd, Suite 150, San Diego, CA 92121, United States.

${ }^{*}$ Correspondence to: Phil S. Baran, pbaran@scripps.edu

\section{SUPPORTING INFORMATION}




\section{Table of Contents}

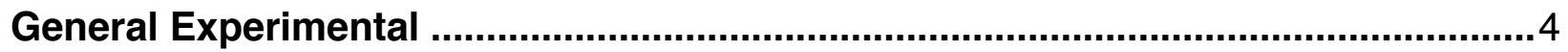

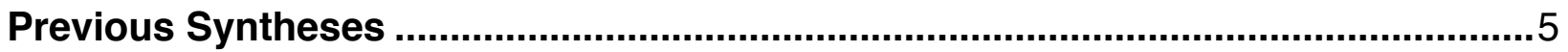

A Summary of Failed Approaches and the Final Optimizations ...............................16

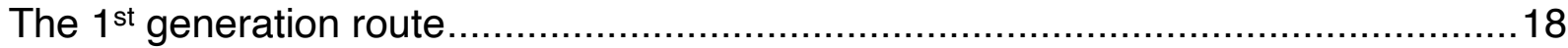

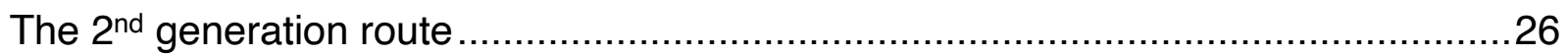

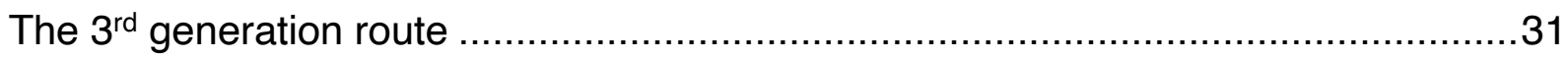

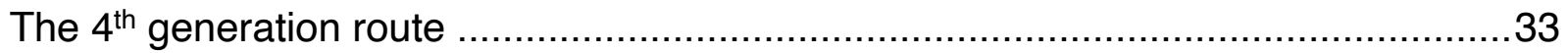

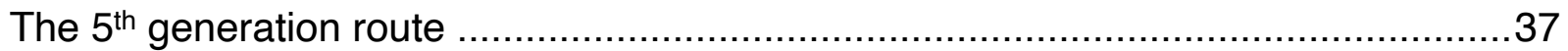

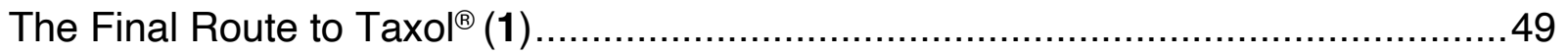

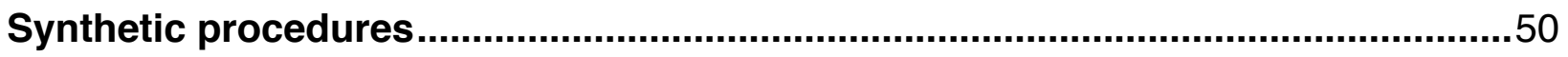

Compound SI3

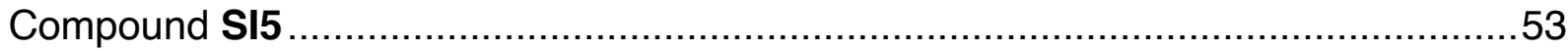

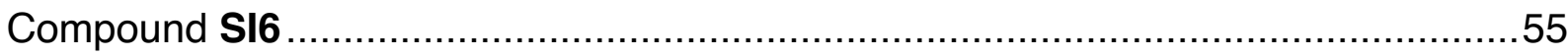

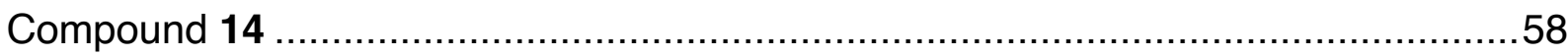

Summary of the Cyclase Phase Optimization ......................................................63

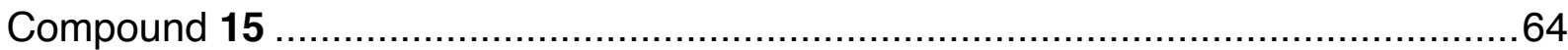

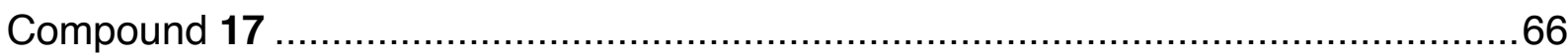

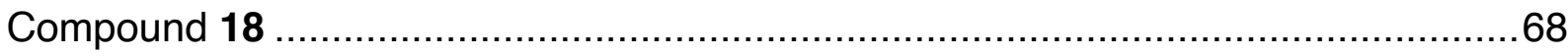

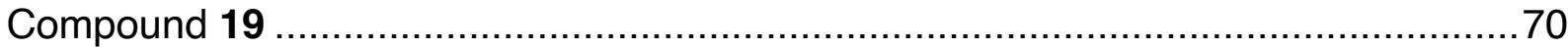

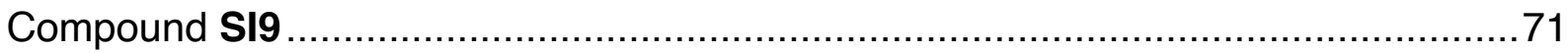

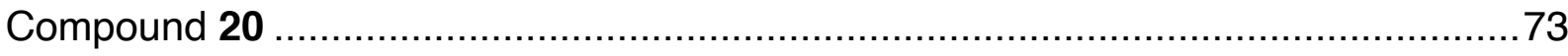

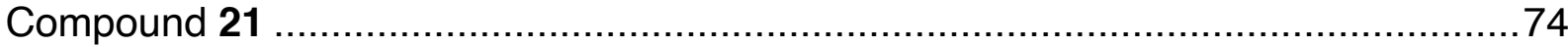

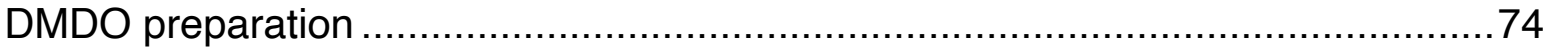

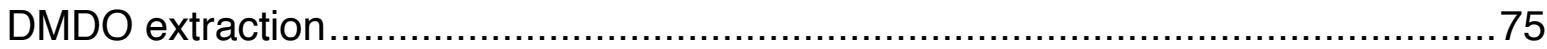

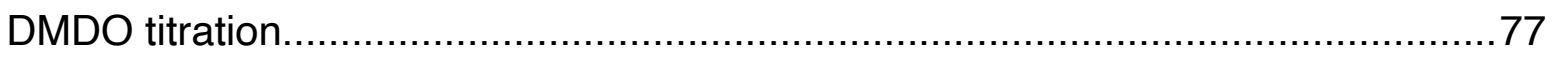

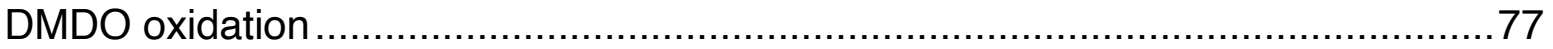

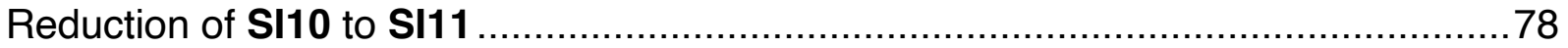

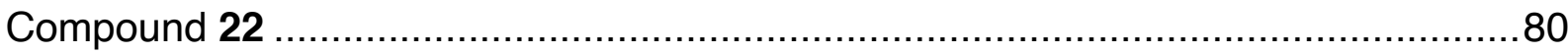

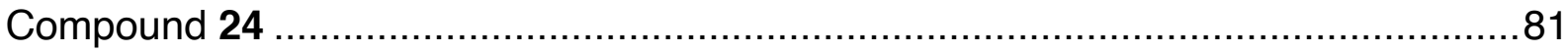

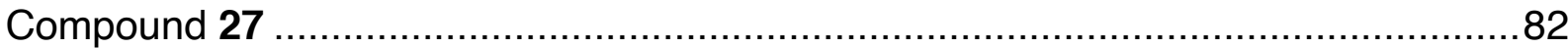

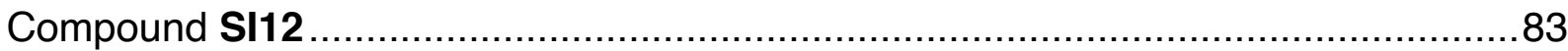

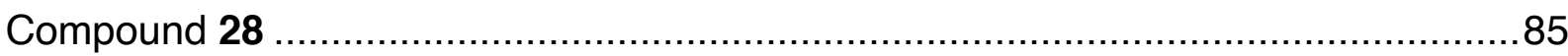

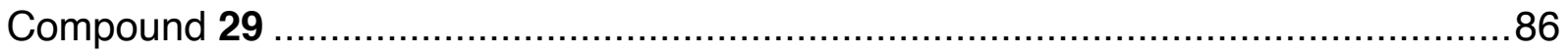




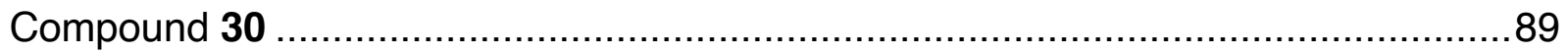

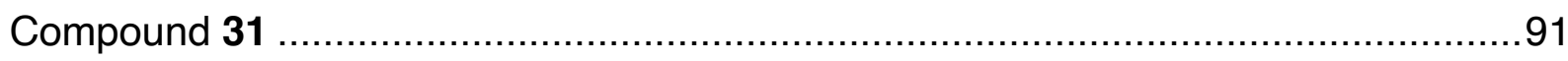

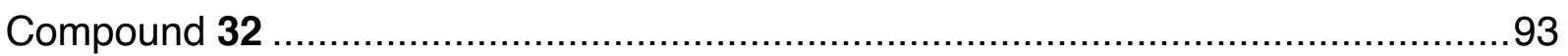

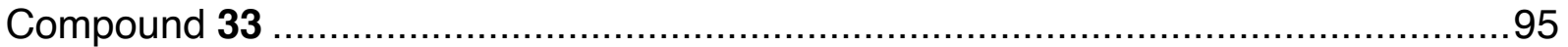

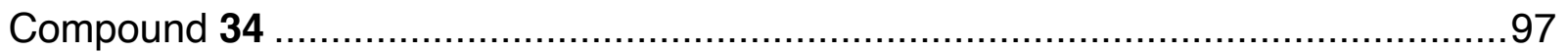

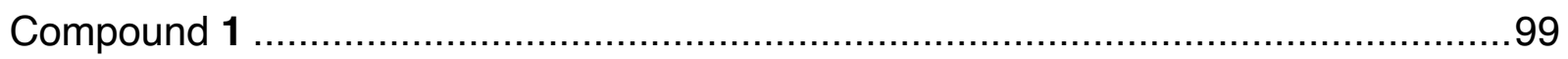

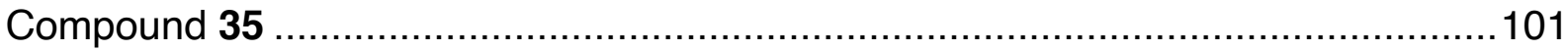

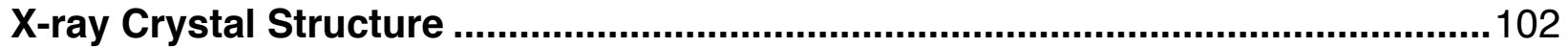

Radiocarbon dating Experiment ................................................................... 110

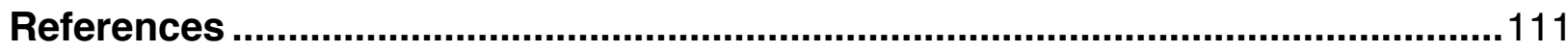

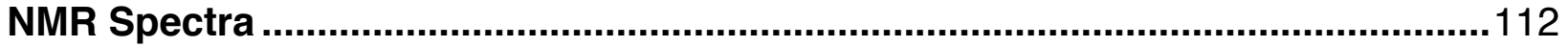




\section{General Experimental}

All reactions were carried out under an inert argon atmosphere with dry solvents under anhydrous conditions unless otherwise stated. Dry acetonitrile ( $\mathrm{MeCN})$, dichloromethane $(\mathrm{DCM})$, diethyl ether $\left(\mathrm{Et}_{2} \mathrm{O}\right)$, tetrahydrofuran $(\mathrm{THF})$, toluene $(\mathrm{PhMe})$, dimethylformamide (DMF), and benzene were obtained by passing the previously degassed solvents through activated alumina columns. Reagents were purchased at the highest commercial quality and used without further purification, unless otherwise stated. Yields refer to chromatographically and spectroscopically $\left({ }^{1} \mathrm{H}\right.$ NMR) homogeneous material, unless otherwise stated. Reactions were monitored by thin layer chromatography (TLC) carried out on $0.25 \mathrm{~mm}$ E. Merck silica plates (60F254), using UV light as the visualizing agent and/or phosphomolybdic acid and $\mathrm{Ce}\left(\mathrm{SO}_{4}\right)_{2}$, acidic ethanolic anisaldehyde, or $\mathrm{KMnO}_{4}$ and heat as developing agents. Flash silica gel chromatography was performed using $\mathrm{E}$. Merck silica gel (60, particle size $0.043-0.063 \mathrm{~mm}$ ). NMR spectra were recorded on Bruker DRX600, DRX-500 and AMX-400 instruments and were calibrated using residual undeuterated solvent as an internal reference $\left(\mathrm{CHCl}_{3}:{ }^{1} \mathrm{H}\right.$ NMR $\delta=7.26 \mathrm{ppm},{ }^{13} \mathrm{C} \mathrm{NMR}$ $\delta=77.16 \mathrm{ppm}$ ). The following abbreviations were used to explain NMR peak multiplicities: $\mathrm{s}=$ singlet, $\mathrm{d}=$ doublet, $\mathrm{t}=$ triplet, $\mathrm{q}=$ quartet, $\mathrm{m}=$ multiplet, $\mathrm{br}=$ broad. High-resolution mass spectra (HRMS) were recorded on an Agilent LC/MSD TOF mass spectrometer by electrospray ionization time-of-flight (ESI-TOF) reflectron experiments. Optical rotations were obtained on a MCP 100 modular circular polarimeter.

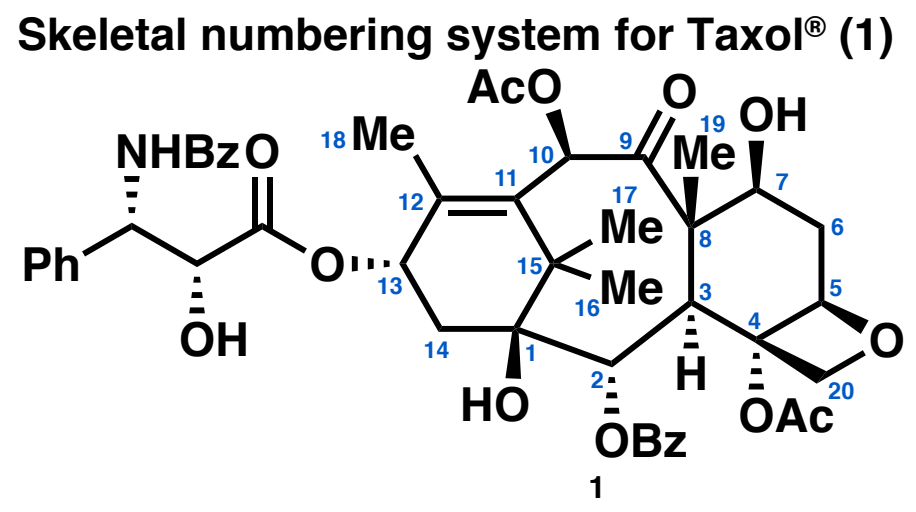


Previous Syntheses

1. Holton's Total Synthesis

J. Am. Chem. Soc. 1994, 116, 1597. J. Am. Chem. Soc. 1994, 116, 1599.
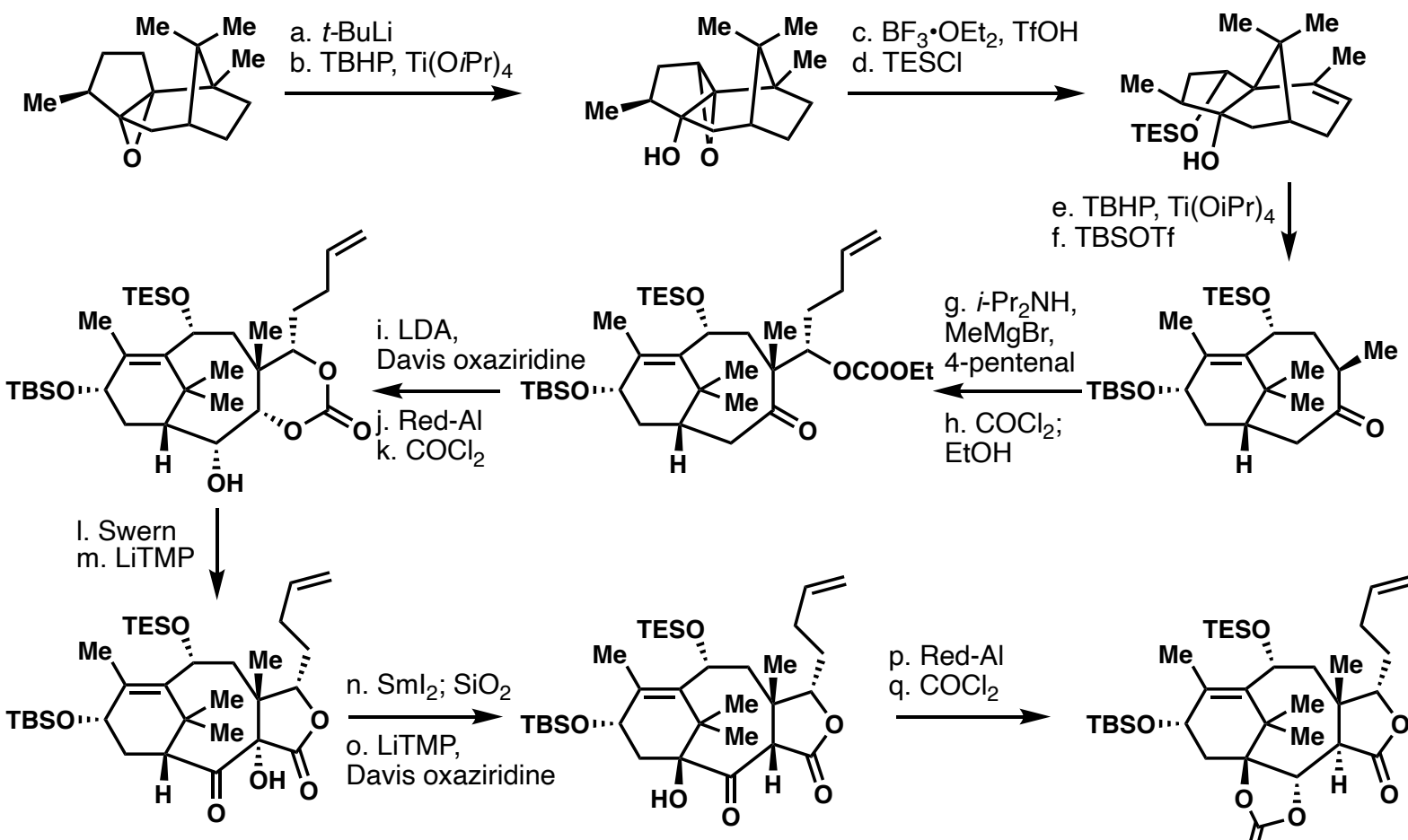

\section{p. Red-Al}

q. $\mathrm{COCl}_{2}$

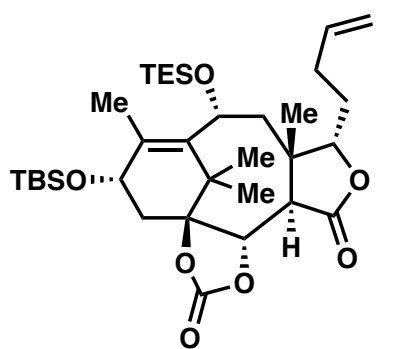

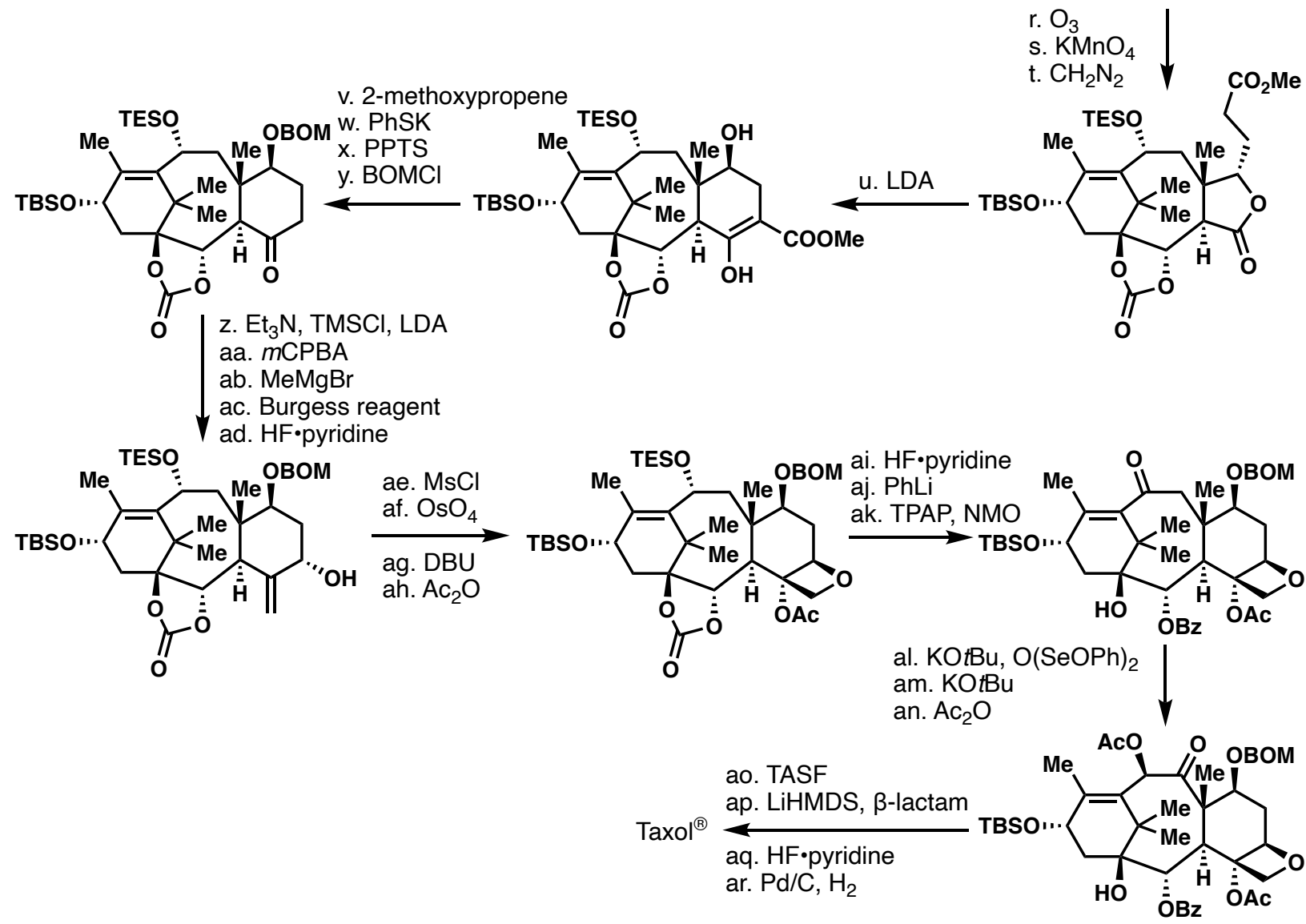


2. Nicolaou's Total Synthesis Nature 1994, 367, 630.
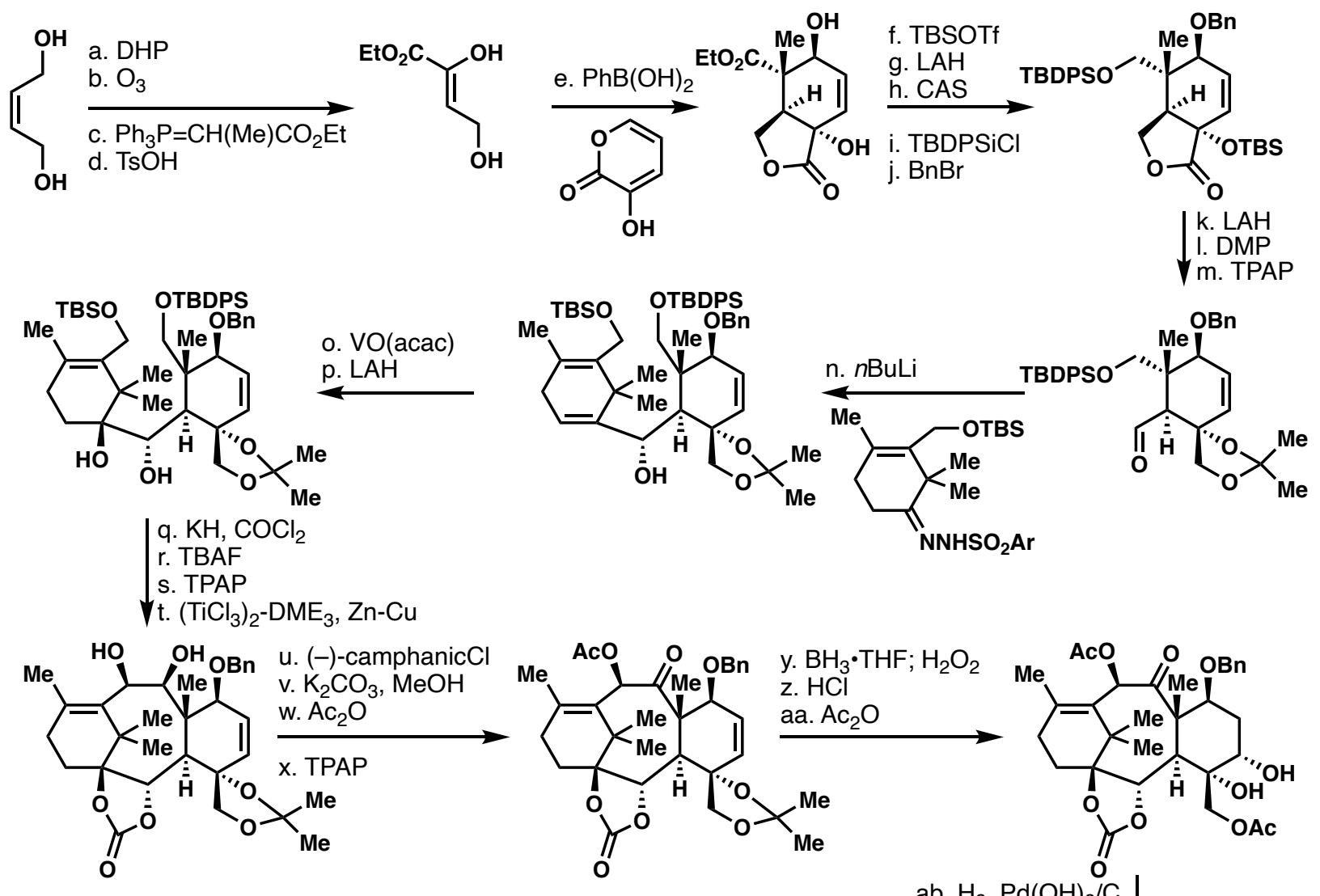

ab. $\mathrm{H}_{2}, \mathrm{Pd}(\mathrm{OH})_{2} / \mathrm{C}$ ac. TESCI

ad. $\mathrm{K}_{2} \mathrm{CO}_{3}, \mathrm{MeOH}$

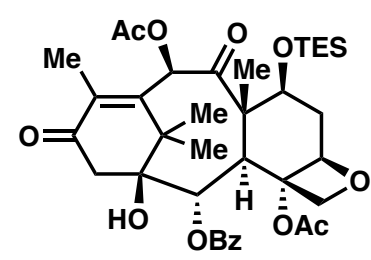

ak. $\mathrm{NaBH}_{4}$ al. NaHMDS, $\beta$-lactam $\checkmark$ am. HF•py

Taxol $^{\circledR}$ ah. $\mathrm{Ac}_{2} \mathrm{O}$ ai. $\mathrm{PhLi}$ aj. PCC

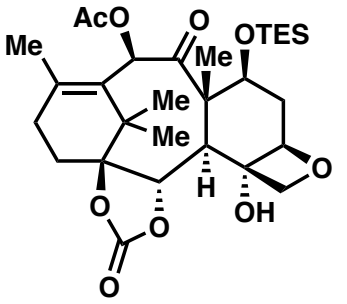
ae. $\mathrm{TMSCl}$ af. $\mathrm{Tf}_{2} \mathrm{O}$ ag. CSA, $\mathrm{SiO}_{2}$

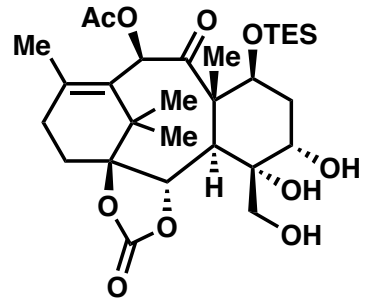


3. Danishefsky's Total Synthesis Angew. Chem. Int. Ed. 1995, 34, 1725.

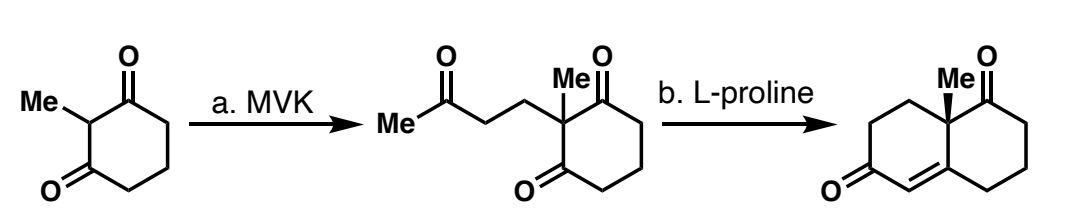

c. $\mathrm{NaBH}_{4}$

d. $\mathrm{Ac}_{2} \mathrm{O}$

e. $\left(\mathrm{HOCH}_{2}\right)_{2}$

f. $\mathrm{NaOMe}$

g. TBSOTf

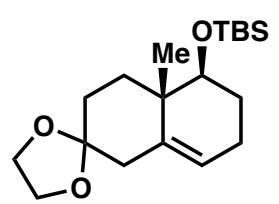

h. $\mathrm{BH}_{3} \cdot \mathrm{THF}, \mathrm{H}_{2} \mathrm{O}_{2}$

i. PDC

j. $\mathrm{NaOMe}$

k. $\mathrm{Me}_{3} \mathrm{SI}, \mathrm{KHMDS}$<smiles>COCC[C@]1(C)[C@@H]([OH2+])C[C@@H]2OC[C@]2(O)[C@@H]1CC=O</smiles>

r. $\mathrm{TsOH}$

s. TMSOTf

t. DMDO

u. $\mathrm{Pb}(\mathrm{OAc})_{4}, \mathrm{MeOH}$

m. $\mathrm{OsO}_{4}$

n. $\mathrm{TMSCl}$

$\checkmark$ I. $\mathrm{Al}(\mathrm{O} i \mathrm{Pr})_{3}$
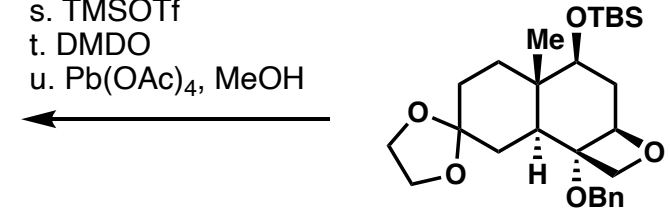

p. $\left(\mathrm{CH}_{2} \mathrm{O}\right.$

q. $\mathrm{BnBr}$
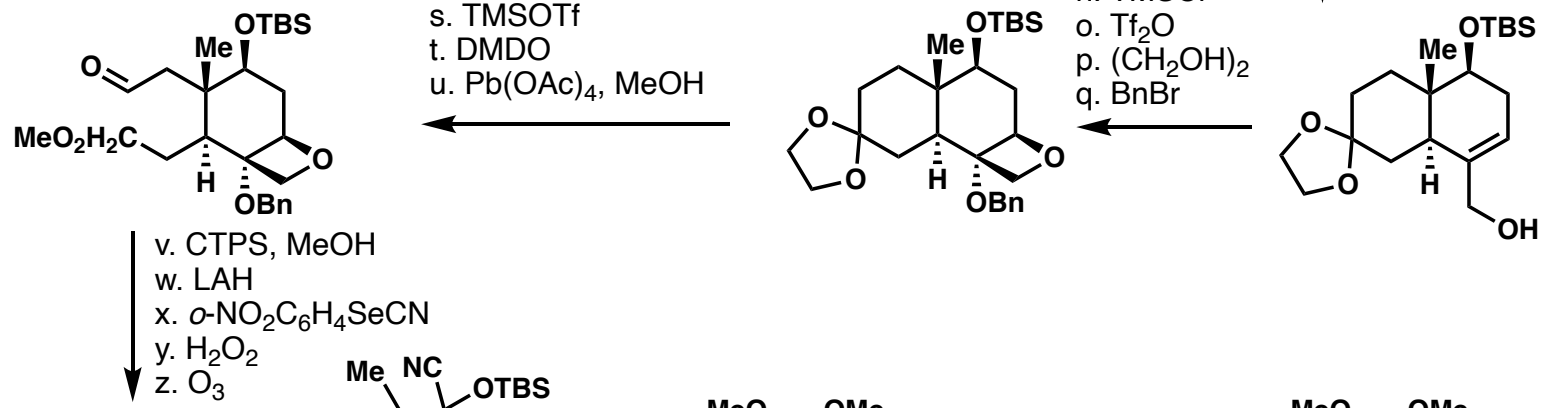

$\mathrm{MeO}$<smiles>COC(C)CC1(C)[C@H](C=O)C[C@H]2OC[C@]2(Cc2ccccc2)[C@@H]1OC</smiles>

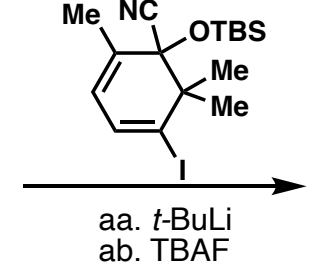

ab. TBAF

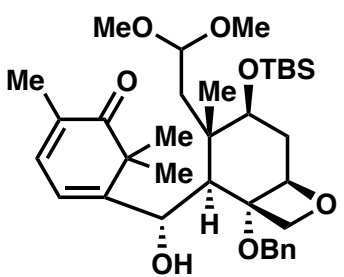

ac. $m C P B A$

ad. $\mathrm{H}_{2}, \mathrm{Pd} / \mathrm{C}$ ae. CDI

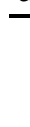

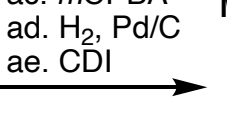<smiles>CC/C=C(/C)CC</smiles>

$\mathrm{MeO} \quad \mathrm{OMe}$

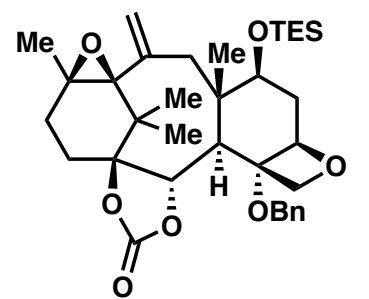

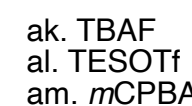

am. mCPBA
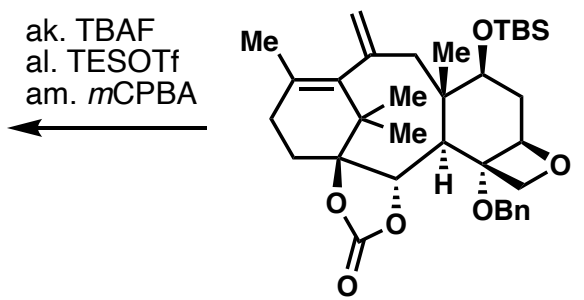

aj. $\mathrm{Pd}\left(\mathrm{PPh}_{3}\right)_{4}$

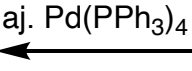

$$
\mid \begin{aligned}
& \text { an. } \mathrm{H}_{2}, \mathrm{Pd} / \mathrm{C} \\
& \text { ao. } \mathrm{Ac}_{2} \mathrm{O} \\
& \text { ap. } \mathrm{PhLi}
\end{aligned}
$$

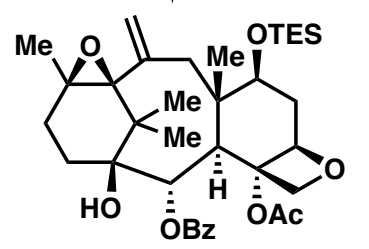

aq. $\mathrm{OsO}_{4}$
ar. $\mathrm{Pb}(\mathrm{OAc})_{4}$
as. $\mathrm{Sml}_{2}, \mathrm{Ac}_{2} \mathrm{O}$
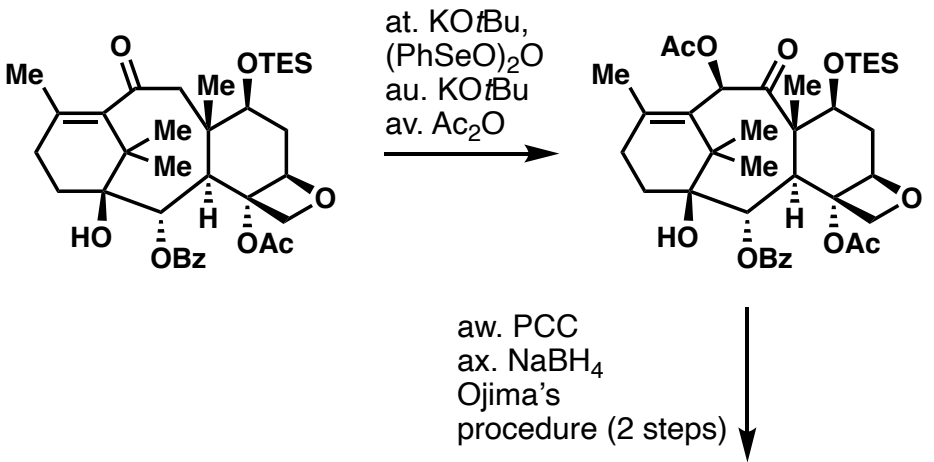

Taxol $^{\circledR}$ 
4. Wender's Total Synthesis

J. Am. Chem. Soc. 1997, 119, 2755. J. Am. Chem. Soc. 1997, 119, 2757.
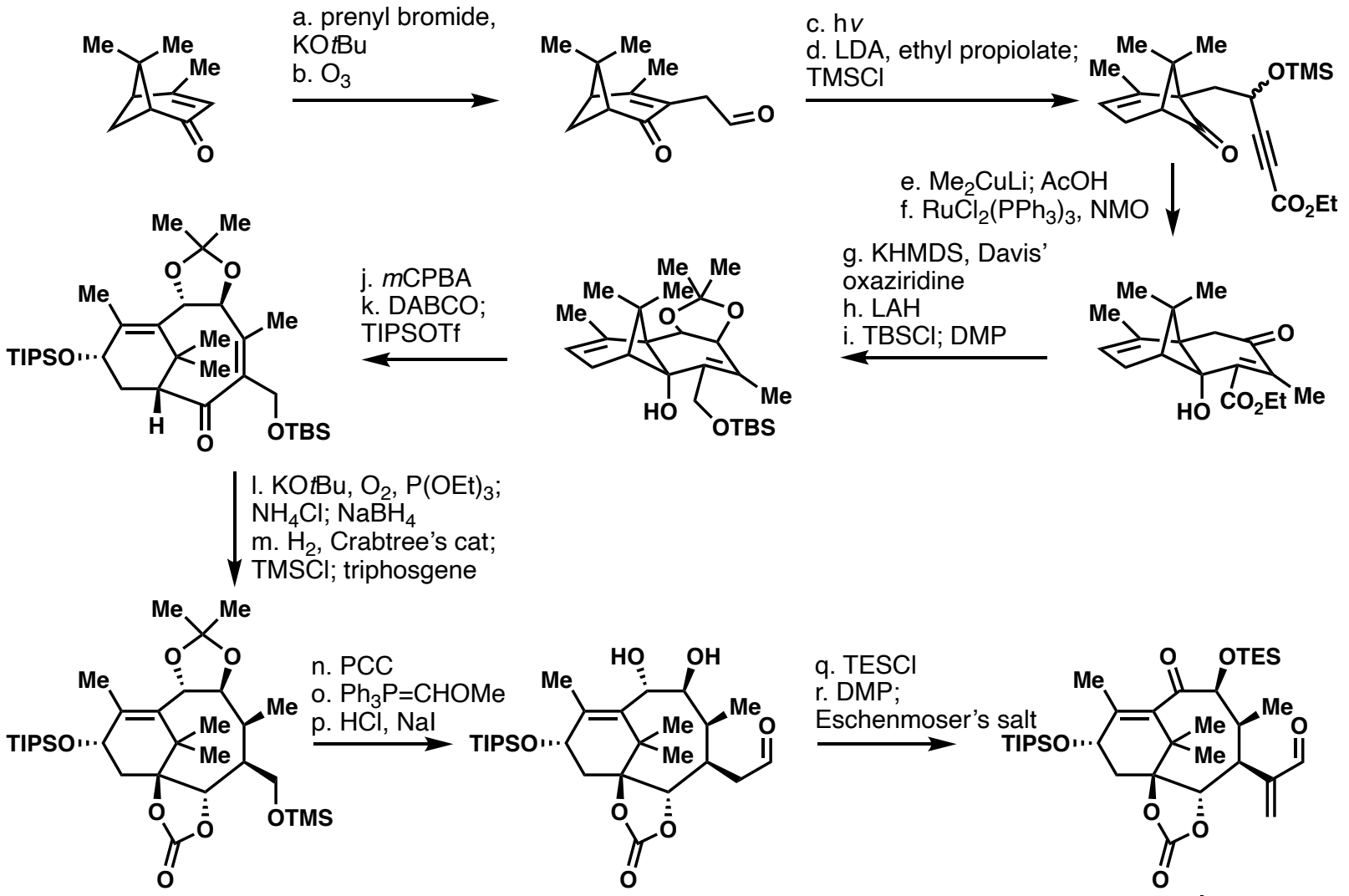

g. KHMDS, Davis'
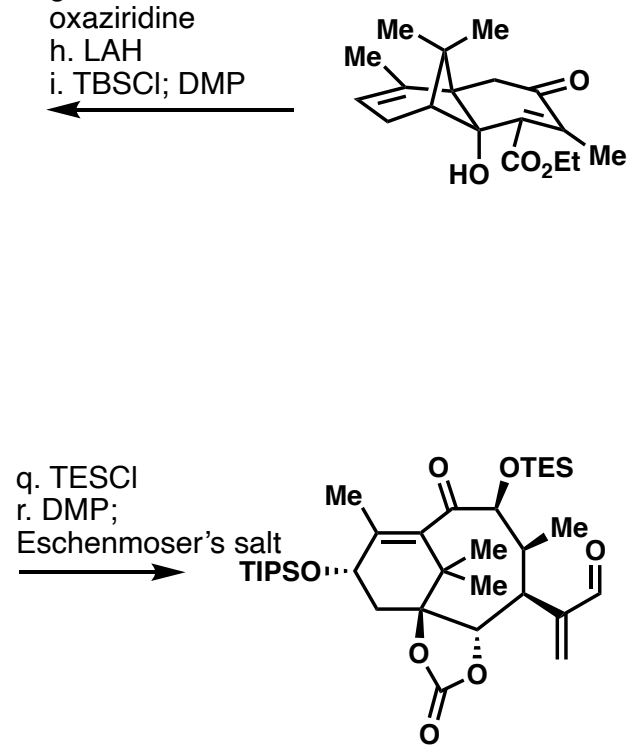

s. allyMgBr, $\mathrm{ZnCl}_{2}$
t. $\mathrm{BOMCl}$

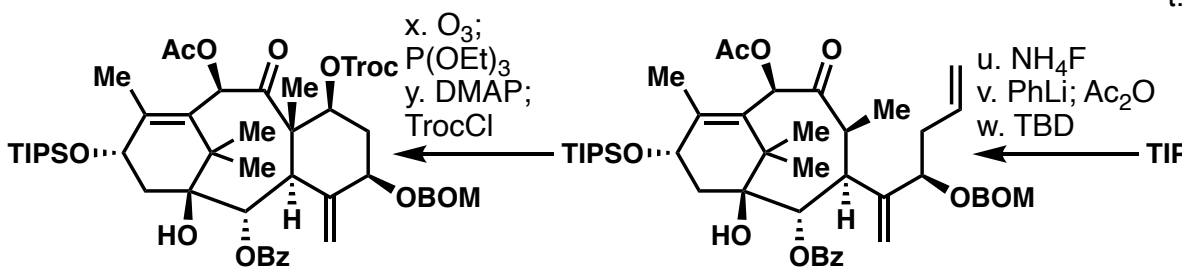

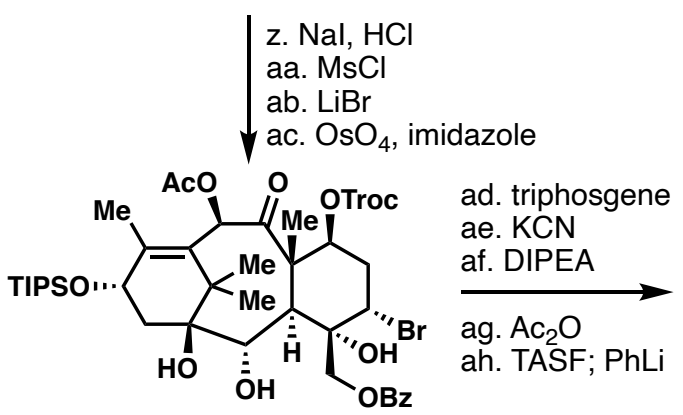

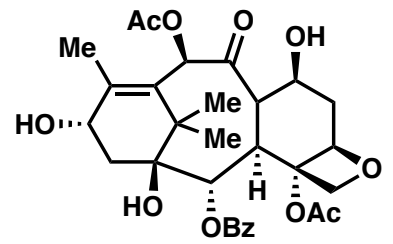

Ojima's procedure (3 steps) Taxol $^{\circledR}$ 
5. Kuwajima's Total Synthesis J. Am. Chem. Soc. 1998, 120, 12980.

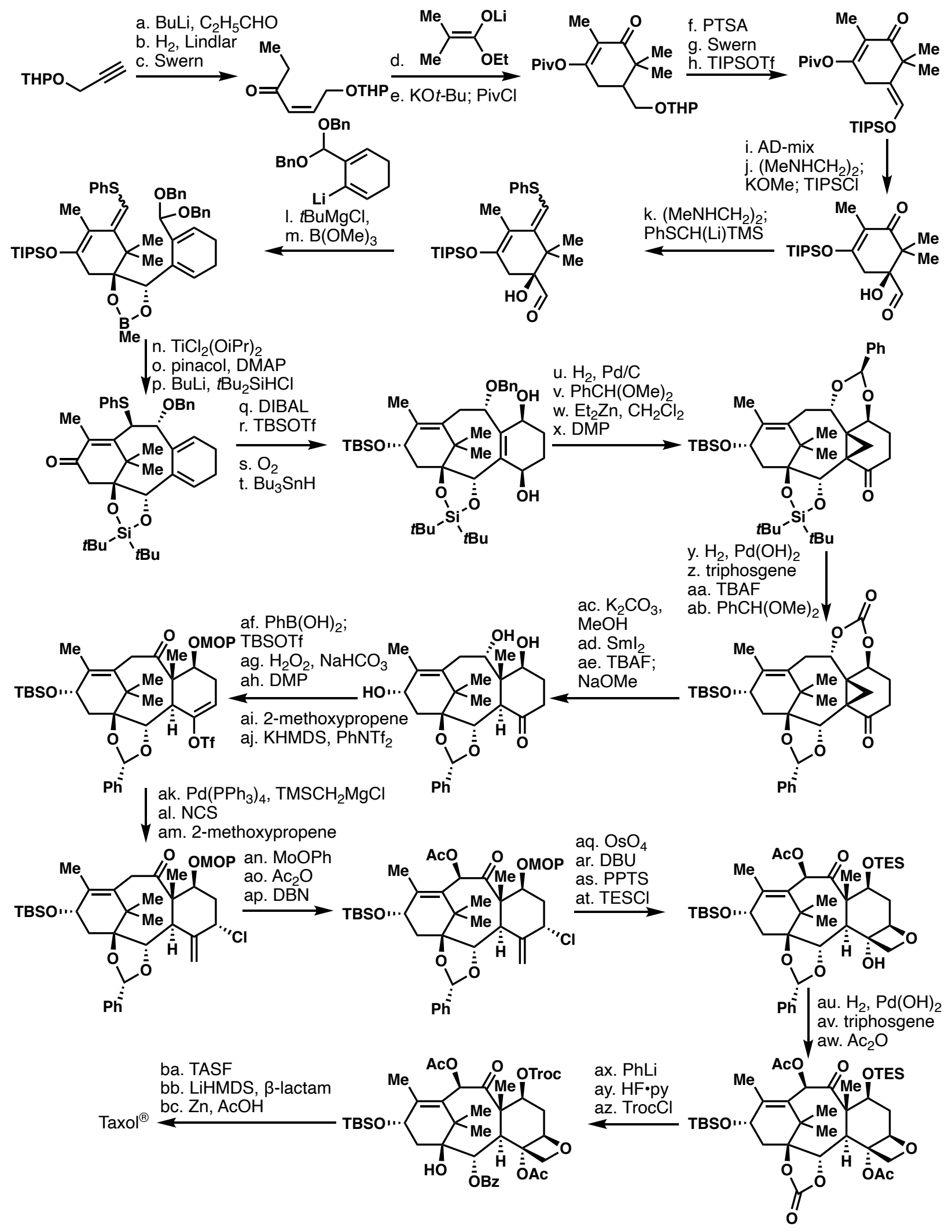


6. Mukaiyama's Total Synthesis Chem. Eur. J. 1999, 5, 121.
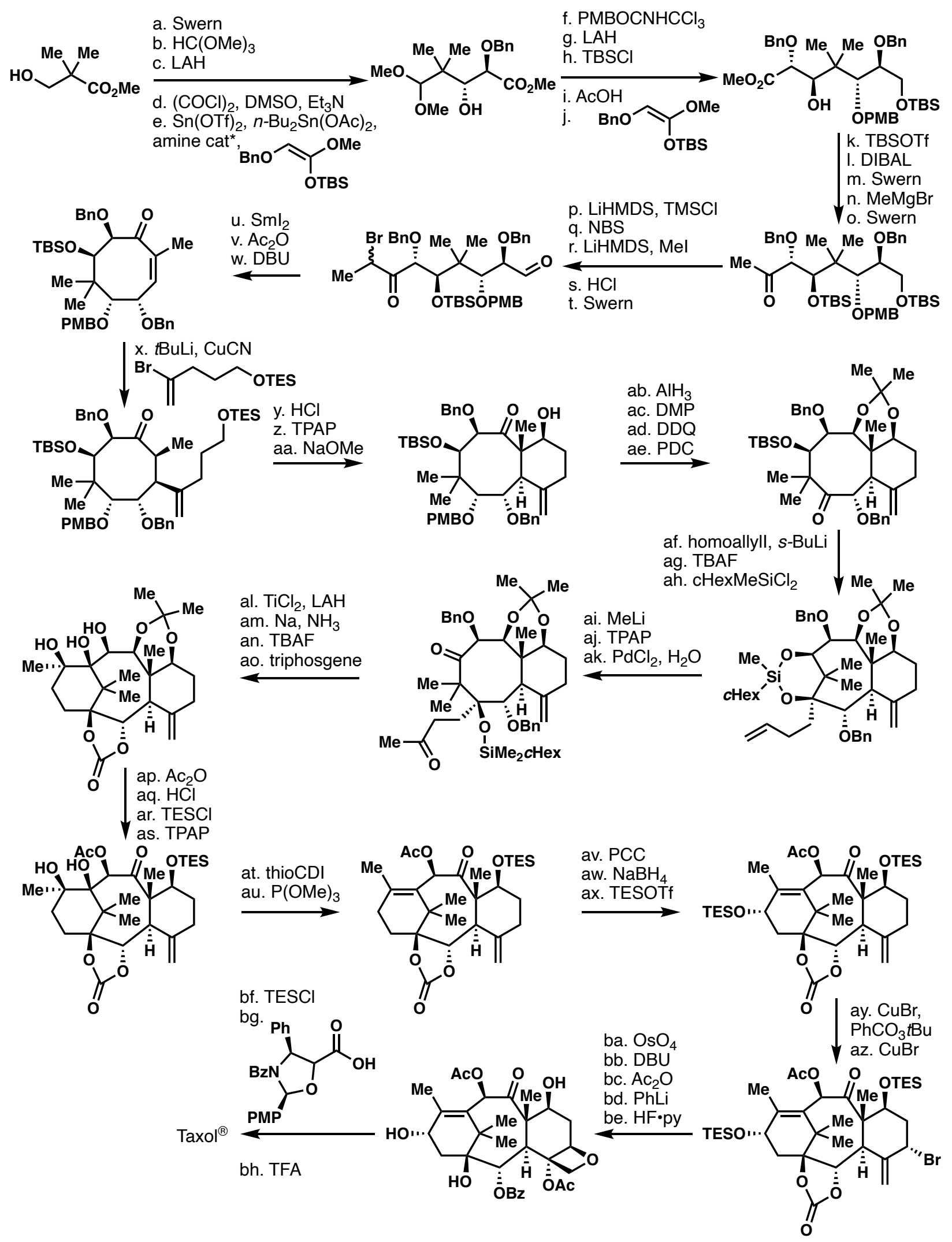
J. Lim, A Total Synthesis of Taxol. Ph.D. thesis, Harvard University, 2000.
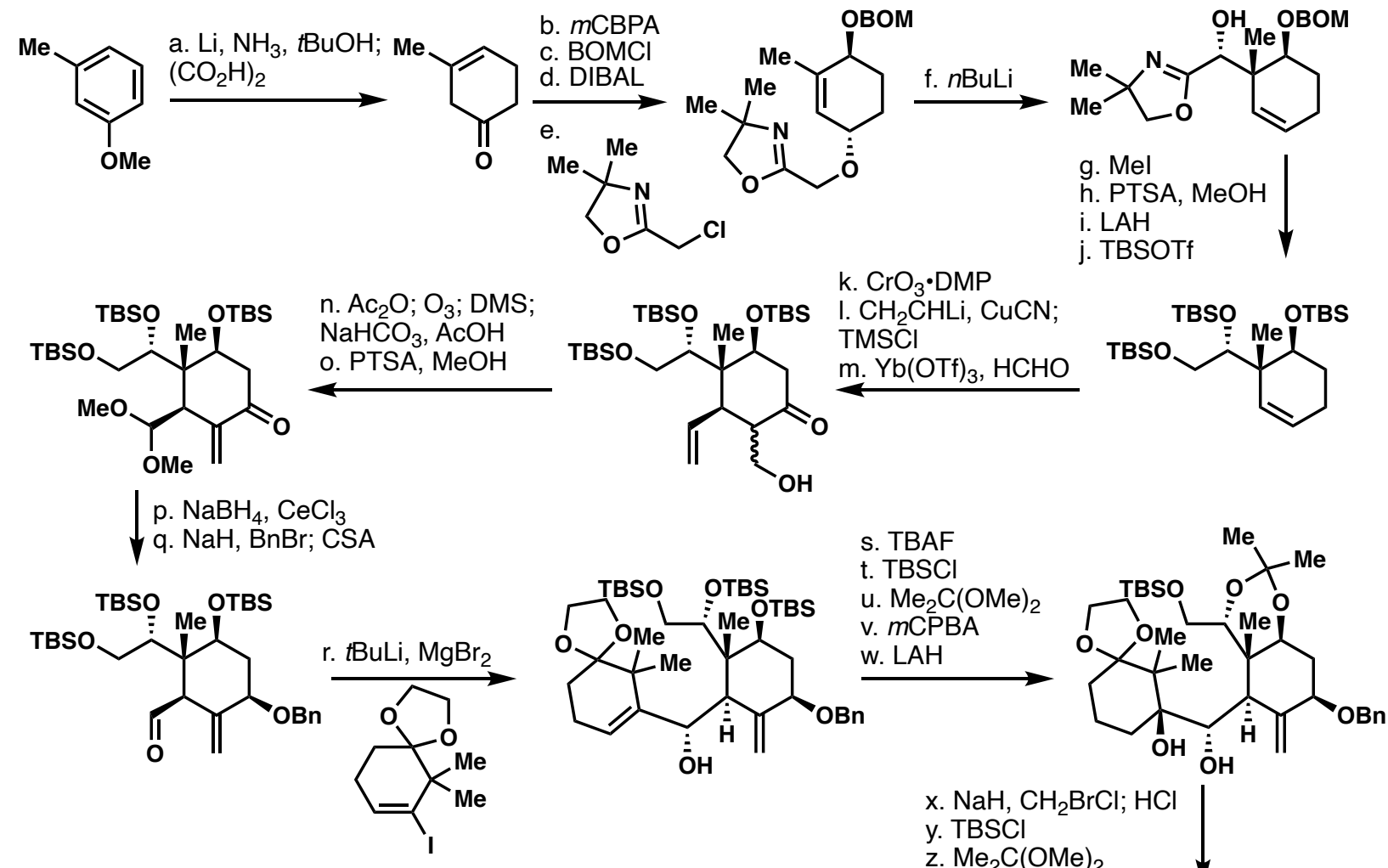

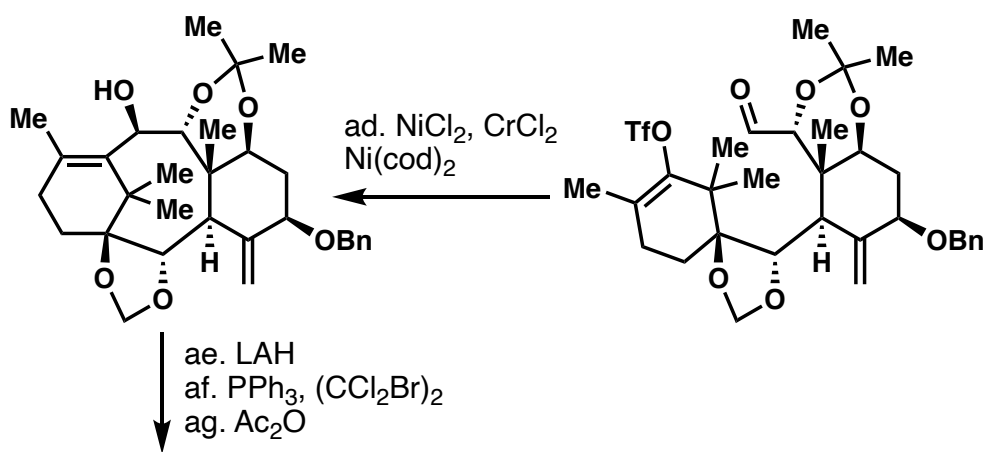

x. $\mathrm{NaH}, \mathrm{CH}_{2} \mathrm{BrCl} ; \mathrm{HCl}$
y. TBSCl
z. $\mathrm{Me}_{2} \mathrm{C}(\mathrm{OMe})_{2}$
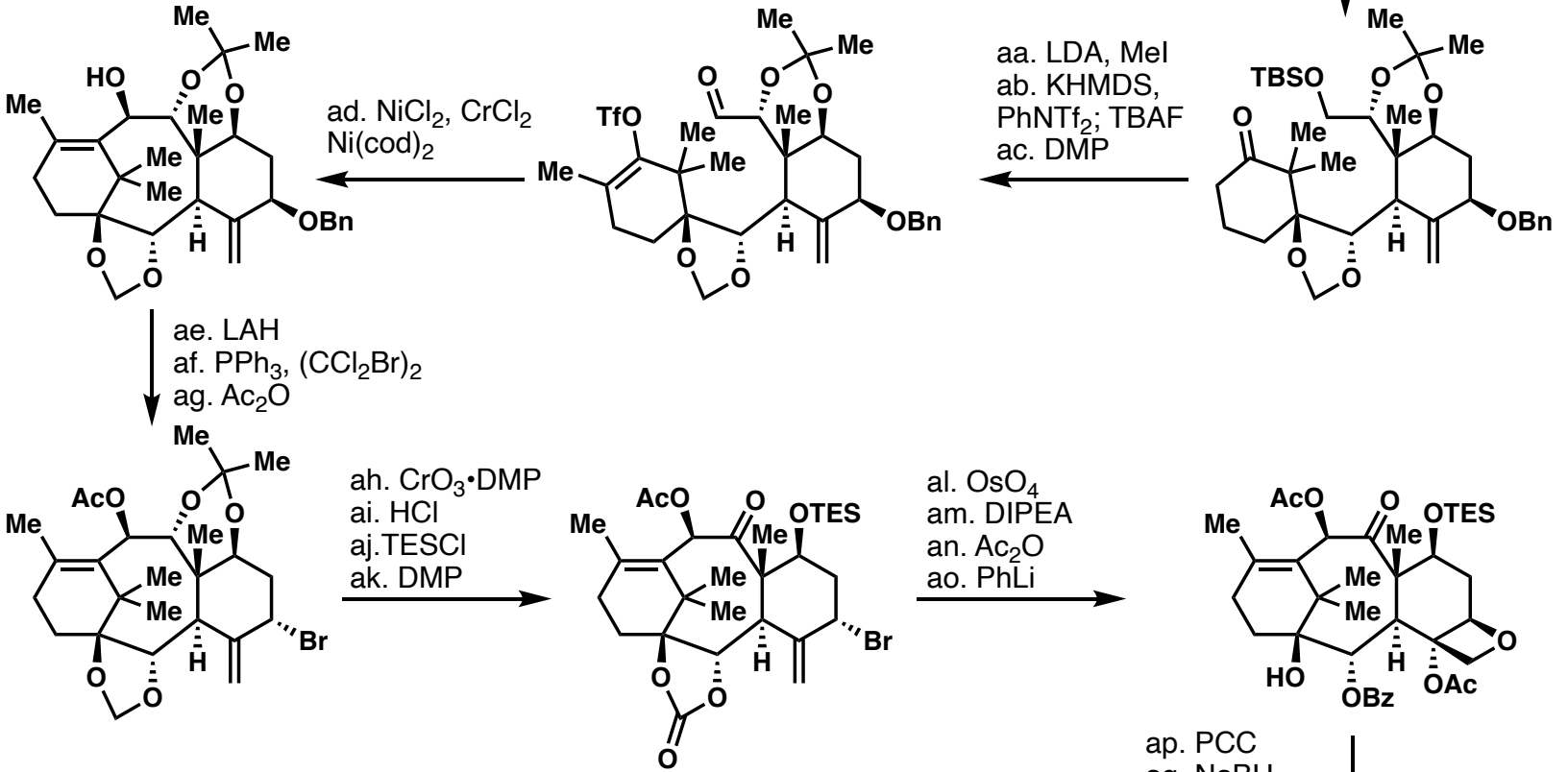

al. $\mathrm{OsO}_{4}$ am. DIPEA an. $\mathrm{Ac}_{2} \mathrm{O}$ ao. $\mathrm{PhLi}$

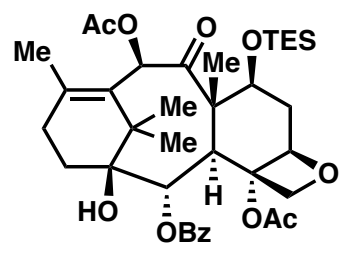

ap. PCC aq. $\mathrm{NaBH}_{4}$ ar. NaHMDS, $\beta$-lactam as. HF•py 
8. Takahashi's Formal Synthesis Chem. Asian J. 2006, 1, 370.
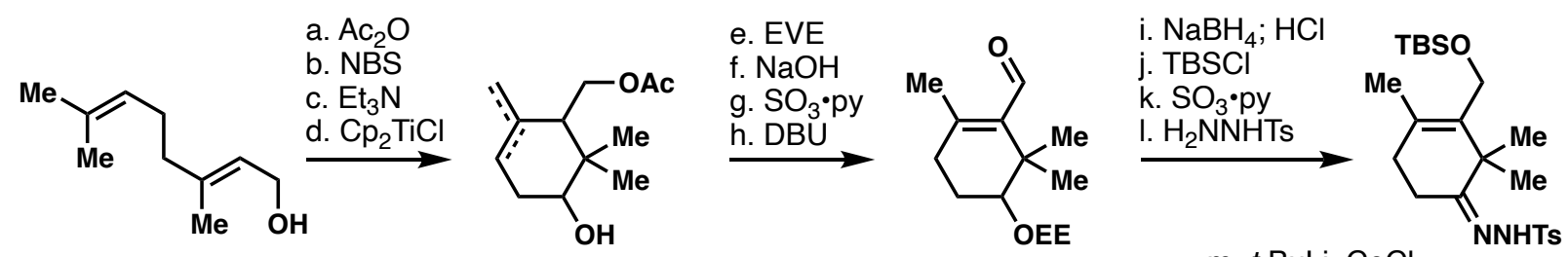

m. $t$-BuLi, $\mathrm{CeCl}_{3}$,

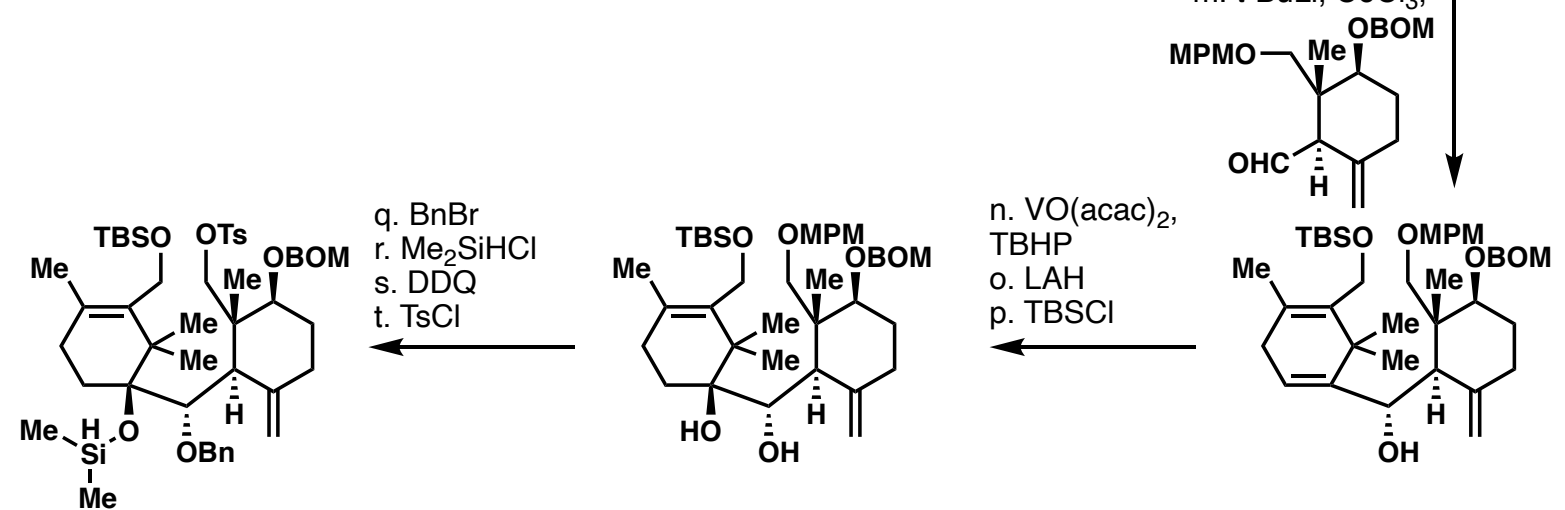

u. TBAF
v. TMSOTf
w. TBAF
x. TPAP
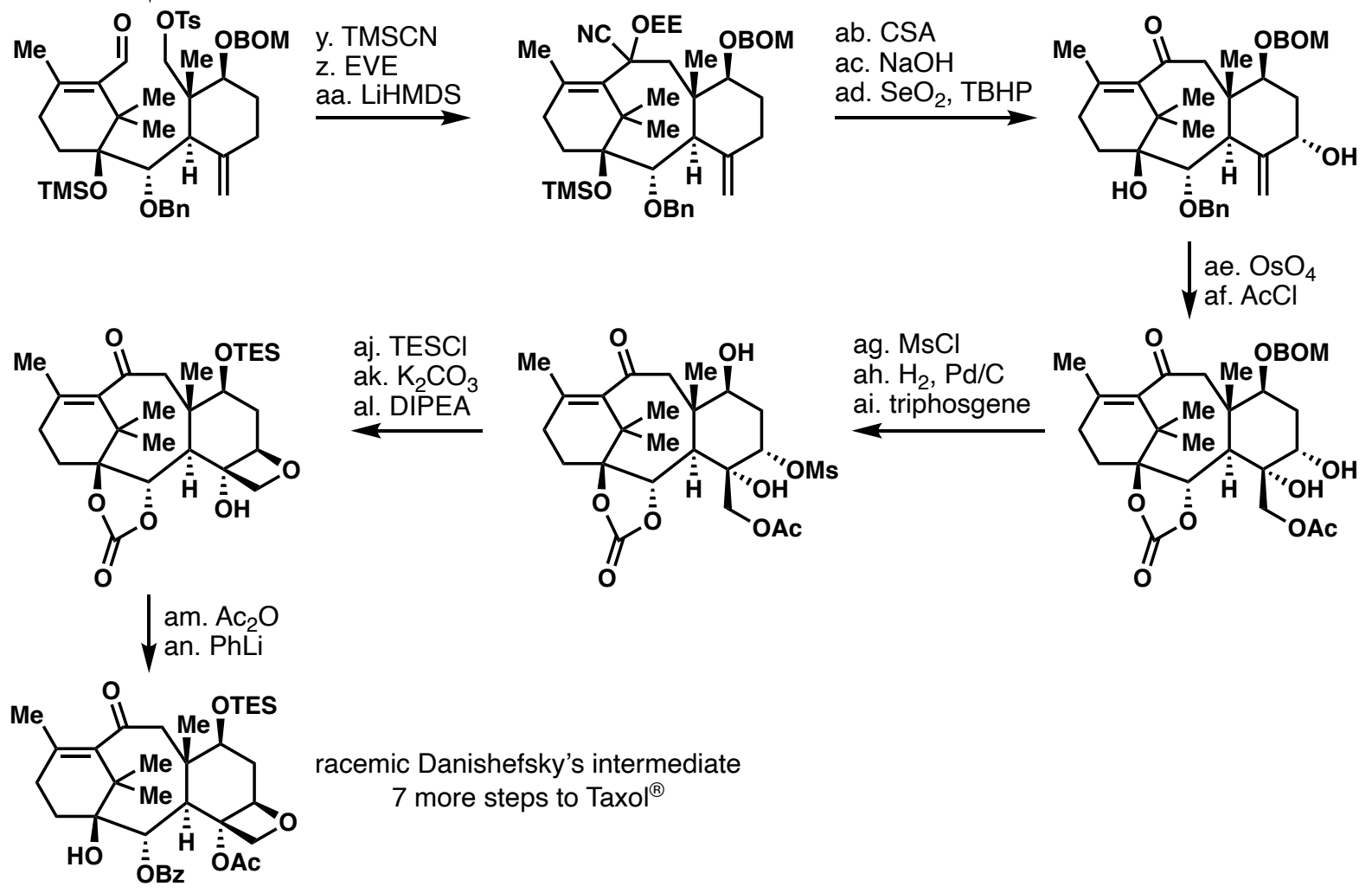
9. Nakada's Formal Synthesis Chem. Eur. J. 2015, 21, 355.

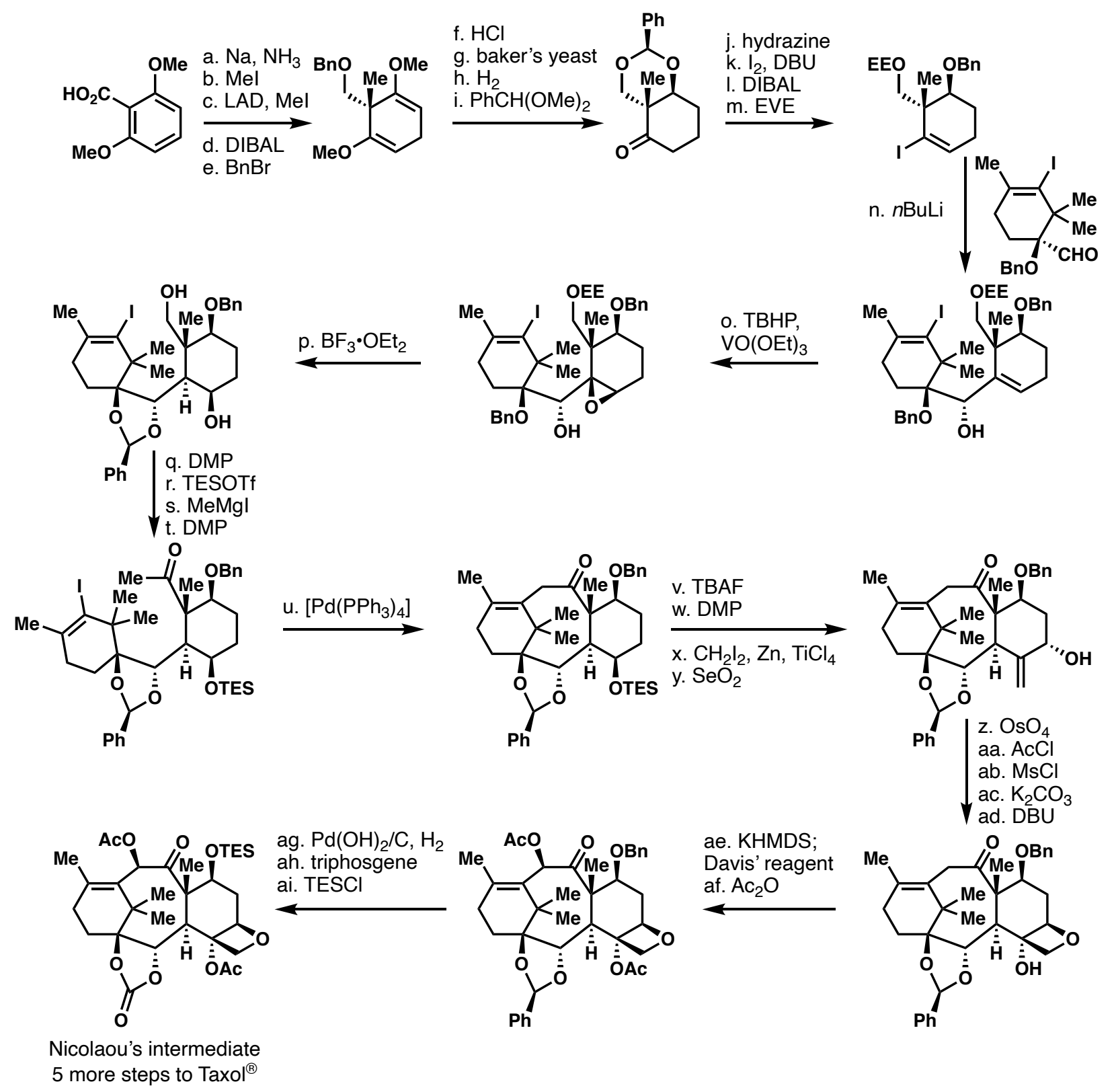


10. Chida's Formal Synthesis Org. Lett. 2015, 17, 2570. Org. Lett. 2015, 17, 2574.

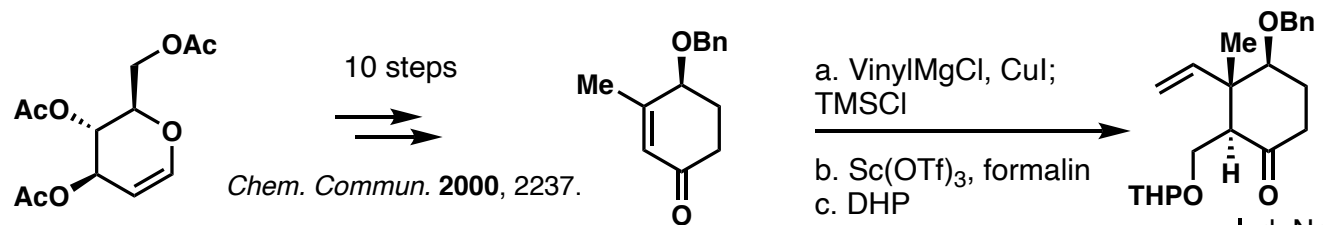
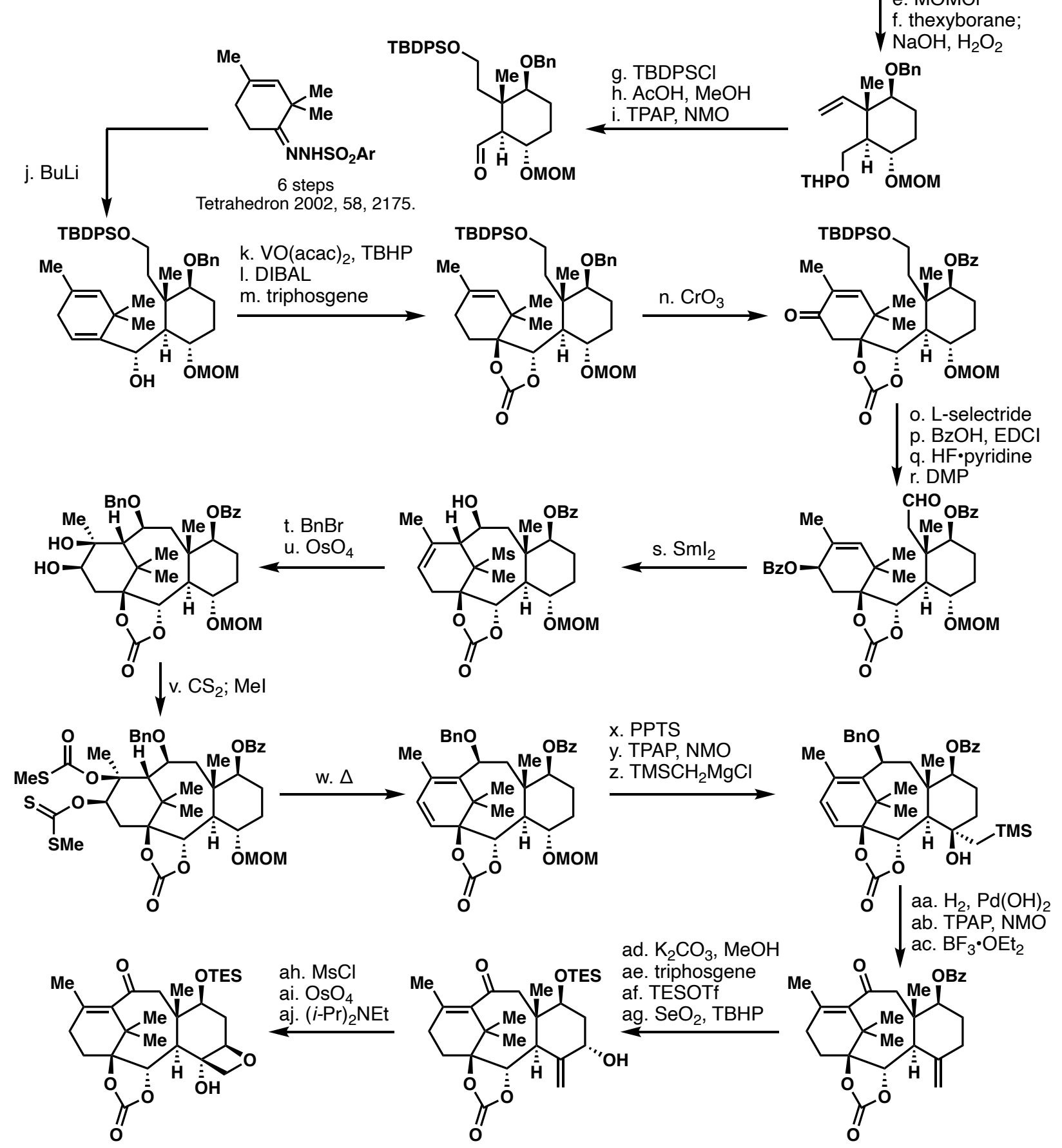

Takahashi's intermediate 9 more steps to Taxol $^{\circledR}$ 


\begin{tabular}{|c|c|c|}
\hline & Synthesized $(\mathrm{mg})$ & Comments \\
\hline Holton & 11.9 & Chiral pool, Synthesized ent \\
\hline Nicolaou & 13.9 & Chiral resolution \\
\hline Danishefsky & No report & - \\
\hline Wender & No report & Chiral pool \\
\hline Kuwajima & 7.4 & - \\
\hline Mukaiyama & 2.9 & - \\
\hline Kishi & 2.9 & Chiral resolution \\
\hline Takahashi & 0 & Formal, racemic \\
\hline Nakada & 0 & Formal \\
\hline Chida & 0 & Formal \\
\hline
\end{tabular}




\section{A Summary of Failed Approaches and the Final Optimizations}

\section{0-1. Strategic Timeline}

Holton, Nicolaou,

Danishefsky,

Wender, Kuwajima,

Mukaiyama, Kishi,

Takahashi, Chida,

Nakada

1994-
${ }_{M e}^{M e}=<_{M e}^{M e}$

Two-Phase project started 2007

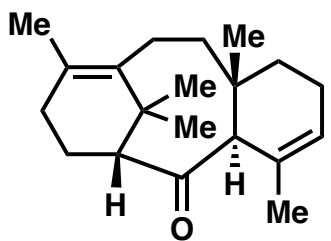

taxadienone

- completion of cyclase phase Nat. Chem. 2012, 4, 21.

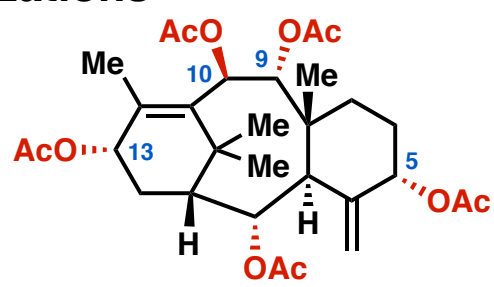

taxabaccatin III

- C9 oxidation

Angew. Chem. Int. Ed. 2016, 55, 8280.
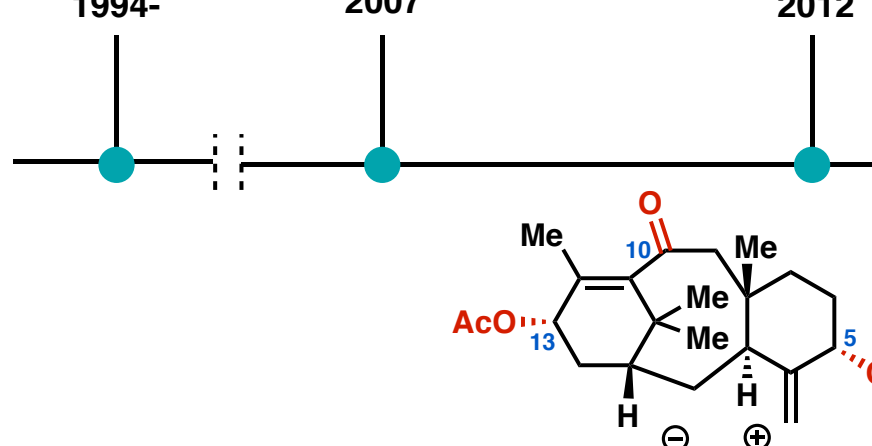

2016

Prior Work

- C5, C10, C13 allylic oxidations

- C9 a-oxidations

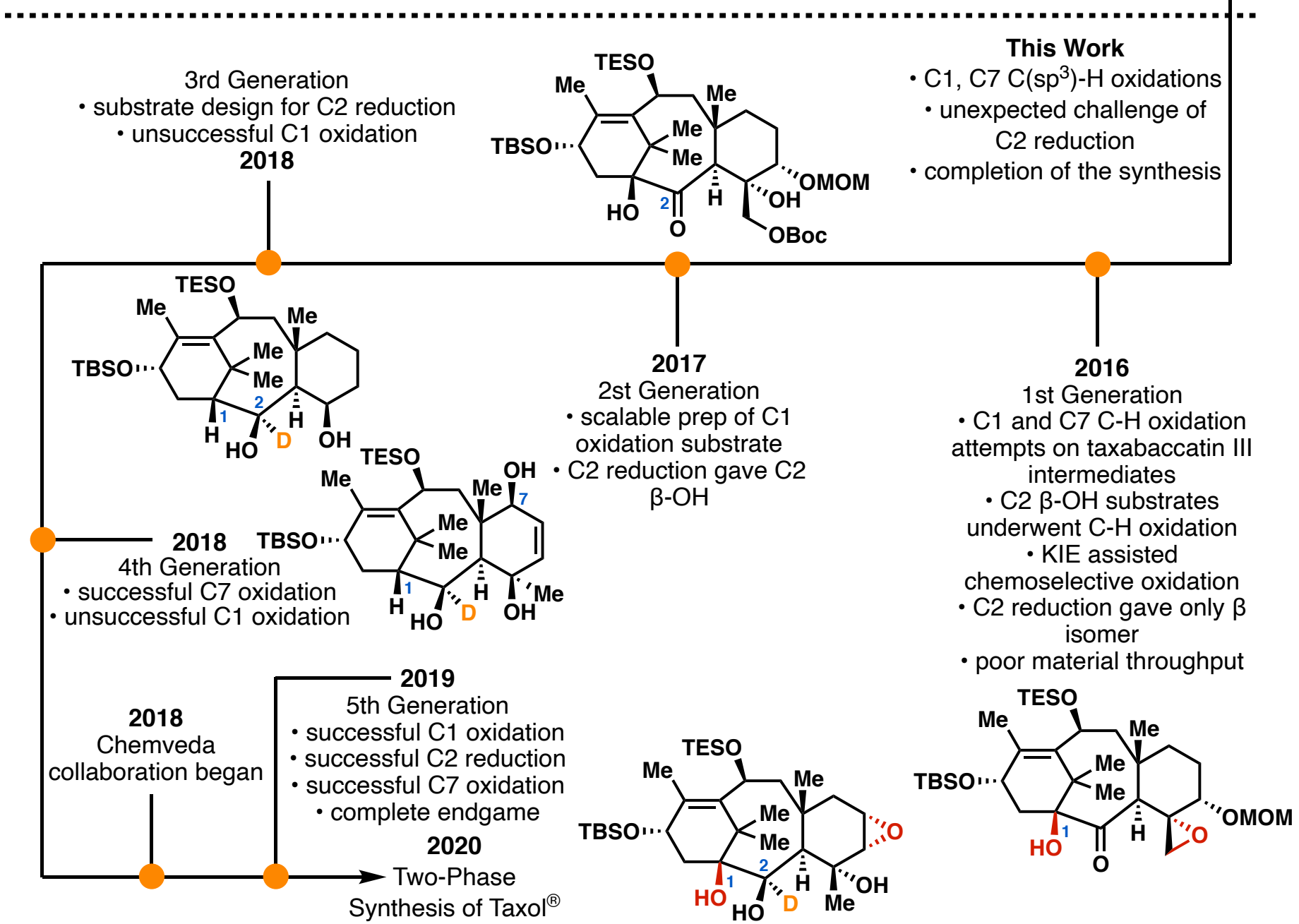


<smiles>CCOC1=CC(=O)CCC1CC=CC1=CC(=O)CCC1</smiles>

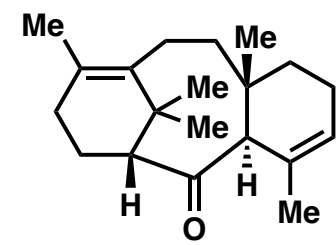

taxadienone cyclase phase endpoint
$\mathrm{Me}_{2} \mathrm{Zn}, \mathrm{Pd}\left(\mathrm{PPh}_{3}\right)_{4}$

Cyclase Phase

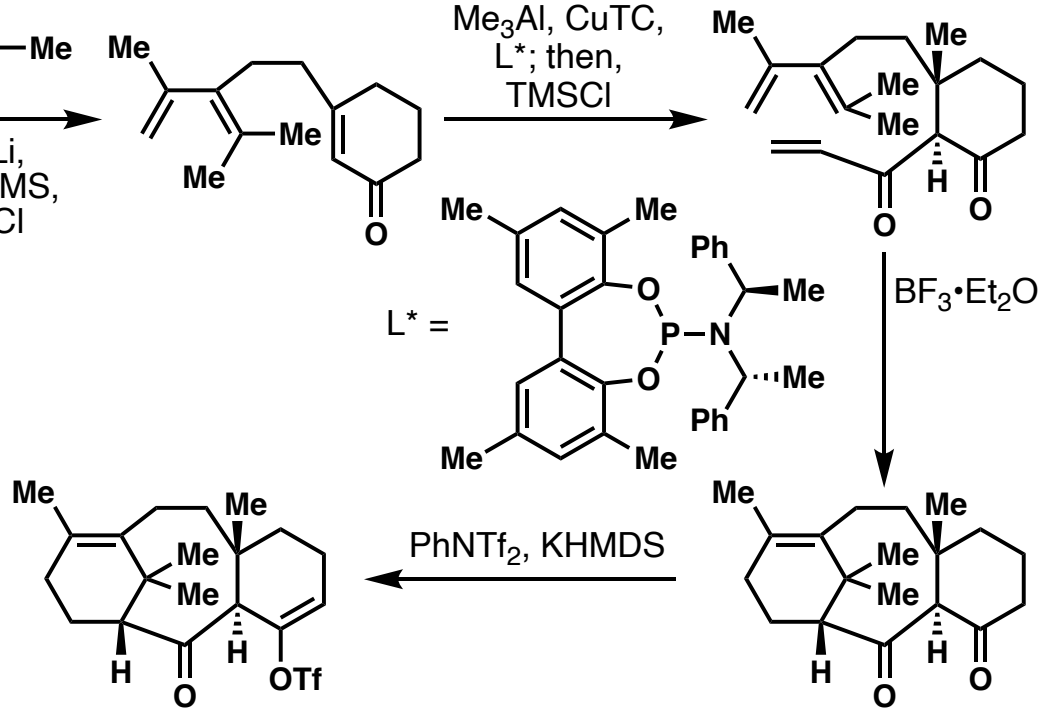

Nat. Chem. 2012, 4, 21.

Angew. Chem. Int. Ed. 2016, 55, 8280.

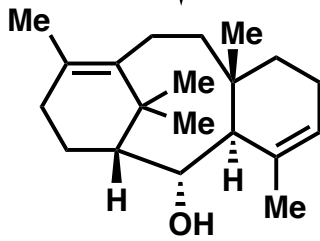
TBHP, VO(acac) $)_{2}$;

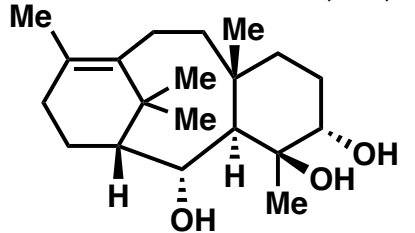

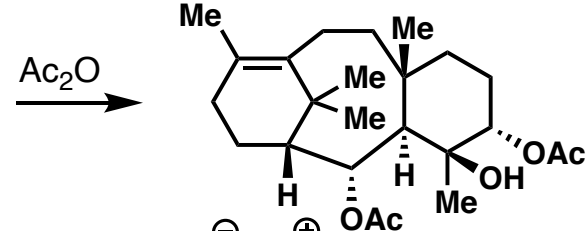

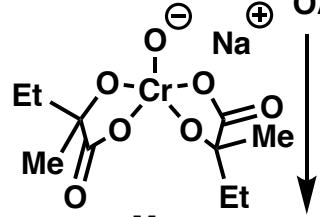

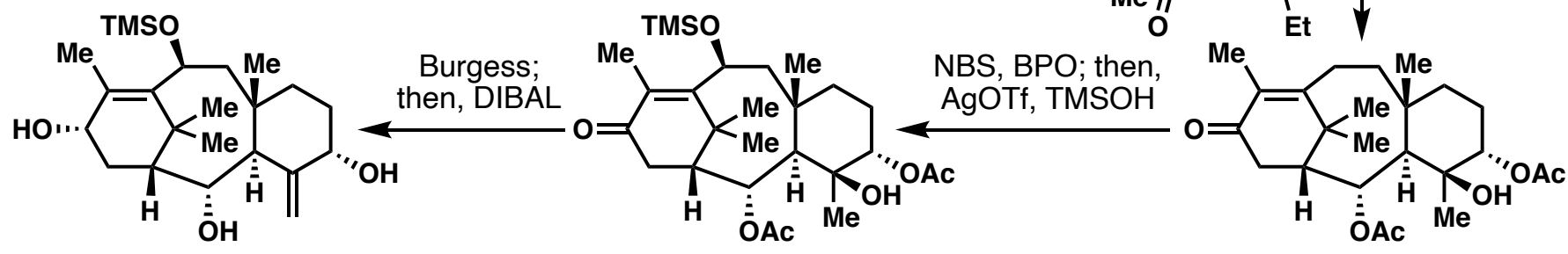

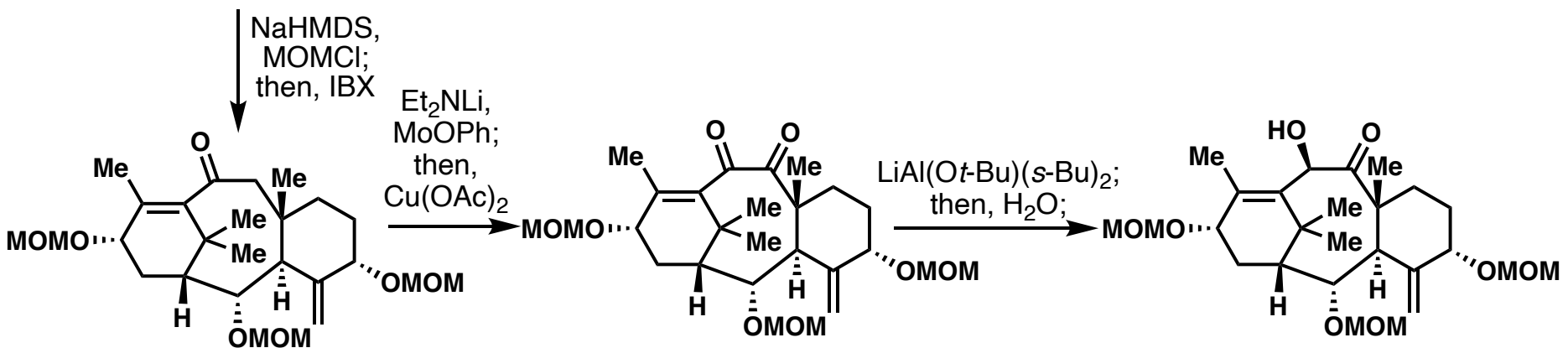

Our previous two approaches to taxanes established robust methods for early and middle oxidation choreography. For instance, $\mathrm{Cr}(\mathrm{V})$ reagent 16 was the only one reagent that gave good mass balance in the presence of the electron-rich bridgehead olefin. Even though it required additional optimization, this novel oxidation tool streamlined our early stage oxidation campaign. Indeed, $\mathrm{C}-10$ and $\mathrm{C}-13$ oxidation methods discovered during the previous syntheses were used in most routes presented below to rapidly access the key $\mathrm{C}-\mathrm{H}$ oxidation precursor. 
1st Generation

Strategic hypothesis: Lead taxabaccatin III intermediate to $\mathrm{Taxol}^{\circledR}$ using late stage $\mathrm{C}\left(\mathrm{sp}^{3}\right)-\mathrm{H}$ functionalizations 1-1. Directed C7 oxidation attempts

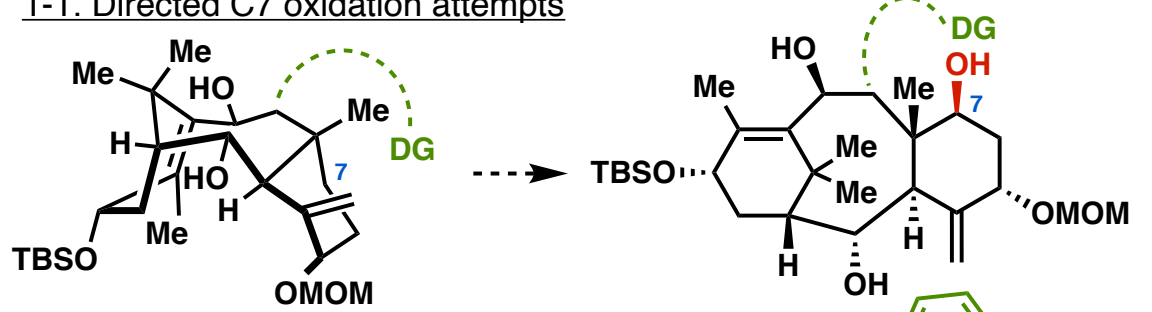

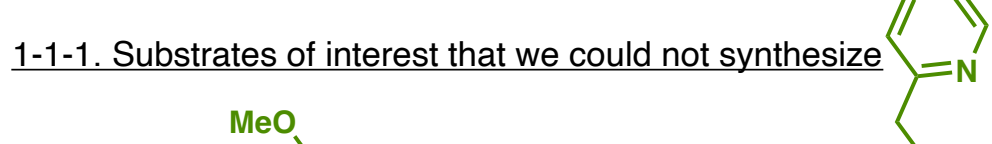

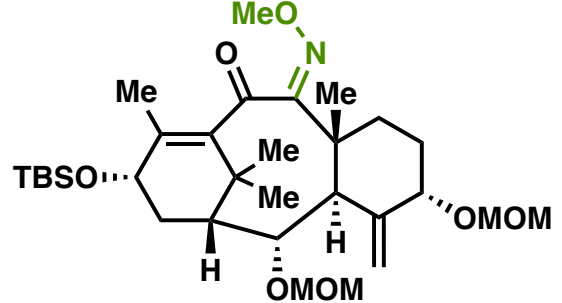

Baldwin oxidation no reaction

J. Chem. Soc., Chem. Commun. 1985, 126.

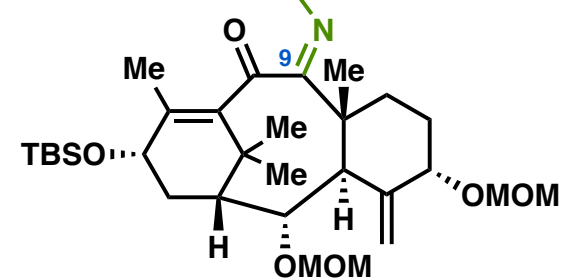

Schönecker oxidation no reaction

Angew. Chem., Int. Ed. 2003, 42, 3240.

neither condensation nor $a$-amination gave no reaction

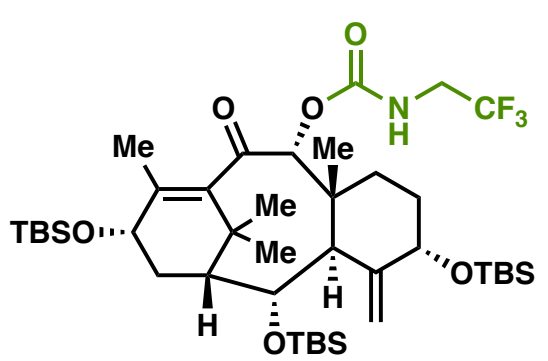

C-H oxidation no reaction

J. Am. Chem. Soc. 2008, 130, 7247.

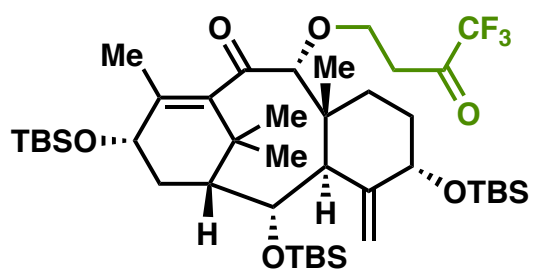

Intramolecular TFDO decomposition

Org. Lett. 2009, 11, 3630

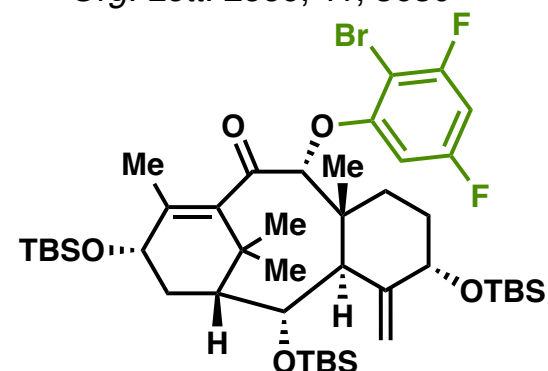

Intramolecular $\mathrm{C}-\mathrm{H}$ abstraction no reaction

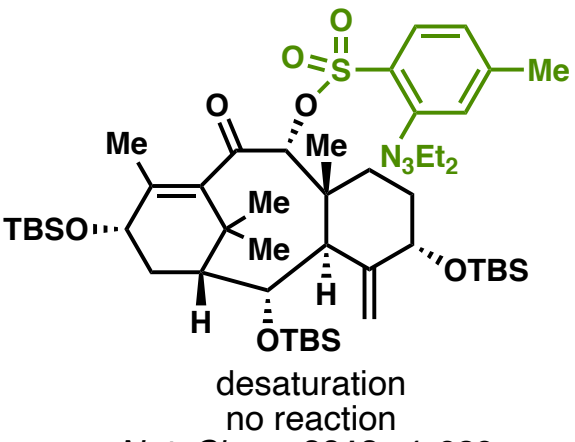

Nat. Chem. 2012, 4, 629.

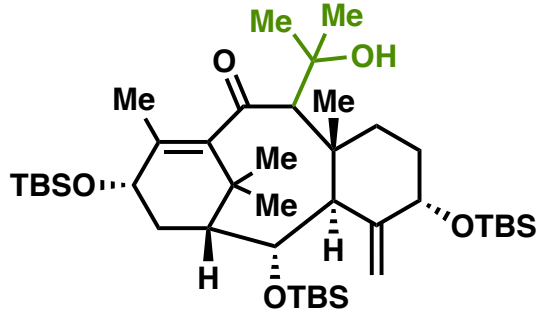

no reaction

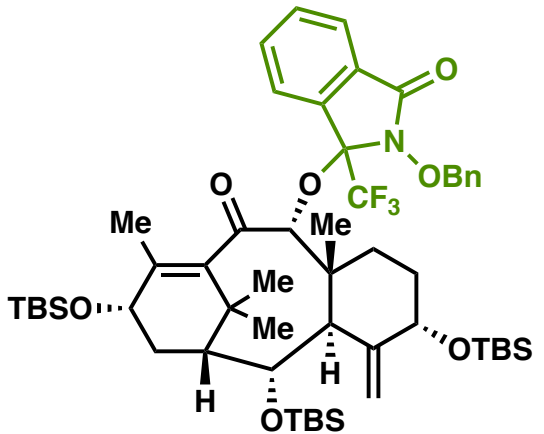

$\mathrm{C}-\mathrm{H}$ oxidation no reaction Org. Biomol. Chem. 2016, 14, 4378.

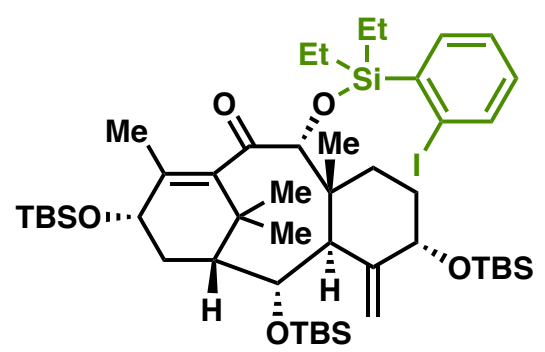

Pd catalyzed desaturation no reaction

J. Am. Chem. Soc. 2016, 138, 6340.

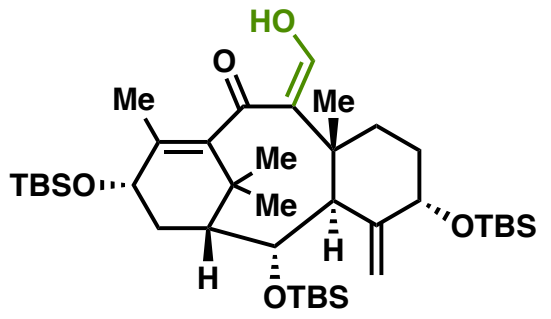

no reaction 


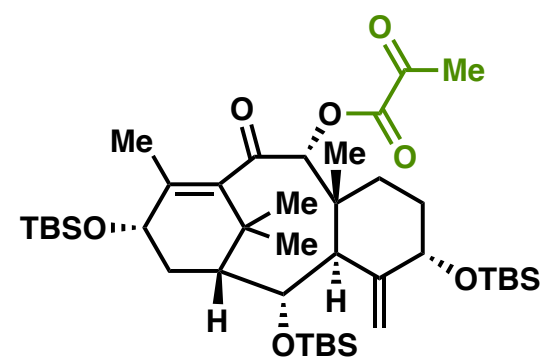

intramolecular DMDO no reaction

J. Org. Chem. 2003, 68, 6321.

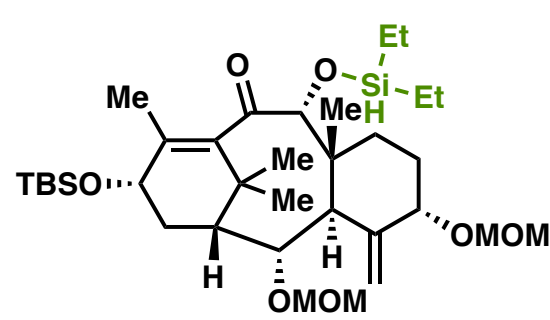

Hartwig C-H silylation decomposition

J. Am. Chem. Soc. 2014, 136, 6586.

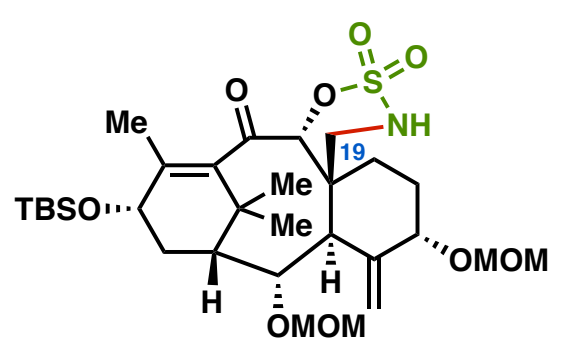

Du Bois-Breslow nitrene insertion C19 oxidation

J. Am. Chem. Soc. 2001, 123, 6935.

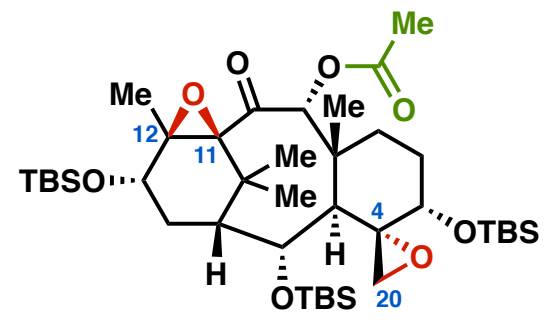

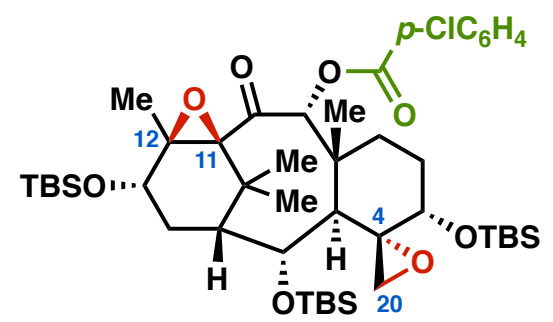

directed DMDO/TFDO

epoxidations of C4-20 and C11-12 olefins

J. Org. Chem. 1996, 61, 5564.

1-1-3. Directed C7 oxidation from C5

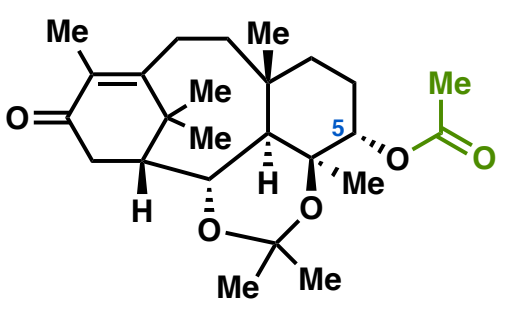

directed DMDO/TFDO decomposition photolytic or Rh catalyzed nitrene insertion decomposition

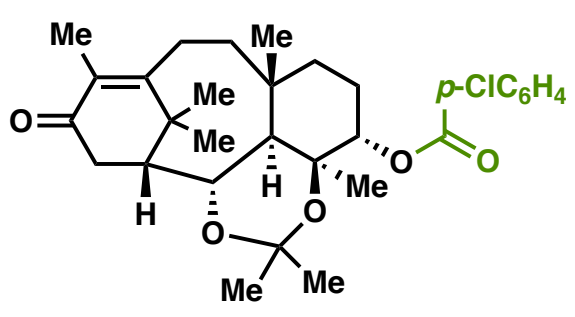

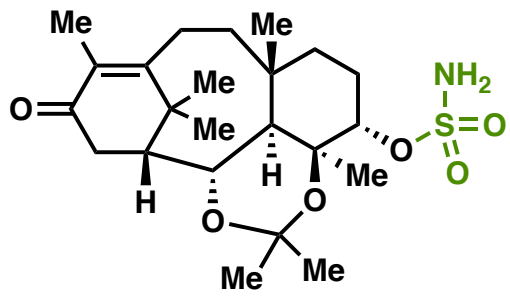

Du Bois-Breslow nitrene insertion decomposition

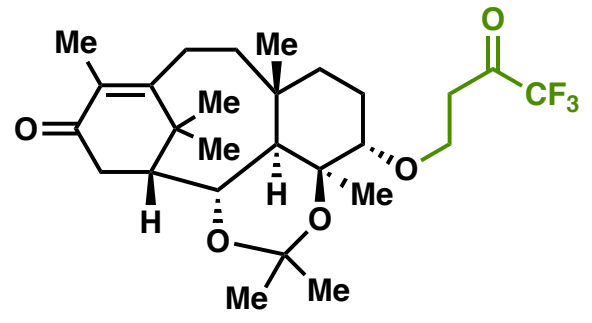

intramolecular TFDO C5 ketone formation

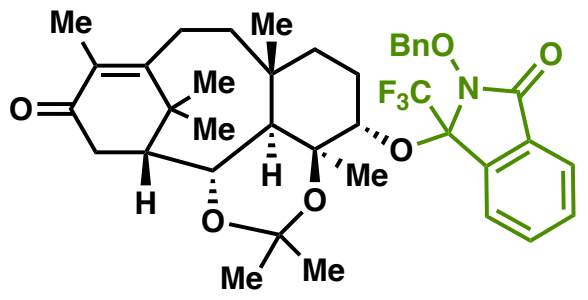

$\mathrm{C}-\mathrm{H}$ oxidation no reaction

This strategy was abandoned due to

- steric hindrance around C9 ketone/alcohol preventing condensation

- steric hindrance around C7 preventing directing groups and oxidants to approach

- substrate instability to harsh oxidation conditions

- poor material throughput

- directing groups on $\mathrm{C} 5 \mathrm{OH}$ taking equatorial conformation

- substrates in high oxidation states were more electron poor, and thus might not be good substrates for $\mathrm{C}-\mathrm{H}$ oxidation 


\section{1-2-1. Substrates of interest that we could not synthesize}
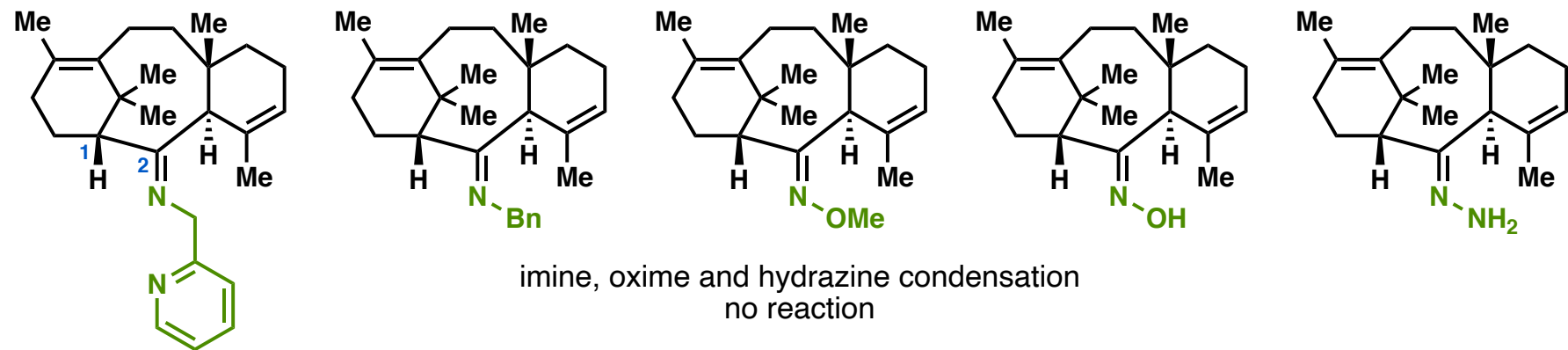

imine, oxime and hydrazine condensation no reaction

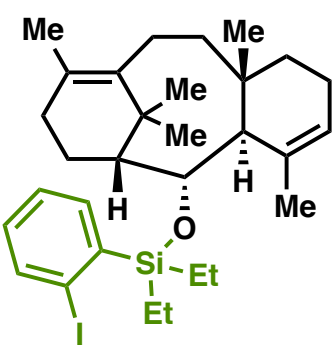

Pd-catalyzed desaturation no reaction

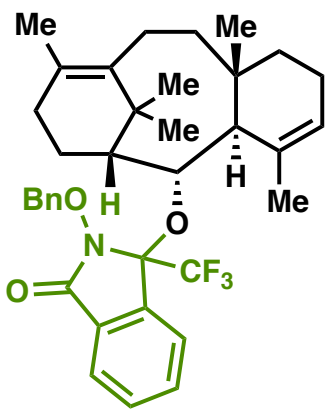

C-H oxidation no reaction

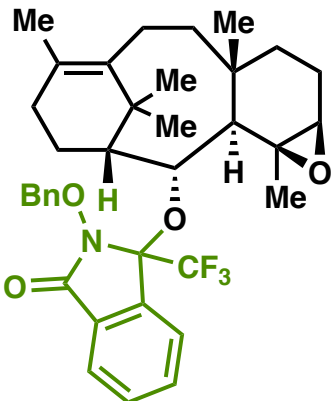

$=C_{1}^{\mathrm{C}}$

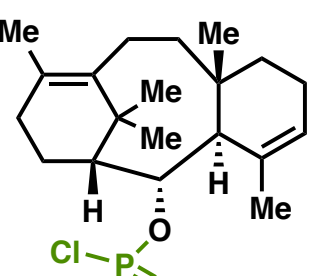

, $>0$

phosphate nitrene insertion decomposition

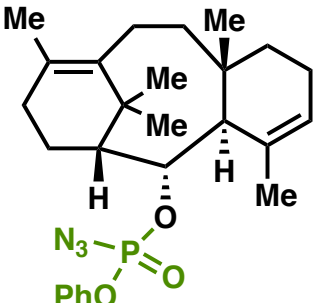

sulfone nitrene insertion decomposition

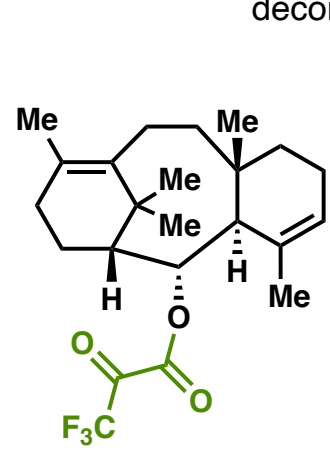

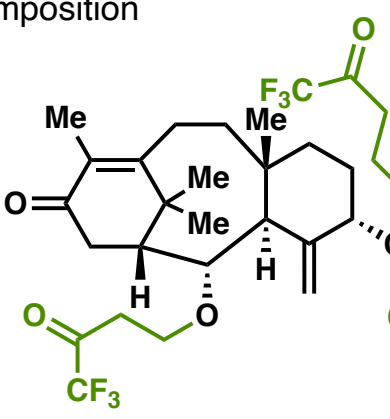

intramolecular TFDO no reaction

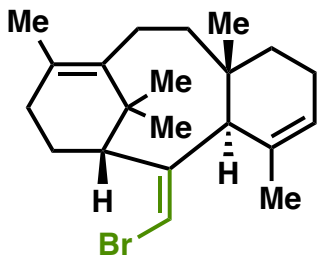

Takai olefination decomposition

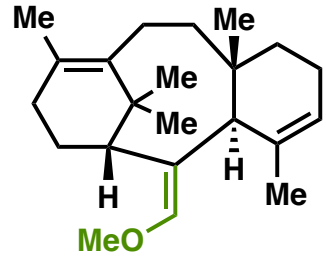

Wittig no reaction<smiles>CCCCOCCC1(C)CCC2OC2(C)[C@H]1[C@H](OCCC(=O)C(F)(F)F)C1(C)CCC2OC21C</smiles>

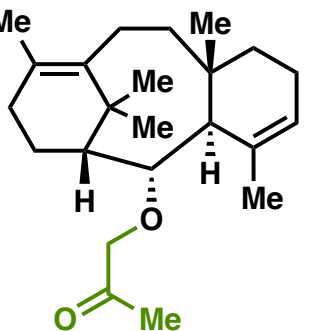

intramolecular Norrish no reaction

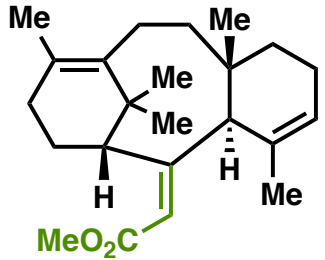

HWE no reaction

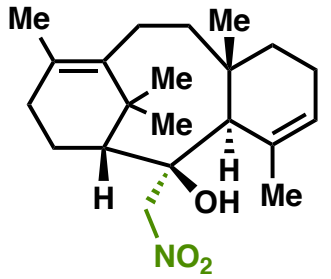

Henry no reaction

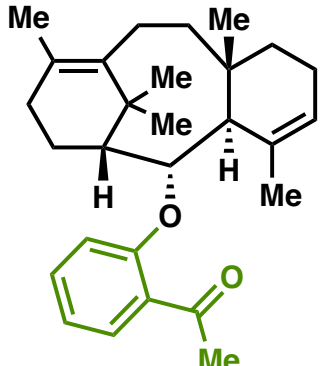

intramolecular Norrish no reaction

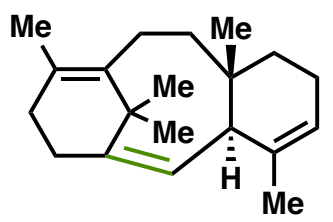

Burgess decomposition

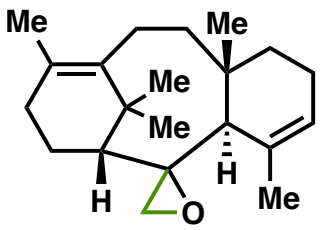

Corey-Chaykovsky no reaction 
<smiles>CC1=CCC[C@]2(C)CCC3=C(C)CC[C@H]([C@H](OC(=O)NCC(F)(F)F)C3(C)C)[C@]12C</smiles>

C-H oxidation decomposition<smiles>CC1=CCCC(C)(C)[C@H]1[C@H](O[Si](C)(C)CCl)[C@H]1CCC(C)=C2CC[C@H](C)[C@H](C)C21C</smiles>

Breslow type C-H abstraction decomposition

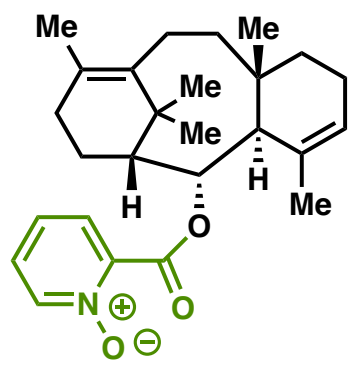

photolytic oxaziridine generation produced 2-pyridone

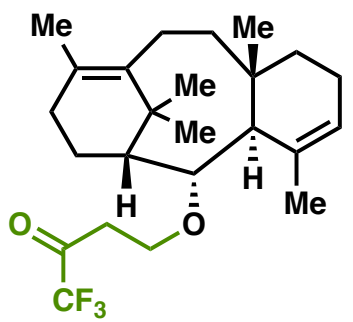

intramolecular TFDO no reaction<smiles>CC1=CCC[C@]2(C)CCC3=C(C)CC[C@H](C)[C@]3(C)[C@H](OC(=O)C(F)(F)F)[C@H]12</smiles>

Norrish type reaction no reaction

Synth. Commun. 1977, 7, 515.<smiles></smiles>

intramolecular DMDO decomposition

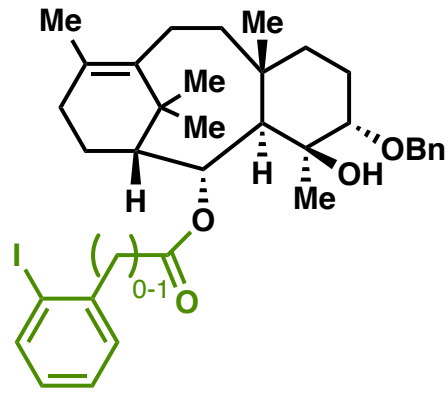

Pd-catalyzed C-H abstraction decomposition

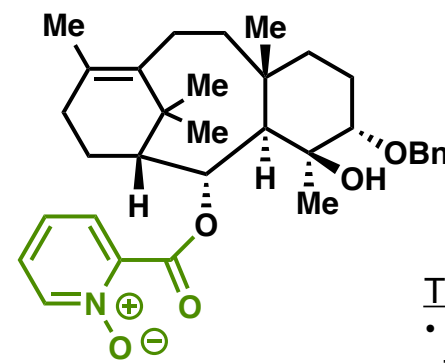

This strategy was abandoned due to

steric hindrance around C2 ketone/alcohol making its functionalization very challenging

- substrate instability to harsh oxidation conditions

1-2-3. C1 aminations

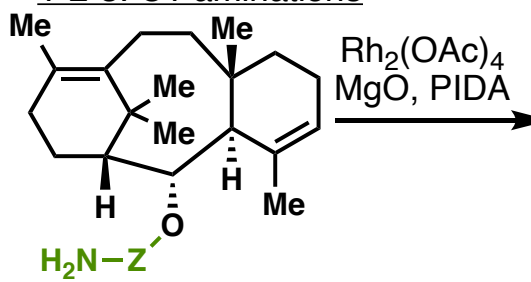

$\mathrm{Z}=\mathrm{CO}$ or $\mathrm{SO}_{2}$

$\mathrm{PG}=\mathrm{PMB}, \mathrm{Ac}$ no protection with

Tf, Cbz, Boc,

Ts, o-Nos, p-Nos, DNos,

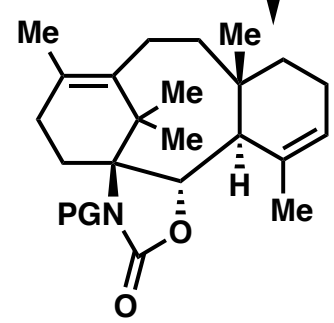<smiles>CC1=CCC[C@@]2(C)CCC3=C(C)[C@@H](C)C[C@H]4NS(=O)(=O)O[C@H]3C4(C)C12C</smiles>

Du Bois-Breslow nitrene insertion sulfamate formation had reproducibility issues

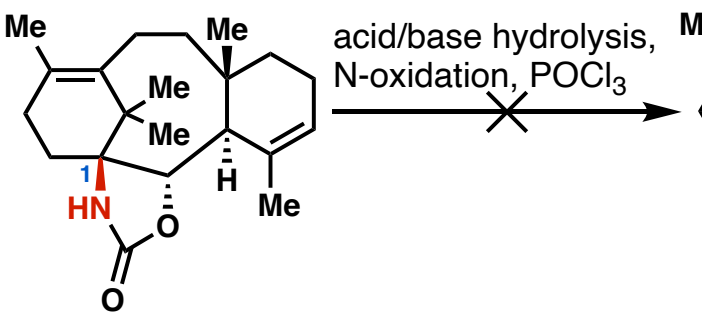<smiles>CC1=CCC[C@@]2(C)CCCC3=C(C)CC[C@](N)([C@@H](O)[C@@H]12)C3(C)C</smiles>

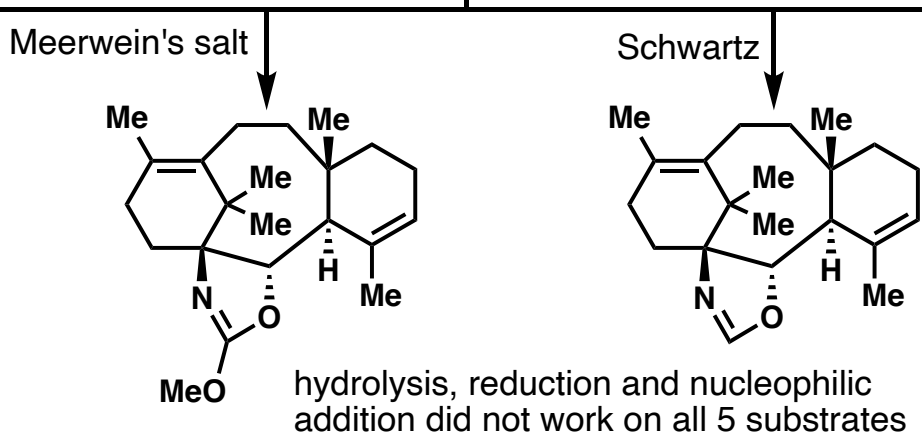

1. Lawesson's reagent 2. allyliodide

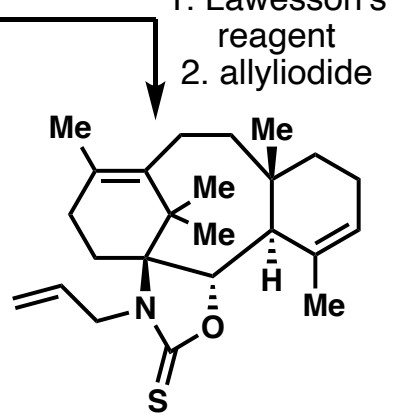


1-3-1. Previous work

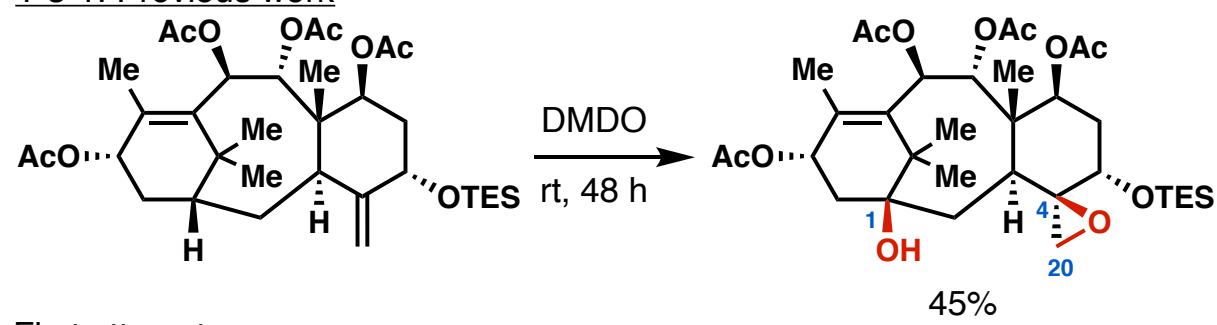

First attempt

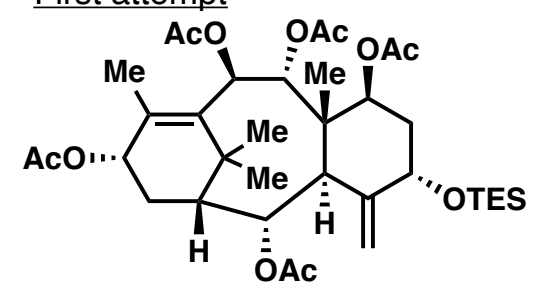

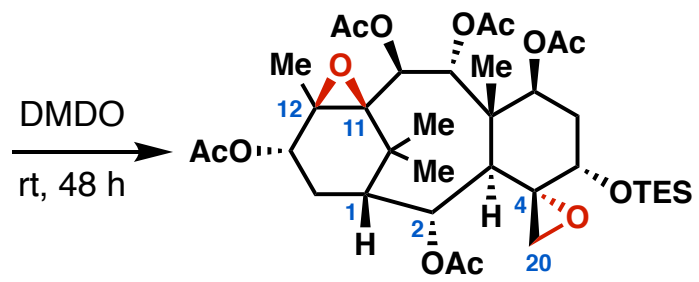

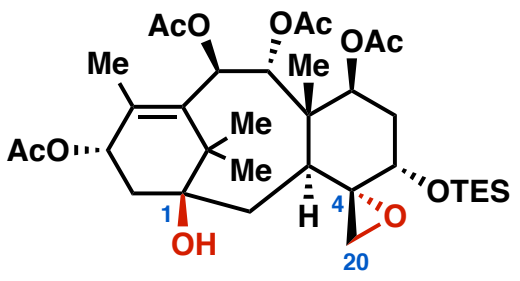

$51 \%$

Heterocycles. 2000, 53, 2629.

- no $\mathrm{C} 1$ oxidation

- overoxidation at $\mathrm{C} 11-12$

- single diastereomer

- differ from the literature precedent in $\mathrm{C} 2$ oxidation pattern

1-3-2. Attempts to electronically supress $\mathrm{C} 11-12$ overoxidation

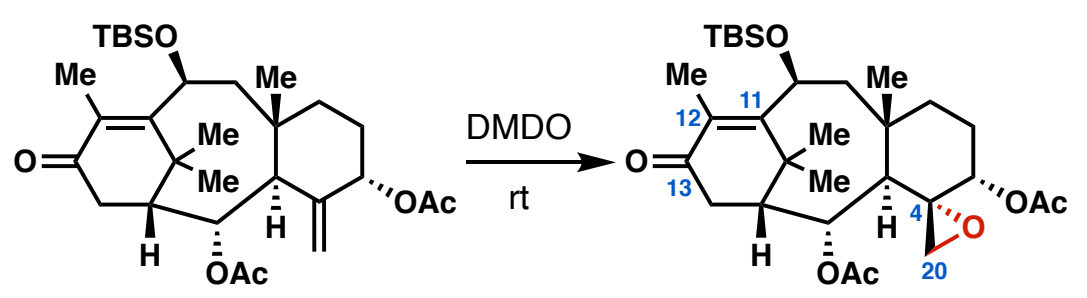

- C13 enone differentiated C4-20 and C11-12 olefins

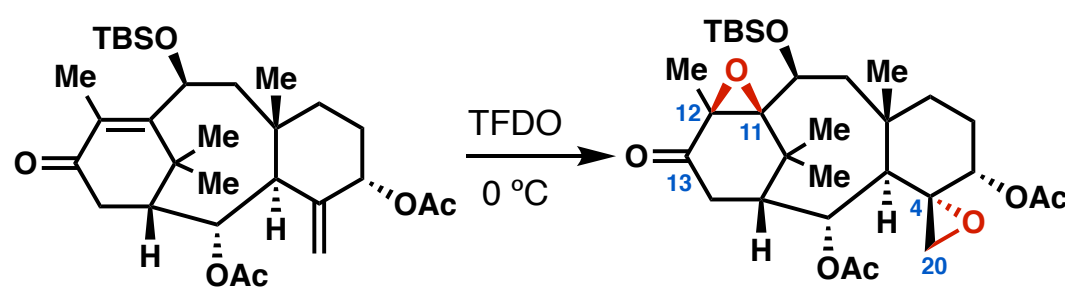

- C4-20 epoxidation took place in $<5$ min - C11-12 epoxidation took place in $6 \mathrm{~h}$ despite its sterics, electron poor character and extra stabilization effect as a hyperstable bridgehead olefin.

- extended reaction time led to substrate decomposition

- TFDO was too reactive

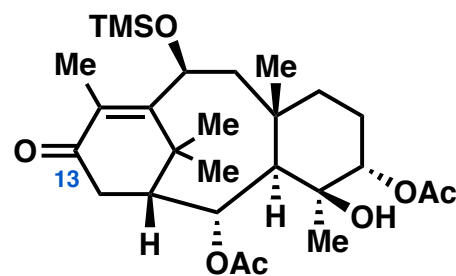

TFDO, $0^{\circ} \mathrm{C}$

C11-12 epoxidation

DMDO, rt

no reaction

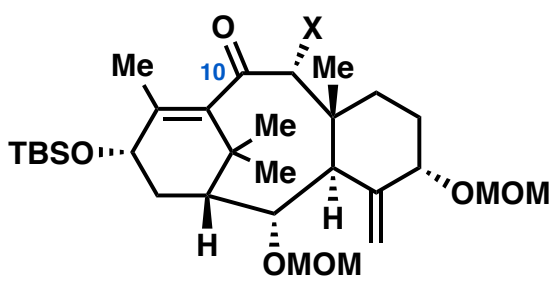

$\mathrm{X}=\mathrm{H}, \mathrm{OH}$

DMDO, rt

C4-20 epoxidation

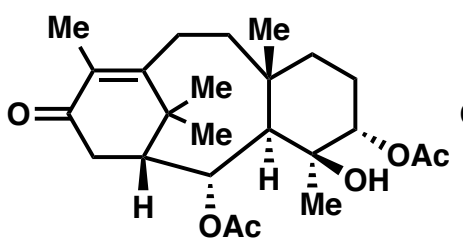

TFDO, $0^{\circ} \mathrm{C}$

C11-12 epoxidation

DMDO, rt

no reaction

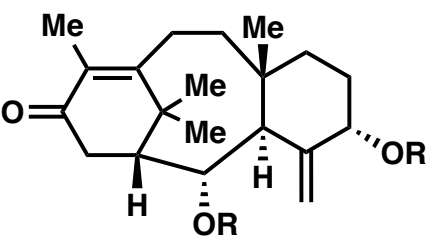

$\mathrm{R}=\mathrm{H}, \mathrm{Ac}$

TFDO, $0^{\circ} \mathrm{C}$

C4-20 epoxidation

then $\mathrm{C} 11-12$ epoxidation

DMDO, rt

C4-20 epoxidation

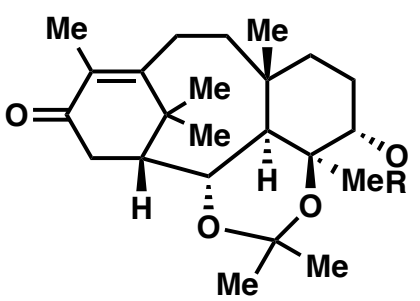

$\mathrm{R}=\mathrm{Ac}, \mathrm{TBS}, \mathrm{Bz}$

TFDO, $0{ }^{\circ} \mathrm{C}$

C11-12 epoxidation

DMDO, rt

no reaction

- TFDO was strong enough to epoxidize the hindered enone but DMDO was not

- protecting groups on C2, 4 and 5 seemed to have little effect on reactivity

- C4-20 epoxidation was very facile with either DMDO or TFDO

- C11-12 epoxidation might inductively deactivate $\mathrm{C} 1 \mathrm{C}-\mathrm{H}$

- enone might inductively deactivate $\mathrm{C} 1 \mathrm{C}-\mathrm{H}$

- both $\mathrm{C} 13$ and $\mathrm{C} 10$ enone seemed to have similar reactivity

- there were material throughput issues to prepare C10 enone substrates 


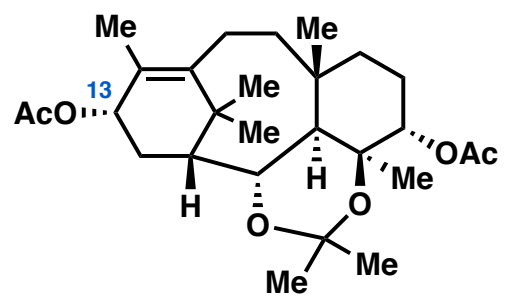

TFDO, $-50^{\circ} \mathrm{C}$

C4-20 epoxidation then $\mathrm{C} 11-12$ epoxidation

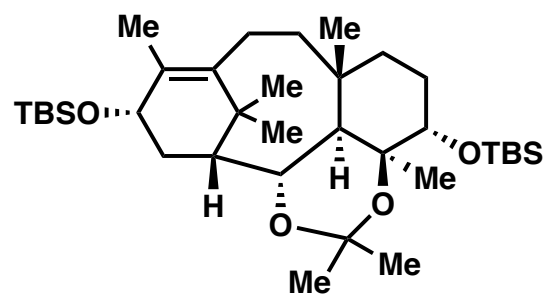

TFDO, $-78^{\circ} \mathrm{C}$ decomposition
- C13 protecting group seemed to inductively affect $\mathrm{C} 11-12$ electron density

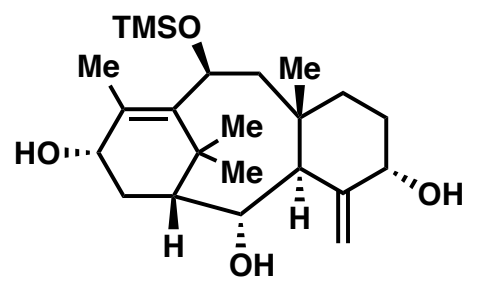

TFDO, $0^{\circ} \mathrm{C}$ decomposition

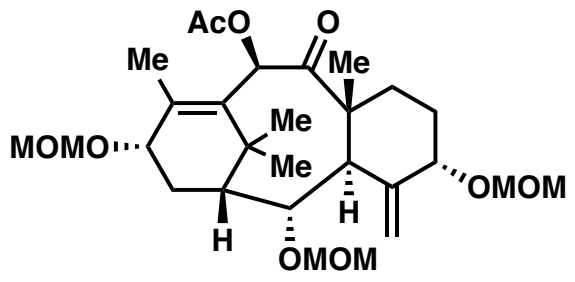

in situ TFDO, $0^{\circ} \mathrm{C}$<smiles></smiles>

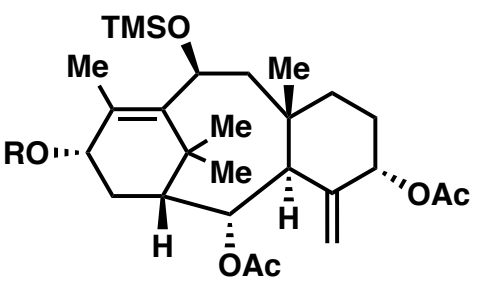

$\mathrm{R}=\mathrm{H}, \mathrm{Ac}$

TFDO, $0^{\circ} \mathrm{C}$ decomposition

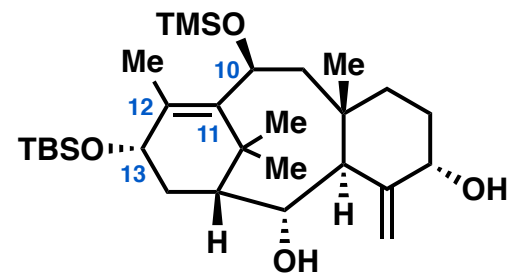

in situ TFDO, $0^{\circ} \mathrm{C}$ C4-20 epoxidation

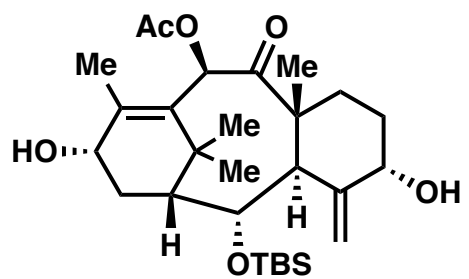

TFDO, $0^{\circ} \mathrm{C}$ decomposition
- C13, 10 bis(silyl)protected substrate did not undergo C11-12 epoxidation despite its electron richness

- TFDO was too harsh for substrates

- C11-12 olefin could survive low concentration of TFDO

TFDO was abandoned at this point for the following reasons

- overall TFDO seemed to be harsh for this substrate class

- in situ TFDO prep generated very low concentration of TFDO which is detrimental for C-H oxidations

- Oritani's example showed that DMDO was strong enough oxidant to do $\mathrm{C} 1 \mathrm{C}-\mathrm{H}$ oxidation

- could not increase reaction temperature higher than $0^{\circ} \mathrm{C}$ while Oritani's example was done at room temperature for $48 \mathrm{~h}$ 


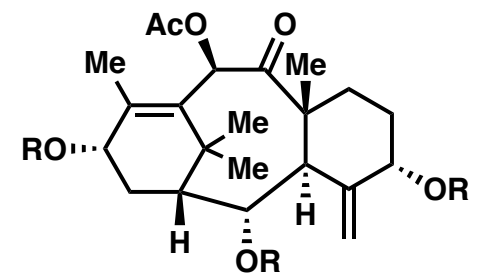

$\mathrm{R}=\mathrm{MOM}, \mathrm{TBS}$

C4-20 epoxidation

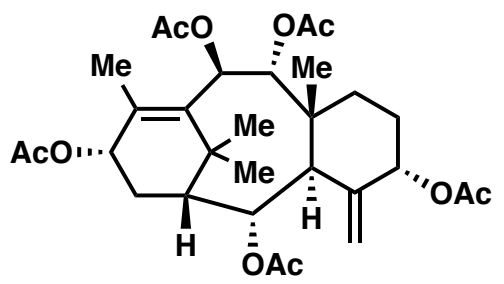

C4-20 epoxidation

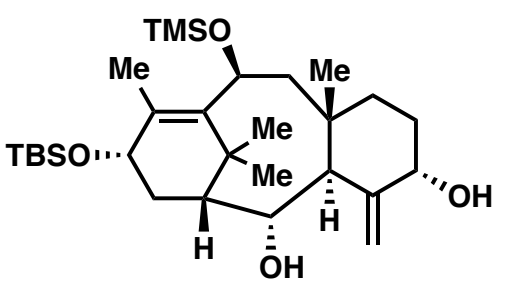

C4-20 epoxidation

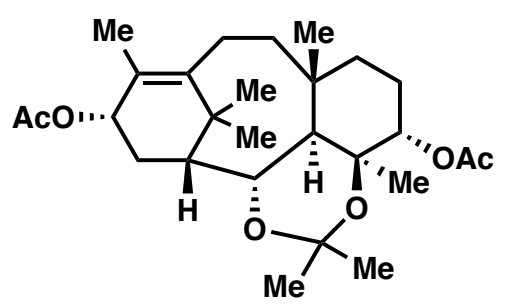

no reaction

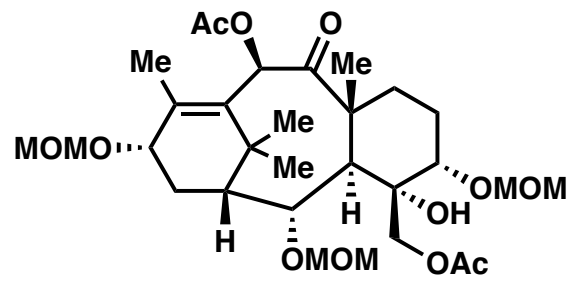

no reaction

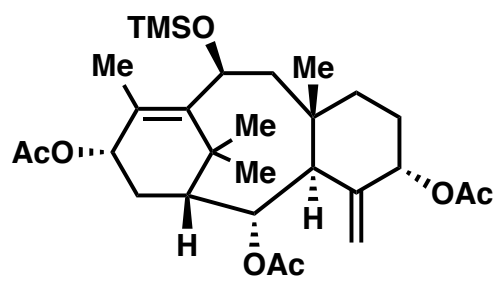

C4-20 epoxidation

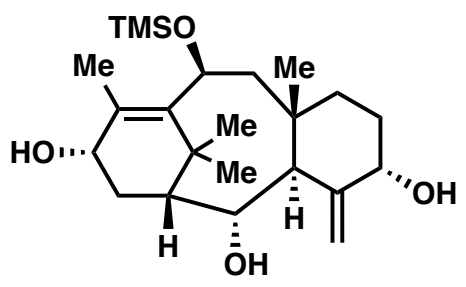

C4-20 epoxidation

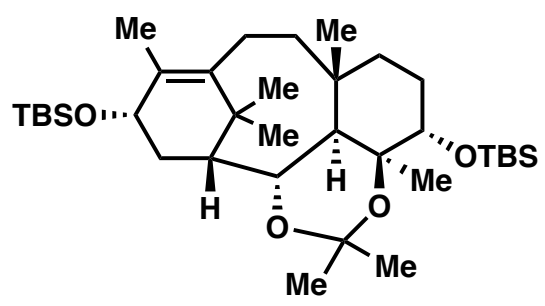

no reaction

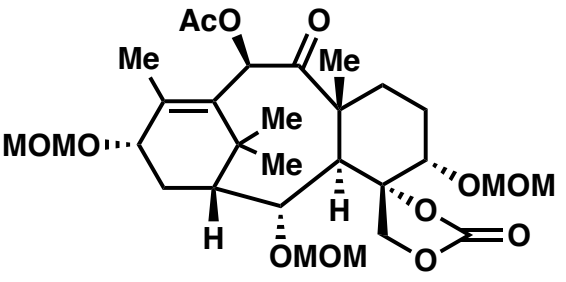

no reaction

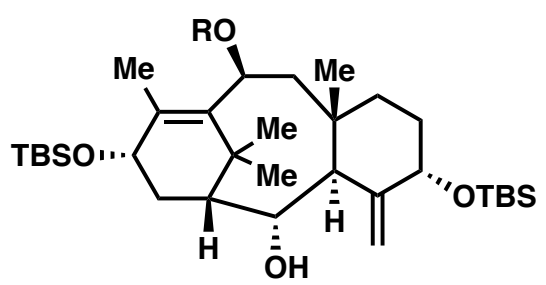

$\mathrm{R}=\mathrm{TMS}$, TES

C4-20 epoxidation

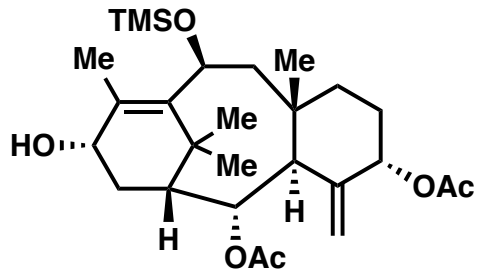

C4-20 epoxidation

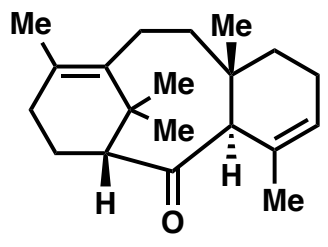

C4-5 epoxidation

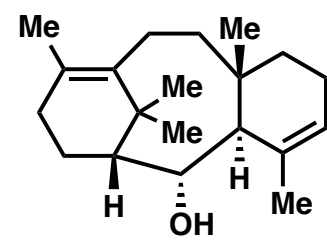

C4-5 epoxidation
- no C11-12 epoxidation

- electronics on A ring did not affect outcomes

- $\mathrm{C} 1$ oxidation did not take place regardless of protecting group on $\mathrm{C} 2 \alpha-\mathrm{OH}$, indicating neither sterics nor electronics around C2 do not significantly affect $\mathrm{C} 1$ reactvity or free $\mathrm{C} 2 \mathrm{a}-\mathrm{OH}$ is sterically or electronically detrimental enough to retard $\mathrm{C} 1$ reactivity 

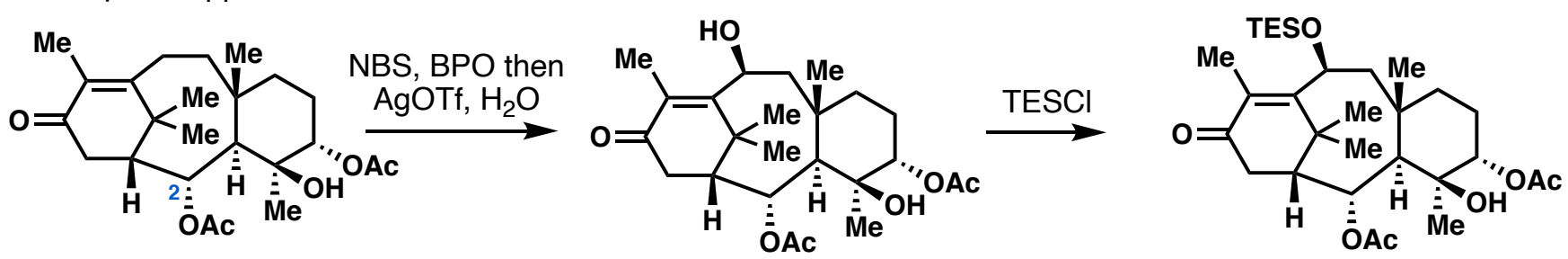

taxabaccation III intermediate
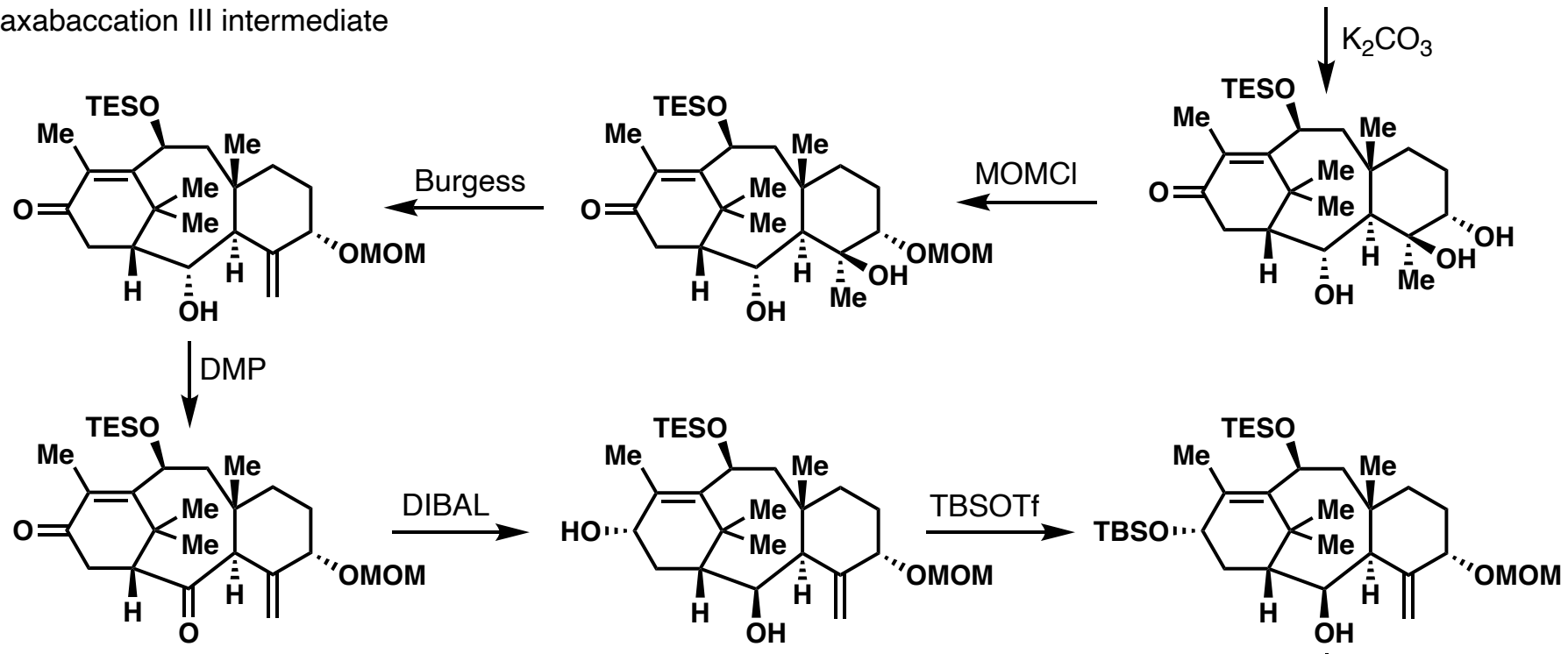

TBSO

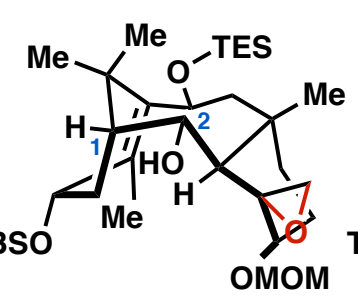

C2a

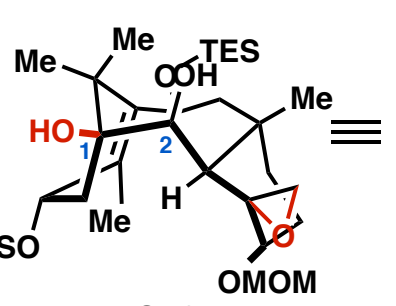

$\mathrm{C} 2 \beta$

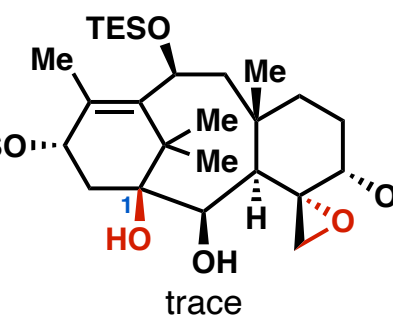

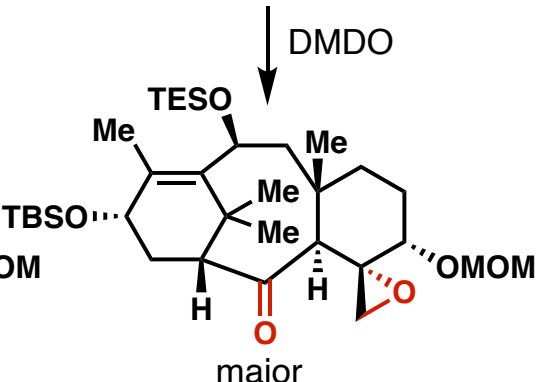

- $\mathrm{a}-\mathrm{OH}$ seemed to create more sterics around $\mathrm{C} 1 \mathrm{C}-\mathrm{H}$

- hyperconjugation effect could affect the reactivity but dihedral angles looked very similar in both diastereomers

- C2 a-OH might have misdirected DMDO via H-bonding (J. Phys. Org. Chem. 1996, 9, 751)

\section{1-3-6. Structure reactivity relationship examination (all substrates were subjected to DMDO at rt)}

TESO

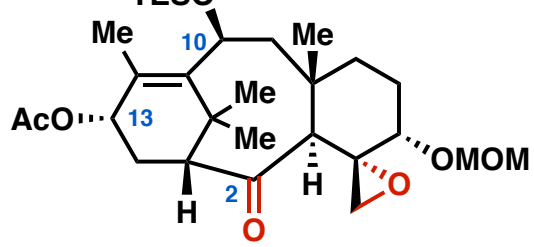

- $\mathrm{C} 2 \mathrm{\beta}-\mathrm{OH}$ substrates consistently gave trace $\mathrm{C} 1$ oxidiz

- $\mathrm{C} 13 \mathrm{OH}$ had to be protected

- C13 enone did not undergo C1 oxidation

- C13 and C10 substitution prevented C11-12 epoxidation

- $\mathrm{C} 13$ OAc might inductively deactivate $\mathrm{C} 1$ reactivity

1st generation route was abandoned for the following reasons

- redundant redox manipulations at C2

- protecting group swapping at C5

- unsuccessful C5 MOM deprotection

- unsuccessful epoxide opening (see 2nd generation for more discussion)

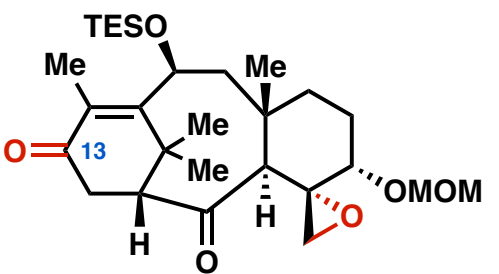


Strategic hypothesis: Scalable synthesis of $\mathbf{C} 1$ oxidation precursor

2-1. Synthetic route

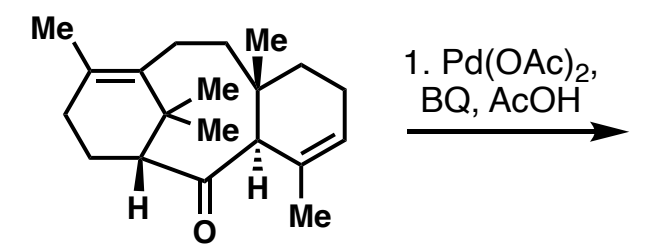<smiles>C=C1[C@H](OC(C)=O)CCC(C)(C)[C@H]1C(=O)C1CCC2=C(C)CC[C@H]1C2(C)C</smiles>

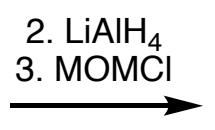

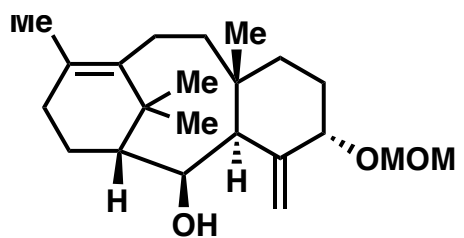

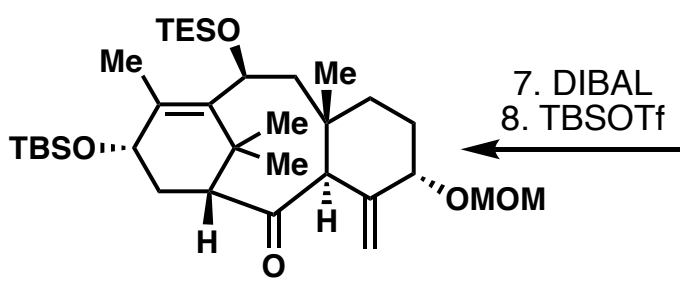

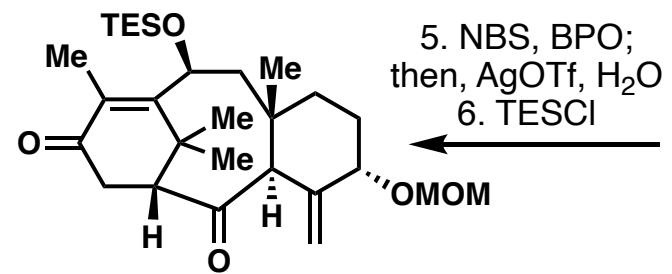

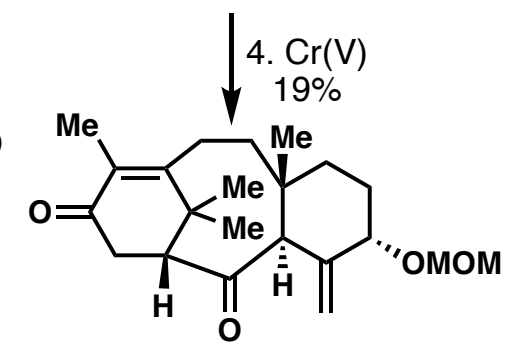

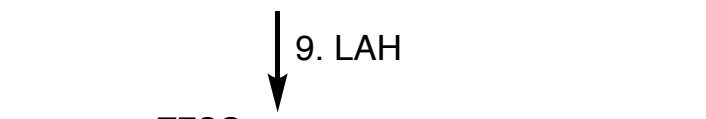

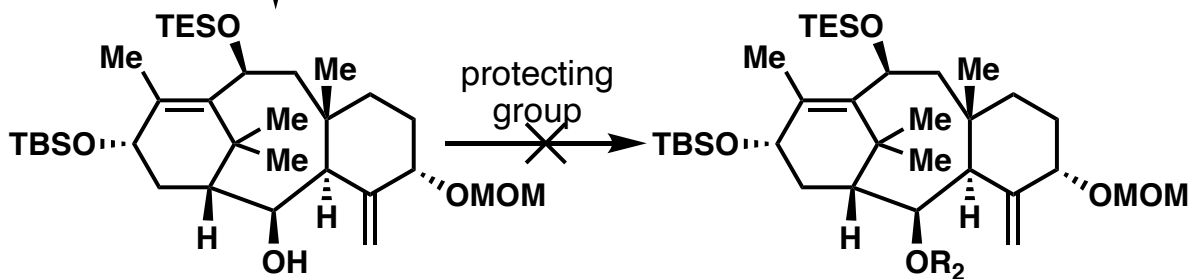

- $\mathrm{C} 2 \beta-\mathrm{OH}$ was too hindered to react with any electrophiles examined protecting groups include $\mathrm{Me}, \mathrm{Ac}, \mathrm{TMS}, \mathrm{TBS}, \mathrm{HSiMe}_{2}, \mathrm{MOM}, \mathrm{Bn}$

intermediate from 1st generation route

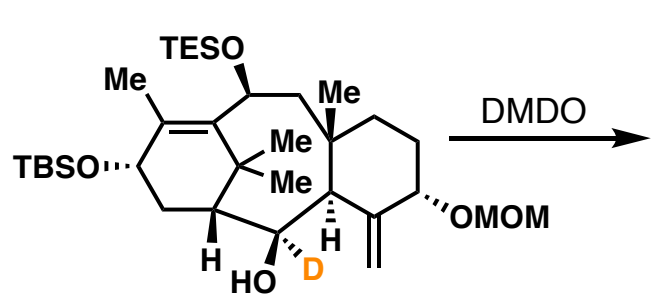

with $\mathrm{LiAID}_{4}$ instead of $\mathrm{LAH}$ from $\mathrm{C} 2$ ketone

- isolable quantity of $\mathrm{C} 1$ oxizided product

- KIE assisted chemoselective oxidation

- only $10 \%$ ketone recovery

- TFDO gave decomposition

Tetrahedron. Lett. 2001, 42, 3753.
TBSO

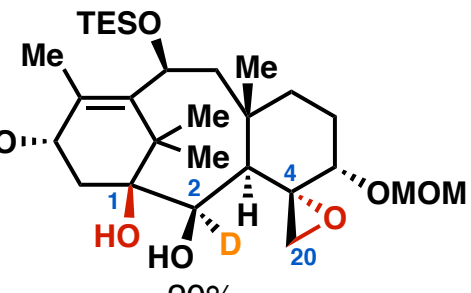

epoxide opening was examined on this product but did not tolerate most conditions

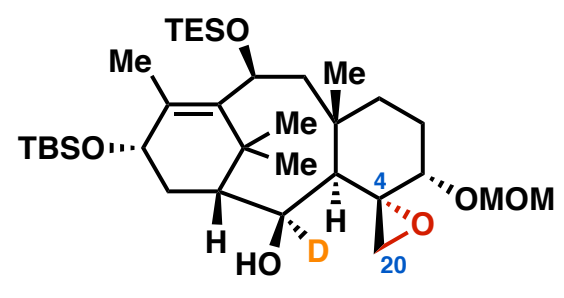

$20 \%$

$+10 \%$ C2 ketone

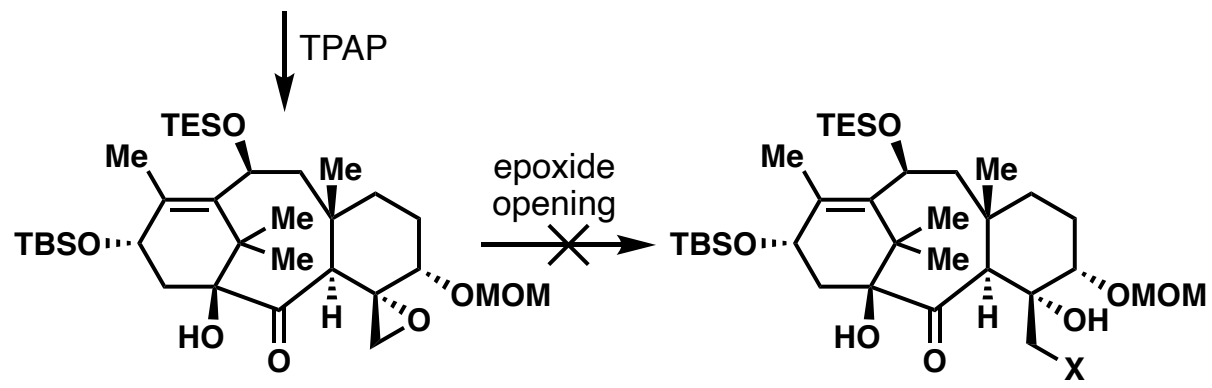

\begin{tabular}{cc}
\hline reagent & result \\
\hline $\mathrm{BF}_{3} \cdot \mathrm{OEt}_{2}, \mathrm{H}_{2} \mathrm{O}_{2}$ & no reaction \\
$\mathrm{LHMDS}, \mathrm{H}_{2} \mathrm{O}_{2}$ & no reaction \\
$\mathrm{BnOH}, \mathrm{WOCl}{ }_{2}, \mathrm{AgOTf}$ & no reaction \\
allylOH, $\mathrm{Bu} \mathrm{S}_{2} \mathrm{SnO}$ & no reaction \\
$\mathrm{H}_{2} \mathrm{O}, \mathrm{Co}(\mathrm{III}) t-\mathrm{Bu}_{2} \mathrm{salen}$ & no reaction \\
$\mathrm{Ac}_{2} \mathrm{O}, \mathrm{Bu} \mathrm{NOAc}_{2} \mathrm{NOA}$ & no reaction \\
$\mathrm{MoO}_{2}(\mathrm{acac})_{2}, \mathrm{H}_{2} \mathrm{O}_{2}$ & no reaction \\
\hline
\end{tabular}

$\mathrm{X}=\mathrm{H}, \mathrm{OH}$, halogen 


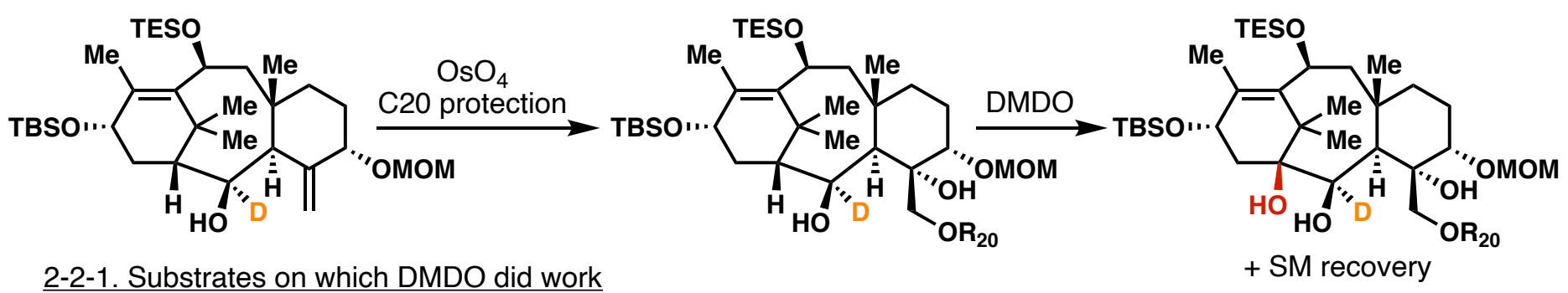

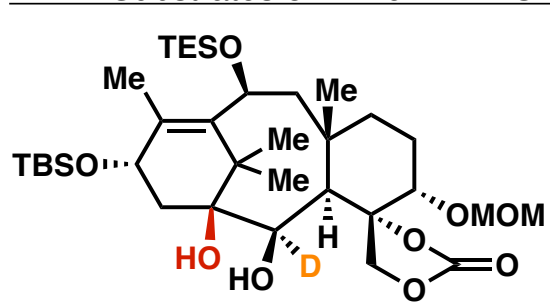

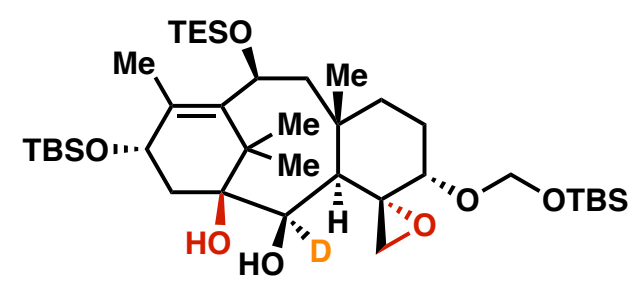

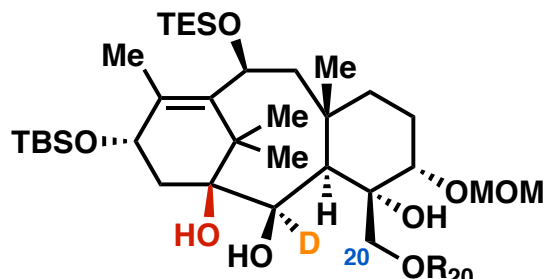
$\mathrm{R}_{20}=\mathrm{Ac} \quad \cdot$ cyclic acetonide did not form before $\mathrm{C} 1$ oxidation Piv $\quad$ OMe acetal substrate did not undergo the following Boc (cleanest) C2 oxidation

2-2-2. Substrates on which DMDO did not work

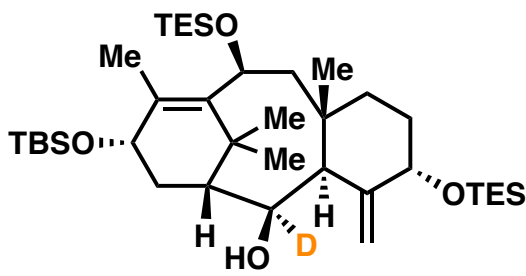

decomposition

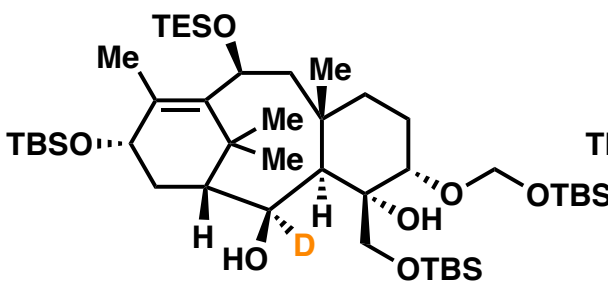

decomposition

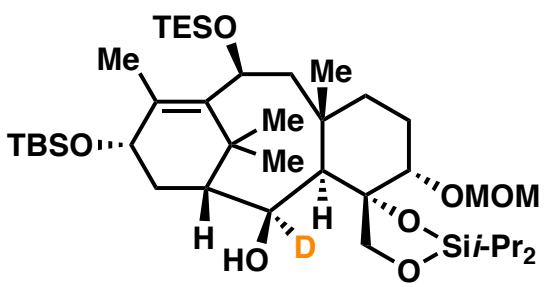

no reaction

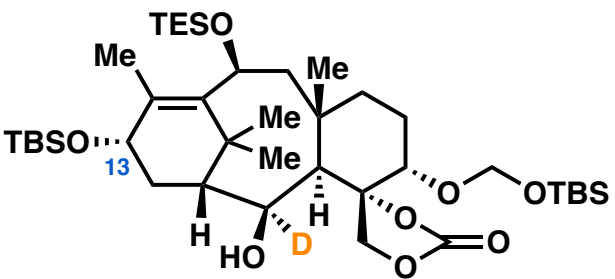

C13 enone formation

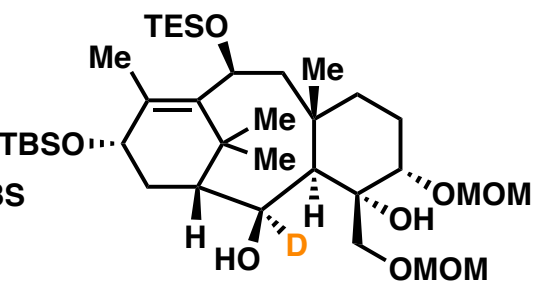

decomposition

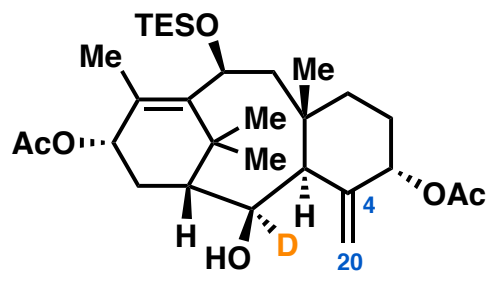

C4-20 epoxidation

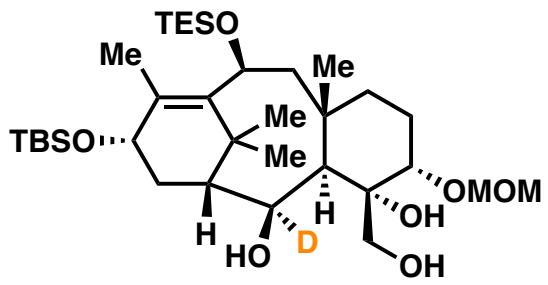

no reaction

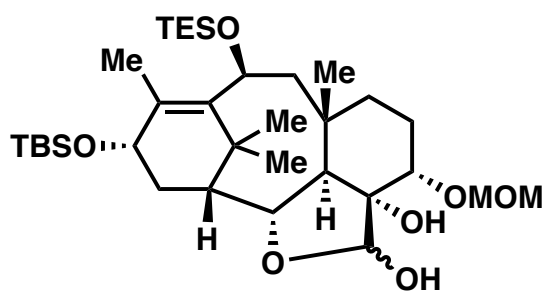

no reaction

- C13 needs electron-donating protecting group

- gradual C13 enone formation was observed for most substrates with extended reaction time (>15 h)

- $\mathrm{C} 1$ oxidation has to be faster than $\mathrm{C} 13$ enone formation (about 30\% conversion after $12 \mathrm{~h}$ )

- C5 OTES substrate adopts boat conformation on $\mathrm{C}$ ring which could be detrimental for $\mathrm{C} 1$ oxidation, while others adopt chair conformation

- it is unclear why C5 TOM substrate did not undergo C1 oxidation even though it is almost identical to MOM.

Methylene oxidation could be an issue

- C20 seemed to require electron withdrawing protecting group 
Or $2 \mathrm{e}^{-}$then, $\mathrm{H}^{+}$

hydride and proton came from a face

2-3-1. C2 reduction substrate and condition screening: Bulkiness and coordination effect from C20

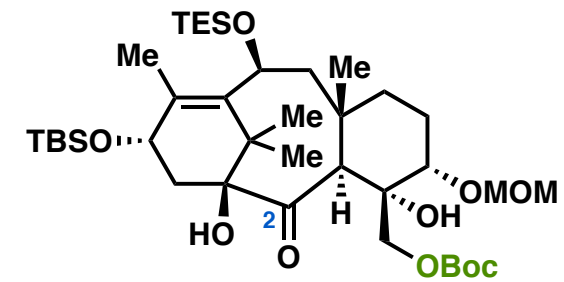

\begin{tabular}{|c|c|}
\hline reagent & result \\
\hline $\begin{array}{c}\mathrm{LiBH}_{4}, \mathrm{ZnCl}_{2} \\
\mathrm{Noyori} \\
\mathrm{CBS} \\
\mathrm{Sml}_{2}, \mathrm{Et}_{3} \mathrm{~N}, \mathrm{H}_{2} \mathrm{O} \\
\mathrm{Sml}_{2}, \mathrm{LiCl} \\
\mathrm{Na}, \mathrm{i}-\mathrm{PrOH}, \mathrm{PhMe} \\
\mathrm{Na}, \mathrm{NH}_{3} \\
\mathrm{Na}_{2} \mathrm{~S}_{2} \mathrm{O}_{4} \\
\mathrm{HSnBu}_{3} \\
\mathrm{EtSH}_{3} \mathrm{hv} \\
\mathrm{Et}_{3} \mathrm{~N}, \mathrm{hv} \\
(\mathrm{TMS})_{3} \mathrm{SiH}, \mathrm{C}_{12} \mathrm{H}_{25} \mathrm{SH} \\
\mathrm{LiNaph} \\
\text { Lawesson's reagent } \\
\mathrm{Cp}_{2} \mathrm{TiCl}\end{array}$ & $\begin{array}{c}\text { no reaction } \\
\text { TES deprotection } \\
\text { no reaction } \\
\text { C2 } \beta-O H \\
\text { C2 } \beta-O H \\
\text { Boc deprotection } \\
\text { decomposition } \\
\text { C2 } \beta-O H \\
\text { no reaction } \\
\text { decomposition } \\
\text { decomposition } \\
\text { no reaction } \\
\text { decomposition } \\
\text { decomposition } \\
\text { no reaction }\end{array}$ \\
\hline
\end{tabular}

TBSO

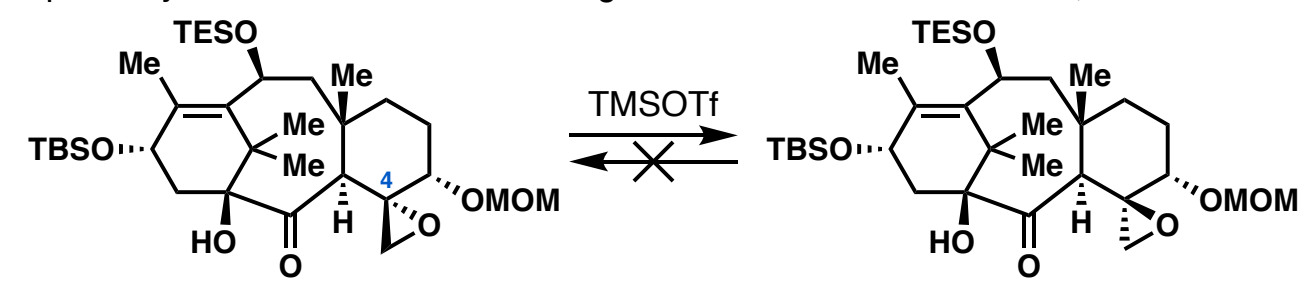

TBSO

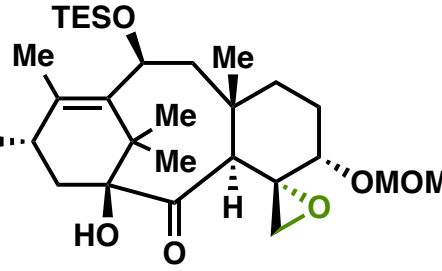

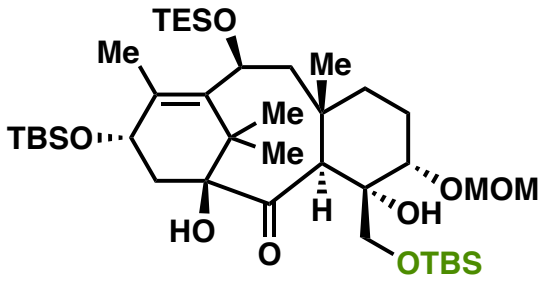

\begin{tabular}{cc}
\hline reagent & result \\
\hline $\mathrm{Sml}_{2}, \mathrm{Et}_{3} \mathrm{~N}, \mathrm{H}_{2} \mathrm{O}$ & $\mathrm{C} 2 \beta-\mathrm{OH}$ \\
$\mathrm{Sml}_{2}, \mathrm{LiCl}$ & $\mathrm{C} 2 \beta-\mathrm{OH}$
\end{tabular} $\mathrm{Al}(\mathrm{O} i-\mathrm{Pr})_{3}, i-\mathrm{PrOH}$ no reaction

$\mathrm{ml}_{2}, \mathrm{Et}_{3} \mathrm{~N}, \mathrm{H}_{2} \mathrm{O} \quad \mathrm{C} 2 \mathrm{\beta}-\mathrm{OH}$

$\mathrm{Sml}_{2}, \mathrm{H}_{2} \mathrm{O}, \mathrm{HMPA}$ no reaction $\mathrm{Na}, \mathrm{i}-\mathrm{PrOH}$ decomposition $i-\mathrm{PrMgBr} \quad \mathrm{C} 2 \mathrm{\beta}-\mathrm{OH}$ NaNaph no reaction LiDBB $\mathrm{C} 2 \mathrm{\beta}-\mathrm{OH}$

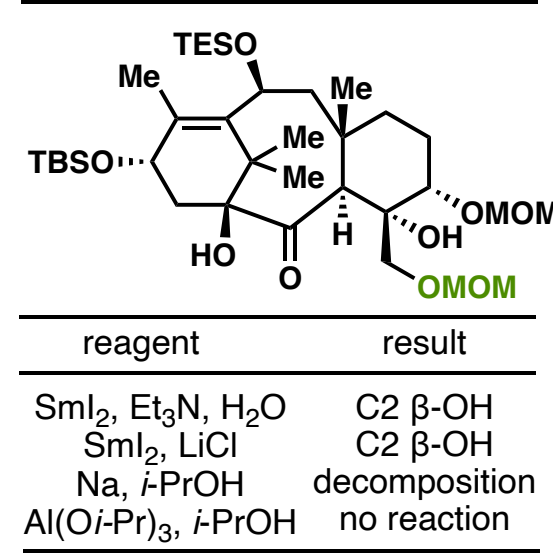

- epoxides could be configurationally unstable to both basic and acidic conditions

- probably underwent cationic rearrangement under acidic conditions;

- probably underwent retro aldol under basic conditions;

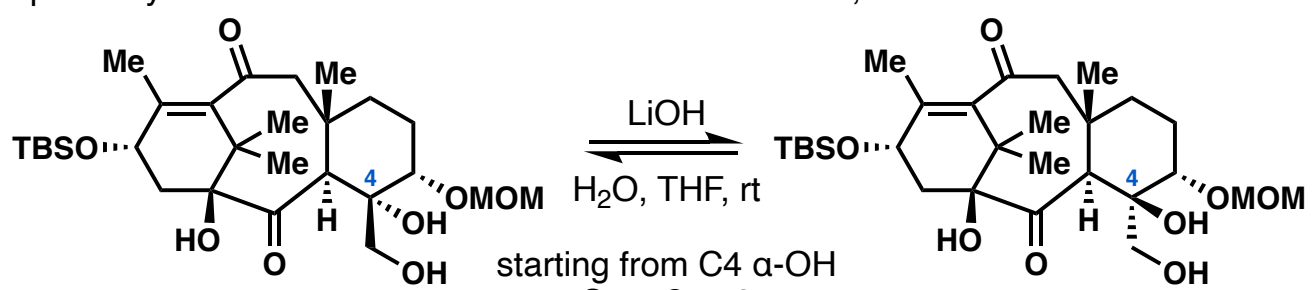

C4 $a: \beta=1: 3$ 


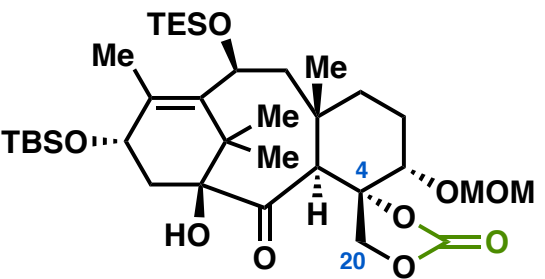

\begin{tabular}{cc}
\hline reagent & result \\
\hline $\begin{array}{c}\mathrm{Sml}_{2}, \mathrm{Et}_{3} \mathrm{~N}, \mathrm{H}_{2} \mathrm{O} \\
\mathrm{Sml}_{2}, \mathrm{LiCl}\end{array}$ & $\begin{array}{c}\text { decomposition } \\
\text { no reaction }\end{array}$ \\
\hline
\end{tabular}

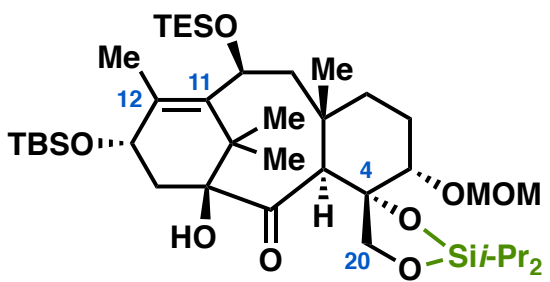

\begin{tabular}{cc}
\hline reagent & result \\
\hline $\mathrm{LAH}_{2}$ & decomposition \\
$\mathrm{Sml}_{2}, \mathrm{Et}_{3} \mathrm{~N}, \mathrm{H}_{2} \mathrm{O}$ & decomposition \\
$\mathrm{Sml}_{2}, \mathrm{LiCl}$ & decomposition \\
$\mathrm{Na}, i-\mathrm{PrOH}, \mathrm{PhMe} \mathrm{C} 11-12$ olefin reduction
\end{tabular}

- cyclic carbonate underwent hydrolysis under basic conditions

- diisopropylsilane were too unstable

- diol protecting groups created too much sterics around C2 ketone and retarded its reactivity

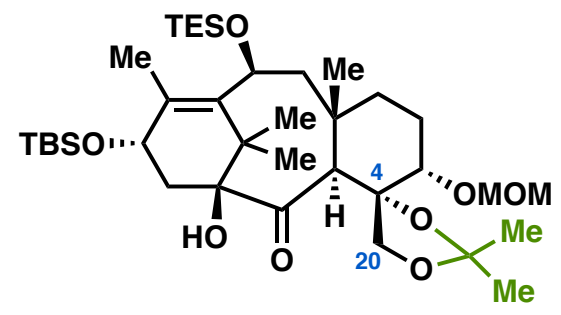

\begin{tabular}{cc}
\hline reagent & result \\
\hline $\mathrm{NaBH}_{4}, \mathrm{CeCl}_{3}$ & no reaction
\end{tabular}

$\mathrm{NaHB}(\mathrm{OAc})_{3}$ no reaction

$\mathrm{LiBH}_{4}, \mathrm{ZnCl}_{2}$ no reaction

DIBAL

$\mathrm{LAH}$

$\mathrm{LiEt}_{3} \mathrm{BH}$

$\mathrm{Al}(\mathrm{O} i-\mathrm{Pr})_{3}, i-\mathrm{PrOH}$ $i-\mathrm{PrMgBr}$

no reaction

no reaction

no reaction

no reaction

no reaction

$\mathrm{Sml}_{2}, \mathrm{Et}_{3} \mathrm{~N}, \mathrm{H}_{2} \mathrm{O}$ $\mathrm{Sml}_{2}, \mathrm{LiCl}$

$\mathrm{Na}, i-\mathrm{PrOH}$

$\mathrm{C} 2 \mathrm{\beta}-\mathrm{OH}$

no reaction

no reaction

$\mathrm{Na}, i-\mathrm{PrOH}, \mathrm{PhMe} \quad \mathrm{C} 2 \beta-\mathrm{OH}$

$\mathrm{Na}, i-\mathrm{PrOH}, \mathrm{Et}_{2} \mathrm{O} \quad \mathrm{C} 2 \beta-\mathrm{OH}$

$\mathrm{Li}, \mathrm{NH}_{3}$

$\mathrm{Na}, \mathrm{NH}_{3}$
$\mathrm{C} 2 \mathrm{\beta}-\mathrm{OH}$

2-3-3. C2 reduction substrate and condition
J. Chem. Soc. Chem. Commun. 1986, 831 .

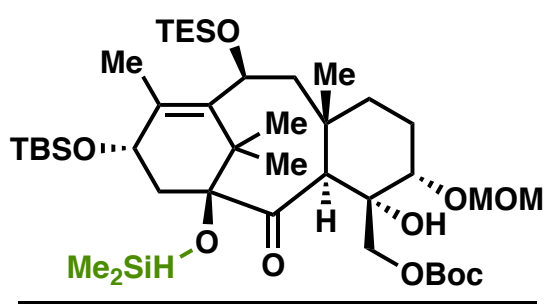

\begin{tabular}{cc}
\hline reagent & \multicolumn{1}{c}{ result } \\
\hline $\mathrm{ZnCl}_{2}$ & decomposition \\
$\mathrm{BF}_{3} \cdot \mathrm{OEt}_{2}$ & decomposition \\
$\mathrm{Sml}_{2}, \mathrm{Et}_{3} \mathrm{~N}, \mathrm{H}_{2} \mathrm{O}$ & $\mathrm{C} 2 \mathrm{\beta}-\mathrm{OH}$ \\
{$[\mathrm{Ir}(\mathrm{cod}) \mathrm{Cl}]_{2}$} & decomposition \\
{$[\mathrm{Rh}(\operatorname{cod}) \mathrm{Cl}]_{2}$} & decomposition \\
\hline
\end{tabular}

2-3-4. Literature precedents for a selective C2 reduction

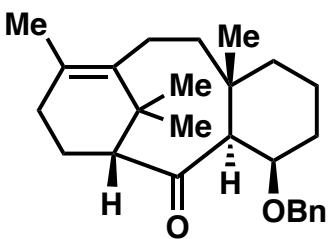

Williams

$\mathrm{Na}, \mathrm{EtOH}$

J. Org. Chem. 2000, $65,7865$.

All substrates above seemed to have chair-chair B ring conformation

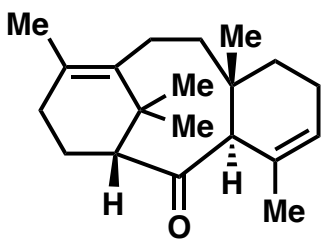

Baran

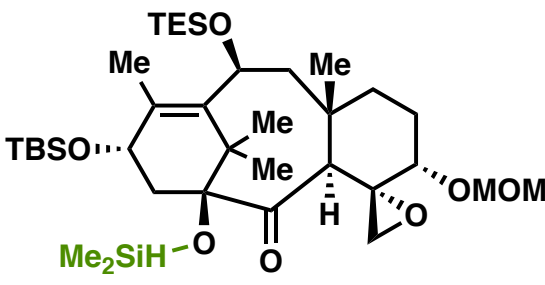

- $\mathrm{C} 1 \mathrm{Me}_{2} \mathrm{SiH}$ substrates did not survive conditions but $\mathrm{C} 1 \mathrm{OH}$ substrates did - cationic rearrangement might be operant

\begin{tabular}{cc}
\hline reagent & result \\
\hline $\mathrm{SnCl}_{4}$ & decomposition \\
$\mathrm{BF}_{3} \cdot \mathrm{OEt}_{2}$ & decomposition \\
$\mathrm{TFA}_{\mathrm{ZnCl}}$ & decomposition \\
$\mathrm{ZnCl}_{2}$ & decomposition \\
{$[\mathrm{Ir}(\mathrm{cod}) \mathrm{Cl}]_{2}$} & decomposition \\
{$[\mathrm{Rh}(\mathrm{cod}) \mathrm{Cl}]_{2}$} & decomposition \\
$\mathrm{Sml}, \mathrm{H}_{2} \mathrm{O}, \mathrm{Et}_{3} \mathrm{~N}$ & decomposition \\
$\mathrm{Na}, i-\mathrm{PrOH}$ & decomposition \\
\hline &
\end{tabular}
$\mathrm{Zn}\left(\mathrm{BH}_{4}\right)_{2}$ WO1993020036 A1, 1993. Angew. Chem. Int. Ed. 2016, 55, 8280.

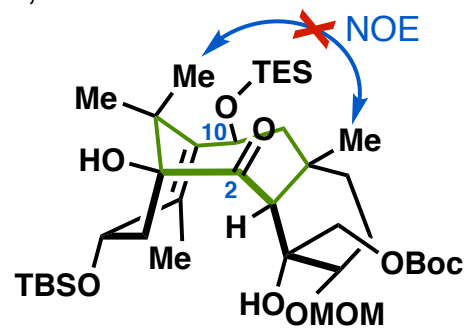

twisted boat
C10 oxidation

changes $B$ ring conformation?

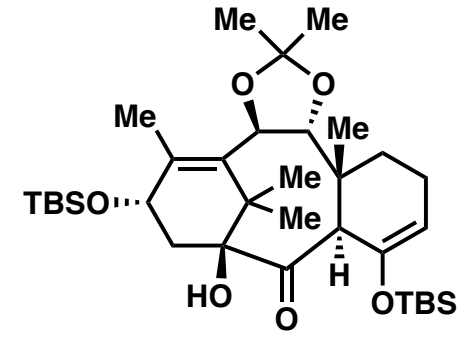

Kuwajima $\mathrm{Na}, \mathrm{CF}_{3} \mathrm{CH}_{2} \mathrm{OH}$ Tennen Yuki Kagobutsu Toronkai Koen Yoshishu. 1999, 41. 463. 


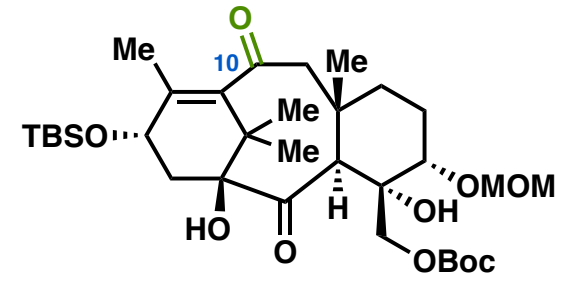

\begin{tabular}{cc}
\hline reagent & result \\
\hline $\mathrm{Zn}\left(\mathrm{BH}_{4}\right)_{2}$ & no reaction \\
$\mathrm{Sml}_{2}, \mathrm{Et}_{3} \mathrm{~N}, \mathrm{H}_{2} \mathrm{O}$ & decomposition \\
$\mathrm{Sml}_{2}, \mathrm{LiCl}$ & decomposition \\
$\mathrm{Na}, i-\mathrm{PrOH}, \mathrm{PhMe}$ & decomposition \\
\hline
\end{tabular}

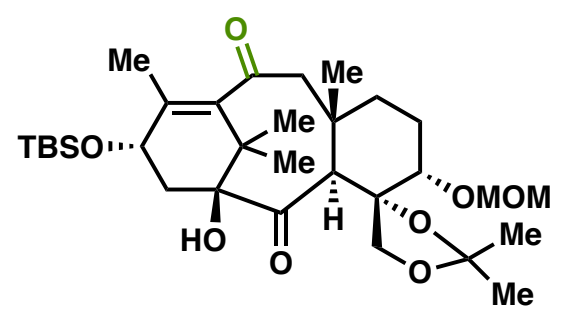

\begin{tabular}{cc}
\hline reagent & result \\
\hline $\mathrm{Sml}_{2}, \mathrm{Et}_{3} \mathrm{~N}, \mathrm{H}_{2} \mathrm{O}$ & decomposition \\
$\mathrm{Sml}_{2}, \mathrm{LiCl}$ & decomposition \\
$\mathrm{LiEt}_{3} \mathrm{BH}$ & decomposition \\
$\mathrm{Na}, i-\mathrm{PrOH}, \mathrm{PhMe}$ & decomposition \\
$\mathrm{Na}, \mathrm{NH}_{3}$ & decomposition \\
\hline
\end{tabular}

- C10 ketone seemed to make the substrate unstable to reductive conditions

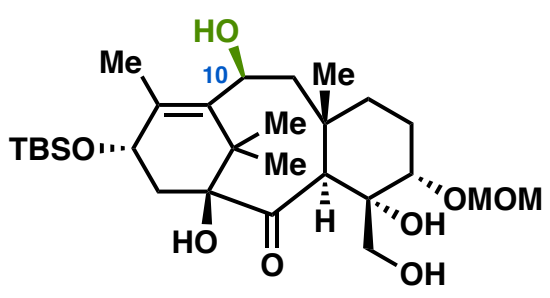

\begin{tabular}{cc}
\hline reagent & result \\
\hline $\mathrm{Sml}_{2}, \mathrm{Et}_{3} \mathrm{~N}, \mathrm{H}_{2} \mathrm{O}$ & $\mathrm{C} 2 \beta-\mathrm{OH}$ \\
$\mathrm{Sml}_{2}, \mathrm{LiCl}$ & $\mathrm{C} 2 \beta-\mathrm{OH}$ \\
\hline
\end{tabular}

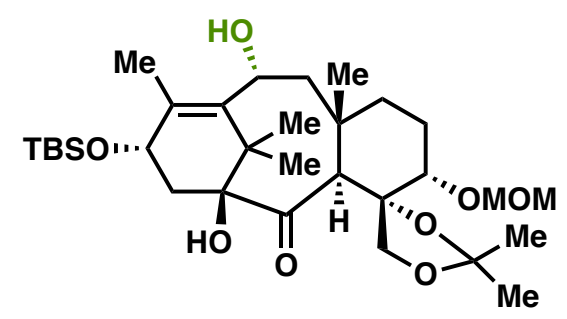

\begin{tabular}{cc}
\hline reagent & result \\
\hline $\mathrm{Sml}_{2}, \mathrm{Et}_{3} \mathrm{~N}, \mathrm{H}_{2} \mathrm{O}$ & decomposition \\
$\mathrm{Sml}_{2}, \mathrm{LiCl}$ & decomposition \\
$\mathrm{Na}, i-\mathrm{PrOH}, \mathrm{PhMe}$ & decomposition \\
\hline
\end{tabular}

- $\mathrm{C} 10 \mathrm{a}-\mathrm{OH}$ seemed to make the substrate unstable to reductive condition - might radically deoxygenate under radical conditions

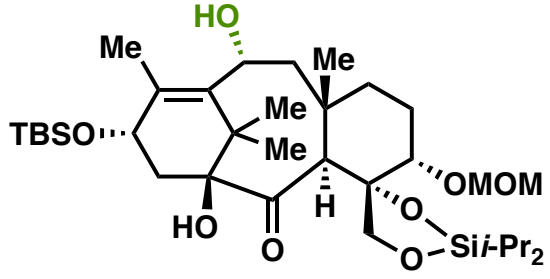

\begin{tabular}{cc}
\hline reagent & result \\
\hline $\mathrm{LAH}$ & decomposition \\
$\mathrm{Sml}_{2}, \mathrm{Et}_{3} \mathrm{~N}, \mathrm{H}_{2} \mathrm{O}$ & decomposition \\
$\mathrm{Sml}_{2}, \mathrm{LiCl}$ & decomposition \\
$\mathrm{Na}, i-\mathrm{PrOH}, \mathrm{PhMe}$ & decomposition \\
$\mathrm{Na}, \mathrm{NH}_{3}$ & decomposition \\
\hline
\end{tabular}

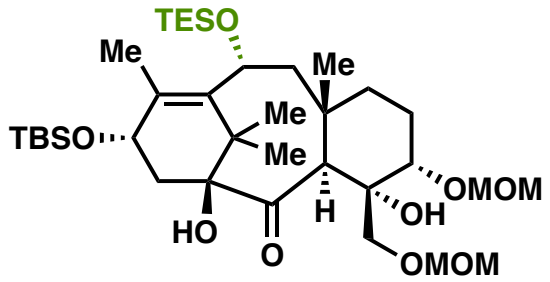

\begin{tabular}{cc}
\hline reagent & result \\
\hline $\mathrm{Sml}_{2}, \mathrm{Et}_{3} \mathrm{~N}, \mathrm{H}_{2} \mathrm{O}$ & $\mathrm{C} 2 \beta-\mathrm{OH}$ \\
$\mathrm{Sml}_{2}, \mathrm{LiCl}$ & $\mathrm{C} 2 \beta-\mathrm{OH}$ \\
\hline
\end{tabular}

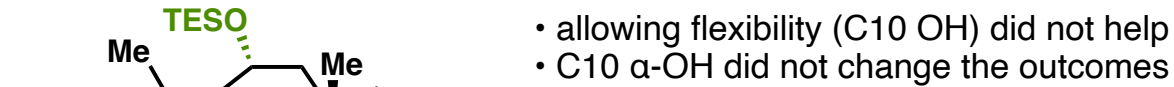

TBSO

- ring conformation might not be a problem

2nd generation route was abandoned for the following reasons

- unsuccessful stereoselective $\mathrm{C} 2$ reduction

- C20 substitution pattern could be a reason for unsuccessful C2 reduction

- unsuccessful C5 MOM deprotection

- lack of $\mathrm{C} 7$ oxidation method

- poor material throughput (C13 allylic oxidation 19\%) 
3rd Generation

Strategic hypothesis: Designed for stereoselective $\mathrm{C} 2$ reduction and late stage installation of $\mathrm{C5}$ oxidation

3-1 Synthetic route<smiles>CC1=C2CC[C@]3(C)CCCC(=O)[C@H]3C(=O)[C@H]2CC1</smiles>
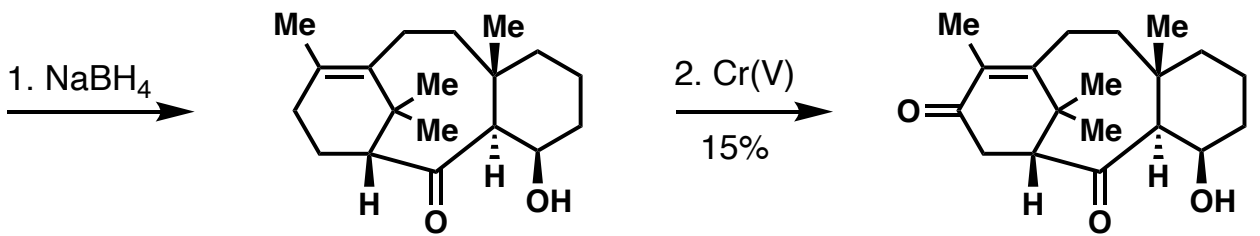

- goal: target substrates similar to literature precedents that had succeeded in C2 reduction

- they had $\mathrm{sp}^{2}$ or trisubstitution at $\mathrm{C} 4$, no tetrasubstituted
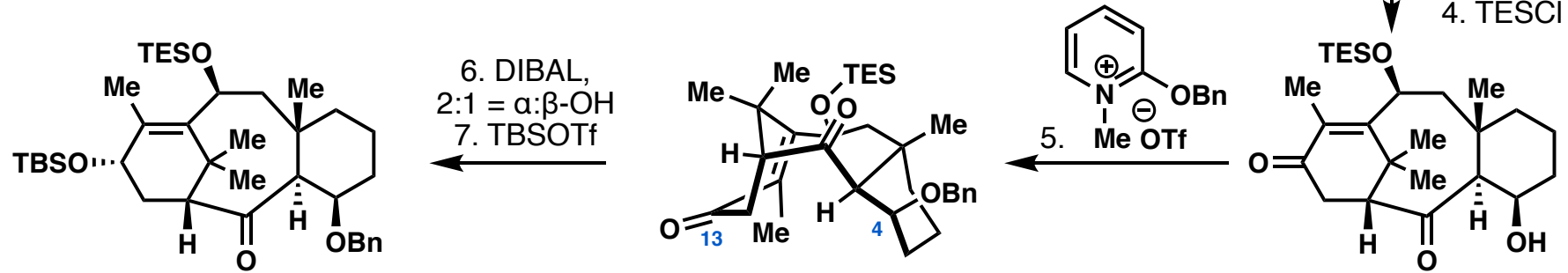

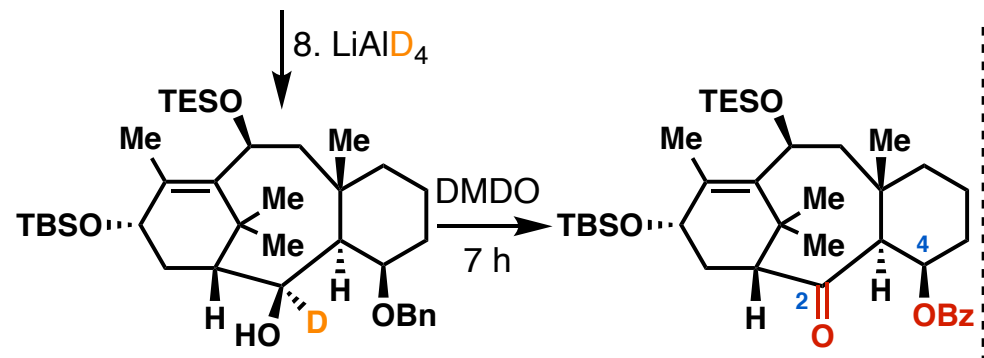

- little sterics around $\mathrm{C} 2$ resulted in rapid $\mathrm{C} 2$ oxidation - needed to balance sterics around C2 to achieve both $\mathrm{C} 1$ oxidation and $\mathrm{C} 2$ reduction

3-2. C2 reduction model study looking for appropriate sterics around C2

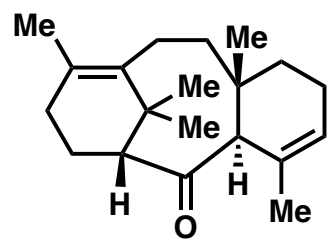

$$
\begin{gathered}
\text { reagent } \\
\mathrm{Sml}_{2}, \mathrm{Et}_{3} \mathrm{~N}, \mathrm{H}_{2} \mathrm{O} \\
\mathrm{Sml}_{2}, \mathrm{LiCl}
\end{gathered}
$$

$\mathrm{Sml}_{2}, \mathrm{PhSH}$, HMPA

$\mathrm{Sml}_{2}$, LiCl, PhSH, HMPA

$\mathrm{Na}, i-\mathrm{PrOH}, \mathrm{PhMe}$
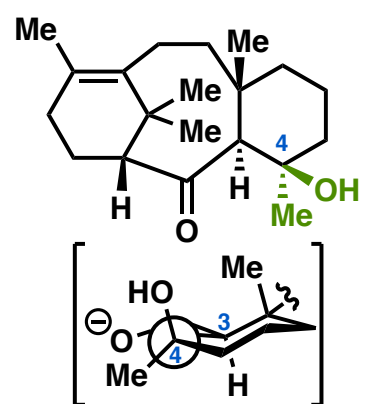

the key staggered configuration

- 1:1 solvent ratio was use for

Bouveault-Blanc reduction

- the first C4 tetrasubstituted

substrate gave $\mathrm{C} 2 \mathrm{a}-\mathrm{OH}$
3-3. Revisiting substrates from 2 nd generation

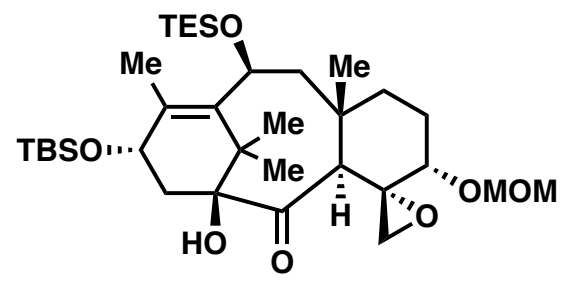

$\mathrm{Na}, i-\mathrm{PrOH}, \mathrm{Et}_{2} \mathrm{O}$ exclusively $\mathrm{C} 2 \beta-\mathrm{OH}$

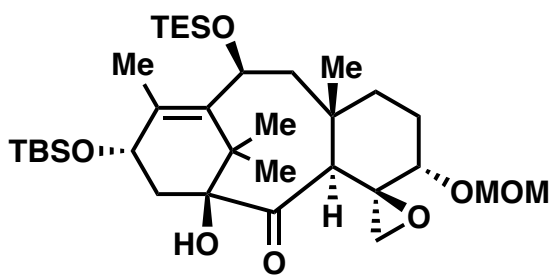

$\mathrm{Na}, i-\mathrm{PrOH}, \mathrm{Et}_{2} \mathrm{O}$ no reaction not condition but substrate issue

\begin{tabular}{|c|c|}
\hline ion & esult \\
\hline $\mathrm{Sml}_{2}, \mathrm{Et}_{3} \mathrm{~N}, \mathrm{H}_{2} \mathrm{O}$ & nor \\
\hline $\mathrm{Sml}_{2}, \mathrm{LiCl}$ & Ction \\
\hline $\mathrm{Na}, i-\mathrm{PrOH}, 80^{\circ} \mathrm{C}$ & $a: f$ \\
\hline $\mathrm{Na}, i-\mathrm{PrOH}, \mathrm{PhMe}$, rt & $2: 1$ \\
\hline $\mathrm{Na}, t-\mathrm{BuOH}, \mathrm{PhMe}, 50^{\circ} \mathrm{C}$ & $a: \beta=1: 1$ \\
\hline $\mathrm{Na}, t$-amylOH, $\mathrm{PhMe}$, rt & $=1: 4$ \\
\hline $\mathrm{Na}, \mathrm{MeOH}, \mathrm{PhMe}, 50^{\circ} \mathrm{C}$ & $2: 1$ \\
\hline $\mathrm{Na}, n-\mathrm{BuOH}, \mathrm{PhMe}$, rt & $a:$ \\
\hline $\mathrm{Na}, \mathrm{C}_{8} \mathrm{H}_{17} \mathrm{OH}, \mathrm{PhMe}$, rt & $a: \beta=2: 1$ \\
\hline $\begin{array}{c}\mathrm{Na}, \mathrm{PhSH}, \mathrm{PhMe}, 50^{\circ} \mathrm{C} \\
\mathrm{Na}, \mathrm{BuNH}_{2}, \mathrm{PhMe}, \mathrm{rt}\end{array}$ & $\begin{array}{c}\text { no reaction } \\
\alpha: \beta=1: 5\end{array}$ \\
\hline Ellman's amine, PhMe, $\mathrm{rt}$ & no reaction \\
\hline $\mathrm{Na}, i$-PrMeNH, PhMe, $50^{\circ} \mathrm{C}$ & $1: 15$ \\
\hline , pyrrolidine, $\mathrm{PhMe}, 50^{\circ} \mathrm{C}$ & $a: \beta$ \\
\hline $\mathrm{Na}, \mathrm{AcNH}_{2}, \mathrm{PhMe}$, rt & $a: f$ \\
\hline $\mathrm{Na}, \mathrm{C}_{2} \mathrm{H}_{4}\left(\mathrm{NH}_{2}\right)_{2}$, PhMe, rt & $\begin{array}{l}a: \beta=0: 1 \\
a: \beta=0: 1\end{array}$ \\
\hline $\mathrm{Na}, \mathrm{CF}_{3} \mathrm{CH}_{2} \mathrm{NH}_{2}, \mathrm{PhMe}$, rt & no $r$ \\
\hline Me, & no $\mathrm{r}$ \\
\hline $\mathrm{Na}, \mathrm{CF}_{3} \mathrm{CH}_{2} \mathrm{OH}, \mathrm{PhMe}$, rt & no reactio \\
\hline $\mathrm{OH}, \mathrm{PhMe}, \mathrm{rt}$ & no re \\
\hline "ni & \\
\hline $\mathrm{Na}, i-\mathrm{PrOH}$, & $2: 1$ \\
\hline$i-\operatorname{PrC}$ & \\
\hline $\mathrm{Na}, i$-PrOr & $=1: 1$ \\
\hline $\mathrm{Na}, i-\mathrm{PrOH}$, dioxane, rt & $a: \beta=1: 2$ \\
\hline $\mathrm{Na}, i-\operatorname{PrC}$ & $a: \beta=2: 1$ \\
\hline $\mathrm{IE}, \mathrm{rt}$ & $\alpha: \beta=2$ \\
\hline
\end{tabular}




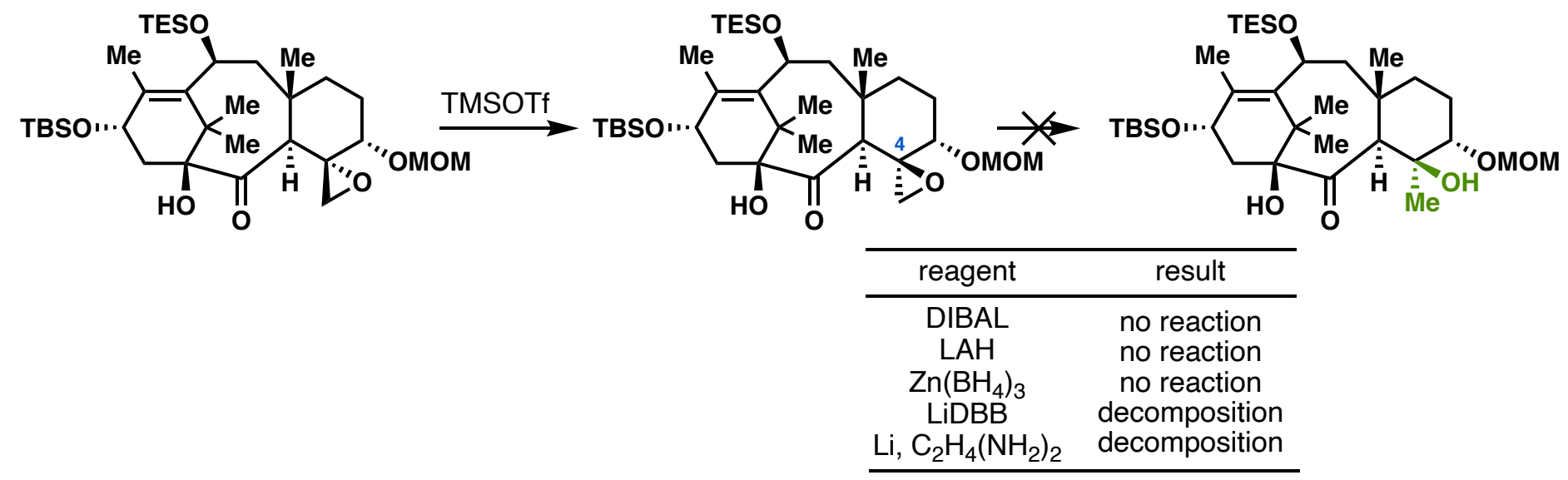

3-4. Attempts to recover material from 3rd generation route
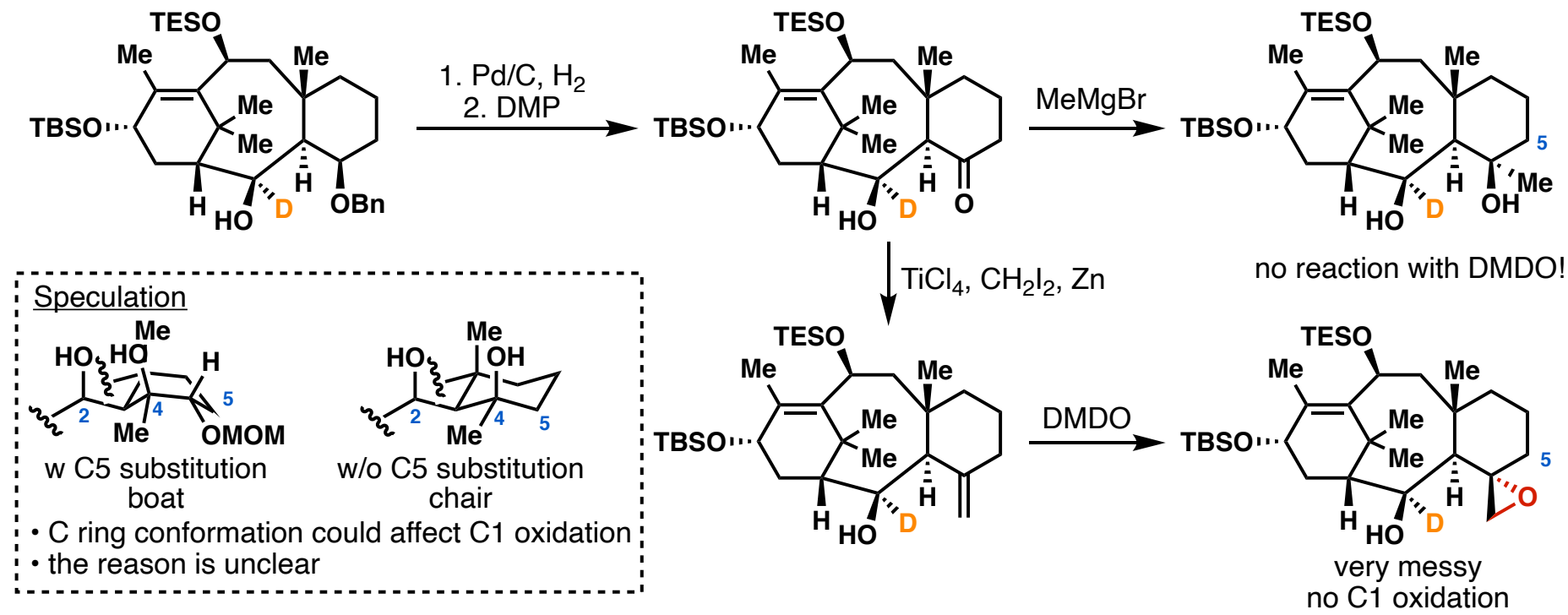

$\underline{3 r d}$ generation route was abandoned for the following reasons

- unsuccessful C1 oxidation

- lack of $\mathrm{C} 7$ oxidation method

- poor material throughput (C13 allylic oxidation 15\%) 


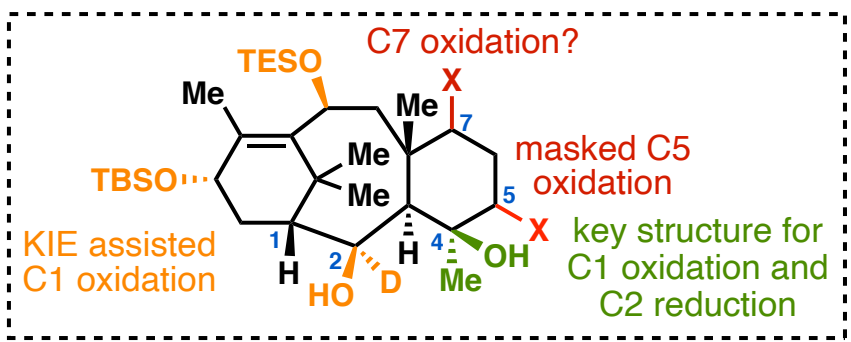

\section{4-1. C7 allylic oxidation attempts}

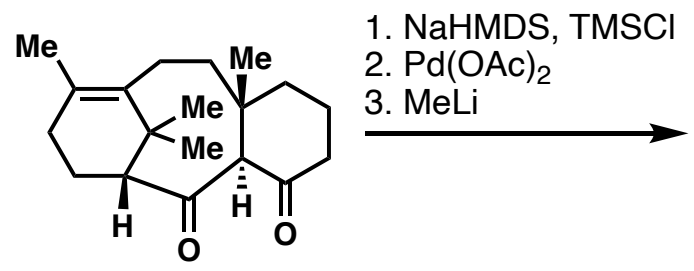

$$
\text { reagent }
$$

$\mathrm{SeO}_{2}$, py

$\mathrm{SeO}_{2}$, dioxane

$\mathrm{SeO}_{2}, \mathrm{HCO}_{2} \mathrm{H}$, dioxane

$\mathrm{SeO}_{2}, \mathrm{TBHP}, \mathrm{DCM}$

$\mathrm{CrO}_{3}$, DMP, $0^{\circ} \mathrm{C}$

$\mathrm{CrO}_{3}, \mathrm{DMP}, \mathrm{rt}$

$\mathrm{CrO}_{3}, \mathrm{HMPA}$

$$
\text { PCC }
$$

$\mathrm{PCC}, \mathrm{NaOAC}$

PCC, TBHP

PDC, TBHP

$\mathrm{Cr}(\mathrm{CO})_{6}, \mathrm{TBHP}$

TCNHPI, $\mathrm{LiClO}_{4}, \mathrm{TBHP}$, acetone, py, e-

quinuclidine, $\mathrm{Me}_{4} \mathrm{NBF}_{4}$,

$\mathrm{MeCN}, \mathrm{HFIP}$, e-

NBS, BPO

Cul, TBHP

$\mathrm{CuBr}$, TBHP

$\mathrm{Mn}(\mathrm{OAc})_{3} \cdot 2 \mathrm{H}_{2} \mathrm{O}$, TBHP

$\mathrm{Pd}(\mathrm{OAc})_{2}$, TBHP

$\mathrm{Pd}(\mathrm{OH})_{2} / \mathrm{C}, \mathrm{TBHP}$

$\mathrm{FeSO}_{4}$, TBHP

$\mathrm{Rh}_{2}(\text { cap })_{4}$, TBHP

$\mathrm{BiCl}_{3}, \mathrm{TBHP}$

$\mathrm{Pb}(\mathrm{OAc})_{4}$

$\mathrm{RuCl}_{3}, \mathrm{TBHP}$ decomposition

e

decomposition

a $(15 \%)+\mathbf{e}(15 \%)$

$f$

no reaction

a $(12 \%)$

a $(24 \%)$

decomposition

decomposition

decomposition

decomposition

decomposition

decomposition

decomposition

decomposition

decomposition

d

decomposition

decomposition

decomposition

decomposition

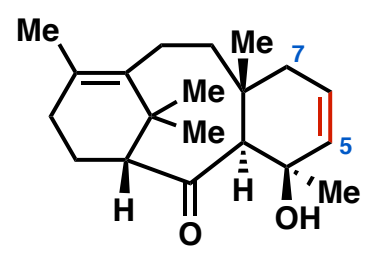

double allylic oxidation

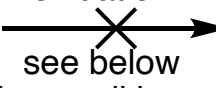

- masked C5 oxidation - potential oxidation relay to $\mathrm{C} 7$

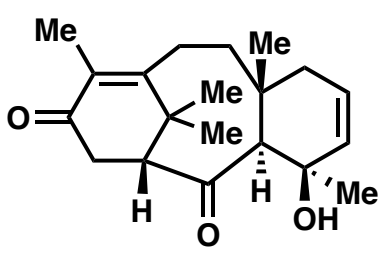

a

desired

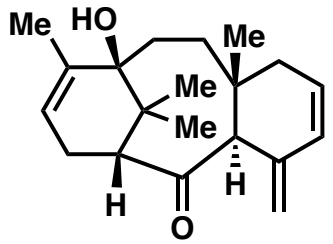

C

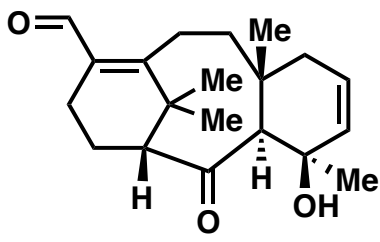

e

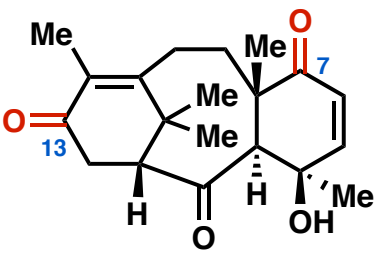

Me HO

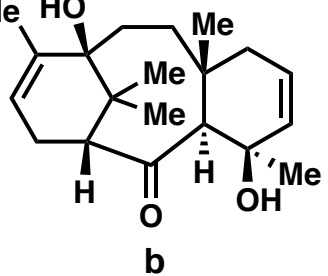

HO

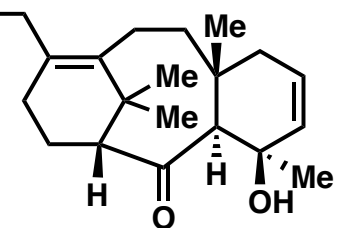

d

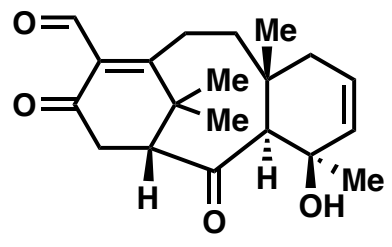

- no C7 oxidation was observed<smiles>CC1=C(C)[C@@]2(C)C(=C(C)CC1)CCC[C@@H]2C(=O)[C@H]1C(=O)C=CCC1(C)C</smiles>

\begin{tabular}{cc}
\hline reagent & result \\
\hline $\mathrm{Cul}, \mathrm{TBHP}$ & decomposition \\
$\mathrm{CuBr}, \mathrm{TBHP}$ & decomposition \\
$\mathrm{Mn}(\mathrm{OAc})_{3} \cdot \mathrm{HH}_{2} \mathrm{O}, \mathrm{TBHP}$ & decomposition \\
$\mathrm{Pd}(\mathrm{OAc})_{2}, \mathrm{TBHP}$ & decomposition \\
$\mathrm{Pd}(\mathrm{OH})_{2} / \mathrm{C}, \mathrm{TBHP}$ & decomposition \\
$\mathrm{FeSO} \mathrm{S}_{4}, \mathrm{TBHP}$ & decomposition
\end{tabular}

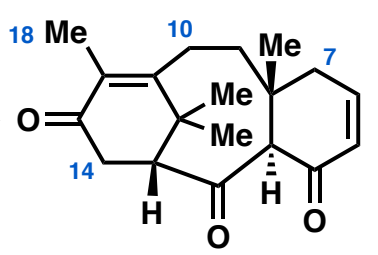

double $\gamma$-oxidation<smiles>CCC(C)(C)C</smiles>

for conditions;

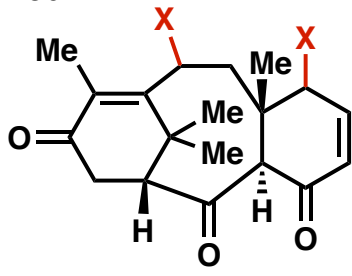

\begin{tabular}{cc}
\hline reagent & result \\
\hline $\mathrm{Rh}_{2}(\mathrm{cap})_{4}, \mathrm{TBHP}$ & decomposition \\
$\mathrm{BiCl}_{3}, \mathrm{TBHP}$ & decomposition \\
$\mathrm{SeO}_{2}$, TBHP, DCM & decomposition \\
$\mathrm{PCC}$, TBHP & decomposition \\
$\mathrm{PDC}, \mathrm{TBHP}$ & decomposition \\
$\mathrm{CuBr}_{2}$ & $\mathrm{C} 10$ and C14 bromination \\
\hline \multicolumn{3}{c}{ SI-33 }
\end{tabular}

\begin{tabular}{cc}
\hline reagent & result \\
\hline $\mathrm{O}_{2}$, pyridine & no reaction \\
NBS, BPO & $\begin{array}{c}\text { statistic mixture } \\
\text { of } \mathrm{C} 10 \text { and } 18 \\
\text { bromination }\end{array}$ \\
\hline
\end{tabular}


<smiles>CC1=C2CCC(C)(C)[C@@H](C(=O)[C@H]3CC=CC(=O)[C@@H]23)C1(C)C</smiles>

a<smiles>CC(=O)OC1(C)C=CC(=O)[C@H]2C(=O)C3CC(=O)C(C)=C1C=CC32C</smiles>

b

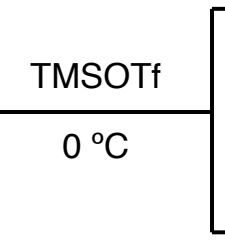

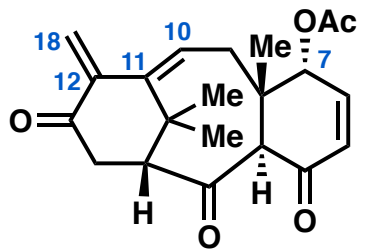

C

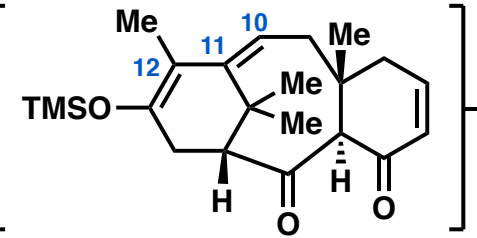

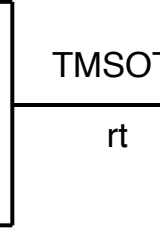

rt

conditions<smiles></smiles>

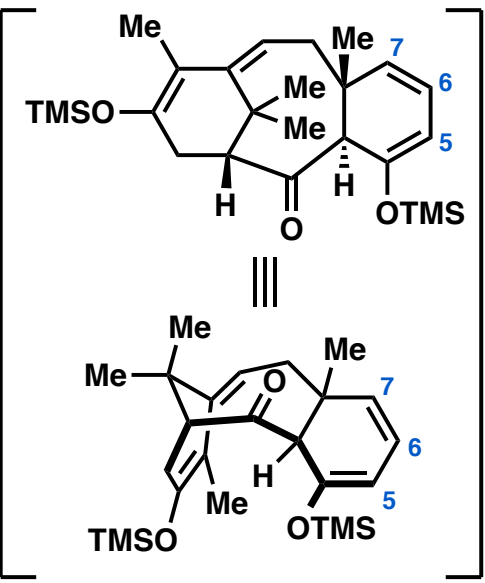

\begin{tabular}{|c|c|c|c|c|}
\hline reagent & result & \multicolumn{3}{|c|}{ Control experiments w/o TMS enol ether formation } \\
\hline \multirow{3}{*}{$\begin{array}{c}\mathrm{Pd}(\mathrm{OAc})_{2}, \mathrm{NaHCO}_{3} \\
\mathrm{Pd}(\mathrm{OAc})_{2}, \mathrm{NaOAc} \\
\mathrm{Pd}(\mathrm{OAc})_{2}, \mathrm{PPh}_{3}, \mathrm{NaOAc} \\
\mathrm{PdCl}_{2}, \mathrm{NaOAc} \\
\mathrm{PdCl}_{2}\left(\mathrm{PPh}_{3}\right)_{2}, \mathrm{NaOAc} \\
\mathrm{Pd}(\mathrm{TFA})_{2}, \mathrm{NaOAc} \\
\mathrm{Pd}(\mathrm{TFA})_{2}, \mathrm{PPh}_{3}, \mathrm{NaOAc}\end{array}$} & 5.16 & reagent & result & \multirow{3}{*}{$\begin{array}{l}\text { - bridgehead olefin dislocation } \\
\text { - interrupted Saegusa oxidation } \\
\text { - triple oxidation } \\
\text { - first C7 oxidation } \\
\text { - undesired C7 stereochemistry }\end{array}$} \\
\hline & $\begin{array}{c}5.16+\text { trace } 5.17 \\
5.16 \\
\text { decomposition } \\
5.16+\mathbf{5 . 1 8}\end{array}$ & $\begin{array}{c}\mathrm{Pd}(\mathrm{OAc})_{2}, \mathrm{NaHCO}_{3} \\
\mathrm{Pd}(\mathrm{OAc})_{2}, \mathrm{DBU} \\
\mathrm{Pd}(\mathrm{OAc})_{2}, \mathrm{BQ}, \mathrm{AcOH} \\
\end{array}$ & $\begin{array}{l}\text { decomposition } \\
\text { decomposition } \\
\text { no reaction }\end{array}$ & \\
\hline & $\begin{array}{c}\mathbf{5 . 1 6} \\
\text { ecomposition }\end{array}$ & & & \\
\hline
\end{tabular}

\section{Triple oxidation attempts}<smiles>CC1=C2CCC3(C)CCCC(=O)[C@@H]3C(=O)C2CC1=O</smiles>

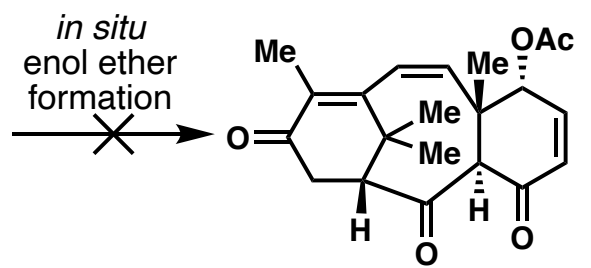

result

decomposition decomposition decomposition

$\mathrm{Pd}(\mathrm{OAc})_{2}$, TMSOTf, DTBP

$\mathrm{Pd}(\mathrm{OAC})_{2}$, TMSOTf, DBU

$\mathrm{Pd}(\mathrm{OAC})_{2}$, TMSOTf, DABCO

$\mathrm{Pd}(\mathrm{TFA})_{2}(\mathrm{DMSO})_{2}, \mathrm{AcOH}, \mathrm{O}_{2}$ $\mathrm{Pd}(\mathrm{TFA})_{2}$, DAF, TsOH, DMSO, $\mathrm{O}_{2}$ $\mathrm{Pd}(\mathrm{TFA})_{2}, 2-\mathrm{Me}_{2} \mathrm{Npy}, \mathrm{TsOH}$,

direct enone formation: J. Am. Chem. Soc. 2011, 133, 14566. DMSO, $\mathrm{O}_{2}$

J. Am. Chem. Soc. 2013, 135, 8205. J. Am. Chem. Soc. 2013, 135, 8213.

\section{4-2-2. C9-10 olefin oxidation attempts}

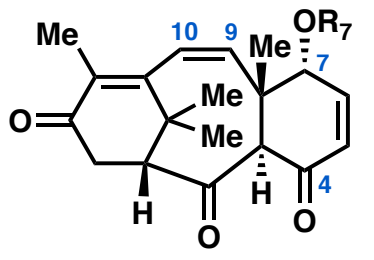

a $\mathrm{R}_{7}=\mathrm{Ac}$

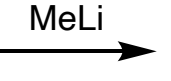

${ }^{1} \mathrm{H} \quad \mathrm{C} 10 \mathrm{C} 9(\mathrm{ppm})$

a 5.286 .36

b 5.146 .35

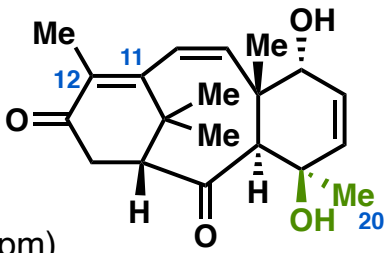

Me

$\mathrm{H}$

\section{result}

no reaction no reaction no reaction no reaction no reaction no reaction decomposition decomposition

\begin{tabular}{cc}
\hline reagent & result \\
\hline $\mathrm{NaOH}, \mathrm{H}_{2} \mathrm{O}_{2}$ & $\mathrm{C} 11-12$ epoxidation \\
$\mathrm{VO}(\mathrm{acac})_{2}, \mathrm{TBHP}$ & no reaction \\
$\mathrm{OsO}_{4}$ & decomposition \\
$\mathrm{RuCl}_{3}, \mathrm{NaBrO}_{3}$ & decomposition \\
\hline
\end{tabular}

- two olefins are not conjugated - C9-10 olefin seemed to be too hindered to react
1. DIBAL
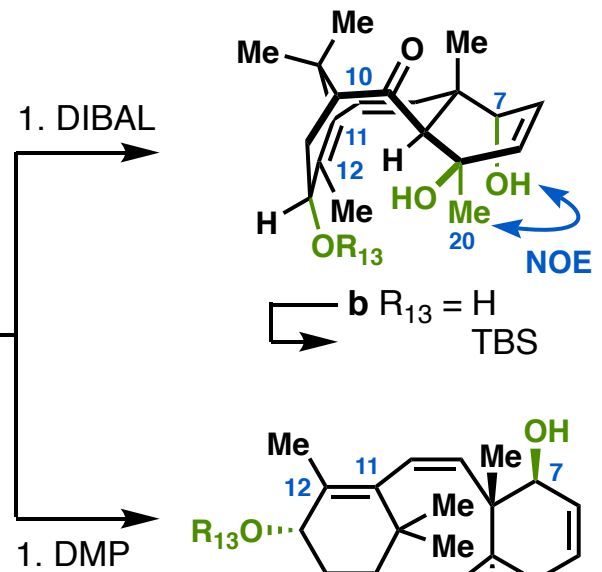

2. DIBAL

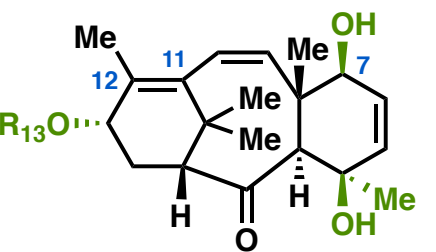

\begin{tabular}{|c|c|}
\hline$\Gamma$ & $\mathrm{R}_{13}=\underset{T B S}{\mathrm{H}}$ \\
\hline reagent & result \\
\hline $\begin{array}{c}\mathrm{VO}(\mathrm{acac})_{2}, \mathrm{TBHP} \\
\mathrm{DMDO} \\
\mathrm{RuCl}_{3}, \mathrm{NaBrO} \\
\mathrm{MeReO}_{3}, \text { urea } \mathrm{H}_{2} \mathrm{O}_{2}\end{array}$ & $\begin{array}{c}\text { no reaction } \\
\text { C11-12 epoxidation } \\
\text { decomposition } \\
\text { decomposition }\end{array}$ \\
\hline
\end{tabular}



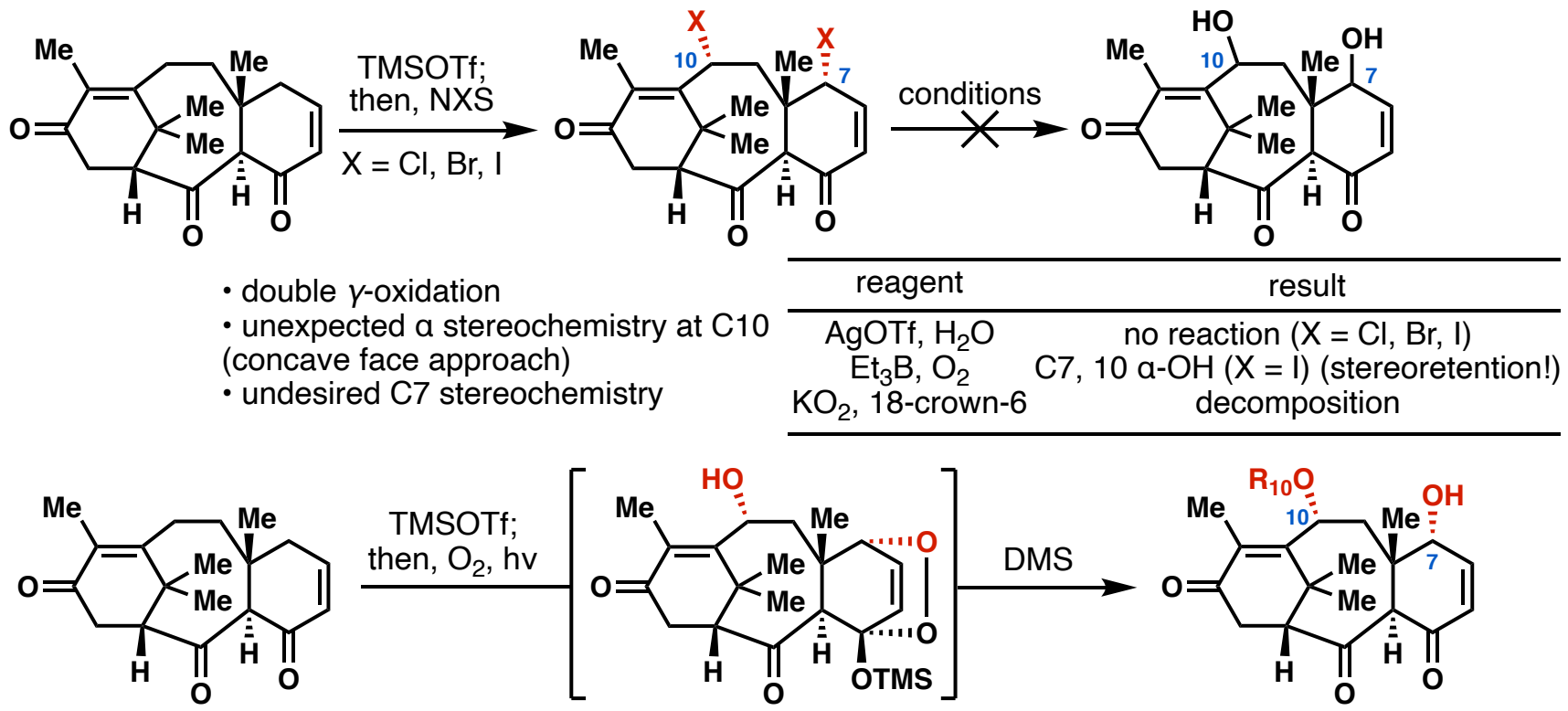

- double $\gamma$-hydroxylation

- peroxide intermediate was stable enough for isolation

- unexpected a stereochemistry at $\mathrm{C} 10$

- undesired $\mathrm{C} 7$ stereochemistry

- other attempted $\gamma$-oxidation conditions:

Shi, Mn(salen), $\mathrm{Fe}(\text { phen })_{3}\left(\mathrm{PF}_{6}\right)_{3}, \mathrm{CAN}, \mathrm{DMDO}$, all led to decomposition

4-3-2. C20 methylation conditions (examined on a and

\begin{tabular}{cc}
\hline reagent & result (c:d) \\
\hline $\mathrm{MeMgBr}, \mathrm{DCM},-78^{\circ} \mathrm{C}$ & no reaction \\
$\mathrm{MeMgBr}, \mathrm{DCM},-50^{\circ} \mathrm{C}$ & $2: 3$ \\
$\mathrm{MeMgBr}, \mathrm{DCM}, \mathrm{CeCl}_{3}, 0^{\circ} \mathrm{C}$ & $0: 1$ \\
$\mathrm{MeMgBr}, \mathrm{THF}, \mathrm{reflux}^{\prime}$ & $0: 1$ \\
$\mathrm{LaCl}_{3} \cdot 2 \mathrm{LiCl}, \mathrm{MeMgBr}$ & $0: 1$ \\
$\mathrm{MeLi}$ & $0: 1$ \\
$\mathrm{MeLi} \cdot \mathrm{LiBr}$ & $0: 1$ \\
\hline
\end{tabular}

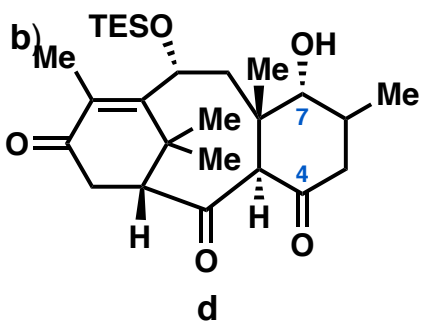

$$
\begin{aligned}
& \text { a } \mathrm{R}_{10}=\begin{array}{r}
\mathrm{H} \\
\text { b }
\end{array} \text { TESCI } \\
& \qquad \begin{array}{l}
\text { 1. conditions } \\
\text { (when } \mathrm{R}_{10}=\mathrm{H} \\
\text { 2. TESCI) }
\end{array}
\end{aligned}
$$
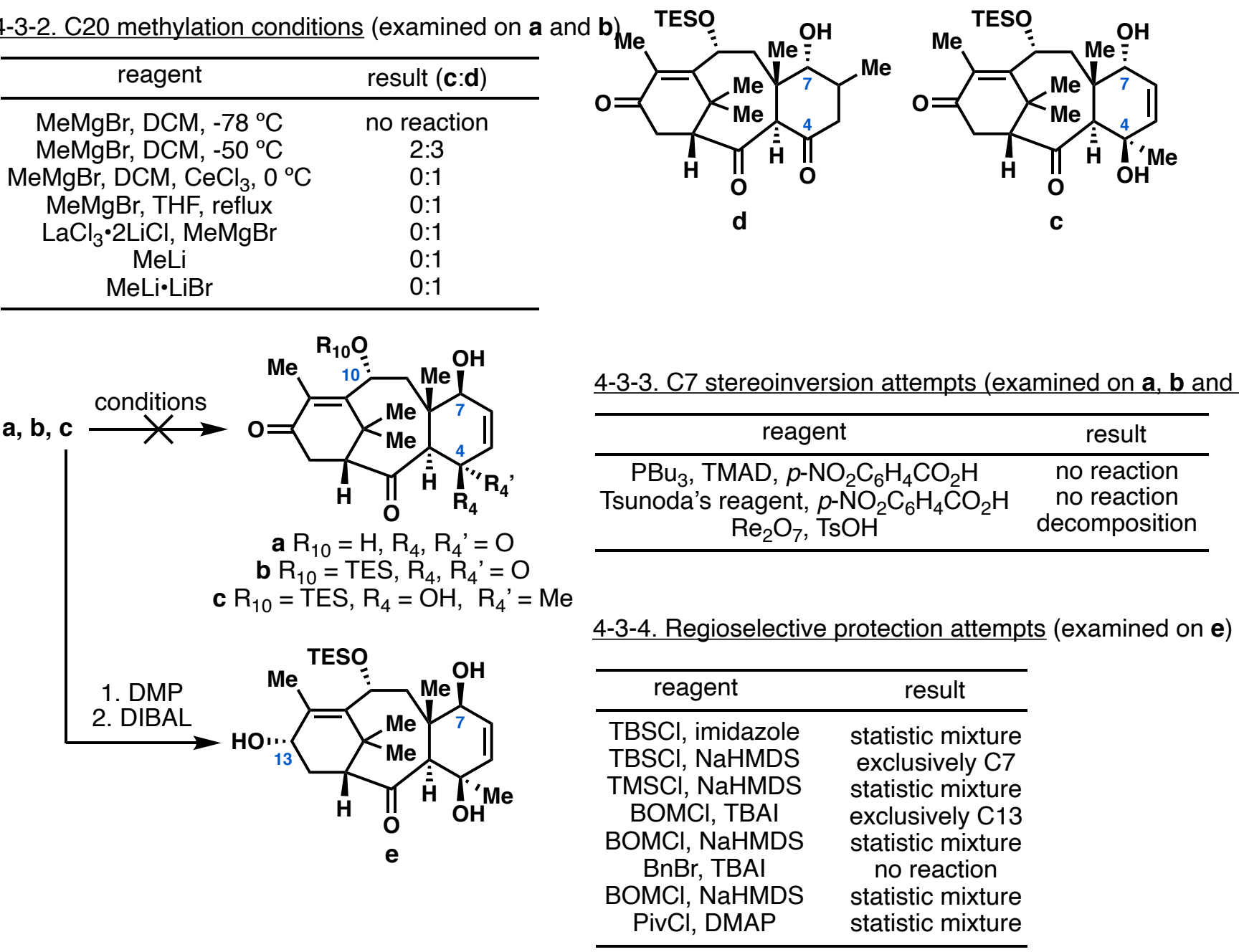


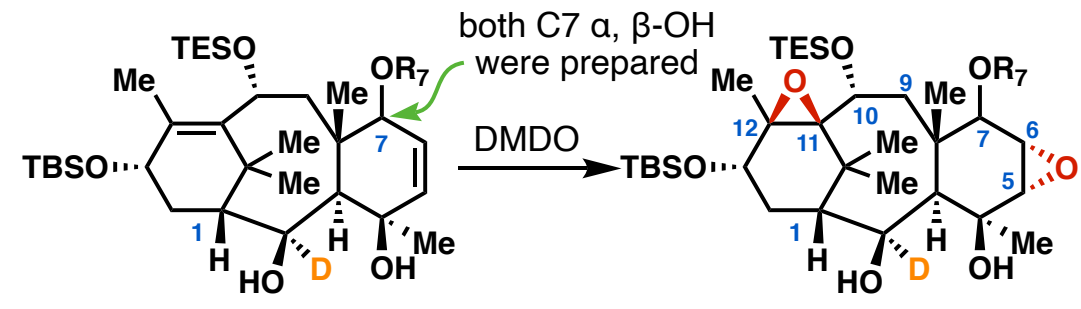

$\mathrm{R}_{7}=\mathrm{H}$, BOM, Piv, TES, TBS

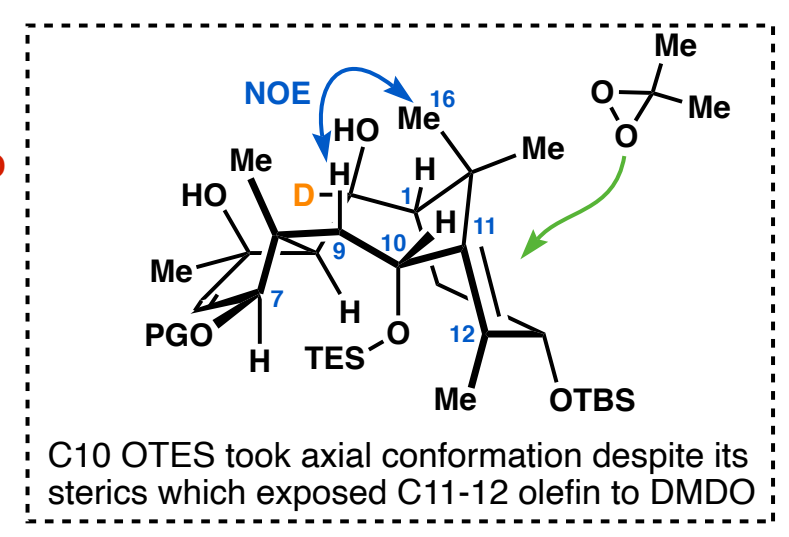

4 th generation route was abandoned for the following reasons

- poor material throughput

- C13 allylic oxidation 30\%

- poor dr for C20 Me installation

- non selective C7 protection

- no suitable PGs for $\mathrm{C7}$

- orthogonal to [Si]

- tolerate DMDO, $\mathrm{Na}, \mathrm{LiAID}_{4}$

- needed stereoinversion at C7

- unsuccessful $\mathrm{C} 1$ oxidation 


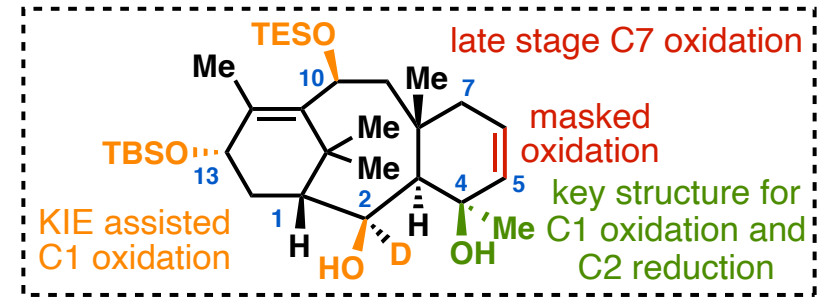

$5-1$. Examine the order of $C 5,10,13$ oxidations

$\mathrm{C} 5, \mathrm{C} 10, \mathrm{C} 13$ oxidations were necessary for $\mathrm{C} 1$ oxidation

\section{5-1-1. $\mathrm{C} 5 \Rightarrow \mathrm{C} 13=>\mathrm{C} 10$}

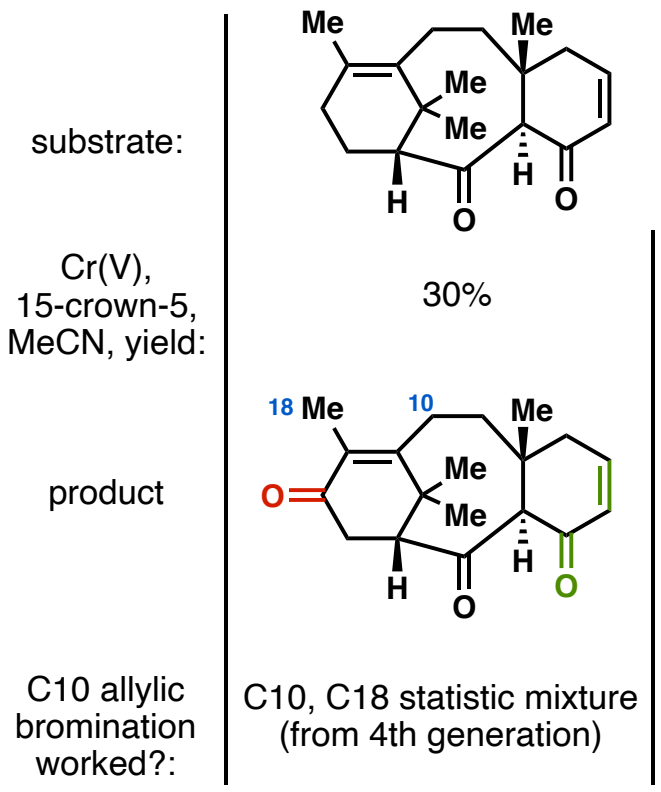

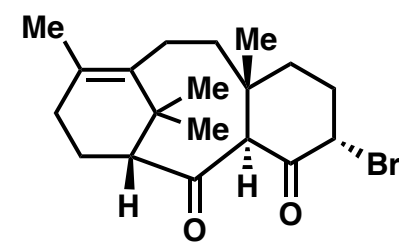

$25 \%$

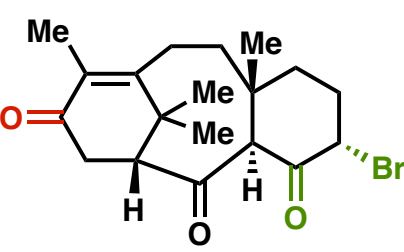

desired product (single product)

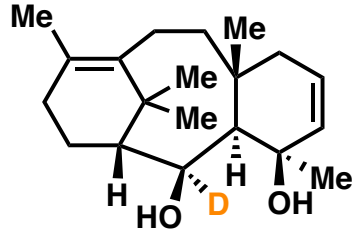

$42 \%$ (32\% w/ C2 H) (22\% when C2 ketone)<smiles>CC1=C2CC[C@]3(C)CC=C[C@](C)(O)[C@H]3C(=O)[C@H]2CC1=O</smiles>

decomposition

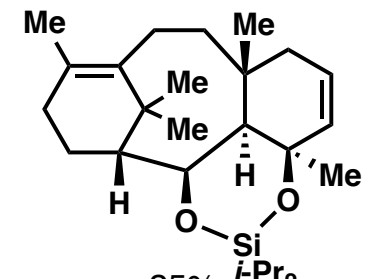

$25 \%$ i-Pr

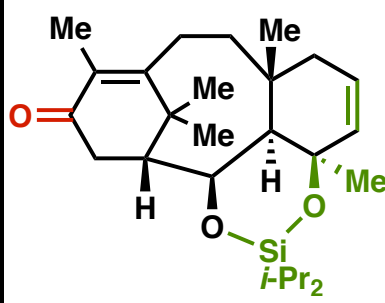

decomposition

\section{5-1-2. C13 $=>C 10 \Rightarrow C 5$}

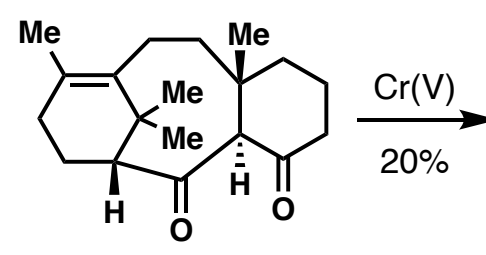

5-1-3. $C 13=>C 5 \Rightarrow C 10$

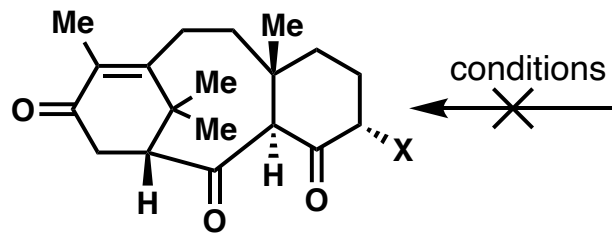

\begin{tabular}{|c|c|}
\hline condition & result \\
\hline $\begin{array}{l}\text { TMSOTf; then, NBS } \\
\text { TMSOTf } \text { then }\end{array}$ & decomposition \\
\hline $\begin{array}{c}\text { TMSOTf; then, DMDO } \\
\text { NBS BPO }\end{array}$ & decomposition \\
\hline $\begin{array}{l}\text { NBS, BPO } \\
\text { CtOAc }\end{array}$ & C10, C18 dibromination \\
\hline CuB & 14 dibrc \\
\hline
\end{tabular}

NBS, BPO<smiles>CCC(C)(C)C1=C2[C@@H](Br)C[C@]3(C)CCCC(=O)[C@H]3C(=O)[C@H]2CC1=O</smiles>

i) $\mathrm{AgOTf}, \mathrm{H}_{2} \mathrm{O}$

ii) TESCl

5-1-4. C13 $=>C 5=>C 10$

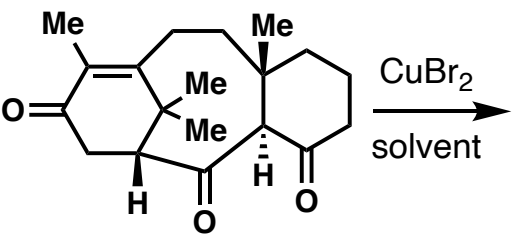

$20 \%$ from dione

- $\mathrm{C} 13=>$ C5 => C10 route gave the best throughput

- C13 allylic oxidation had to be optimized
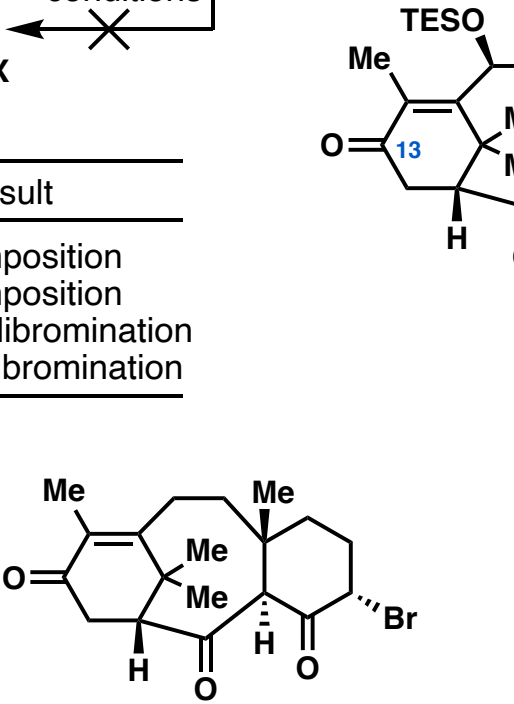

about 1:1:1 = product:SM:C18 bromination

in $\mathrm{DCE}, \mathrm{CHCl}_{3}, \mathrm{CCl}_{4}$, benzene, $\mathrm{PhCl}$,

$\mathrm{MeCN}$, EtOAc, pyridine

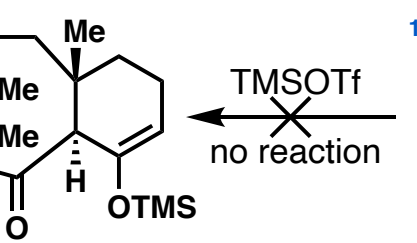

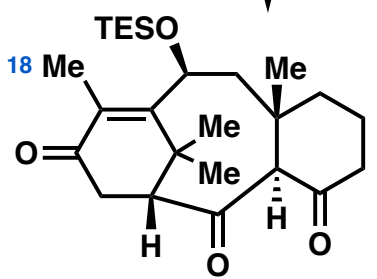




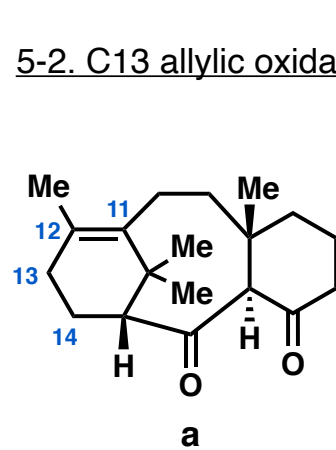

5-2-1. General trend

- $\mathrm{Cr}(\mathrm{V})$ is milder than $\mathrm{Cr}(\mathrm{VI})$ which cleaved or epoxidized $\mathrm{C} 11-12$ olefin

- mass balance is good

- major product is normally C11 tertiary alcohol (c)

- $\mathrm{C} 14$ allylic oxidation from $\mathbf{c}$ to $\mathbf{d}$ is more facile than $\mathrm{C} 13$ allylic oxidation from $\mathbf{a}$ to $\mathbf{b}$

- $\mathrm{Cr}(\mathrm{V})$ is paramagnetic which makes NMR study not feasible

- requires heat (very sluggish at $<80^{\circ} \mathrm{C}$. goes to completion at $>80^{\circ} \mathrm{C}$ )

- proposed mechanism:<smiles>CC1=C(C)C(C)(C)C(C)(C)CC1</smiles>

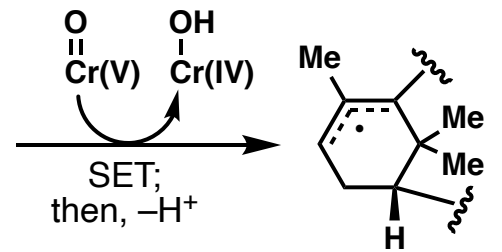

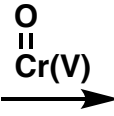

- reaction color turned green at the end which indicated generation of $\mathrm{Cr}$ (III)

- the intermediate allylic alcohol leading to $\mathbf{b}$ was never observed

5-2-1. Initial screening

\begin{tabular}{|c|c|c|c|}
\hline & solvent & temperature $\left[{ }^{\circ} \mathrm{C}\right]$ & result $(\mathbf{a} / \mathbf{b} / \mathbf{c} / \mathbf{d})$ \\
\hline $\begin{array}{c}\mathrm{Cr}(\mathrm{V}), 15-\text { crown-5 } \\
\mathrm{Na}_{2} \mathrm{Cr}_{2} \mathrm{O}_{7}, 15-\mathrm{crown}_{7} \text {, } \\
\text { PDC } \\
\text { TCNHPI, LiClO } \\
\text { quinuclidine, } \mathrm{Et}_{4} \mathrm{NBF}_{4} \\
\text { quinuclidine, } \mathrm{Et}_{4} \mathrm{NBF}_{4} \\
\mathrm{CrO}_{3}, \mathrm{Bu}_{4} \mathrm{NIO}_{4} \\
\mathrm{CrO}_{3}, \mathrm{H}_{5} \mathrm{IO}_{6}, \mathrm{Ac}_{2} \mathrm{O} \\
\mathrm{PCC} \mathrm{Celite}, \mathrm{NaOAc} \\
\text { PCC, Celite, } \mathrm{NaOAC}\end{array}$ & $\begin{array}{c}\text { DMF } \\
\text { acetone } \\
\text { MeCN, THIP } \\
\text { MeCN, THIP } \\
\text { MeCN, DCM } \\
\text { DCM } \\
\text { DCM } \\
\text { benzene }\end{array}$ & $\begin{array}{c}80 \\
80 \\
80 \\
r t \\
r t \\
r t \\
-40 \\
-40 \text { to } 0 \\
40 \\
40\end{array}$ & $\begin{array}{c}\text { 0/20/22/45 } \\
\text { no reaction } \\
\text { no reaction } \\
\text { decomposition } \\
\text { decomposition } \\
\text { decomposition } \\
\text { decomposition } \\
\text { decomposition } \\
5 / 15 / 0 / 0 \\
21 / 3 / 0 / 0\end{array}$ \\
\hline
\end{tabular}

5-2-2. Other attempted substrates
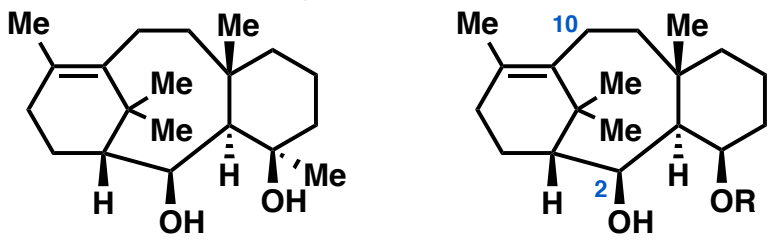

$\mathrm{R}=\mathrm{H}, 13 \%$, accompanied $\mathrm{C} 2$ oxidation

$10 \%$

$R=A c, 17 \%$

$R=$ TES, $28 \%$

the following $\mathrm{C} 10$ oxidation did not work - could not find any substrates or conditions that gave

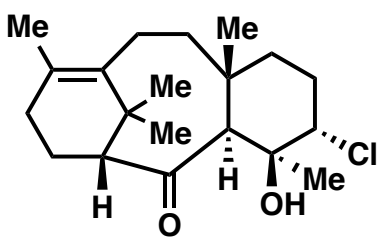

exclusively $t-\mathrm{OH}$

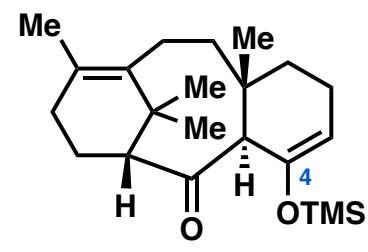

$18 \%$

accompanied C4 enone formation

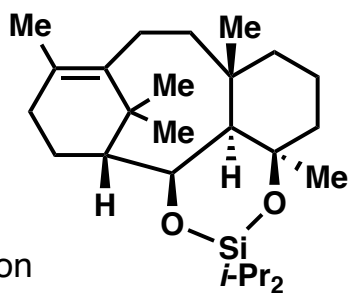

$22 \%$
Inorg. Chem. 1985, 24, 617.

J. Am. Chem. Soc. 1976, 98, 872.

J. Am. Chem. Soc. 2014, 136, 4909.

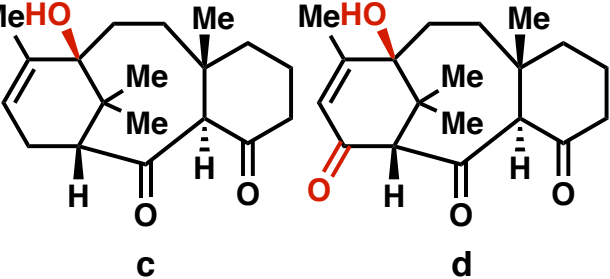




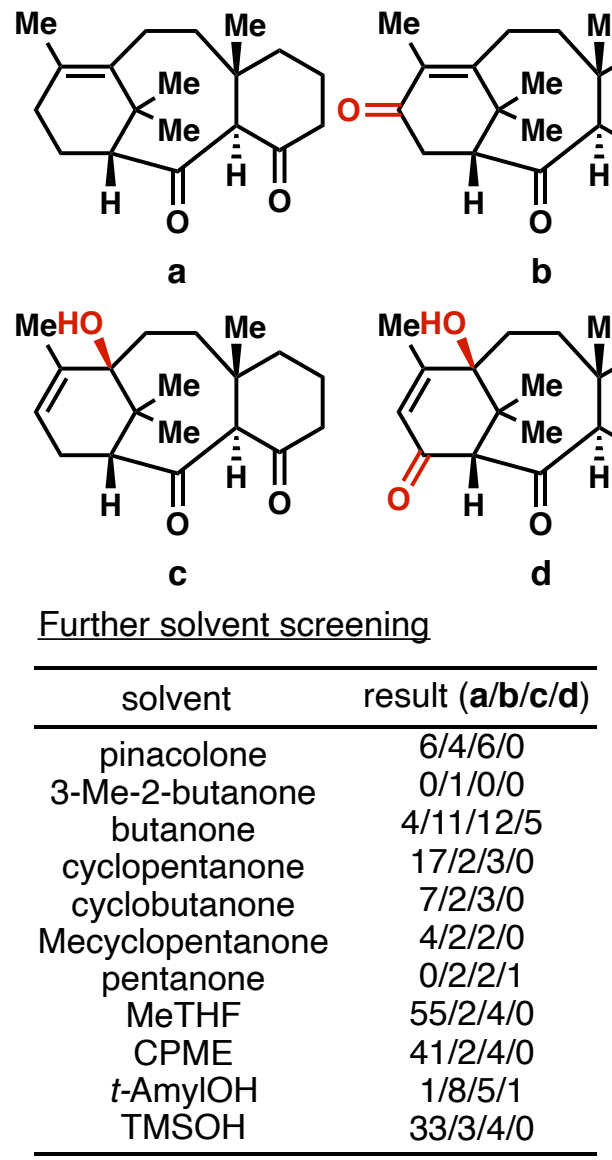

Solvent screening w/ $\mathrm{Cr}(\mathrm{V})(7.0 \mathrm{eq}), 15-\mathrm{crown}-5$ at $80^{\circ} \mathrm{C}$

\begin{tabular}{cccc}
\hline solvent & result $(\mathbf{a} / \mathbf{b} / \mathbf{c} / \mathbf{d})$ & solvent & result $(\mathbf{a} / \mathbf{b} / \mathbf{c} / \mathbf{d})$ \\
\hline$t$-BuOH & $0 / 1 / 1 / 0$ & $\mathrm{THF}$ & $3 / 0 / 1 / 0$ \\
$\mathrm{DMF}$ & $1 / 0 / 1 / 0$ & dioxane & $1 / 0 / 1 / 0$ \\
$\mathrm{DMSO}$ & $0 / 0 / 1 / 0$ & $\mathrm{MTBE}$ & $1 / 1 / 2 / 0$ \\
$\mathrm{DMPU}$ & $5 / 0 / 0 / 1$ & acetone & $0 / 1 / 1 / 0$ \\
$\mathrm{NO}_{2} \mathrm{Me}$ & $1 / 0 / 0 / 0$ & $\mathrm{AcOH}$ & $2 / 0 / 0 / 0$ \\
$\mathrm{EtOAc}$ & $0 / 1 / 1 / 0$ & $\mathrm{Et}_{3} \mathrm{~N}$ & $4 / 0 / 0 / 0$ \\
$\mathrm{DCE}$ & $1 / 0 / 0 / 0$ & pyridine $^{2}$ & $2 / 0 / 1 / 0$ \\
$\mathrm{CCl}_{4}$ & $0 / 0 / 1 / 0$ & $\mathrm{PhH}$ & $1 / 0 / 24 / 0$ \\
$\mathrm{CHCl}_{3}$ & $2 / 0 / 0 / 0$ & cyclohexane & $2 / 0 / 0 / 0$ \\
$\mathrm{PhCF}_{3}$ & $1 / 0 / 0 / 0$ & $\mathrm{C}_{6} \mathrm{~F}_{14}$ & $2 / 1 / 1 / 0$ \\
& & $\mathrm{HFIP}$ & $0 / 2 / 1 / 0$ \\
\hline
\end{tabular}

- $t$-BuOH, MTBE, acetone and HFIP gave b/c ratio $>0.7$

- solvents listed above showed somewhat stirrable mixture

- given the fact mass balance was good in MeCN, we set increasing b/c ratio as the direction of this optimization

- $\mathrm{C}_{6} \mathrm{~F}_{14}$ was excluded because of poor solubility of $\mathrm{Cr}(\mathrm{V})$

- HFIP probably stabilizes a carbocation i.e. intermediate

- alcohol, ether, ketone based solvents were worth investigating

- most solvents showed stirrable mixture

- $b / c$ ratio was generally better than the initial screening

- MeTHF, CPME and TMSOH showed exceptionally good mass balance

Mixed solvent system in 1:1 = solvent:HFIP $\cdot$ dramatic increase in yield with $\mathrm{TMSOH}$

\begin{tabular}{cc}
\hline solvent & result $(\mathbf{a} / \mathbf{b} / \mathbf{c} / \mathbf{d})$ \\
\hline MeCN & $0 / 12 / 9 / 3$ \\
acetone & $0 / 28 / 10 / 8$ \\
pinacolone & $0 / 32 / 18 / 9$ \\
3-Me-2-butanone & $0 / 14 / 6 / 5$ \\
butanone & $0 / 27 / 14 / 7$ \\
cyclopentanone & $5 / 22 / 19 / 6$ \\
cyclobutanone & $7 / 17 / 15 / 11$ \\
Mecyclopentanone & $0 / 27 / 14 / 11$ \\
pentanone & $0 / 23 / 11 / 11$ \\
MeTHF & $25 / 9 / 10 / 0$ \\
CPME & $8 / 16 / 10 / 0$ \\
$t$-BuOH & $0 / 28 / 10 / 8$ \\
$t$-AmylOH & $0 / 26 / 9 / 5$ \\
TMSOH & $0 / 42 / 16 / 7$ \\
\hline
\end{tabular}

- small amount of ketone or alcohol based solvents made the reaction mixture almost homogeneous - a separate experiment showed $5 \% t$-BuOH was enough to solubilize $\mathrm{Cr}(\mathrm{V})$ and did not affect the yield
- $t$-AmylOH gave comparable $\mathbf{b} / \mathbf{c}$ ratio but abandoned due to inferior mass balance

- HFIP/TMSOH mixture was not the most solubilizing solvent for $\mathrm{Cr}(\mathrm{V})$

Three solvent system

\begin{tabular}{ccc}
\hline solvent & solvent ratio & result $(\mathbf{a} / \mathbf{b} / \mathbf{c} / \mathbf{d})$ \\
\hline HFIP/TMSOH/pinacolone & $3: 6: 1$ & $12 / 18 / 8 / 2$ \\
& $1: 1: 1$ & $7 / 19 / 10 / 3$ \\
$6: 1: 3$ & $4 / 24 / 13 / 0$ \\
& $6: 2: 2$ & $5 / 24 / 12 / 0$ \\
HFIP/TMSOH/acetone & $6: 3: 1$ & $7 / 26 / 12 / 5$ \\
& $8: 1: 1$ & $19 / 23 / 15 / 1$ \\
& $3: 6: 1$ & $12 / 23 / 12 / 0$ \\
& $1: 1: 1$ & $5 / 21 / 15 / 4$ \\
HFIP/TMSOH/t-BuOH & $6: 2: 2$ & $3 / 23 / 15 / 5$ \\
& $6: 3: 1$ & $2 / 22 / 12 / 6$ \\
& $8: 1: 1$ & $3 / 39 / 12 / 5$ \\
& $3: 6: 1$ & $12 / 25 / 17 / 2$ \\
& $1: 1: 1$ & $13 / 22 / 10 / 0$ \\
& $6: 1: 3$ & $5 / 25 / 12 / 5$ \\
& $6: 2: 2$ & $9 / 27 / 12 / 5$ \\
& $6: 3: 1$ & $9 / 30 / 13 / 5$ \\
HFIP/TMSOH/t-AmylOH & $8: 1: 1$ & $18 / 23 / 5 / 1$ \\
& $3: 6: 1$ & $12 / 24 / 11 / 4$ \\
& $1: 1: 1$ & $10 / 18 / 8 / 3$ \\
& $6: 1: 3$ & $8 / 23 / 11 / 4$ \\
& $6: 2: 2$ & $7 / 26 / 12 / 5$ \\
& $6: 3: 1$ & $6 / 28 / 12 / 5$ \\
& $8: 1: 1$ & $18 / 22 / 12 / 1$ \\
\hline
\end{tabular}




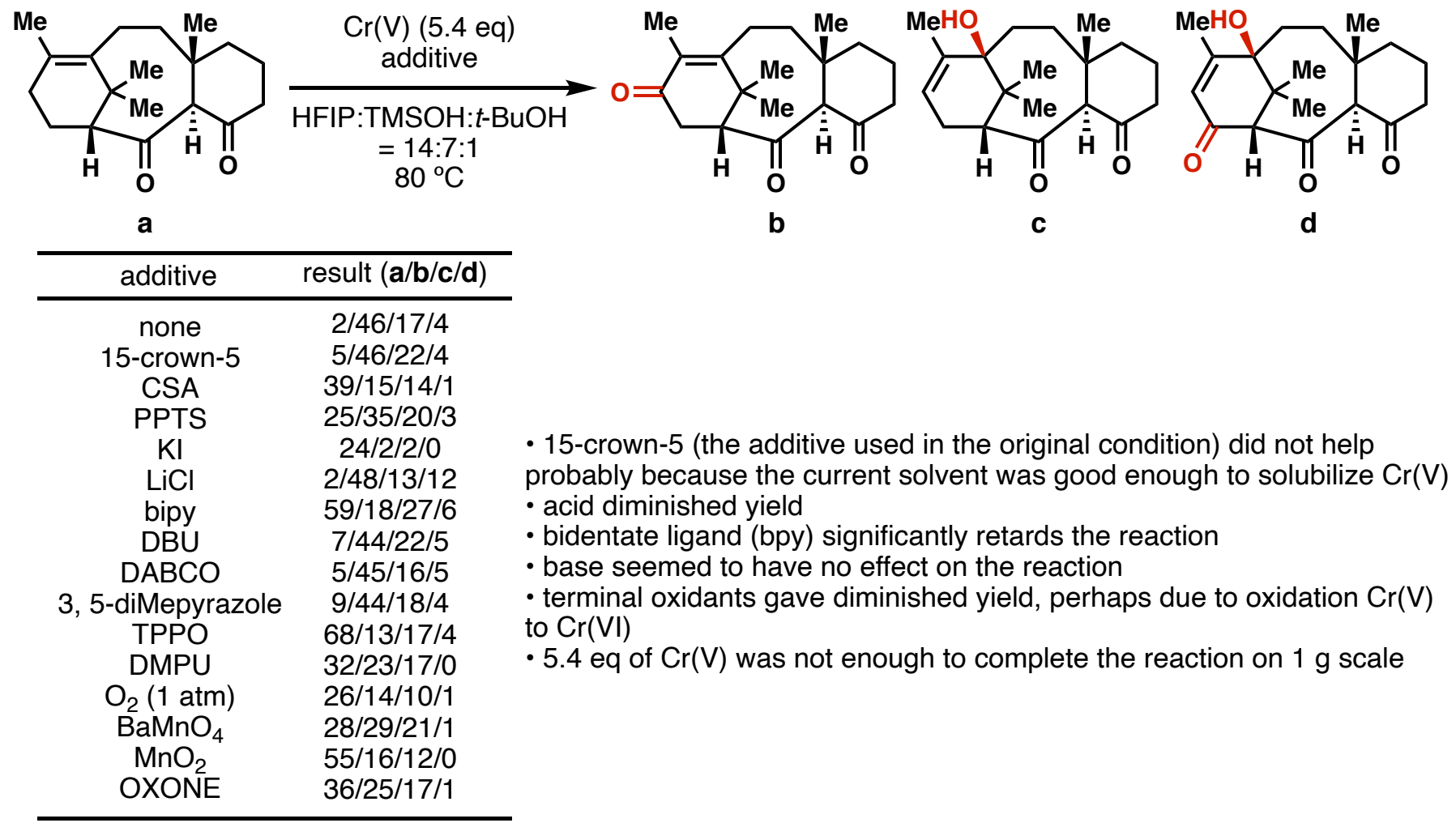

\section{5-2-4. C5, 10,13 oxidation choreography summary}<smiles>CC1=C2CCC[C@]3(C)C(C(=O)[C@@H]2C(=O)CCC[C@@H]3C)C1(C)C</smiles>

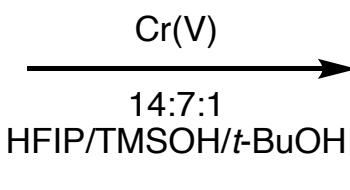<smiles>CC1=C2CC[C@]3(C)CCCC(=O)[C@H]3C(=O)[C@H]2CC1=O</smiles><smiles>CC(C)(Br)CC(F)(F)Br</smiles><smiles>CC1=C2CC[C@]3(C)CC[C@@H](Br)C(=O)[C@H]3C(=O)[C@H]2CC1=O</smiles>

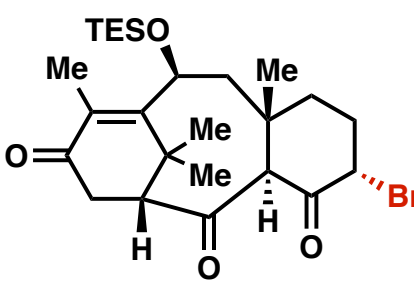
NBS, BPO, $\mathrm{CCl}_{4}$ then TESOH, AgOTf, DTBMP, PhMe
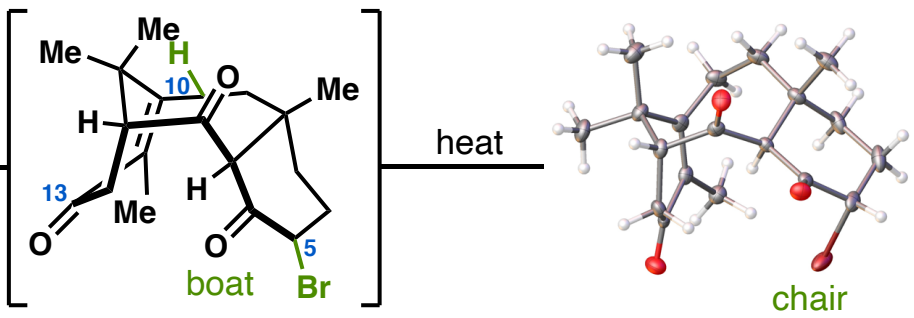

Hypothesis

C5 $\mathrm{Br}$ allowed $\mathrm{C}$ ring to adopt boat conformation upon heating and allowed C10 hydrogen to have better orbital overlap with the bridgehead olefin 


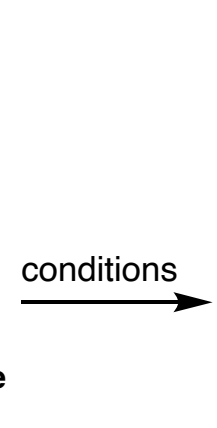

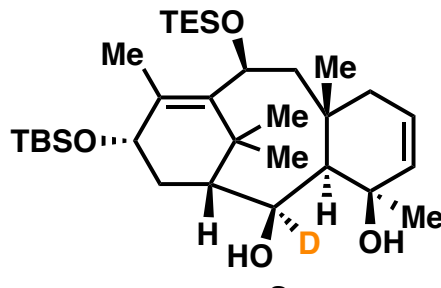

a

(desired)

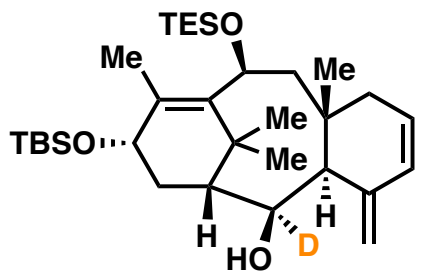

C

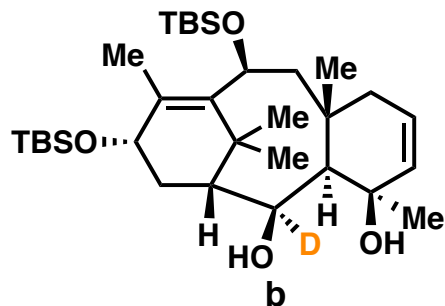

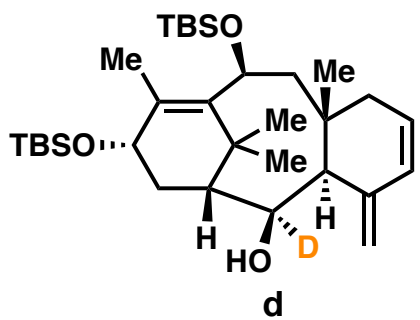

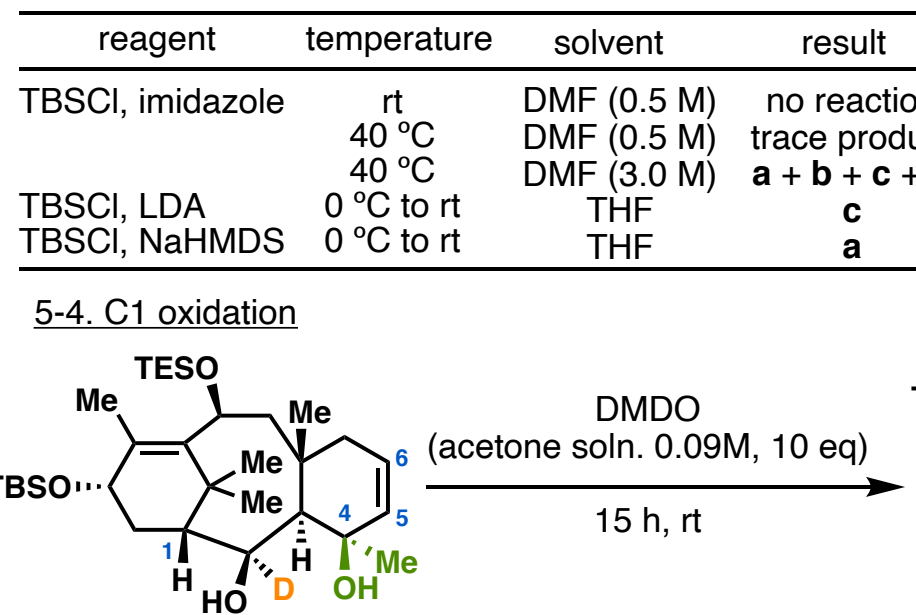

5-4-1. Additive screening

\begin{tabular}{cc}
\hline variation & result (e/f/g) [\%] \\
\hline$+\mathrm{NaHCO}_{3}$ & no change \\
$+\mathrm{CaCO}_{3}$ & no change \\
$+\mathrm{K}_{2} \mathrm{HPO}_{4}$ & no change
\end{tabular}

DMDO: phosphate buffer $(\mathrm{pH}=7.5)=1: 1$ poor conversion

DMDO: phosphate buffer $(\mathrm{pH}=7.5)=1: 2$ poor conversion under $\mathrm{N}_{2}$ portionwise addition ( 1.0 eq every $2 \mathrm{~h}$ ) DMDO (40 eq) $21 \mathrm{~h}$ $\mathrm{C} 2 \mathrm{H}$ instead of $\mathrm{D}$ no change no change $15 / 15 / 10$ $20 / 31 / 11$ $9 / 12 / 37$
- C4 tetrasubstituted carbon seemed to protect C2 from undesired oxidation

- C5-6 epoxide might force $\mathrm{C}$ ring to take boat conformation

- $f$ was isolated with impurities as inseparable mixture - yield of $\mathbf{f}$ was probably overestimated

\section{5-4-2. Cosolvent screening}

\begin{tabular}{cc}
\hline $\begin{array}{c}\text { cosolvent:DMDO (acetone) } \\
=1: 1\end{array}$ & result $(\mathbf{e} / \mathbf{f} / \mathbf{g})[\%]$ \\
\hline EtOAc & no change \\
$\mathrm{DCM}$ & no change \\
$\mathrm{PhCl}$ & no change \\
$\mathrm{CCl}_{4}$ & no change \\
$\mathrm{CHCl}_{3}$ & poor conversion \\
$t-\mathrm{BuOH}$ & poor conversion \\
$\mathrm{TFE}_{\mathrm{PhCF}}$ & poor conversion \\
$\mathrm{TMSOH}_{3}$ & poor conversion \\
$\mathrm{HFIP}$ & no reaction \\
$\mathrm{CD}_{3} \mathrm{OD}$ & decomposition \\
sulfolane & decomposition \\
\hline
\end{tabular}

5-4-3. DMDO extraction: Tetrahedron. 1997, 53 (25), 8643.

\begin{tabular}{ccc}
\hline solvent and variation & conc [M] & result $(\mathbf{e} / \mathbf{f} / \mathbf{g})[\%]$ \\
\hline $\mathrm{DCM}$ & 0.21 & $29 / 11 / 12$ \\
$\mathrm{CHCl}_{3}$ & 0.19 & $34 / 15 / 10$ \\
$\mathrm{CCl}_{4}$ & 0.13 & decomposition \\
$\mathrm{PhCF}_{3}$ & 0.15 & $11 / 0 / 20$ \\
$\mathrm{CHCl}_{3}$ (no buffer wash) & 0.10 & poor conversion \\
$\mathrm{CHCl}_{3}\left(\mathrm{Na}_{2} \mathrm{SO}_{4}\right.$ dried before use) & 0.20 & decomposition \\
$\mathrm{CHCl}_{3}(\mathrm{DMDO}): \mathrm{CHCl}_{3}=1: 1$ & 0.08 & $32 /$ trace/11 \\
$\mathrm{CHCl}_{3}$ (3 extractions), $\mathrm{h}$ & 0.30 & $45 / 9 / 11$ \\
$\mathrm{CHCl}_{3}(3$ extractions), 0 & $\circ$ \\
\hline
\end{tabular}

- DMDO was extracted 4 times then washed with buffer 4 times unless otherwise noted

- reactions were run at room temperature unless otherwise noted SI-41 


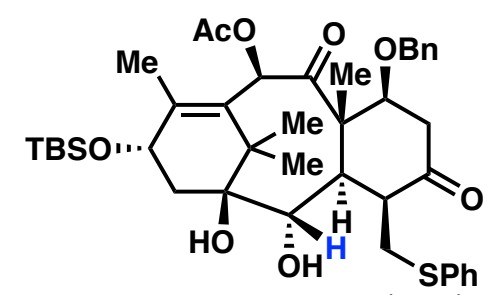

Danishefsky w/ $\mathrm{Zn}\left(\mathrm{BH}_{4}\right)_{2}$ W01993020036 A1, 1993.

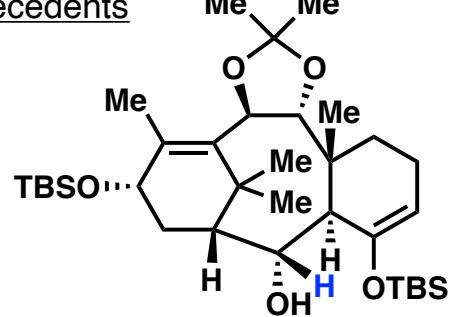

Kuwajima w/ $\mathrm{Na}$, TFE Symposium on the Chemistry of Natural Products, symposium papers, 1999, 41, 463.

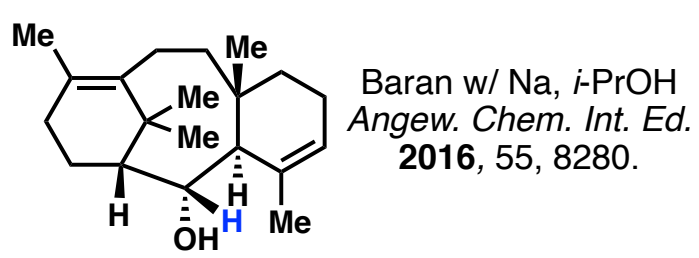

3e

Williams w/ Na, EtOH J. Org. Chem. 2000, 65, 7865.

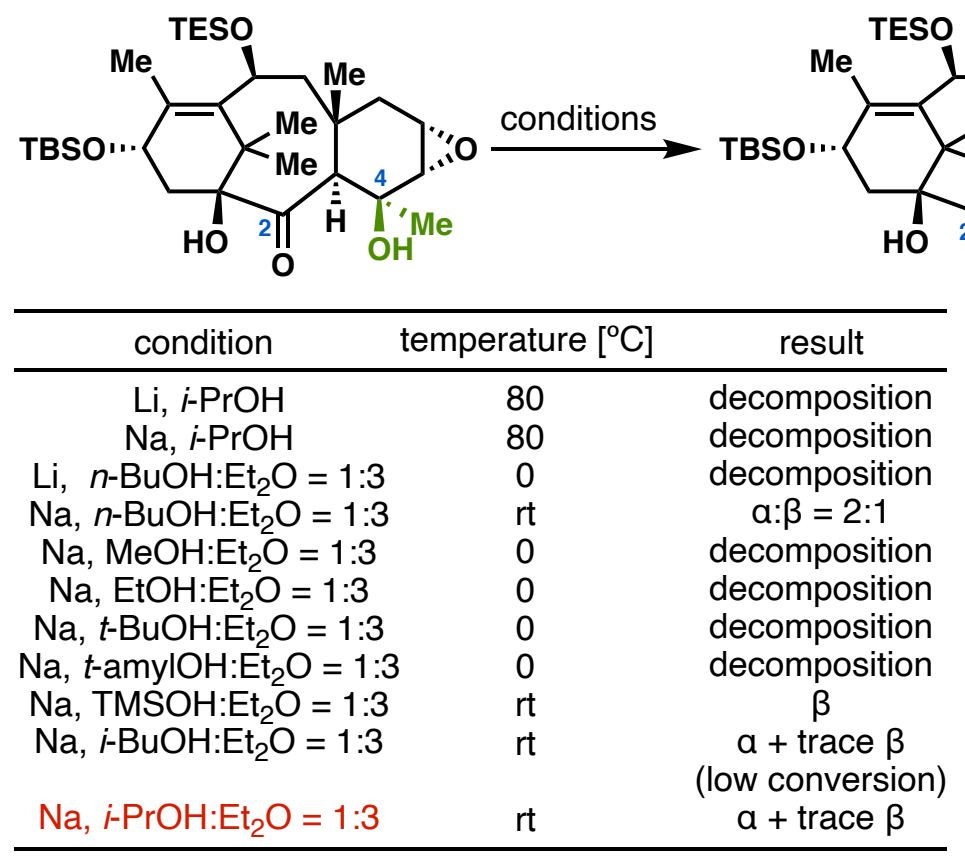

5-6. C5-6 epoxide opening attempts

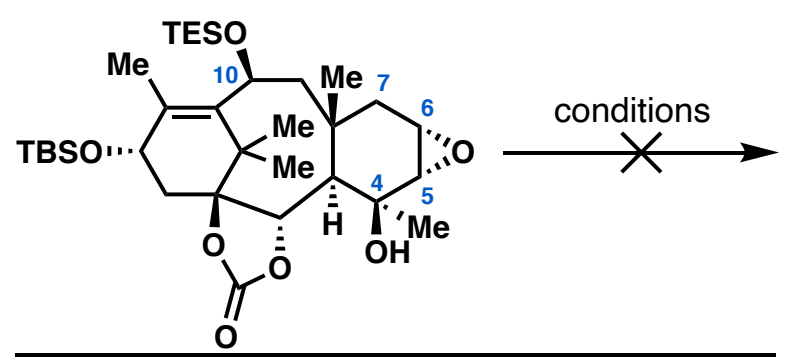

\begin{tabular}{|c|c|}
\hline condition & result \\
\hline 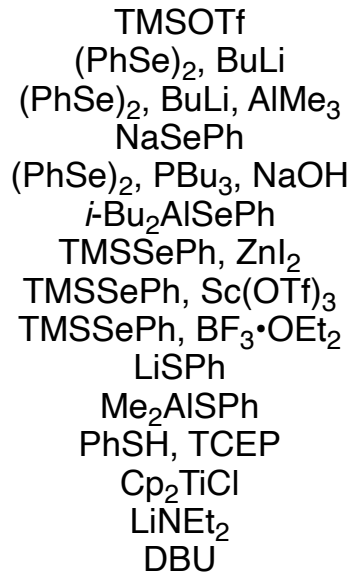 & $\begin{array}{c}\mathbf{c} \\
\text { no reaction } \\
\text { no reaction } \\
\text { carbonate deprotection } \\
\text { carbonate deprotection } \\
\mathbf{d} \\
\text { no reaction } \\
\text { no reaction } \\
\text { decomposition } \\
\text { decomposition } \\
\text { decomposition } \\
\text { no reaction } \\
\mathbf{e} \\
\text { carbonate deprotection } \\
\text { decomposition }\end{array}$ \\
\hline
\end{tabular}

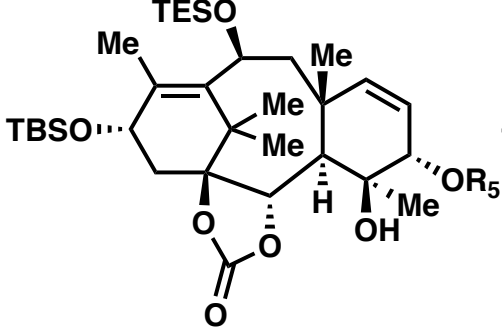

(desired)

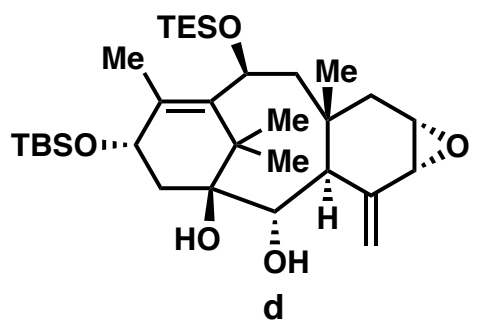

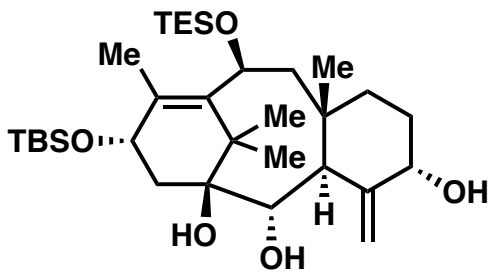

- $\mathrm{C} 7$ was too hindered to be deprotonated

- C7 $\beta$ proton probably took equatorial conformation which was almost perpendicular to $\mathrm{C} 6 \mathrm{C}-\mathrm{O}$ bond 

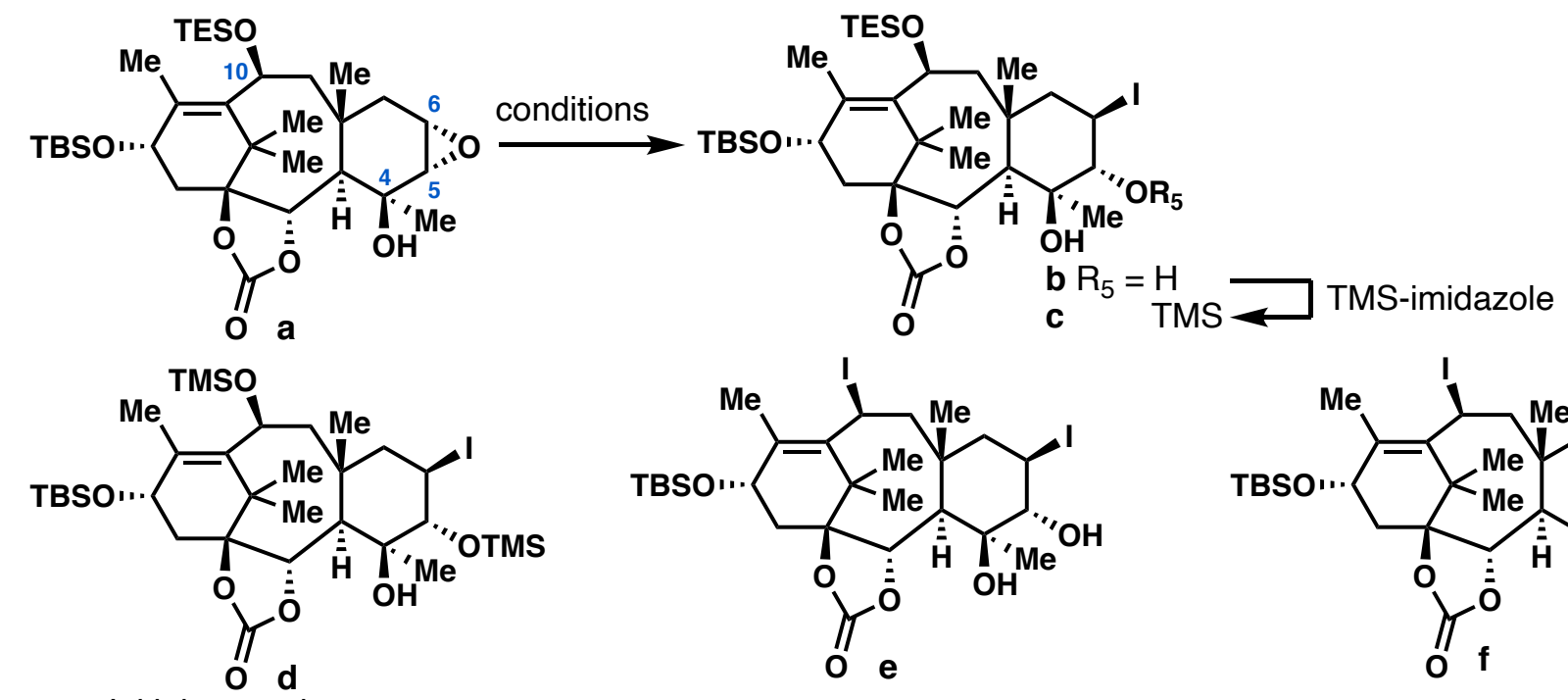

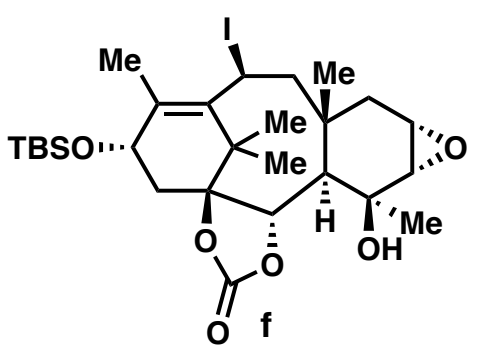

5-7-1. Initial screening

\begin{tabular}{|c|c|c|}
\hline conc & temperature $\left[{ }^{\circ} \mathrm{C}\right]$ & result \\
\hline $\begin{array}{c}\text { TMSI, DCM } \\
\text { TESI, DCM } \\
\text { TMSCI, Nal, DCM } \\
\text { TMSCI, TBAI, DCM } \\
\text { TESCI, TBAI, DCM } \\
\text { TIPSCI, TBAI, PhCI } \\
\mathrm{I}_{2}, \mathrm{PPh}_{3}, \mathrm{DCM} \\
\mathrm{I}_{2}, \mathrm{PPh}_{3}, \mathrm{DIPEA}, \mathrm{DCM} \\
\mathrm{I}_{2}, \mathrm{PPh}_{3}, \mathrm{DTBP}, \mathrm{DCM} \\
\mathrm{PPh}_{3}, 2-\mathrm{Me}_{2}-\mathrm{butene}, \mathrm{DCM} \\
\mathrm{PP}_{2}, \mathrm{PPh}_{3}, \text { thiourea, DCM } \\
\mathrm{I}_{2}, \mathrm{PBu}_{3}, \mathrm{DCM} \\
\text { thiourea, } \mathrm{I}_{2}, \mathrm{MeCN}\end{array}$ & $\begin{array}{l}-78 \\
\text { rt } \\
\text { rt } \\
-20 \\
\text { rt } \\
0 \text { to rt } \\
0 \text { to rt } \\
0 \text { to rt } \\
0 \text { to rt } \\
0 \text { to rt } \\
0 \text { to rt } \\
0 \text { to rt } \\
80\end{array}$ & $\begin{array}{c}\text { decomposition } \\
\mathbf{e} \\
\text { no reaction } \\
\mathbf{f} \\
\mathbf{b}+\mathbf{e}+\mathbf{f} \\
\mathbf{b}+\text { inseparable inpurities } \\
\mathbf{a}+\mathbf{b} \\
\text { same as above } \\
\text { same as above } \\
\text { same as above } \\
\text { same as above } \\
\mathbf{g} \\
\text { no reaction }\end{array}$ \\
\hline
\end{tabular}

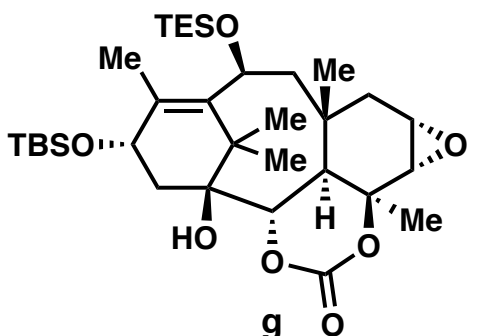

- in situ TMS protection did not work

- $\mathrm{PPh}_{3}+\mathrm{I}_{2}$ looked good but there were problems:

- inconsistent yield

- incomplete reaction

- C5-6 epoxide reclosure

- decomposition through workup

- addition of acid scavenger did not help

\section{5-7-2. Strong Lewis acid approach}

\begin{tabular}{ccc}
\hline condition & temperature $\left[{ }^{\circ} \mathrm{C}\right]$ & result \\
\hline $\mathrm{BF}_{3} \cdot \mathrm{OEt}_{2}$, TBAI, DCM & -78 & $\mathbf{b}(73 \%)$ \\
$\mathrm{Zn}(\mathrm{OTf})_{2}$, TBAI, DCM & $\mathrm{rt}$ & no reaction \\
$\mathrm{In}(\mathrm{OTf})_{3}$, TBAl, DCM & $\mathrm{rt}$ & decomposition \\
$\mathrm{Gd}(\mathrm{OTf})_{3}$, TBAI, DCM & $\mathrm{rt}$ & no reaction \\
$\mathrm{Sc}(\mathrm{OTf})_{3}$, TBAI, DCM & $\mathrm{rt}$ & decomposition \\
$\mathrm{La}(\mathrm{OTf})_{3}$, TBAI, DCM & $\mathrm{rt}$ & $\mathbf{e}$ \\
$\mathrm{Yb}(\mathrm{OTf})_{3}$, TBAI, DCM & $\mathrm{rt}$ & $\mathbf{b}(52 \%)+\mathbf{a}(10 \%)$ \\
\hline
\end{tabular}

- $\mathbf{b}$ is unstable by itself and went back to a resulting in inconsistent yield

\section{5-7-3. One pot protection attempts}

\begin{tabular}{|c|c|c|}
\hline condition & temperature $\left[{ }^{\circ} \mathrm{C}\right]$ & result \\
\hline 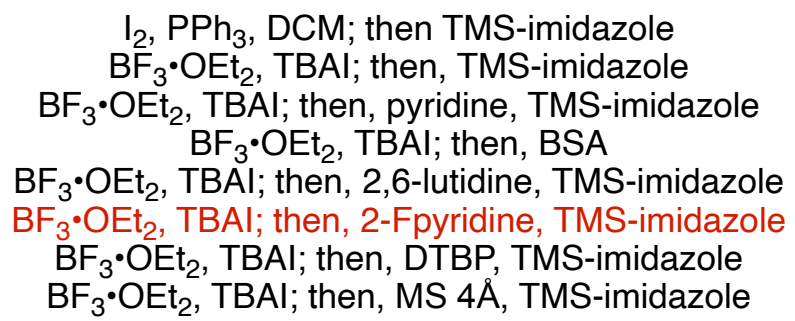 & $\begin{array}{l}0 \text { to rt } \\
-78 \text { to rt } \\
-78 \text { to rt } \\
-78 \text { to rt } \\
-78 \text { to rt } \\
-78 \text { to rt } \\
-78 \text { to rt } \\
-78 \text { to rt }\end{array}$ & $\begin{array}{c}\mathbf{c}: \mathbf{d}=6: 4 \\
\mathbf{a}(63 \%)+\mathbf{c}(24 \%) \\
\mathbf{a}+\mathbf{b}+\mathbf{c} \\
\mathbf{a}+\mathbf{b} \\
\mathbf{a} \\
\mathbf{c} \\
\mathbf{c}+\operatorname{trace} \mathbf{a} \\
\mathbf{a}+\mathbf{b}+\mathbf{c}\end{array}$ \\
\hline
\end{tabular}

- $\mathrm{BF}_{3} \cdot \mathrm{OEt}_{2}$ had to be quenched with base

- 2-Fpyridine was basic enough to quench $\mathrm{BF}_{3} \cdot \mathrm{OEt}_{2}$

-2-Fpyridine was not too basic to induce $\mathrm{C5}-6$ epoxide reclosure 


\section{5-8. Oxidation relay to $\mathrm{C} 7$}

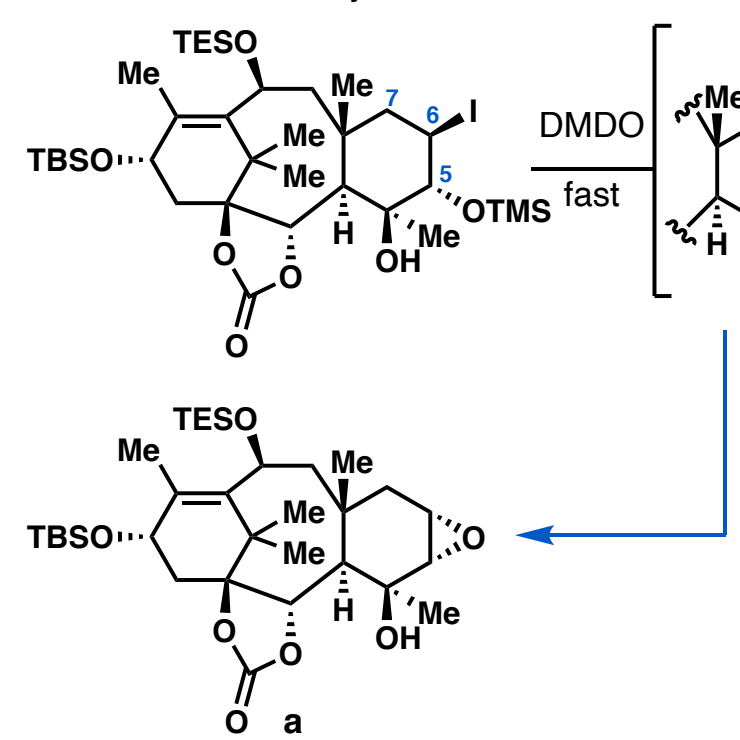

- iodoso species underwent rapid syn elimination

- this result was consistent with inability of anti elimination

- only DMDO could do both iodine oxidation and epoxidation to completion

- DMDO gave clean and high yield on small scale (1 mg)

- inconsistent yield was obtained on scale

- major product was almost always a

- it seemed the substrate had low solubility to acetone and acetone/buffer mixture and brown insolubles were observed on scale - reverse addition of substrate solution to mixture of DMDO and buffer lead to clean conversion to $\mathbf{c}$ without formation of $\mathbf{a}$

\begin{tabular}{|c|c|c|}
\hline condition & temperature $\left[{ }^{\circ} \mathrm{C}\right]$ & result \\
\hline 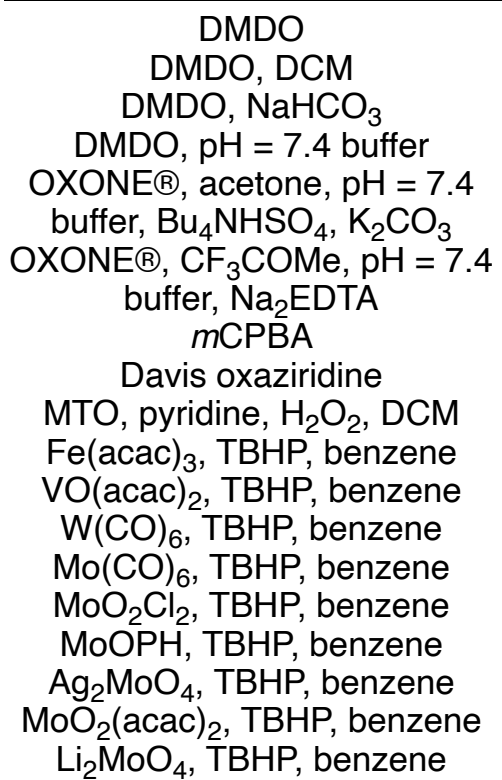 & $\begin{array}{c}-78 \text { to } 0 \\
-78 \text { to } 0 \\
0 \text { to rt } \\
0 \text { to rt } \\
0 \text { to rt }\end{array}$ & $\begin{array}{c}\mathbf{a}+\mathbf{b}+\mathbf{c} \text { (messy) } \\
\text { same as above } \\
\text { same as above } \\
\mathbf{a}+\mathbf{b}+\mathbf{c} \text { (cleaner) } \\
\text { decomposition } \\
\text { decomposition } \\
\mathbf{a} \\
\text { no reaction } \\
\text { decomposition } \\
\text { no reaction } \\
\text { no reaction } \\
\mathbf{b} \\
\mathbf{b}+\mathbf{c} \\
\text { decomposition } \\
\text { decomposition } \\
\mathbf{b} \\
\text { decomposition } \\
\mathbf{b}\end{array}$ \\
\hline
\end{tabular}

\section{5-9. C6-7 epoxide opening}

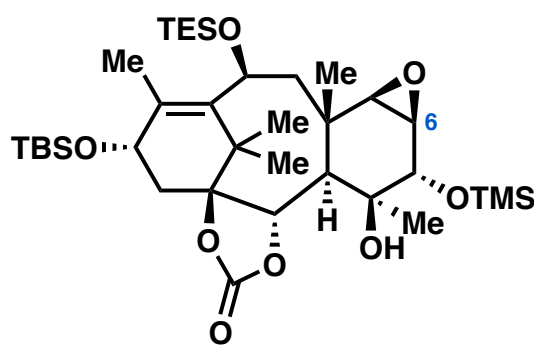

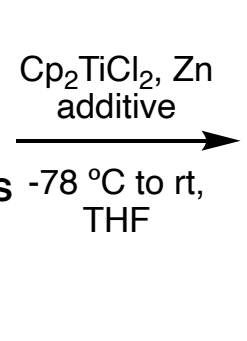

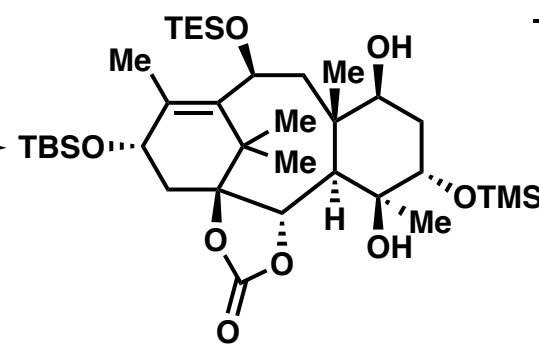

- epoxide reduction in presence of carbonate, TMS, $t-\mathrm{OH}$

- hypothesized slow $\mathrm{C} 6$ radical quenching lead to decomposition

- $\mathrm{Et}_{3} \mathrm{SiH}$ gave the cleanest TLC and was employed as the final condition
Tetrahedron. 2002, 58, (35), 7017.

Angew. Chem. Int. Ed. 2006, 45, (33), 5522.

J. Am. Chem. Soc. 2016, 138 (14), 4962. 

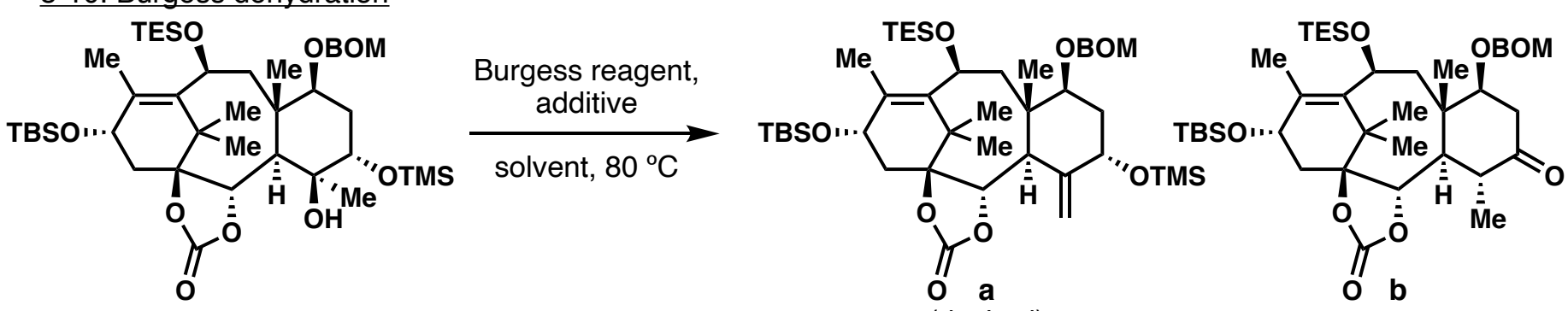

\begin{tabular}{cc}
\hline solvent & result $(\mathbf{a}: \mathbf{b})$ \\
\hline EtOAc & no reaction \\
butanone & $1.2: 1.0$ \\
DMC & $1.7: 1.0$ \\
THF & $1.3: 1.0$ \\
dioxane & $1.9: 1.0$ \\
$\mathrm{Bu}_{2} \mathrm{O}$ & $1.0: 1.0$ \\
$\mathrm{glyme}$ & no reaction \\
diethyl glycol & no reaction \\
$\mathrm{DMF}$ & decomposition \\
$\mathrm{CHCl}_{3}$ & no reaction \\
$\mathrm{MeCCl}_{3}$ & $1.3: 1.0$ \\
$\mathrm{DCE}$ & $1.3: 1.0$ \\
$\mathrm{CCl}_{4}$ & $1.0: 1.0$ \\
$\mathrm{PhCF}_{3}$ & $1.4: 1.0$ \\
$\mathrm{MeNO}_{2}$ & no reaction \\
$\mathrm{MeCN}$ & $1.0: 1.0$ \\
$i-\mathrm{PrCN}$ & no reaction \\
\hline
\end{tabular}

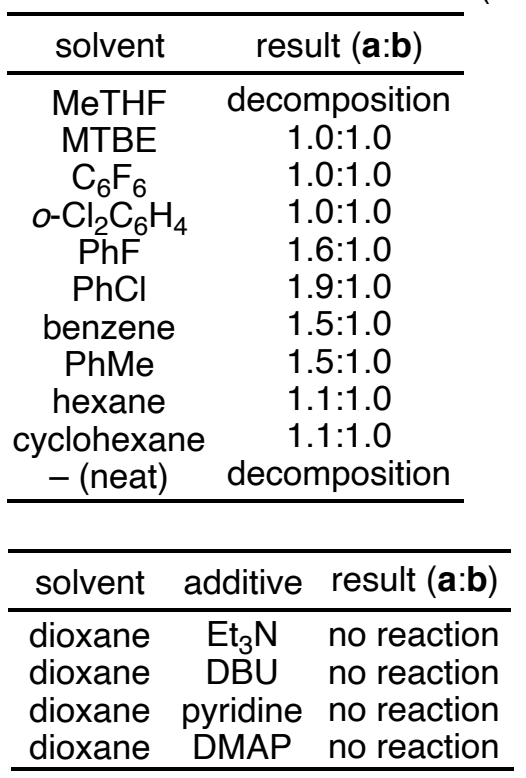

\begin{tabular}{cc}
\hline solvent $(1: 1)$ & result $(\mathbf{a}: \mathbf{b})$ \\
\hline dioxane:DCE & $2.0: 1.0$ \\
dioxane:benznene & $2.1: 1.0$ \\
dioxane:PhMe & $2.0: 1.0$ \\
dioxane:PhCl & $1.7: 1.0$ \\
dioxane:DMC & $1.7: 1.0$ \\
dioxane:CCl & $1.0: 1.0$ \\
dioxane:cyclohexane & $2.0: 1.0$ \\
PhCl:DMC & $1.5: 1.0$ \\
benzene:DCE & no reaction \\
PhMe:DCE & no reaction \\
\hline
\end{tabular}

- Martin sulfurane gave no reaction

- $\mathrm{SOCl}_{2}$, pyridine gave exclusively $\mathbf{b}$

- $\mathrm{Et}_{3} \mathrm{NSO}_{2} \mathrm{NBoc}$ gave the same ratio as Burgess but messier

- burgess adduct was observed around $40^{\circ} \mathrm{C}$

- dehydration did not happen below about $70^{\circ} \mathrm{C}$

- higher temperature gave messier NMR trace

\section{5-11. C5 TMS deprotection}

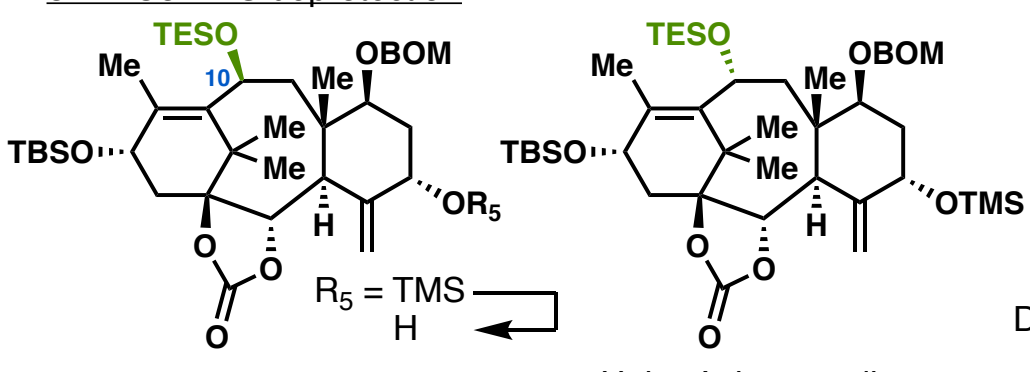

Holton's intermediate

\begin{tabular}{cc}
\hline condition & result \\
\hline CSA, MeOH & C5, 10 double deprotection \\
PPTS, MeOH & C5, 10 double deprotection \\
CSA, EtOH & C5, 10 double deprotection \\
PPTS, EtOH & C5, 10 double deprotection \\
KF, MeOH & decomposition \\
KF, HFIP & decomposition \\
CsF, DMF & decomposition \\
CsF, DME & no reaction \\
DBN, BzF, HFIP, TBME & decomposition \\
TBAF, - $78^{\circ} \mathrm{C}$ & $\mathrm{C5}, 10$ double deprotection
\end{tabular}

- despite the similarities of our substrate and Holton's intermediate, Holton's condition ( $\mathrm{HF}, \mathrm{H}_{2} \mathrm{O}, \mathrm{MeCN}$, pyridine, $0^{\circ} \mathrm{C}$ ) gave no reaction - deprotection started working at rt

\section{5-12. C5 mesylation}

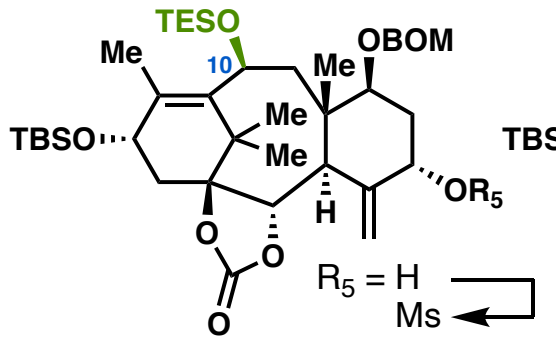

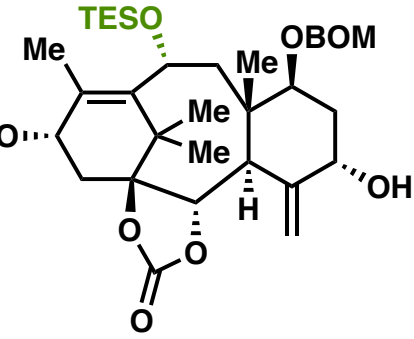

Holton's intermediate
BzF condition:

J. Am. Chem. Soc. 2010, 132, 3268.
- despite the similarities of our substrate and Holton's intermediate, Holton's condition ( $\mathrm{MsCl}$, pyridine, $0{ }^{\circ} \mathrm{C}$ ) gave no reaction

- mesylation started working at rt 


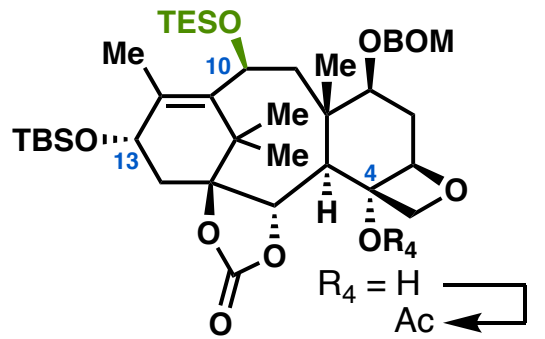

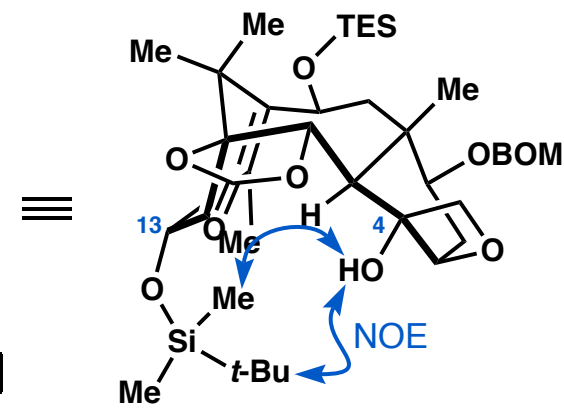

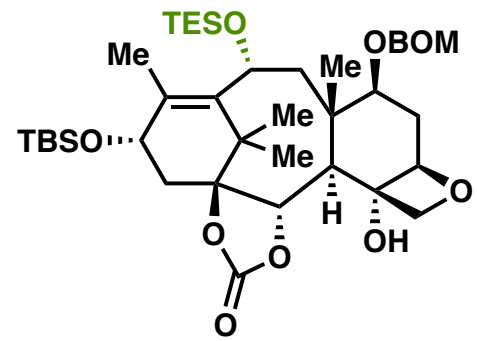

Holton's intermediate

- despite the similarities of our substrate and Holton's intermediate, Holton's condition

$\left(\mathrm{Ac}_{2} \mathrm{O}\right.$, DMAP, pyridine, rt) gave no desired product

- trace product was isolated when heated to $40^{\circ} \mathrm{C}$

- $\mathrm{C} 13$ TBS and $\mathrm{C} 4 \mathrm{OH}$ were close to each other which retarded $\mathrm{C} 4 \mathrm{OH}$ nucleophilicity

5-13-1. Solvent screening

\begin{tabular}{|c|c|c|}
\hline cono & solvent & result \\
\hline 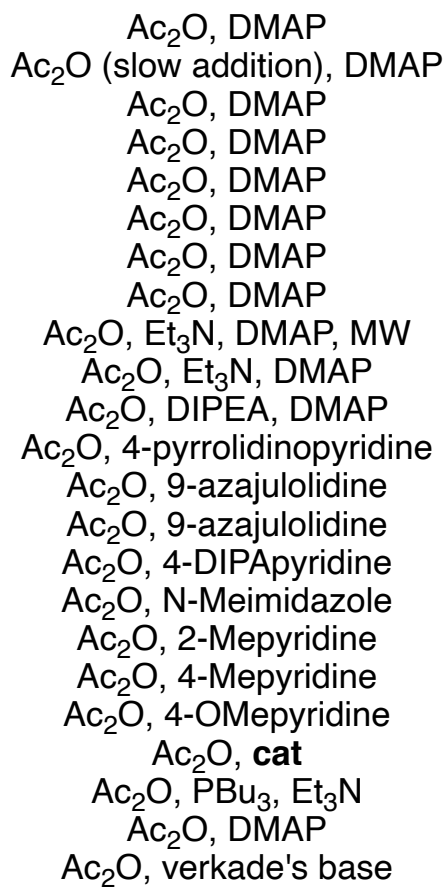 & $\begin{array}{l}\text { pyridine } \\
\text { pyridine } \\
\text { DCM } \\
\text { DCE } \\
\text { PhCF }_{3} \\
\mathrm{PhMe} \mathrm{Et}_{3} \mathrm{~N} \\
\mathrm{TMSNEt}_{2} \\
\mathrm{MeCN} \\
\mathrm{PhMe} \\
\mathrm{PhMe} \\
\text { pyridine } \\
\text { pyridine } \\
\text { DCE } \\
\text { pyridine } \\
\text { pyridine } \\
\text { pyridine } \\
\text { pyridine } \\
\text { pyridine } \\
\text { pyridine } \\
\text { MeCN } \\
\text { pyrimidine } \\
\text { MeCN }\end{array}$ & $\begin{array}{c}3 \% \\
\text { same as above } \\
\text { decomposition } \\
\text { no reaction } \\
\text { no reaction } \\
\text { decomposition } \\
\text { decomposition } \\
\text { decomposition } \\
\text { no reaction } \\
\text { no reaction } \\
\text { a } \\
\text { b } \\
\text { decomposition } \\
\text { no reaction } \\
\text { no reaction } \\
\text { no reaction } \\
\text { decomposition } \\
\text { no reaction } \\
\text { no reaction } \\
\text { no reaction } \\
\text { no reaction } \\
\text { decomposition } \\
\text { decomposition }\end{array}$ \\
\hline
\end{tabular}

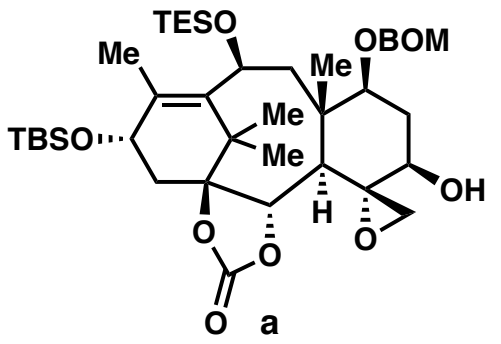

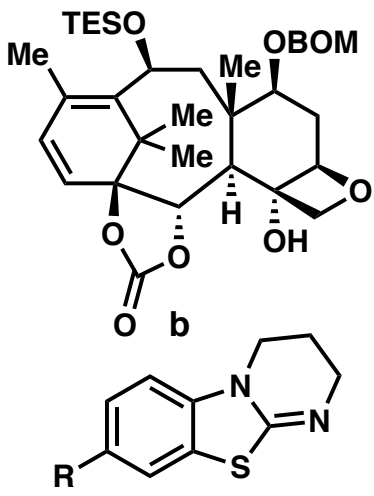

cat: $\mathrm{R}=\mathrm{H}$ or $\mathrm{OMe}$

Org. Lett. 2006, 8, 1351.

Tetrahedron Lett. 2006, 47, 4347.

Tetrahedron Lett. 2014, 55, 1909.

J. Org. Chem. 1996, 61, 2963.

\section{5-13-2. Hard deprotonation}

\begin{tabular}{ccc}
\hline condition & solvent & result \\
\hline AcCl, DIPEA, DMAP & PhMe & decomposition \\
LiHMDS, AcCl & THF & decomposition \\
NHMDS, Ac $\mathrm{C}_{2} \mathrm{O}$ & THF & decomposition \\
$\mathrm{NHMDS}, \mathrm{Ac}_{2} \mathrm{O}$ & $\mathrm{THF}$ & decomposition \\
$\mathrm{NaH}, \mathrm{AcCl}$ & $\mathrm{DMF}$ & decomposition \\
\hline
\end{tabular}

5-13-3. Acetylpyridinium pregeneration

\begin{tabular}{ccc}
\hline condition & solvent & result \\
\hline AcCl, DMAP (pre-mix) & pyridine & no reaction \\
AcCl, DMAP (pre-mix) & $\mathrm{Et}_{3} \mathrm{~N}$ & no reaction \\
AcCl, DMAP (pre-mix) & $\mathrm{CHCl}_{3}$ & no reaction \\
AcCl, DMAP (pre-mix) & $\mathrm{DCE}^{\text {no reaction }}$ & no reaction \\
\hline AcCl, DMAP (pre-mix) & $\mathrm{DMF}$ & no reaction \\
\hline
\end{tabular}

5-13-4. Stronger conjugate base: Chem. Eur. J. 2005, 11, 4751.

\begin{tabular}{|c|c|c|}
\hline condition & solvent & result \\
\hline $\begin{array}{c}\text { AcNHS, DMAP, Sc(OTf) } \\
\text { AcNHS, DMAP, Sc(OTf) } \\
\text { AcNHS, DMAP } \\
\text { AcNHS, 9-azajulolidine } \\
\text { AcCN, 9-azajulolidine } \\
\text { AcHFIP, DMAP } \\
\text { AcOCOAd, DMAP } \\
\text { AcN } \\
\text { Br DIPEA, PivOH } \\
\text { AgCN }\end{array}$ & $\begin{array}{l}\text { DCM } \\
\text { MeCN } \\
\text { pyridine } \\
\text { pyridine } \\
\text { pyridine } \\
\text { pyridine } \\
\text { pyridine } \\
\text { pyridine } \\
\mathrm{DMF}^{\mathrm{CHCl}} \\
\mathrm{CHCE}_{3} \\
\mathrm{DHCl}_{3} \\
\mathrm{PhMe}^{\mathrm{PhM}}\end{array}$ & $\begin{array}{c}\text { no reaction } \\
\text { no reaction } \\
\text { no reaction } \\
\text { decomposition } \\
\text { decomposition } \\
\text { no reaction } \\
\text { no reaction } \\
\text { no reaction } \\
\text { decomposition } \\
\text { no reaction } \\
\text { no reaction } \\
\text { no reaction } \\
\text { no reaction }\end{array}$ \\
\hline
\end{tabular}

Ac-imidolinium: Chem. Pharm. Bull. 1982, 30, 4242. SI-46 


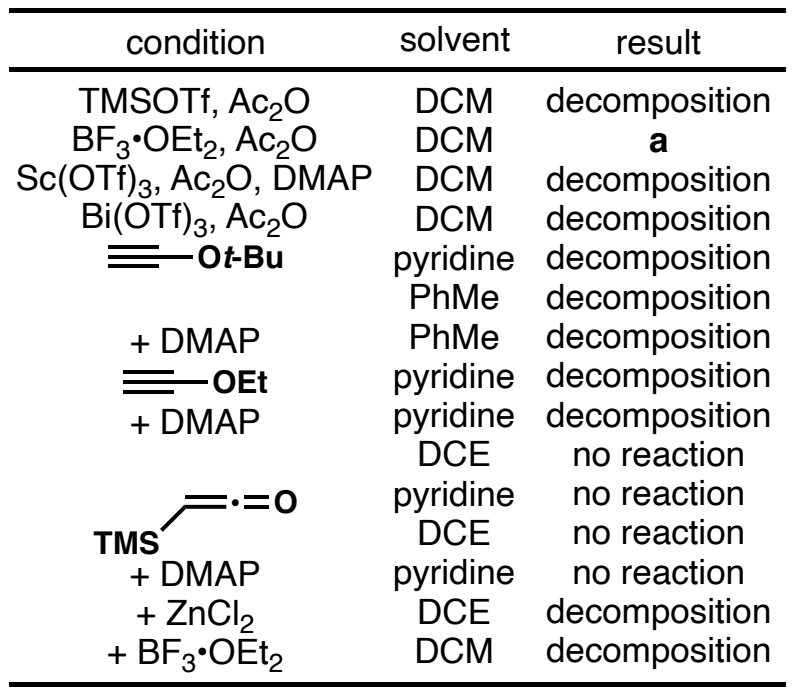

J. Org. Chem. 1998, 63, 2342.

J. Org. Chem. 1996, 61, 4560.

Angew. Chem. Int. Ed. 2000, 39, 2877.

TMS ketene:

J. Org. Chem. 1974, 39, 3607

J. Org. Chem. 1990, 55, 1108.

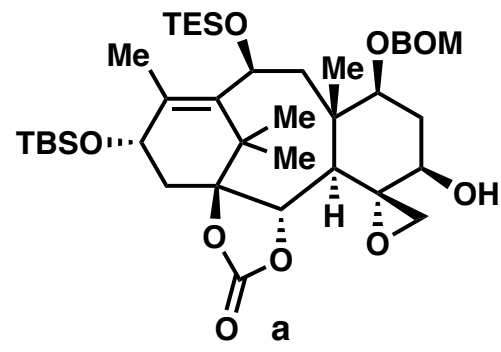

- ketene might not be the reactive species

5-13-6. Direct acetylation attempts

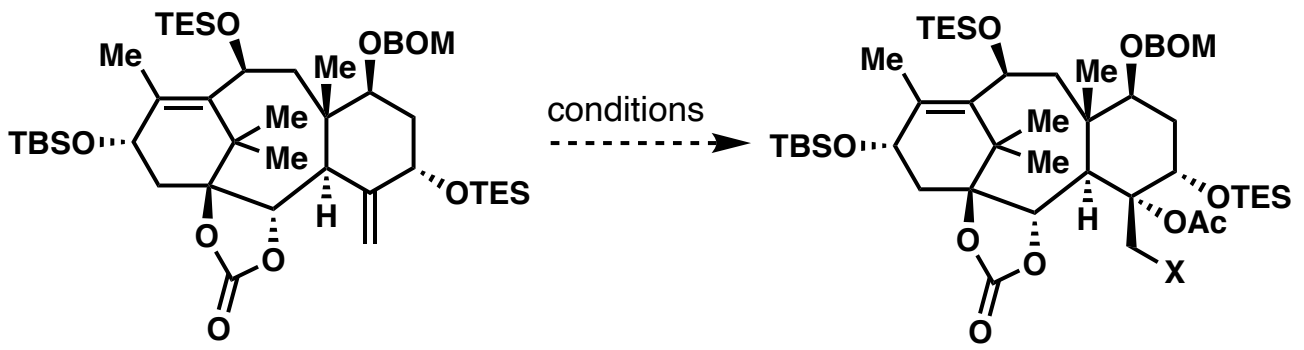

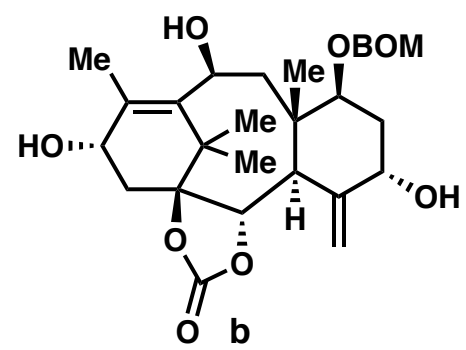

\begin{tabular}{ccc}
\hline condition & solvent & result \\
\hline $\mathrm{Phl}(\mathrm{OAc})_{2}, \mathrm{Sc}(\mathrm{OTf})_{3}$ & $\mathrm{DCM}$ & no reaction \\
$\mathrm{Phl}(\mathrm{OAc})_{2}, \mathrm{Sc}(\mathrm{OTf})_{3}, \mathrm{AcOH}$ & $\mathrm{DCM}$ & $\mathbf{b}$ \\
$\mathrm{Phl}(\mathrm{OAc})_{2}, \mathrm{Sc}(\mathrm{OTf})_{3}, \mathrm{HFIP}$ & $\mathrm{DCM}$ & decomposition \\
$\mathrm{NBS}, \mathrm{AcOH}$ & $\mathrm{DCM}$ & decomposition \\
$\mathrm{NBS}, \mathrm{AcOH}$ & $\mathrm{THF}$ & $\mathbf{b}$ \\
$\mathrm{NBS}, \mathrm{AcOH}$ & $\mathrm{MeCN}$ & $\mathbf{c}$ \\
\hline
\end{tabular}

J. Org. Chem. 2011, 76, 9997. J. Am. Chem. Soc. 2008, 130, 2962.

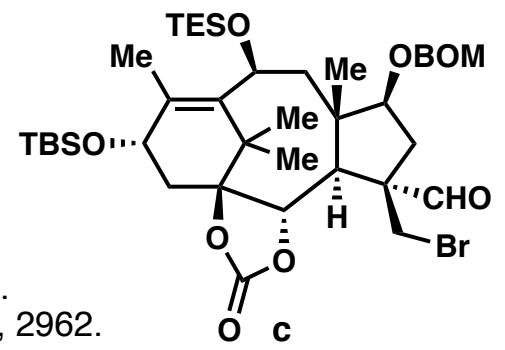

5-13-7. Biomimetic attempts

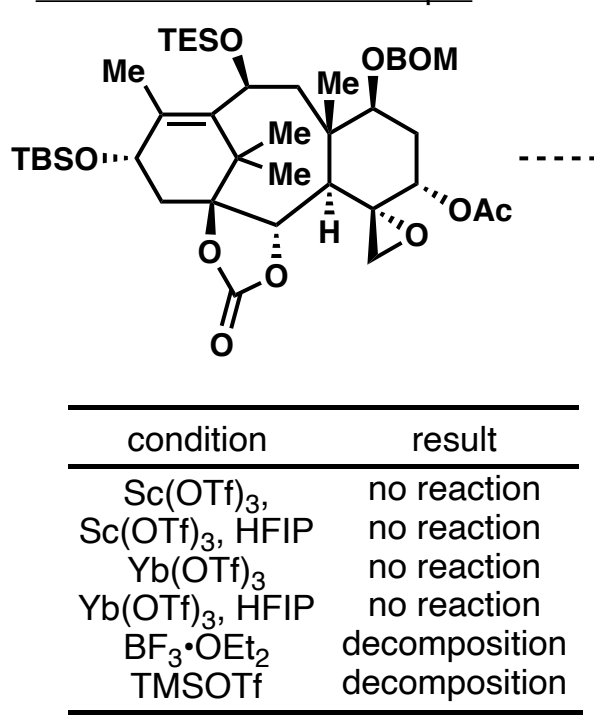<smiles>Cc1ccc(F)cc1OCCCl</smiles>

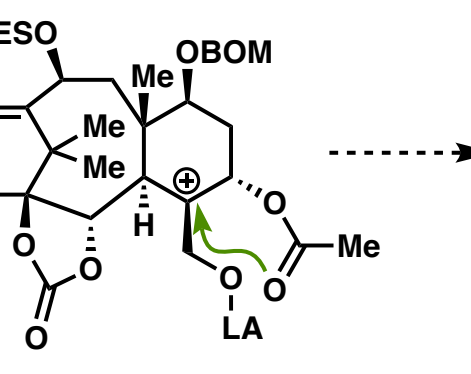

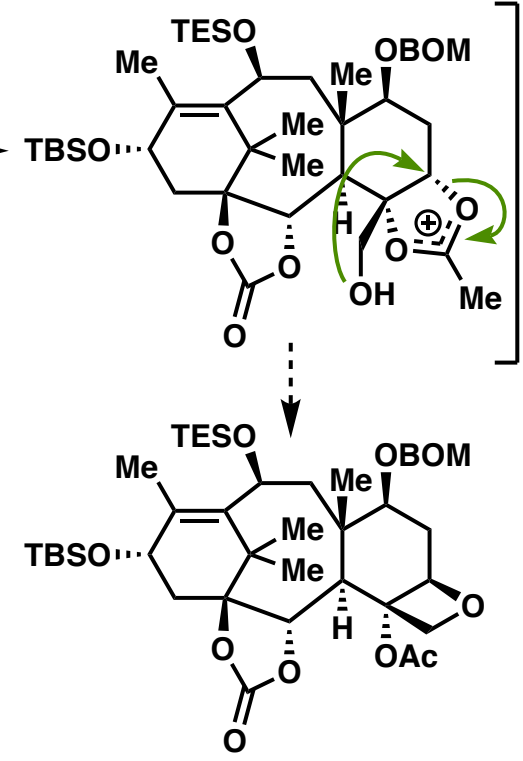




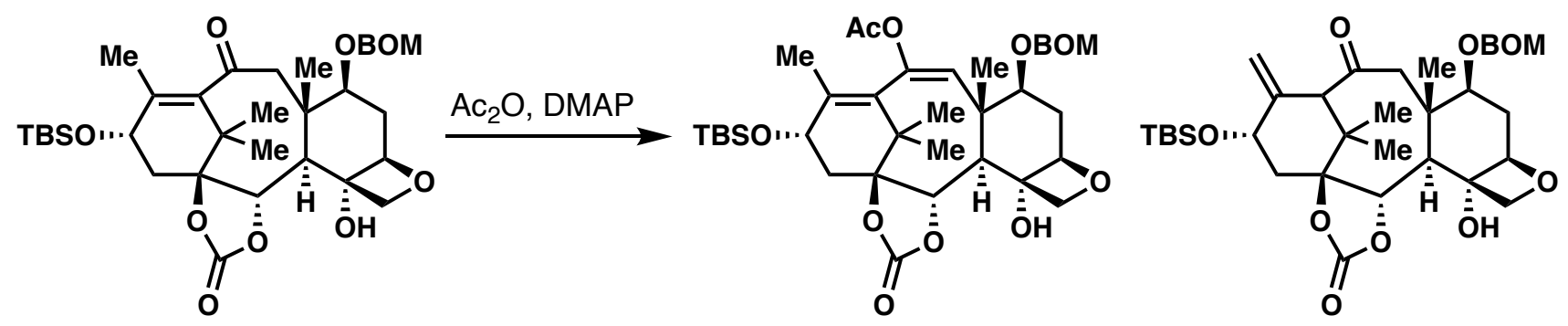

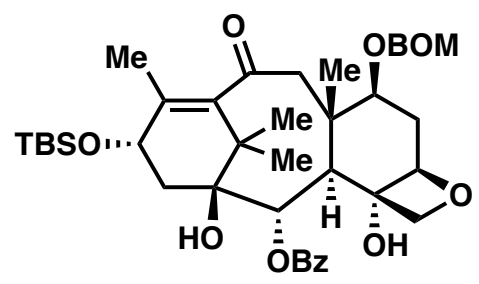

$\mathrm{Ac}_{2} \mathrm{O}$, DMAP no reaction

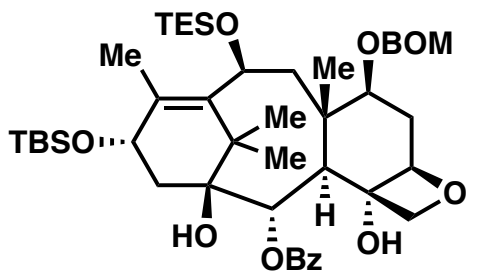

$\mathrm{Ac}_{2} \mathrm{O}$, DMAP LiHMDS, AcCl decomposition no reaction

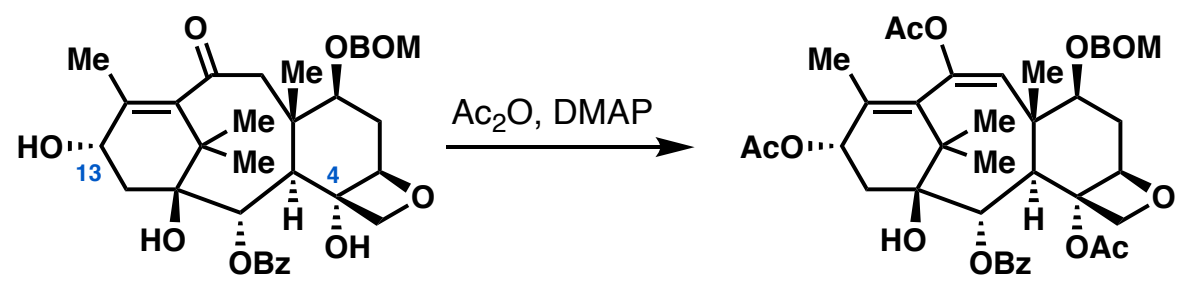

- there are $\mathrm{C} 4$ acetylation precedents under kinetic deprotonation conditions with $\mathrm{C} 2$ benzoate but not with $\mathrm{Ac}_{2} \mathrm{O}$, DMAP condition which was the only successful one in the present case

- C4 acetylation was possible without C13 TBS

- this steric issue was inherent to this intermediate and could not be overcome

- order of operations were reorganized

\section{5-13-8. Discovery of the successful substrate}

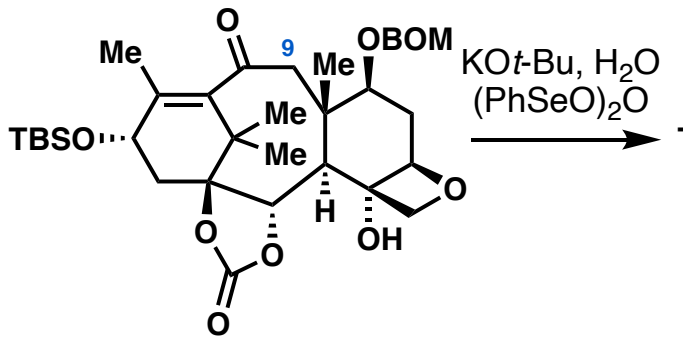

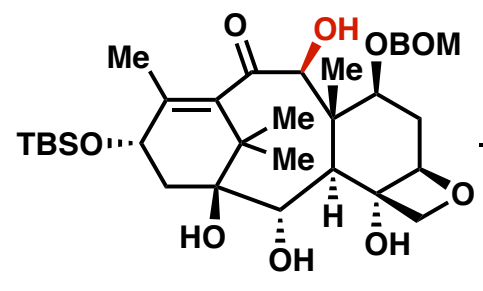
$\mathrm{KO} t-\mathrm{Bu}$; then, triphosgene; then, $\mathrm{Ac}_{2} \mathrm{O}$

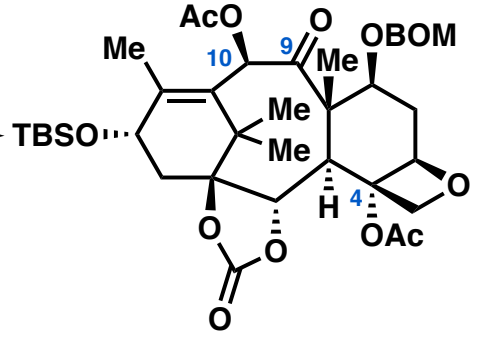



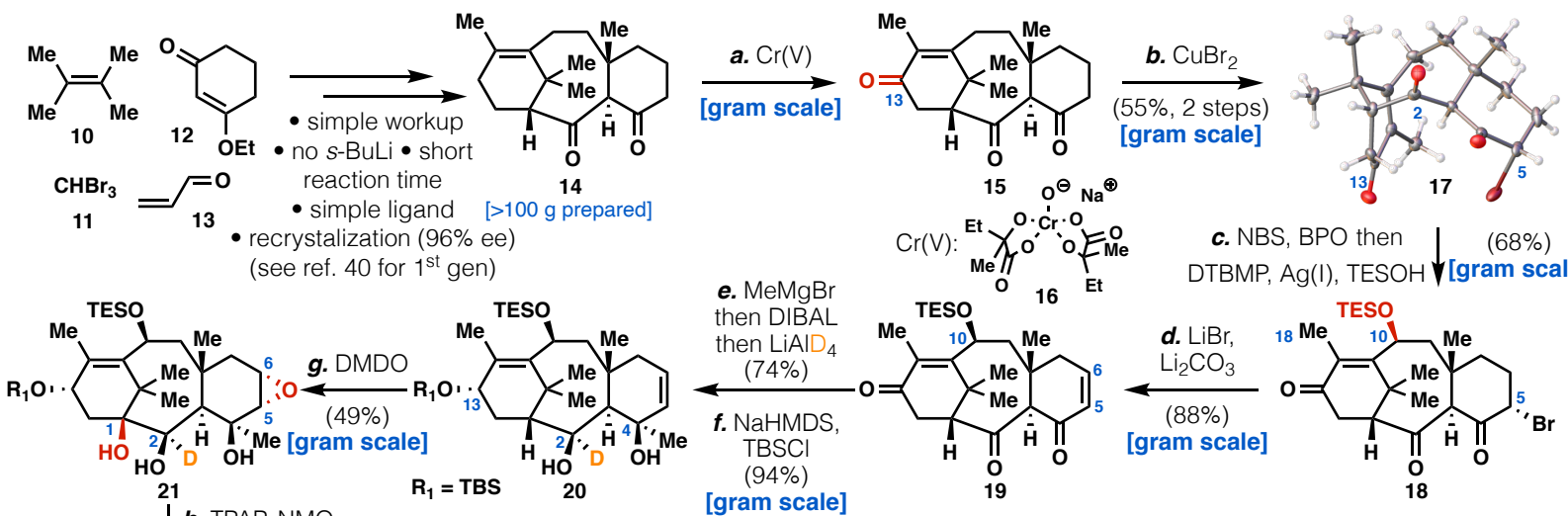
DTBMP, Ag(I), TESOH $\downarrow$ [gram scale]

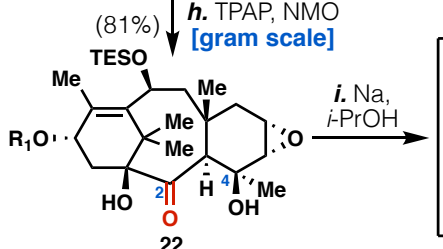
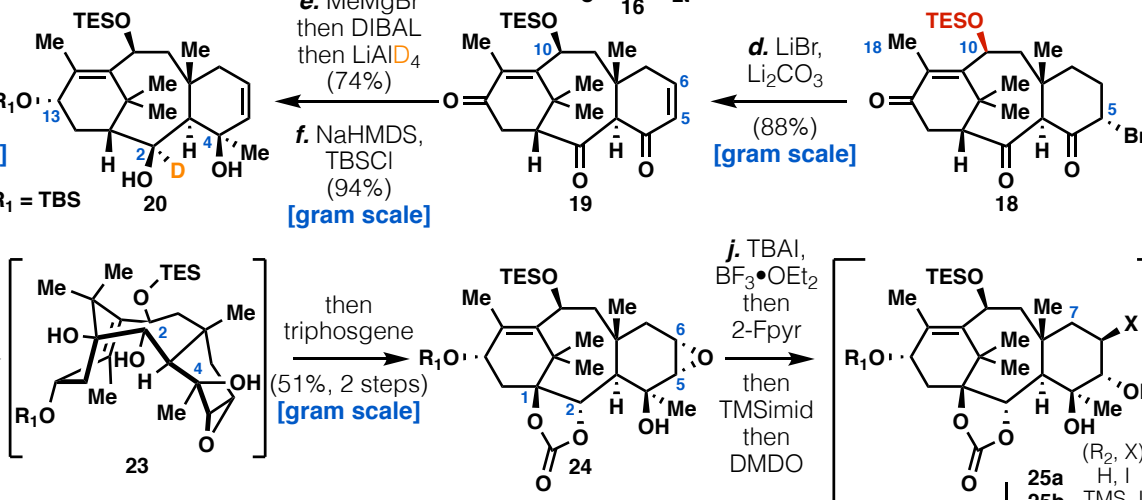

23

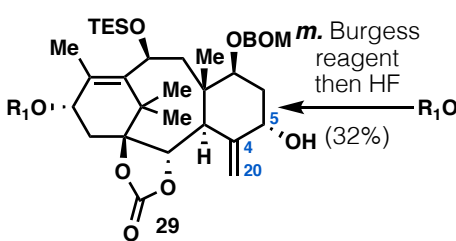
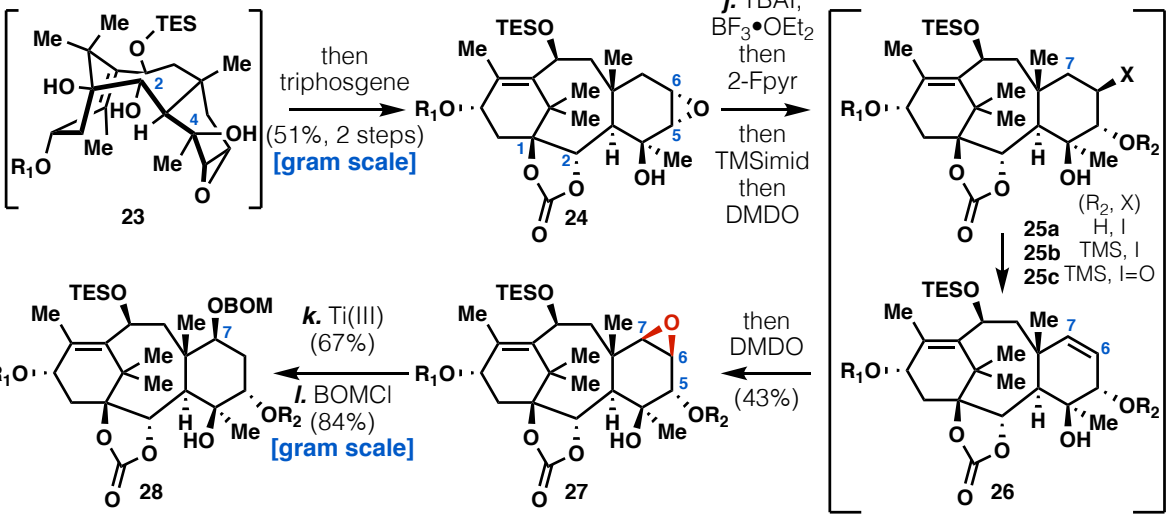

$(68 \%) \downarrow_{\text {then } \mathrm{OsO}_{4}}$

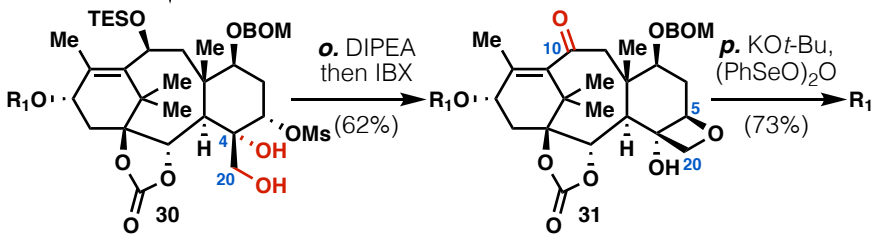
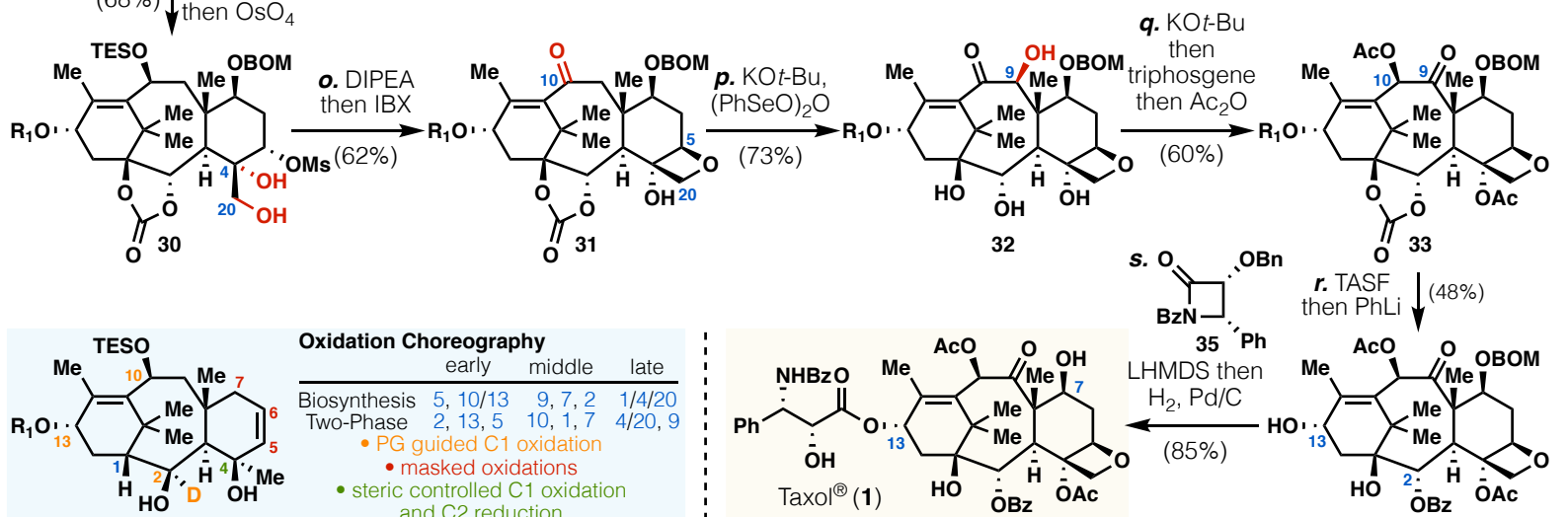

Oxidation Choreography
\begin{tabular}{llrr} 
early & middle & late \\
\hline Biosynthesis & $5,10 / 13$ & $9,7,2$ & $1 / 4 / 20$ \\
Two-Phase & $2,13,5$ & $10,1,7$ & $4 / 20,9$
\end{tabular}

$\begin{array}{lrr}\text { Two-Phase 2, 13, } 5 & 10,1,7 & 4 / 20,9\end{array}$

- PG guided C1 oxidatio

steric controlled C1 oxidation and $\mathrm{C} 2$ reduction

\begin{tabular}{cr} 
step a & \\
Solvent & Yield [\%] \\
\hline $\mathrm{PhCF}_{3}$ & trace \\
MeCN & 20 \\
HFIP & trace \\
TMSOH & trace \\
HFIP, TMSOH & 50
\end{tabular}

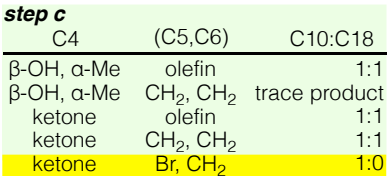

\begin{tabular}{cr} 
step $g$ Solvent [M] & Yield [\%] \\
\hline acetone (0.09) & $20-25$ \\
$\mathrm{DCM}(0.21)$ & 29 \\
$\mathrm{CHCl}_{3}(0.19)$ & 34 \\
acetone: $\mathrm{CHCl}_{3}=1: 1(0.05)$ & 22 \\
$\mathrm{CHCl}_{3}(0.30)$ & 49
\end{tabular}

$\mathrm{BF}_{3}$ quencher Result

$\begin{array}{cc}\text { BSA } & \mathbf{2 5 a}+\mathbf{2 4} \\ \text { pyridine } & 25 b+24\end{array}$

$\begin{array}{cr}\text { pyridine } & \mathbf{2 5 b}+\mathbf{2 4} \\ 2 \text { 6-lutidine } & \mathbf{2 5 a}\end{array}$

$\begin{array}{ll}\text { 2,6-lutidine } & \mathbf{2 5 a} \\ \text { DTBP } & \mathbf{2 5 a}\end{array}$

2-Fpyridine 25b

$\boldsymbol{r}$ TASF
then PhLi
$(48 \%)$

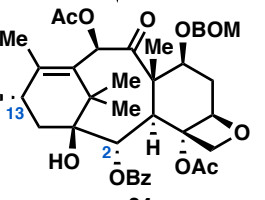

\begin{tabular}{cr} 
step $\boldsymbol{k}$ & \\
Additive & Yield [\%] \\
\hline none & 42 \\
collidine, TMSCl & low conv. \\
$\mathrm{H}_{2} \mathrm{O}$ & low conv. \\
$\mathrm{i}-\mathrm{PrOH}$ & ca. 35 \\
$\mathrm{Et}_{3} \mathrm{SiH}$ & 67
\end{tabular} 


\section{Synthetic procedures}

\section{Compound SI3}

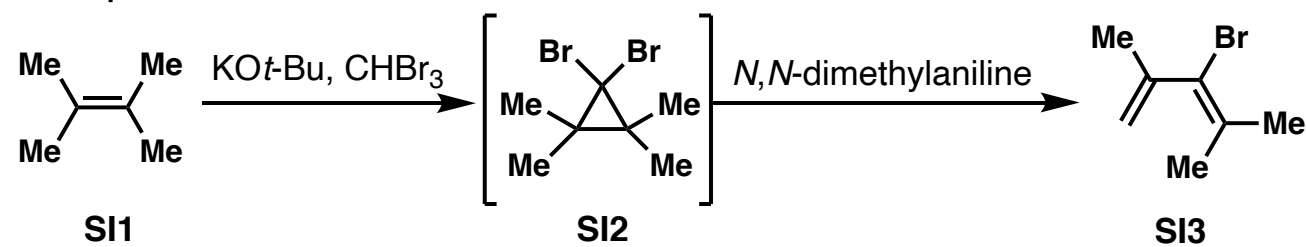

SI1

$\mathrm{SI} 2$

SI3

\section{One-pot procedure}

An oven dried round bottom flask was charged with $\mathrm{KO} t \mathrm{Bu}(170 \mathrm{~g}, 1.52 \mathrm{~mol}, 1.1 \mathrm{eq})$, hexane (1.5 L) and 2,3-dimethyl-2-butene SI1 (300 mL, $3.56 \mathrm{~mol}, 1.98 \mathrm{eq})$ under nitrogen atmosphere. The reaction flask was cooled to $0{ }^{\circ} \mathrm{C}$ and wrapped in aluminum foil, then bromoform (347 g, $1.37 \mathrm{~mol}, 1.0 \mathrm{eq}$ ) was added over a period of $1 \mathrm{~h}$ through a dropping funnel. After the addition was complete, the reaction flask was removed from the icewater bath and was warmed to room temperature over $2 \mathrm{~h}$. The volatile components were removed removed under reduced pressure, upon which $N, N$-dimethylaniline $(650 \mathrm{~mL}$, $5.13 \mathrm{~mol}, 3.74 \mathrm{eq}$ ) was added. A reflux condenser was placed atop the reaction flask, and this reaction was heated to $150^{\circ} \mathrm{C}$. A small aliquot was taken, and the reaction progress was monitored by ${ }^{1} \mathrm{H}$ NMR. On complete consumption of $S \mid 2$ in $1 \mathrm{~h}$, it was cooled to room temperature. $\mathrm{Et}_{2} \mathrm{O}(2 \mathrm{~L})$ and aqueous hydrochloric acid $(3 \mathrm{M}, 1 \mathrm{~L})$ were then added. The phases were separated, and the organic phase was washed with additional hydrochloric acid $(3 \mathrm{M}, 2 \times 1 \mathrm{~L})$, followed by water $(1 \mathrm{~L})$ and brine $(1 \mathrm{~L})$. The organic phase was dried over $\mathrm{MgSO}_{4}$ and filtered. The volatile components were removed under reduced pressure to yield a brown oil. The spectroscopic data for this compound were identical to those reported previously. ${ }^{1}$

\section{Two-step procedure}

To a flame-dried 4-neck $20 \mathrm{~L}$ round-bottom flask equipped with a mechanical stirrer was added with $\mathrm{KOt}$-Bu (1.33 kg, $1.19 \mathrm{~mol}, 1.0 \mathrm{eq})$, $n$-heptane $(8 \mathrm{~L})$, and 2,3-dimethyl-2butene SI1 $(1.00 \mathrm{~kg}, 119 \mathrm{mmol}, 1.0 \mathrm{eq})$ under nitrogen atmosphere. The reaction was cooled to $-10^{\circ} \mathrm{C}$ and then treated with bromoform $(2.70 \mathrm{~kg}, 1.07 \mathrm{~mol}, 0.9 \mathrm{eq})$ over a period of $2 \mathrm{~h}$ through a dropping funnel (Caution: vigorous exotherm occurred during addition). The mixture was allowed to warm to $\mathrm{rt}$, and stirred for $2 \mathrm{~h}$, with the progress of the reaction was monitored by TLC. The following step could be performed in the same pot but was performed in a different reaction vessel for convenience. ${ }^{1}$ After completion of the reaction, $\mathrm{NaHCO}_{3}$ (saturadted aq., $6 \mathrm{~L}$ ) was slowly added into the reaction while temperature was maintained below $35^{\circ} \mathrm{C}$. The aqueous layer was separated and extracted with heptanes $(2 \mathrm{~L})$. The combined organics were dried $\left(\mathrm{Na}_{2} \mathrm{SO}_{4}\right)$ and evaporated under reduced pressure to yield SI2 $(2.31 \mathrm{~kg}, 76 \%)$ as an off-white solid.

A flame-dried 4-neck $10 \mathrm{~L}$ round bottom flask equipped with a mechanical stirrer under nitrogen atmosphere was charged with $\mathrm{S} 12(800 \mathrm{~g}, 312 \mathrm{mmol}), \mathrm{N}, \mathrm{N}$-dimethyl aniline (1.55 $\mathrm{kg}, 1.28 \mathrm{~mol}, 4.1 \mathrm{eq}$ ) and stirred for $15 \mathrm{~min}$ at rt. The reaction mixture was then heated to $160^{\circ} \mathrm{C}$ (where frothing was observed at outer temp $140^{\circ} \mathrm{C}$ while inner temperature was $155^{\circ} \mathrm{C}$ ) and maintained for $1 \mathrm{~h}$. The reaction was completed after frothing disappeared (as a small aliquot was taken and monitored by ${ }^{1} \mathrm{H}$ NMR). It was cooled down to rt slowly and MTBE $(4 \mathrm{~L})$ was added followed by $\mathrm{HCl}(4 \mathrm{M}, 4 \mathrm{~L})$. The layers were separated and the organic layer was washed with additional $\mathrm{HCl}(4 \mathrm{M}, 4 \mathrm{~L})$. The organic layer was dried $\left(\mathrm{Na}_{2} \mathrm{SO}_{4}\right)$, and solvent was evaporated completely under reduced pressure $\left(40{ }^{\circ} \mathrm{C}\right)$ to 
produce SI3 (480 g, 88\%) as a thick residue. The spectroscopic data for this compound were identical to those reported previously. ${ }^{1}$

\section{Compound SI}

${ }^{1} \mathrm{H}$ NMR (400 MHz, $\left.\mathrm{CDCl}_{3}\right) \delta 1.25(\mathrm{~s}, 12 \mathrm{H})$.

Compound SI3

${ }^{1} \mathrm{H}$ NMR $\left(400 \mathrm{MHz}, \mathrm{CDCl}_{3}\right) \delta 5.05-5.03(\mathrm{~m}, 1 \mathrm{H}), 4.92-4.91(\mathrm{~m}, 1 \mathrm{H}), 1.89-1.88(\mathrm{~m}$, $6 \mathrm{H}), 1.81(\mathrm{~s}, 3 \mathrm{H})$.

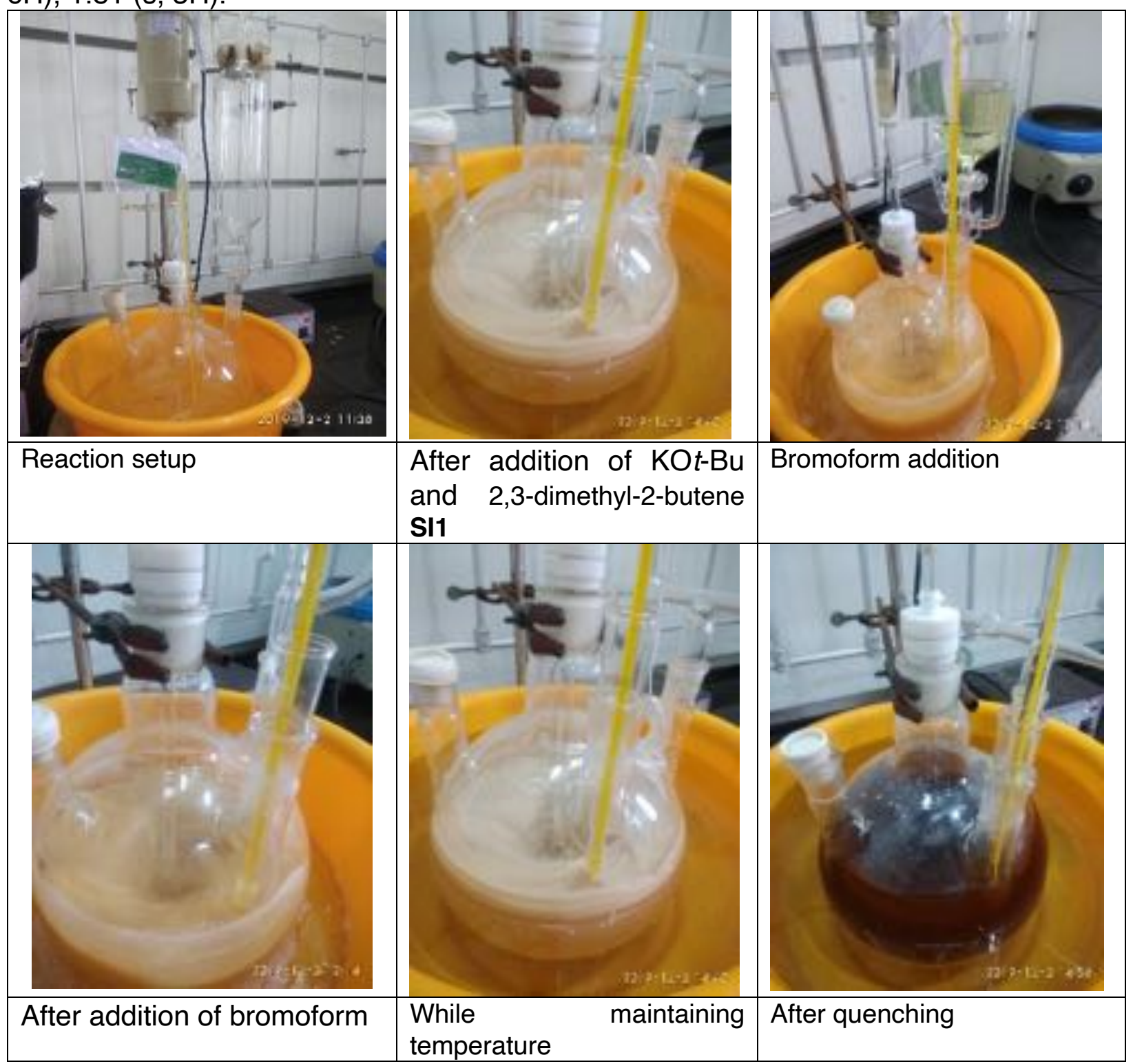



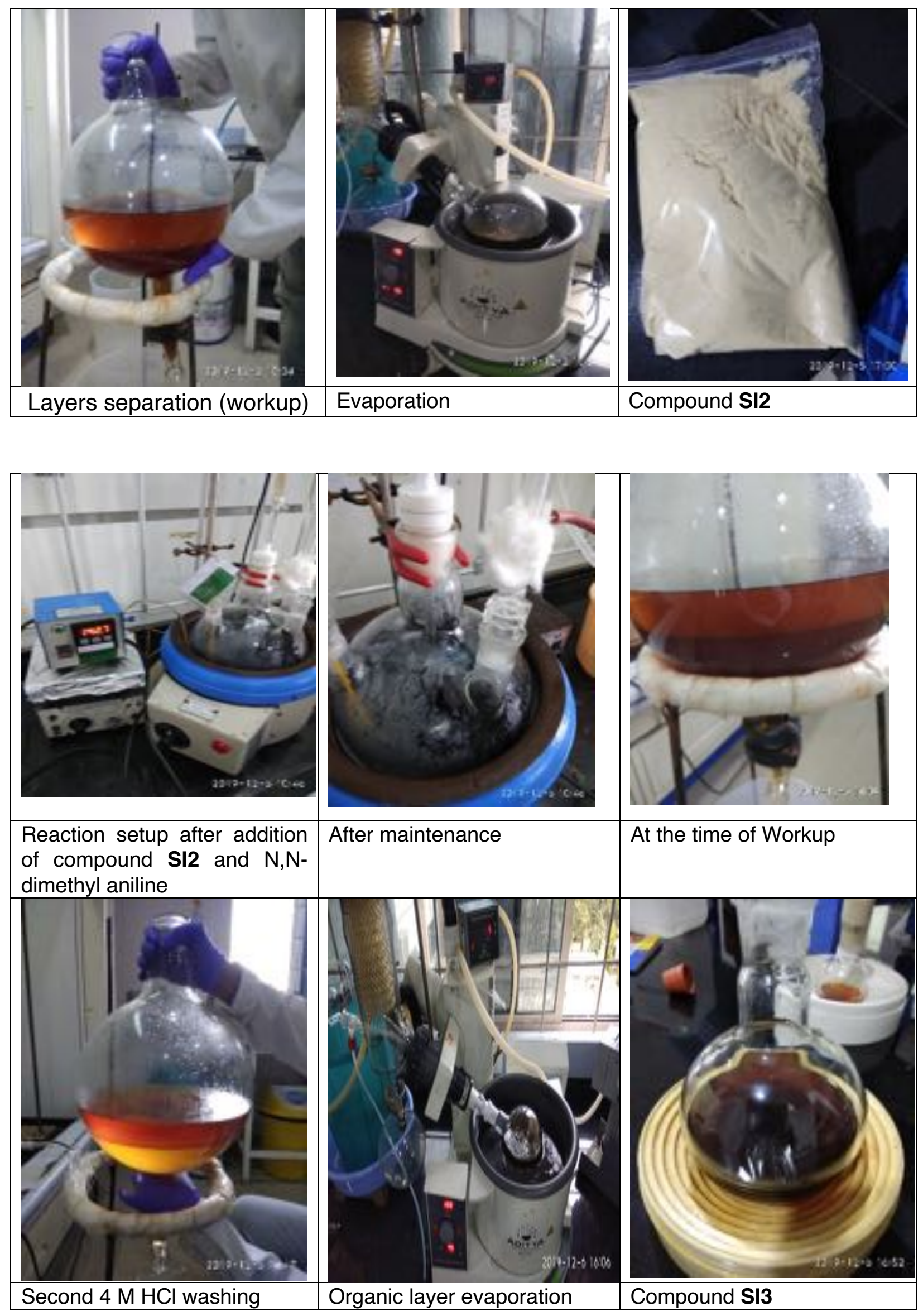
Compound SI5

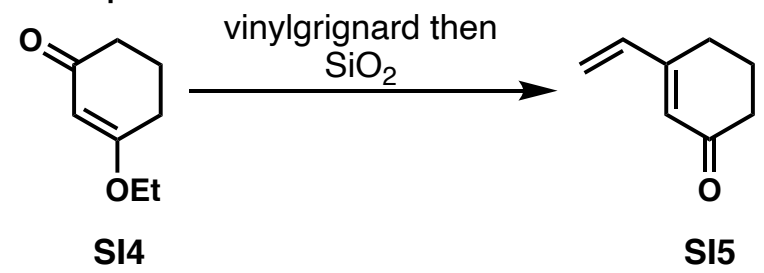

To a flame-dried 4-neck $5 \mathrm{~L}$ round-bottom flask equipped with a mechanical stirrer and an addition funnel was added a mixture of S14 (100 g, $71 \mathrm{mmol}, 1.00 \mathrm{eq})$ and MTBE (800 $\mathrm{mL})$ at $0{ }^{\circ} \mathrm{C}$ under continuous stream of nitrogen. Vinyl magnesium bromide $(1070 \mathrm{~mL}$, $106.9 \mathrm{mmol}, 1.0 \mathrm{M}$ in THF, $1.5 \mathrm{eq}$ ) was transferred via cannula into the addition funnel and added over $1 \mathrm{~h}$ at $0{ }^{\circ} \mathrm{C}$. The reaction mixture was warmed to rt and stirred for $1 \mathrm{~h}$. After completion of the reaction, the mixture was cooled to $15^{\circ} \mathrm{C}$, and $\mathrm{NH}_{4} \mathrm{Cl}$ (saturated aq., $1000 \mathrm{~mL}$ ) was added. The aqueous layer was separated and extracted with MTBE $(300 \mathrm{~mL})$. The combined organics were concentrated under reduced pressure, and passed through a silica plug (100-200 mesh, 2\% EtOAc in hexane). The fractions containing product were concentrated under reduced pressure to obtain SI5 (70 g, 80\%) as a pale yellow liquid. The spectroscopic data for this compound were identical to those previously reported. ${ }^{1}$

${ }^{1} \mathrm{H}$ NMR (400 MHz, $\left.\mathrm{CDCl}_{3}\right) \delta 6.46(\mathrm{dd}, \mathrm{J}=17.6,6.8 \mathrm{~Hz}, 1 \mathrm{H}), 5.88(\mathrm{~s}, 1 \mathrm{H}), 5.64(\mathrm{~d}, \mathrm{~J}=$ $17.6 \mathrm{~Hz}), 5.41(\mathrm{~d}, \mathrm{~J}=10.4 \mathrm{~Hz}), 2.42-2.34(\mathrm{~m}, 3 \mathrm{H}), 2.06-2.01(\mathrm{~m}, 2 \mathrm{H})$. 


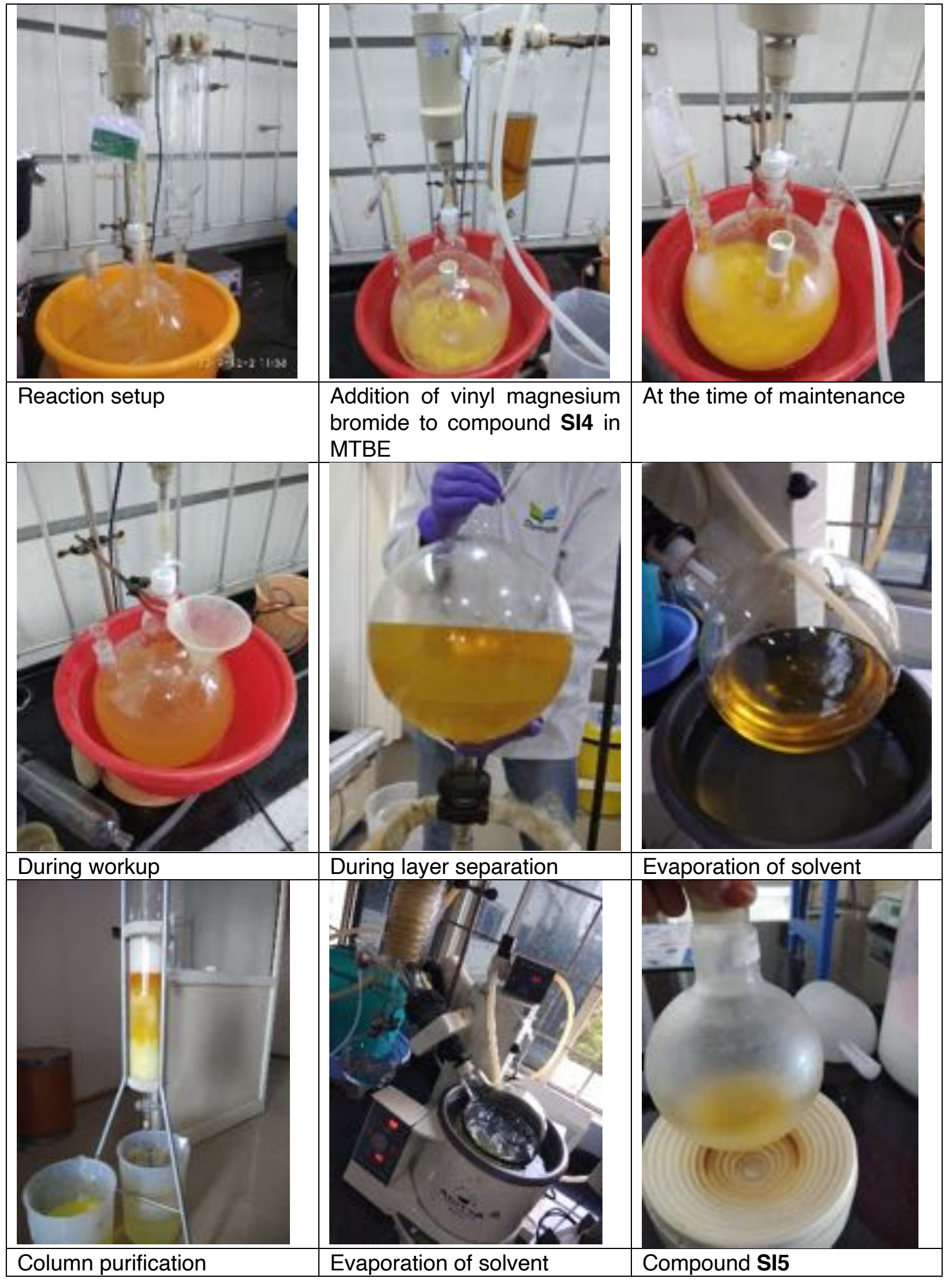


Compound SI6

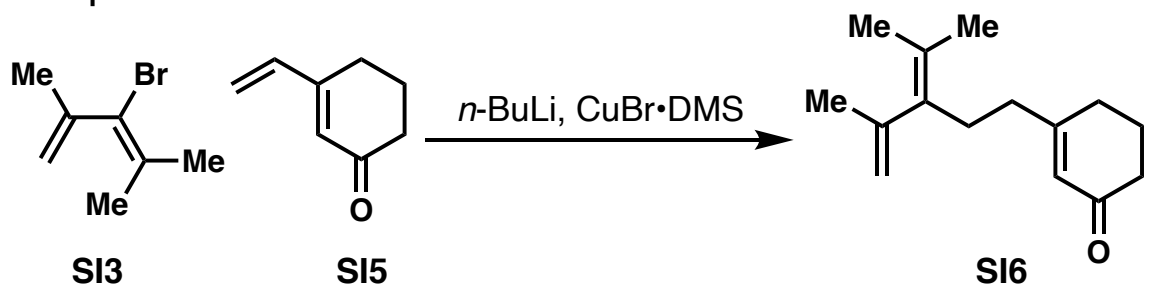

To a solution of SI3 $(110 \mathrm{~g}, 90 \mathrm{mmol}, 1.0 \mathrm{eq})$ in THF $(3.300 \mathrm{~L})$ at $-78^{\circ} \mathrm{C}$ was added $n$ BuLi ( $844 \mathrm{~mL}, 135 \mathrm{mmol}, 1.6 \mathrm{M}$ in hexane, $1.5 \mathrm{eq}$ ) over $40 \mathrm{~min}$ under nitrogen atmosphere. The reaction was stirred for $45 \mathrm{~min}$, then CuBr-DMS (204 g, $99 \mathrm{mmol}, 1.8 \mathrm{eq})$ was added portionwise over $30 \mathrm{~min}$ at the same temperature. The reaction was stirred for $0.5 \mathrm{~h}$ and treated with TMSCl $(229 \mathrm{~mL}, 196 \mathrm{~g}, 180 \mathrm{mmol}, 2.0 \mathrm{eq})$ over $40 \mathrm{~min}$, maintaining the temperature within -70 to $-78{ }^{\circ} \mathrm{C}$. The reaction was stirred for $0.5 \mathrm{~h}$ and was then treated with a solution of SI5 (282 g, $162 \mathrm{mmol}, 1.8 \mathrm{eq})$ in THF $(320 \mathrm{~mL})$ over a $1 \mathrm{~h}$ period at the same temperature range. The reaction was stirred for $2 \mathrm{~h}$ and completion of the reaction was monitored by TLC (EtOAc/hexane 3:7). The reaction was quenched by slow addition of acetic acid/water $(2: 1,188 \mathrm{~mL})$ and stirred for an additional $20 \mathrm{~min}$. The contents of the flask were transferred to a $5 \mathrm{~L}$ beaker, containing $\mathrm{NaHCO}_{3}$ (saturated aq., $4.4 \mathrm{~L}$ ), and stirred for $0.5 \mathrm{~h}$. After this time, the mixture was filtered, and the layers allowed to separate. The aqueous layer was extracted with MTBE $(2 \times 100 \mathrm{~mL})$. The organics were combined, concentrated under reduced pressure, and passed through a silica plug (100-200 mesh, $0-2 \%$ EtOAc in hexane). The fractions containing product were concentrated under reduced pressure to afford SI6 (150 g, 76\% yield) as a yellow oil. The spectroscopic data for this compound were identical to those previously reported. ${ }^{1}$

${ }^{1} \mathrm{H}$ NMR $\left(400 \mathrm{MHz}, \mathrm{CDCl}_{3}\right) \delta 5.88(\mathrm{br}, 1 \mathrm{H}), 4.97-4.95(\mathrm{~m}, 1 \mathrm{H}), 4.57-4.56(\mathrm{~m}, 1 \mathrm{H}), 2.31$ $-2.25(\mathrm{~m}, 2 \mathrm{H}), 2.23-2.19(\mathrm{~m}, 6 \mathrm{H}), 2.04-1.95(\mathrm{~m}, 2 \mathrm{H}), 1.76(\mathrm{~s}, 3 \mathrm{H}), 1.69(\mathrm{~s}, 6 \mathrm{H})$.

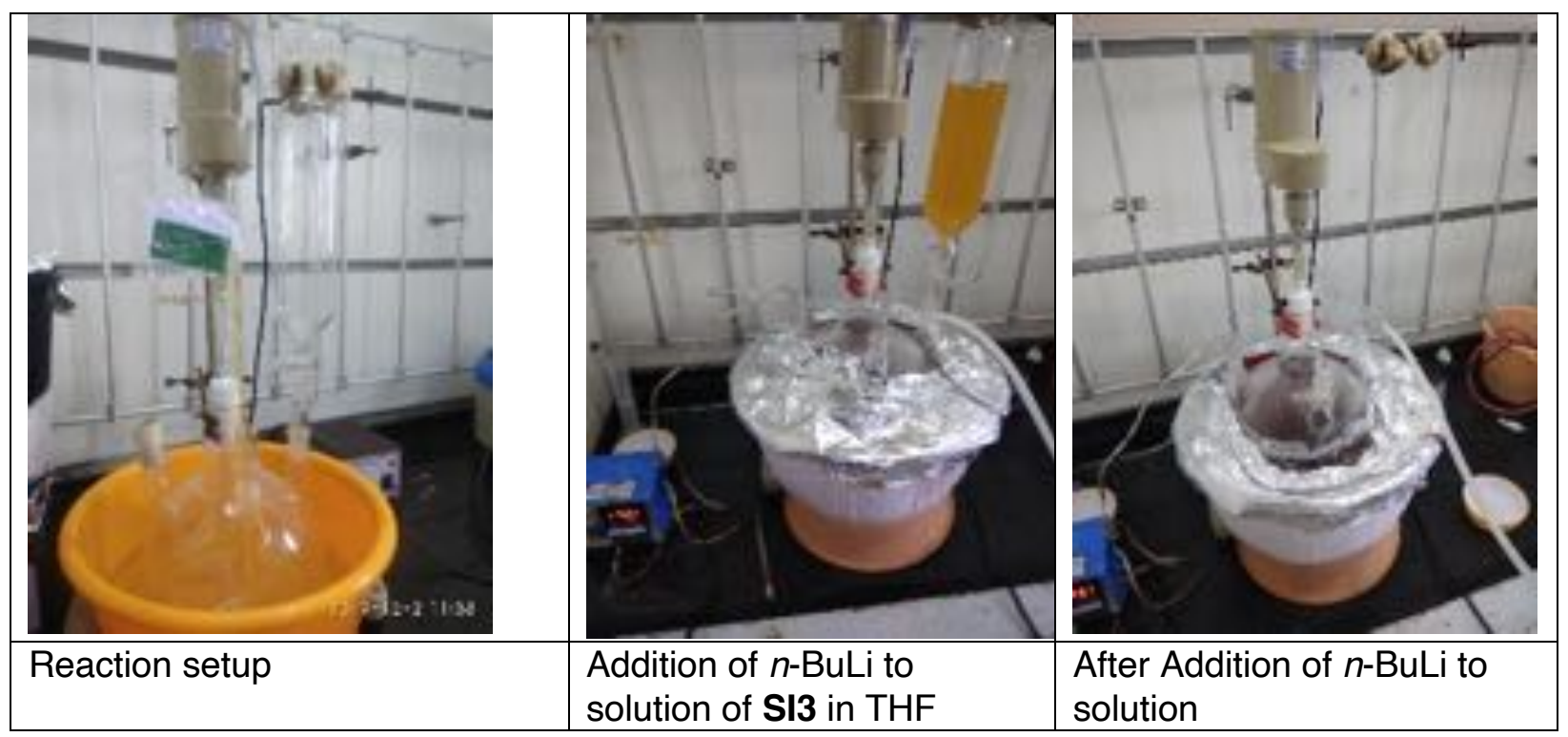




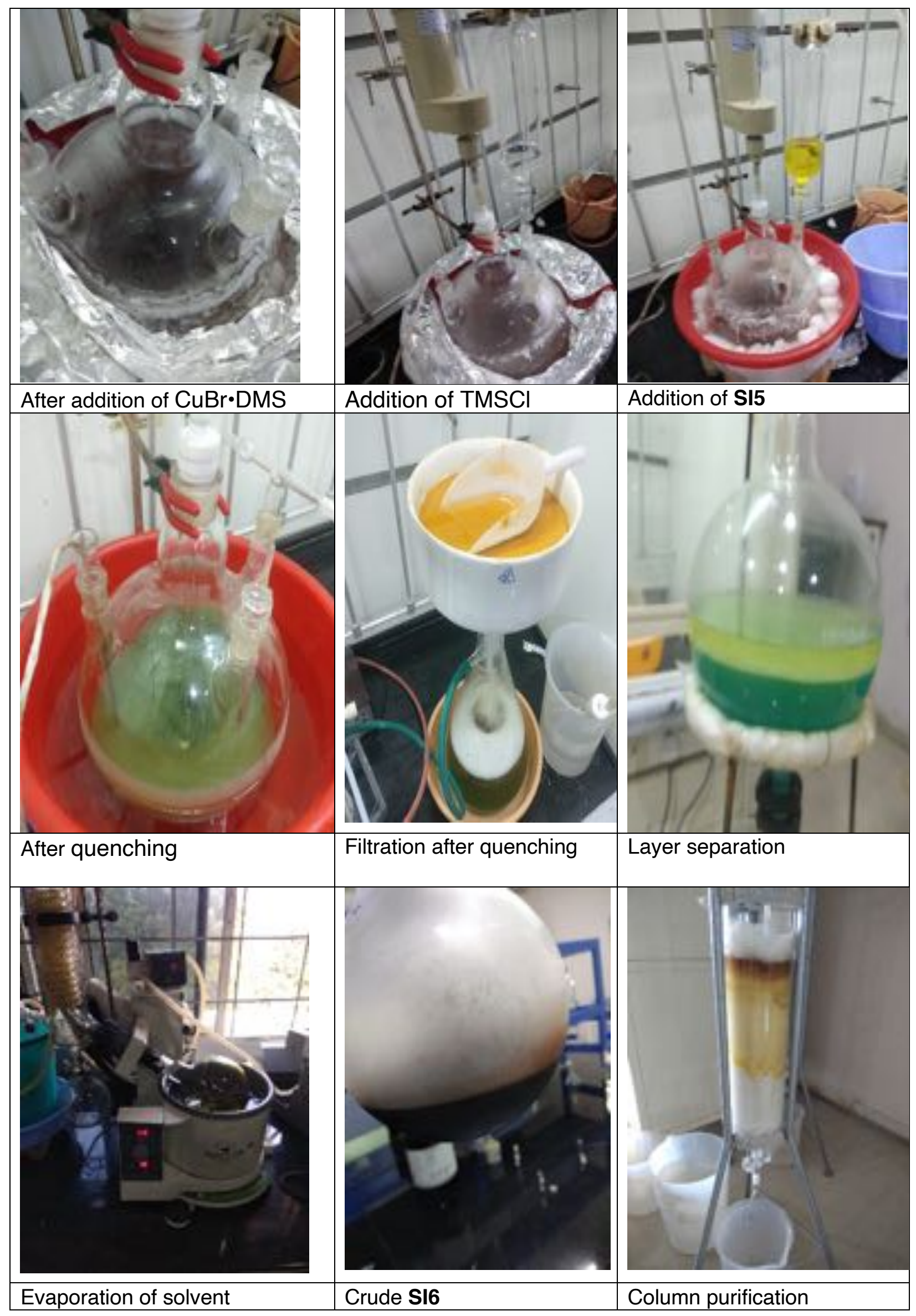




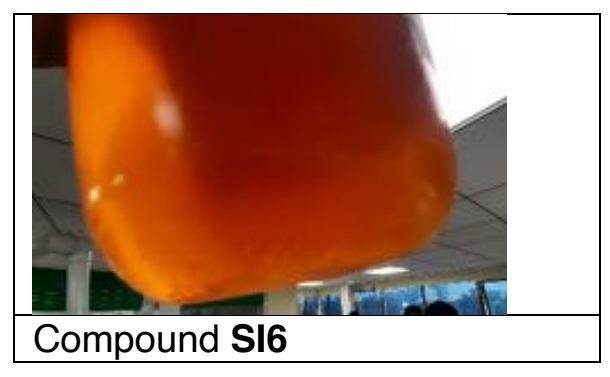


Compound 14<smiles>C=CC(=O)[C@H]1C(=O)CCC[C@@]1(C)CCC(C(=C)C)=C(C)C(C)=O</smiles>

To an oven-dried 2-neck $1 \mathrm{~L}$ round-bottom flask equipped with an addition funnel under nitrogen atmosphere was added with $\mathrm{Cu}(\mathrm{OTf})_{2}(2.6 \mathrm{~g}, 7.32 \mathrm{mmol}, 16 \mathrm{~mol} \%)$ and dried $\mathrm{PhMe}(125 \mathrm{~mL})$. Then NHC ligand $(2.8 \mathrm{~g}, 6.87 \mathrm{mmol}, 15 \mathrm{~mol} \%$, prepared according to ref. $\left.{ }^{2}\right)$ was added and the mixture was stirred for $45 \mathrm{~min}$ at rt. $\mathrm{MeMgBr}(61 \mathrm{~mL}, 183.2 \mathrm{mmol}$, $3 \mathrm{M}$ in $\left.\mathrm{Et}_{2} \mathrm{O}, 4 \mathrm{eq}\right)$ was added dropwise $(20 \mathrm{~min})$ to the above solution at $-40{ }^{\circ} \mathrm{C}$. After 5 $10 \mathrm{~min}$ a solution of SI6 $(10 \mathrm{~g}, 45.8 \mathrm{mmol})$ in PhMe $(100 \mathrm{~mL})$ was added dropwise at the same temperature (40 min) and stirring continued for another $30 \mathrm{~min}$. Progress of the reaction was monitored by TLC (EtOAc/hexane 1:9). The reaction mixture was cooled to $-78^{\circ} \mathrm{C}$ and freshly distilled acrolein $(15 \mathrm{~mL}, 229 \mathrm{mmol}, 5 \mathrm{eq})$ was added and stirred for another $1 \mathrm{~h}$. After completion of reaction, the mixture was quenched with $\mathrm{NH}_{4} \mathrm{Cl}$ (saturated aq., $20 \mathrm{~mL}$ ) at $-78^{\circ} \mathrm{C}$ and warmed to rt. Solvent was evaporated under reduced pressure to $1 / 3$ volume of the reaction mixture at $35-40{ }^{\circ} \mathrm{C}$. It was further diluted with DCM (100 $\mathrm{mL}, 10 \mathrm{v} / \mathrm{v} \%$ to crude) at it and cooled to $0{ }^{\circ} \mathrm{C}$. Dess-Martin periodinane $(17 \mathrm{~g}, 41.3 \mathrm{mmol}$, $1.2 \mathrm{eq})$ was added portion wise (20-30 $\mathrm{min})$ and stirred for $2 \mathrm{~h}$. After completion of the reaction the reaction mass was diluted with $\mathrm{DCM}(100 \mathrm{~mL})$ and washed with $\mathrm{NaHCO}_{3}$ (saturated aq., $150 \mathrm{~mL}$ ) followed by brine $(150 \mathrm{~mL})$. The crude was purified by flash column chromatography $\left(\mathrm{SiO}_{2}, 2 \%\right.$ EtOAc in hexane) to afford $\mathbf{S I 7}(6.9 \mathrm{~g})$ as a semi-pure pale-yellow oil. The spectroscopic data for this compound were identical to those previously reported. The next step was performed without further purification.

A mixture of $\mathrm{BF}_{3} \cdot \mathrm{OEt}_{2}(10.3 \mathrm{~mL}, 83.85 \mathrm{mmol}, 3.5 \mathrm{eq})$ and $\mathrm{DCM}(1400 \mathrm{~mL})$ at $0{ }^{\circ} \mathrm{C}$ was treated with a solution of semi-pure S17 $(6.9 \mathrm{~g}, 23.95 \mathrm{mmol}, 1.00 \mathrm{eq})$ in DCM (120 mL) over $2 \mathrm{~h}$ using an addition funnel at $0-5^{\circ} \mathrm{C}$. The reaction was stirred for $1 \mathrm{~h}$ at $0{ }^{\circ} \mathrm{C}$ and then was quenched with $\mathrm{NaHCO}_{3}$ (saturated aq., $800 \mathrm{~mL}$ ), and stirred for $15 \mathrm{~min}$. The layers were separated, and aqueous layer was extracted with DCM $(2 \times 500 \mathrm{~mL})$. The organic layers were combined, concentrated under reduced pressure, and passed through a silica plug (100-200 mesh, 2-3\% EtOAc in hexane). The product-containing fractions were concentrated under reduced pressure to produce $3.5 \mathrm{~g}$ of the titled compound. The material was suspended in $52 \mathrm{~mL}$ heptane and leftover solids were removed by filtration. The filtrate was evaporated to yield crude residue, which was triturated again by the same procedure ( $35 \mathrm{~mL}$ heptane). The resultant filtrate was evaporated to obtain $2 \mathrm{~g}$ of dione 14 with $94 \% e e$. To enrich further chiral purity, the above process is performed a third time. The filtrate was evaporated to obtain $1.4 \mathrm{~g}$ of dione 14 with $96 \%$ ee. The spectroscopic data for this compound were identical to those previously reported. ${ }^{1}$ 
Compound SI7

${ }^{1} \mathrm{H}$ NMR (400 MHz, $\left.\mathrm{CDCl}_{3}\right) \delta 6.40-6.36(\mathrm{dd}, \mathrm{J}=7.2,2 \mathrm{~Hz}, 1 \mathrm{H}), 6.36-6.21(\mathrm{dt}, \mathrm{J}=8.0$, $13.6 \mathrm{~Hz}, 1 \mathrm{H}), 5.83-5.79(\mathrm{~m}, 1 \mathrm{H}), 4.91-4.86(\mathrm{~m}, 1 \mathrm{H}), 4.53-4.47(\mathrm{dd}, \mathrm{J}=2.0,0.8 \mathrm{~Hz}$, $1 \mathrm{H}), 3.76(\mathrm{~d}, \mathrm{~J}=5.6 \mathrm{~Hz}, 1 \mathrm{H}), 2.64-2.68(\mathrm{~m}, 1 \mathrm{H}), 2.35-2.25(\mathrm{~m}, 1 \mathrm{H}), 2.20-1.80(\mathrm{~m}$, $5 \mathrm{H}), 1.69-1.64(\mathrm{~m}, 5 \mathrm{H}), 1.64-1.5(\mathrm{~m}, 11 \mathrm{H}), 1.45-1.49(\mathrm{~m}, 1 \mathrm{H}), 1.30-1.2(\mathrm{~m}, 3$ $\mathrm{H}), 0.95(\mathrm{~s}, 2 \mathrm{H}), 0.93(\mathrm{~s}, 2 \mathrm{H}) \mathrm{ppm}$.

Compound 14

${ }^{1} \mathrm{H}$ NMR (400 MHz, $\left.\mathrm{CDCl}_{3}\right) \delta 4.22(\mathrm{~s}, 1 \mathrm{H}), 2.93$ (ddd, J = 8.0, 14, $5.6 \mathrm{~Hz}, 1 \mathrm{H}$ ), $2.52-$ $2.44(\mathrm{~m}, 2 \mathrm{H}), 2.38(\mathrm{~d}, \mathrm{~J}=14.8 \mathrm{~Hz}, 1 \mathrm{H}), 2.23-2.19(\mathrm{~m}, 1 \mathrm{H}), 2.22-2.11(\mathrm{~m}, 2 \mathrm{H}), 2.12$ $-1.95(\mathrm{~m}, 5 \mathrm{H}), 1.88(\mathrm{~s}, 3 \mathrm{H}), 1.81-1.62(\mathrm{~m}, 2 \mathrm{H}), 1.51-1.41(\mathrm{~m}, 1 \mathrm{H}), 1.39-1.35(\mathrm{~m}$, $1 \mathrm{H}), 1.26(\mathrm{~s}, 3 \mathrm{H}), 1.12(\mathrm{~s}, 3 \mathrm{H}), 1.03(\mathrm{~s}, 3 \mathrm{H})$.

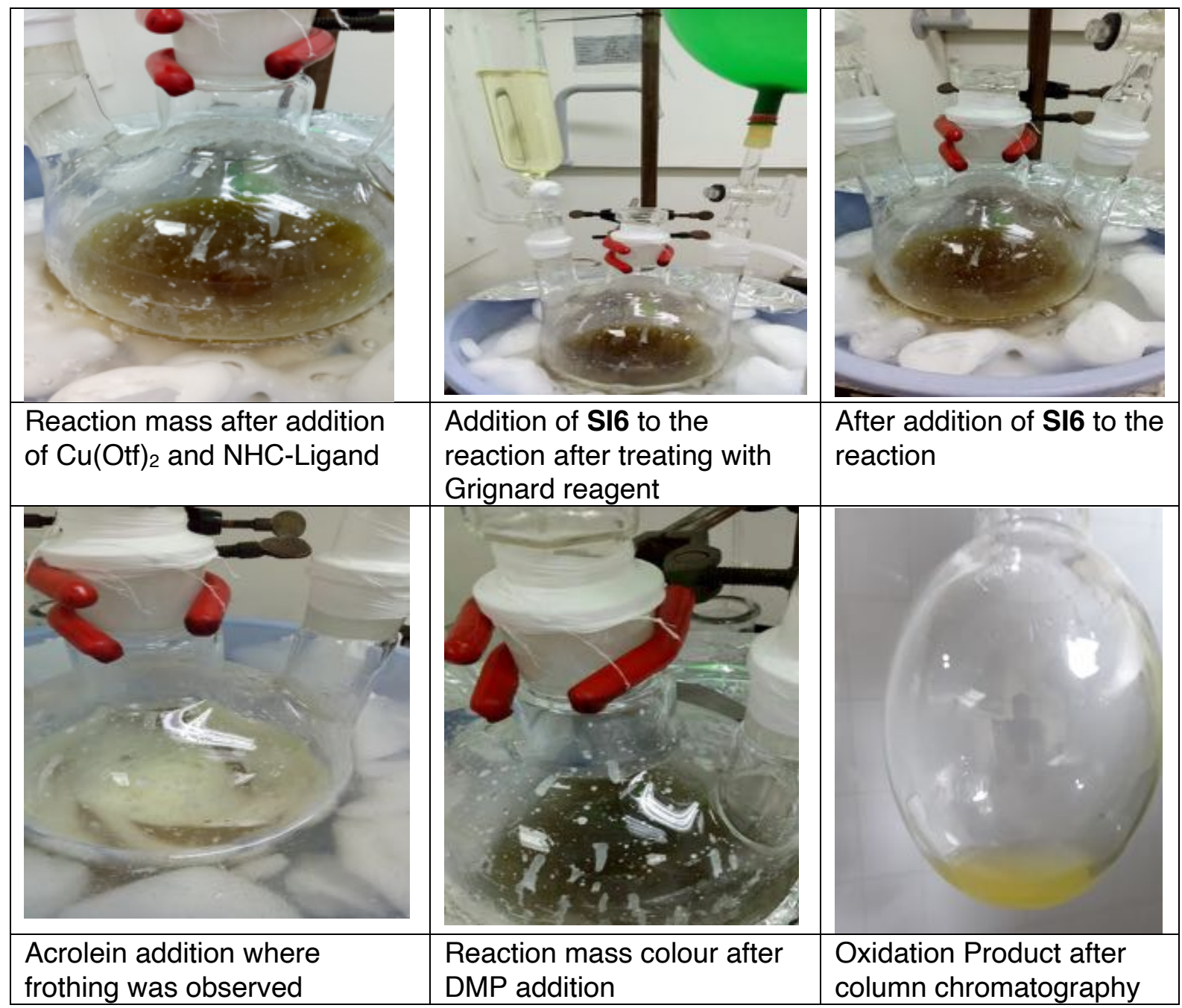



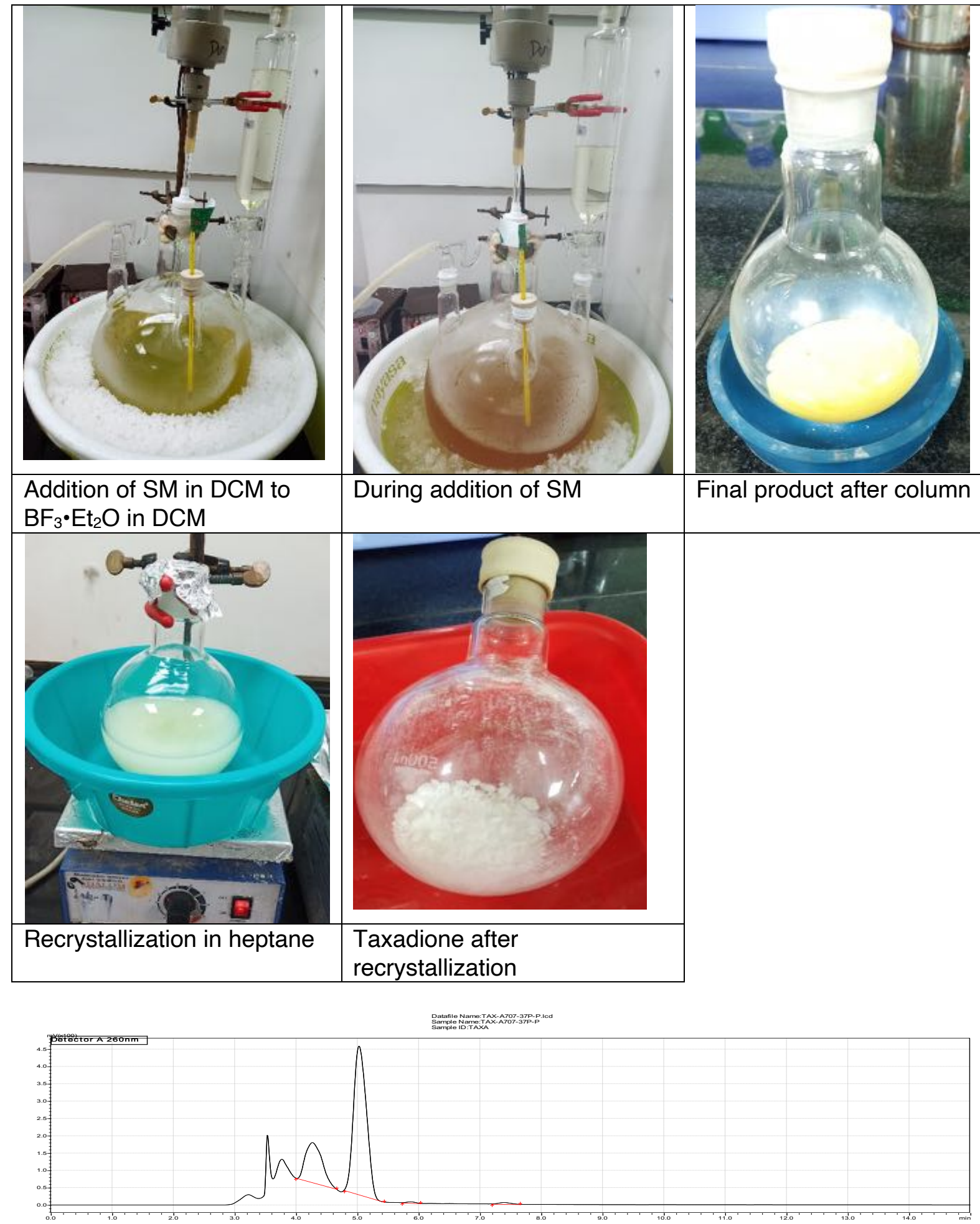

Chromatogram of crude (+)-SI7 on a chiral column.

Detector A 260nm

\begin{tabular}{|r|r|r|r|}
\hline \multicolumn{1}{|c|}{ Reak\# } & \multicolumn{1}{|c|}{ Ret. Time } & \multicolumn{1}{c|}{ Area } & \multicolumn{1}{c|}{ Area\% } \\
\hline 1 & 4.268 & 2188051 & 25.571 \\
\hline 2 & 5.029 & 6280807 & 73.403 \\
\hline 3 & 5.870 & 31460 & 0.368 \\
\hline 4 & 7.396 & 563188556636 & 0.658 \\
\hline Total & & 8556636 & 100.000 \\
\hline
\end{tabular}




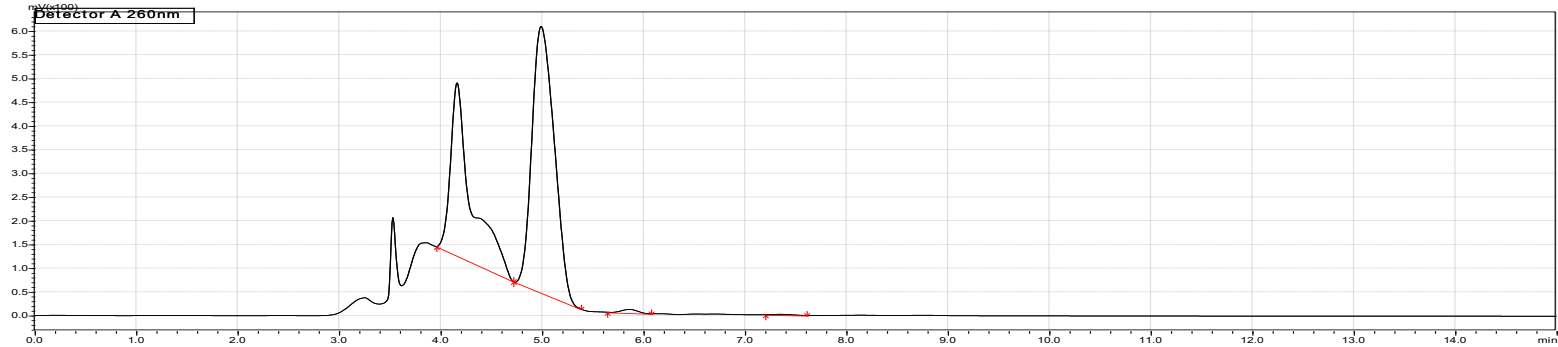

Chromatogram of crude racemic-SI7 on a chiral column.

Detector A 260nm

\begin{tabular}{|c|c|c|c|}
\hline Peak\# & Ret. Time & Area & Area\% \\
\hline 1 & 4.165 & 5225807 & 37.543 \\
\hline 2 & 4.994 & 8596831 & 61.760 \\
\hline 3 & 5.862 & 80065 & 0.575 \\
\hline 4 & 7.347 & 16972 & 0.122 \\
\hline Total & & 13919675 & 100.000 \\
\hline
\end{tabular}

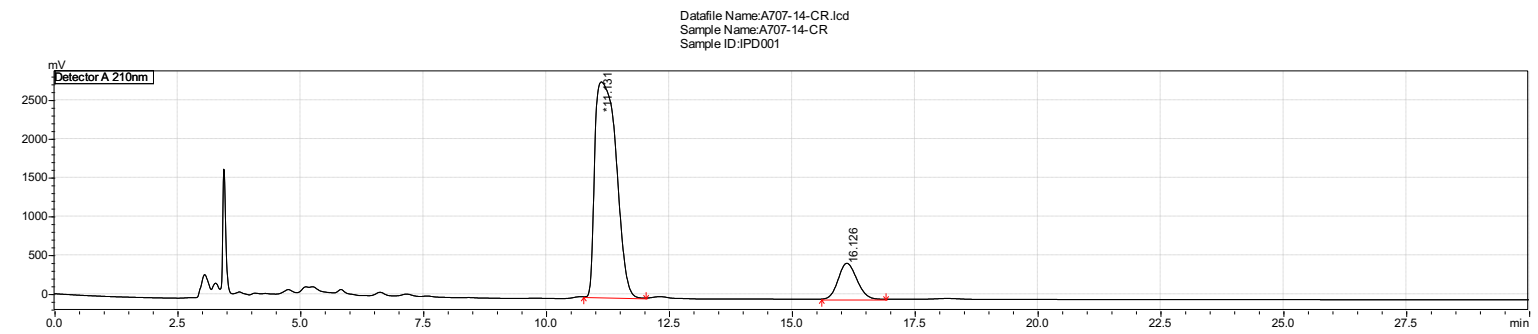

Chromatogram of crude (+)-14 on a chiral column.

Detector A 210nm

\begin{tabular}{|r|r|r|r|}
\hline \multicolumn{1}{|c|}{ Peak\# } & \multicolumn{1}{|c|}{ Ret. Time } & \multicolumn{1}{c|}{ Area } & \multicolumn{1}{|c|}{ Area\% } \\
\hline 1 & 11.131 & 83840712 & 87.336 \\
\hline 2 & 16.126 & 12157265 & 12.664 \\
\hline Total & & 95997977 & 100.000 \\
\hline
\end{tabular}

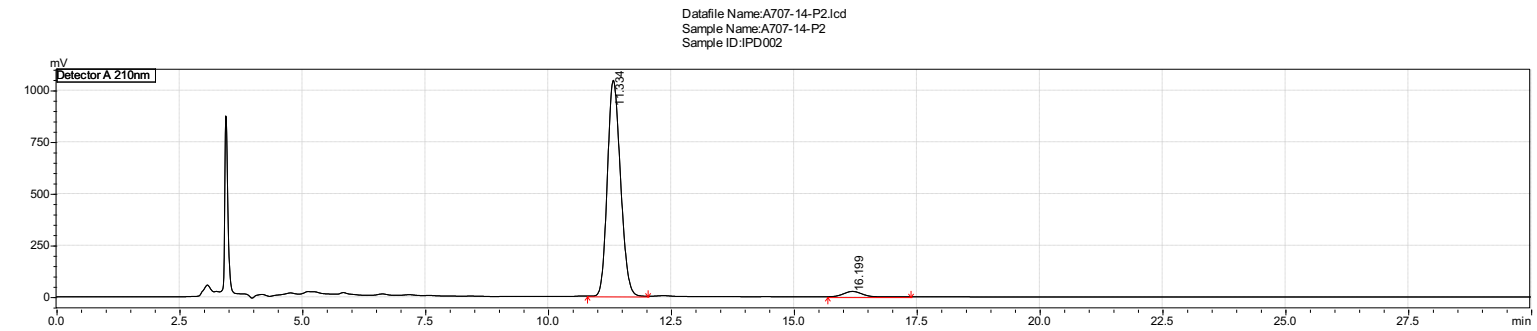

Chromatogram of (+)-14 after the first trituration on a chiral column.

Detector A 210nm

\begin{tabular}{|r|r|r|r|}
\hline \multicolumn{1}{|c|}{ Peak\# } & \multicolumn{1}{|c|}{ Ret. Time } & \multicolumn{1}{c|}{ Area } & \multicolumn{1}{c|}{ Area\% } \\
\hline 1 & 11.334 & 19357110 & 96.694 \\
\hline 2 & 16.199 & 661797 & 3.306 \\
\hline Total & & 20018907 & 100.000 \\
\hline
\end{tabular}




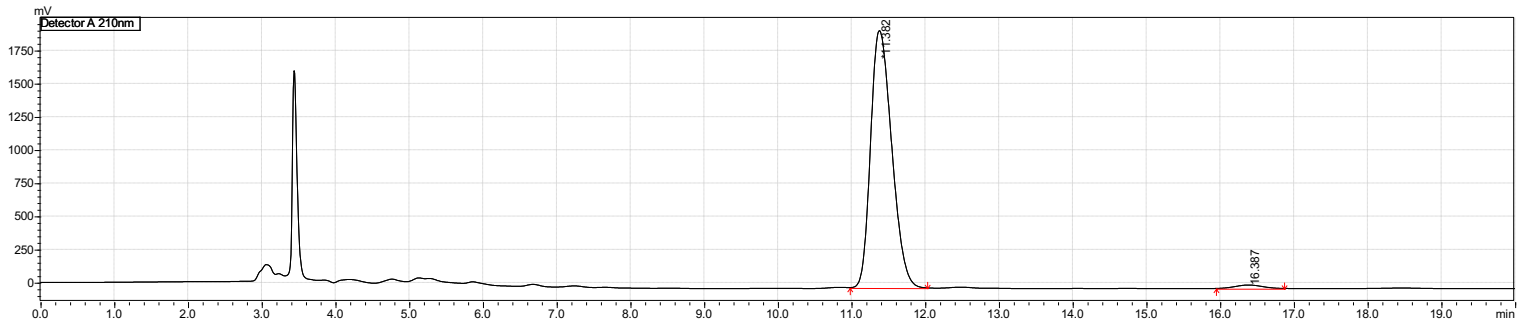

Chromatogram of (+)-14 after the second trituration on a chiral column.

Detector A 210nm

\begin{tabular}{|r|r|r|r|}
\hline Peak\# & Ret. Time & \multicolumn{1}{|c|}{ Area } & \multicolumn{1}{c|}{ Area\% } \\
\hline 1 & 11.382 & 38583667 & 98.333 \\
\hline 2 & 16.387 & 653928 & 1.667 \\
\hline Total & & 39237595 & 100.000 \\
\hline
\end{tabular}

Datafile Name:A653-75-RACEMIC-A.ICd
Sample Name:A653-75-RACEMIC

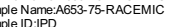

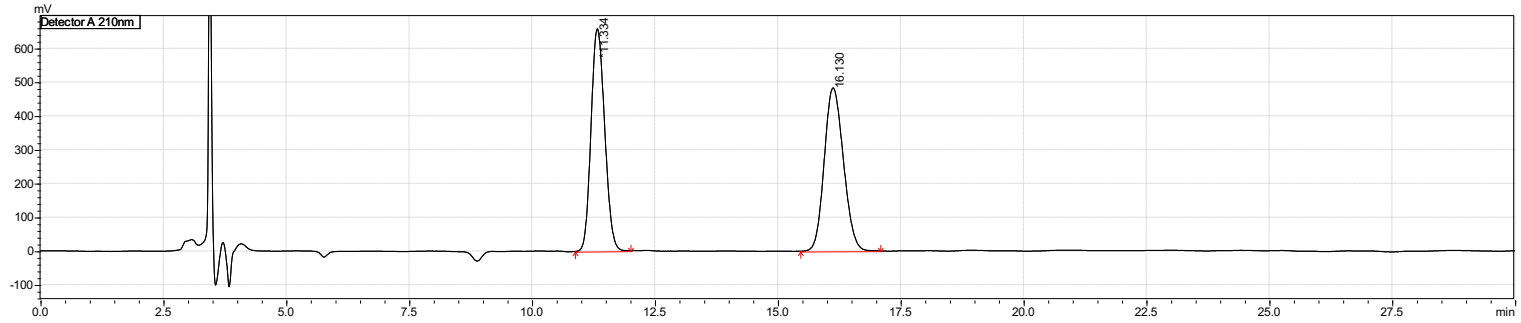

Chromatogram of racemic 14 on a chiral column.

Detector A 210nm

\begin{tabular}{|r|r|r|r|}
\hline Peak\# & Ret. Time & Area & \multicolumn{1}{|c|}{ Area\% } \\
\hline 1 & 11.334 & 12726614 & 49.241 \\
\hline 2 & 16.130 & 13118778 & 50.759 \\
\hline Total & & 25845392 & 100.000 \\
\hline
\end{tabular}

Datafile Name:A653-75-RACEMIC-A.Ic

Sample Name:A653-75-RACEMIC

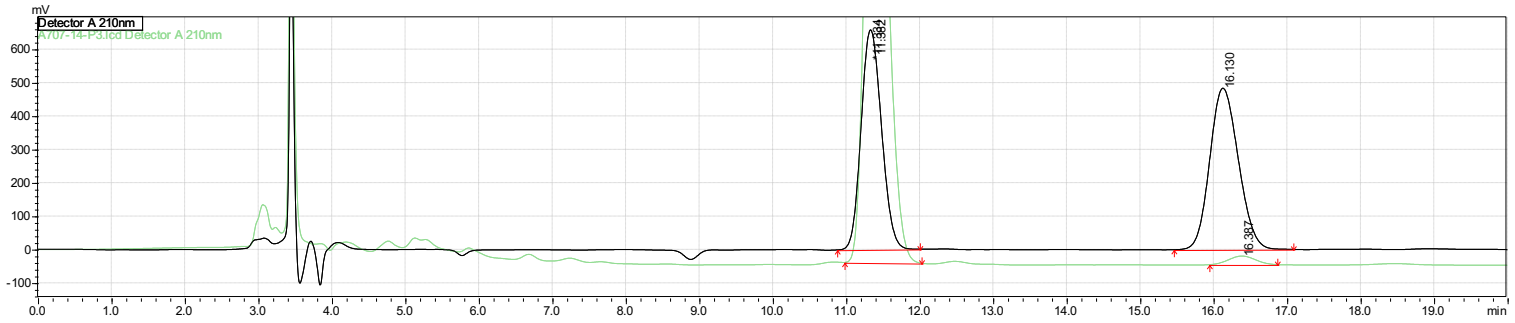

Overlay of racemic 14 and enantioenriched (+)-14.

Chiral HPLC: 98.33 [11.38 min]. 96.67 ee\%.

Column: LUX I-CELLULOSE $\left(250 * 4.6 \mathrm{~mm}{ }^{\star} 5.0 \mu\right)$

Mobile: $0.1 \%$ DEA in hexane:IPA (60:40)

Flow rate: $1.0 \mathrm{~mL} / \mathrm{min}$ 
Summary of the Cyclase Phase Optimization

Mukaiyama aldol route

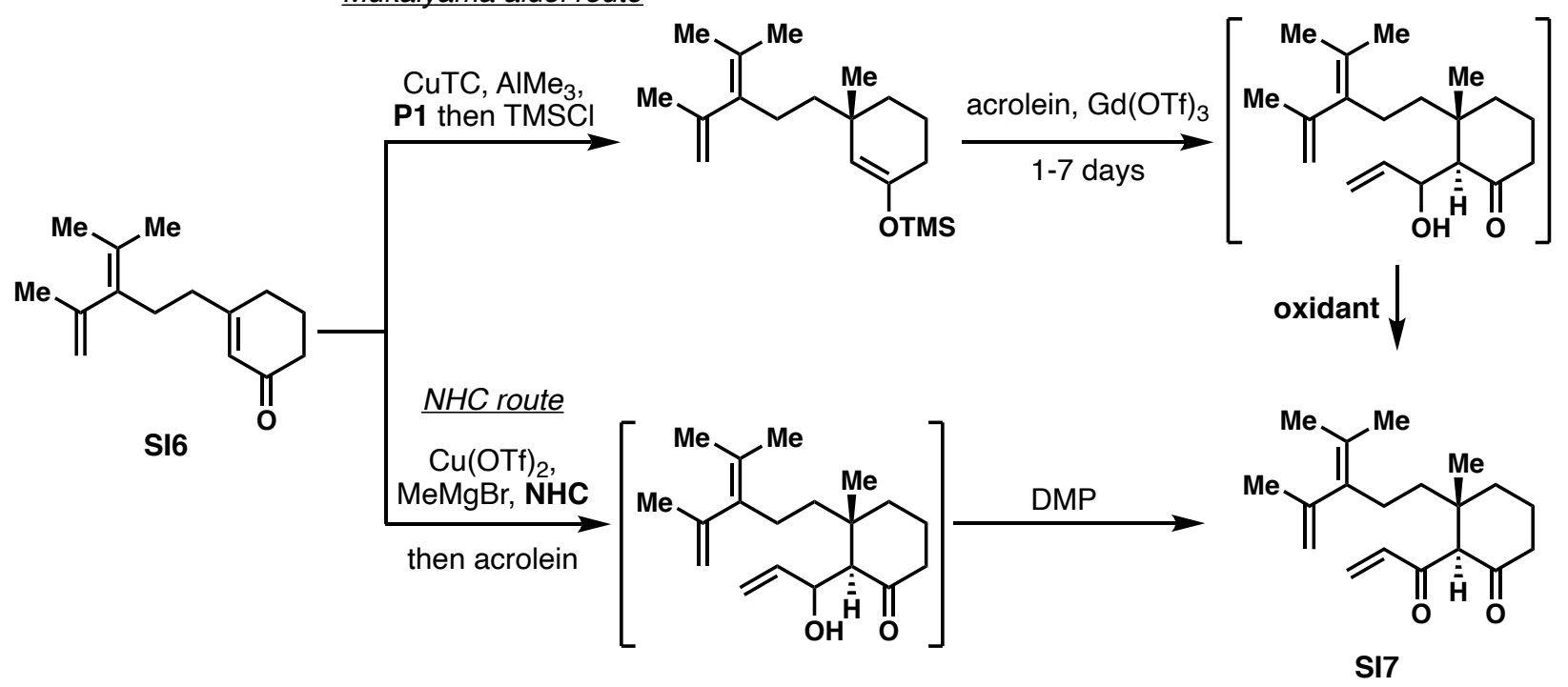

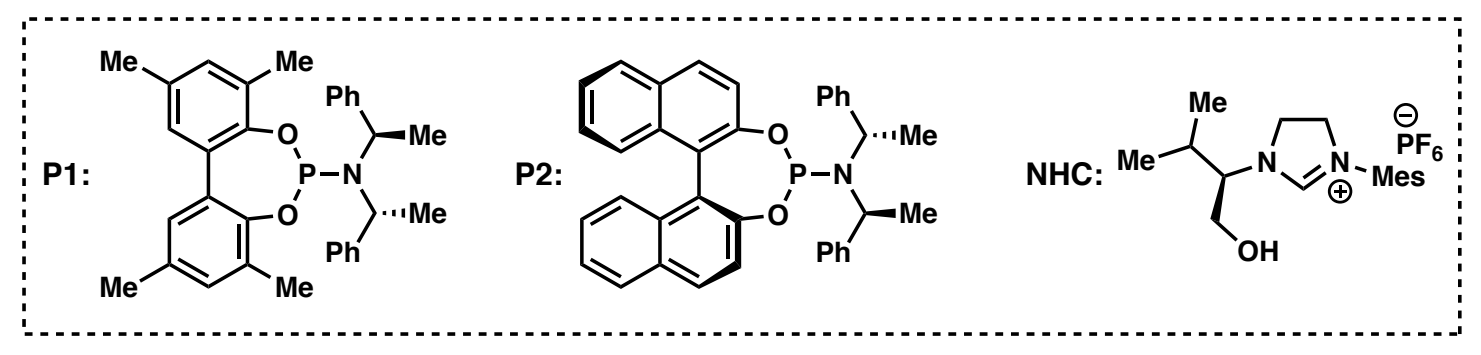

\begin{tabular}{|c|c|c|c|c|c|c|}
\hline Route to SI7 & $\begin{array}{c}\text { Isolated } \\
\text { intermediates }\end{array}$ & Ligand & Oxidant & $\begin{array}{l}\text { Reaction } \\
\text { time from } \\
\text { SI6 to SI7 }\end{array}$ & $\begin{array}{l}\text { Overall yield } \\
\text { from SI6 to } 14\end{array}$ & Comments \\
\hline $\begin{array}{c}\text { Mukaiyama } \\
\text { (original } \\
\text { prep) }\end{array}$ & 3 & P1 & $\begin{array}{l}\text { Jones } \\
\text { reagent }\end{array}$ & 2-8 days & $7.6 \%$ & $\begin{array}{c}\text { - unstable intermediates } \\
\text { - irreproducible aldol } \\
\text { • large scale Cr(VI) } \\
\text { • costly ligand } \\
\end{array}$ \\
\hline Mukaiyama & 2 & P2 & DMP & 2-3 days & $6.1 \%$ & $\begin{array}{c}\text { - unstable TMS enolate } \\
\text { - irreproducible aldol } \\
\text { - accessible ligand }\end{array}$ \\
\hline $\mathrm{NHC}$ & 2 & NHC & DMP & 2 days & $4.8 \%$ & $\begin{array}{c}\text { - reproducible } \\
\cdot \text { accessible ligand }\end{array}$ \\
\hline $\mathrm{NHC}$ & 1 & NHC & DMP & 1 day & $10.6 \%$ & $\begin{array}{c}\cdot \text { short reaction time } \\
\text { - concise operation } \\
\cdot \text { minimum material loss }\end{array}$ \\
\hline
\end{tabular}

Comparison of yields from different procedures: (1) Original report from ref. ${ }^{1}$. (2) Michael addition and aldol in one-pot then DMP oxidation in a separate pot. (3) Michael addition, aldol and DMP all in one-pot. 


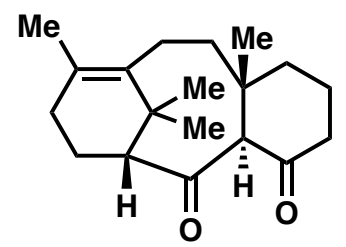

14

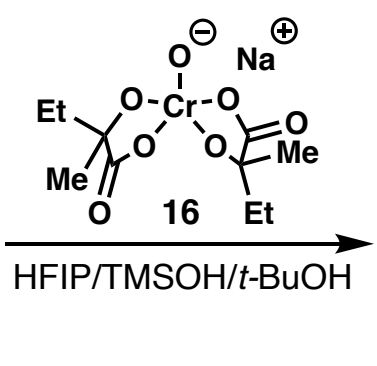

$$
\text { (1) }
$$

Dione $14(21.7 \mathrm{~g}, 75.3 \mathrm{mmol})$, the $\mathrm{Cr}(\mathrm{V})$ reagent $(16)^{3}(171 \mathrm{~g}, 527 \mathrm{mmol}, 7 \mathrm{eq})$ were placed in a round-bottom flask. $t$ - BuOH $(34 \mathrm{~mL}), \mathrm{TMSOH}(240 \mathrm{~mL})$ and HFIP $(480 \mathrm{~mL})$ were added, and the mixture was then stirred for $6 \mathrm{~h}$ at $80^{\circ} \mathrm{C}$, open to air. On complete consumption of 14, $\mathrm{SiO}_{2}(513 \mathrm{~g}, 300 \mathrm{wt}$. \% to $\mathrm{Cr}(\mathrm{V}))$ was added. Solvent was completely removed under reduced pressure. The resulting blackish-green powder was loaded on a pad of $\mathrm{SiO}_{2}(855 \mathrm{~g}, 500$ wt.\% to $\mathrm{Cr}(\mathrm{V}))$ and eluted with hexane/EtOAc (2:1) to obtain a pale yellow solid $(19.4 \mathrm{~g})$. The next step was performed without further purification.

\section{Compound 15}

Physical state: white solid;

TLC: $R_{f}=0.42$ (DCM/acetone 19:1, UV active on TLC, stains orange upon $p$ anisaldehyde staining);

${ }^{1} \mathrm{H}$ NMR $\left(600 \mathrm{MHz}, \mathrm{CDCl}_{3}\right) \delta 4.01$ (s, 1H), 2.97 (ddd, $J=14.3,10.7,5.1 \mathrm{~Hz}, 1 \mathrm{H}$ ), 2.89 (dd, $J=19.8,6.9 \mathrm{~Hz}, 1 \mathrm{H}), 2.78(\mathrm{~d}, J=7.0 \mathrm{~Hz}, 1 \mathrm{H}), 2.50-2.43(\mathrm{~m}, 2 \mathrm{H}), 2.43-2.37(\mathrm{~m}$, $1 \mathrm{H}$ ), 2.13 (dddd, $J=15.6,11.0,8.2,1.2 \mathrm{~Hz}, 1 \mathrm{H}), 2.02(\mathrm{~s}, 3 \mathrm{H}), 2.00-1.81(\mathrm{~m}, 4 \mathrm{H}), 1.66$ (dt, $J=15.3,5.7 \mathrm{~Hz}, 1 \mathrm{H}$ ), 1.46 (dddd, $J=13.4,4.4,2.8,1.2 \mathrm{~Hz}, 1 \mathrm{H}$ ), 1.34 (s, 3H), 1.21 (s, 3H), $1.09(\mathrm{~s}, 3 \mathrm{H})$.

${ }^{13} \mathrm{C}$ NMR (151 MHz, $\left.\mathrm{CDCl}_{3}\right) \delta 206.7,205.8,196.7,160.7,134.2,64.0,58.4,42.9,38.8$, 38.6, 38.0, 37.7, 34.8, 34.7, 25.9, 24.3, 22.9, 19.6, 13.9.

HRMS (ESI-TOF): calc'd for $\mathrm{C}_{19} \mathrm{H}_{27} \mathrm{O}_{3}\left[\mathrm{M}+\mathrm{H}^{+}\right]$: 303.1955 , found: 303.1965 .

$[\alpha]^{25} \mathrm{D}:+60.1\left(c=1.0, \mathrm{CHCl}_{3}\right)$

\section{Compound SI8}

Physical state: white solid;

TLC: $R_{f}=0.40$ (DCM/acetone 19:1, UV active on TLC, stains orange upon $p$ anisaldehyde staining);

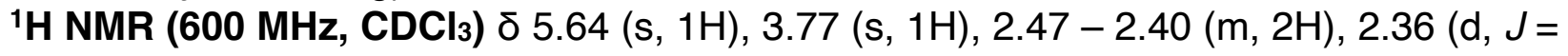
$5.8 \mathrm{~Hz}, 1 \mathrm{H}$ ), 2.20 (ddd, $J=15.9,12.9,7.6 \mathrm{~Hz}, 1 \mathrm{H}$ ), $2.13(\mathrm{dd}, J=19.4,4.5 \mathrm{~Hz}, 1 \mathrm{H}), 2.04$ (dd, $J=16.2,11.3 \mathrm{~Hz}, 1 \mathrm{H}), 1.95-1.80(\mathrm{~m}, 5 \mathrm{H}), 1.65-1.47(\mathrm{~m}, 5 \mathrm{H}), 1.34(\mathrm{~s}, 1 \mathrm{H}), 1.19$ $(\mathrm{s}, 3 \mathrm{H}), 1.13(\mathrm{~s}, 3 \mathrm{H}), 0.97(\mathrm{~s}, 3 \mathrm{H})$.

${ }^{13} \mathrm{C}$ NMR (151 MHz, $\mathrm{CDCl}_{3}$ ) $\delta$ 209.4, 207.4, 139.7, 121.5, 77.7, 63.4, 57.7, 41.6, 41.4, 41.0, 39.8, 30.2, 27.1, 24.4, 20.8, 19.7, 19.6, 19.1.

HRMS (ESI-TOF): calc'd for $\mathrm{C}_{19} \mathrm{H}_{29} \mathrm{O}_{3}\left[\mathrm{M}+\mathrm{H}^{+}\right]$: 305.2111 , found: 305.2120 . $[\alpha]^{25} \mathrm{D}:+6.2\left(c=0.5, \mathrm{CHCl}_{3}\right)$ 


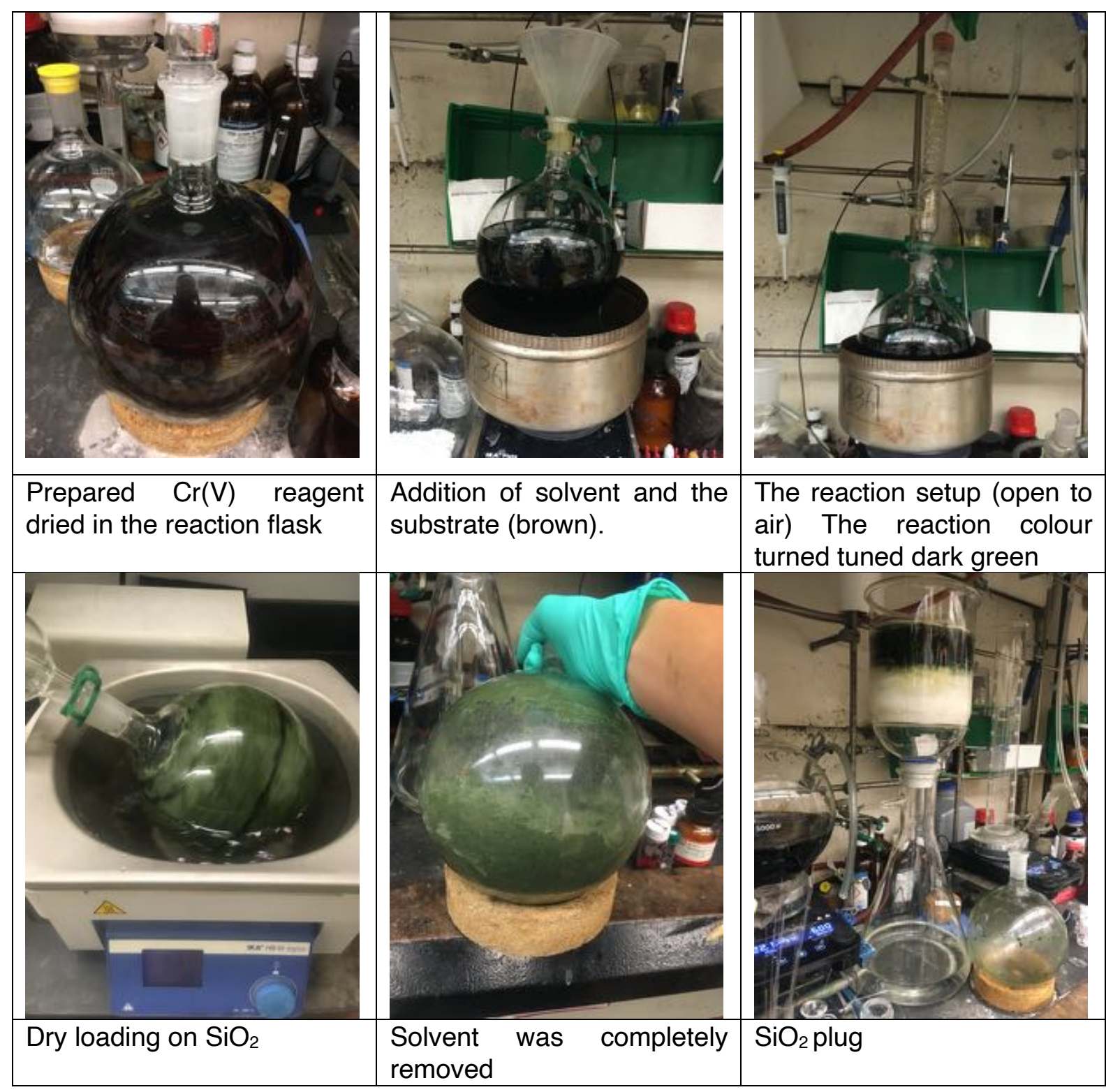


Compound 17

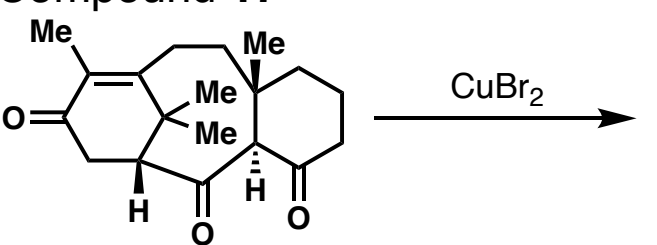

15

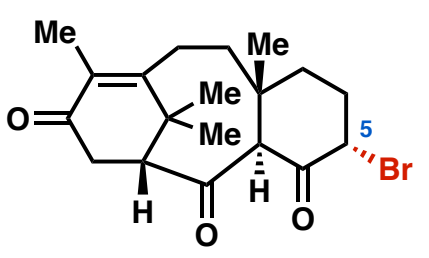

17

The crude mixture of 15 (30.4 g) was dissolved in THF (330 mL). Upon addition of $\mathrm{CuBr}_{2}$ ( $45.0 \mathrm{~g} 201 \mathrm{mmol}, 2.0 \mathrm{eq}$ based on crude mass) at rt open to air, the pale yellow solution turned to dark brown. As reaction progressed, the reaction mixture became a green suspension. On complete consumption of $15(1 \mathrm{~h})$, the reaction mixture was poured on Celite ${ }^{\circledR}(450 \mathrm{~g})$ over a pad of $\mathrm{SiO}_{2}\left(900 \mathrm{~g}, 2000 \mathrm{wt} \%\right.$ to $\left.\mathrm{CuBr}_{2}\right)$ and eluted with hexane/EtOAc (1:1). The solvent was removed under reduced pressure. The crude product was purified by flash column chromatography $\left(\mathrm{SiO}_{2}, \mathrm{DCM} /\right.$ acetone gradient 1:0 to $60: 1)$ to yield compound $\alpha$-bromotrione 17 (20.4 g, $53.7 \mathrm{mmol}, 55 \%$ over 2 steps).

Physical state: white solid;

TLC: $R_{f}=0.69$ (DCM/acetone 19:1, UV active on TLC, stains green upon $p$-anisaldehyde staining);

1H NMR (600 MHz, CDCl 3 ) ס 5.36 (s, 1H), 4.29 (d, J=3.1 Hz, 1H), 3.09 (td, J = 13.5, 5.4 $\mathrm{Hz}, 1 \mathrm{H}), 2.89$ (dd, $J=19.7,7.1 \mathrm{~Hz}, 1 \mathrm{H}), 2.76(\mathrm{~d}, J=7.1 \mathrm{~Hz}, 1 \mathrm{H}), 2.53-2.44(\mathrm{~m}, 2 \mathrm{H})$, $2.41-2.30(\mathrm{~m}, 2 \mathrm{H}), 2.19-2.13(\mathrm{~m}, 4 \mathrm{H}), 1.95$ (ddd, J=15.7, 13.2, $5.4 \mathrm{~Hz}, 1 \mathrm{H}), 1.57$ (ddd, $J=15.8,5.4,3.4 \mathrm{~Hz}, 1 \mathrm{H}), 1.34(\mathrm{~s}, 3 \mathrm{H}), 1.30$ (ddd, $J=13.7,4.8,2.4 \mathrm{~Hz}, 1 \mathrm{H}), 1.21(\mathrm{~s}, 3 \mathrm{H})$, $1.00(\mathrm{~s}, 3 \mathrm{H})$.

${ }^{13} \mathrm{C}$ NMR (151 MHz, $\left.\mathrm{CDCl}_{3}\right) \delta$ 205.6, 198.5, 196.8, 159.9, 135.3, 58.7, 57.3, 49.9, 45.4, 38.6, 37.9, 35.7, 35.6, 32.8, 30.5, 27.0, 24.2, 23.6, 14.4 .

HRMS (ESI-TOF): calc'd for $\mathrm{C}_{19} \mathrm{H}_{26} \mathrm{BrO}_{3}\left[\mathrm{M}+\mathrm{H}^{+}\right]$: 381.1060 , found: 381.1065 .

$[\alpha]^{25} \mathrm{D}:+286.7\left(c=1.0, \mathrm{CHCl}_{3}\right)$

X-Ray Crystallography: Solved structure; see page SI-102

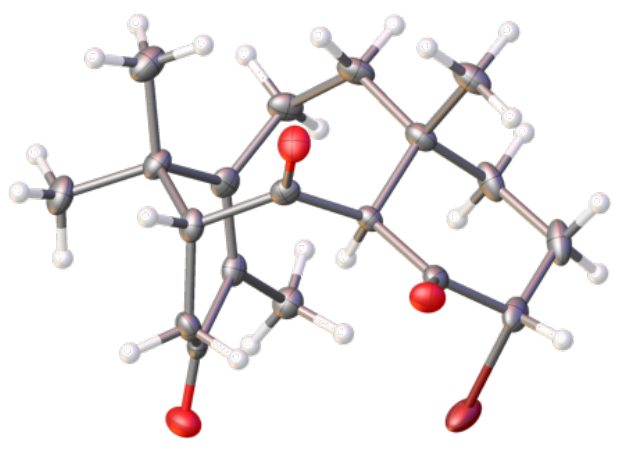



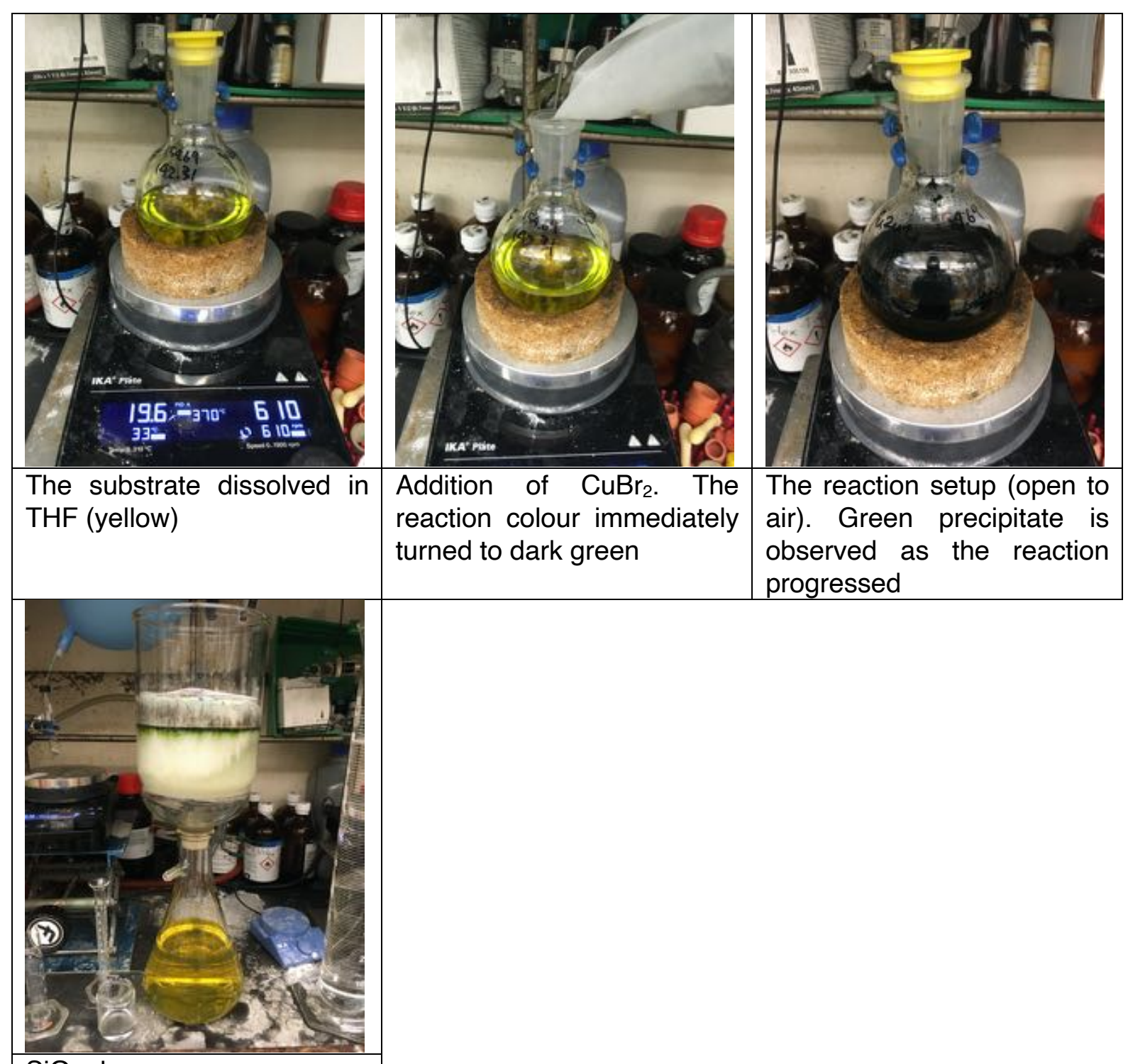

$\mathrm{SiO}_{2}$ plug 
Compound 18

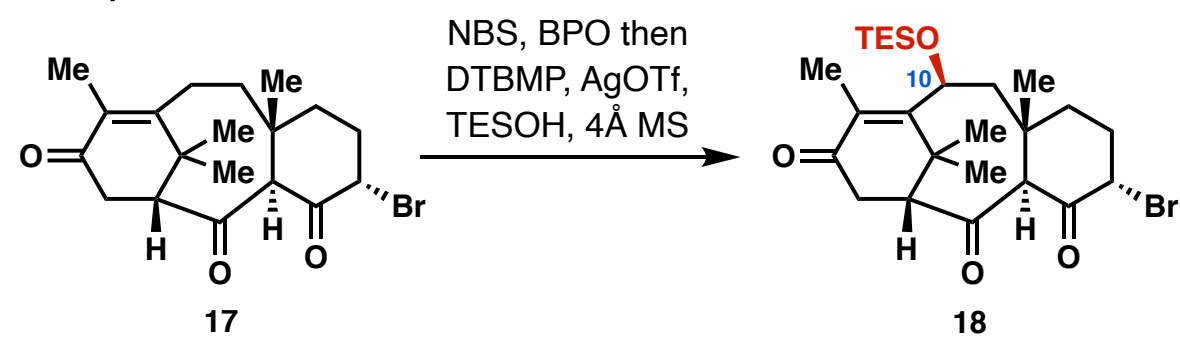

An oven dried round bottom flask was charged with $\alpha$-bromotrione $17(8.82 \mathrm{~g}, 23.1 \mathrm{mmol})$, NBS (4.32 g, $24.3 \mathrm{mmol}, 1.05 \mathrm{eq})$, BPO (866 mg, $3.58 \mathrm{mmol}, 0.3 \mathrm{eq}$ ) and $\mathrm{CCl}_{4}(461 \mathrm{~mL})$. This suspension was degassed for 30 min via sparging with argon under sonication. The resulting suspension was placed in a pre-heated oil bath at $80^{\circ} \mathrm{C}$ and stirred for $20 \mathrm{~min}$. The reaction mixture was concentrated under reduced pressure to give a pale yellow oil.

The crude oil, $4 \AA$ molecular sieves (8.82 g, 100 wt.\% to 17) and 2,6-ditert-butyl-4methylpyridine (DTBMP) $(40.8 \mathrm{~g}, 199 \mathrm{mmol}, 8.6 \mathrm{eq})$ were placed in a round bottom flask. The flask was subjected to three vacuum/argon cycles quickly (DTBMP sublimes under reduced pressure), then PhMe (461 mL) was added. To this yellow suspension was added TESOH $(29.8 \mathrm{~mL}, 194 \mathrm{mmol}, 8.4 \mathrm{eq})$. The reaction flask was wrapped with aluminum foil and $\operatorname{AgOTf}(30.3 \mathrm{~g}, 118 \mathrm{mmol}, 5.1 \mathrm{eq})$ was added in one portion. White solid immediately crashed out. The resulting suspension was stirred for $2 \mathrm{~h}$ at $\mathrm{rt}$ in the dark. On completion of the reaction, the gray suspension was poured over Celite ${ }^{\circledR}$ (110 g) and a pad of $\mathrm{SiO}_{2}(220 \mathrm{~g}, 2500$ wt.\% to 17). The filtrate was concentrated under reduced pressure to give a yellow solid. The resulting residue was purified by flash column chromatography $\left(\mathrm{SiO}_{2}\right.$, hexane/EtOAc $0: 1$ to 6:1) to yield TES trione 18 (8.04 g, 15.7 $\mathrm{mmol}, 68 \%)$.

Physical state: white solid;

TLC: $R_{f}=0.63$ (hexane/EtOAc 2:1, UV active on TLC, stains blue upon $p$-anisaldehyde staining);

'H NMR (600 MHz, $\left.\mathrm{CDCl}_{3}\right) \delta 5.24(\mathrm{dd}, J=11.4,5.8 \mathrm{~Hz}, 1 \mathrm{H}), 5.20(\mathrm{~s}, 1 \mathrm{H}), 4.29(\mathrm{~d}, J=$ $2.8 \mathrm{~Hz}, 1 \mathrm{H}$ ), 2.92 (dd, $J=19.8,7.2 \mathrm{~Hz}, 1 \mathrm{H}$ ), $2.71(\mathrm{~d}, J=7.2 \mathrm{~Hz}, 1 \mathrm{H}), 2.49$ (ddt, $J=16.2$, 13.1, $4.7 \mathrm{~Hz}, 1 \mathrm{H}), 2.37(\mathrm{~d}, J=19.8 \mathrm{~Hz}, 1 \mathrm{H}), 2.29-2.22(\mathrm{~m}, 4 \mathrm{H}), 2.18-2.11(\mathrm{~m}, 2 \mathrm{H})$, $1.68(\mathrm{dd}, J=15.2,5.8 \mathrm{~Hz}, 1 \mathrm{H}), 1.48(\mathrm{~s}, 3 \mathrm{H}), 1.40(\mathrm{ddd}, J=13.6,4.8,2.5 \mathrm{~Hz}, 1 \mathrm{H}), 1.23$ (s, 3H), $1.03(\mathrm{~s}, 3 \mathrm{H}), 0.97$ (t, $J=7.9 \mathrm{~Hz}, 9 \mathrm{H}), 0.62$ (q, $J=7.9 \mathrm{~Hz}, 6 \mathrm{H}$ ).

${ }^{13} \mathrm{C}$ NMR (151 MHz, CDCl 3$) \delta$ 204.8, 198.7, 198.2, 158.7, 134.6, 68.9, 59.0, 56.2, 50.0, 47.7, 43.2, 38.2, 36.4, 35. 6, 33.5, 30.4, 24.1, 23.5, 14.6, 7.0, 5.1.

HRMS (ESI-TOF): calc'd for $\mathrm{C}_{25} \mathrm{H}_{40} \mathrm{BrO}_{4} \mathrm{Si}\left[\mathrm{M}+\mathrm{H}^{+}\right]$: 511.1874 , found: 511.1880 . $[\alpha]^{25} \mathrm{D}:+227.3\left(c=1.0, \mathrm{CHCl}_{3}\right)$ 


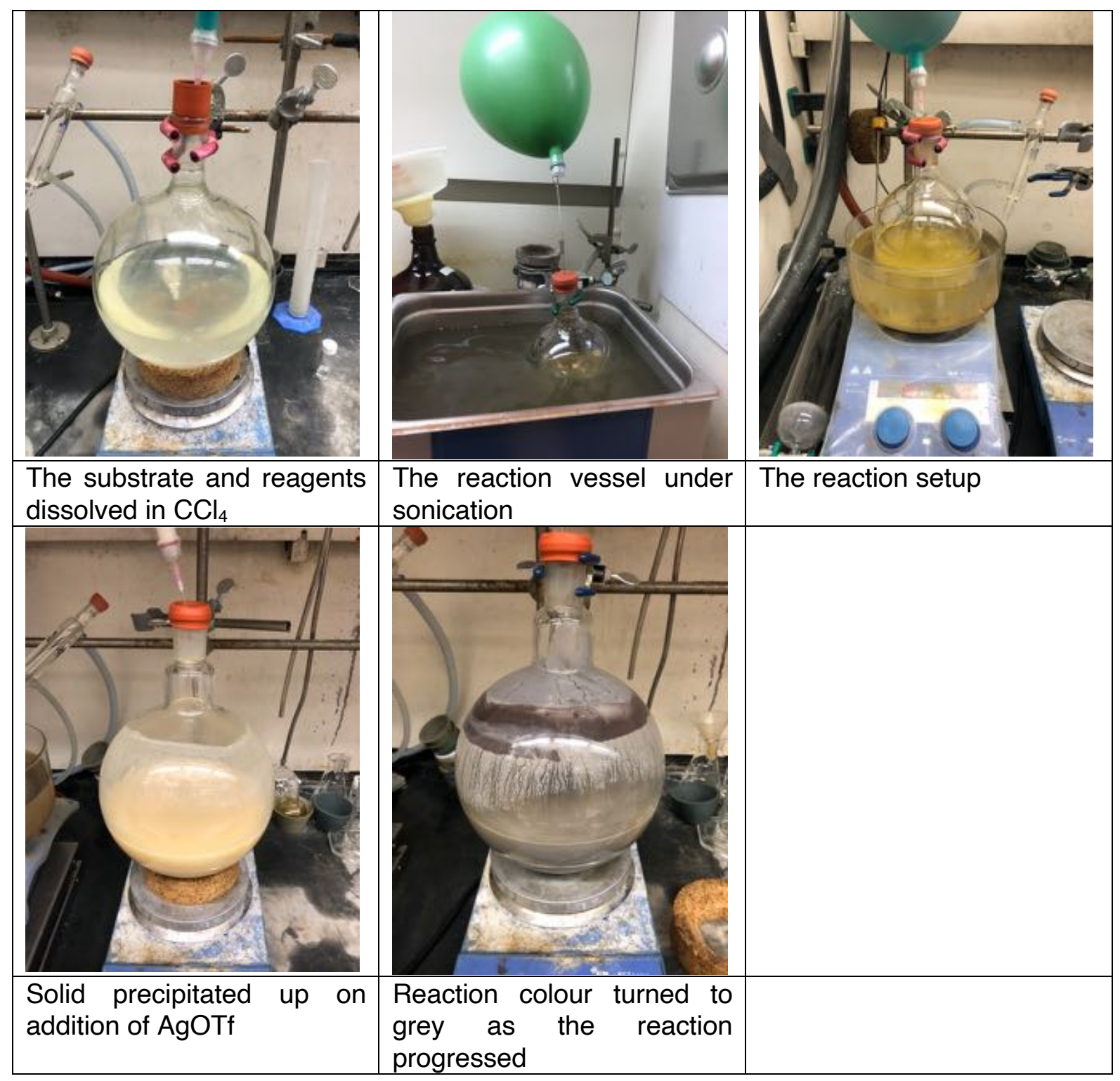


Compound 19

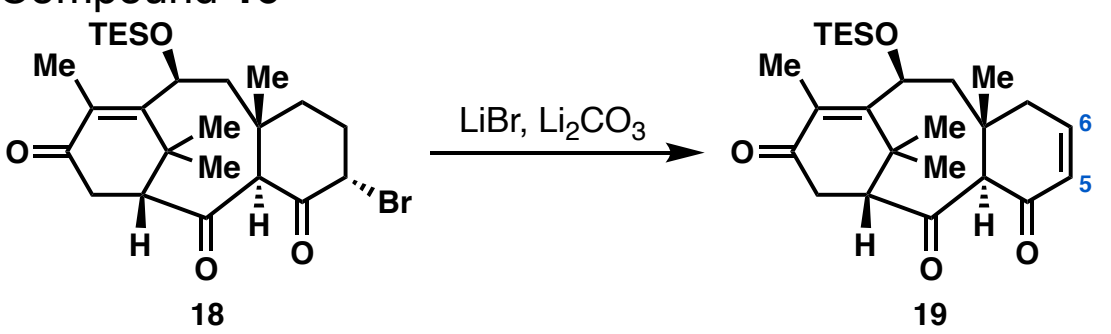

An oven-dried round bottom flask was charged with TES trione $18(5.67 \mathrm{~g}, 11.1 \mathrm{mmol})$, $\mathrm{LiBr}(2.89 \mathrm{~g}, 33.3 \mathrm{mmol}, 3 \mathrm{eq}), \mathrm{Li}_{2} \mathrm{CO}_{3}(4.10 \mathrm{~g}, 55.5 \mathrm{mmol}, 5 \mathrm{eq})$ and DMF (100 mL). The mixture was heated to $100{ }^{\circ} \mathrm{C}$ and stirred for $6 \mathrm{~h}$. After cooling to rt, to the brown mixture was added hexane/EtOAc ( $4: 1)$ and $\mathrm{LiCl}$ solution (5\% aq.) The mixture was extracted with hexane/EtOAc 4:1 (x 3). The organic phase was washed with brine, then dried over $\mathrm{MgSO}_{4}$. The solvent was removed under reduced pressure and the resulting residue was purified by flash column chromatography $\left(\mathrm{SiO}_{2}\right.$, hexane/EtOAc $10: 1$ to $\left.3: 1\right)$ to yield dienone 19 (4.21 g, $9.79 \mathrm{mmol}, 88 \%)$.

Physical state: white solid;

TLC: $R_{f}=0.44$ (hexane/EtOAc 2:1, UV active on TLC, stains orange upon $p$-anisaldehyde staining);

${ }^{1} \mathrm{H}$ NMR $\left(600 \mathrm{MHz}, \mathrm{CDCl}_{3}\right) \delta 6.76$ (ddd, $J=10.1,6.0,2.2 \mathrm{~Hz}, 1 \mathrm{H}$ ), 5.97 (dd, $J=10.1,2.0$ $\mathrm{Hz}, 1 \mathrm{H}$ ), 5.14 (dd, $J=11.2,5.9 \mathrm{~Hz}, 1 \mathrm{H}), 4.00(\mathrm{~s}, 1 \mathrm{H}), 2.91(\mathrm{dd}, J=20.0,7.1 \mathrm{~Hz}, 1 \mathrm{H}), 2.74$ $(\mathrm{d}, J=7.1 \mathrm{~Hz}, 1 \mathrm{H}), 2.57(\mathrm{dt}, J=18.8,2.7 \mathrm{~Hz}, 1 \mathrm{H}), 2.42(\mathrm{~d}, J=20.0 \mathrm{~Hz}, 1 \mathrm{H}), 2.22(\mathrm{dd}, J$ $=15.3,11.3 \mathrm{~Hz}, 1 \mathrm{H}$ ), 2.07 (ddd, $J=18.8,6.1,1.2 \mathrm{~Hz}, 1 \mathrm{H}$ ), $1.99(\mathrm{~s}, 3 \mathrm{H}), 1.68$ (dd, $J=15.4$, $6.0 \mathrm{~Hz}, 1 \mathrm{H}), 1.50(\mathrm{~s}, 3 \mathrm{H}), 1.23(\mathrm{~s}, 3 \mathrm{H}), 1.14(\mathrm{~s}, 3 \mathrm{H}), 0.96(\mathrm{t}, J=8.0 \mathrm{~Hz}, 9 \mathrm{H}), 0.61$ (q, $J=$ $8.0 \mathrm{~Hz}, 6 \mathrm{H})$.

${ }^{13} \mathrm{C}$ NMR (151 MHz, $\left.\mathrm{CDCl}_{3}\right) \delta 207.0,198.7,195.1,158.4,146.3,134.0,129.4,68.9,61.2$, 58.9, 47.1, 40.6, 40.1, 37.9, 36.2, 35.2, 24.2, 23.4, 14.0, 6.9.

HRMS (ESI-TOF): calc'd for $\mathrm{C}_{25} \mathrm{H}_{39} \mathrm{O}_{4} \mathrm{Si}\left[\mathrm{M}+\mathrm{H}^{+}\right]$: 431.2612 , found: 431.2614 . $[\alpha]^{25} \mathrm{D}:+154.6\left(c=1.0, \mathrm{CHCl}_{3}\right)$ 
Compound SI9
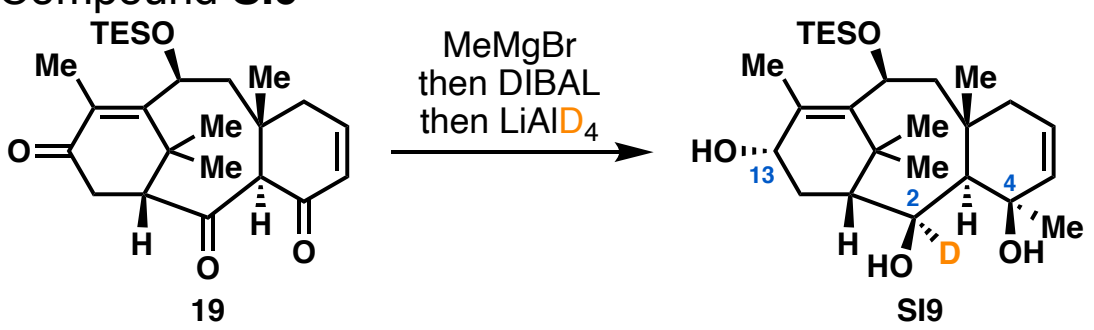

To a colorless solution of dienone $19(1.01 \mathrm{~g}, 2.34 \mathrm{mmol})$ in DCM $(50 \mathrm{~mL})$ at $0{ }^{\circ} \mathrm{C}$ was added $\mathrm{MeMgBr}\left(1.44 \mathrm{~mL}, 4.32 \mathrm{mmol}, 3.0 \mathrm{M}\right.$ in $\left.\mathrm{Et}_{2} \mathrm{O}, 1.8 \mathrm{eq}\right)$ dropwise, and the mixture was stirred for $1 \mathrm{~h}$. The reaction color turned yellow upon addition of the reagent. The reaction was quenched with $\mathrm{TESOH}(0.72 \mathrm{~mL})$ at $0^{\circ} \mathrm{C}$ and stirred for $10 \mathrm{~min}$. The volatiles were removed in vacuo. The residue was further dried via azeotrope with PhMe four times. The crude residue was dissolved in DCM $(50 \mathrm{~mL})$ and the yellow solution was cooled to $-78^{\circ} \mathrm{C}$. DIBAL (7.50 mL, $7.50 \mathrm{mmol}, 1.0 \mathrm{M}$ in PhMe, 3.2 eq) was added dropwise and the yellow mixture was stirred for $1 \mathrm{~h}$. The reaction was quenched with $\mathrm{MeOH}(5 \mathrm{~mL})$ at -78 ${ }^{\circ} \mathrm{C}$ then warmed to rt. The volatiles were removed in vacuo, and the residue was dried via azeotrope with PhMe four times. TLC indicated that small amount of unreacted substrate remained. The crude was thus dissolved in DCM $(50 \mathrm{~mL})$ and the yellow solution was cooled to $-78{ }^{\circ} \mathrm{C}$. DIBAL $(1.17 \mathrm{~mL}, 1.17 \mathrm{mmol}, 1.0 \mathrm{M}$ in PhMe, $0.50 \mathrm{eq})$ was added dropwise and the yellow reaction mixture was stirred for $1 \mathrm{~h}$. The reaction was quenched with $\mathrm{MeOH}(1 \mathrm{~mL})$ at $-78^{\circ} \mathrm{C}$ then warmed up to rt. The volatiles were removed in vacuo. The residue was further dried via azeotrope with PhMe four times. THF $(50 \mathrm{~mL})$ was added to the crude residue, followed by LiAlD 4 (285 mg, $6.95 \mathrm{mmol}, 3.0 \mathrm{eq}$ ) and this gray suspension was stirred at $\mathrm{rt}$ for $1 \mathrm{~h}$. TLC indicated that small amount of unreacted substrate remained. Additional $\mathrm{LiAlD}_{4}(106 \mathrm{mg}, 2.59 \mathrm{mmol}, 1.1 \mathrm{eq})$ was added and the reaction was heated to $60^{\circ} \mathrm{C}$ for $30 \mathrm{~min}$. The reaction mixture was then cooled to $-78^{\circ} \mathrm{C}$ and quenched with $\mathrm{NH}_{4} \mathrm{Cl}$ (saturated aq.). The aqueous layer was extracted with DCM (x 3). The combined organic phase was dried over $\mathrm{MgSO}_{4}$ and concentrated under reduced pressure. The resulting residue was purified by flash column chromatography $\left(\mathrm{SiO}_{2}\right.$, hexane/EtOAc 1:1) to yield triol SI9 (783 mg, $1.73 \mathrm{mmol}, 74 \%)$.

Physical state: white solid;

TLC: $R_{f}=0.28$ (hexane/EtOAc 2:1, slightly UV active on TLC, stains blue upon $p$ anisaldehyde staining);

${ }^{1} \mathrm{H}$ NMR (600 MHz, CDCl $\left.)_{3}\right) \delta 5.65$ (ddd, $J=9.9,6.2,1.8 \mathrm{~Hz}, 1 \mathrm{H}$ ), 5.53 (dd, J = 9.8, 1.9 $\mathrm{Hz}, 1 \mathrm{H}), 4.95$ (dd, $J=11.6,5.6 \mathrm{~Hz}, 1 \mathrm{H}), 4.42(\mathrm{~d}, J=8.7 \mathrm{~Hz}, 1 \mathrm{H}), 3.01$ (dd, J=14.7, 11.6 $\mathrm{Hz}, 1 \mathrm{H}$ ), 2.82 (ddd, $J=15.5,10.3,9.3 \mathrm{~Hz}, 1 \mathrm{H}$ ), 2.27 (dt, $J=16.9,2.5 \mathrm{~Hz}, 1 \mathrm{H}$ ), 2.14 (s, $1 \mathrm{H}), 1.96(\mathrm{~d}, J=9.2 \mathrm{~Hz}, 1 \mathrm{H}), 1.83(\mathrm{~d}, J=1.3 \mathrm{~Hz}, 3 \mathrm{H}), 1.80(\mathrm{~s}, 3 \mathrm{H}), 1.54$ (dd, J=17.0, 6.3 $\mathrm{Hz}, 1 \mathrm{H}$ ), 1.43 (dd, $J=14.7,5.6 \mathrm{~Hz}, 1 \mathrm{H}), 1.34$ (dd, $J=15.6,3.5 \mathrm{~Hz}, 1 \mathrm{H}), 1.28(\mathrm{~s}, 3 \mathrm{H}), 1.26$ (s, 3H), $1.00-0.94(\mathrm{~m}, 12 \mathrm{H}), 0.60$ (qd, J=7.9, $1.3 \mathrm{~Hz}, 6 \mathrm{H})$.

${ }^{13} \mathrm{C}$ NMR (25 of 26 observed, $151 \mathrm{MHz}, \mathrm{CDCl}_{3}$ ) $\delta$ 141.9, 134.3, 130.8, 126.2, 74.0, 68.40, 67.6, 48.3, 48.1, 45.0, 42.5, 38.7, 35.8, 35.4, 34.2, 31.8, 27.5, 26.3, 15.6, 7.1, 5.3.

HRMS (ESI-TOF): calc'd for $\mathrm{C}_{26} \mathrm{H}_{46} \mathrm{DO}_{4} \mathrm{Si}\left[\mathrm{M}+\mathrm{H}^{+}\right]$: 452.3301 , found: 452.3313 . $[\alpha]^{25} \mathrm{D}:+71.0\left(c=1.0, \mathrm{CHCl}_{3}\right)$ 


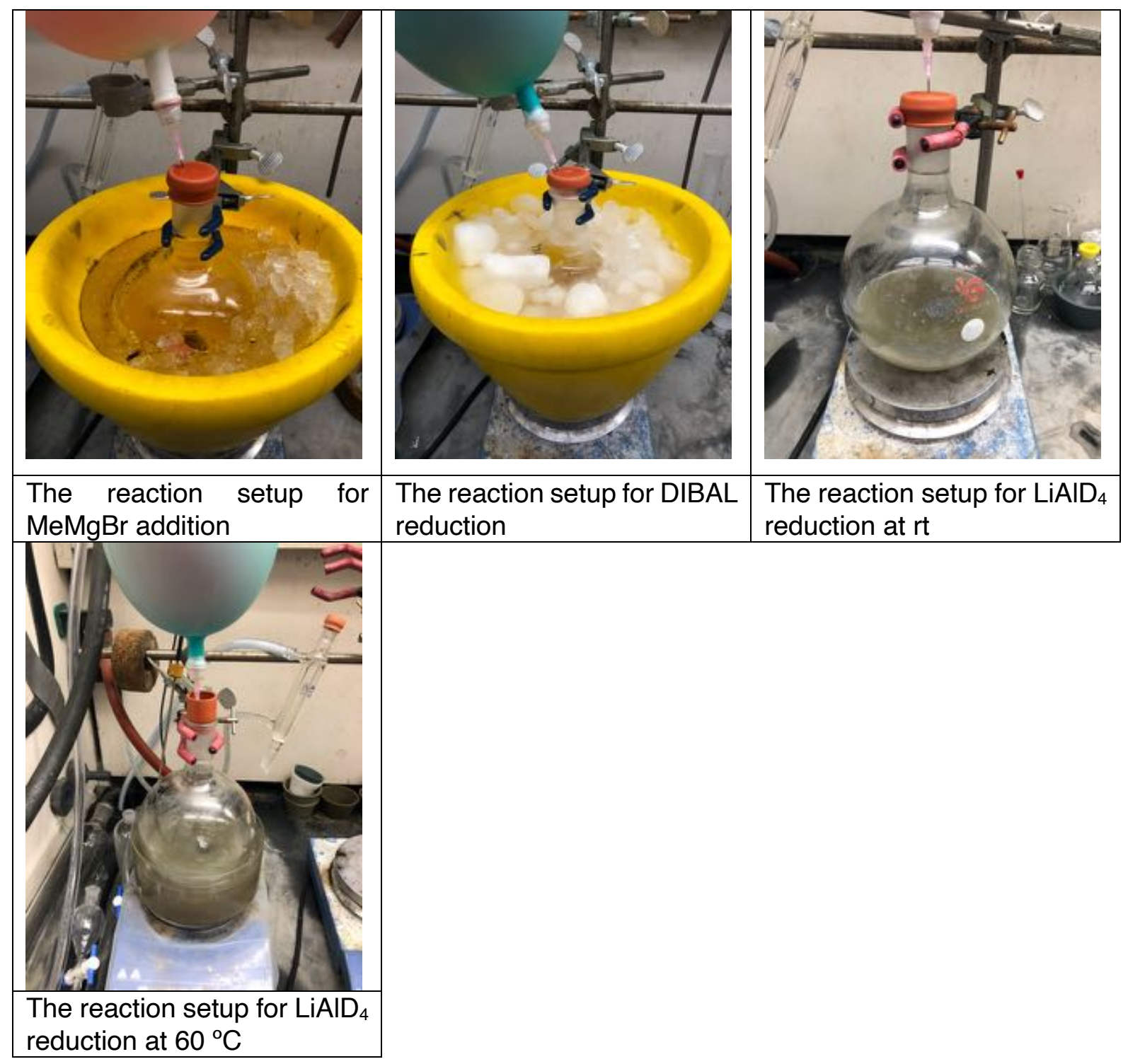


Compound 20

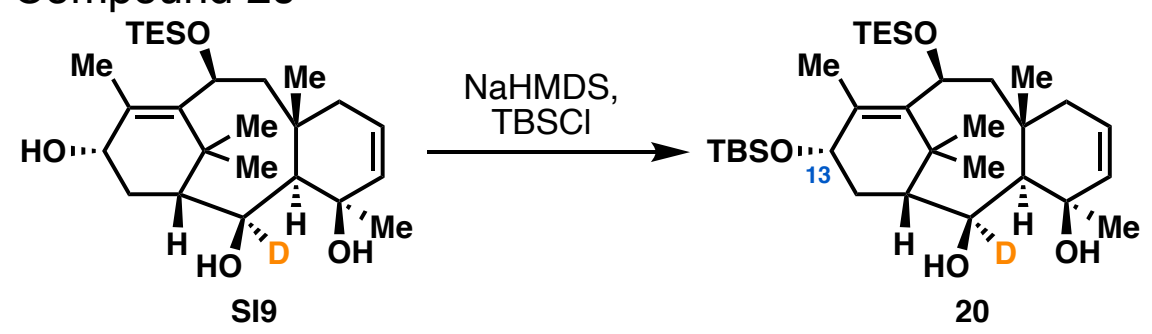

An oven-dried round-bottom flask was charged with triol SI9 $(7.75 \mathrm{~g}, 17.2 \mathrm{mmol})$ and THF $(200 \mathrm{~mL})$. The mixture was cooled to $0{ }^{\circ} \mathrm{C}$ and NaHMDS $(48 \mathrm{~mL}, 48.1 \mathrm{mmol}, 1.0 \mathrm{M}$ in THF, 2.8 eq.) was added dropwise. The yellow mixture was stirred for $30 \mathrm{~min}$, then a THF solution of TBSCl ( $8.79 \mathrm{~g}, 58.3 \mathrm{mmol}, 3.4 \mathrm{eq}$, in $37 \mathrm{~mL}$ of THF) was added dropwise. The mixture was warmed up to rt and stirred for $1 \mathrm{~h}$. The reaction was quenched with $\mathrm{NH}_{4} \mathrm{Cl}$ (saturated aq.). The aqueous layer was extracted with DCM (x 3$)$. The combined organic phase was dried over $\mathrm{MgSO}_{4}$ and concentrated under reduced pressure, and the resulting residue was purified by flash column chromatography $\left(\mathrm{SiO}_{2}\right.$, hexane/EtOAc 10:1 to $6: 1)$ to obtain diol $20(9.12 \mathrm{~g}, 16.1 \mathrm{mmol}, 94 \%)$.

Physical state: white foam;

TLC: $R_{f}=0.49$ (hexane/EtOAc 4:1, slightly UV active on TLC, stains blue upon $p$ anisaldehyde staining);

${ }^{1} \mathrm{H}$ NMR (400 MHz, CDCl 3 ) $\delta 5.64$ (ddd, $J=9.9,6.2,1.7 \mathrm{~Hz}, 1 \mathrm{H}$ ), 5.52 (dd, $J=9.9,2.5$ $\mathrm{Hz}, 1 \mathrm{H}), 4.96(\mathrm{dd}, J=11.6,5.6 \mathrm{~Hz}, 1 \mathrm{H}), 4.41(\mathrm{dd}, J=10.1,2.3 \mathrm{~Hz}, 1 \mathrm{H}), 2.99(\mathrm{dd}, J=$ 14.7, $11.6 \mathrm{~Hz}, 1 \mathrm{H}), 2.70(\mathrm{dt}, J=15.2,9.8 \mathrm{~Hz}, 1 \mathrm{H}), 2.27(\mathrm{~d}, J=16.9 \mathrm{~Hz}, 1 \mathrm{H}), 2.11(\mathrm{~d}, J=$ $9.4 \mathrm{~Hz}, 1 \mathrm{H}), 1.93(\mathrm{~d}, J=9.4 \mathrm{~Hz}, 1 \mathrm{H}), 1.80(\mathrm{~s}, 3 \mathrm{H}), 1.75(\mathrm{~s}, 3 \mathrm{H}), 1.52(\mathrm{dd}, J=16.9,6.3 \mathrm{~Hz}$, $1 \mathrm{H}), 1.41(\mathrm{dd}, J=14.7,5.6 \mathrm{~Hz}, 1 \mathrm{H}), 1.35-1.28(\mathrm{~m}, 1 \mathrm{H}), 1.25(\mathrm{~s}, 6 \mathrm{H}), 1.01-0.93(\mathrm{~m}$, $12 \mathrm{H}), 0.86(\mathrm{~s}, 9 \mathrm{H}), 0.59(\mathrm{q}, J=7.7 \mathrm{~Hz}, 6 \mathrm{H}), 0.07(\mathrm{~s}, 3 \mathrm{H}), 0.04(\mathrm{~s}, 3 \mathrm{H})$.

${ }^{13} \mathrm{C}$ NMR (151 MHz, $\left.\mathrm{CDCl}_{3}\right) \delta 140.3,134.3,132.4,132.4,126.1,73.9,68.6,68.3,48.5$, 48.4, 45.0, 42.4, 38.8, 36.4, 33.5, 32.0, 27.4, 26.4, 26.1, 18.3, 16.4, 7.1, 5.3, -3.9, -5.0.

HRMS (ESI-TOF): calc'd for $\mathrm{C}_{32} \mathrm{H}_{60} \mathrm{DO}_{4} \mathrm{Si}\left[\mathrm{M}+\mathrm{Na}^{+}\right]$: 588.3991, found: 588.3990 . $[\alpha]^{25} \mathrm{D}:+63.2\left(c=0.5, \mathrm{CHCl}_{3}\right)$ 


\section{Compound 21}

DMDO preparation

A $12 L$ four-neck flask equipped with a mechanical stirrer, a condenser and a receiving flask were assembled as a picture below. The flask was charged with $\mathrm{NaHCO}_{3}(1.0 \mathrm{~kg})$, deionized water $(1.85 \mathrm{~L})$ and acetone $(1.35 \mathrm{~L})$, and stirred at $150 \mathrm{rpm}$. The condenser and the receiving flask were cooled to $-78^{\circ} \mathrm{C}$. OXONE ${ }^{\circledR}(2.0 \mathrm{~kg})$ was slowly added to the flask in six portions over $30 \mathrm{~min}$. (Vigorous gas evolution was observed on addition of OXONE ${ }^{\circledR}$. After addition of each portion, the flask was sealed and pressure was gradually reduced (150-400 $\mathrm{mmHg}$ ) such that a steady stream of distillate condensed. Pressure had to be carefully controlled to avoid the reaction mixture frothing over into the receiving flask. The entire system was allowed to return to atmospheric pressure to add next portion of OXONE ${ }^{\circledR}$, then was set to reduced pressure again. This process was repeated until all OXONE ${ }^{\circledR}$ was added. Pressure had to be carefully controlled even after addition of all $\mathrm{OXONE}^{\circledR}$. This suspension was stirred under reduced pressure for $1.5 \mathrm{~h}$ until it stopped generating bubbles. The reaction was allowed to return to atmospheric pressure to obtain about $800 \mathrm{~mL}$ of DMDO as yellow solution. The concentration of this solution was determined by the DMDO titration procedure described below, with typical concentrations ranging from $0.09-0.085 \mathrm{M}$. This DMDO solution could be stored in a $-20^{\circ} \mathrm{C}$ freezer for six months without substantial change to concentration. 


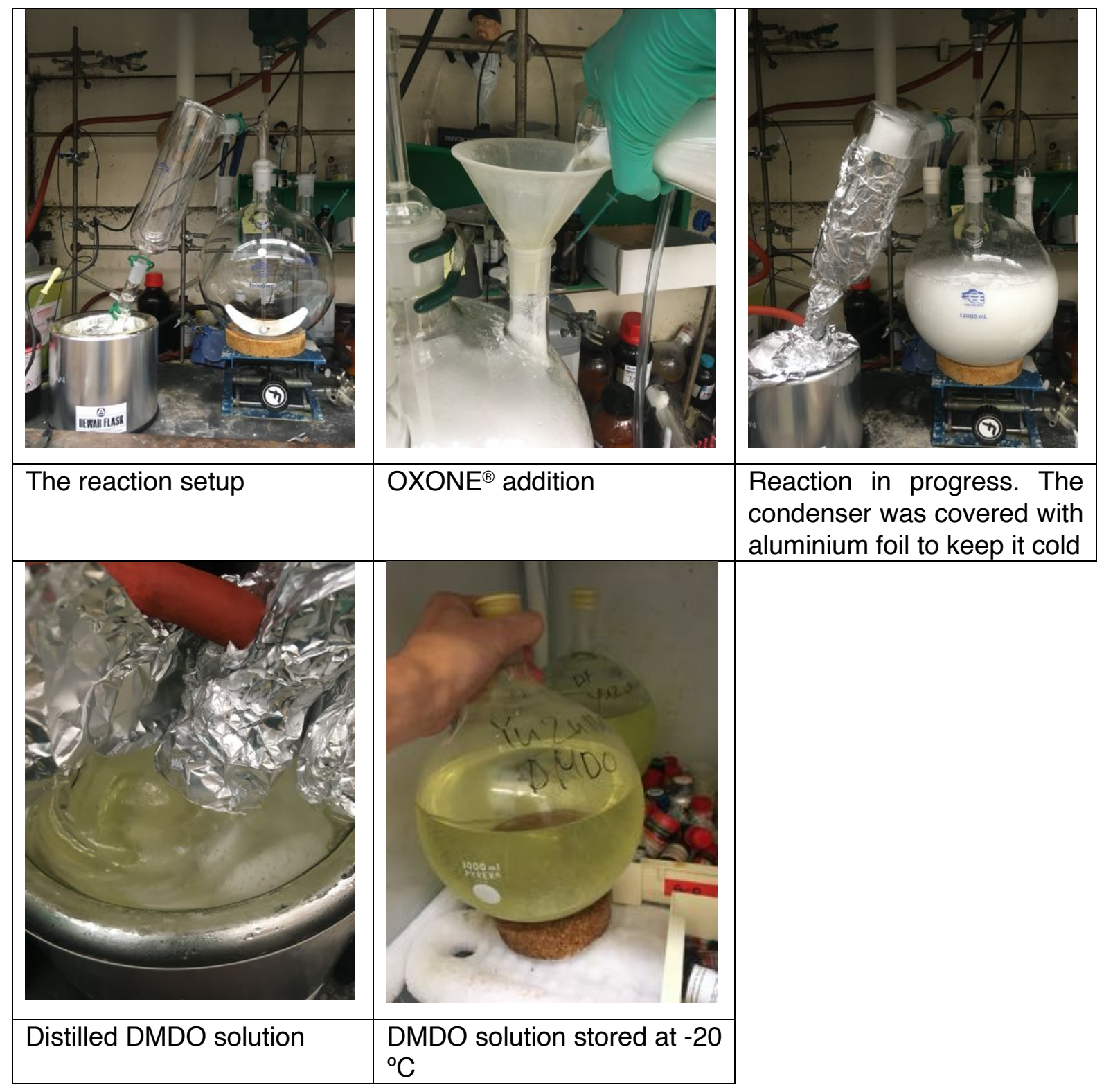

DMDO extraction ${ }^{4}$

To a $4 \mathrm{~L}$ separatory funnel which had been precooled in a $-20^{\circ} \mathrm{C}$ freezer, DMDO solution $(1.7 \mathrm{~L}, 153 \mathrm{mmol}, 0.09 \mathrm{M}$ in acetone, prepared according to DMDO preparation experimental above and stored in a $-20^{\circ} \mathrm{C}$ freezer), deionized water $(1.7 \mathrm{~L}$, precooled in a $4{ }^{\circ} \mathrm{C}$ refrigerator) and $\mathrm{CHCl}_{3}(85 \mathrm{~mL})$ was added. DMDO was quickly extracted with $\mathrm{CHCl}_{3}(85 \mathrm{~mL} \times 3)$ while it was still cold which gave a DMDO solution in acetone and $\mathrm{CHCl}_{3}$ (about $800 \mathrm{~mL}$ ). To a $2 \mathrm{~L}$ separatory funnel, which had been precooled in $-20^{\circ} \mathrm{C}$ freezer, was added this obtained DMDO solution and $\mathrm{pH}=7.0 \quad \mathrm{Na}_{2} \mathrm{HPO}_{4} / \mathrm{NaH}_{2} \mathrm{PO}_{4}$ aqueous buffer solution $\left(0.01 \mathrm{M}, 100 \mathrm{v} / \mathrm{v} \%\right.$ to DMDO solution, pre-cooled in a $4{ }^{\circ} \mathrm{C}$ fridge). The organic phase was washed with the buffer $(100 \mathrm{v} / \mathrm{v} \% \times 4)$. This process reduced acetone concentration in the organic phase. The Obtained yellow solution $(0.30 \mathrm{M})$ was transferred to a flask precooled at $0{ }^{\circ} \mathrm{C}$. The concentration of this solution was determined (normally $0.30 \mathrm{M}$ ) by the DMDO titration procedure described below. This DMDO solution was immediately used after titration. NOTE: Significant drop-offs in DMDO concentration are observed if the glassware is not pre-cooled and/or this procedure is not carried out sufficiently quickly. 


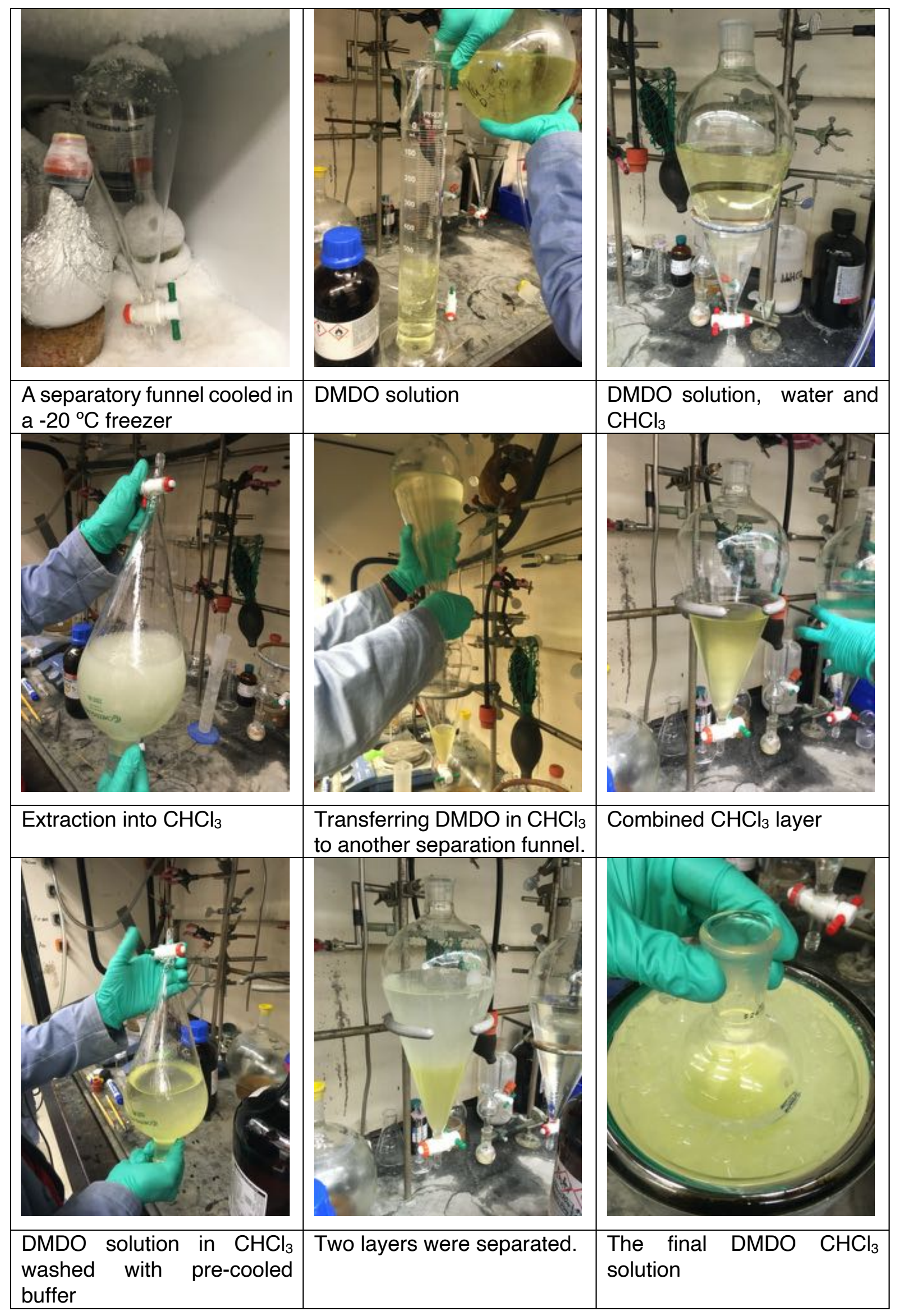


DMDO titration

Thioanisole $(15.0 \mathrm{mg}, 0.12 \mathrm{mmol})$ was placed in a test tube. DMDO $(0.2 \mathrm{~mL}$, in acetone or $\mathrm{CHCl}_{3}$ ) was added at rt open to air. DMDO is fully consumed spontaneously. An aliquot of this mixture was analyzed by ${ }^{1} \mathrm{H}$ NMR where thioanisole, methyl phenyl sulfoxide and methyl phenyl sulfone were observed. Concentration of DMDO solution was determined based on relative ${ }^{1} \mathrm{H}$ NMR integrations of each species.

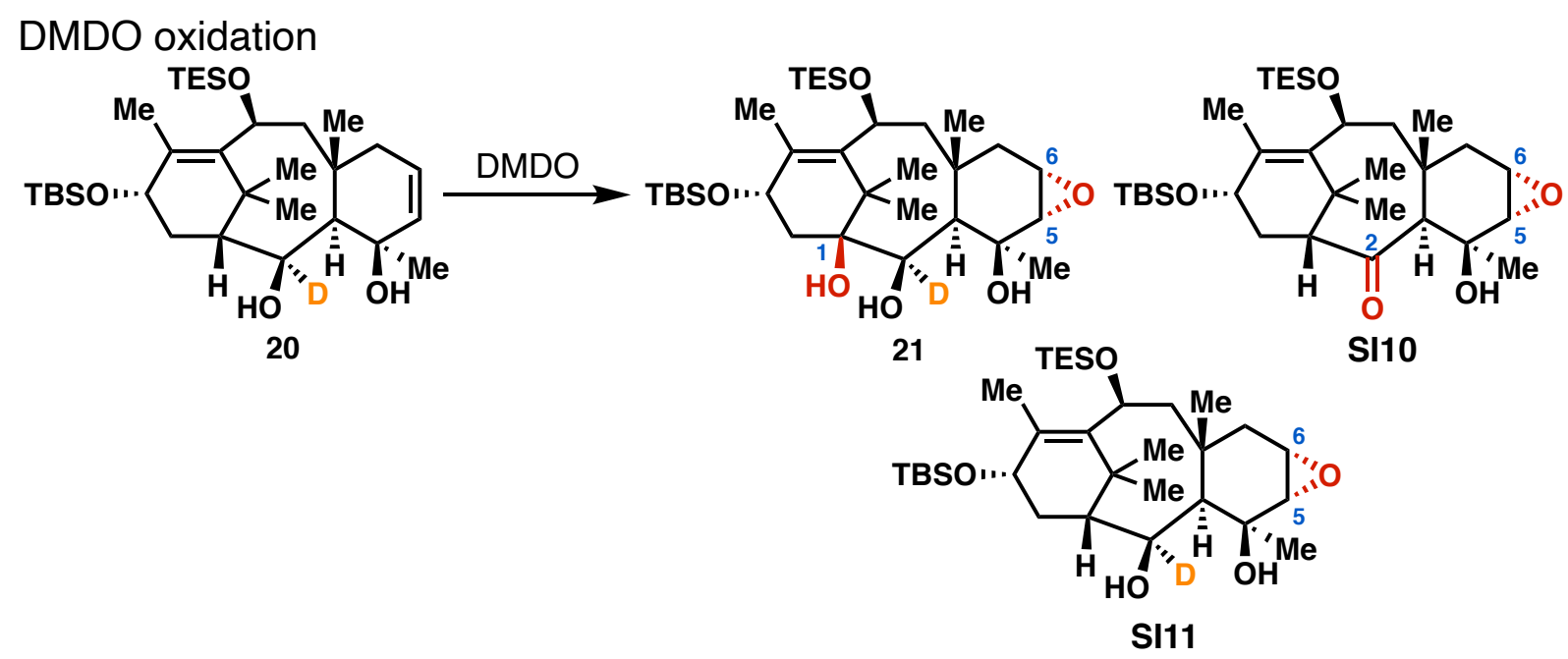

DMDO solution (prepared according to DMDO extraction experimental above, $126 \mathrm{~mL}$, $37.9 \mathrm{mmol}, 10$ eq., $0.30 \mathrm{M}$ in $\left.\mathrm{CHCl}_{3}\right)$ was added to $20(2.14 \mathrm{~g}, 3.79 \mathrm{mmol})$ and stirred at 0 ${ }^{\circ} \mathrm{C}$ for $24 \mathrm{~h}$ open to air. The reaction was quenched with 2-methyl-2-butene (12 mL, 114 $\mathrm{mmol}, 30 \mathrm{eq}$ ) and the yellow color disappeared. The reaction mixture was stirred for 5 min before being warmed to rt and stirred an additional 15 min. The mixture was concentrated under reduced pressure. The resulting residue was purified by flash column chromatography $\left(\mathrm{SiO}_{2}\right.$, hexane/EtOAc 8:1 to 2:1) to obtain triol $21(1.11 \mathrm{~g}, 1.86 \mathrm{mmol}$, $49 \%$ ) accompanying ketone SI10 (263 mg, $0.455 \mathrm{mmol}, 12 \%)$. Ketone SI10 could be recycled to diol SI11 by the following procedure and then subjected to DMDO oxidation to obtain more triol 21.

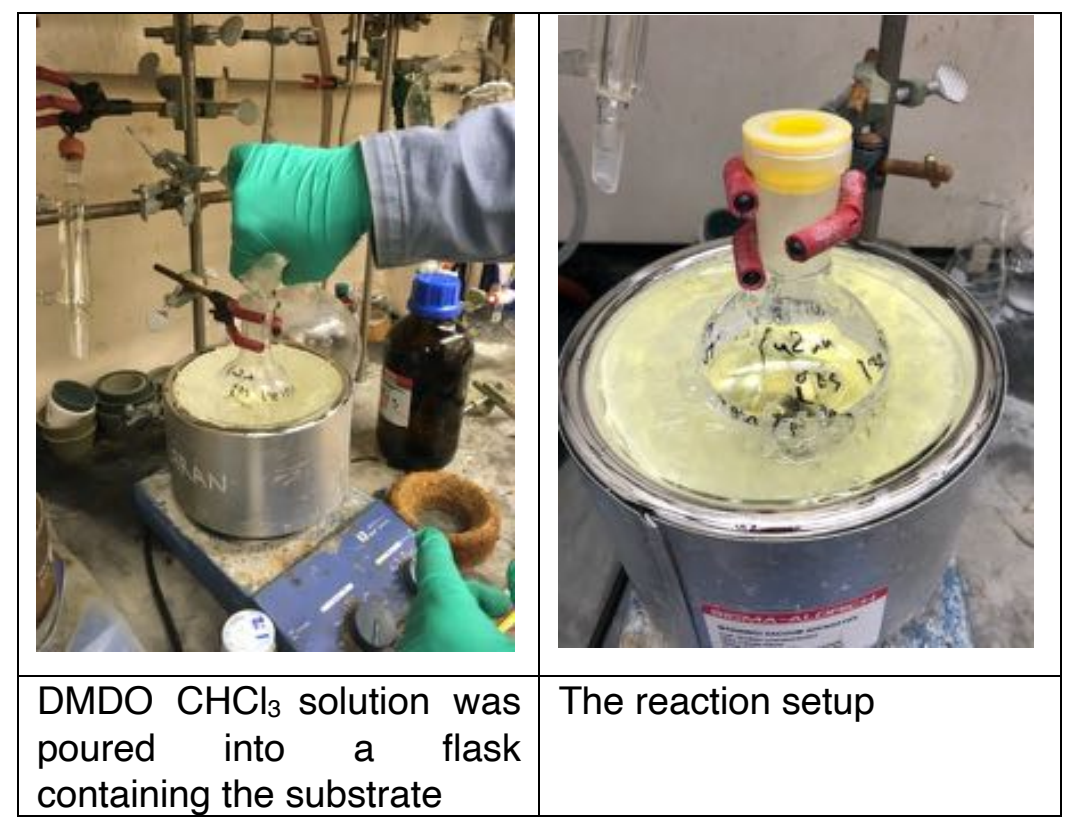


Reduction of SI10 to SI11

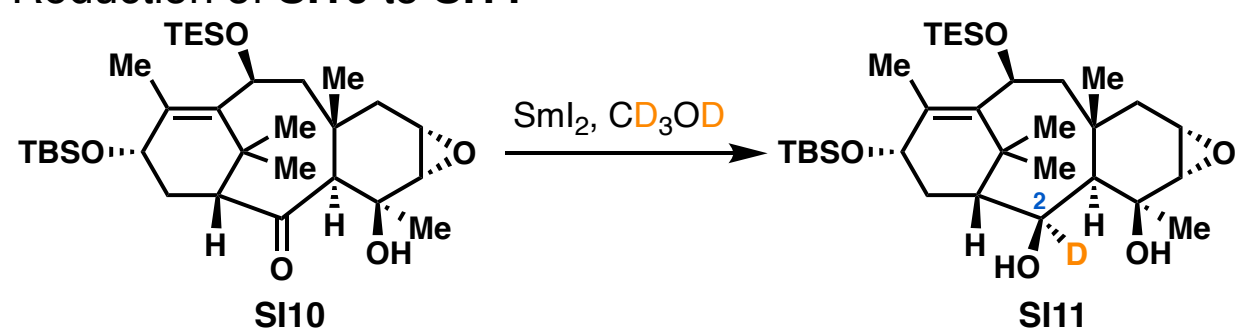

An oven dried round bottom flask was charged with SI10 (597 mg, $1.03 \mathrm{mmol}$ ), LiCl (43.7 $\mathrm{mg}, 10.3 \mathrm{mmol}, 10.0 \mathrm{eq})$, and $\mathrm{CD}_{3} \mathrm{OD}(20 \mathrm{~mL})$. This mixture was degassed for $15 \mathrm{~min}$ via sparging with argon under sonication. $\mathrm{Sml}_{2}(41.3 \mathrm{~mL}, 4.13 \mathrm{mmol}, 0.1 \mathrm{M}$ in THF, $4.0 \mathrm{eq})$ was added at rt. The resulting blue suspension was stirred at the same temperature for 2 $\mathrm{h}$. The mixture was concentrated under reduced pressure. The resulting residue was purified by flash column chromatography $\left(\mathrm{SiO}_{2}\right.$, hexane/EtOAc 6:1 to 4:1) to obtain SI11 (558 mg, $0.960 \mathrm{mmol}, 93 \%$ ).

\section{Compound 21}

Physical state: colorless wax;

TLC: $R_{f}=0.20$ (hexane/EtOAc 4:1, not UV active on TLC, stains blue upon $p$ anisaldehyde staining);

${ }^{1} \mathrm{H}$ NMR $\left(600 \mathrm{MHz}, \mathrm{CDCl}_{3}\right) \delta 4.86(\mathrm{dd}, J=11.8,5.4 \mathrm{~Hz}, 1 \mathrm{H}), 4.54(\mathrm{dd}, J=10.0,2.0 \mathrm{~Hz}$, $1 \mathrm{H}), 3.26(\mathrm{dd}, J=6.4,3.7 \mathrm{~Hz}, 1 \mathrm{H}), 2.96-2.87(\mathrm{~m}, 2 \mathrm{H}), 2.50(\mathrm{dd}, J=15.2,10.0 \mathrm{~Hz}, 1 \mathrm{H})$, $1.97(\mathrm{~s}, 1 \mathrm{H}), 1.90(\mathrm{~d}, J=14.6 \mathrm{~Hz}, 1 \mathrm{H}), 1.76(\mathrm{~d}, J=1.3 \mathrm{~Hz}, 3 \mathrm{H}), 1.68(\mathrm{~s}, 3 \mathrm{H}), 1.59$ (dd, $J$ $=15.2,3.7 \mathrm{~Hz}, 1 \mathrm{H}), 1.47-1.43(\mathrm{~m}, 4 \mathrm{H}), 1.28-1.22(\mathrm{~m}, 4 \mathrm{H}), 1.00(\mathrm{~s}, 3 \mathrm{H}), 0.94(\mathrm{t}, J=8.0$ $\mathrm{Hz}, 9 \mathrm{H}), 0.88(\mathrm{~s}, 9 \mathrm{H}), 0.60-0.54(\mathrm{~m}, 6 \mathrm{H}), 0.07(\mathrm{~s}, 3 \mathrm{H}), 0.04(\mathrm{~s}, 3 \mathrm{H})$.

${ }^{13} \mathrm{C}$ NMR (151 MHz, $\left.\mathrm{CDCl}_{3}\right) \delta 139.8,134.3,77.4,74.2,69.3,68.5,60.2,54.1,47.9,44.5$, 43.6, 42.7, 40.9, 36.3, 29.6, 29.4, 28.9, 26.0, 20.5, 18.3, 16.7, 7.1, 5.2, -3.9, -5.1.

HRMS (ESI-TOF): calc'd for $\mathrm{C}_{32} \mathrm{H}_{60} \mathrm{DO}_{6} \mathrm{Si}_{2}\left[\mathrm{M}+\mathrm{Na}^{+}\right]$: 620.3889 , found: 620.3900 .

$[\alpha]^{25} \mathrm{D}:+67.0\left(c=1.0, \mathrm{CHCl}_{3}\right)$

\section{Compound SI10}

Physical state: colorless wax;

TLC: $R_{f}=0.70$ (hexane/EtOAc 4:1, not UV active on TLC, stains orange upon $p$ anisaldehyde staining);

${ }^{1} \mathrm{H}$ NMR $\left(600 \mathrm{MHz}, \mathrm{CDCl}_{3}\right) \delta 4.95$ (dd, $\left.J=11.4,5.9 \mathrm{~Hz}, 1 \mathrm{H}\right), 4.56(\mathrm{~s}, 1 \mathrm{H}), 4.50$ (dd, $J=$ 9.9, $2.4 \mathrm{~Hz}, 1 \mathrm{H}$ ), 3.22 (dd, $J=6.1,3.7 \mathrm{~Hz}, 1 \mathrm{H}), 2.96(\mathrm{~s}, 1 \mathrm{H}), 2.93(\mathrm{~d}, J=3.7 \mathrm{~Hz}, 1 \mathrm{H}), 2.54$ (ddd, $J=15.8,10.0,8.3 \mathrm{~Hz}, 1 \mathrm{H}$ ), $2.33(\mathrm{~d}, J=8.2 \mathrm{~Hz}, 1 \mathrm{H}$ ), 2.09 (dd, $J=15.3,11.4 \mathrm{~Hz}$, 1H), $2.05-1.97(\mathrm{~m}, 2 \mathrm{H}), 1.93(\mathrm{~d}, J=1.5 \mathrm{~Hz}, 3 \mathrm{H}), 1.53(\mathrm{dd}, J=15.0,6.2 \mathrm{~Hz}, 1 \mathrm{H}), 1.43$ $(\mathrm{s}, 3 \mathrm{H}), 1.34-1.28(\mathrm{~m}, 4 \mathrm{H}), 1.17(\mathrm{~s}, 3 \mathrm{H}), 1.03(\mathrm{~s}, 3 \mathrm{H}), 0.97-0.91(\mathrm{~m}, 18 \mathrm{H}), 0.57(\mathrm{q}, J=$ $8.1 \mathrm{~Hz}, 6 \mathrm{H}), 0.13(\mathrm{~s}, 3 \mathrm{H}), 0.08(\mathrm{~s}, 3 \mathrm{H})$.

${ }^{13} \mathrm{C}$ NMR (126 MHz, $\left.\mathrm{CDCl}_{3}\right) \delta$ 221.8, 138.1, 135.3, 71.4, 68.1, 67.2, 61.7, 58.9 52.9, 49.6, 48.8, 38.4, 37.6, 37.2, 31.4, 31.4, 30.3, 27.9, 26.0, 25.5, 18.2, 16.7, 7.0, 5.1, -3.8, -5.1.

HRMS (ESI-TOF): calc'd for $\mathrm{C}_{32} \mathrm{H}_{59} \mathrm{O}_{5} \mathrm{Si}_{2}\left[\mathrm{M}+\mathrm{H}^{+}\right]$: 579.3896 , found: 579.3886 .

$[\alpha]^{25} \mathrm{D}:+42.6\left(c=0.25, \mathrm{CHCl}_{3}\right)$

Compound SI11

Physical state: colorless wax; 
TLC: $R_{f}=0.40$ (hexane/EtOAc 4:1, not UV active on TLC, stains blue upon $p$ anisaldehyde staining);

${ }^{1} \mathrm{H}$ NMR (600 MHz, CDCl 3$) \delta 4.87$ (dd, $J=11.7,5.6 \mathrm{~Hz}, 1 \mathrm{H}$ ), $4.36(\mathrm{dd}, J=10.1,2.0 \mathrm{~Hz}$, $1 \mathrm{H}), 3.26(\mathrm{dd}, J=6.5,3.7 \mathrm{~Hz}, 1 \mathrm{H}), 2.97-2.89(\mathrm{~m}, 2 \mathrm{H}), 2.65(\mathrm{dt}, J=15.1,9.8 \mathrm{~Hz}, 1 \mathrm{H})$, $1.97-1.89(\mathrm{~m}, 2 \mathrm{H}), 1.82(\mathrm{~d}, J=9.2 \mathrm{~Hz}, 1 \mathrm{H}), 1.79(\mathrm{~d}, J=1.5 \mathrm{~Hz}, 3 \mathrm{H}), 1.74(\mathrm{~s}, 3 \mathrm{H}), 1.45$ (dd, $J=14.7,6.6 \mathrm{~Hz}, 1 \mathrm{H}), 1.39(\mathrm{~s}, 3 \mathrm{H}), 1.31-1.21(\mathrm{~m}, 5 \mathrm{H}), 0.97-0.92(\mathrm{~m}, 12 \mathrm{H}), 0.89$ (s, 9H), 0.57 (qd, $J=7.9,1.4 \mathrm{~Hz}, 6 \mathrm{H}), 0.08$ (s, 3H), 0.04 (s, 3H).

${ }^{13} \mathrm{C}$ NMR (151 MHz, $\left.\mathrm{CDCl}_{3}\right) \delta$ 139.6, 132.8, 74.4, 68.4, 68.0, 60.1, 54.1, 48.9, 48.4, 41.0, 41.0, 38.5, 36.1, 36.0, 33.5, 29.5, 28.8, 26.4, 26.1, 18.3, 16.7, 7.1, 5.2, -3.8, -5.1. HRMS (ESI-TOF): calcd for $\mathrm{C}_{32} \mathrm{H}_{60} \mathrm{DO}_{5} \mathrm{Si}_{2}\left[\mathrm{M}+\mathrm{H}^{+}\right]$: 582.4120 , found: 582.4115 .

$[\alpha]^{25} \mathrm{D}:+80.6\left(c=0.5, \mathrm{CHCl}_{3}\right)$ 
Compound 22

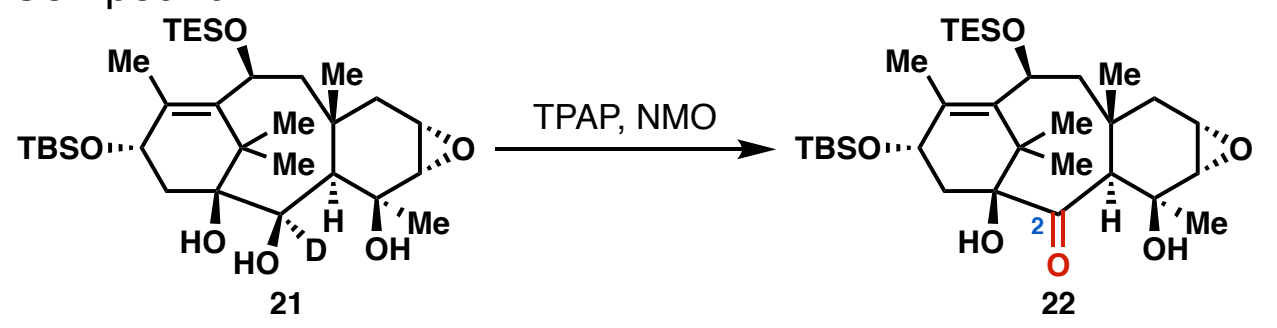

An oven-dried round-bottom flask was charged with triol $21(6.65 \mathrm{~g}, 11.1 \mathrm{mmol})$, NMO (5.20 g, $44.4 \mathrm{mmol}, 4.0 \mathrm{eq})$ and DCM (150 mL). TPAP (780 mg, $2.22 \mathrm{mmol}, 20 \mathrm{~mol} \%)$ was added at rt and the reaction color turned black. After $2 \mathrm{~h}$, the mixture was directly loaded onto flash column chromatography and purified $\left(\mathrm{SiO}_{2}\right.$, hexane/EtOAc 10:1 to 6:1) to obtain keto alcohol 22 (5.34 g, $8.59 \mathrm{mmol}, 81 \%)$.

Physical state: colorless wax;

TLC: $R_{f}=0.64$ (hexane/EtOAc 4:1, slightly UV active on TLC, stains blue upon $p$ anisaldehyde staining);

${ }^{1} \mathrm{H}$ NMR (600 MHz, $\left.\mathbf{C D C l}_{3}\right) \delta 4.96$ (dd, $\left.J=11.5,5.7 \mathrm{~Hz}, 1 \mathrm{H}\right), 4.73$ (dd, $J=9.7,2.3 \mathrm{~Hz}$, $1 \mathrm{H}), 3.30(\mathrm{~s}, 1 \mathrm{H}), 3.23(\mathrm{dd}, J=6.0,3.8 \mathrm{~Hz}, 1 \mathrm{H}), 2.96(\mathrm{~d}, J=3.7 \mathrm{~Hz}, 1 \mathrm{H}), 2.51(\mathrm{dd}, J=$ 15.9, $9.8 \mathrm{~Hz}, 1 \mathrm{H}), 2.23(\mathrm{dd}, J=15.8,3.9 \mathrm{~Hz}, 1 \mathrm{H}), 2.06-1.99(\mathrm{~m}, 2 \mathrm{H}), 1.91(\mathrm{~d}, J=1.5$ $\mathrm{Hz}, 3 \mathrm{H}), 1.55$ (dd, $J=15.0,6.1 \mathrm{~Hz}, 1 \mathrm{H}), 1.34-1.28(\mathrm{~m}, 4 \mathrm{H}), 1.22(\mathrm{~s}, 3 \mathrm{H}), 1.19(\mathrm{~s}, 3 \mathrm{H})$, $1.10(\mathrm{~s}, 3 \mathrm{H}), 0.97-0.91(\mathrm{~m}, 18 \mathrm{H}), 0.57(\mathrm{q}, J=8.0 \mathrm{~Hz}, 6 \mathrm{H}), 0.13(\mathrm{~s}, 3 \mathrm{H}), 0.08(\mathrm{~s}, 3 \mathrm{H})$.

${ }^{13} \mathrm{C}$ NMR $\left(151 \mathrm{MHz}, \mathrm{CDCl}_{3}\right) \delta 221.7,138.7,136.9,84.3,71.6,69.0,68.2,58.9,52.7,51.4$, 48.4, 42.2, 39.3, 38.2, 37.7, 30.3, 27. 8, 26.0, 19.9, 18.2, 17.0, 7.0, 5.1, -3.8, -5.1s.

HRMS (ESI-TOF): calcd for $\mathrm{C}_{33} \mathrm{H}_{59} \mathrm{O}_{7} \mathrm{Si}_{2}\left[\mathrm{M}+\mathrm{H}^{+}\right]$: 595.3845 , found: 595.3838 .

$[\alpha]^{25} \mathrm{D}:+63.8\left(c=0.5, \mathrm{CHCl}_{3}\right)$ 
Compound 24

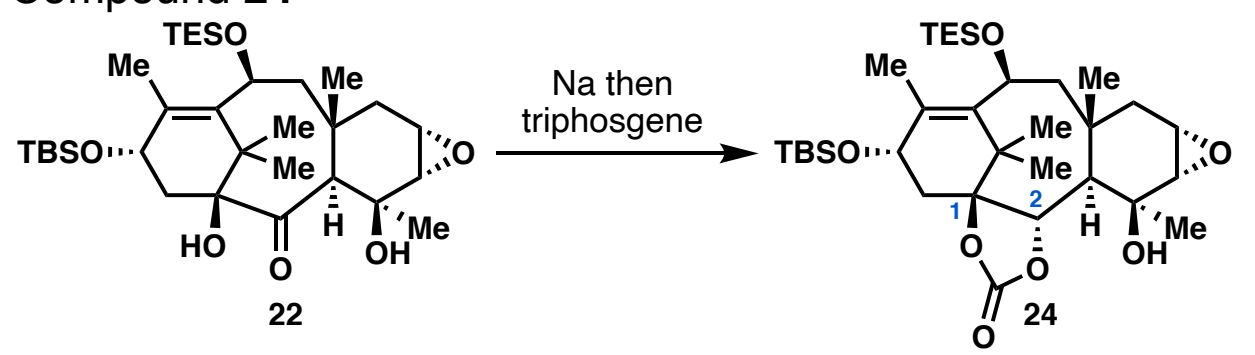

An oven-dried round-bottom flask was charged with keto alcohol $22(1.99 \mathrm{~g}, 3.34 \mathrm{mmol})$, $i$-PrOH $(72 \mathrm{~mL})$ and $\mathrm{Et}_{2} \mathrm{O}(216 \mathrm{~mL})$. Na $(5.40 \mathrm{~g}, 235 \mathrm{mmol}, 70.3 \mathrm{eq})$ was added at $\mathrm{rt}$ and the mixture was vigorously stirred for $4 \mathrm{~h}$. The reaction was quenched with $\mathrm{NH}_{4} \mathrm{Cl}$ (saturated aq.). The mixture was neutralized with $\mathrm{pH}=7.4 \mathrm{~K}_{2} \mathrm{HPO}_{4} / \mathrm{KH}_{2} \mathrm{PO}_{4}$ aqueous buffer solution $(0.2 \mathrm{M})$. Volatiles were removed under reduced pressure and the residue was further dried via azeotrope with $\mathrm{PhMe}$ four times. The crude residue was dissolved in DCM (200 mL), and DMAP (934 mg, $7.65 \mathrm{mmol}, 2.3 \mathrm{eq})$ and pyridine $(5.2 \mathrm{~mL}, 64.6$ $\mathrm{mmol}, 19 \mathrm{eq})$ was added. The reaction was cooled to $-78^{\circ} \mathrm{C}$ before triphosgene $(1.05 \mathrm{~g}$, $3.54 \mathrm{mmol}$, in $35 \mathrm{~mL}$ of DCM, $1.05 \mathrm{eq}$ ) was added dropwise. On completion of addition, the mixture was warmed to $0{ }^{\circ} \mathrm{C}$ and stirred for $3 \mathrm{~h}$. The reaction was quenched with $\mathrm{NaHCO}_{3}$ (saturated aq.). The aqueous layer was extracted with DCM (x 3). The combined organic phase was dried over $\mathrm{MgSO}_{4}$ and concentrated under reduced pressure. The resulting residue was purified by flash column chromatography $\left(\mathrm{SiO}_{2}\right.$, hexane/EtOAc 6:1) to obtain carbonate $24(1.06 \mathrm{~g}, 1.71 \mathrm{mmol}, 51 \%)$ accompanied by residual starting material 22 (159 mg, $0.268 \mathrm{mmol}, 8 \%)$.

Physical state: white solid;

TLC: $R_{f}=0.47$ (hexane/EtOAc 4:1, not UV active on TLC, stains blue upon $p$ anisaldehyde staining);

${ }^{1} \mathrm{H}$ NMR $\left(600 \mathrm{MHz}, \mathrm{CDCl}_{3}\right) \delta 4.84(\mathrm{dd}, J=11.4,5.9 \mathrm{~Hz}, 1 \mathrm{H}), 4.66$ (dd, $J=9.6,2.5 \mathrm{~Hz}$, $1 \mathrm{H}), 4.61(\mathrm{~d}, J=5.9 \mathrm{~Hz}, 1 \mathrm{H}$ ), $3.21(\mathrm{dd}, J=6.0,3.6 \mathrm{~Hz}, 1 \mathrm{H}), 2.93(\mathrm{~d}, J=3.7 \mathrm{~Hz}, 1 \mathrm{H}), 2.50$ (dd, $J=15.2,9.7 \mathrm{~Hz}, 1 \mathrm{H}$ ), $2.34(\mathrm{dd}, J=15.2,4.0 \mathrm{~Hz}, 1 \mathrm{H}$ ), 2.04 (dd, $J=15.7,11.3 \mathrm{~Hz}$, $1 \mathrm{H}), 1.95(\mathrm{~d}, J=5.9 \mathrm{~Hz}, 1 \mathrm{H}), 1.86(\mathrm{~d}, J=15.0 \mathrm{~Hz}, 1 \mathrm{H}), 1.82(\mathrm{~d}, J=1.6 \mathrm{~Hz}, 3 \mathrm{H}), 1.58(\mathrm{~m}$, 4H), $1.53(\mathrm{~s}, 3 \mathrm{H}), 1.35(\mathrm{dd}, J=15.7,5.8 \mathrm{~Hz}, 1 \mathrm{H}), 1.14(\mathrm{~s}, 3 \mathrm{H}), 1.09(\mathrm{~s}, 3 \mathrm{H}), 0.96-0.87$ $(\mathrm{m}, 18 \mathrm{H}), 0.55(\mathrm{q}, J=8.2 \mathrm{~Hz}, 6 \mathrm{H}), 0.10(\mathrm{~s}, 3 \mathrm{H}), 0.07(\mathrm{~s}, 3 \mathrm{H})$.

${ }^{13} \mathrm{C}$ NMR (151 MHz, CDCl 3$) \delta$ 153.1, 139.0, 136.1, 91.8, 82.4, 72.0, 68.4, 67.3, 60.6, 53.0, 49.0, 41.7, 37.9, 37.0, 36.5, 30.4, 27.6, 27.3, 25.9, 20.3, 18.2, 16.9, 6.9, 5.0, -3.9, -5.2.

HRMS (ESI-TOF): calc'd for $\mathrm{C}_{33} \mathrm{H}_{59} \mathrm{O}_{7} \mathrm{Si}_{2}\left[\mathrm{M}+\mathrm{H}^{+}\right]$: 623.3794, found: 623.3809. $[\alpha]^{25} \mathrm{D}:+55.4\left(c=0.5, \mathrm{CHCl}_{3}\right)$ 
Compound 27

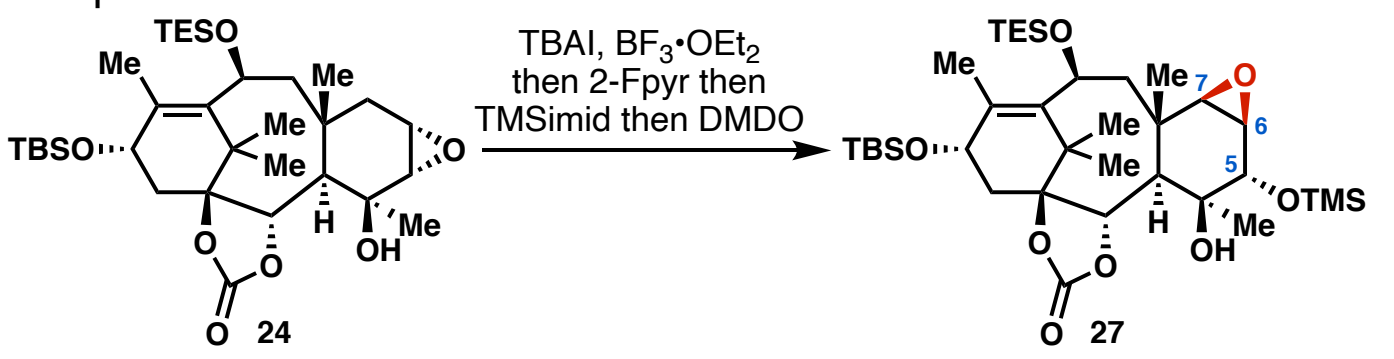

An oven-dried round-bottom flask was charged with carbonate 24 (46.2 mg, $74.2 \mu \mathrm{mol})$, TBAI (54.7 mg, $0.148 \mathrm{mmol}, 2.0 \mathrm{eq})$ and DCM $(5 \mathrm{~mL})$. The mixture was cooled to $-78{ }^{\circ} \mathrm{C}$ and $\mathrm{BF}_{3} \cdot \mathrm{OEt}_{2}(28 \mu \mathrm{L}, 0.223 \mathrm{mmol}, 3.0 \mathrm{eq})$ was added dropwise. The reaction was maintained at the same temperature for $1 \mathrm{~h}$ then quenched with 2-fluoropyridine $(80 \mu \mathrm{l})$ and stirred for additional $10 \mathrm{~min}$. TMS-imidazole ( $33 \mu \mathrm{L}, 0.223 \mathrm{mmol}, 3.0 \mathrm{eq}$ ) was added and the mixture was stirred for $5 \mathrm{~min}$ before the reaction was warmed up to rt. After 4.5 $\mathrm{h}$, the reaction was cooled to $-78^{\circ} \mathrm{C}$, and a mixture of $\mathrm{pH}=7.4 \mathrm{~K}_{2} \mathrm{HPO}_{4} / \mathrm{KH}_{2} \mathrm{PO}_{4}$ aqueous buffer solution $(2.0 \mathrm{~mL}, 0.2 \mathrm{M})$ and DMDO $(6.0 \mathrm{~mL}, 0.540 \mathrm{mmol}, 0.09 \mathrm{M}$ in acetone, 7.2 eq) at $-78^{\circ} \mathrm{C}$ was added in one portion. The reaction was warmed up to rt and stirred for $1 \mathrm{~h}$. To this mixture was added extra DMDO $(5.5 \mathrm{~mL}, 0.495 \mathrm{mmol}, 0.09 \mathrm{M}$ in acetone, 6.7 eq). The reaction was stirred for $1 \mathrm{~h}$ before volatiles were removed under reduced pressure. The resulting residue was dissolved in a solution of DMDO $(20 \mathrm{~mL}, 1.8 \mathrm{mmol}$, $0.09 \mathrm{M}$ in acetone, $24 \mathrm{eq}$ ) at rt and stirred for $7.5 \mathrm{~h}$. TLC indicated that a small amount of unreacted substrate remained. Volatiles were removed under reduced pressure and the residue was dissolved in DMDO (40 mL, $3.6 \mathrm{mmol}, 0.09 \mathrm{M}$ in acetone, $48 \mathrm{eq})$. After $7 \mathrm{~h}$, the reaction was quenched with 2-methyl-2-butene $(10 \mathrm{~mL}, 94.4 \mathrm{mmol}, 1272 \mathrm{eq})$. Volatiles were removed under reduced pressure. The residue was dissolved in DCM and water. The aqueous layer was extracted with DCM (x 3). The combined organic phase was dried over $\mathrm{MgSO}_{4}$. The solvent was removed under reduced pressure and the resulting residue was purified by preparative TLC $\left(\mathrm{SiO}_{2}\right.$, hexane/EtOAc 6:1) to yield epoxide 27 (22.9 mg, $32.2 \mu \mathrm{mol}, 43 \%)$.

Physical state: white solid;

TLC: $R_{f}=0.53$ (hexane/EtOAc 6:1, not UV active on TLC, stains blue upon $p$ anisaldehyde staining);

${ }^{1} \mathrm{H}$ NMR $\left(600 \mathrm{MHz}, \mathrm{CDCl}_{3}\right) \delta 5.06(\mathrm{dd}, J=10.3,6.0 \mathrm{~Hz}, 1 \mathrm{H}), 4.72(\mathrm{dd}, J=9.4,3.1 \mathrm{~Hz}$, $1 \mathrm{H}), 4.66(\mathrm{~d}, J=5.7 \mathrm{~Hz}, 1 \mathrm{H}), 3.82(\mathrm{~d}, J=2.4 \mathrm{~Hz}, 1 \mathrm{H}), 3.11(\mathrm{~d}, J=4.5 \mathrm{~Hz}, 1 \mathrm{H}), 2.90(\mathrm{dd}$, $J=4.5,2.4 \mathrm{~Hz}, 1 \mathrm{H}), 2.59(\mathrm{~s}, 1 \mathrm{H}), 2.52(\mathrm{dd}, J=15.4,9.5 \mathrm{~Hz}, 1 \mathrm{H}), 2.45$ (dd, $J=15.3,4.4$ $\mathrm{Hz}, 1 \mathrm{H}), 2.11-2.03(\mathrm{~m}, 2 \mathrm{H}), 1.94(\mathrm{dd}, J=15.5,6.0 \mathrm{~Hz}, 1 \mathrm{H}), 1.77(\mathrm{~d}, J=1.5 \mathrm{~Hz}, 3 \mathrm{H})$, $1.58(\mathrm{~s}, 3 \mathrm{H}), 1.37(\mathrm{~s}, 3 \mathrm{H}), 1.19(\mathrm{~s}, 3 \mathrm{H}), 1.06(\mathrm{~s}, 3 \mathrm{H}), 0.95(\mathrm{t}, J=8.0 \mathrm{~Hz}, 9 \mathrm{H}), 0.90(\mathrm{~s}, 9 \mathrm{H})$, 0.59 (q, $J=8.0 \mathrm{~Hz}, 6 \mathrm{H}), 0.16(\mathrm{~s}, 9 \mathrm{H}), 0.12(\mathrm{~s}, 3 \mathrm{H}), 0.09$ (s, 3H).

${ }^{13} \mathrm{C}$ NMR (151 MHz, CDCl 3$) \delta$ 153.0, 138.2, 136.3, 91.9, 82.1, 77.9, 68.6, 68.1, 59.9, 56.0, $48.6,47.2,40.9,38.0,38.0,28.0,27.7,26.2,22.8,20.9,18.4,16.6,7.0,5.0,0.1$, -3.8, 5.0 .

HRMS (ESI-TOF): calc'd for $\mathrm{C}_{36} \mathrm{H}_{67} \mathrm{O}_{8} \mathrm{Si}_{3}\left[\mathrm{M}+\mathrm{H}^{+}\right]$: 711.4138 , found: 711.4137 . $[\alpha]^{25} \mathrm{D}:+37.4\left(c=0.5, \mathrm{CHCl}_{3}\right)$ 
Compound Sl12

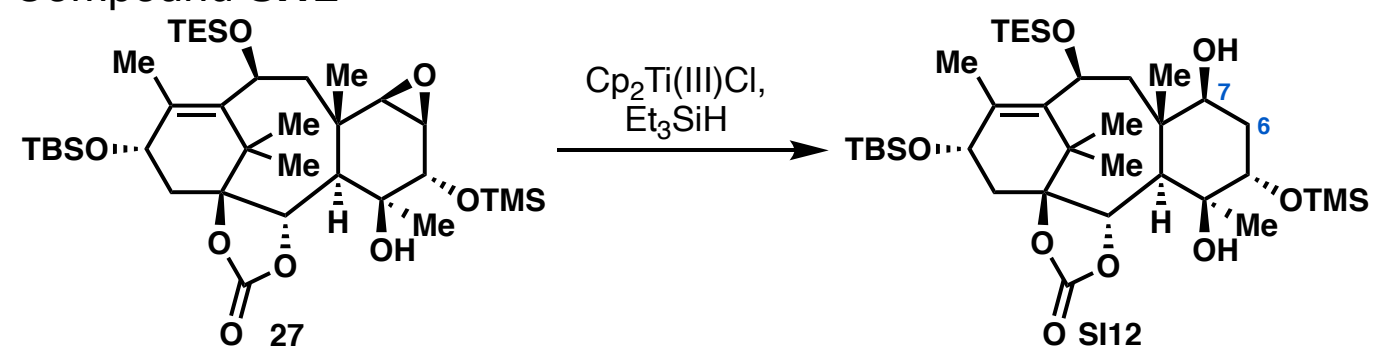

An oven-dried round-bottom flask was charged with $\mathrm{Cp}_{2} \mathrm{TiCl}_{2}(2.00 \mathrm{~g}, 8.03 \mathrm{mmol})$, zinc (707 mg, $10.8 \mathrm{mmol})$ and THF $(75 \mathrm{~mL})$. This suspension was stirred at rt for $40 \mathrm{~min}$. The reaction color turned from red to green upon putative formation of $\mathrm{Cp}_{2} \mathrm{Ti}(\mathrm{III}) \mathrm{Cl}$.

An oven-dried round-bottom flask was charged with epoxide $27(1.16 \mathrm{~g}, 1.63 \mathrm{~mol}), \mathrm{Et}_{3} \mathrm{SiH}$ $(5.96 \mathrm{~mL}, 37.5 \mathrm{mmol}, 23 \mathrm{eq})$ and THF $(89 \mathrm{~mL})$, and cooled to $-78^{\circ} \mathrm{C}$. The freshly prepared $\mathrm{Cp}_{2} \mathrm{TiCl}(38.0 \mathrm{~mL}, 4.08 \mathrm{mmol}, 0.11 \mathrm{M}$ in THF, $2.5 \mathrm{eq}$ ) was added to the mixture. The reaction was warmed up to $\mathrm{rt}$ and stirred for $40 \mathrm{~min}$. Volatiles were removed under reduced pressure and the resulting residue was purified by flash column chromatography $\left(\mathrm{SiO}_{2}\right.$, hexane/EtOAc 8:1 to $\left.4: 1\right)$ to yield diol SI12 (783 mg, $\left.1.10 \mathrm{mmol}, 67 \%\right)$.

Physical state: white solid;

TLC: $R_{f}=0.20$ (hexane/EtOAc 4:1, not UV active on TLC, stains blue upon $p$ anisaldehyde staining);

${ }^{1} \mathrm{H}$ NMR (600 MHz, $\left.\mathrm{CDCl}_{3}\right) \delta 4.93$ (dd, $\left.J=9.3,6.0,1.7 \mathrm{~Hz}, 1 \mathrm{H}\right), 4.86(\mathrm{dd}, J=11.6,5.7$ $\mathrm{Hz}, 1 \mathrm{H}$ ), $4.74(\mathrm{~d}, J=4.3 \mathrm{~Hz}, 1 \mathrm{H}$ ), 3.77 (ddd, $J=11.6,7.3,3.9 \mathrm{~Hz}, 1 \mathrm{H}), 3.45(\mathrm{t}, J=2.9 \mathrm{~Hz}$, $1 \mathrm{H}), 2.51-2.39(\mathrm{~m}, 2 \mathrm{H}), 2.34(\mathrm{~d}, J=4.4 \mathrm{~Hz}, 1 \mathrm{H}), 2.31(\mathrm{dd}, J=16.0,5.7 \mathrm{~Hz}, 1 \mathrm{H}), 2.11-$ $2.02(\mathrm{~m}, 2 \mathrm{H}), 1.85(\mathrm{~d}, J=1.5 \mathrm{~Hz}, 3 \mathrm{H}), 1.70(\mathrm{dt}, J=13.1,3.6 \mathrm{~Hz}, 1 \mathrm{H}), 1.68-1.60(\mathrm{~m}, 4 \mathrm{H})$, $1.38(\mathrm{~s}, 3 \mathrm{H}), 1.25(\mathrm{~s}, 3 \mathrm{H}), 1.17(\mathrm{~d}, J=7.6 \mathrm{~Hz}, 1 \mathrm{H}), 1.08(\mathrm{~s}, 3 \mathrm{H}), 0.94(\mathrm{t}, J=8.0 \mathrm{~Hz}, 9 \mathrm{H})$, $0.92(\mathrm{~s}, 9 \mathrm{H}), 0.58(\mathrm{qd}, J=7.9,2.4 \mathrm{~Hz}, 6 \mathrm{H}), 0.13(\mathrm{~s}, 3 \mathrm{H}), 0.12(\mathrm{~s}, 9 \mathrm{H}), 0.10(\mathrm{~s}, 3 \mathrm{H})$.

${ }^{13} \mathrm{C}$ NMR (151 MHz, $\mathrm{CDCl}_{3}$ ) ठ 153.6, 139.8, 136.3, 91.9, 83.2, 76.6, 75.6, 69.0, 68.5, 67.1, $44.1,42.0,42.0,41.4,38.1,35.6,29.6,26.8,26.3,21.6,18.8,18.5,15.1,7.0,5.1,0.5$, 4.3.

HRMS (ESI-TOF): calc'd for $\mathrm{C}_{36} \mathrm{H}_{69} \mathrm{O}_{8} \mathrm{Si}_{3}\left[\mathrm{M}+\mathrm{H}^{+}\right]$: 713.4295 , found: 713.4313 . $[\alpha]^{25} \mathrm{D}:+51.2\left(c=0.5, \mathrm{CHCl}_{3}\right)$

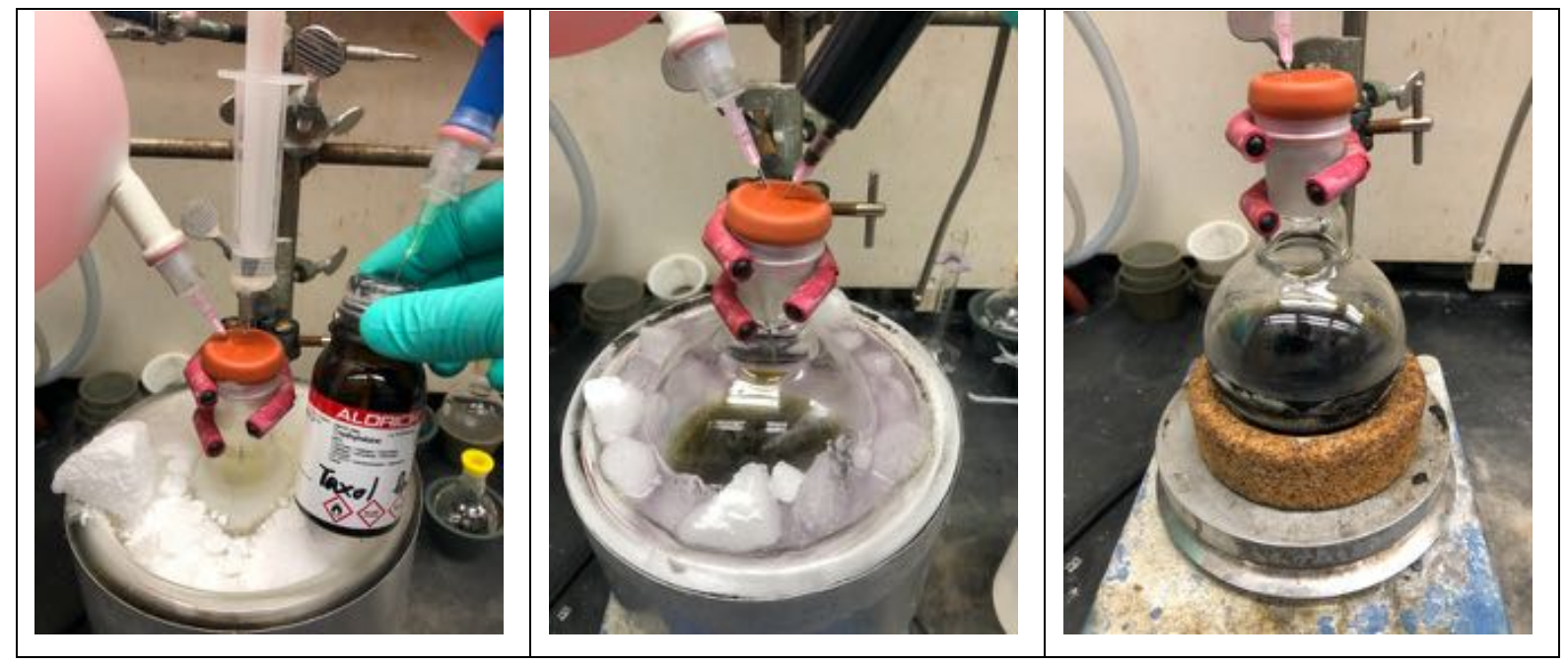


The substrate and reagents Addition of $\mathrm{Cp}_{2} \mathrm{TiCl}$ solution

The reaction at $\mathrm{rt}$ dissolved in THF 
Compound 28

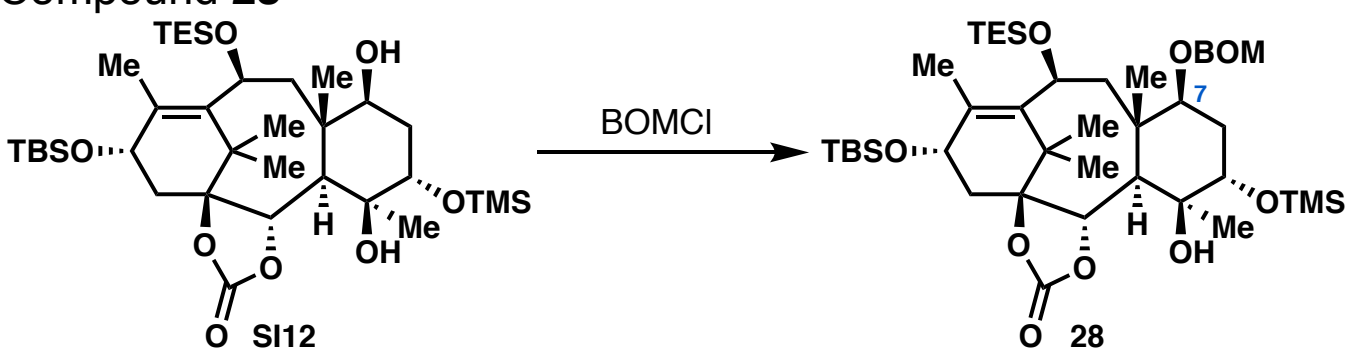

An oven-dried round-bottom flask was charged with diol SI12 (1.34 g, $1.88 \mathrm{mmol})$, DIPEA (23.0 mL, $132 \mathrm{mmol}, 70 \mathrm{eq})$, TBAl (1.67 g, $4.51 \mathrm{mmol}, 2.4 \mathrm{eq})$, BOMCl (4.69 mL, 33.8 mmol, $18 \mathrm{eq})$ and DCE $(150 \mathrm{~mL})$. The yellow mixture was stirred at $45^{\circ} \mathrm{C}$ for $18 \mathrm{~h}$. The reaction was quenched with $i-\mathrm{PrOH}(15 \mathrm{~mL})$ and stirred at $45^{\circ} \mathrm{C}$ for $1 \mathrm{~h}$. The reaction was cooled to rt and $\mathrm{NaHCO}_{3}$ (saturated aq.) was added. After stirring for $15 \mathrm{~min}$, the aqueous layer was extracted with hexane/EtOAc 2:1 (x 3). The combined organics were dried over $\mathrm{MgSO}_{4}$. The solvent was removed under reduced pressure and the resulting residue was purified by flash column chromatography $\left(\mathrm{SiO}_{2}\right.$, hexane/EtOAc 1:0 to 10:1) to yield $\mathrm{BOM}$ alcohol 28 (1.34 g, $1.61 \mathrm{mmol}, 84 \%)$.

Physical state: white solid;

TLC: $R_{f}=0.75$ (hexane/EtOAc 3:1, slightly UV active on TLC, stains blue upon $p$ anisaldehyde staining);

${ }^{1} \mathrm{H}$ NMR $\left(600 \mathrm{MHz}, \mathrm{CDCl}_{3}\right) \delta 7.36-7.27(\mathrm{~m}, 5 \mathrm{H}), 4.97-4.86(\mathrm{~m}, 3 \mathrm{H}), 4.84(\mathrm{dd}, J=11.6$, $5.8 \mathrm{~Hz}, 1 \mathrm{H}), 4.78(\mathrm{~d}, J=6.7 \mathrm{~Hz}, 1 \mathrm{H}), 4.75(\mathrm{~d}, J=4.4 \mathrm{~Hz}, 1 \mathrm{H}), 4.73(\mathrm{~d}, J=11.9 \mathrm{~Hz}, 1 \mathrm{H})$, $4.51(\mathrm{~d}, J=11.9 \mathrm{~Hz}, 1 \mathrm{H}), 3.70(\mathrm{dd}, J=11.7,4.0 \mathrm{~Hz}, 1 \mathrm{H}), 3.46(\mathrm{t}, J=2.9 \mathrm{~Hz}, 1 \mathrm{H}), 2.52-$ $2.43(\mathrm{~m}, 2 \mathrm{H}), 2.38$ (d, $J=4.4 \mathrm{~Hz}, 1 \mathrm{H}$ ), 2.24 (dd, $J=16.2,5.7 \mathrm{~Hz}, 1 \mathrm{H}$ ), 2.18 (ddd, $J=14.0$, 11.7, $2.6 \mathrm{~Hz}, 1 \mathrm{H}), 2.13(\mathrm{~s}, 1 \mathrm{H}), 1.99(\mathrm{dt}, J=13.5,3.7 \mathrm{~Hz}, 1 \mathrm{H}), 1.88(\mathrm{~d}, J=1.5 \mathrm{~Hz}, 3 \mathrm{H})$, $1.69-1.61(\mathrm{~m}, 4 \mathrm{H}), 1.39(\mathrm{~s}, 3 \mathrm{H}), 1.27(\mathrm{~s}, 3 \mathrm{H}), 1.16(\mathrm{~s}, 3 \mathrm{H}), 0.96-0.90(\mathrm{~m}, 18 \mathrm{H}), 0.55$ (q, $J=8.2 \mathrm{~Hz}, 6 \mathrm{H}), 0.15(\mathrm{~s}, 3 \mathrm{H}), 0.11(\mathrm{~s}, 3 \mathrm{H}), 0.08(\mathrm{~s}, 9 \mathrm{H})$.

${ }^{13} \mathrm{C}$ NMR (151 MHz, $\left.\mathrm{CDCl}_{3}\right) \delta$ 153.6, 139.7, 137.9, 136.2, 128.5, 127.9, 127.8, 95.9, 91.8, 83.2, 77.5, 76.7, 75.4, 69.7, 69.0, 67.4, 43.8, 42.1, 42.0, 41.6, 38.1, 32.9, 29.7, 26.7, 26.3, 21.6, 19.6, 18.8, 15.1, 7.0, 5.0, 0.4, -4.3.

HRMS (ESI-TOF): calc'd for $\mathrm{C}_{44} \mathrm{H}_{77} \mathrm{O}_{9} \mathrm{Si}_{3}\left[\mathrm{M}+\mathrm{H}^{+}\right]$: 833.4870, found: 833.4875.

$[\alpha]^{25} \mathrm{D}:+26.6\left(c=0.5, \mathrm{CHCl}_{3}\right)$ 
Compound 29

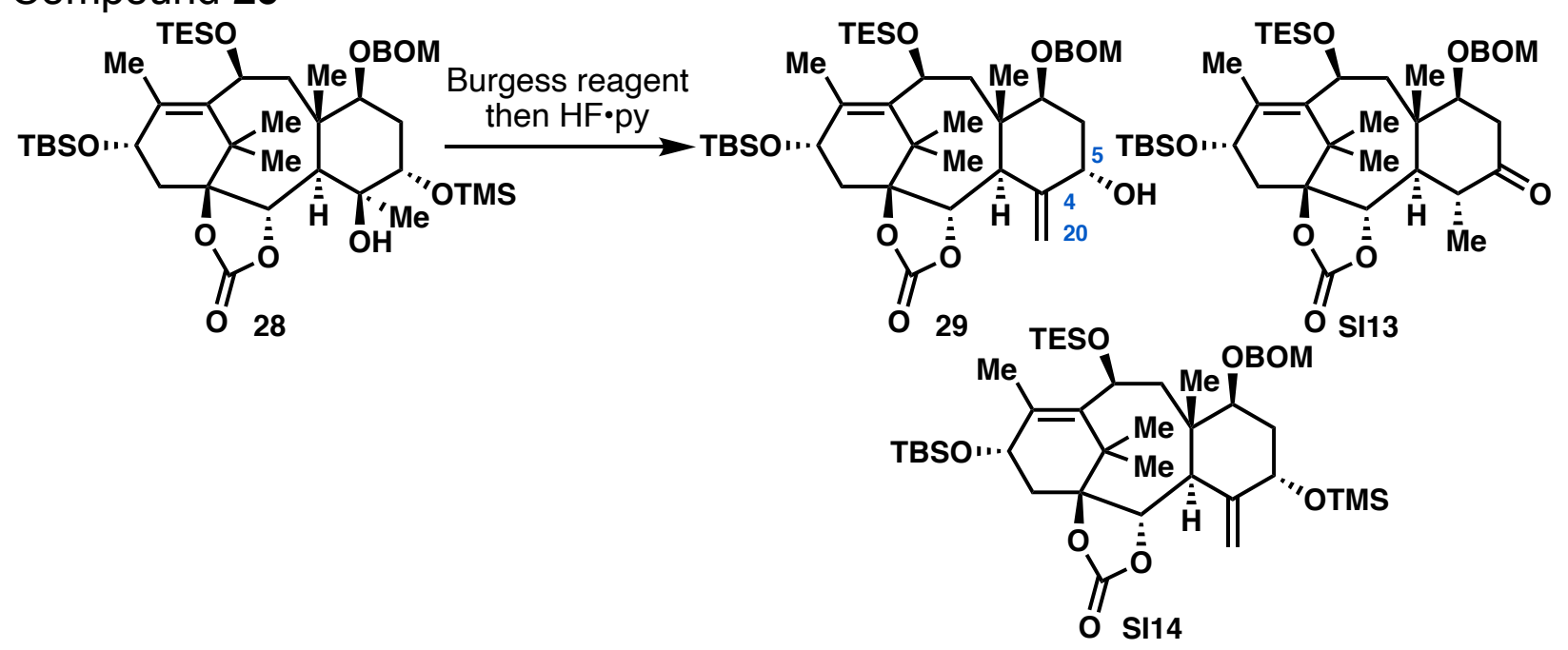

A plastic centrifuge tube was charged with BOM carbonate 28 (157 mg, $0.189 \mathrm{mmol})$, Burgess reagent $(90.0 \mathrm{mg}, 0.378 \mathrm{mmol}, 2.0 \mathrm{eq})$, benzene $(2.5 \mathrm{~mL})$ and dioxane $(2.5 \mathrm{~mL})$. The mixture was heated to $80^{\circ} \mathrm{C}$ for $2 \mathrm{~h}$. The volatiles were removed under reduced pressure. The resulting residue was dissolved in wet $\mathrm{MeCN}(5.0 \mathrm{~mL})$ and a solution of $\mathrm{HF}$ (5.0 mL, $6.85 \mathrm{mmol}$, HF aq. (48\%):MeCN:pyridine=1:10:10, $36 \mathrm{eq}$ ) was added dropwise at rt open to air. After $2 \mathrm{~h}$, the reaction was quenched with $\mathrm{NaHCO}_{3}$ (saturated aq.). The aqueous layer was extracted with EtOAc $(x 3)$. The combined organic phase was washed with brine then dried over $\mathrm{MgSO}_{4}$. The solvent was removed under reduced pressure and the resulting residue was purified by flash column chromatography $\left(\mathrm{SiO}_{2}\right.$, hexane/EtOAc 10:1 to $6: 1)$ to yield allylic alcohol $29(45.3 \mathrm{mg}, 60.5 \mu \mathrm{mol}, 32 \%)$ accompanying C5-ketone Sl13 (38.3 mg, $51.0 \mu \mathrm{mol}, 27 \%$ ) and allylic OTMS SI14 (7.6 mg, $9.3 \mu \mathrm{mol}, 5 \%)$. Allylic OTMS SI14 could be re-subjected to HF-py to obtain allylic alcohol 29. Allylic alcohol 29 was unstable for storage and was utilized in the next step immediately.

\section{Compound Sl13}

Physical state: colorless oil;

TLC: $R_{f}=0.75$ (hexane/EtOAc 3:1, slightly UV active on TLC, stains blue upon $p$ anisaldehyde staining);

${ }^{1} \mathrm{H}$ NMR $\left(600 \mathrm{MHz}, \mathrm{CDCl}_{3}\right) \delta 7.40-7.28(\mathrm{~m}, 5 \mathrm{H}), 4.93(\mathrm{dd}, J=11.2,6.0 \mathrm{~Hz}, 1 \mathrm{H}), 4.90-$ $4.86(\mathrm{~m}, 1 \mathrm{H}), 4.77(\mathrm{~d}, J=6.8 \mathrm{~Hz}, 1 \mathrm{H}), 4.73(\mathrm{~d}, J=11.9 \mathrm{~Hz}, 1 \mathrm{H}), 4.71-4.67(\mathrm{~m}, 2 \mathrm{H}), 4.54$ (d, $J=11.9 \mathrm{~Hz}, 1 \mathrm{H}$ ), 3.84 (dd, $J=8.4,4.9 \mathrm{~Hz}, 1 \mathrm{H}), 2.87$ (dd, $J=16.9,8.4 \mathrm{~Hz}, 1 \mathrm{H}$ ), 2.61 $-2.49(\mathrm{~m}, 3 \mathrm{H}), 2.31-2.18(\mathrm{~m}, 3 \mathrm{H}), 1.87-1.79(\mathrm{~m}, 4 \mathrm{H}), 1.60(\mathrm{~s}, 3 \mathrm{H}), 1.26(\mathrm{~d}, J=6.9 \mathrm{~Hz}$, $3 \mathrm{H}), 1.18(\mathrm{~s}, 3 \mathrm{H}), 0.94(\mathrm{t}, J=8.0 \mathrm{~Hz}, 9 \mathrm{H}), 0.90(\mathrm{~s}, 12 \mathrm{H}), 0.57(\mathrm{q}, J=7.8 \mathrm{~Hz}, 6 \mathrm{H}), 0.13(\mathrm{~s}$, $3 \mathrm{H}), 0.09(\mathrm{~s}, 3 \mathrm{H})$.

${ }^{13} \mathrm{C}$ NMR (151 MHz, $\left.\mathrm{CDCl}_{3}\right) \delta 212.0,153.7,138.4,137.7,137.3,128.7,128.1,128.1$, $95.5,90.8,80.5,77.5,70.3,68.7,67.4,43.8,43.3,43.0,42.9,42.1,40.8,38.3,27.8,26.1$, $20.4,19.0,18.5,18.3,17.3,7.0,5.0,-3.9,-5.0$.

HRMS (ESI-TOF): calc'd for $\mathrm{C}_{41} \mathrm{H}_{67} \mathrm{O}_{8} \mathrm{Si}_{2}\left[\mathrm{M}+\mathrm{H}^{+}\right]$: 743.4369 , found: 743.4386 . $[\alpha]^{25} \mathrm{D}:+17.9\left(c=5.0, \mathrm{CHCl}_{3}\right)$

\section{Compound SI14}

Physical state: white foam; 
TLC: $R_{f}=0.56$ (hexane/EtOAc 6:1, slightly UV active on TLC, stains blue upon $p$ anisaldehyde staining);

${ }^{1} \mathrm{H}$ NMR (600 MHz, CDCl 3$) \delta 7.40-7.26(\mathrm{~m}, 5 \mathrm{H}), 5.41(\mathrm{~s}, 1 \mathrm{H}), 5.18(\mathrm{t}, J=8.4 \mathrm{~Hz}, 1 \mathrm{H})$, $5.09(\mathrm{~s}, 1 \mathrm{H}), 4.94(\mathrm{dd}, J=11.5,5.8 \mathrm{~Hz}, 1 \mathrm{H}), 4.91(\mathrm{~d}, J=6.6 \mathrm{~Hz}, 1 \mathrm{H}), 4.84(\mathrm{~d}, J=6.6 \mathrm{~Hz}$, $1 \mathrm{H}), 4.75(\mathrm{~d}, J=11.9 \mathrm{~Hz}, 1 \mathrm{H}), 4.72(\mathrm{~d}, J=6.1 \mathrm{~Hz}, 1 \mathrm{H}), 4.51(\mathrm{~d}, J=11.9 \mathrm{~Hz}, 1 \mathrm{H}), 4.13(\mathrm{t}$, $J=3.0 \mathrm{~Hz}, 1 \mathrm{H}), 3.82(\mathrm{dd}, J=11.3,4.8 \mathrm{~Hz}, 1 \mathrm{H}), 3.25(\mathrm{~d}, J=6.0 \mathrm{~Hz}, 1 \mathrm{H}), 2.39-2.32(\mathrm{~m}$, 2H), 2.24 (dd, $J=14.7,8.5 \mathrm{~Hz}, 1 \mathrm{H}$ ), 2.16 (ddd, $J=13.3,4.9,2.8 \mathrm{~Hz}, 1 \mathrm{H}$ ), 1.98 (d, $J=1.4$ $\mathrm{Hz}, 3 \mathrm{H}), 1.81(\mathrm{dd}, J=15.8,11.5 \mathrm{~Hz}, 1 \mathrm{H}), 1.73(\mathrm{ddd}, J=13.3,11.3,3.4 \mathrm{~Hz}, 1 \mathrm{H}), 1.69(\mathrm{~s}$, $3 \mathrm{H}), 1.41(\mathrm{~s}, 3 \mathrm{H}), 0.97-0.93(\mathrm{~m}, 18 \mathrm{H}), 0.86(\mathrm{~s}, 3 \mathrm{H}), 0.58(\mathrm{q}, J=8.1 \mathrm{~Hz}, 6 \mathrm{H}), 0.17(\mathrm{~s}$, $3 \mathrm{H}), 0.13(\mathrm{~s}, 9 \mathrm{zH}), 0.11(\mathrm{~s}, 3 \mathrm{H})$.

${ }^{13} \mathrm{C}$ NMR (151 MHz, $\left.\mathrm{CDCl}_{3}\right) \delta$ 154.3, 144.6, 140.1, 137.9, 135.5, 128.6, 127.9, 127.8, 115.3, 95.5, 92.2, 82.5, 77.5, 76.0, 69.7, 69.1, 67.5, 44.7, 42.2, 41.4, 39.8, 38.9, 38.3, $28.1,26.4,22.9,19.2,17.9,15.2,7.0,5.0,0.5,-4.2,-4.3$.

HRMS (ESI-TOF): calc'd for $\mathrm{C}_{44} \mathrm{H}_{75} \mathrm{O}_{8} \mathrm{Si}_{3}\left[\mathrm{M}+\mathrm{H}^{+}\right]$: 815.4764 , found: 815.4763 .

$[\alpha]^{25} \mathrm{D}: 14.4\left(c=1.0, \mathrm{CHCl}_{3}\right)$

\section{Compound 29}

Physical state: colorless oil;

TLC: $R_{f}=0.23$ (hexane/EtOAc 6:1, slightly UV active on TLC, stains blue upon $p$ anisaldehyde staining);

${ }^{1} \mathrm{H}$ NMR (600 MHz, $\left.\mathrm{CDCl}_{3}\right) \delta 7.37-7.24(\mathrm{~m}, 5 \mathrm{H}), 5.51(\mathrm{t}, J=1.4 \mathrm{~Hz}, 1 \mathrm{H}), 5.17(\mathrm{~s}, 1 \mathrm{H})$, $4.99(\mathrm{dd}, J=11.2,5.9 \mathrm{~Hz}, 1 \mathrm{H}), 4.89(\mathrm{~d}, J=6.5 \mathrm{~Hz}, 1 \mathrm{H}), 4.81(\mathrm{~d}, J=6.5 \mathrm{~Hz}, 1 \mathrm{H}), 4.77$ (dd, $J=9.6,4.8 \mathrm{~Hz}, 1 \mathrm{H}), 4.75-4.69(\mathrm{~m}, 2 \mathrm{H}), 4.49(\mathrm{~d}, J=11.9 \mathrm{~Hz}, 1 \mathrm{H}), 4.20(\mathrm{~s}, 1 \mathrm{H}), 3.87$ (dd, $J=11.2,4.9 \mathrm{~Hz}, 1 \mathrm{H}), 3.53(\mathrm{~d}, J=5.1 \mathrm{~Hz}, 1 \mathrm{H}), 2.54(\mathrm{dd}, J=15.3,9.5 \mathrm{~Hz}, 1 \mathrm{H}), 2.38$ (dt, $J=13.5,4.4 \mathrm{~Hz}, 1 \mathrm{H}$ ), 2.31 (dd, $J=15.7,5.9 \mathrm{~Hz}, 1 \mathrm{H}$ ), $2.07(\mathrm{dd}, J=15.2,4.7 \mathrm{~Hz}, 1 \mathrm{H}$ ), $1.96(\mathrm{~d}, J=1.4 \mathrm{~Hz}, 3 \mathrm{H}), 1.74-1.51(\mathrm{~m}, 5 \mathrm{H}), 1.18(\mathrm{~s}, 3 \mathrm{H}), 0.94$ (t, $J=8.0 \mathrm{~Hz}, 9 \mathrm{H}), 0.91$ (s, 9H), $0.84(\mathrm{~s}, 3 \mathrm{H}), 0.57(\mathrm{q}, J=8.1 \mathrm{~Hz}, 6 \mathrm{H}), 0.13(\mathrm{~s}, 3 \mathrm{H}), 0.11(\mathrm{~s}, 3 \mathrm{H})$.

${ }^{13} \mathrm{C}$ NMR (151 MHz, $\left.\mathrm{CDCl}_{3}\right) \delta$ 154.1, 144.7, 139.1, 137.9, 137.8, 128.6, 127.9, 127.8, 116.0, 95.6, 91.3, 81.4, 75.9, 69.8, 69.5, 67.6, 44.9, 41.8, 40.7, 38.9, 38.6, 36. 8, 27.8, 26.0, 20.6, 18.4, 17.0, 7.0, 5.0, -4.3, -4.8.

HRMS (ESI-TOF): calc'd for $\mathrm{C}_{41} \mathrm{H}_{67} \mathrm{O}_{8} \mathrm{Si}_{2}\left[\mathrm{M}+\mathrm{H}^{+}\right]$: 743.4369 , found: 743.4365 . $[\alpha]^{25} \mathrm{D}:+15.6(c=0.5$, benzene $)$ 


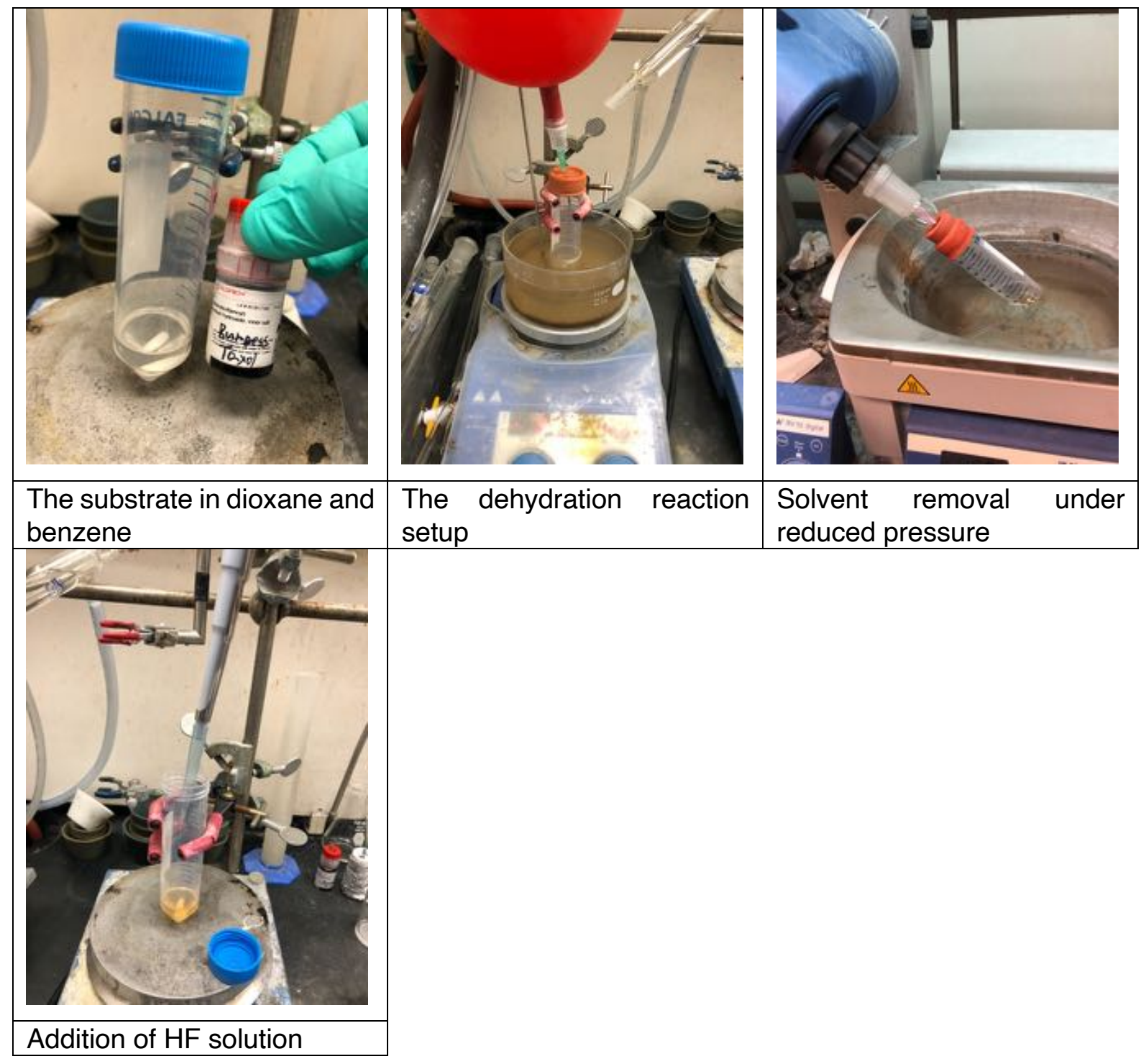


Compound $\mathbf{3 0}$

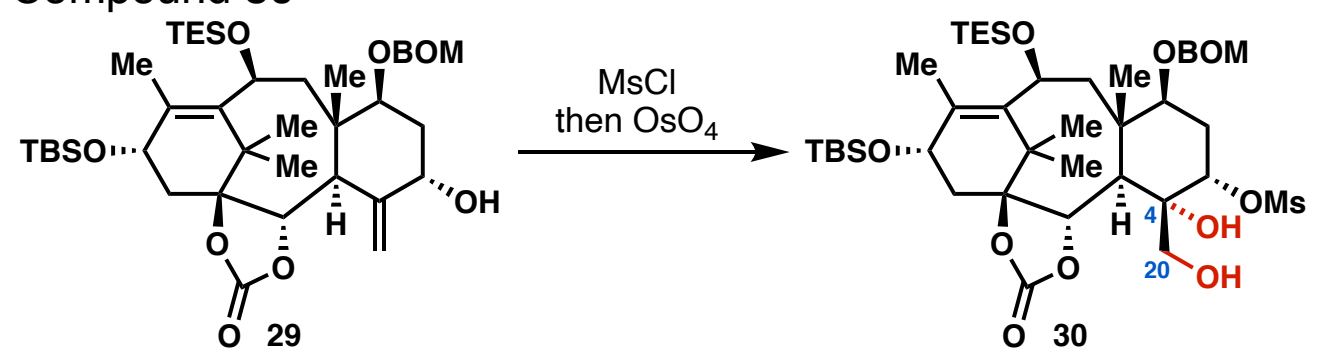

An oven-dried round-bottom flask was charged with semi-pure allylic alcohol 29 (303 mg, $0.408 \mathrm{mmol}$ ) and pyridine $(15.6 \mathrm{~mL})$ and cooled to $0{ }^{\circ} \mathrm{C}$. Freshly distilled $\mathrm{MsCl}(0.54 \mathrm{~mL}$, $6.93 \mathrm{mmol}, 17 \mathrm{eq}$ ) was added dropwise, and the mixture was then warmed up to rt. After $2.5 \mathrm{~h}$, the resulting yellow solution was cooled to $0{ }^{\circ} \mathrm{C}$ and diluted with THF $(15.6 \mathrm{~mL})$. $\mathrm{OsO}_{4}(3.8 \mathrm{~mL}, 0.589 \mathrm{mmol}, 0.157 \mathrm{M}$ in THF, $1.5 \mathrm{eq})$ was added dropwise and the reaction color turned brown. The mixture was warmed up to rt and stirred for $3 \mathrm{~h}$. The reaction was cooled to $0{ }^{\circ} \mathrm{C}$ and quenched with aqueous solution of $\mathrm{NaHSO}_{3}(4.77 \mathrm{~g})$ in water $(27 \mathrm{~mL})$. The resulting brown suspension was warmed up to rt and vigorously stirred for $9 \mathrm{~h}$. Complete consumption of osmate ester $\left(R_{f}=0.26\right.$ in EtOAc) was confirmed by TLC. The reaction was diluted with EtOAc and cooled to $0{ }^{\circ} \mathrm{C}$, then quenched by dropwise addition of $\mathrm{NaHCO}_{3}$ (saturated aq.). The resulting mixture was extracted with EtOAc (x 3). The combined organic phase was washed with brine then dried over $\mathrm{MgSO}_{4}$. The solvent was removed under reduced pressure and the resulting residue was purified by flash column chromatography $\left(\mathrm{SiO}_{2}\right.$, hexane/EtOAc $4: 1$ to $\left.1: 1\right)$ to yield diol $30(237 \mathrm{mg}, 0.278 \mathrm{mmol}$, $68 \%)$.

Physical state: white foam;

TLC: $R_{f}=0.62$ (hexane/EtOAc 1:1, slightly UV active on TLC, stains blue upon $p$ anisaldehyde staining);

${ }^{1}$ H NMR (600 MHz, CDCl 3 ) $\delta 7.38-7.27(\mathrm{~m}, 5 \mathrm{H}), 4.99(\mathrm{t}, J=8.0 \mathrm{~Hz}, 1 \mathrm{H}), 4.89(\mathrm{dd}, J=$ $11.5,5.8 \mathrm{~Hz}, 1 \mathrm{H}), 4.86(\mathrm{~d}, J=6.5 \mathrm{~Hz}, 1 \mathrm{H}), 4.81(\mathrm{t}, J=2.8 \mathrm{~Hz}, 1 \mathrm{H}), 4.78(\mathrm{~d}, J=6.5 \mathrm{~Hz}$, $1 \mathrm{H}), 4.72(\mathrm{~d}, J=12.0 \mathrm{~Hz}, 1 \mathrm{H}), 4.60(\mathrm{~d}, J=4.8 \mathrm{~Hz}, 1 \mathrm{H}), 4.52(\mathrm{~d}, J=12.0 \mathrm{~Hz}, 1 \mathrm{H}), 4.11(\mathrm{~d}$, $J=11.3 \mathrm{~Hz}, 1 \mathrm{H}), 3.72(\mathrm{dd}, J=11.5,4.4 \mathrm{~Hz}, 1 \mathrm{H}), 3.53(\mathrm{dd}, J=11.3,6.1 \mathrm{~Hz}, 1 \mathrm{H}), 3.33-$ $3.29(\mathrm{~m}, 1 \mathrm{H}), 3.10-3.02(\mathrm{~m}, 4 \mathrm{H}), 2.86(\mathrm{~d}, J=4.8 \mathrm{~Hz}, 1 \mathrm{H}), 2.54(\mathrm{dt}, J=15.1,4.0 \mathrm{~Hz}, 1 \mathrm{H})$, $2.37(\mathrm{dd}, J=15.0,9.2 \mathrm{~Hz}, 1 \mathrm{H}), 2.18(\mathrm{dd}, J=16.1,5.8 \mathrm{~Hz}, 1 \mathrm{H}), 1.99-1.91(\mathrm{~m}, 4 \mathrm{H}), 1.74$ $-1.63(\mathrm{~m}, 2 \mathrm{H}), 1.60(\mathrm{~s}, 3 \mathrm{H}), 1.29(\mathrm{~s}, 3 \mathrm{H}), 0.95-0.90(\mathrm{~m}, 18 \mathrm{H}), 0.86(\mathrm{~s}, 3 \mathrm{H}), 0.55(\mathrm{q}, J=$ $8.0 \mathrm{~Hz}, 6 \mathrm{H}), 0.18(\mathrm{~s}, 3 \mathrm{H}), 0.12(\mathrm{~s}, 3 \mathrm{H})$.

${ }^{13} \mathrm{C}$ NMR $\left(151 \mathrm{MHz}, \mathrm{CDCl}_{3}\right) \delta 154.0,141.0,137.5,135.6,128.7,128.0,127.9,95.6,92.2$, 82.1, 81.6, 75.7, 74.6, 70.1, 69.3, 67.2, 63.3, 43.1, 42.7, 42.3, 41.7, 38.7, 37.4, 32.1, 27.3, 26.3, 21.8, 20.0, 19.0, 15.5, 7.0, 5.0, -4.4, -4.5.

HRMS (ESI-TOF): calcd for $\mathrm{C}_{42} \mathrm{H}_{71} \mathrm{O}_{12} \mathrm{Ssi}_{2}\left[\mathrm{M}+\mathrm{H}^{+}\right]$: 855.4199, found: 855.4201. $[\alpha]^{25} \mathrm{D}:+13.1\left(c=0.21, \mathrm{CHCl}_{3}\right)$ 


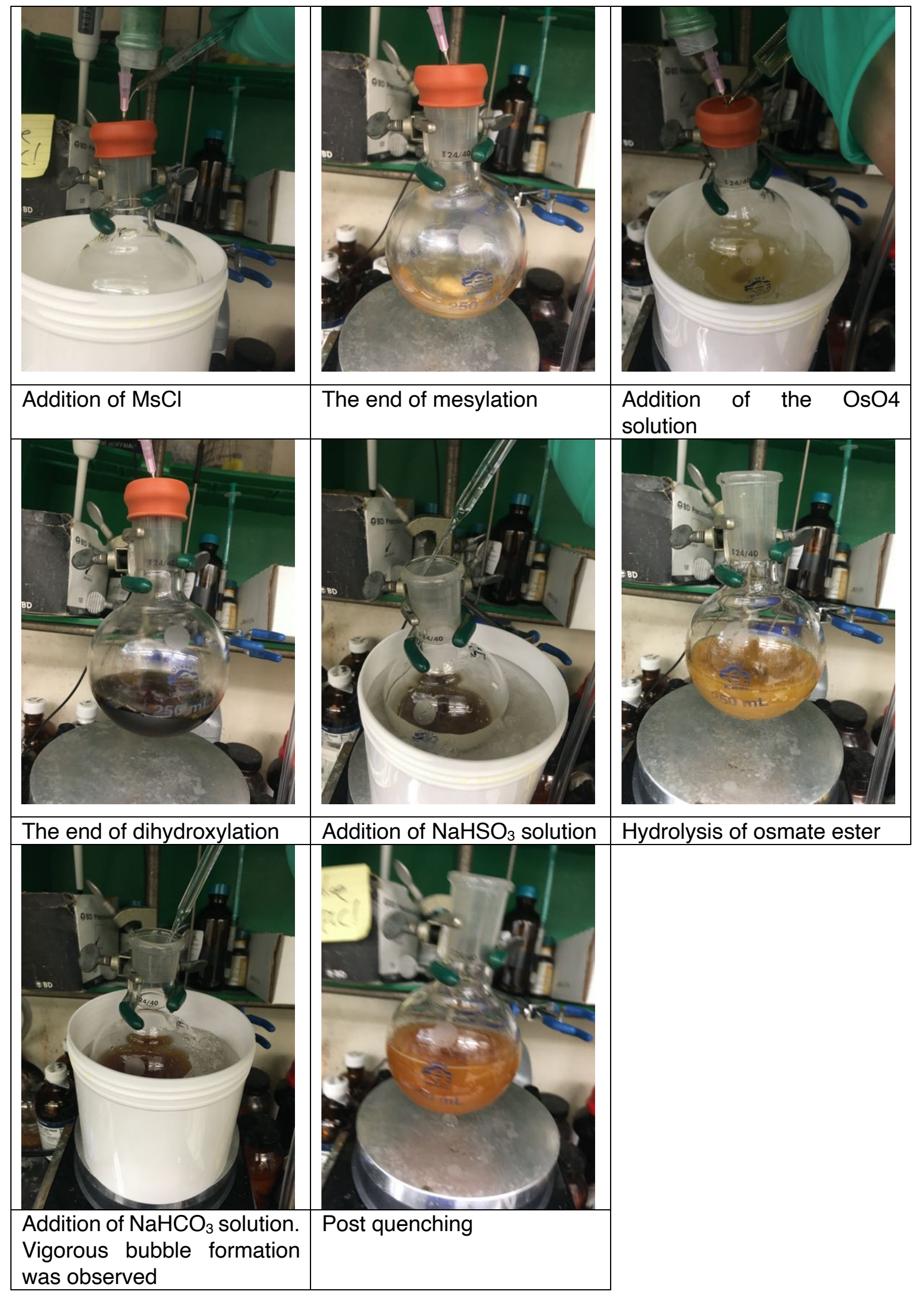




\section{Compound 31}

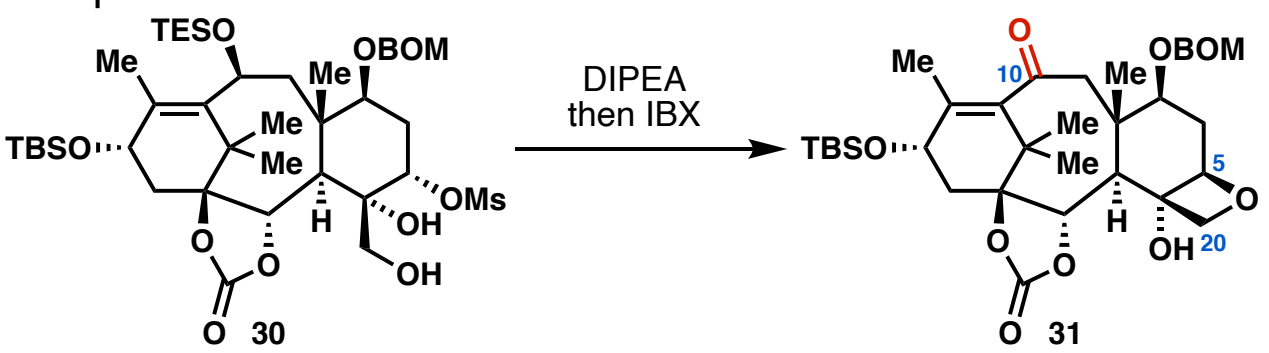

An oven-dried round-bottom flask was charged with diol $30(214 \mathrm{mg}, 0.251 \mathrm{mmol})$ and PhMe $(31.7 \mathrm{~mL})$. DIPEA $(0.63 \mathrm{~mL}, 3.63 \mathrm{mmol}, 14 \mathrm{eq})$ was added and the reaction was stirred at $110^{\circ} \mathrm{C}$ for $15 \mathrm{~h}$. The volatiles were removed in vacuo. The residue was further dried via azeotrope with $\mathrm{PhMe}$ four times. The crude residue was dissolved in DMSO/water 10:1 (17.5 mL) and IBX (3.51 g, $12.5 \mathrm{mmol}, 50 \mathrm{eq})$ was added. The resulting pale yellow suspension was heated to $80^{\circ} \mathrm{C}$ for $30 \mathrm{~min}$ open to air. On complete of the reaction, the reaction mixture was diluted with $\mathrm{Et}_{2} \mathrm{O}$ then water. The resulting mixture was extracted with $\mathrm{Et}_{2} \mathrm{O}$ (x 5). The combined organic phase was washed with brine then dried over $\mathrm{MgSO}_{4}$. The solvent was removed under reduced pressure and the resulting residue was purified by flash column chromatography $\left(\mathrm{SiO}_{2}\right.$, hexane/EtOAc $4: 1$ to $\left.2: 1\right)$ to yield keto-oxetane 31 (100 mg, $0.155 \mathrm{mmol}, 62 \%$ ).

Physical state: white foam;

TLC: $R_{f}=0.43$ (hexane/EtOAc 2:1, UV active on TLC, stains blue upon $p$-anisaldehyde staining);

${ }^{1} \mathrm{H}$ NMR $\left(600 \mathrm{MHz}, \mathrm{CDCl}_{3}\right) \delta 7.37-7.27(\mathrm{~m}, 5 \mathrm{H}), 4.88(\mathrm{~d}, J=7.3 \mathrm{~Hz}, 1 \mathrm{H}), 4.83(\mathrm{~d}, J=$ $5.2 \mathrm{~Hz}, 1 \mathrm{H}), 4.77-4.70(\mathrm{~m}, 3 \mathrm{H}), 4.69-4.63(\mathrm{~m}, 3 \mathrm{H}), 4.52(\mathrm{dd}, J=8.7,1.1 \mathrm{~Hz}, 1 \mathrm{H}), 3.41$ (dd, $J=10.2,7.1 \mathrm{~Hz}, 1 \mathrm{H}$ ), $3.16(\mathrm{~d}, J=16.9 \mathrm{~Hz}, 1 \mathrm{H}), 2.89$ (dd, $J=15.4,3.8 \mathrm{~Hz}, 1 \mathrm{H}), 2.63$ $-2.49(\mathrm{~m}, 4 \mathrm{H}), 2.35(\mathrm{~d}, J=16.8 \mathrm{~Hz}, 1 \mathrm{H}), 1.97$ (ddd, $J=15.3,10.2,2.8 \mathrm{~Hz}, 1 \mathrm{H}), 1.71(\mathrm{~d}$, $J=1.4 \mathrm{~Hz}, 3 \mathrm{H}), 1.41(\mathrm{~s}, 3 \mathrm{H}), 1.36(\mathrm{~s}, 3 \mathrm{H}), 1.23(\mathrm{~s}, 3 \mathrm{H}), 0.92(\mathrm{~s}, 9 \mathrm{H}), 0.12(\mathrm{~s}, 3 \mathrm{H}), 0.11(\mathrm{~s}$, $3 \mathrm{H})$.

${ }^{13} \mathrm{C}$ NMR (151 MHz, $\left.\mathrm{CDCl}_{3}\right) \delta 202.5,153.2,142.7,142.0,137.6,128.7,128.0,94.5,90.1$, 87.6, 80.2, 79.8, 77.9, 74.7, 70.3, 68.4, 52.8, 46.3, 44.2, 38.6, 36.8, 35.0, 26.8, 26.0, 21.3, $18.3,16.9,16.7,-4.3,-4.8$.

HRMS (ESI-TOF): calc'd for $\mathrm{C}_{35} \mathrm{H}_{51} \mathrm{O}_{9} \mathrm{Si}\left[\mathrm{M}+\mathrm{H}^{+}\right]$: 643.3297 , found: 643.3314 . $[\alpha]^{25} \mathrm{D}:+4.8\left(c=0.5, \mathrm{CHCl}_{3}\right)$ 


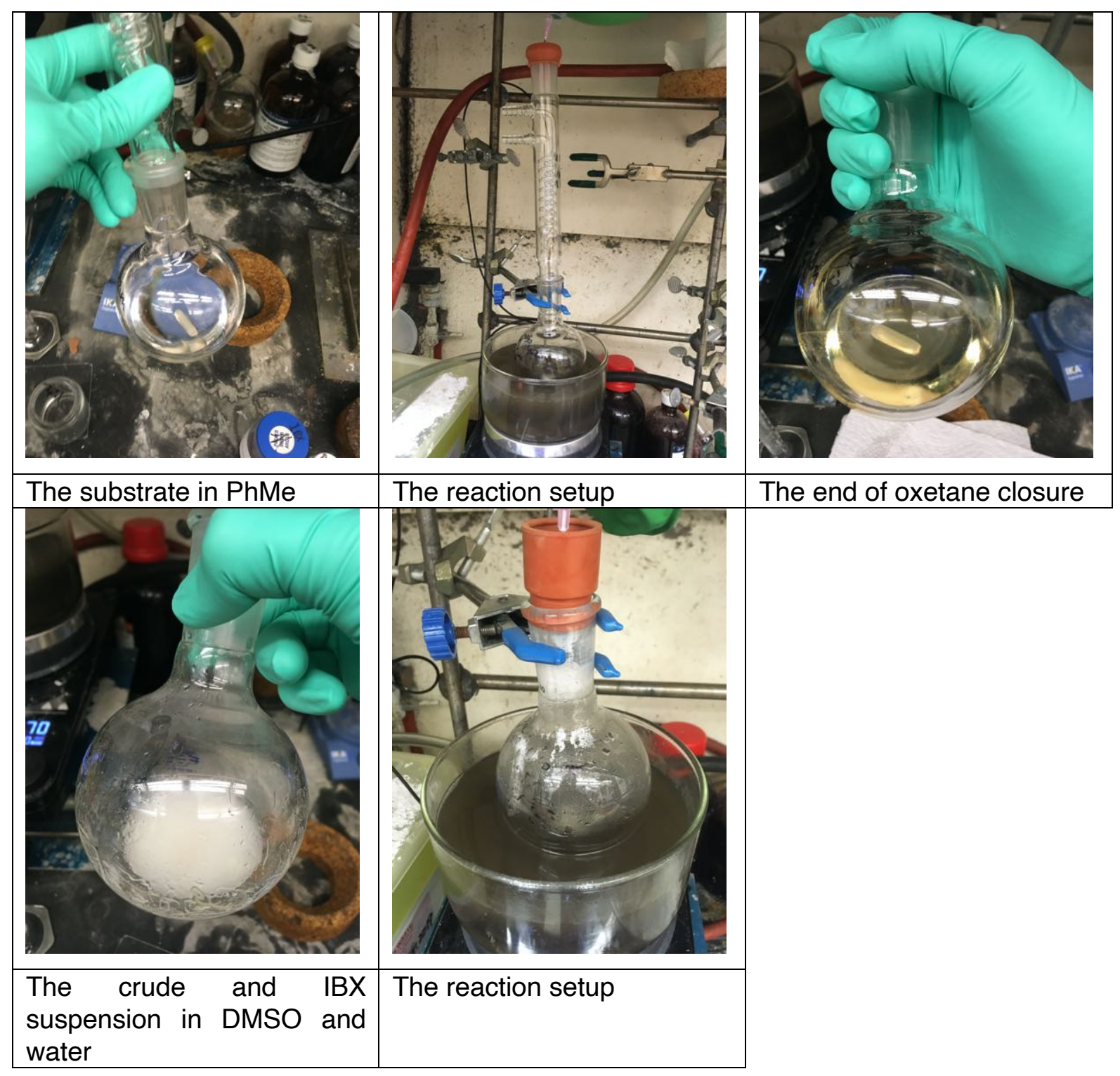


Compound 32
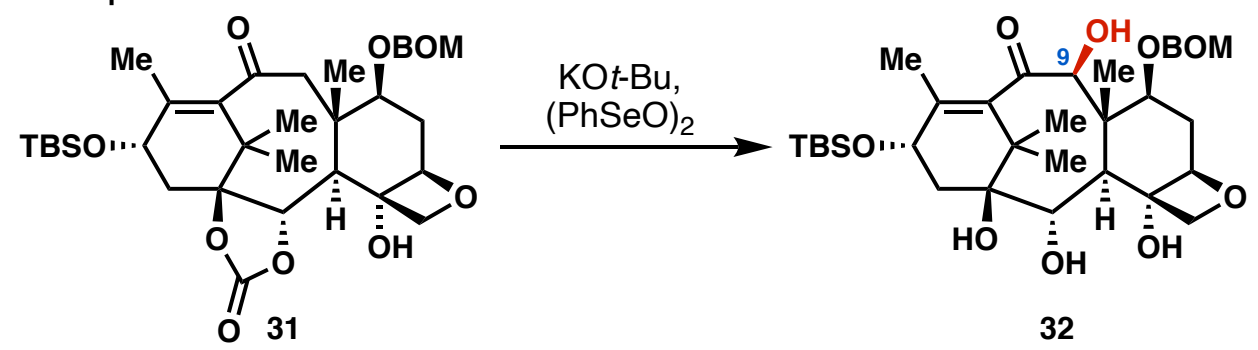

An oven-dried round-bottom flask was charged with enone $\mathbf{3 1}(35.8 \mathrm{mg}, 55.8 \mu \mathrm{mol})$ and THF $(3.0 \mathrm{~mL})$ and water $(5.0 \mathrm{mg}, 0.279 \mathrm{mmol}, 5 \mathrm{eq})$. The solution was cooled to $-78^{\circ} \mathrm{C}$ and $\mathrm{Kot}$ - $\mathrm{Bu}(0.56 \mathrm{~mL}, \mathrm{mmol}, 1.0 \mathrm{M}$ in THF, $10 \mathrm{eq})$ was added dropwise. The reaction was warmed to $0{ }^{\circ} \mathrm{C}$ and stirred for $1.5 \mathrm{~h}$ before being added to a suspension of $(\mathrm{PhSeO})_{2} \mathrm{O}$ $(401 \mathrm{mg}, 1.12 \mathrm{mmol}, 20 \mathrm{eq})$ in THF $(3 \mathrm{~mL})$ at $0^{\circ} \mathrm{C}$. The reaction vessel was washed with THF $(0.2 \mathrm{~mL} \times 2)$ and the rinse was also added to the suspension. The reaction was quenched with $\mathrm{NH}_{4} \mathrm{Cl}$ (saturated aq.) in 30 min. The aqueous layer was extracted with $\mathrm{Et}_{2} \mathrm{O}$ (x 5). The combined organic phase was dried over $\mathrm{MgSO}_{4}$ and concentrated under reduced pressure. The resulting residue was suspended in DCM and solid was removed. The combined yellow DMC solution was concentrated under reduced pressure. The resulting residue was purified by preparative TLC $\left(\mathrm{SiO}_{2}\right.$, hexane/EtOAc 1:1) to obtain tetraol $32(25.8 \mathrm{mg}, 40.8 \mu \mathrm{mol}, 73 \%)$. The product is unstable for storage and was utilized in the next step immediately.

Physical state: white foam

TLC: $R_{f}=0.57$ (hexane/EtOAc 1:1, UV active on TLC, stains blue upon $p$-anisaldehyde staining);

${ }^{1} \mathrm{H}$ NMR (600 MHz, CDCl 3$) \delta 7.39-7.26(\mathrm{~m}, 5 \mathrm{H}), 5.03(\mathrm{~d}, J=7.4 \mathrm{~Hz}, 1 \mathrm{H}), 4.94(\mathrm{~d}, J=$ $7.0 \mathrm{~Hz}, 1 \mathrm{H}), 4.78(\mathrm{~d}, J=7.0 \mathrm{~Hz}, 1 \mathrm{H}), 4.74(\mathrm{~d}, J=11.6 \mathrm{~Hz}, 1 \mathrm{H}), 4.69(\mathrm{~d}, J=11.5 \mathrm{~Hz}, 1 \mathrm{H})$, $4.66-4.61(\mathrm{~m}, 2 \mathrm{H}), 4.45(\mathrm{~s}, 1 \mathrm{H}), 4.36(\mathrm{~d}, J=7.5 \mathrm{~Hz}, 1 \mathrm{H}), 4.31(\mathrm{~d}, J=11.2 \mathrm{~Hz}, 1 \mathrm{H}), 4.15$ $(\mathrm{d}, J=4.0 \mathrm{~Hz}, 1 \mathrm{H}), 4.01(\mathrm{~d}, J=4.0 \mathrm{~Hz}, 1 \mathrm{H}), 3.95(\mathrm{dd}, J=11.3,4.6 \mathrm{~Hz}, 1 \mathrm{H}), 3.86(\mathrm{~d}, J=$ $1.7 \mathrm{~Hz}, 1 \mathrm{H}), 3.72(\mathrm{~s}, 1 \mathrm{H}), 2.90(\mathrm{~d}, J=4.6 \mathrm{~Hz}, 1 \mathrm{H}), 2.50-2.41(\mathrm{~m}, 2 \mathrm{H}), 2.31$ (dd, $J=15.8$, $2.4 \mathrm{~Hz}, 1 \mathrm{H}$ ), 1.72 (d, $J=1.4 \mathrm{~Hz}, 3 \mathrm{H}), 1.63$ (ddd, $J=16.3,4.0,2.3 \mathrm{~Hz}, 1 \mathrm{H}), 1.17(\mathrm{~s}, 3 \mathrm{H}$ ), $1.16(\mathrm{~s}, 3 \mathrm{H}), 1.09(\mathrm{~s}, 3 \mathrm{H}), 0.97(\mathrm{~s}, 9 \mathrm{H}), 0.20(\mathrm{~s}, 3 \mathrm{H}), 0.16(\mathrm{~s}, 3 \mathrm{H})$.

${ }^{13} \mathrm{C}$ NMR (151 MHz, $\left.\mathrm{CDCl}_{3}\right) \delta 206.33,143.71,141.02,138.18,128.54,128.16,127.75$, $93.98,87.75,82.11,79.33,76.60,75.72,74.15,69.79,69.63,50.36,47.93,41.44,39.22$, $29.85,29.05,27.64,26.16,20.62,20.25,18.44,16.66,-4.21,-4.60$.

HRMS (ESI-TOF): calc'd for $\mathrm{C}_{34} \mathrm{H}_{53} \mathrm{O}_{9} \mathrm{Si}\left[\mathrm{M}+\mathrm{H}^{+}\right]$: 633.3453, found: 655.3283 . $[\alpha]^{25} \mathrm{D}:+129.1(c=0.21$, benzene $)$ 


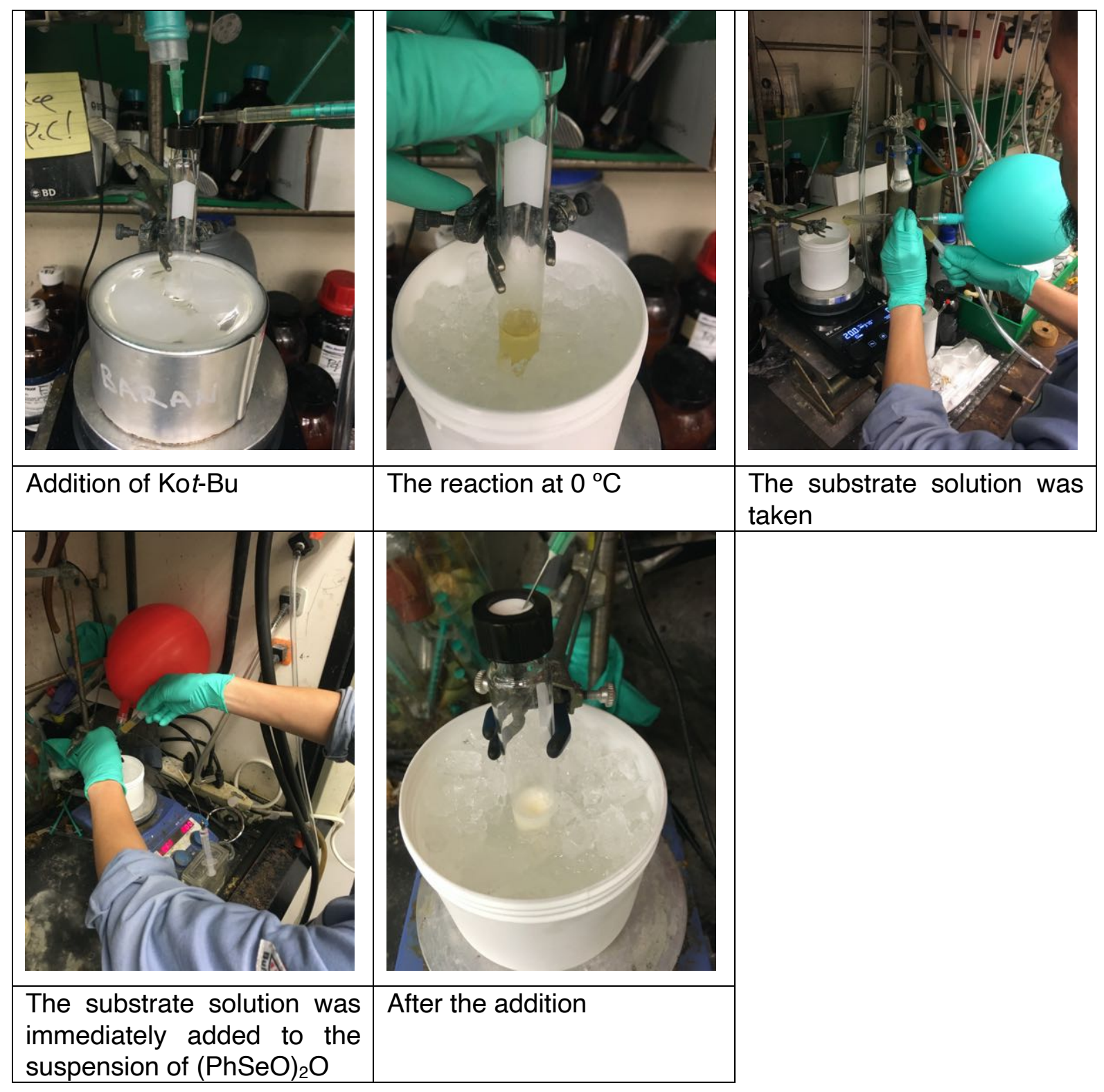


Compound 33

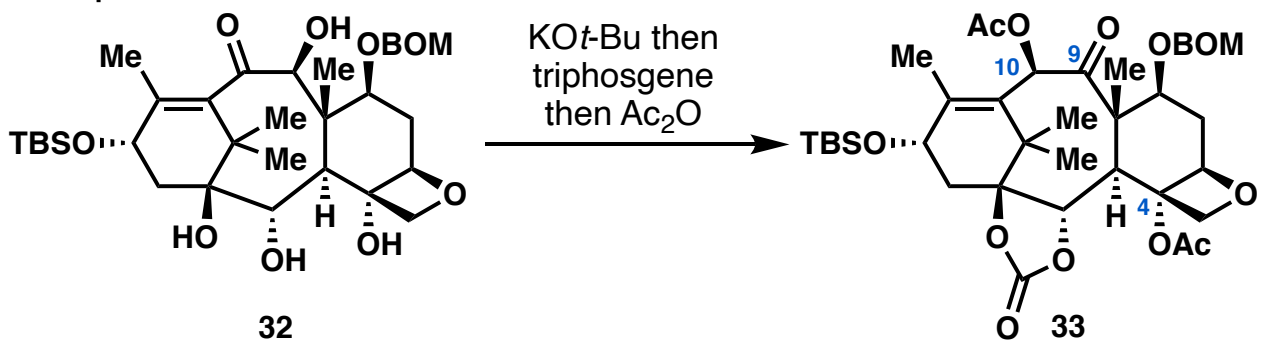

An oven-dried round-bottom flask was charged with tetraol $32(24.9 \mathrm{mg}, 39.4 \mu \mathrm{mol})$ and THF $(2.4 \mathrm{~mL})$. The solution was cooled to $-78^{\circ} \mathrm{C}$ and $\mathrm{Kot}-\mathrm{Bu}(0.32 \mathrm{~mL}, 0.32 \mathrm{mmol}, 1.0 \mathrm{M}$ in THF, $8.0 \mathrm{eq}$ ) was added dropwise. The reaction was stirred for $3 \mathrm{~h}$ at the same temperature before $\mathrm{AcOH}$ solution $(0.50 \mathrm{~mL}, 0.8 \mathrm{M}$, in THF) was added. The reaction was maintained at $-78{ }^{\circ} \mathrm{C}$ for 5 min then warmed up to rt. The reaction was neutralized with $\mathrm{pH}=7.0 \mathrm{Na}_{2} \mathrm{HPO}_{4} / \mathrm{NaH}_{2} \mathrm{PO}_{4}$ aqueous buffer solution $(0.1 \mathrm{M})$. Volatiles were removed under reduced pressure and the residue was further dried via azeotrope with PhMe three times. The resulting white powder was charged with DCM $(0.8 \mathrm{~mL})$, DMAP $(2.4 \mathrm{mg}, 19.7$ $\mu \mathrm{mol}, 0.5 \mathrm{eq})$ and pyridine ( $60 \mu \mathrm{L}, 0.749 \mathrm{mmol}, 19 \mathrm{eq})$. The reaction was cooled to -78 ${ }^{\circ} \mathrm{C}$ before triphosgene solution (12.3 mg, $41.4 \mu \mathrm{mol}$, in $0.3 \mathrm{~mL}$ of DCM, $1.05 \mathrm{eq}$ ) was added dropwise. On completion of addition, the mixture was warmed to $0{ }^{\circ} \mathrm{C}$ and stirred for $30 \mathrm{~min}$. The reaction was quenched with $\mathrm{pH}=7.0 \mathrm{Na}_{2} \mathrm{HPO}_{4} / \mathrm{NaH}_{2} \mathrm{PO}_{4}$ aqueous buffer solution $(0.1 \mathrm{M})$. Volatiles were removed under reduced pressure and the residue was further dried via azeotrope with $\mathrm{PhMe}$ three times. The resulting white powder was charged with pyridine $(0.16 \mathrm{~mL})$ and DMAP $(5 \mathrm{mg}, 40.9 \mu \mathrm{mol}, 1.0 \mathrm{eq}) . \mathrm{Ac}_{2} \mathrm{O}(37 \mu \mathrm{L}, 40$ $\mathrm{mg}, 0.39 \mathrm{mmol}, 10 \mathrm{eq}$ ) was added at rt open to air, and this orange suspension was stirred at the same temperature for $36 \mathrm{~h}$. The reaction was diluted with hexane/EtOAc (3:1) then quenched with $\mathrm{NaHCO}_{3}$ (saturated aq.). The aqueous layer was extracted with hexane/EtOAc 3:1 (x 3). The combined organic phase was dried over $\mathrm{MgSO}_{4}$ and concentrated under reduced pressure. The resulting residue was purified by preparative TLC $\left(\mathrm{SiO}_{2}\right.$, hexane/EtOAc 3:1) to obtain carbonate 33 (17.4 mg, $\left.23.5 \mu \mathrm{mol}, 60 \%\right)$.

Physical state: white foam;

TLC: $R_{f}=0.28$ (hexane/EtOAc 2:1, UV active on TLC, stains blue upon $p$-anisaldehyde staining);

${ }^{1} \mathrm{H}$ NMR $\left(600 \mathrm{MHz}, \mathrm{CDCl}_{3}\right) \delta 7.35-7.23(\mathrm{~m}, 5 \mathrm{H}), 6.38(\mathrm{~s}, 1 \mathrm{H}), 5.10-5.07(\mathrm{t}, J=8.3 \mathrm{~Hz}$, $1 \mathrm{H}), 4.96(\mathrm{dd}, J=9.3,1.5 \mathrm{~Hz}, 1 \mathrm{H}), 4.83-4.76(\mathrm{~m}, 2 \mathrm{H}), 4.66(\mathrm{~d}, J=12.1 \mathrm{~Hz}, 1 \mathrm{H}), 4.62$ $(\mathrm{d}, J=9.0 \mathrm{~Hz}, 1 \mathrm{H}), 4.49-4.44(\mathrm{~m}, 2 \mathrm{H}), 4.21(\mathrm{dd}, J=9.8,7.1 \mathrm{~Hz}, 1 \mathrm{H}), 3.47$ (dd, $J=5.9$, $1.3 \mathrm{~Hz}, 1 \mathrm{H}$ ), 2.87 (dddd, $J=14.8,9.4,7.1,5.5 \mathrm{~Hz}, 1 \mathrm{H}$ ), 2.44 (dd, $J=15.2,8.8 \mathrm{~Hz}, 1 \mathrm{H}$ ), 2.15 (s, 3H), 2.04 (s, 3H), 2.00 (ddd, $J=14.8,9.8,1.6 \mathrm{~Hz}, 1 \mathrm{H}), 1.80(\mathrm{~s}, 3 \mathrm{H}), 1.30(\mathrm{~s}, 3 \mathrm{H})$, $1.19(\mathrm{~s}, 3 \mathrm{H}), 0.94(\mathrm{~s}, 9 \mathrm{H}), 0.16(\mathrm{~s}, 3 \mathrm{H}), 0.13(\mathrm{~s}, 3 \mathrm{H})$.

${ }^{13} \mathrm{C}$ NMR (151 MHz, $\left.\mathrm{CDCl}_{3}\right) \delta 202.9,170.1,169.4,153.1,148.9,137.8,129.1,128.5$, $127.7127 .7,96.1,90.6,84.3,81.6,79.6,79.0,76.6,76.2,69.9,68.3,58.6,43.9,41.4$, 37.0, 36.0, 26.3, 25.6, 22.6, 21.2, 20.9, 19.1, 16.0, 10.8, 7.9, 4.9, -4.5.

HRMS (ESI-TOF): calcd for $\mathrm{C}_{39} \mathrm{H}_{55} \mathrm{O}_{12} \mathrm{Si}\left[\mathrm{M}+\mathrm{H}^{+}\right]$: 743.3457 , found: 743.3472 . $[\alpha]^{25} \mathrm{D}:-91.0\left(c=2.7, \mathrm{CHCl}_{3}\right)$ 


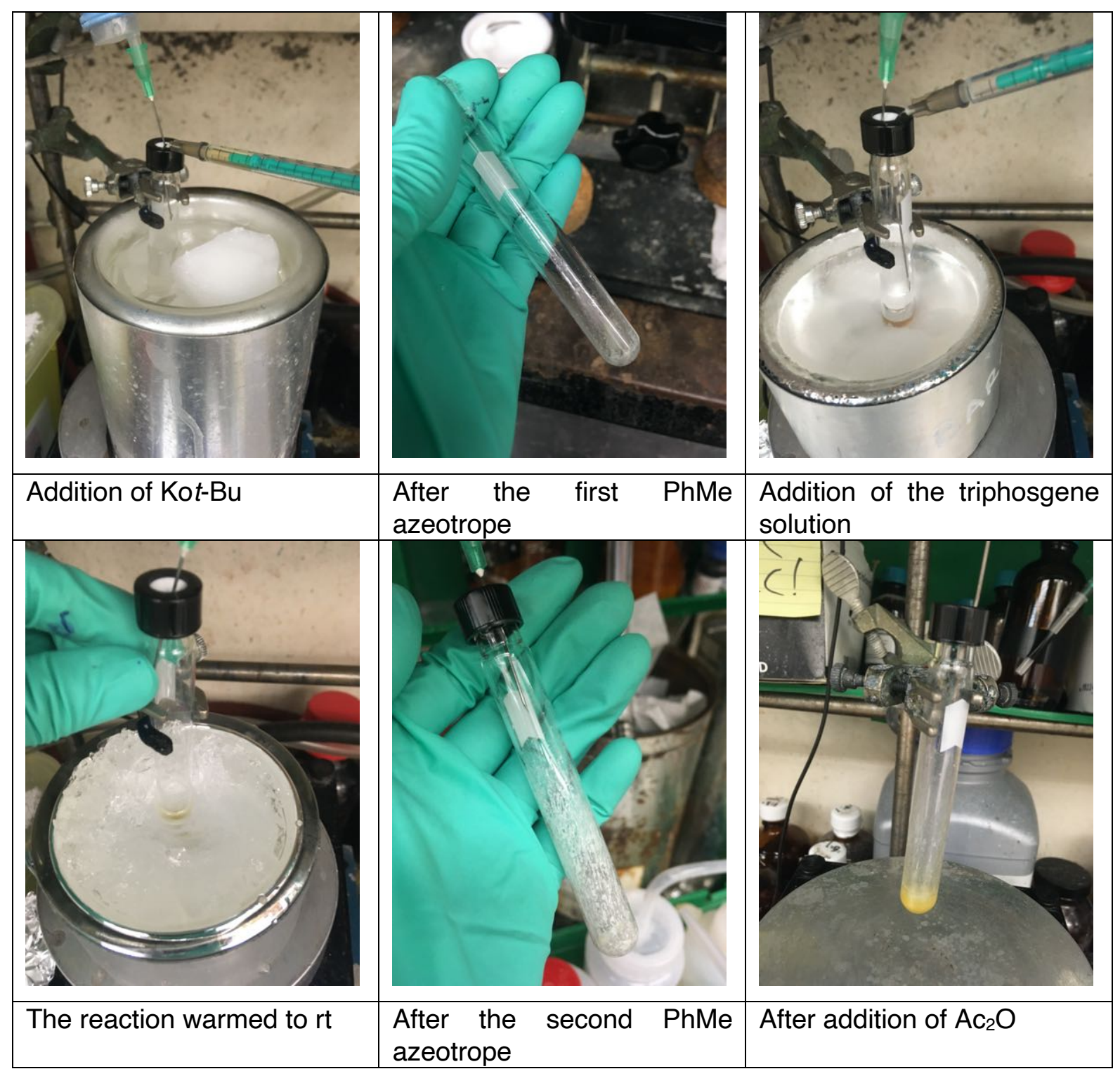




\section{Compound 34}

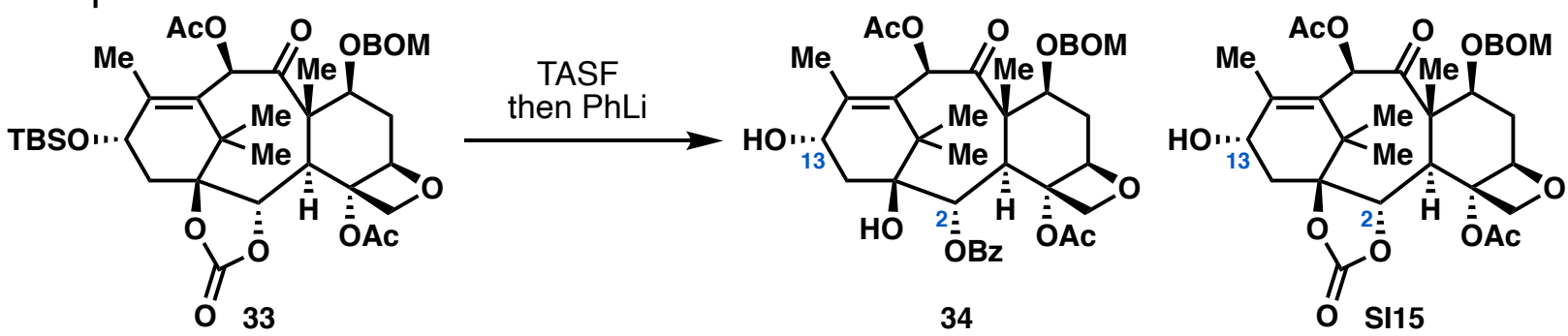

An oven-dried round-bottom flask was charged with carbonate $33(46.6 \mathrm{mg}, 62.7 \mu \mathrm{mol})$ and THF $(3.0 \mathrm{~mL})$. TASF $(19.0 \mathrm{mg}, 69 \mu \mathrm{mol}, 1.1 \mathrm{eq})$ was added at $\mathrm{rt}$ and the mixture was stirred for $1 \mathrm{~h}$. The reaction was cooled to $-78^{\circ} \mathrm{C}$ and $\mathrm{PhLi}(0.75 \mathrm{~mL}, 0.236 \mathrm{mmol}, 0.317$ $\mathrm{M}$ in $5 \mathrm{~mL}$ of THF and $1.0 \mathrm{~mL}$ of $\mathrm{Bu}_{2} \mathrm{O}, 3.75 \mathrm{eq}$ ) was slowly added through a $22 \mathrm{G}$ needle with its tip immersed in the reaction solution. The reaction was quenched with $\mathrm{NH}_{4} \mathrm{Cl}$ (saturated aq.) after $3 \mathrm{~h}$. The aqueous layer was extracted with DCM (x 3). The combined organic phase was dried over $\mathrm{MgSO}_{4}$ and concentrated under reduced pressure. The resulting residue was purified by preparative $\mathrm{TLC}\left(\mathrm{SiO}_{2}\right.$, hexane/EtOAc 2:3) to obtain BOM baccatin III 34 (21.2 mg, $30.0 \mu \mathrm{mol}, 48 \%)$ accompanying carbonate SI15 (6.3 mg, $10 \mu \mathrm{mol}, 16 \%)$. Carbonate SI15 could be re-subjected to the PhLi condition to obtain more BOM baccatin III 34. The spectroscopic data for BOM baccatin III 34 were identical to those reported by Holton. ${ }^{5}$

\section{Compound 34}

Physical state: white solid;

TLC: $R_{f}=0.49$ (hexane/EtOAc 2:3, UV active on TLC, stains blue upon $p$-anisaldehyde staining);

${ }^{1} \mathrm{H}-\mathrm{NMR}\left(600 \mathrm{MHz}, \mathrm{CDCl}_{3}\right): \delta 8.11$ (dd, $\left.J=8.0,1.4 \mathrm{~Hz}, 2 \mathrm{H}\right), 7.61$ (t, $\left.J=7.4 \mathrm{~Hz}, 1 \mathrm{H}\right), 7.48$ $(\mathrm{t}, J=7.6 \mathrm{~Hz}, 2 \mathrm{H}), 7.35-7.23(\mathrm{~m}, 5 \mathrm{H}), 6.40(\mathrm{~s}, 1 \mathrm{H}), 5.63(\mathrm{~d}, J=6.9 \mathrm{~Hz}, 1 \mathrm{H}), 4.95(\mathrm{dd}, J$ $=9.7,2.0 \mathrm{~Hz}, 1 \mathrm{H}), 4.90-4.83(\mathrm{~m}, 2 \mathrm{H}), 4.68(\mathrm{~d}, J=11.9 \mathrm{~Hz}, 1 \mathrm{H}), 4.44(\mathrm{~d}, J=11.9 \mathrm{~Hz}$, $1 \mathrm{H}), 4.31(\mathrm{~d}, J=8.4 \mathrm{~Hz}, 1 \mathrm{H}), 4.24(\mathrm{dd}, J=10.5,6.8 \mathrm{~Hz}, 1 \mathrm{H}), 4.16(\mathrm{~d}, J=8.4 \mathrm{~Hz}, 1 \mathrm{H})$, $3.94(\mathrm{~d}, J=6.9 \mathrm{~Hz}, 1 \mathrm{H}), 2.90$ (ddd, $J=14.4,9.7,6.8 \mathrm{~Hz}, 1 \mathrm{H}), 2.32-2.25(\mathrm{~m}, 5 \mathrm{H}), 2.20$ (s, 3H), 2.10 (d, $J=1.4 \mathrm{~Hz}, 3 \mathrm{H}$ ), $2.08(\mathrm{~d}, J=5.2 \mathrm{~Hz}, 1 \mathrm{H}$ ), 2.00 (ddd, $J=14.7,10.5,2.1$ $\mathrm{Hz}, 1 \mathrm{H}), 1.77(\mathrm{~s}, 3 \mathrm{H}), 1.63(\mathrm{~s}, 1 \mathrm{H}), 1.18(\mathrm{~s}, 3 \mathrm{H}), 1.08(\mathrm{~s}, 3 \mathrm{H})$.

${ }^{13}$ C-NMR (150 MHz, $\left.\mathrm{CDCl}_{3}\right): \delta 202.1,170.2,169.1,166.6,143.6,137.4,133.2,131.8$, 129.7, 128.9, 128.2, 127.9, 127.3, 127.1, 96.3, 83.9, 80.4, 80.0, 78.3, 76.0, 75.4, 74.1, 69.5, 67.5, 57.0, 46.9, 42.3, 38.0, 35.1, 26.3, 22.2, 20.5, 19.8, 14.8, 10.2.

HRMS (ESI-TOF): calc'd for $\mathrm{C}_{39} \mathrm{H}_{47} \mathrm{O}_{12}\left[\mathrm{M}+\mathrm{H}^{+}\right]:$: 707.3062, found: 707.3063 . $[\alpha]^{25} \mathrm{D}:-106.2\left(c=1.0, \mathrm{CHCl}_{3}\right)$

\section{Compound SI15}

Physical state: white solid;

TLC: $R_{f}=0.54$ (hexane/EtOAc 2:3, UV active on TLC, stains blue upon $p$-anisaldehyde staining);

${ }^{1} \mathrm{H}-\mathrm{NMR}\left(600 \mathrm{MHz}, \mathrm{CDCl}_{3}\right): \delta 7.34-7.25(\mathrm{~m}, 5 \mathrm{H}), 6.38(\mathrm{~s}, 1 \mathrm{H}), 4.98(\mathrm{dd}, J=9.3,1.4 \mathrm{~Hz}$, $1 \mathrm{H}), 4.92-4.87(\mathrm{~m}, 1 \mathrm{H}), 4.83(\mathrm{~d}, J=7.6 \mathrm{~Hz}, 1 \mathrm{H}), 4.78(\mathrm{~d}, J=7.6 \mathrm{~Hz}, 1 \mathrm{H}), 4.66(\mathrm{~d}, J=$ $12.0 \mathrm{~Hz}, 1 \mathrm{H}), 4.62(\mathrm{~d}, J=8.6 \mathrm{~Hz}, 1 \mathrm{H}), 4.49-4.45(\mathrm{~m}, 2 \mathrm{H}), 4.44(\mathrm{~d}, J=5.7 \mathrm{~Hz}, 1 \mathrm{H}), 4.21$ (dd, $J=9.7,7.2 \mathrm{~Hz}, 1 \mathrm{H}$ ), 3.52 (dd, $J=5.8,1.2 \mathrm{~Hz}, 1 \mathrm{H}$ ), 2.88 (ddd, $J=14.8,9.3,7.2 \mathrm{~Hz}$, 
$1 \mathrm{H}), 2.59-2.52(\mathrm{~m}, 2 \mathrm{H}), 2.18-2.14(\mathrm{~m}, 7 \mathrm{H}), 2.09(\mathrm{~d}, J=1.6 \mathrm{~Hz}, 3 \mathrm{H}), 2.01$ (ddd, $J=$ $14.9,9.7,1.5 \mathrm{~Hz}, 1 \mathrm{H}), 1.81(\mathrm{~s}, 3 \mathrm{H}), 1.21(\mathrm{~s}, 3 \mathrm{H}), 1.18(\mathrm{~s}, 3 \mathrm{H})$.

${ }^{13} \mathrm{C}-N M R\left(150 \mathrm{MHz}, \mathrm{CDCl}_{3}\right): \delta$ 202.8, 171.1, 169.5, 153.2, 147.8, 137.7, 130.2, 128.5, 127.8, 127.8, 96.1, 90.5, 84.3, 81.2, 79.6, 79.1, 76.6, 76.2, 70.1, 67.0, 59.0, 44.4, 41.1, $36.1,35.8,25.8,22.7,20.9,20.0,16.2,10.7$.

HRMS (ESI-TOF): calc'd for $\mathrm{C}_{33} \mathrm{H}_{41} \mathrm{O}_{12}\left[\mathrm{M}+\mathrm{H}^{+}\right]$: 629.2593, found: 629.2592 .

$[\alpha]^{25} \mathrm{D}: \mathrm{XX}\left(c=1.0, \mathrm{CHCl}_{3}\right)$ 
Compound 1

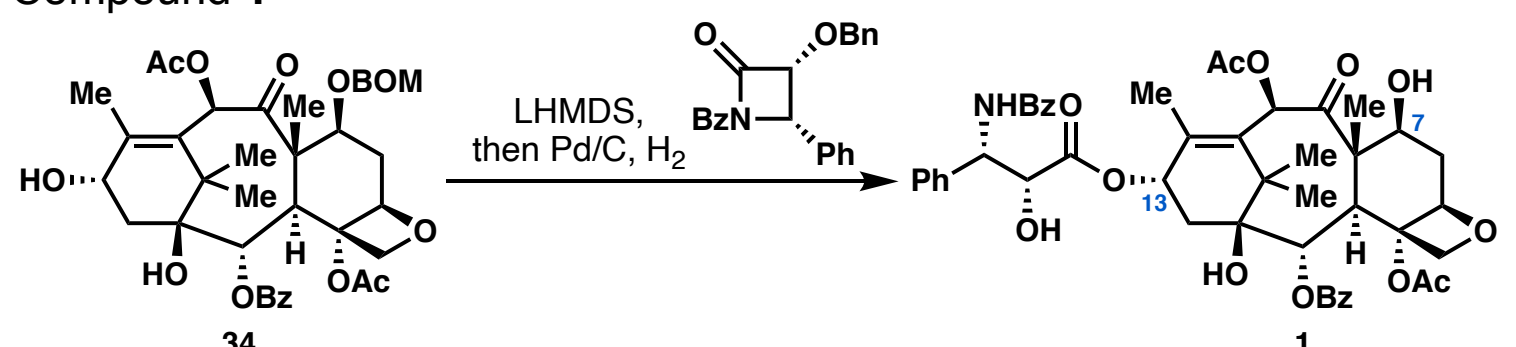

34

An oven-dried round-bottom flask was charged with BOM baccatin III 34 (30.0 mg, 42.4 $\mu \mathrm{mol})$ and THF $(2.0 \mathrm{~mL})$ and cooled to $-45^{\circ} \mathrm{C}$. LiHMDS $(64 \mu \mathrm{L}, 64 \mu \mathrm{mol}, 1.0 \mathrm{M}$ in THF, $1.5 \mathrm{eq})$ was added dropwise and the mixture was then stirred for $1 \mathrm{~h}$. $\beta$-lactam 35 (31.9 $\mathrm{mg}, 89 \mu \mathrm{mol}$, in $2.0 \mathrm{~mL}$ of THF, $2.1 \mathrm{eq}$ ) was added dropwise. The mixture was stirred at $0{ }^{\circ} \mathrm{C}$ for $1.5 \mathrm{~h}$ then warmed up to rt before the reaction was quenched with $\mathrm{AcOH}(0.2$ $\mathrm{mL}$ ). Volatiles were removed under reduced pressure and the residue was further dried via azeotrope with $\mathrm{PhMe}$ three times. The resulting residue was loaded with $\mathrm{Pd} / \mathrm{C}$ (511 $\mathrm{mg} 10$ wt.\%) and $\mathrm{EtOH}(6.0 \mathrm{~mL})$. The flask was subjected to three vacuum $/ \mathrm{H}_{2}$ cycles quickly then heated to reflux for $6.5 \mathrm{~h}$. The mixture was directly poured onto a pad of $\mathrm{SiO}_{2}$ and eluted with EtOAc. The solvent was removed under reduced pressure and the resulting residue was purified by flash column chromatography $\left(\mathrm{SiO}_{2}\right.$, hexane/EtOAc 1:4) to obtain Taxol$^{\circledR} 1$ (30.6 mg, $\left.35.8 \mu \mathrm{mol}, 85 \%\right)$.

Physical state: white solid;

TLC: $R_{f}=0.31$ (hexane/EtOAc 1:2, UV active on TLC, stains blue upon $p$-anisaldehyde staining);

${ }^{1} \mathrm{H}-\mathrm{NMR}\left(600 \mathrm{MHz}, \mathrm{CDCl}_{3}\right): \delta 8.14(\mathrm{~d}, J=7.2 \mathrm{~Hz}, 1 \mathrm{H}), 7.74(\mathrm{~d}, J=6.9 \mathrm{~Hz}, 0 \mathrm{H}), 7.62(\mathrm{t}, J$ $=7.4 \mathrm{~Hz}, \mathrm{OH}), 7.54-7.46(\mathrm{~m}, 2 \mathrm{H}), 7.45-7.37(\mathrm{~m}, 2 \mathrm{H}), 7.35(\mathrm{t}, J=7.2 \mathrm{~Hz}, 0 \mathrm{H}), 6.97(\mathrm{~d}$, $J=8.9 \mathrm{~Hz}, \mathrm{OH}), 6.27(\mathrm{~s}, \mathrm{OH}), 6.24(\mathrm{t}, J=8.9 \mathrm{~Hz}, \mathrm{OH}), 5.79(\mathrm{dd}, J=8.8,2.7 \mathrm{~Hz}, \mathrm{OH}), 5.68$ $(\mathrm{d}, J=7.1 \mathrm{~Hz}, \mathrm{OH}), 4.95(\mathrm{~d}, J=9.4 \mathrm{~Hz}, \mathrm{OH}), 4.79(\mathrm{~s}, \mathrm{OH}), 4.40(\mathrm{~s}, \mathrm{OH}), 4.31(\mathrm{~d}, J=8.5 \mathrm{~Hz}$, OH), $4.20(\mathrm{~d}, J=8.5 \mathrm{~Hz}, \mathrm{OH}), 3.80(\mathrm{~d}, J=7.0 \mathrm{~Hz}, \mathrm{OH}), 3.51(\mathrm{~d}, J=5.0 \mathrm{~Hz}, \mathrm{OH}), 2.55$ (ddd, $J=14.7,9.6,6.7 \mathrm{~Hz}, \mathrm{OH}), 2.44(\mathrm{~s}, \mathrm{OH}), 2.41-2.27(\mathrm{~m}, 2 \mathrm{H}), 2.24(\mathrm{~s}, 1 \mathrm{H}), 1.88$ (ddd, $J=$ $14.3,11.2,2.0 \mathrm{~Hz}, 1 \mathrm{H}), 1.80(\mathrm{~s}, 1 \mathrm{H}), 1.69(\mathrm{~s}, 1 \mathrm{H}), 1.24(\mathrm{~s}, 1 \mathrm{H}), 1.15(\mathrm{~s}, 1 \mathrm{H})$.

${ }^{13}$ C-NMR (150 MHz, CDCl $): \delta 203.79,172.86,171.42,170.52,167.19,167.15,142.15$, 138.11, 133.90, 133.77, 133.34, 132.14, 130.37, 129.28, 129.21, 128.89, 128.87, 128.55, $127.19,84.56,81.31,79.23,76.67,75.72,75.08,73.35,72.55,72.37,58.79,55.17,45.76$, 43.33, 35.83, 35.76, 27.03, 22.80, 21.96, 21.02, 15.02, 9.71.

HRMS (ESI-TOF): calc'd for $\mathrm{C}_{47} \mathrm{H}_{52} \mathrm{NO}_{14}\left[\mathrm{M}+\mathrm{H}^{+}\right]$: 854.3382 , found: 854.3391 .

$[\alpha]^{25} \mathrm{D}:-51.5\left(c=0.2, \mathrm{CHCl}_{3}\right)$

So far, $35.2 \mathrm{mg}$ of synthetic 1 has been prepared:

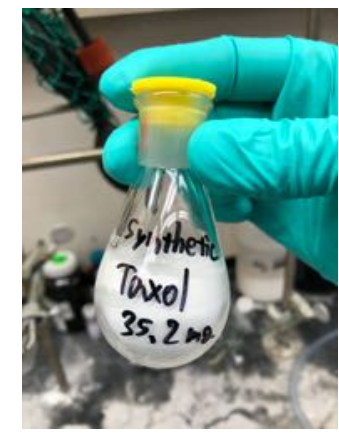




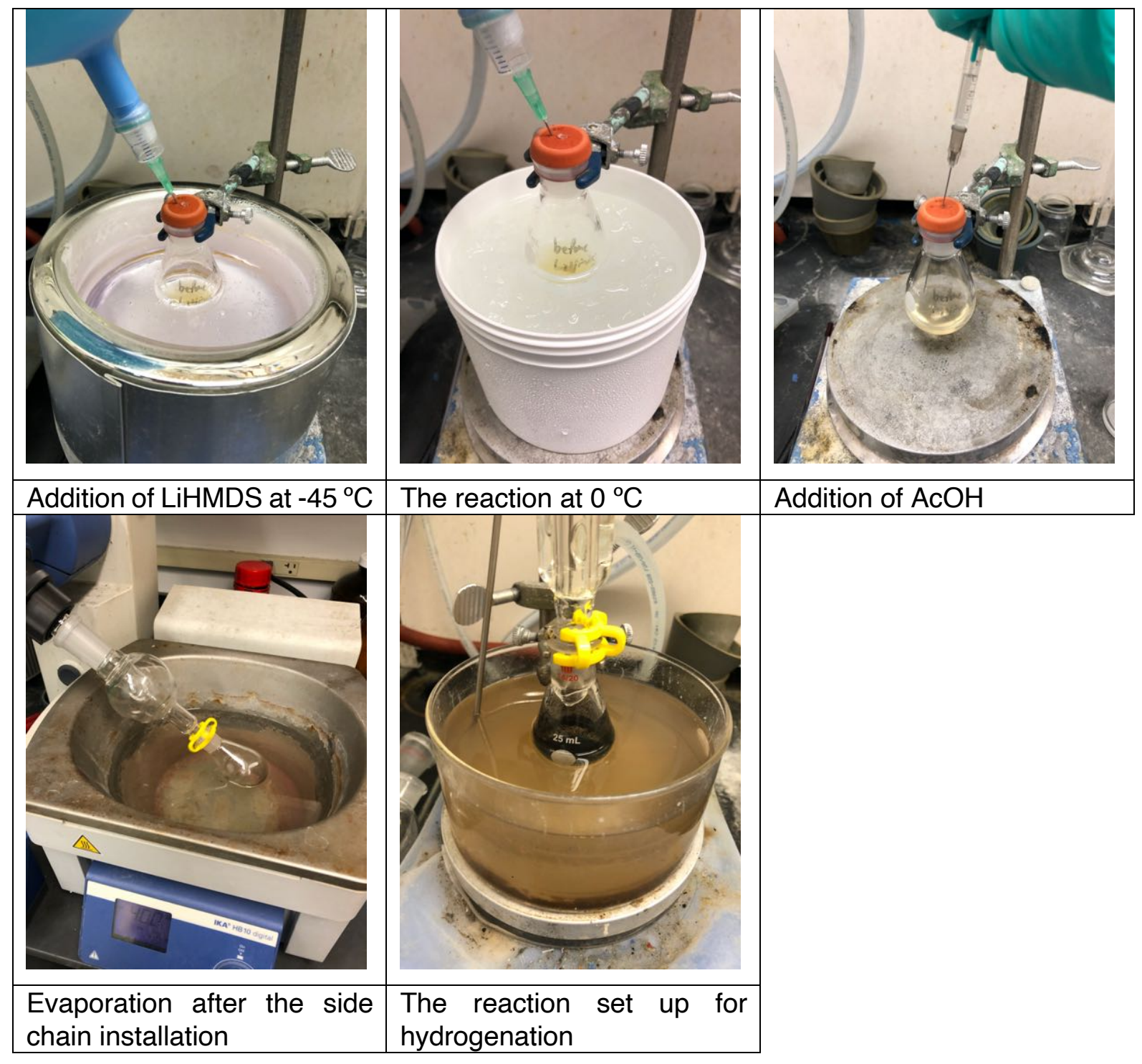


Compound 35

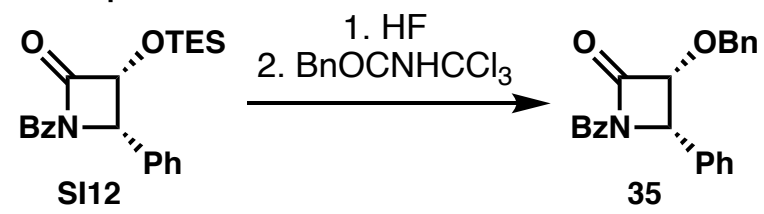

A plastic centrifuge tube was charged with TES $\beta$-lactam SI12 $(500 \mathrm{mg}, 1.31 \mathrm{mmol})$ and MeCN $(5 \mathrm{~mL})$ then cooled to $0{ }^{\circ} \mathrm{C}$. A solution of $\mathrm{HF}(137 \mu \mathrm{L}, 3.93 \mathrm{mmol}, 48 \%$ aqueous solution, $3 \mathrm{eq})$ in pyridine $(274 \mu \mathrm{L})$ and $\mathrm{MeCN}(5 \mathrm{~mL})$ was added dropwise and the reaction was stirred at the same temperature. After $1 \mathrm{~h}$, the reaction was diluted with EtOAc and quenched with $\mathrm{NaHCO}_{3}$ (saturated aq.). The aqueous layer was extracted with EtOAc (x 3). The combined organic phase was washed with brine then dried over $\mathrm{MgSO}_{4}$. The solvent was removed under reduced pressure and the resulting residue was re-dissolved in EtOAc and filtered through a short plug of $\mathrm{SiO}_{2}$. The solvent was removed under reduced pressure and the resulting white solid was subjected to the next step without further purification.

The crude material from the previous step was dissolved in DCM $(5 \mathrm{~mL})$. To this solution benzyl 2,2,2-trichloroacetimidate $(0.42 \mathrm{~mL}, 2.24 \mathrm{mmol}, 1.7 \mathrm{eq})$ and TfOH $(50 \mu \mathrm{L}, 0.560$ $\mathrm{mmol}, 0.43 \mathrm{eq}$ ) was added at $\mathrm{rt}$ and stirred for $20 \mathrm{~min}$. The reaction was diluted with EtOAc and quenched with $\mathrm{NaHCO}_{3}$ (saturated aq.). The aqueous layer was extracted with EtOAc (x 3). The combined organic phase was washed with brine then dried over $\mathrm{MgSO}_{4}$. The solvent was removed under reduced pressure and the resulting residue was purified by flash column chromatography $\left(\mathrm{SiO}_{2}\right.$, hexane/EtOAc 6:1 to 4:1) to yield Benzyl $\beta$-lactam 35 (358 mg, $1.00 \mathrm{mmol}, 76 \%$ ). Benzyl $\beta$-lactam 35 slowly decomposed in frozen benzene at $-20^{\circ} \mathrm{C}$ and was utilized in the next step immediately.

Physical state: white solid;

TLC: $R_{f}=0.39$ (hexane/EtOAc 4:1, UV active on TLC);

${ }^{1} \mathrm{H}$ NMR $\left(600 \mathrm{MHz}, \mathrm{CDCl}_{3}\right) \delta 8.07-7.99(\mathrm{~m}, 2 \mathrm{H}), 7.60(\mathrm{ddt}, J=8.7,7.1,1.3 \mathrm{~Hz}, 1 \mathrm{H})$, $7.51-7.45(\mathrm{~m}, 3 \mathrm{H}), 7.43-7.36(\mathrm{~m}, 3 \mathrm{H}), 7.28-7.24(\mathrm{~m}, 4 \mathrm{H}), 7.02-7.00(\mathrm{~m}, 2 \mathrm{H}), 5.45$ $(\mathrm{d}, J=5.9 \mathrm{~Hz}, 1 \mathrm{H}), 4.99(\mathrm{~d}, J=5.9 \mathrm{~Hz}, 1 \mathrm{H}), 4.43(\mathrm{~d}, J=11.3 \mathrm{~Hz}, 1 \mathrm{H}), 4.36(\mathrm{~d}, J=11.3$ $\mathrm{Hz}, 1 \mathrm{H})$.

${ }^{13} \mathrm{C}$ NMR $\left(151 \mathrm{MHz}, \mathrm{CDCl}_{3}\right) \delta 166.2,163.5,136.1,133.7,133.6,132.0,130.1,128.8$, 128.7, 128. 6, 128.6, 128.4, 128.4, 128.3, 128.1, 81.3, 72.9, 60.0.

HRMS (ESI-TOF): calc'd for $\mathrm{C}_{23} \mathrm{H}_{20} \mathrm{NO}_{3}\left[\mathrm{M}+\mathrm{H}^{+}\right]$: 358.1438, found: 358.1447 . $[\alpha]^{25} \mathrm{D}:+221.4\left(c=0.33, \mathrm{CHCl}_{3}\right)$ 


\section{X-ray Crystal Structure}

Compound 17

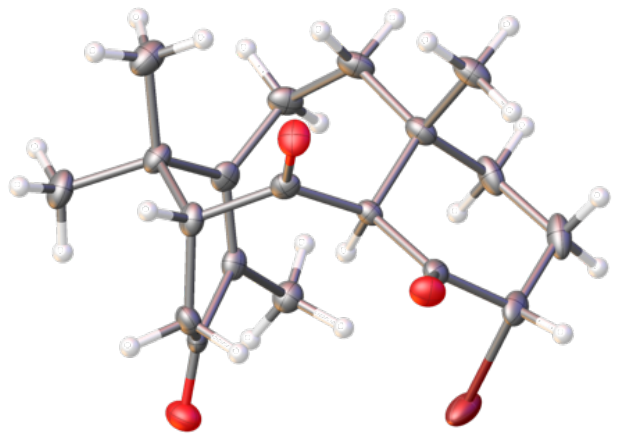

Table 1. Crystal data and structure refinement for Baran 753.

Report date

Identification code

Empirical formula

Molecular formula

Formula weight

Temperature

Wavelength

Crystal system

Space group

Unit cell dimensions

Volume

Z

Density (calculated)

Absorption coefficient

$\mathrm{F}(000)$

Crystal size

Crystal color, habit

Theta range for data collection

Index ranges

Reflections collected

Independent reflections

Completeness to theta $=25.242^{\circ}$

Absorption correction

Max. and min. transmission

Refinement method

Data / restraints / parameters

Goodness-of-fit on $\mathrm{F}^{2}$
2019-12-11

baran 753

C19 H25 Br O3

C19 H25 Br O3

381.30

$100.0 \mathrm{~K}$

$0.71073 \AA$

Orthorhombic

$\mathrm{P} 2{ }_{12}{ }_{1}$

$\mathrm{a}=8.3426(2) \AA$

$\alpha=90^{\circ}$.

$\mathrm{B}=13.7003(4) \AA$

$\beta=90^{\circ}$.

$\mathrm{C}=15.3067(4) \AA$

$\gamma=90^{\circ}$.

1749.50(8) $\AA^{3}$

4

$1.448 \mathrm{Mg} / \mathrm{m}^{3}$

$2.362 \mathrm{~mm}^{-1}$

792

$0.15 \times 0.15 \times 0.115 \mathrm{~mm}^{3}$

colorless block

1.995 to $26.729^{\circ}$.

$-10<=\mathrm{h}<=10,-17<=\mathrm{k}<=17,-18<=\mathrm{k}<=19$

10579

$3670[\mathrm{R}(\mathrm{int})=0.0288]$

$99.1 \%$

Semi-empirical from equivalents

0.6467 and 0.5163

Full-matrix least-squares on $\mathrm{F}^{2}$

$3670 / 0 / 212$

1.049 
Final $\mathrm{R}$ indices $[\mathrm{I}>2 \operatorname{sigma}(\mathrm{I})]$

$$
\begin{aligned}
& \mathrm{R} 1=0.0271, \mathrm{wR} 2=0.0640 \\
& \mathrm{R} 1=0.0301, \mathrm{wR} 2=0.0648 \\
& 0.004(5) \\
& 0.518 \text { and }-0.325 \text { e. } \AA^{-3}
\end{aligned}
$$$$
\mathrm{R} \text { indices (all data) }
$$

\begin{tabular}{|c|c|c|c|c|}
\hline & $\mathrm{x}$ & $\mathrm{y}$ & $\mathrm{z}$ & $\mathrm{U}(\mathrm{eq})$ \\
\hline $\operatorname{Br}(1)$ & 2661(1) & $4633(1)$ & $4340(1)$ & $33(1)$ \\
\hline $\mathrm{O}(1)$ & $5797(2)$ & $6390(2)$ & $4164(1)$ & $24(1)$ \\
\hline $\mathrm{O}(2)$ & $8081(2)$ & $6404(2)$ & $5653(1)$ & $24(1)$ \\
\hline $\mathrm{O}(3)$ & $5809(3)$ & $2450(2)$ & $5180(1)$ & $28(1)$ \\
\hline $\mathrm{C}(1)$ & $7316(3)$ & $5651(2)$ & $5674(2)$ & $17(1)$ \\
\hline$C(2)$ & $5478(3)$ & $5682(2)$ & $5606(2)$ & $15(1)$ \\
\hline$C(3)$ & 4920(3) & $6076(2)$ & $4726(2)$ & $17(1)$ \\
\hline$C(4)$ & $3120(4)$ & $6021(2)$ & 4594(2) & $23(1)$ \\
\hline$C(5)$ & $2160(4)$ & $6364(2)$ & 5381(2) & $29(1)$ \\
\hline$C(6)$ & $2800(3)$ & $5995(2)$ & $6248(2)$ & $24(1)$ \\
\hline$C(7)$ & $4595(4)$ & $6216(2)$ & $6376(2)$ & $19(1)$ \\
\hline $\mathrm{C}(8)$ & 5193(4) & $5887(3)$ & $7286(2)$ & $23(1)$ \\
\hline $\mathrm{C}(9)$ & $5267(4)$ & 4791(2) & $7544(2)$ & $22(1)$ \\
\hline$C(10)$ & $6153(3)$ & $4160(2)$ & $6908(2)$ & $18(1)$ \\
\hline $\mathrm{C}(11)$ & 7974(3) & $4317(2)$ & $6757(2)$ & $20(1)$ \\
\hline$C(12)$ & $8125(3)$ & $4660(2)$ & $5782(2)$ & $19(1)$ \\
\hline$C(13)$ & $7485(4)$ & $3859(2)$ & $5176(2)$ & $20(1)$ \\
\hline$C(14)$ & $6222(3)$ & $3182(2)$ & $5572(2)$ & $18(1)$ \\
\hline$C(15)$ & $5399(3)$ & $3515(2)$ & $6385(2)$ & $17(1)$ \\
\hline$C(16)$ & $3688(3)$ & $3161(2)$ & $6470(2)$ & $21(1)$ \\
\hline $\mathrm{C}(17)$ & $4874(4)$ & $7325(2)$ & $6328(2)$ & $24(1)$ \\
\hline$C(18)$ & $8810(4)$ & $5050(3)$ & $7366(2)$ & $28(1)$ \\
\hline$C(19)$ & $8870(4)$ & $3341(3)$ & $6874(2)$ & $26(1)$ \\
\hline
\end{tabular}

Absolute structure parameter

Table 2. Atomic coordinates $\left(\times 10^{4}\right)$ and equivalent isotropic displacement parameters $\left(\AA^{2} \times 10^{3}\right)$ for Baran753. $U(e q)$ is defined as one third of the trace of the orthogonalized $U^{i j}$ tensor. 
Table 3. Bond lengths $[\AA]$ and angles $\left[{ }^{\circ}\right]$ for Baran 753 .

\begin{tabular}{|c|c|}
\hline $\begin{array}{l}\mathrm{Br}(1)-\mathrm{C}(4) \\
\mathrm{O}(1)-\mathrm{C}(3)\end{array}$ & $\begin{array}{l}1.978(3) \\
1.208(3)\end{array}$ \\
\hline $\mathrm{O}(2)-\mathrm{C}(1)$ & $1.214(3)$ \\
\hline $\mathrm{O}(3)-\mathrm{C}(14)$ & $1.219(4)$ \\
\hline$C(1)-C(2)$ & $1.537(3)$ \\
\hline$C(1)-C(12)$ & $1.525(4)$ \\
\hline $\mathrm{C}(2)-\mathrm{H}(2)$ & 1.0000 \\
\hline $\mathrm{C}(2)-\mathrm{C}(3)$ & $1.524(4)$ \\
\hline $\mathrm{C}(2)-\mathrm{C}(7)$ & $1.571(4)$ \\
\hline$C(3)-C(4)$ & $1.518(4)$ \\
\hline $\mathrm{C}(4)-\mathrm{H}(4)$ & 1.0000 \\
\hline$C(4)-C(5)$ & $1.520(4)$ \\
\hline $\mathrm{C}(5)-\mathrm{H}(5 \mathrm{~A})$ & 0.9900 \\
\hline $\mathrm{C}(5)-\mathrm{H}(5 \mathrm{~B})$ & 0.9900 \\
\hline$C(5)-C(6)$ & $1.517(4)$ \\
\hline $\mathrm{C}(6)-\mathrm{H}(6 \mathrm{~A})$ & 0.9900 \\
\hline $\mathrm{C}(6)-\mathrm{H}(6 \mathrm{~B})$ & 0.9900 \\
\hline$C(6)-C(7)$ & $1.540(4)$ \\
\hline $\mathrm{C}(7)-\mathrm{C}(8)$ & $1.547(4)$ \\
\hline$C(7)-C(17)$ & $1.539(4)$ \\
\hline $\mathrm{C}(8)-\mathrm{H}(8 \mathrm{~A})$ & 0.9900 \\
\hline $\mathrm{C}(8)-\mathrm{H}(8 \mathrm{~B})$ & 0.9900 \\
\hline $\mathrm{C}(8)-\mathrm{C}(9)$ & $1.554(4)$ \\
\hline $\mathrm{C}(9)-\mathrm{H}(9 \mathrm{~A})$ & 0.9900 \\
\hline $\mathrm{C}(9)-\mathrm{H}(9 \mathrm{~B})$ & 0.9900 \\
\hline$C(9)-C(10)$ & $1.497(4)$ \\
\hline$C(10)-C(11)$ & $1.552(4)$ \\
\hline$C(10)-C(15)$ & $1.349(4)$ \\
\hline$C(11)-C(12)$ & $1.569(4)$ \\
\hline$C(11)-C(18)$ & $1.537(4)$ \\
\hline$C(11)-C(19)$ & $1.543(4)$ \\
\hline $\mathrm{C}(12)-\mathrm{H}(12)$ & 1.0000 \\
\hline$C(12)-C(13)$ & $1.533(4)$ \\
\hline $\mathrm{C}(13)-\mathrm{H}(13 \mathrm{~A})$ & 0.9900 \\
\hline C(13)-H(13B) & 0.9900 \\
\hline$C(13)-C(14)$ & $1.529(4)$ \\
\hline$C(14)-C(15)$ & $1.492(4)$ \\
\hline$C(15)-C(16)$ & $1.513(4)$ \\
\hline $\mathrm{C}(16)-\mathrm{H}(16 \mathrm{~A})$ & 0.9800 \\
\hline
\end{tabular}




\begin{tabular}{|c|c|}
\hline $\mathrm{C}(16)-\mathrm{H}(16 \mathrm{~B})$ & 0.9800 \\
\hline $\mathrm{C}(16)-\mathrm{H}(16 \mathrm{C})$ & 0.9800 \\
\hline $\mathrm{C}(17)-\mathrm{H}(17 \mathrm{~A})$ & 0.9800 \\
\hline $\mathrm{C}(17)-\mathrm{H}(17 \mathrm{~B})$ & 0.9800 \\
\hline $\mathrm{C}(17)-\mathrm{H}(17 \mathrm{C})$ & 0.9800 \\
\hline $\mathrm{C}(18)-\mathrm{H}(18 \mathrm{~A})$ & 0.9800 \\
\hline $\mathrm{C}(18)-\mathrm{H}(18 \mathrm{~B})$ & 0.9800 \\
\hline $\mathrm{C}(18)-\mathrm{H}(18 \mathrm{C})$ & 0.9800 \\
\hline $\mathrm{C}(19)-\mathrm{H}(19 \mathrm{~A})$ & 0.9800 \\
\hline $\mathrm{C}(19)-\mathrm{H}(19 \mathrm{~B})$ & 0.9800 \\
\hline C(19)-H(19C) & 0.980 \\
\hline $\mathrm{O}(2)-\mathrm{C}(1)-\mathrm{C}(2)$ & $120.0(3)$ \\
\hline $\mathrm{O}(2)-\mathrm{C}(1)-\mathrm{C}(12)$ & $121.8(2)$ \\
\hline $\mathrm{C}(12)-\mathrm{C}(1)-\mathrm{C}(2)$ & $118.2(2)$ \\
\hline $\mathrm{C}(1)-\mathrm{C}(2)-\mathrm{H}(2)$ & 106.0 \\
\hline $\mathrm{C}(1)-\mathrm{C}(2)-\mathrm{C}(7)$ & $115.4(2)$ \\
\hline$C(3)-C(2)-C(1)$ & $112.0(2)$ \\
\hline $\mathrm{C}(3)-\mathrm{C}(2)-\mathrm{H}(2)$ & 106.0 \\
\hline$C(3)-C(2)-C(7)$ & $110.7(2)$ \\
\hline $\mathrm{C}(7)-\mathrm{C}(2)-\mathrm{H}(2)$ & 106.0 \\
\hline $\mathrm{O}(1)-\mathrm{C}(3)-\mathrm{C}(2)$ & $124.8(3)$ \\
\hline $\mathrm{O}(1)-\mathrm{C}(3)-\mathrm{C}(4)$ & $121.5(3)$ \\
\hline$C(4)-C(3)-C(2)$ & $113.7(2)$ \\
\hline $\mathrm{Br}(1)-\mathrm{C}(4)-\mathrm{H}(4)$ & 109.0 \\
\hline$C(3)-C(4)-B r(1)$ & $105.4(2)$ \\
\hline $\mathrm{C}(3)-\mathrm{C}(4)-\mathrm{H}(4)$ & 109.0 \\
\hline$C(3)-C(4)-C(5)$ & $113.6(3)$ \\
\hline$C(5)-C(4)-B r(1)$ & $110.5(2)$ \\
\hline $\mathrm{C}(5)-\mathrm{C}(4)-\mathrm{H}(4)$ & 109.0 \\
\hline $\mathrm{C}(4)-\mathrm{C}(5)-\mathrm{H}(5 \mathrm{~A})$ & 108.8 \\
\hline $\mathrm{C}(4)-\mathrm{C}(5)-\mathrm{H}(5 \mathrm{~B})$ & 108.8 \\
\hline $\mathrm{H}(5 \mathrm{~A})-\mathrm{C}(5)-\mathrm{H}(5 \mathrm{~B})$ & 107.7 \\
\hline$C(6)-C(5)-C(4)$ & $113.8(2)$ \\
\hline $\mathrm{C}(6)-\mathrm{C}(5)-\mathrm{H}(5 \mathrm{~A})$ & 108.8 \\
\hline $\mathrm{C}(6)-\mathrm{C}(5)-\mathrm{H}(5 \mathrm{~B})$ & 108.8 \\
\hline $\mathrm{C}(5)-\mathrm{C}(6)-\mathrm{H}(6 \mathrm{~A})$ & 109.0 \\
\hline $\mathrm{C}(5)-\mathrm{C}(6)-\mathrm{H}(6 \mathrm{~B})$ & 109.0 \\
\hline$C(5)-C(6)-C(7)$ & $112.8(2)$ \\
\hline $\mathrm{H}(6 \mathrm{~A})-\mathrm{C}(6)-\mathrm{H}(6 \mathrm{~B})$ & 107.8 \\
\hline $\mathrm{C}(7)-\mathrm{C}(6)-\mathrm{H}(6 \mathrm{~A})$ & 109.0 \\
\hline
\end{tabular}




\begin{tabular}{|c|c|}
\hline $\mathrm{C}(7)-\mathrm{C}(6)-\mathrm{H}(6 \mathrm{~B})$ & 109.0 \\
\hline$C(6)-C(7)-C(2)$ & $105.6(2)$ \\
\hline$C(6)-C(7)-C(8)$ & $111.8(3)$ \\
\hline$C(8)-C(7)-C(2)$ & $112.9(2)$ \\
\hline$C(17)-C(7)-C(2)$ & $110.7(2)$ \\
\hline$C(17)-C(7)-C(6)$ & $109.6(3)$ \\
\hline$C(17)-C(7)-C(8)$ & $106.3(3)$ \\
\hline $\mathrm{C}(7)-\mathrm{C}(8)-\mathrm{H}(8 \mathrm{~A})$ & 107.0 \\
\hline $\mathrm{C}(7)-\mathrm{C}(8)-\mathrm{H}(8 \mathrm{~B})$ & 107.0 \\
\hline$C(7)-C(8)-C(9)$ & $121.5(3)$ \\
\hline $\mathrm{H}(8 \mathrm{~A})-\mathrm{C}(8)-\mathrm{H}(8 \mathrm{~B})$ & 106.7 \\
\hline $\mathrm{C}(9)-\mathrm{C}(8)-\mathrm{H}(8 \mathrm{~A})$ & 107.0 \\
\hline $\mathrm{C}(9)-\mathrm{C}(8)-\mathrm{H}(8 \mathrm{~B})$ & 107.0 \\
\hline $\mathrm{C}(8)-\mathrm{C}(9)-\mathrm{H}(9 \mathrm{~A})$ & 108.7 \\
\hline $\mathrm{C}(8)-\mathrm{C}(9)-\mathrm{H}(9 \mathrm{~B})$ & 108.7 \\
\hline $\mathrm{H}(9 \mathrm{~A})-\mathrm{C}(9)-\mathrm{H}(9 \mathrm{~B})$ & 107.6 \\
\hline$C(10)-C(9)-C(8)$ & $114.3(2)$ \\
\hline $\mathrm{C}(10)-\mathrm{C}(9)-\mathrm{H}(9 \mathrm{~A})$ & 108.7 \\
\hline $\mathrm{C}(10)-\mathrm{C}(9)-\mathrm{H}(9 \mathrm{~B})$ & 108.7 \\
\hline$C(9)-C(10)-C(11)$ & $120.0(3)$ \\
\hline$C(15)-C(10)-C(9)$ & $122.3(3)$ \\
\hline$C(15)-C(10)-C(11)$ & $117.3(3)$ \\
\hline $\mathrm{C}(10)-\mathrm{C}(11)-\mathrm{C}(12)$ & $105.2(2)$ \\
\hline$C(18)-C(11)-C(10)$ & $116.4(3)$ \\
\hline$C(18)-C(11)-C(12)$ & $110.2(2)$ \\
\hline$C(18)-C(11)-C(19)$ & $106.0(2)$ \\
\hline$C(19)-C(11)-C(10)$ & $109.7(3)$ \\
\hline$C(19)-C(11)-C(12)$ & $109.4(2)$ \\
\hline$C(1)-C(12)-C(11)$ & $109.5(2)$ \\
\hline $\mathrm{C}(1)-\mathrm{C}(12)-\mathrm{H}(12)$ & 107.6 \\
\hline$C(1)-C(12)-C(13)$ & $114.7(2)$ \\
\hline $\mathrm{C}(11)-\mathrm{C}(12)-\mathrm{H}(12)$ & 107.6 \\
\hline $\mathrm{C}(13)-\mathrm{C}(12)-\mathrm{C}(11)$ & $109.5(2)$ \\
\hline $\mathrm{C}(13)-\mathrm{C}(12)-\mathrm{H}(12)$ & 107.6 \\
\hline $\mathrm{C}(12)-\mathrm{C}(13)-\mathrm{H}(13 \mathrm{~A})$ & 108.3 \\
\hline $\mathrm{C}(12)-\mathrm{C}(13)-\mathrm{H}(13 \mathrm{~B})$ & 108.3 \\
\hline $\mathrm{H}(13 \mathrm{~A})-\mathrm{C}(13)-\mathrm{H}(13 \mathrm{~B})$ & 107.4 \\
\hline $\mathrm{C}(14)-\mathrm{C}(13)-\mathrm{C}(12)$ & $115.7(2)$ \\
\hline $\mathrm{C}(14)-\mathrm{C}(13)-\mathrm{H}(13 \mathrm{~A})$ & 108.3 \\
\hline $\mathrm{C}(14)-\mathrm{C}(13)-\mathrm{H}(13 \mathrm{~B})$ & 108.3 \\
\hline $\mathrm{O}(3)-\mathrm{C}(14)-\mathrm{C}(13)$ & $119.9(3)$ \\
\hline
\end{tabular}




$\begin{array}{ll}\mathrm{O}(3)-\mathrm{C}(14)-\mathrm{C}(15) & 122.1(3) \\ \mathrm{C}(15)-\mathrm{C}(14)-\mathrm{C}(13) & 117.5(3) \\ \mathrm{C}(10)-\mathrm{C}(15)-\mathrm{C}(14) & 118.7(3) \\ \mathrm{C}(10)-\mathrm{C}(15)-\mathrm{C}(16) & 126.8(3) \\ \mathrm{C}(14)-\mathrm{C}(15)-\mathrm{C}(16) & 114.1(3) \\ \mathrm{C}(15)-\mathrm{C}(16)-\mathrm{H}(16 \mathrm{~A}) & 109.5 \\ \mathrm{C}(15)-\mathrm{C}(16)-\mathrm{H}(16 \mathrm{~B}) & 109.5 \\ \mathrm{C}(15)-\mathrm{C}(16)-\mathrm{H}(16 \mathrm{C}) & 109.5 \\ \mathrm{H}(16 \mathrm{~A})-\mathrm{C}(16)-\mathrm{H}(16 \mathrm{~B}) & 109.5 \\ \mathrm{H}(16 \mathrm{~A})-\mathrm{C}(16)-\mathrm{H}(16 \mathrm{C}) & 109.5 \\ \mathrm{H}(16 \mathrm{~B})-\mathrm{C}(16)-\mathrm{H}(16 \mathrm{C}) & 109.5 \\ \mathrm{C}(7)-\mathrm{C}(17)-\mathrm{H}(17 \mathrm{~A}) & 109.5 \\ \mathrm{C}(7)-\mathrm{C}(17)-\mathrm{H}(17 \mathrm{~B}) & 109.5 \\ \mathrm{C}(7)-\mathrm{C}(17)-\mathrm{H}(17 \mathrm{C}) & 109.5 \\ \mathrm{H}(17 \mathrm{~A})-\mathrm{C}(17)-\mathrm{H}(17 \mathrm{~B}) & 109.5 \\ \mathrm{H}(17 \mathrm{~A})-\mathrm{C}(17)-\mathrm{H}(17 \mathrm{C}) & 109.5 \\ \mathrm{H}(17 \mathrm{~B})-\mathrm{C}(17)-\mathrm{H}(17 \mathrm{C}) & 109.5 \\ \mathrm{C}(11)-\mathrm{C}(18)-\mathrm{H}(18 \mathrm{~A}) & 109.5 \\ \mathrm{C}(11)-\mathrm{C}(18)-\mathrm{H}(18 \mathrm{~B}) & 109.5 \\ \mathrm{C}(11)-\mathrm{C}(18)-\mathrm{H}(18 \mathrm{C}) & 109.5 \\ \mathrm{H}(18 \mathrm{~A})-\mathrm{C}(18)-\mathrm{H}(18 \mathrm{~B}) & 109.5 \\ \mathrm{H}(18 \mathrm{~A})-\mathrm{C}(18)-\mathrm{H}(18 \mathrm{C}) & 109.5 \\ \mathrm{H}(18 \mathrm{~B})-\mathrm{C}(18)-\mathrm{H}(18 \mathrm{C}) & 109.5 \\ \mathrm{C}(11)-\mathrm{C}(19)-\mathrm{H}(19 \mathrm{~A}) & 109.5 \\ \mathrm{C}(11)-\mathrm{C}(19)-\mathrm{H}(19 B) & 109.5 \\ \mathrm{C}(11)-\mathrm{C}(19)-\mathrm{H}(19 \mathrm{C}) & 109.5 \\ \mathrm{H}(19 \mathrm{~A})-\mathrm{C}(19)-\mathrm{H}(19 \mathrm{~B}) & 109.5 \\ \mathrm{H}(19 \mathrm{~A})-\mathrm{C}(19)-\mathrm{H}(19 \mathrm{C}) & 109.5 \\ \mathrm{H}(19 \mathrm{~B})-\mathrm{C}(19)-\mathrm{H}(19 \mathrm{C}) & 109.5 \\ & \end{array}$


Table 4. Anisotropic displacement parameters $\left(\AA^{2} \times 10^{3}\right)$ for Baran753. The anisotropic displacement factor exponent takes the form: $-2 \pi^{2}\left[h^{2} a^{* 2} U^{11}+\ldots+2 h k a^{*} b^{*} U^{12}\right]$

\begin{tabular}{|c|c|c|c|c|c|c|}
\hline & $\mathrm{U}^{11}$ & $\mathrm{U}^{22}$ & $\mathrm{U}^{33}$ & $\mathrm{U}^{23}$ & $\mathrm{U}^{13}$ & $\mathrm{U}^{12}$ \\
\hline $\operatorname{Br}(1)$ & $37(1)$ & $21(1)$ & $39(1)$ & $3(1)$ & $-22(1)$ & $-7(1)$ \\
\hline $\mathrm{O}(1)$ & $29(1)$ & $24(1)$ & $18(1)$ & $0(1)$ & $3(1)$ & $-3(1)$ \\
\hline $\mathrm{O}(2)$ & $18(1)$ & $22(1)$ & $31(1)$ & $5(1)$ & $2(1)$ & $-6(1)$ \\
\hline $\mathrm{O}(3)$ & $27(1)$ & $23(1)$ & $32(1)$ & $-8(1)$ & 1(1) & $-1(1)$ \\
\hline $\mathrm{C}(1)$ & $18(1)$ & $21(2)$ & $13(1)$ & $0(1)$ & 2(1) & $-2(1)$ \\
\hline$C(2)$ & $16(1)$ & $13(1)$ & $15(1)$ & $0(1)$ & $3(1)$ & $0(1)$ \\
\hline$C(3)$ & $20(2)$ & $12(2)$ & $19(1)$ & $-1(1)$ & $-1(1)$ & $1(1)$ \\
\hline$C(4)$ & $23(2)$ & $12(2)$ & $33(2)$ & $6(1)$ & $-7(1)$ & $-1(1)$ \\
\hline$C(5)$ & $15(1)$ & $19(2)$ & $53(2)$ & $9(2)$ & $2(1)$ & $2(1)$ \\
\hline$C(6)$ & $17(2)$ & $17(2)$ & $37(2)$ & $2(1)$ & 11(1) & $3(1)$ \\
\hline$C(7)$ & $21(2)$ & $16(2)$ & $20(1)$ & $-2(1)$ & $6(1)$ & $-1(1)$ \\
\hline$C(8)$ & $31(2)$ & $21(2)$ & $18(1)$ & $-4(1)$ & $7(1)$ & $-3(2)$ \\
\hline $\mathrm{C}(9)$ & $30(2)$ & $21(2)$ & $14(1)$ & $1(1)$ & $4(1)$ & $-3(1)$ \\
\hline$C(10)$ & $21(1)$ & $18(2)$ & $14(1)$ & $6(1)$ & 1(1) & $-1(1)$ \\
\hline $\mathrm{C}(11)$ & $17(1)$ & $22(2)$ & $20(1)$ & $3(1)$ & $-4(1)$ & $-3(1)$ \\
\hline $\mathrm{C}(12)$ & $13(1)$ & $21(2)$ & $23(1)$ & $5(1)$ & 2(1) & $0(1)$ \\
\hline $\mathrm{C}(13)$ & $21(1)$ & $18(2)$ & $20(1)$ & $-1(1)$ & $3(1)$ & $5(1)$ \\
\hline$C(14)$ & $16(1)$ & $15(2)$ & $22(1)$ & $-1(1)$ & $-5(1)$ & $5(1)$ \\
\hline$C(15)$ & $16(1)$ & $16(2)$ & $20(1)$ & $7(1)$ & $0(1)$ & $-1(1)$ \\
\hline$C(16)$ & $19(2)$ & $21(2)$ & $22(2)$ & $3(1)$ & $0(1)$ & $-3(1)$ \\
\hline $\mathrm{C}(17)$ & $31(2)$ & $16(2)$ & $25(2)$ & $-3(1)$ & $12(1)$ & $-3(1)$ \\
\hline $\mathrm{C}(18)$ & $28(2)$ & $28(2)$ & $28(2)$ & $7(2)$ & $-8(1)$ & $-7(1)$ \\
\hline$C(19)$ & $16(1)$ & $29(2)$ & $32(2)$ & $7(2)$ & $-4(1)$ & $1(1)$ \\
\hline
\end{tabular}


Table 5. Hydrogen coordinates ( $\left.\times 10^{4}\right)$ and isotropic displacement parameters $\left(\AA^{2} \times 10^{3}\right)$ for Baran753.

\begin{tabular}{|c|c|c|c|c|}
\hline & $\mathrm{x}$ & $\mathrm{y}$ & z & $\mathrm{U}(\mathrm{eq})$ \\
\hline $\mathrm{H}(2)$ & 5114 & 4987 & 5628 & 18 \\
\hline $\mathrm{H}(4)$ & 2819 & 6424 & 4075 & 27 \\
\hline $\mathrm{H}(5 \mathrm{~A})$ & 2155 & 7086 & 5390 & 35 \\
\hline $\mathrm{H}(5 \mathrm{~B})$ & 1036 & 6143 & 5314 & 35 \\
\hline $\mathrm{H}(6 \mathrm{~A})$ & 2630 & 5281 & 6282 & 29 \\
\hline $\mathrm{H}(6 \mathrm{~B})$ & 2185 & 6302 & 6728 & 29 \\
\hline $\mathrm{H}(8 \mathrm{~A})$ & 4509 & 6217 & 7724 & 28 \\
\hline $\mathrm{H}(8 \mathrm{~B})$ & 6288 & 6154 & 7359 & 28 \\
\hline $\mathrm{H}(9 \mathrm{~A})$ & 5787 & 4735 & 8123 & 26 \\
\hline $\mathrm{H}(9 \mathrm{~B})$ & 4158 & 4541 & 7602 & 26 \\
\hline $\mathrm{H}(12)$ & 9291 & 4747 & 5652 & 23 \\
\hline $\mathrm{H}(13 \mathrm{~A})$ & 7019 & 4174 & 4652 & 24 \\
\hline $\mathrm{H}(13 \mathrm{~B})$ & 8402 & 3456 & 4979 & 24 \\
\hline $\mathrm{H}(16 \mathrm{~A})$ & 3264 & 3350 & 7042 & 31 \\
\hline $\mathrm{H}(16 \mathrm{~B})$ & 3661 & 2448 & 6413 & 31 \\
\hline $\mathrm{H}(16 \mathrm{C})$ & 3032 & 3455 & 6009 & 31 \\
\hline $\mathrm{H}(17 \mathrm{~A})$ & 6009 & 7466 & 6434 & 36 \\
\hline $\mathrm{H}(17 \mathrm{~B})$ & 4220 & 7651 & 6773 & 36 \\
\hline $\mathrm{H}(17 \mathrm{C})$ & 4571 & 7564 & 5748 & 36 \\
\hline $\mathrm{H}(18 \mathrm{~A})$ & 8462 & 5713 & 7221 & 42 \\
\hline $\mathrm{H}(18 \mathrm{~B})$ & 9974 & 4999 & 7292 & 42 \\
\hline $\mathrm{H}(18 \mathrm{C})$ & 8527 & 4904 & 7974 & 42 \\
\hline $\mathrm{H}(19 \mathrm{~A})$ & 8835 & 3147 & 7490 & 39 \\
\hline $\mathrm{H}(19 \mathrm{~B})$ & 9988 & 3419 & 6690 & 39 \\
\hline $\mathrm{H}(19 \mathrm{C})$ & 8356 & 2837 & 6516 & 39 \\
\hline
\end{tabular}




\section{Radiocarbon dating Experiment}

Natural sample was purchased from Sigma-Aldrich (part\# T7402-5MG, Paclitaxel, from Taxus brevifolia, $>=95 \%$ (HPLC), powder).

Radiocarbon dating experiment was performed by National Ocean Sciences Accelerator Mass Spectrometry (NOSAMS) facility.

When an organism dies it ceases to replenish carbon in its tissues and lose ${ }^{14} \mathrm{C}$ by radio decay to ${ }^{14} \mathrm{~N}$. Therefore, ${ }^{14} \mathrm{C}$ isotope content measurement tells when the organism that produced sample died, namely how old the sample is. Fraction modern ( $F$ Modern) is the ratio of the activity of the sample from that of a modern standard. As the deviation is bigger (closer to 0 ) the older the sample is, and smaller (close to 1 ) the more modern. ${ }^{6}$ Baran1 was synthetic Taxol $^{\circledR}$ prepared from petroleum feed stocks chemicals (10-13, $\mathrm{MeMgBr}$ ). This resulted in a $\mathrm{F}$ modern value significantly smaller than 1 , proving that this sample is made of aged carbons which do not contain a lot of ${ }^{14} \mathrm{C}$ isotope. On the other hand, natural tree-derived Taxol ${ }^{\circledR}$, Baran2, exhibited $\mathrm{F}$ modern value close to 1 , meaning this sample was made of ${ }^{14} \mathrm{C}$ isotope rich $\mathrm{CO}_{2}$ freshly harvested from air.

\begin{tabular}{|l|l|l|l|r|r|r|r|r|}
\hline $\begin{array}{l}\text { Submitter } \\
\text { Identification }\end{array}$ & Type & Process & Accession \# & F Modern & Fm Err & Age & Age Err & $\boldsymbol{\Delta}$ 13C \\
\hline Baran1 & Compound-specific & (OC) & OS-154085 & 0.01150 & 0.00410 & 35900 & 2900 & -31.89 \\
\hline Baran2 & Compound-specific & (OC) & OS-154086 & 1.00300 & 0.00200 & $>$ Modern & & -29.69 \\
\hline
\end{tabular}

Libby ages were calculated by: Age $=-8033 \ln (\mathrm{Fm})$.

$\tau_{1 / 2}=5568$ following the convention outlined by Stuiver and Polach (1977) and Stuiver (1980). 


\section{References}

1. Mendoza, A., Ishihara, Y. \& Baran, P. S. Scalable enantioselective total synthesis of taxanes. Nat. Chem. 4, 21-25 (2012).

2. Kehrli, S., Martin, D., Rix, D., Mauduit, M. \& Alexakis, A. Formation of quaternary chiral centers by $\mathrm{N}$-Heterocyclic carbene-CuCatalyzed asymmetric conjugate addition reactions with grignard reagents on trisubstituted cyclic enones. Chem. A Eur. J. 16, 9890-9904 (2010).

3. Wilde, N. C., Isomura, M., Mendoza, A. \& Baran, P. S. Two-Phase Synthesis of (-)-Taxuyunnanine D. J. Am. Chem. Soc. 136, 4909-4912 (2014).

4. Gibert, M., Ferrer, M., Sänchez-Baeza, F. \& Messeguer, A. Availability and Reactivity of Concentrated Dimethyldioxirane Solutions in Solvents Other Than Acetone. Tetrahedron 53, 8643-8650 (1997).

5. Holton, R. A., Kim, H-B., Somoza, C., Liang, F., Biediger, R. J., Boatman, P. D., Shindo, M., Smith, C. C., Kim, S., Nadizadeh, H., Suzuki, Y., Tao, C., Vu, P., Tang, S., Zhang, P., Murthi, K. K., Gentile, L. N. \& Liu, J. H. First Total Synthesis of Taxol. 2. Completion of the $C$ and D Rings. J. Am. Chem. Soc. 116, 1599-1600 (1994).

6. Reddy, C. M., Xu, L., Eglinton, T. I., Boon, J. P. \& Faulkner, D. J. Radiocarbon content of synthetic and natural semi-volatile halogenated organic compounds. Environ. Pollut. 120, 163-168 (2002). 


\section{NMR Spectra}

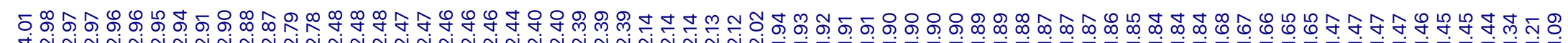

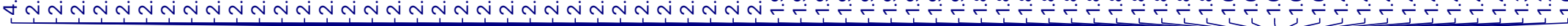
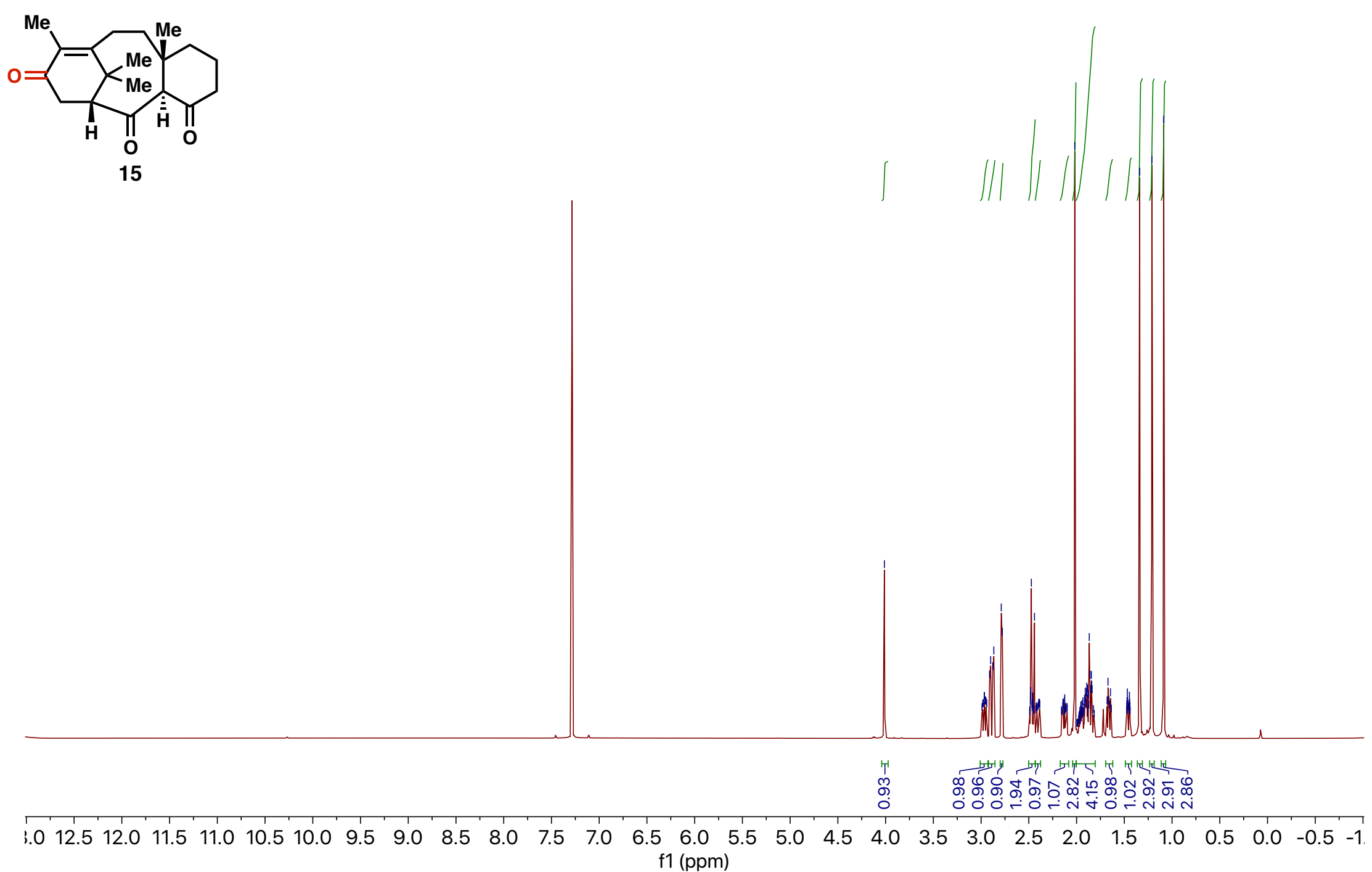


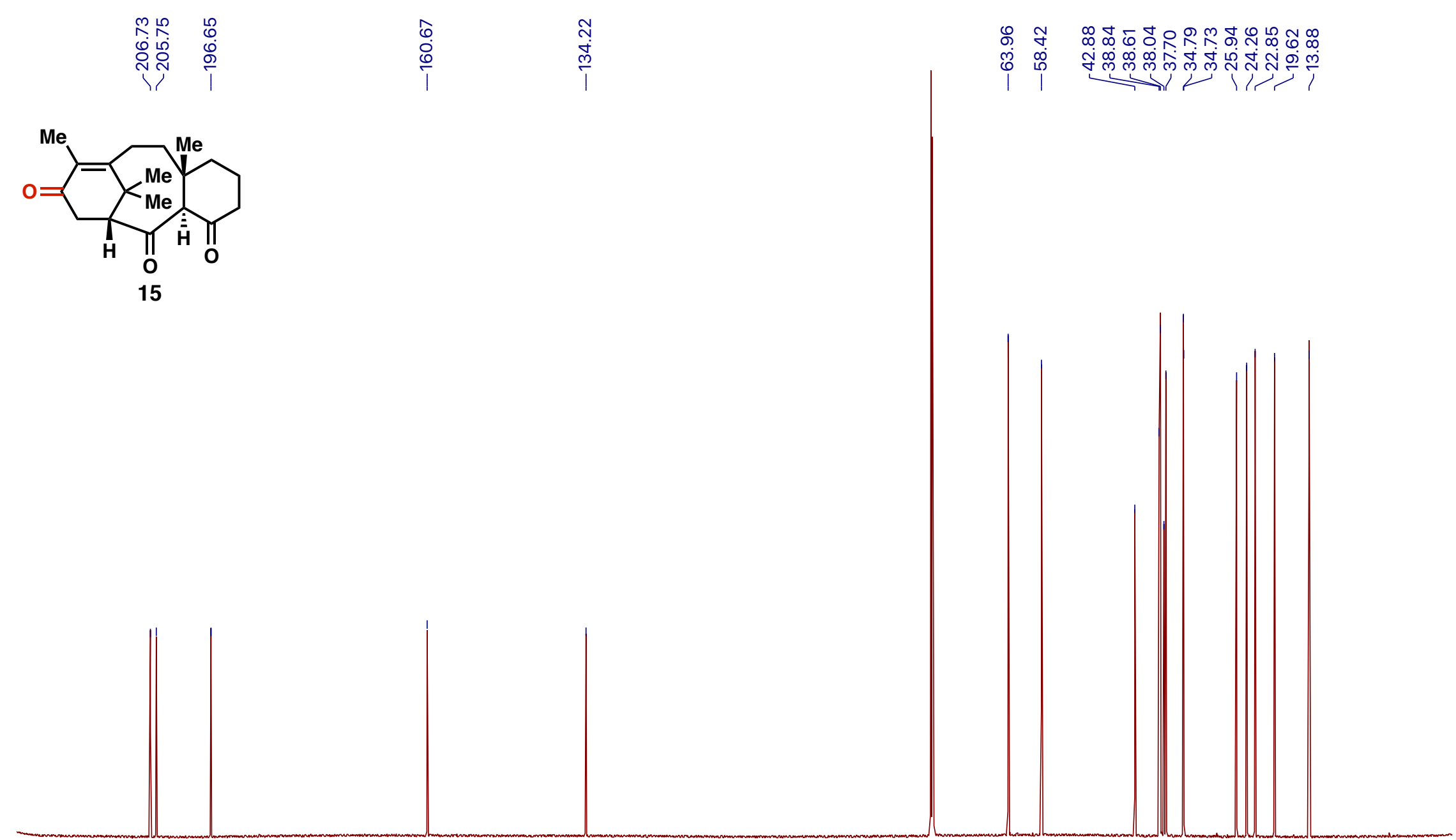

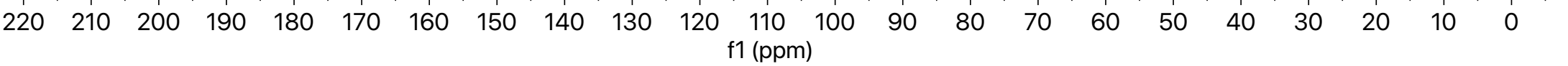




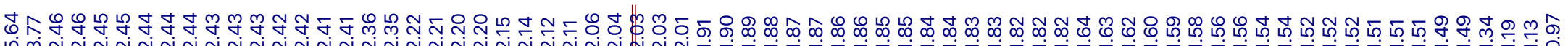

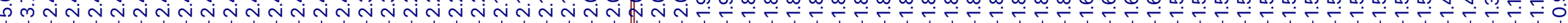
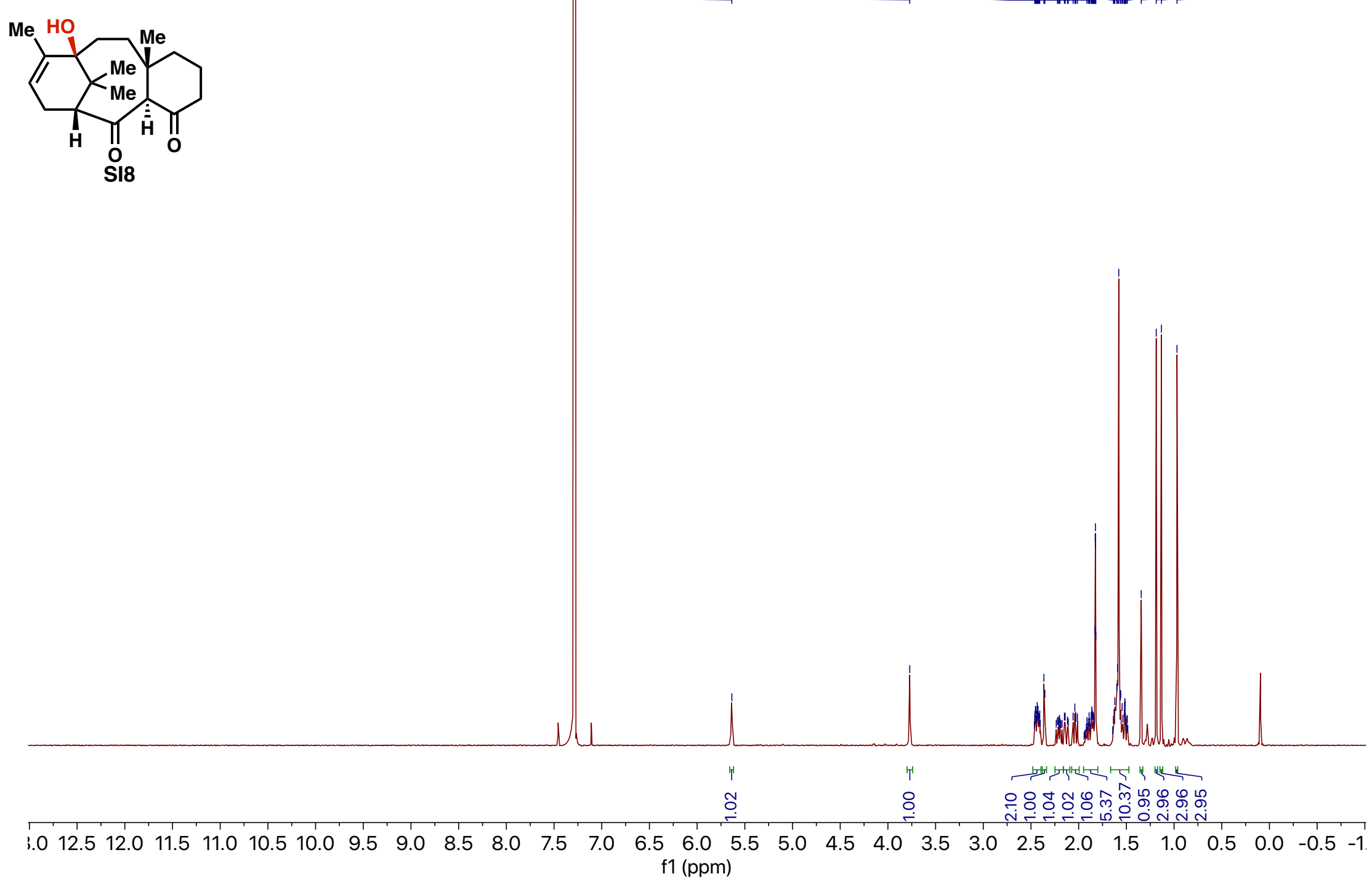


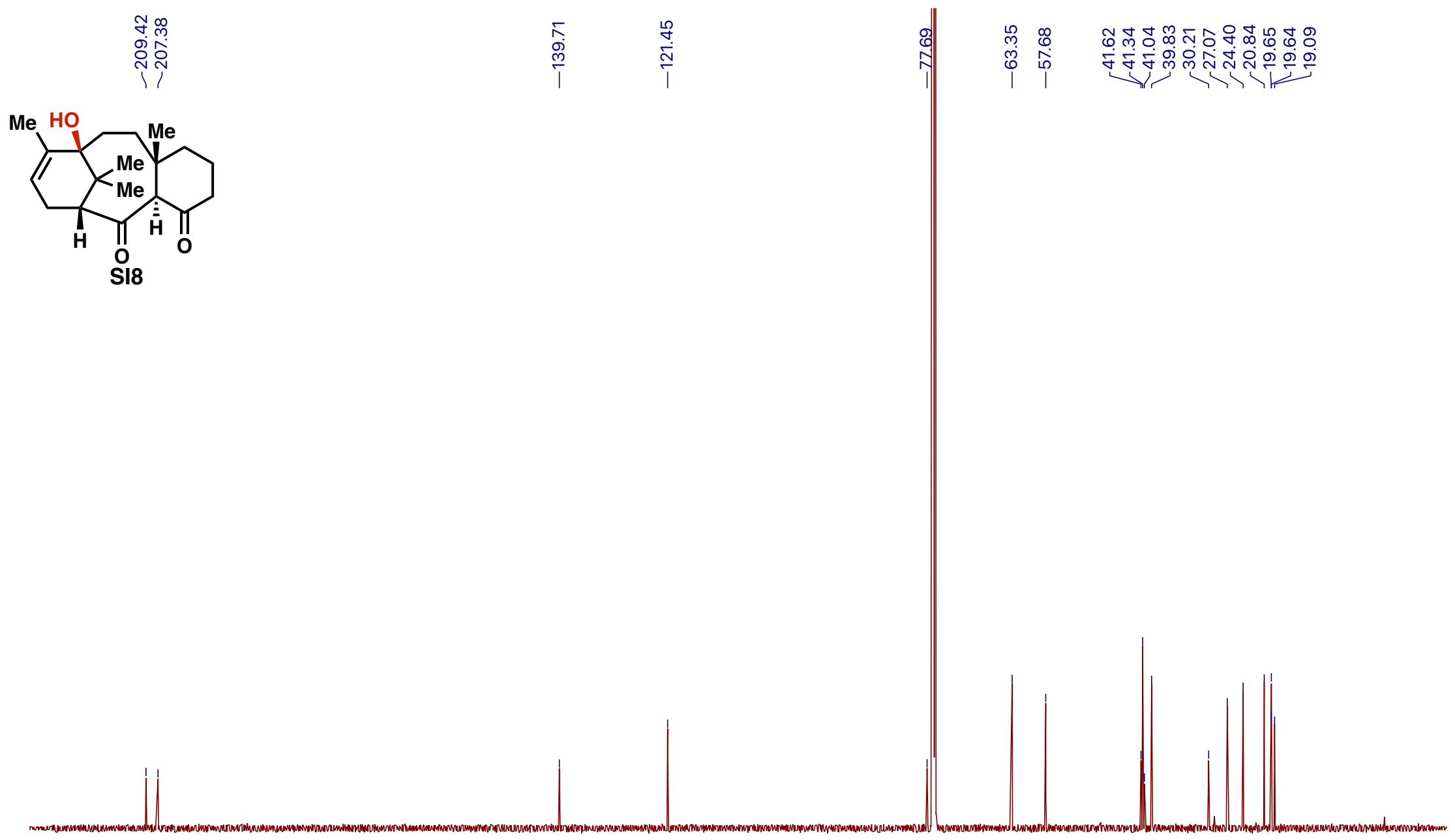

$\begin{array}{lllllll}220 & 210 & 200 & 190 & 180 & 170 & 160\end{array}$ $150140 \quad 130$

$120 \quad 110$

1 (ppm)

$60 \quad 50$

$40 \quad 30 \quad 20$

201

100 


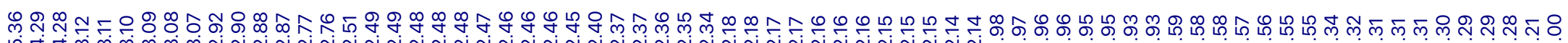

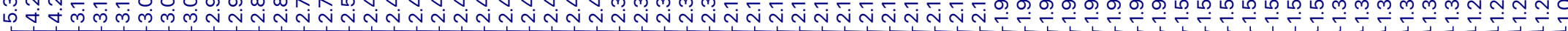
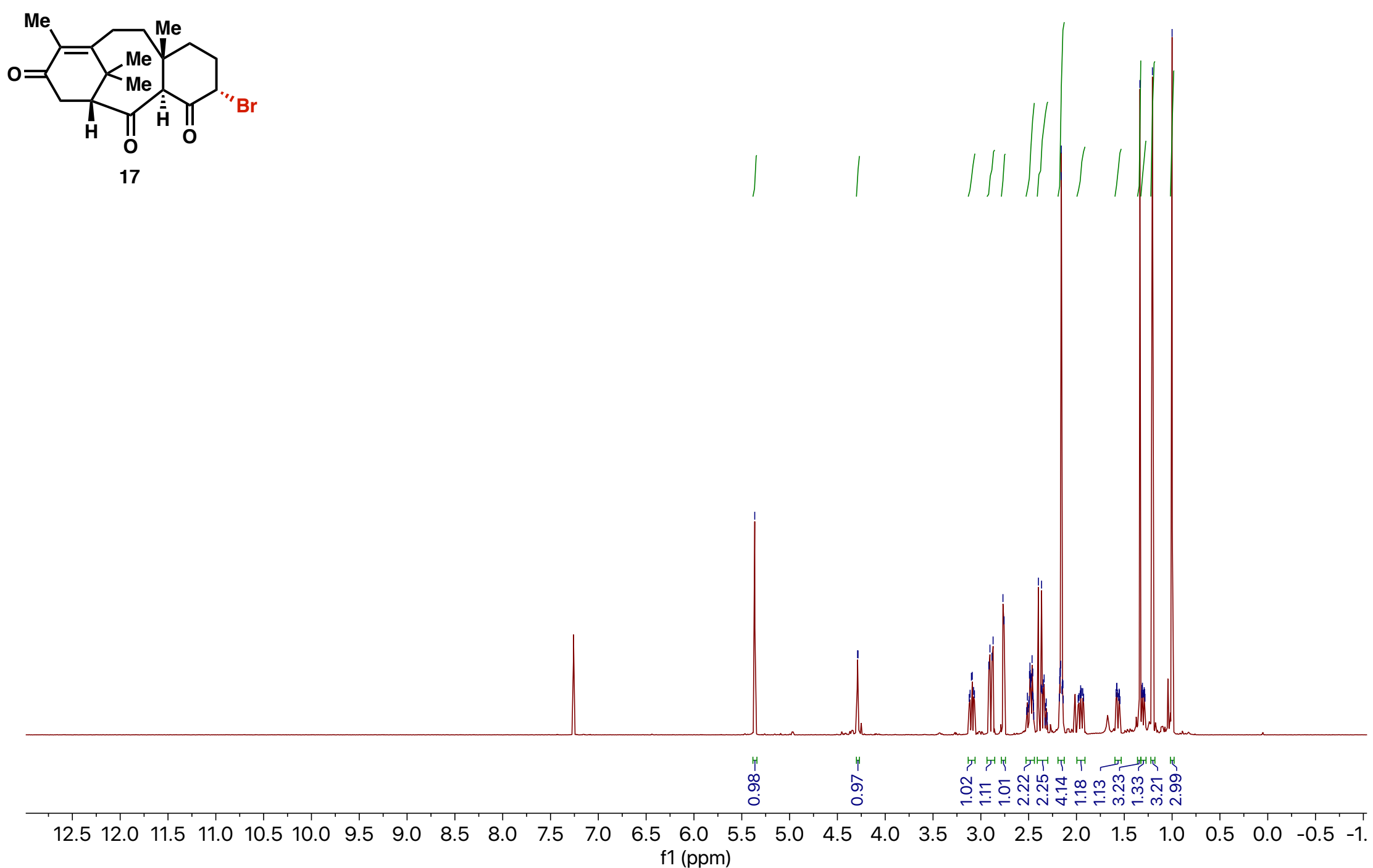


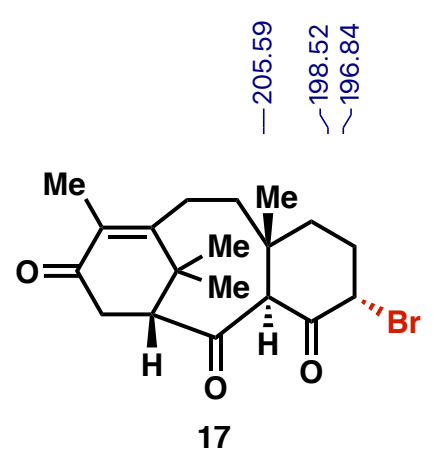

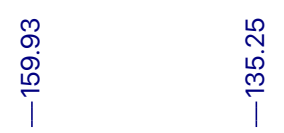

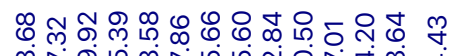

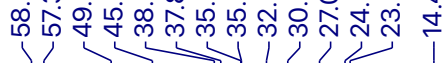

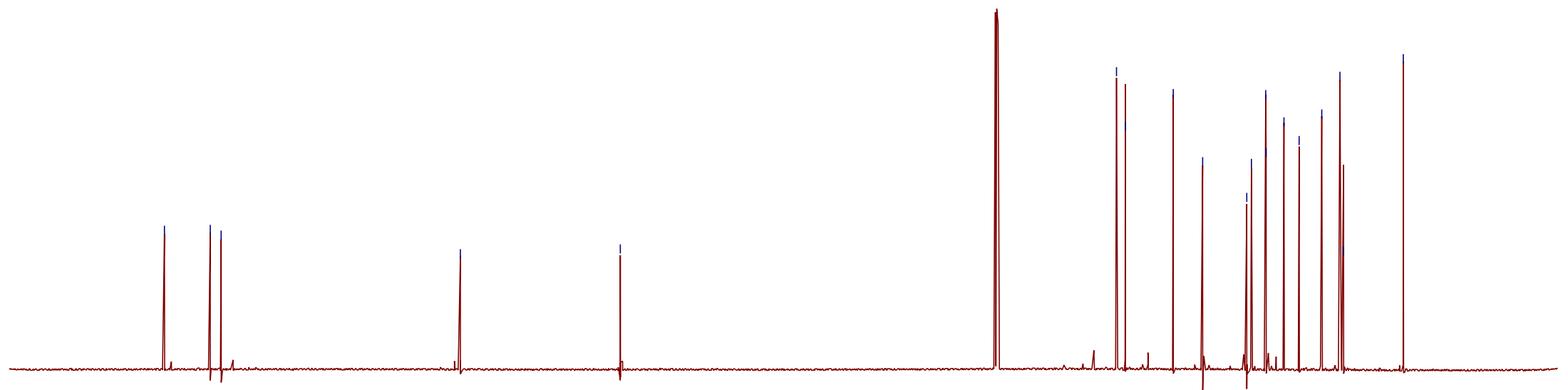

$120 \quad 110$

1 (ppm)

SI-117 


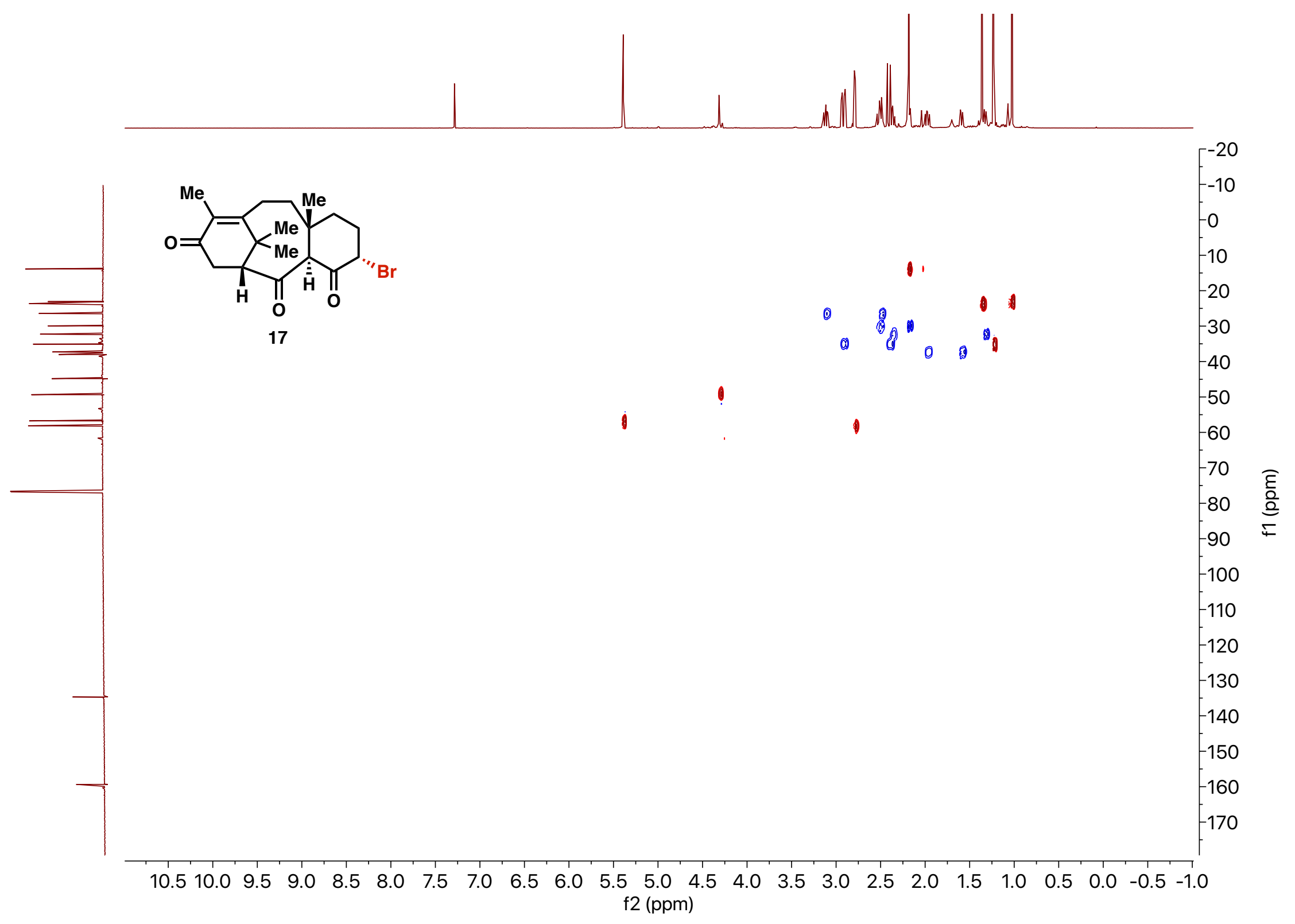

SI-118 


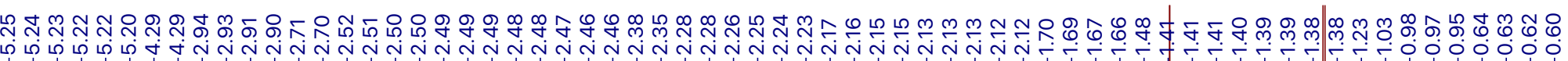
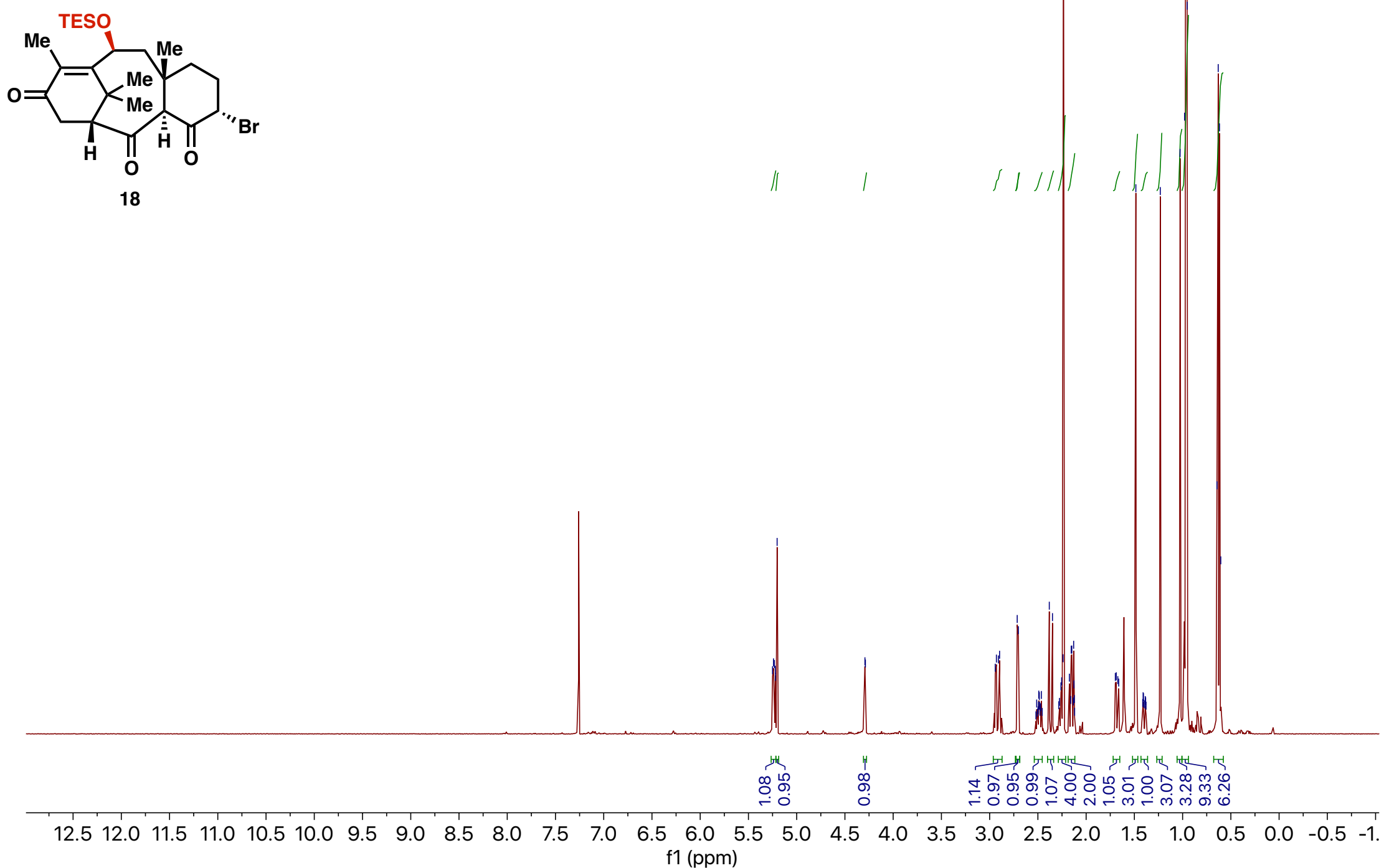


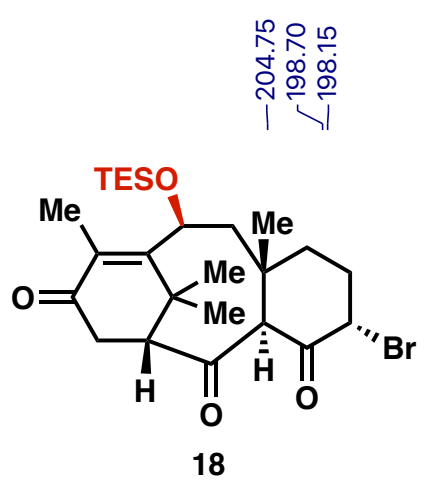

$\begin{array}{ll}8 & 8 \\ 0 & 0 \\ 0 & \stackrel{0}{0} \\ 1 & \stackrel{0}{1}\end{array}$

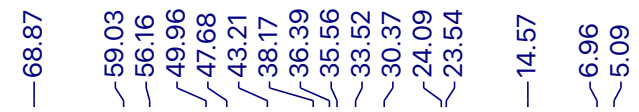

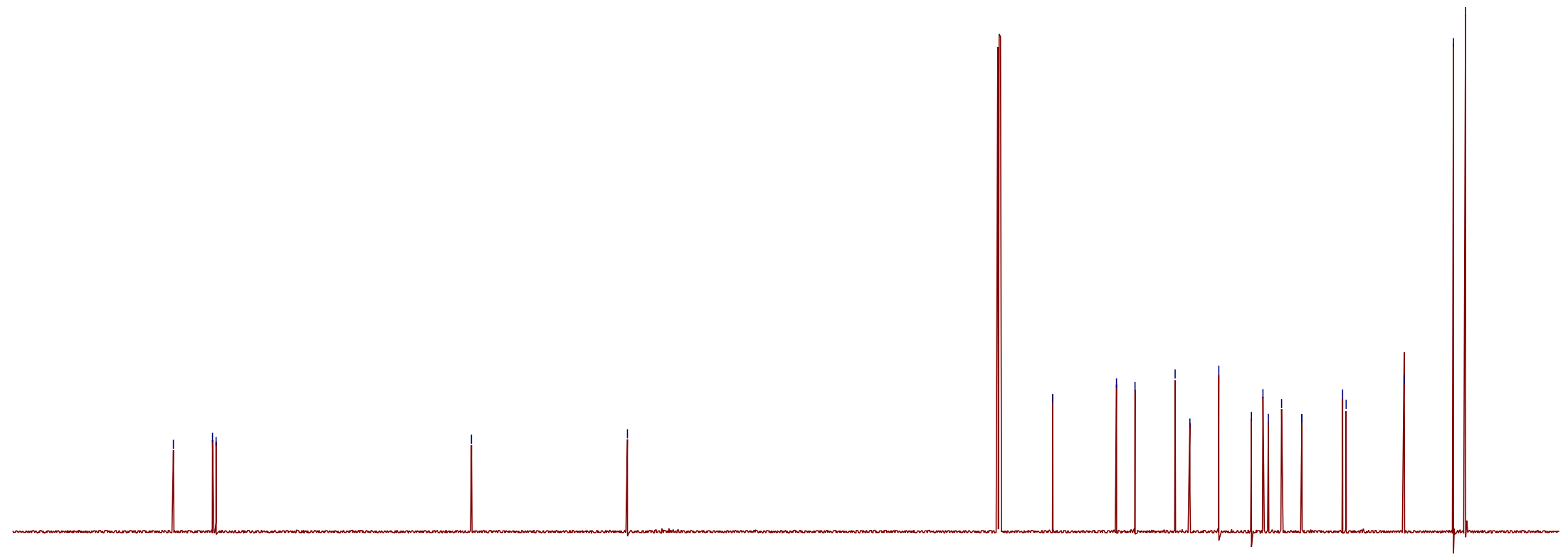

$\begin{array}{llllllllllllllllllllllllll}220 & 210 & 200 & 190 & 180 & 170 & 160 & 150 & 140 & 130 & 120 & 110 & 100 & 90 & 80 & 70 & 60 & 50 & 40 & 30 & 20 & 10 & 0 & 10\end{array}$ 


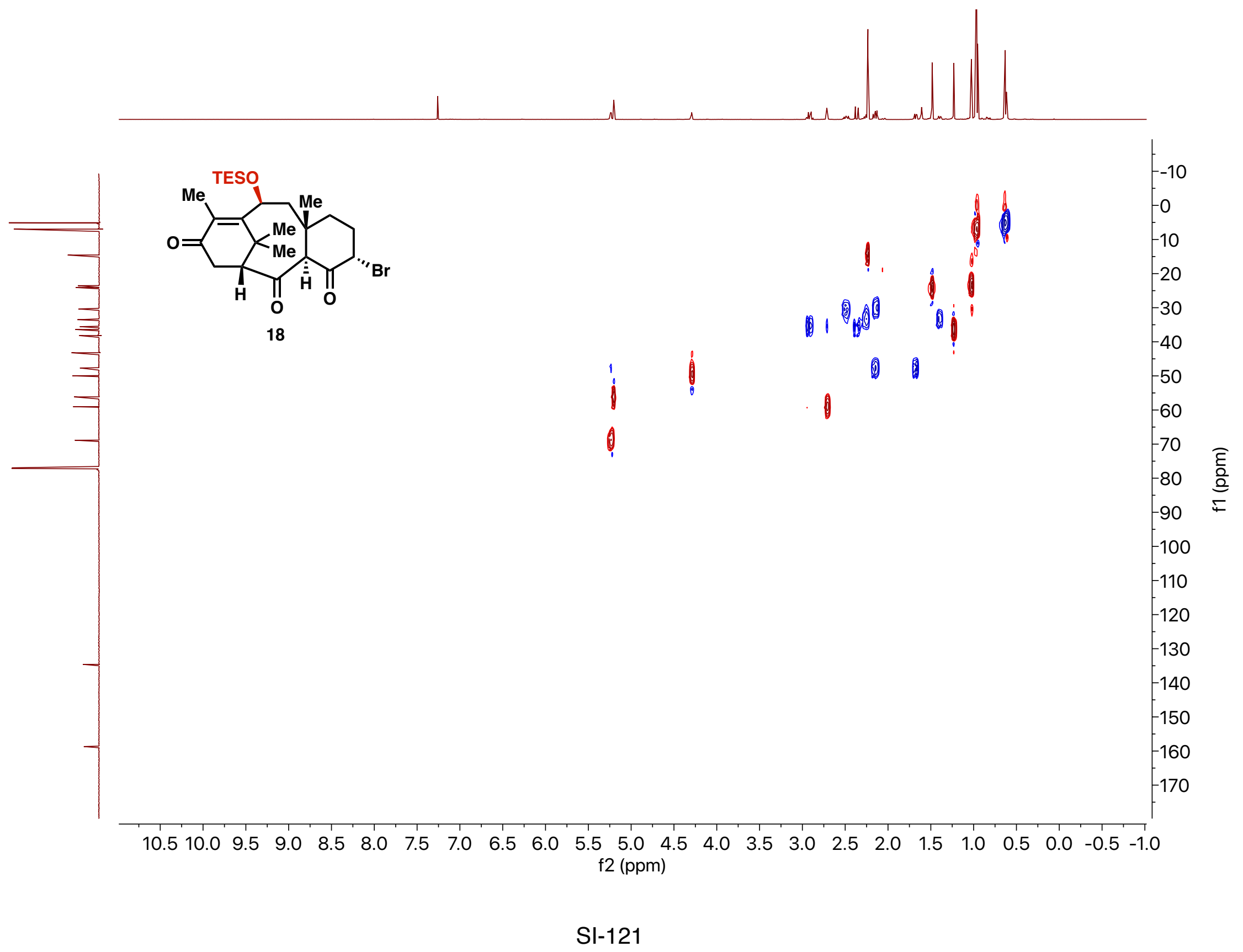




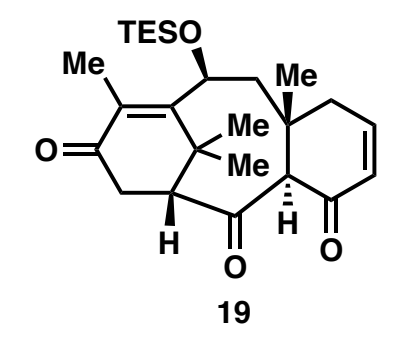

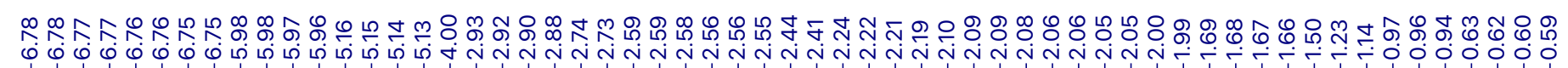
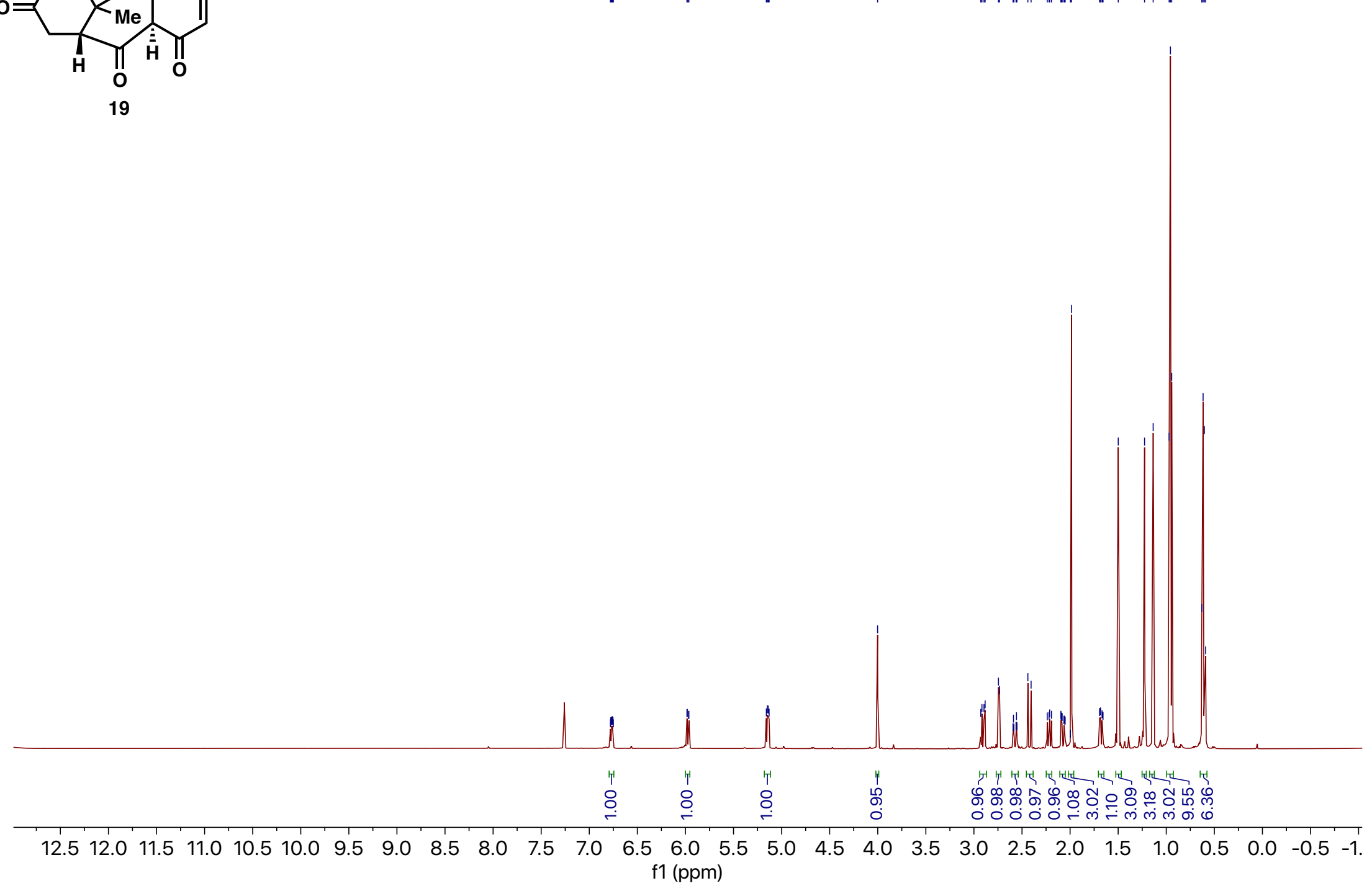


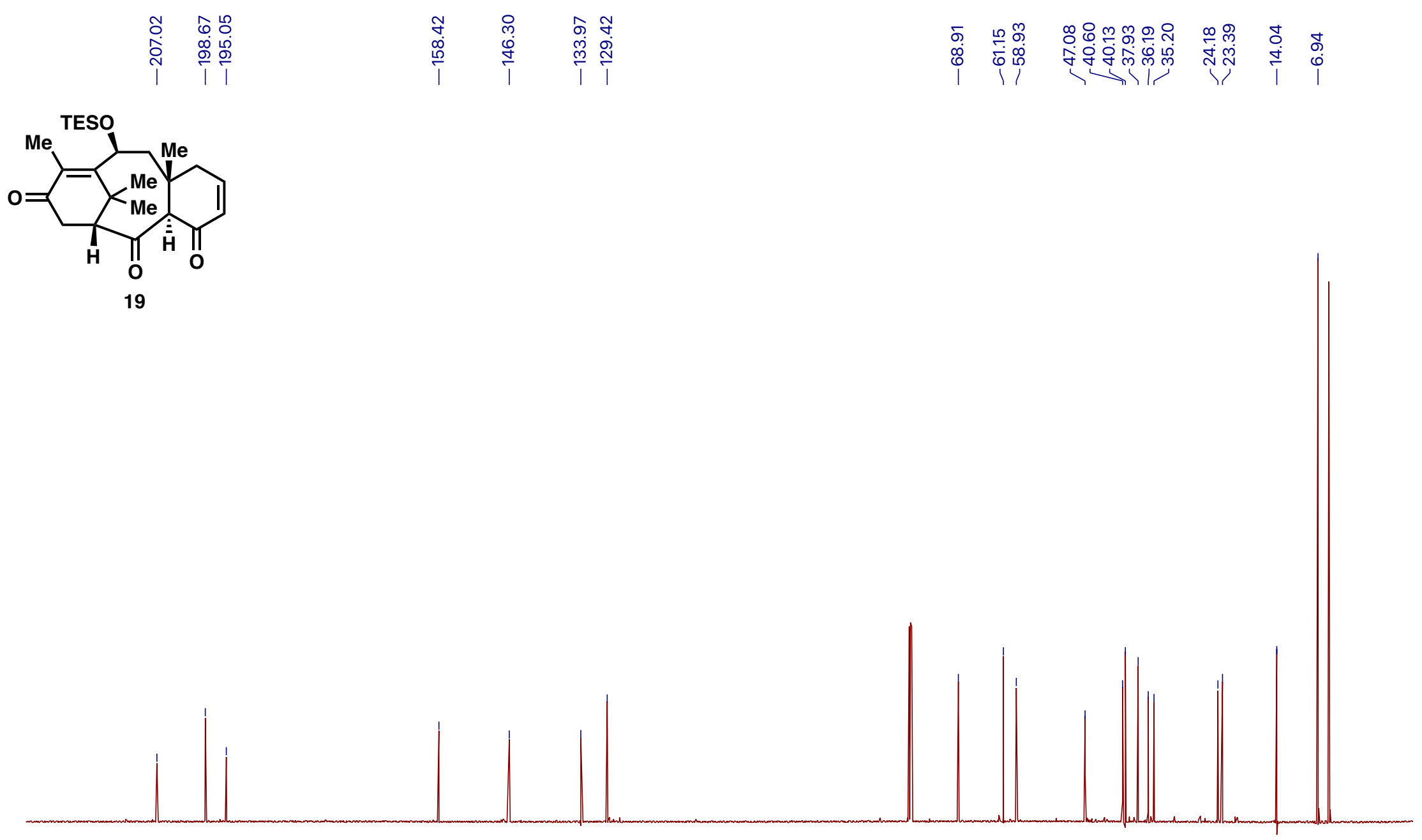

$\begin{array}{lllllllllllllllllllllllllll}220 & 210 & 200 & 190 & 180 & 170 & 160 & 150 & 140 & 130 & 120 & 110 & 100 & 90 & 80 & 70 & 60 & 50 & 40 & 30 & 20 & 10 & 0 & \end{array}$ 
๕

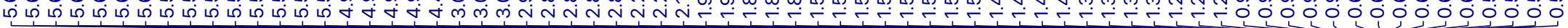
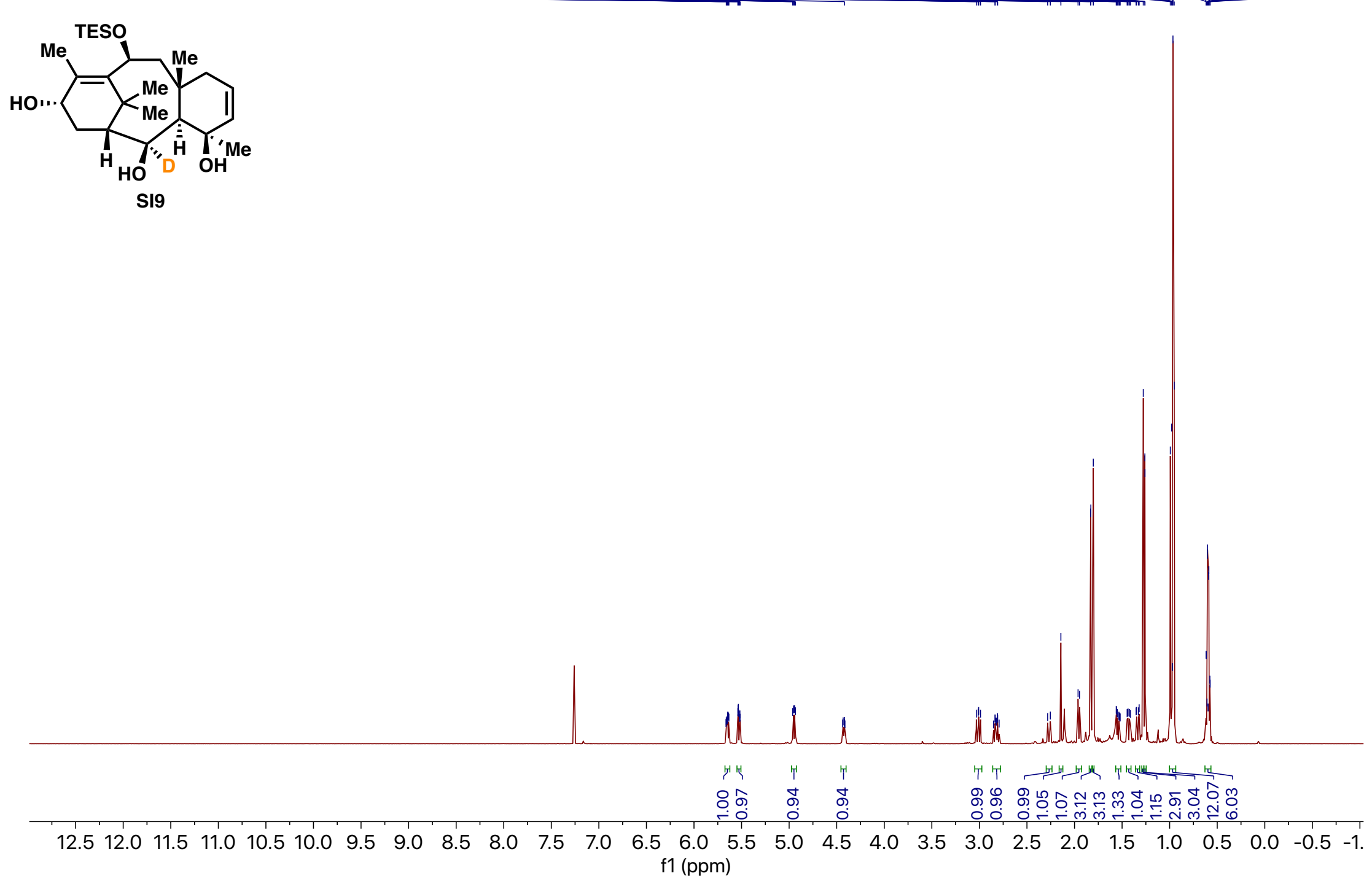

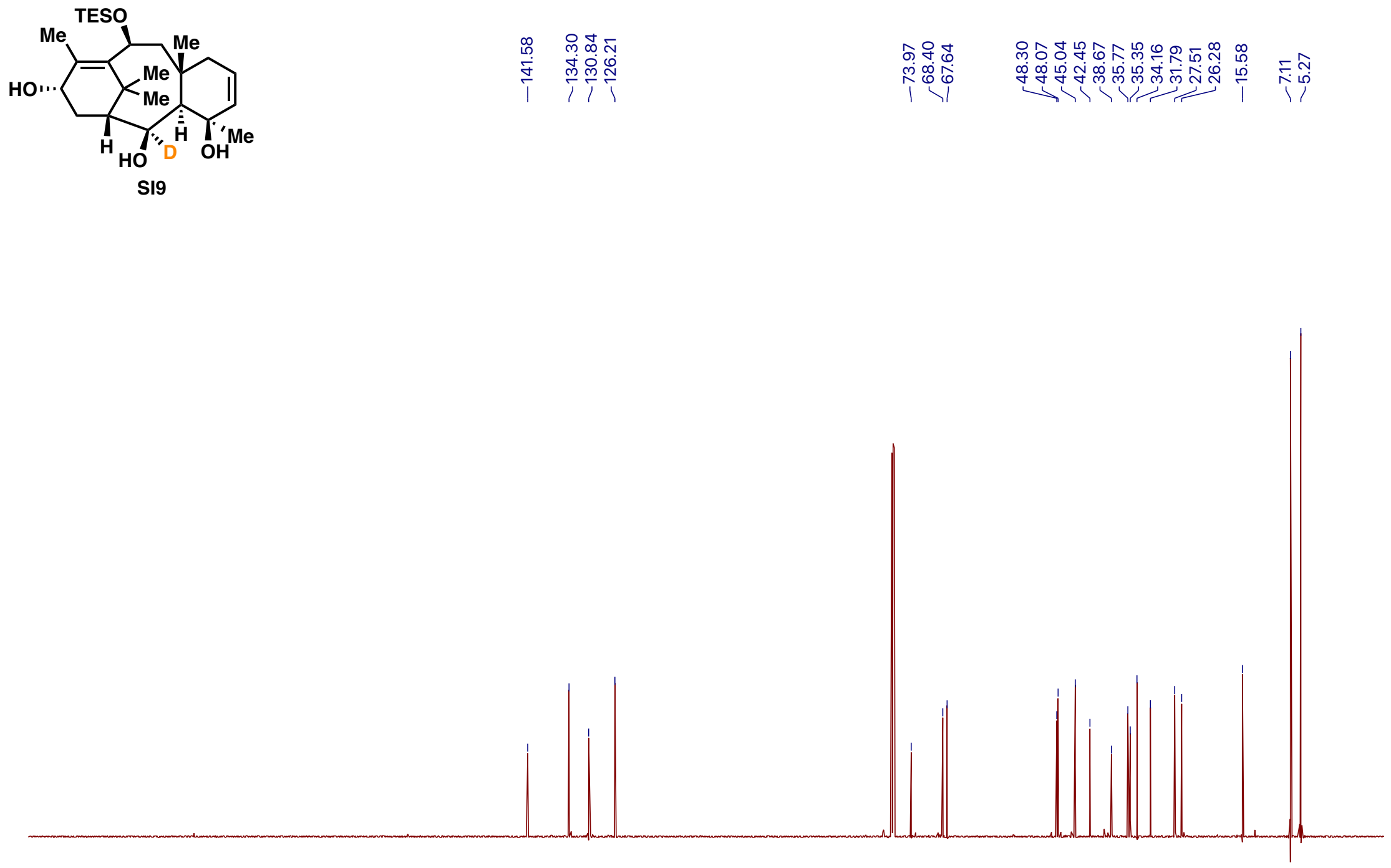

$\begin{array}{llllllllllllllllllllllll}220 & 210 & 200 & 190 & 180 & 170 & 160 & 150 & 140 & 130 & 120 & 110 & 100 & 90 & 80 & 70 & 60 & 50 & 40 & 30 & 20 & 10 & 0 & \end{array}$ 


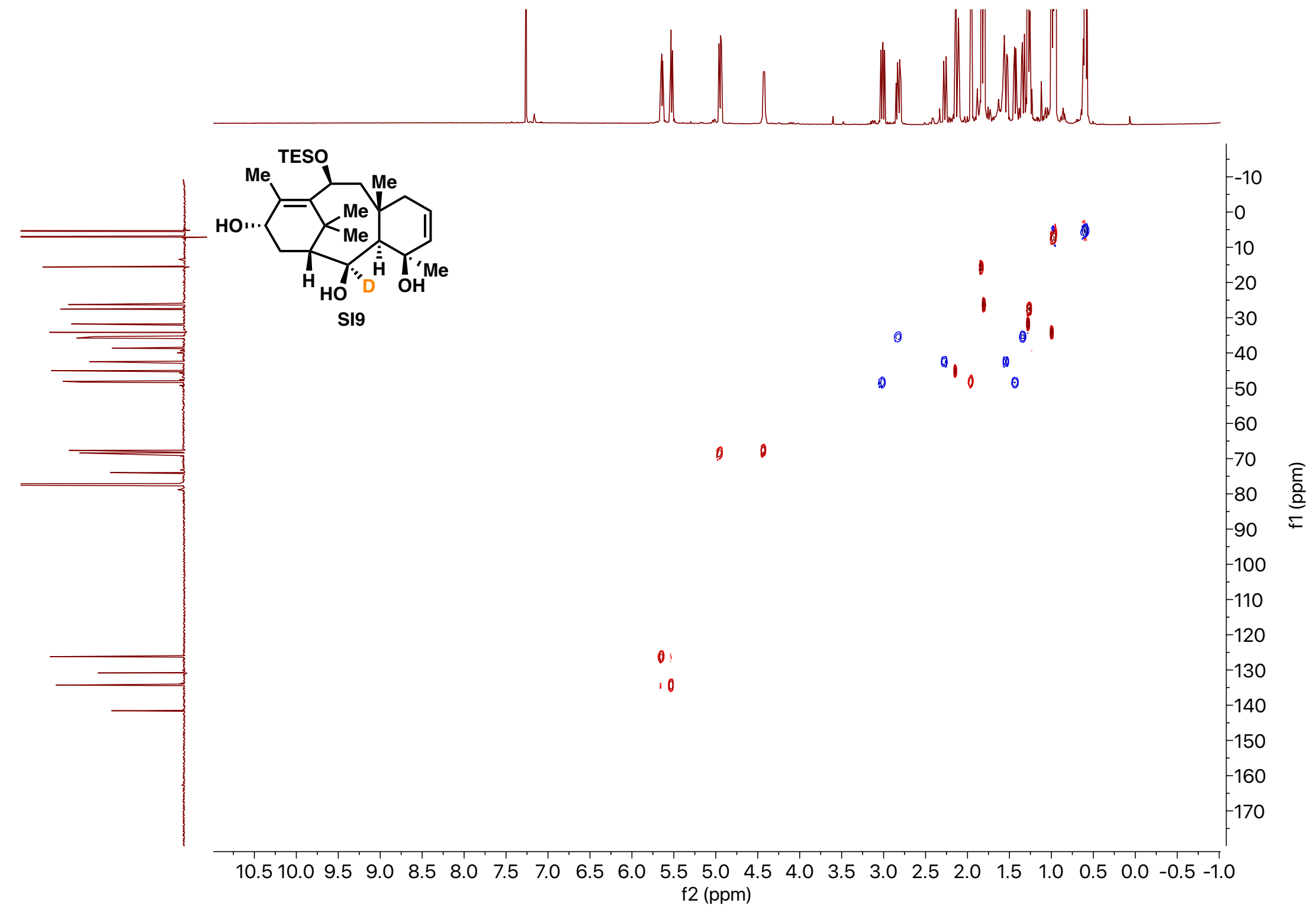

SI-126 


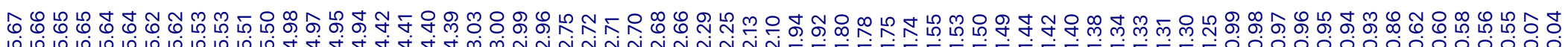

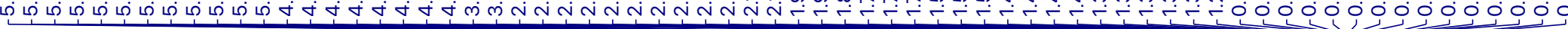

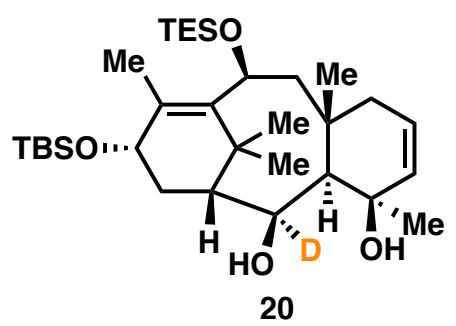

20

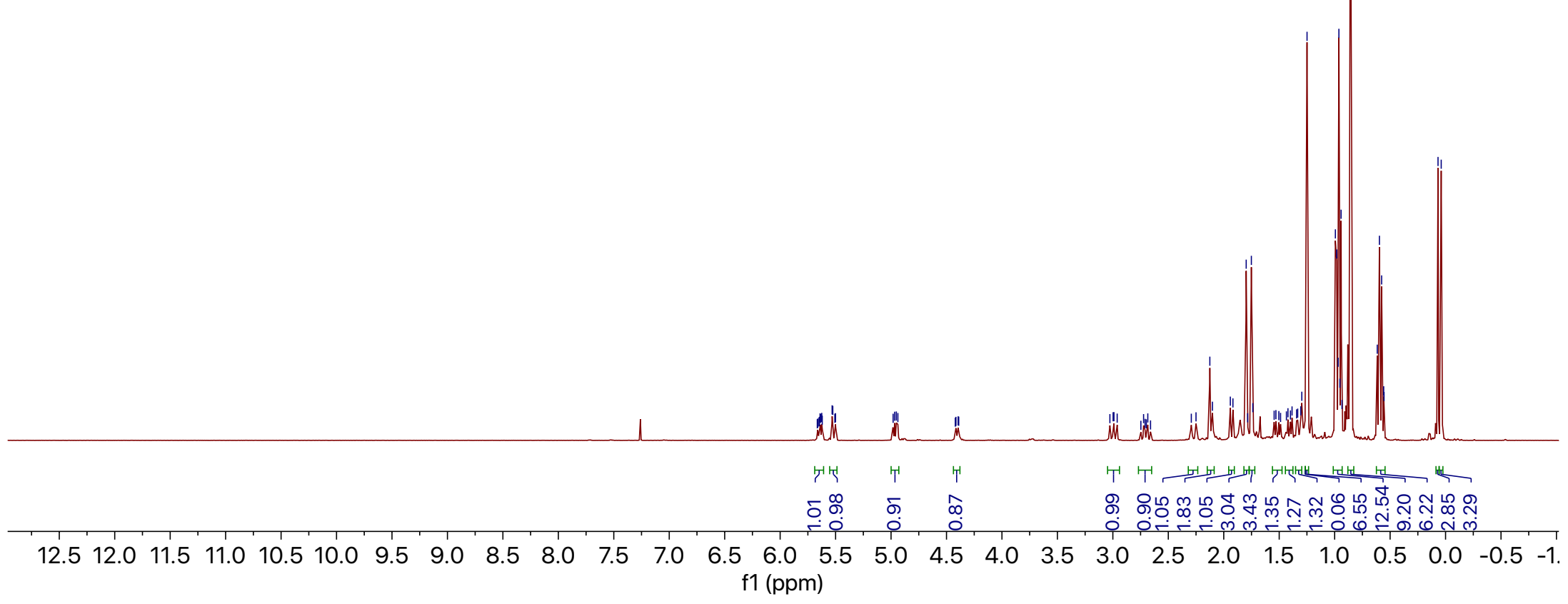



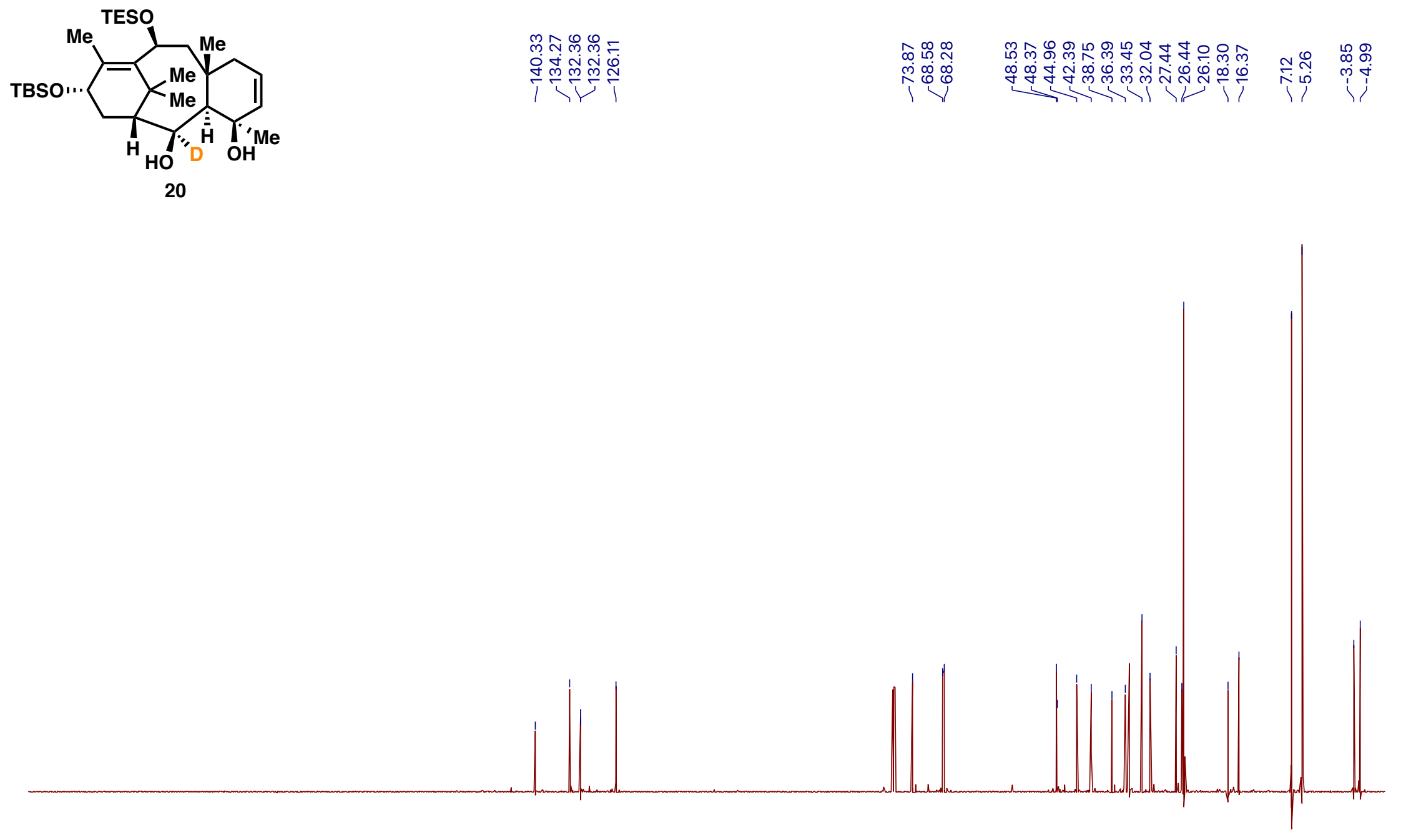

$\begin{array}{llllllllllllllllllllllll}220 & 210 & 200 & 190 & 180 & 170 & 160 & 150 & 140 & 130 & 120 & 110 & 100 & 90 & 80 & 70 & 60 & 50 & 40 & 30 & 20 & 10 & 0 & \end{array}$ 


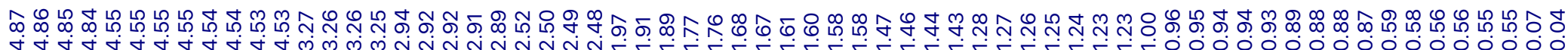
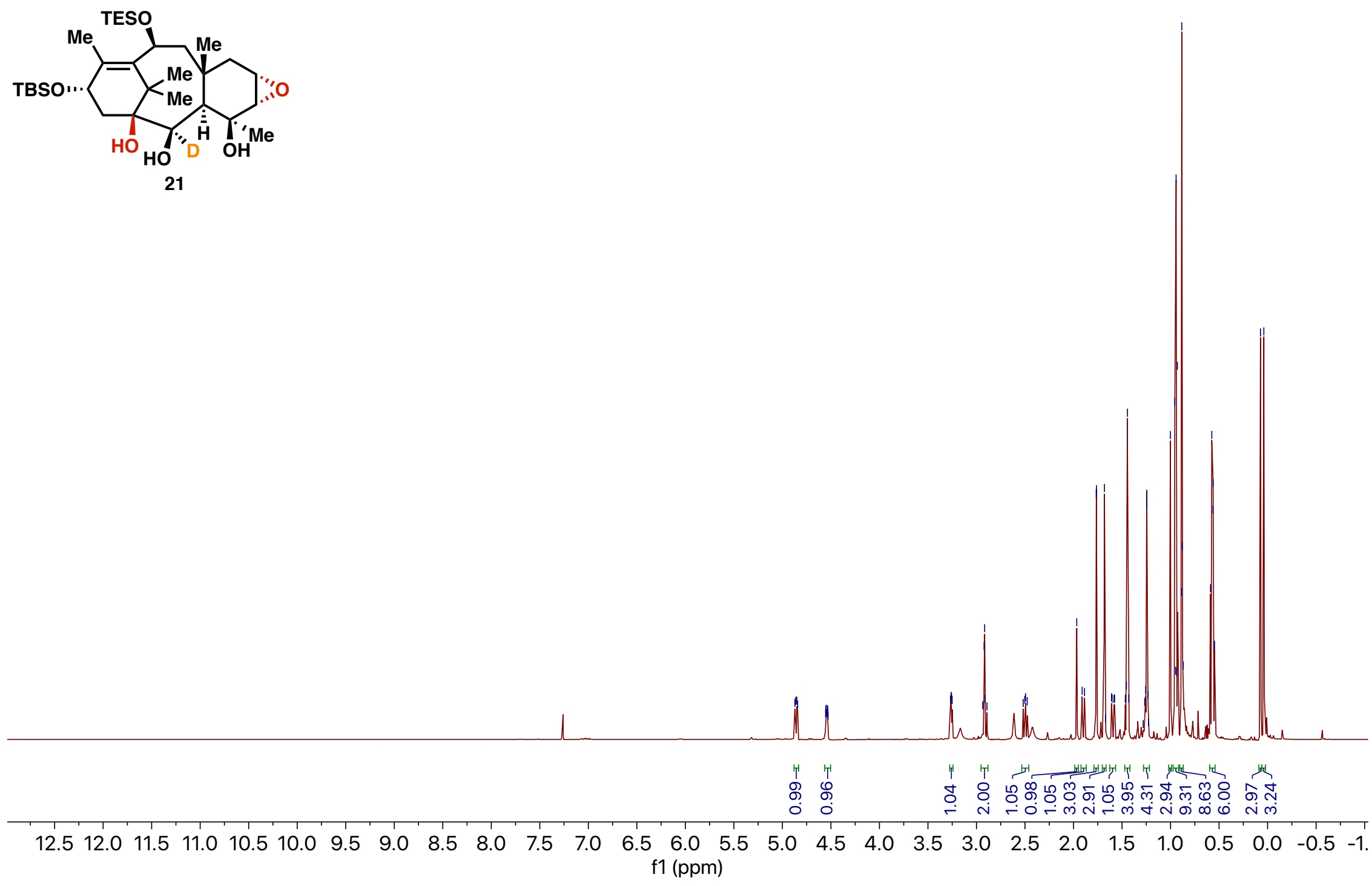


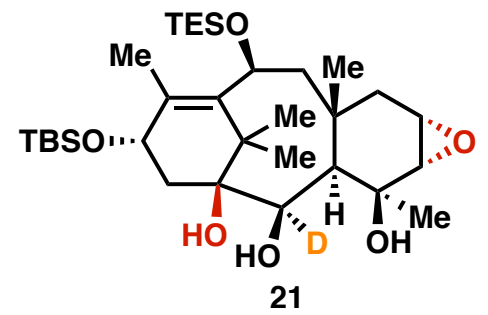

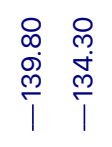

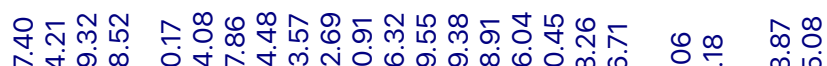

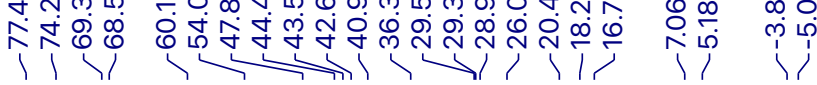

21

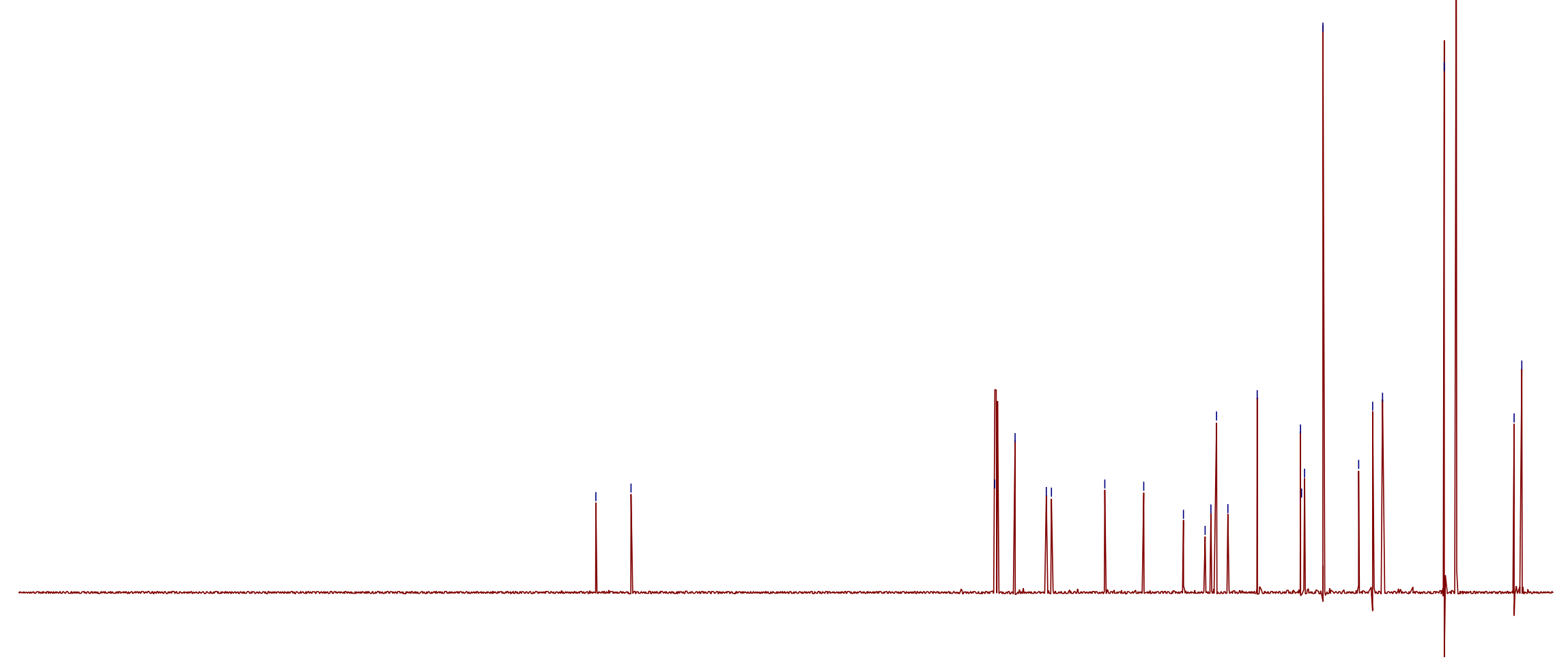

140

120

f1 (ppm)

SI-130 


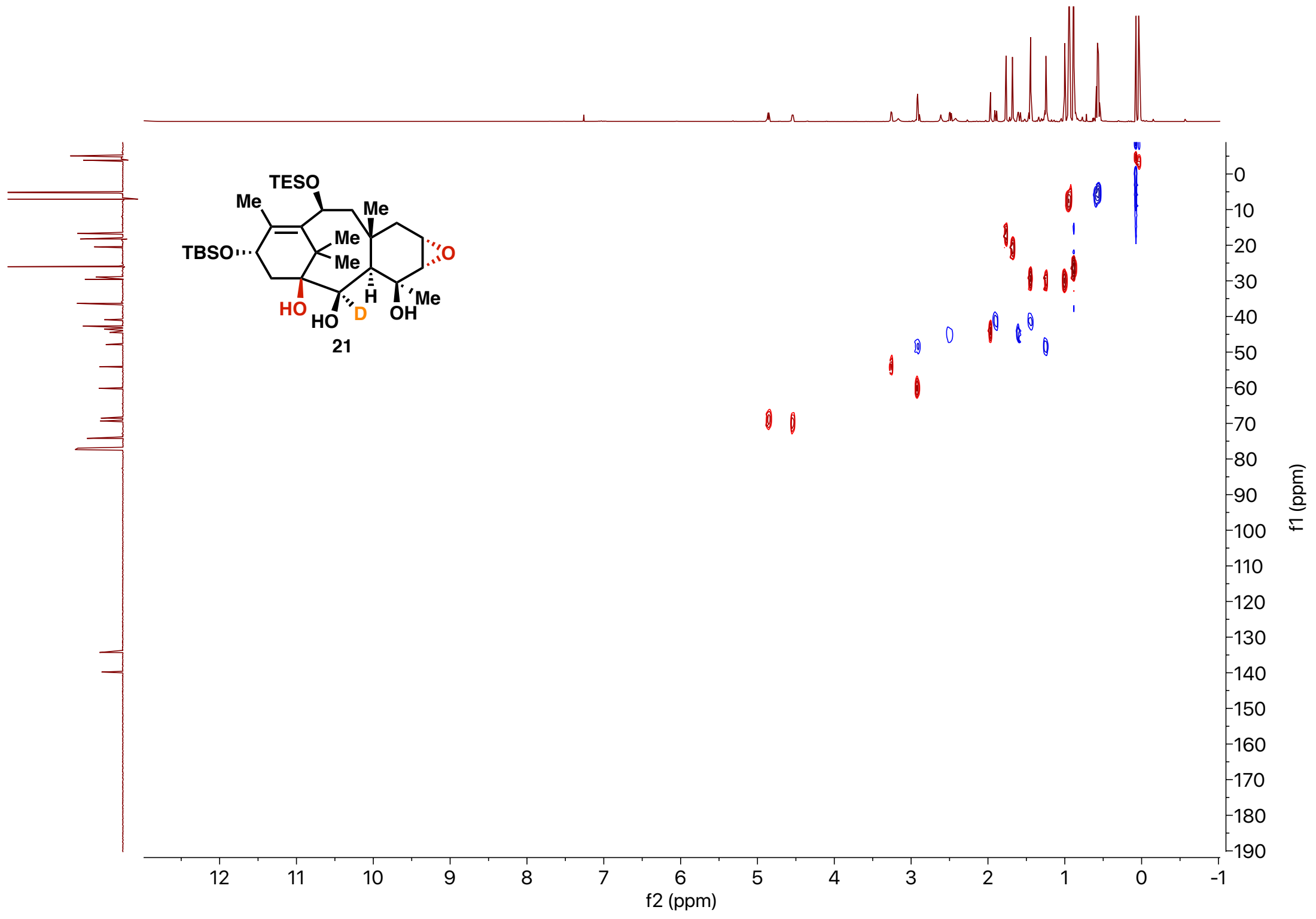

SI-131 


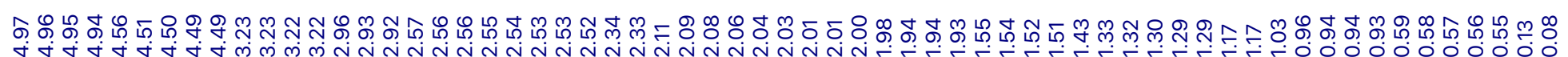

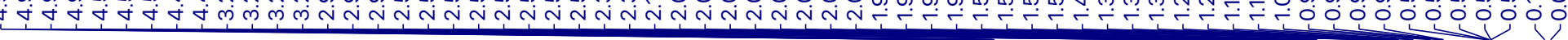
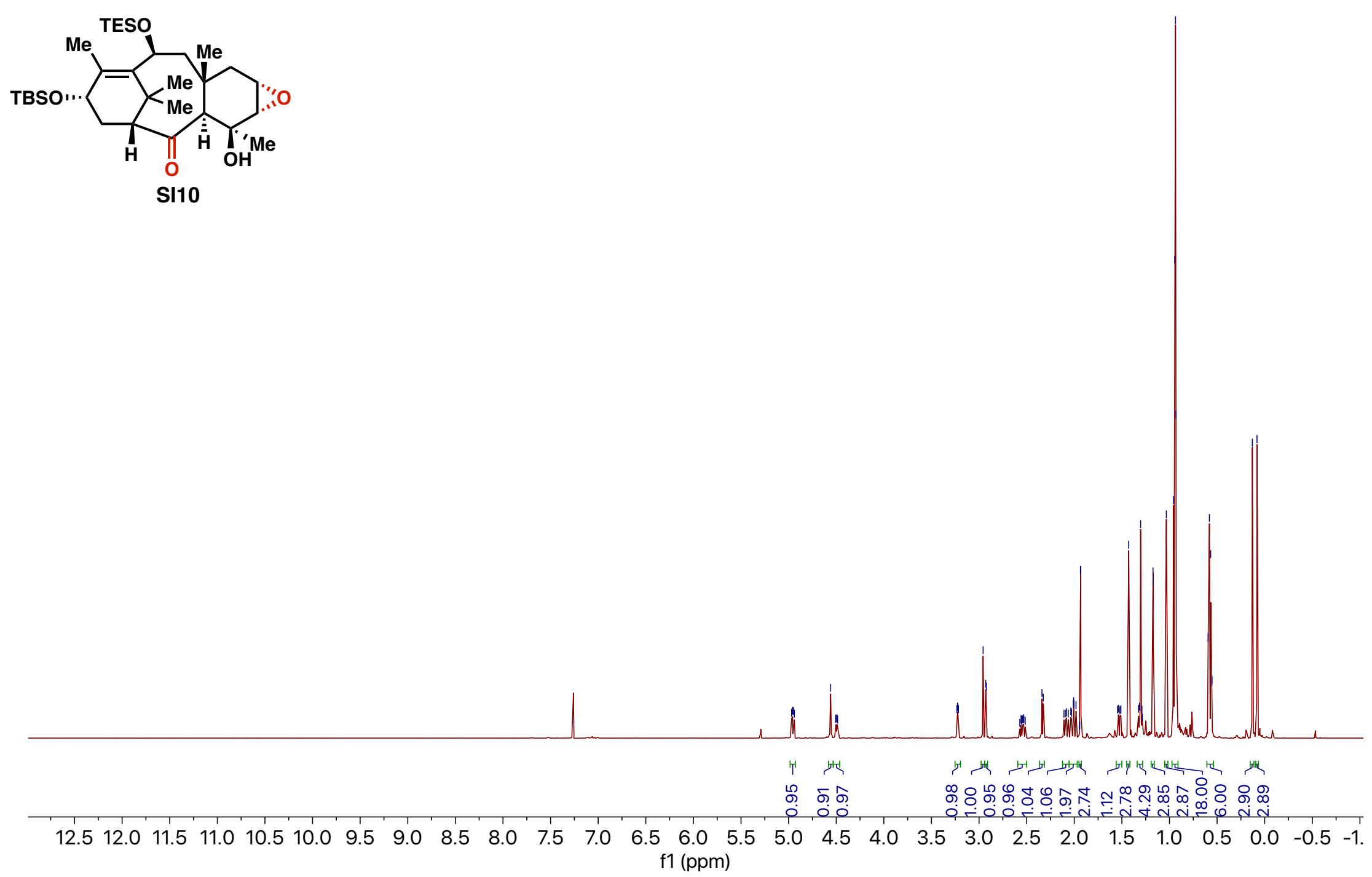


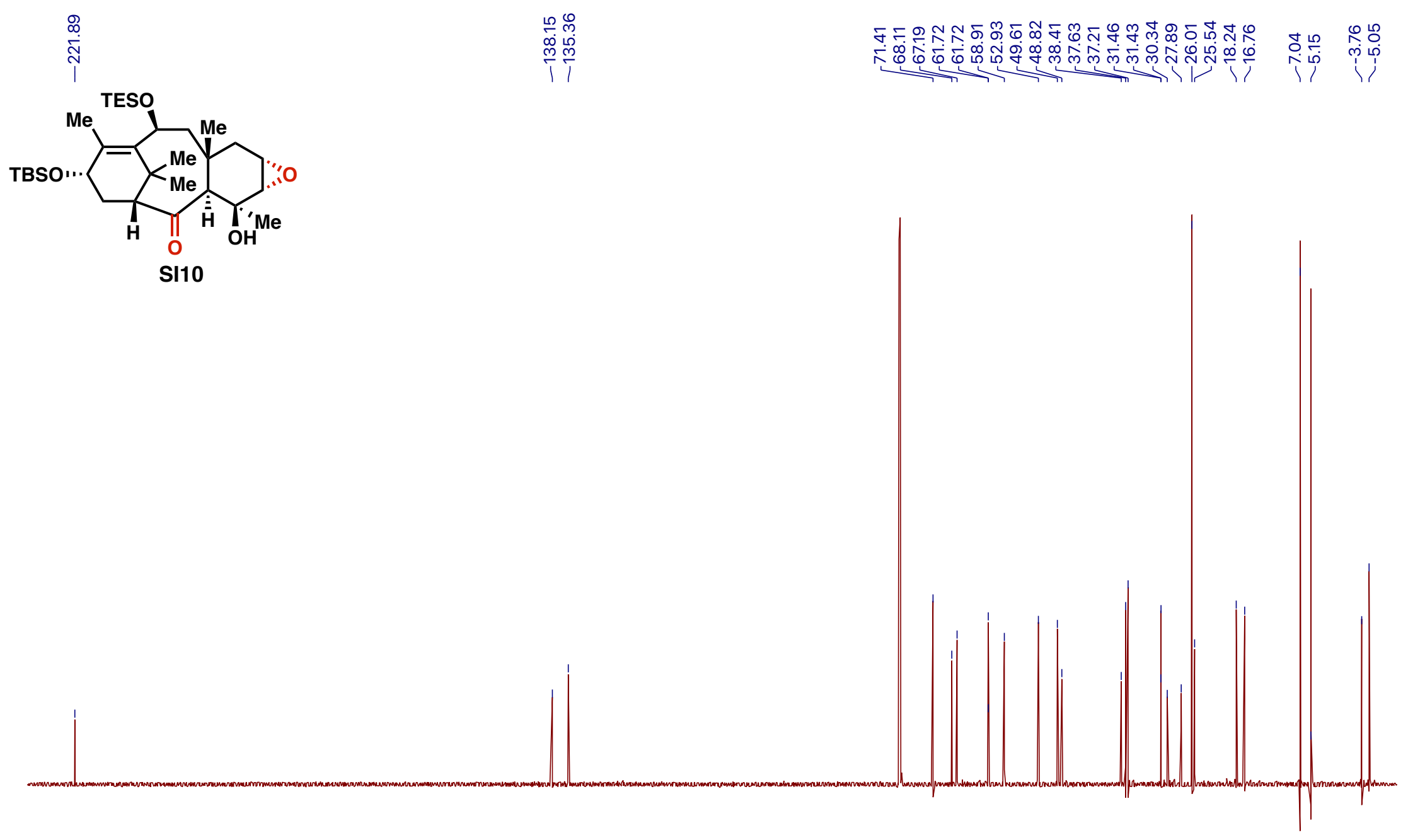

$\begin{array}{llllllllllllllllllllllllllllllllllll}30 & 220 & 210 & 200 & 190 & 180 & 170 & 160 & 150 & 140 & 130 & 120 & \begin{array}{c}110 \\ \mathrm{f} 1(\mathrm{ppm})\end{array} & 100 & 90 & 80 & 70 & 60 & 50 & 40 & 30 & 20 & 10 & 0 & \end{array}$ 


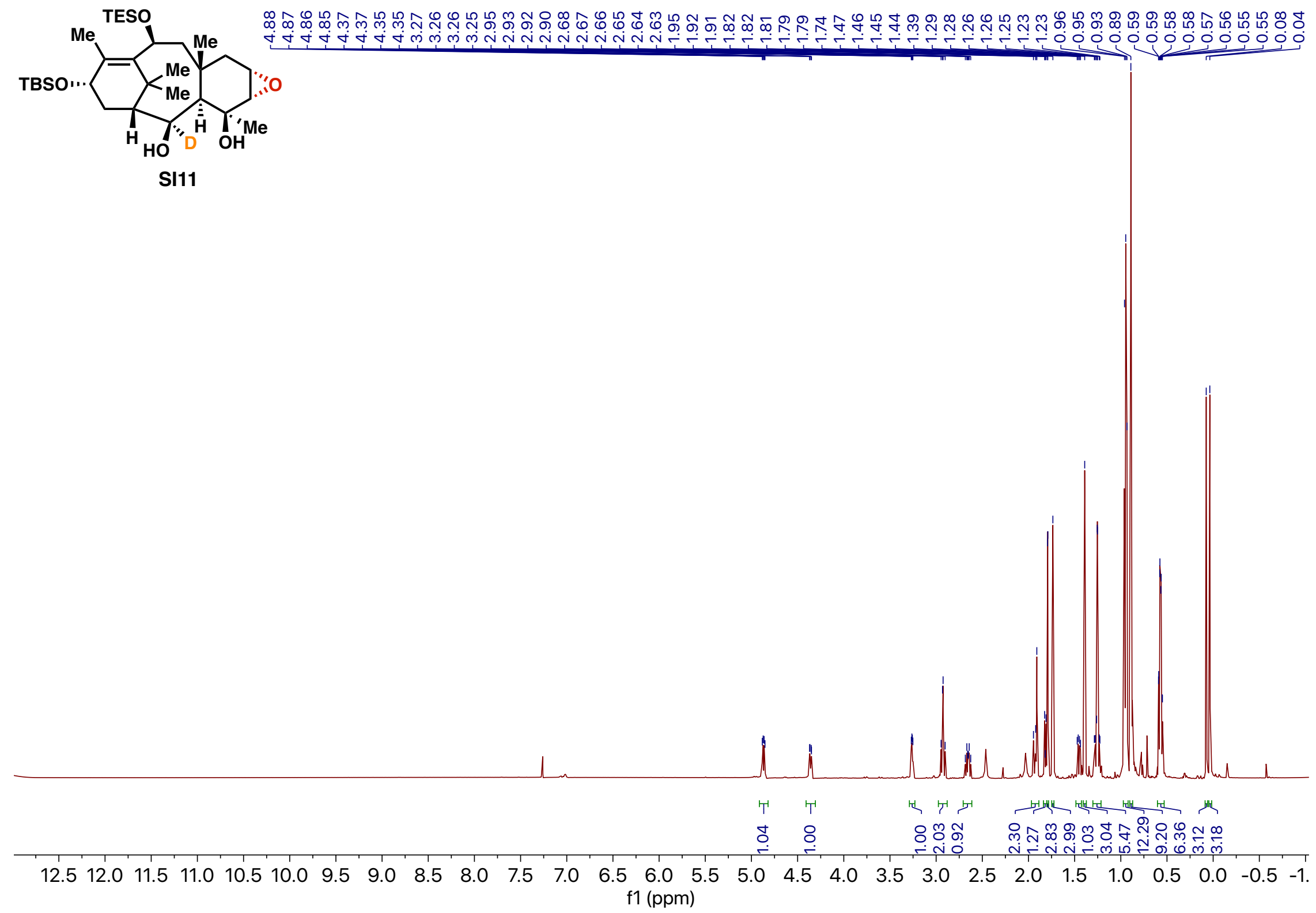



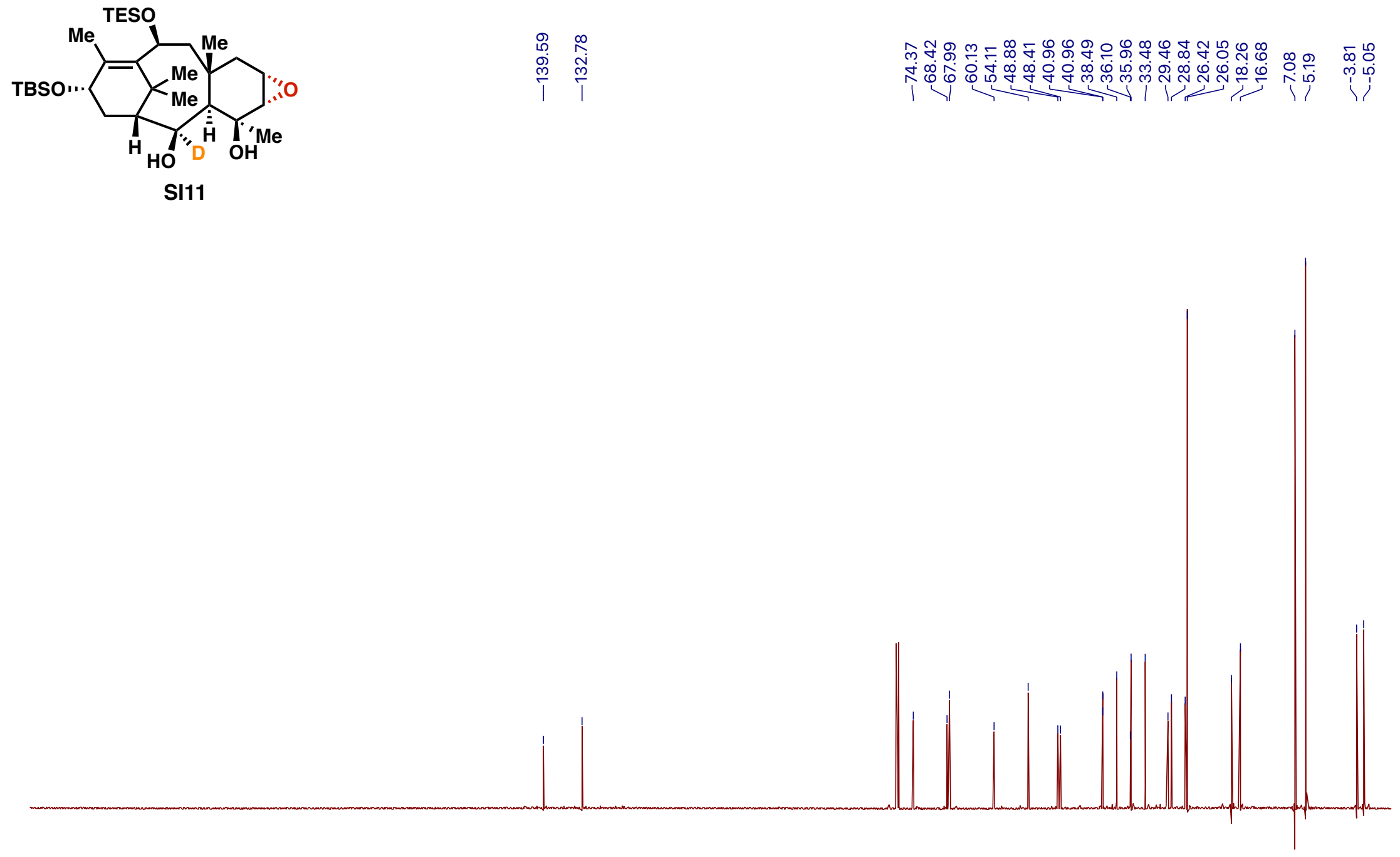

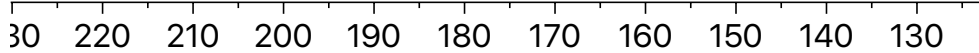

$120 \quad 110 \quad 100$

$80 \quad 70 \quad 60 \quad 50$

$40 \quad 30 \quad 20$

$20 \quad 10 \quad 0$

SI-135 


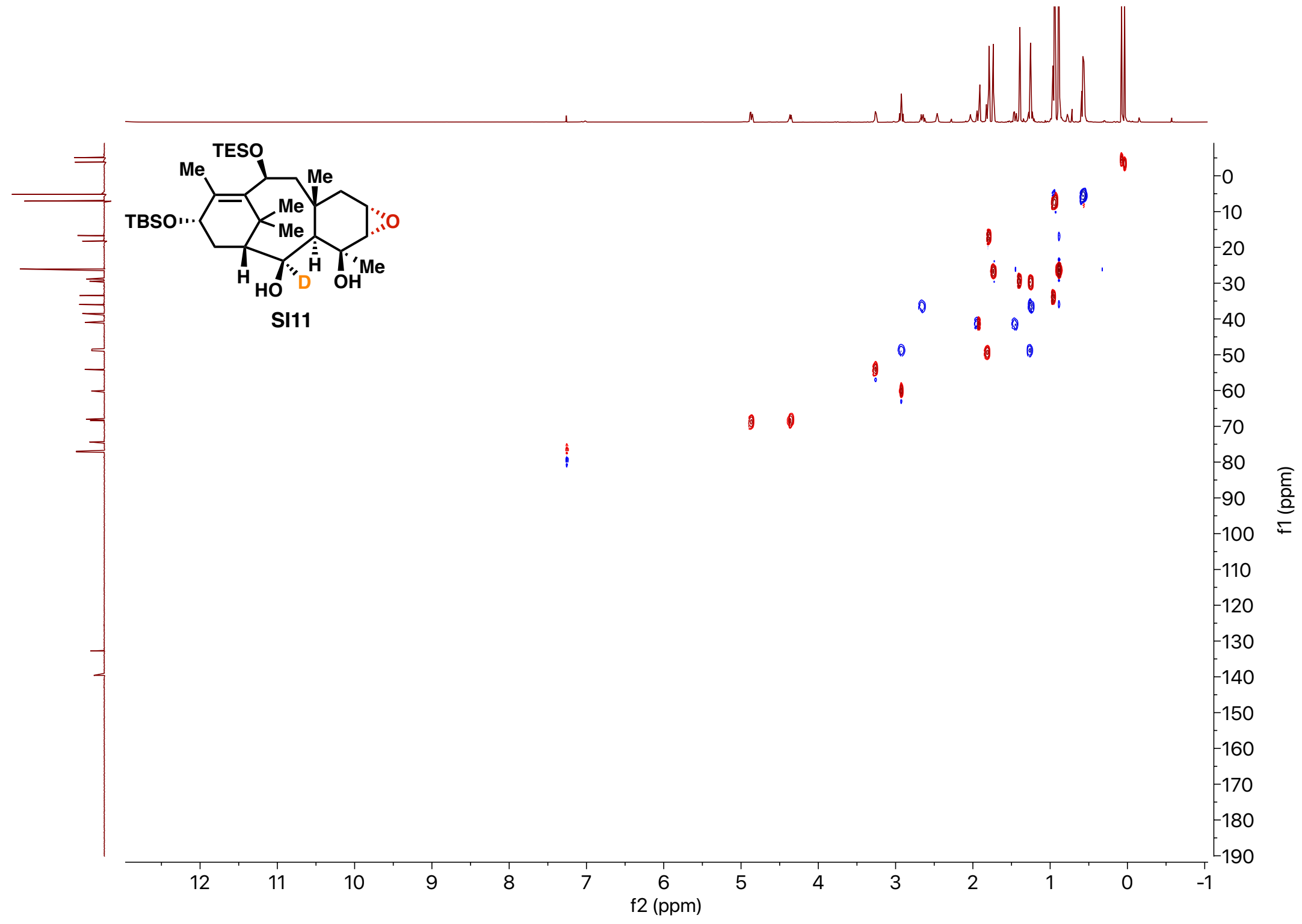

SI-136 

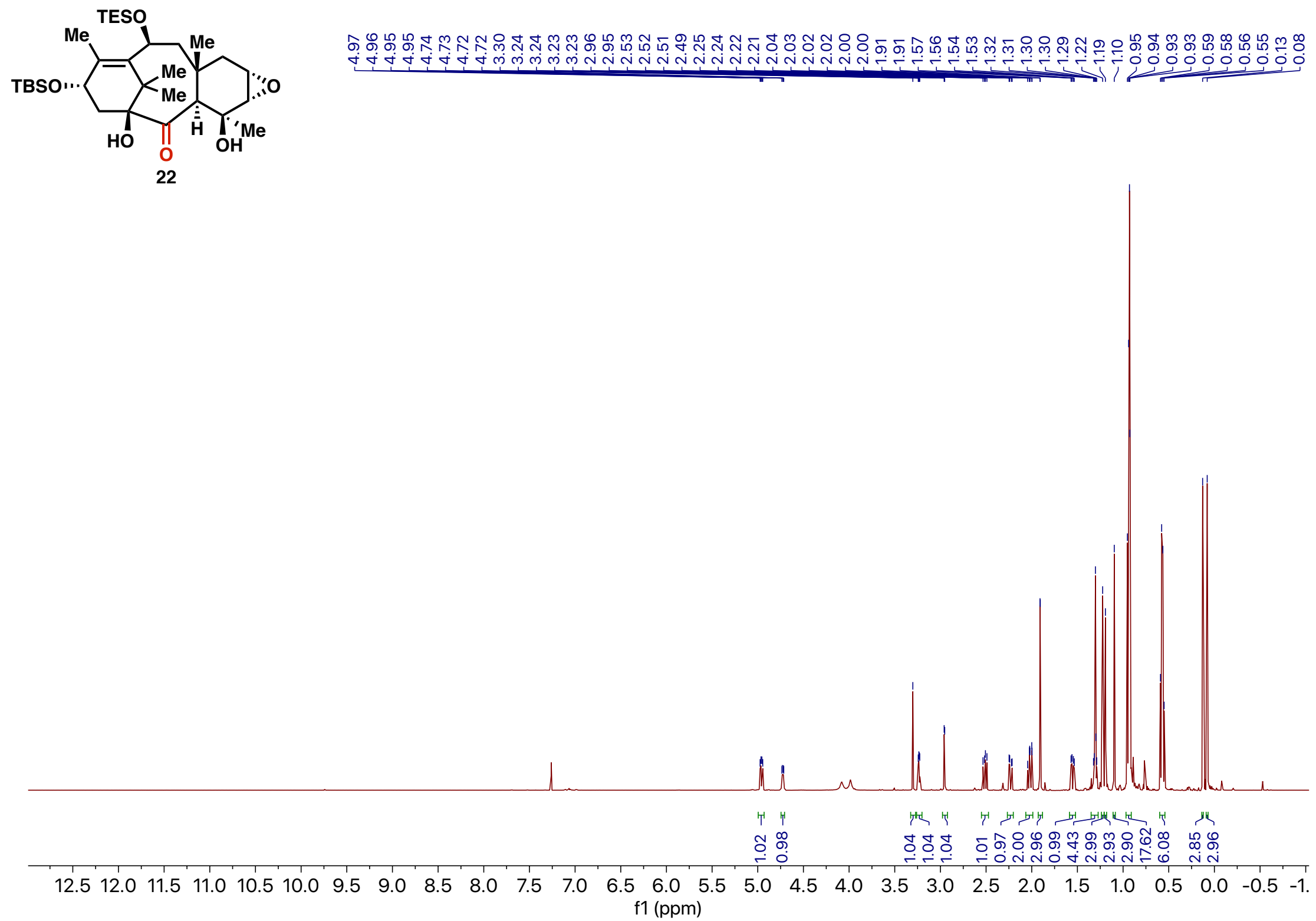

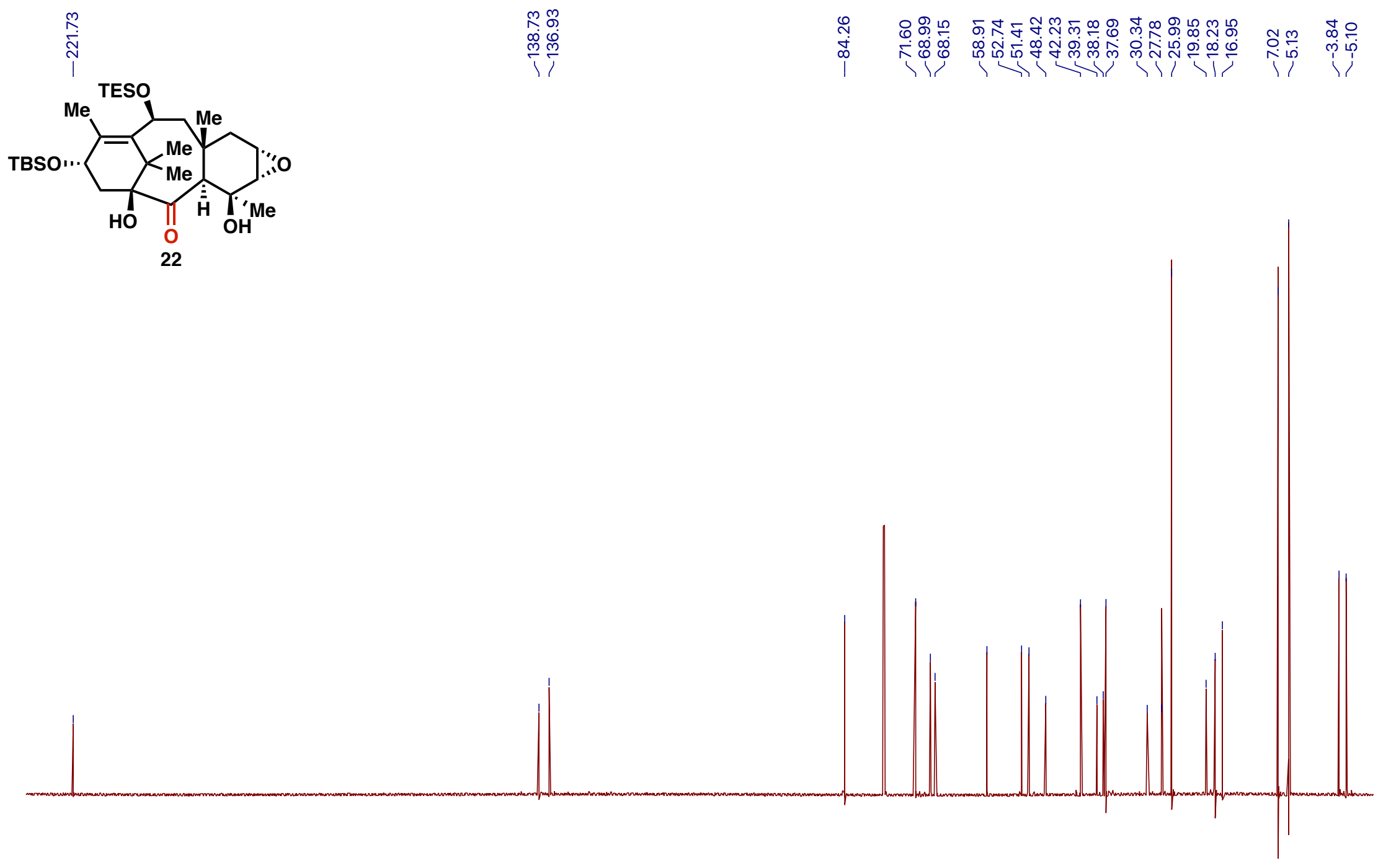

$\begin{array}{llllllll}30 & 220 & 210 & 200 & 190 & 180 & 170 & 160\end{array}$ 1100 $\begin{array}{llll}80 & 70 & 60 & 50\end{array}$ $\begin{array}{lllll}40 & 30 & 20 & 10 & 0\end{array}$ 


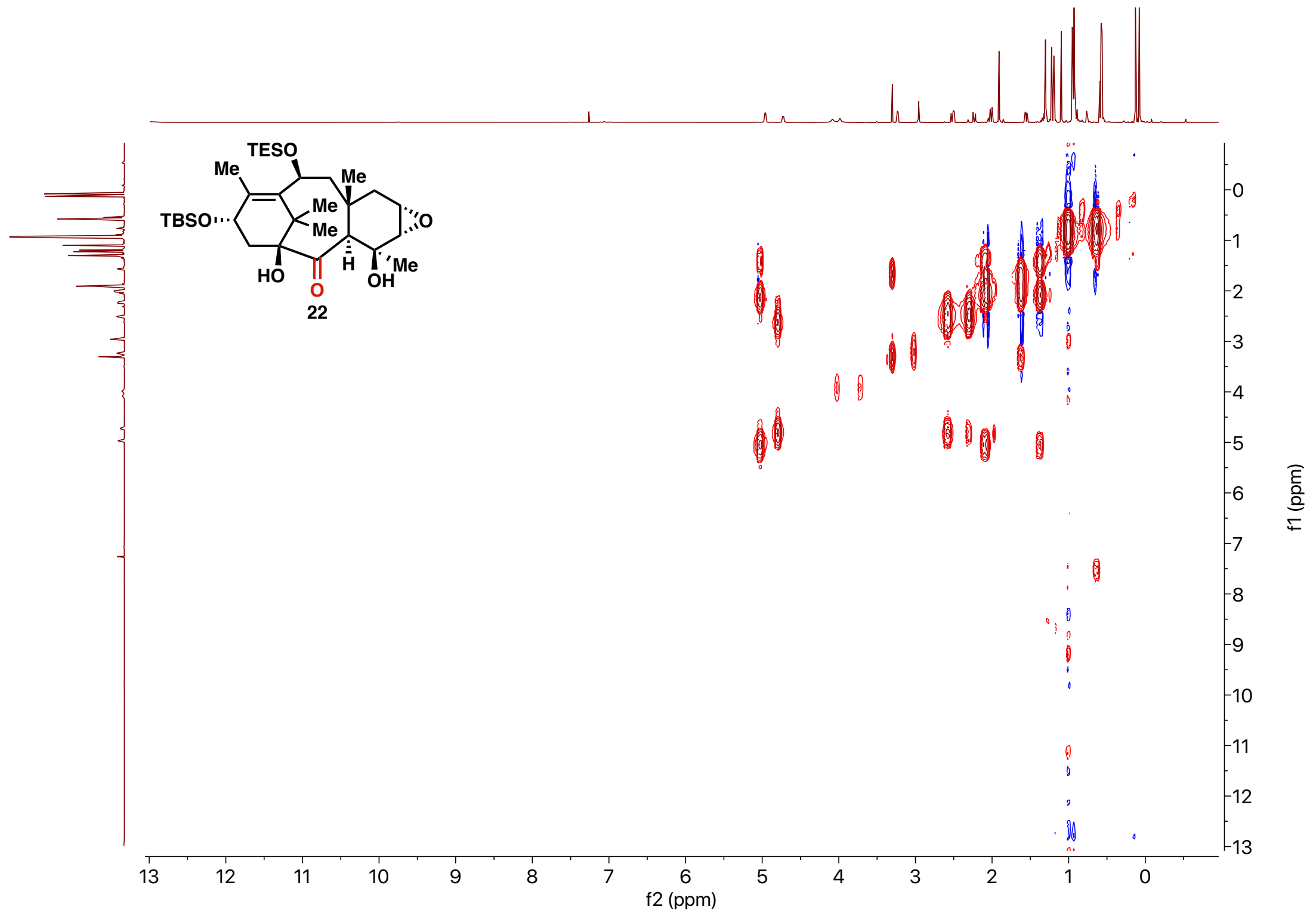




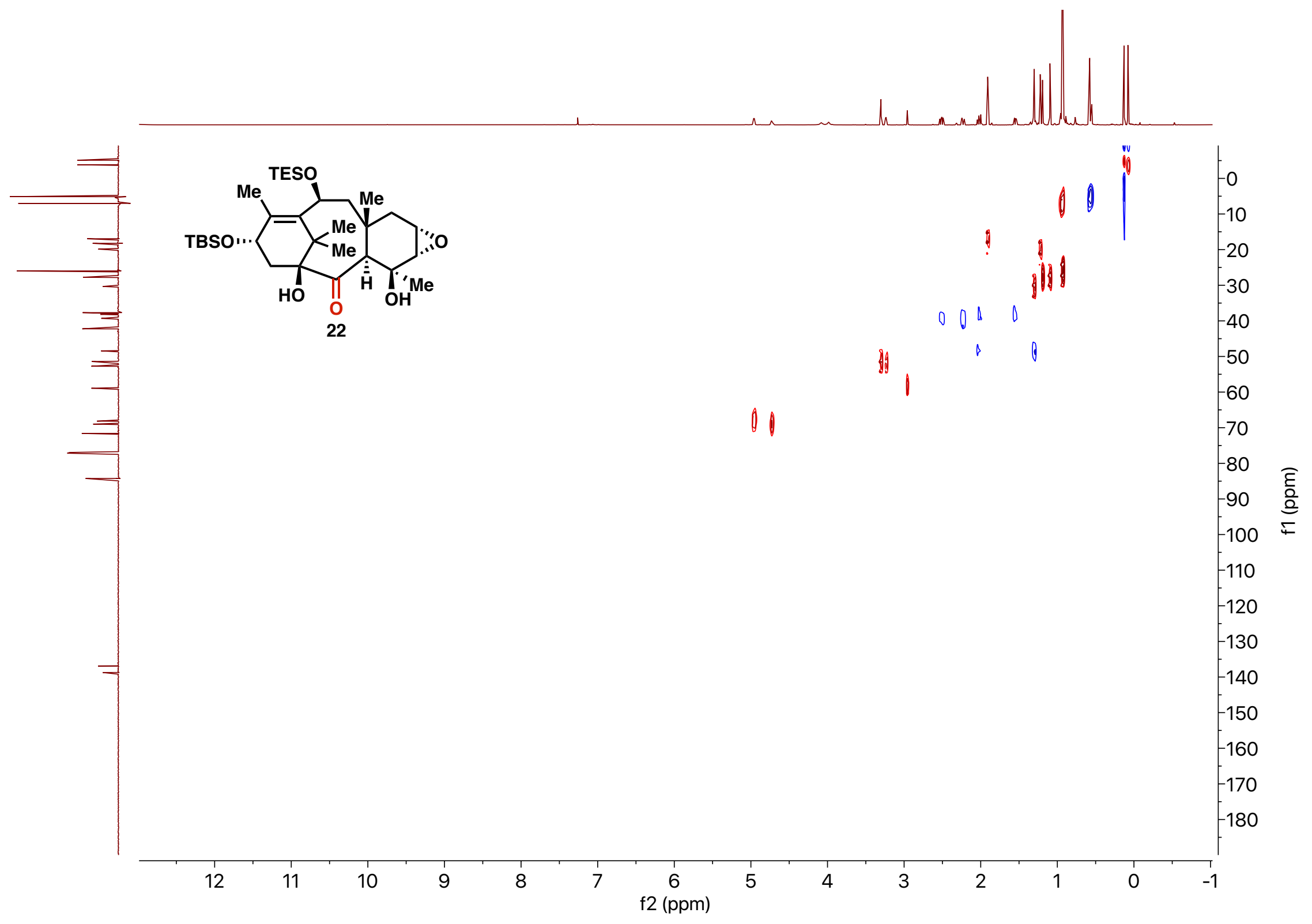

SI-140 


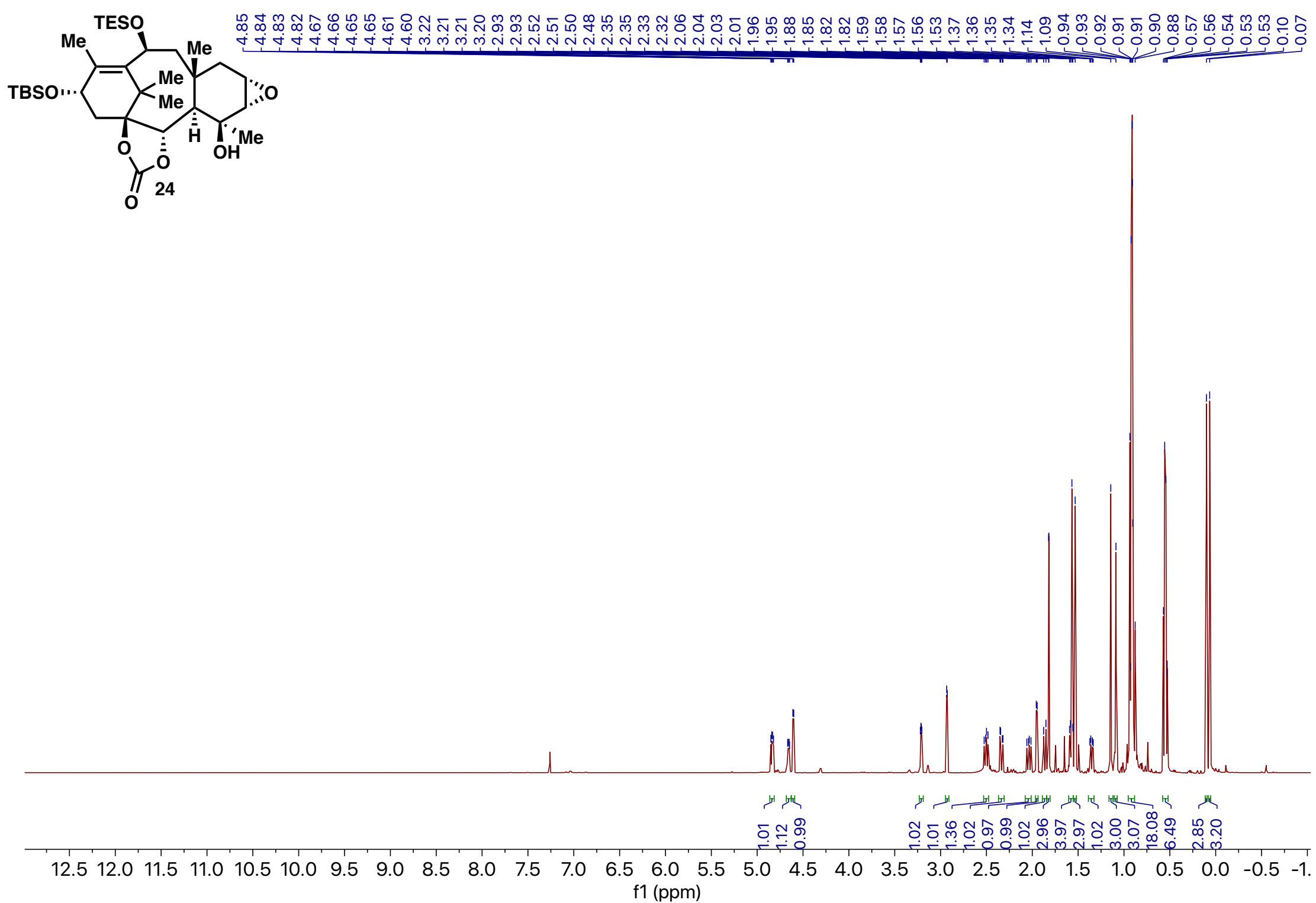




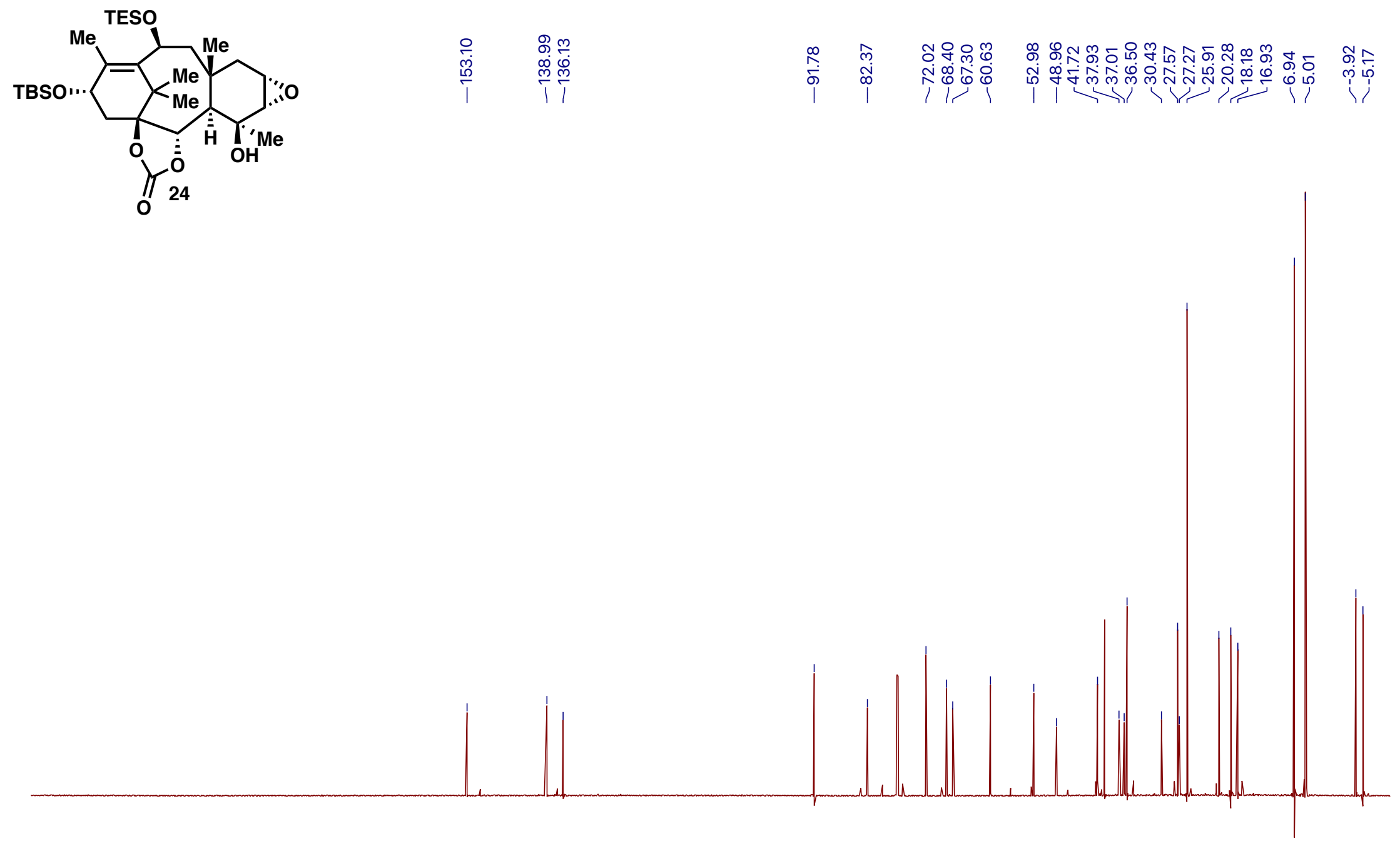

$\begin{array}{llllllllllllllllllllllllllllllllllll}30 & 220 & 210 & 200 & 190 & 180 & 170 & 160 & 150 & 140 & 130 & 120 & \begin{array}{c}110 \\ \mathrm{f} 1(\mathrm{ppm})\end{array} & 100 & 90 & 80 & 70 & 60 & 50 & 40 & 30 & 20 & 10 & 0 & \end{array}$ 


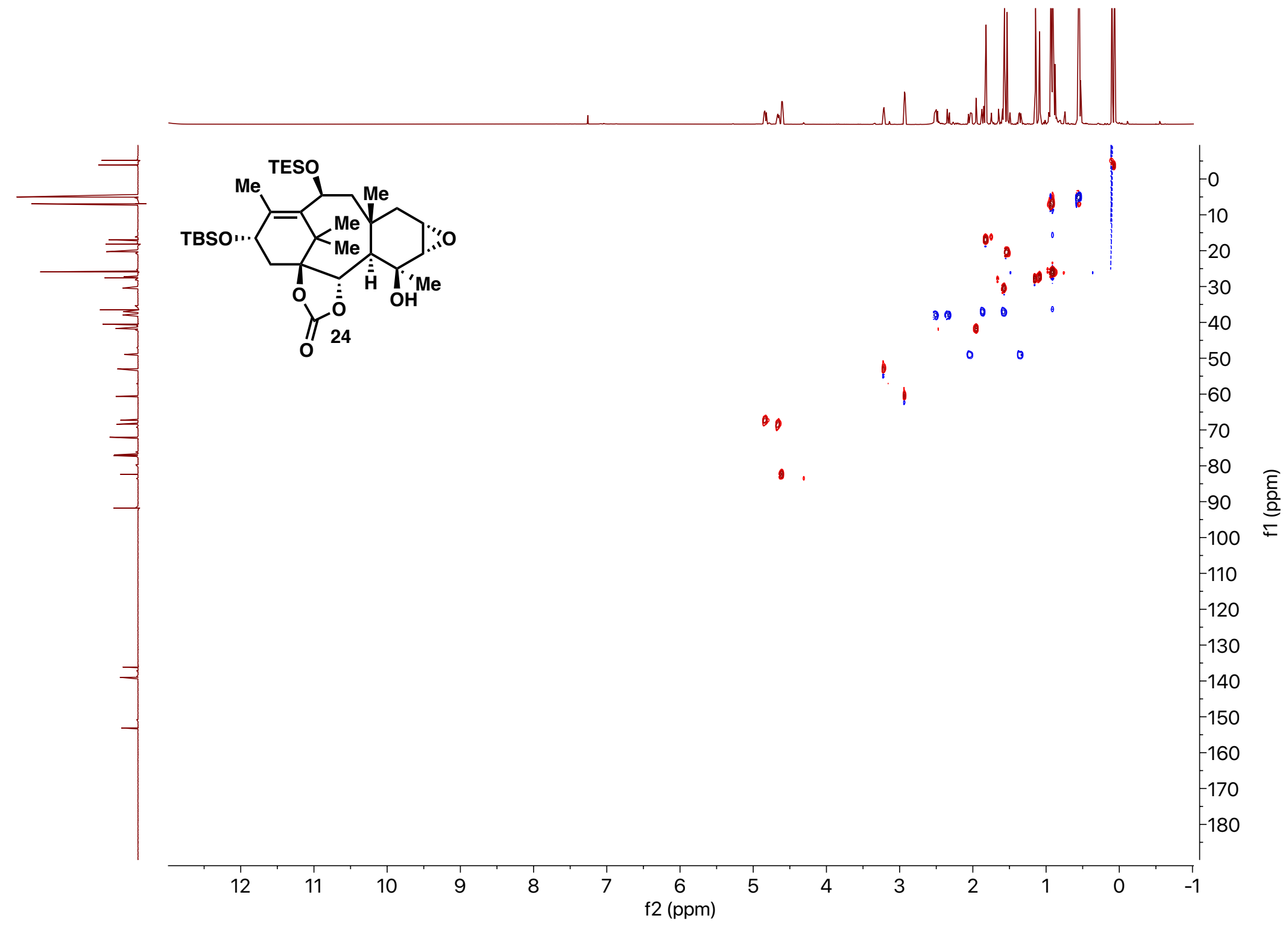

SI-143 


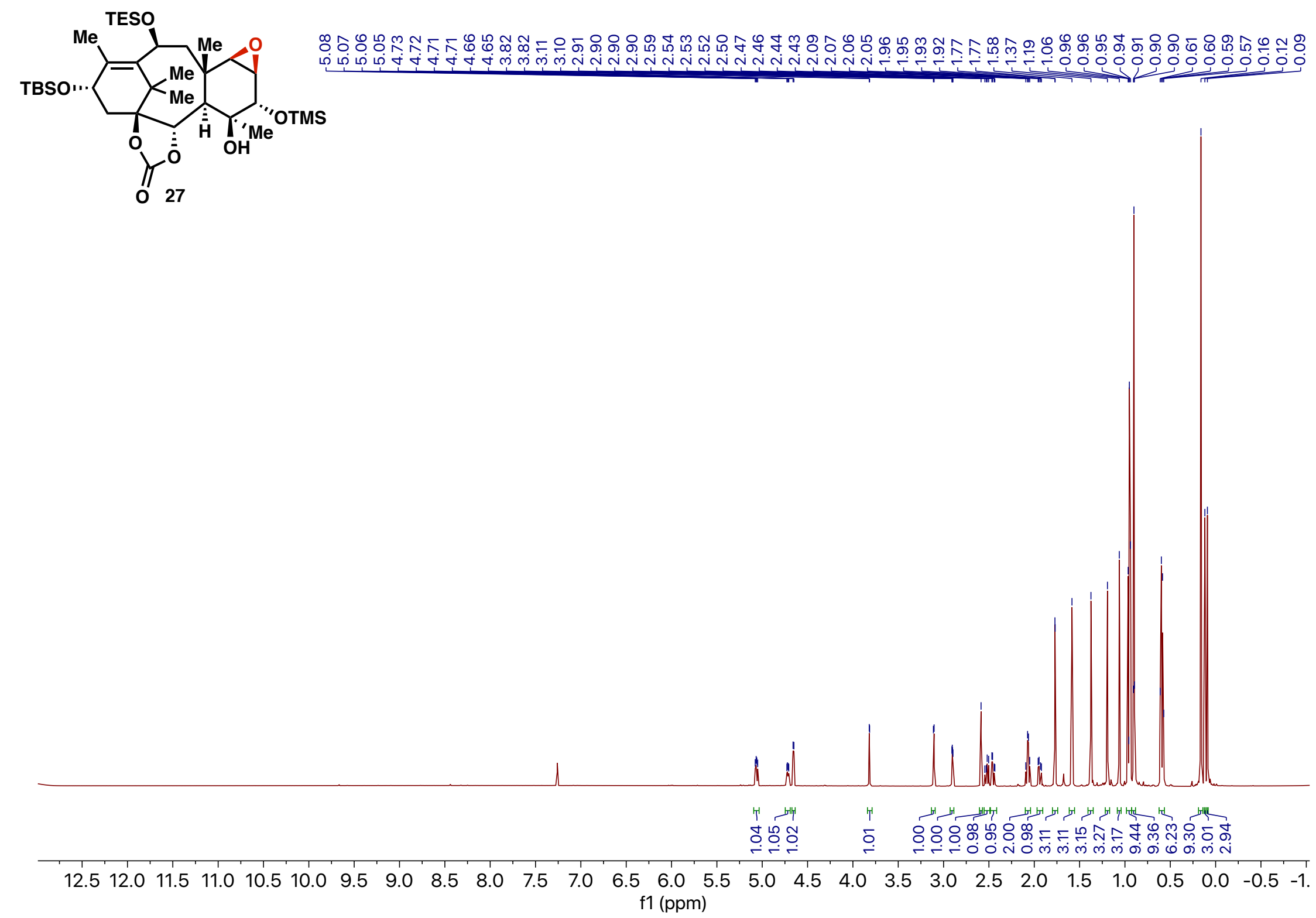



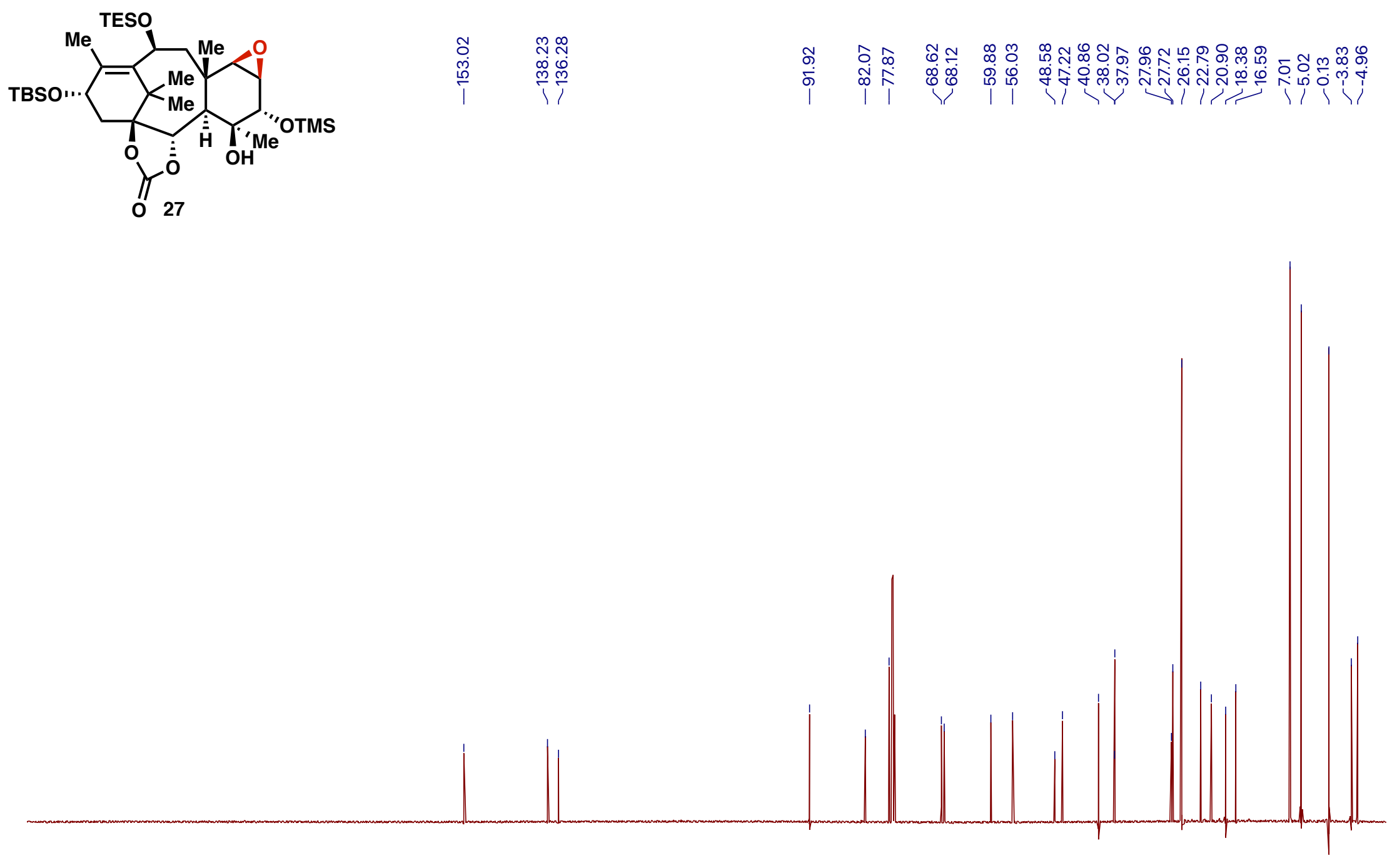

$\begin{array}{llllllllllllllllllllllllllllllllll}30 & 220 & 210 & 200 & 190 & 180 & 170 & 160 & 150 & 140 & 130 & 120 & 110 & 100 & 90 & 80 & 70 & 60 & 50 & 40 & 30 & 20 & 10 & 0 & f 0\end{array}$ 


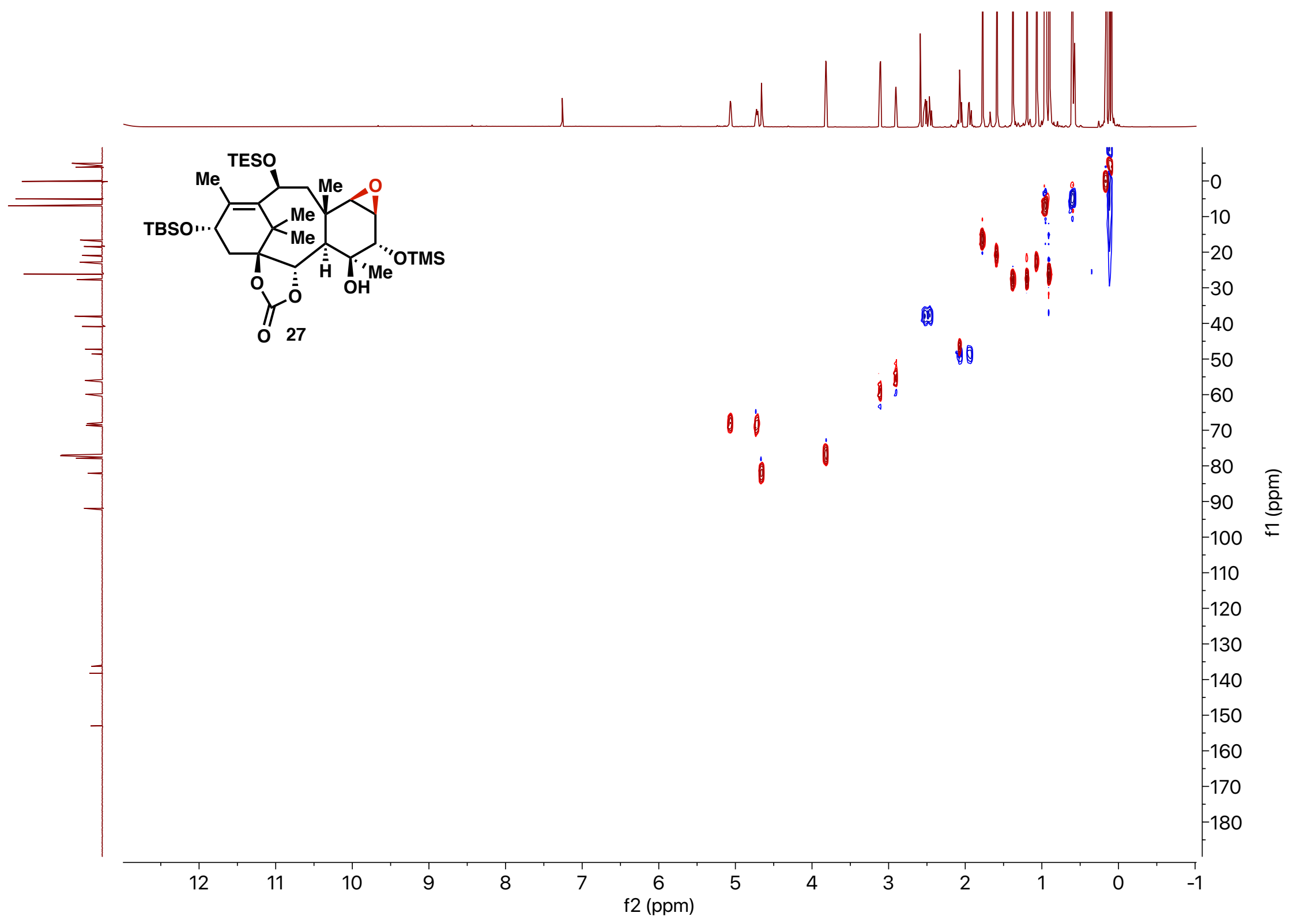

SI-146 


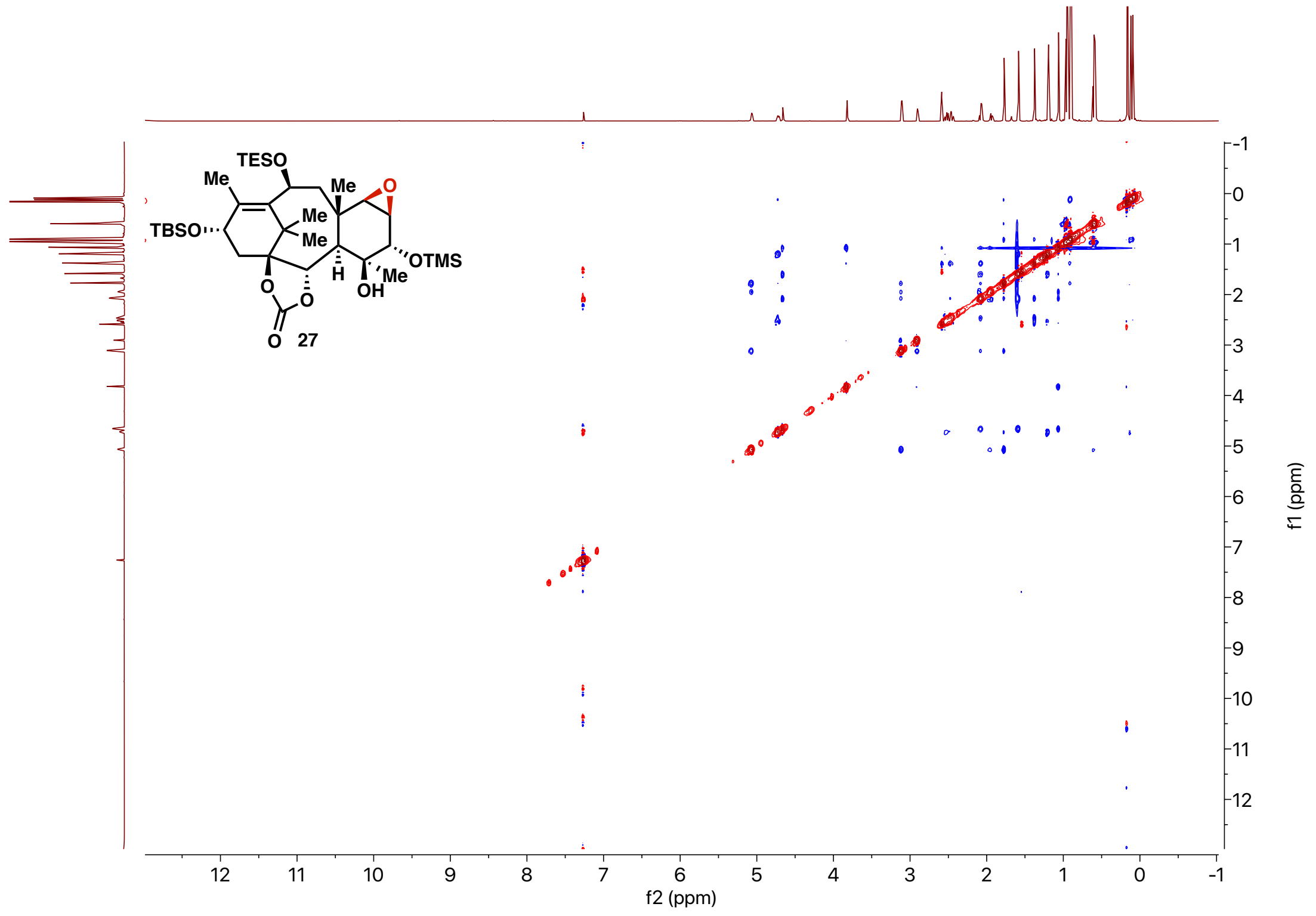

SI-147 


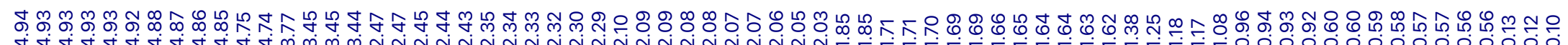

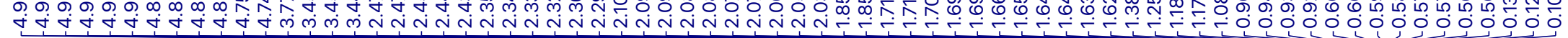

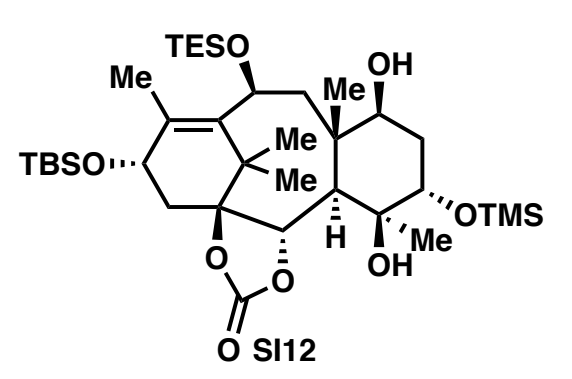

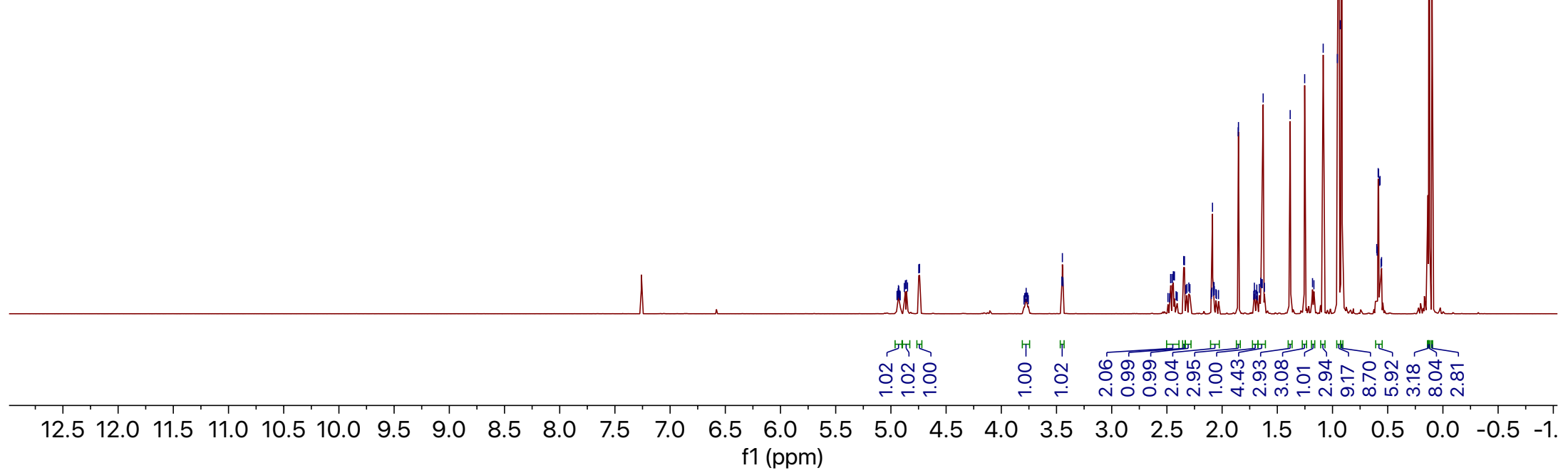



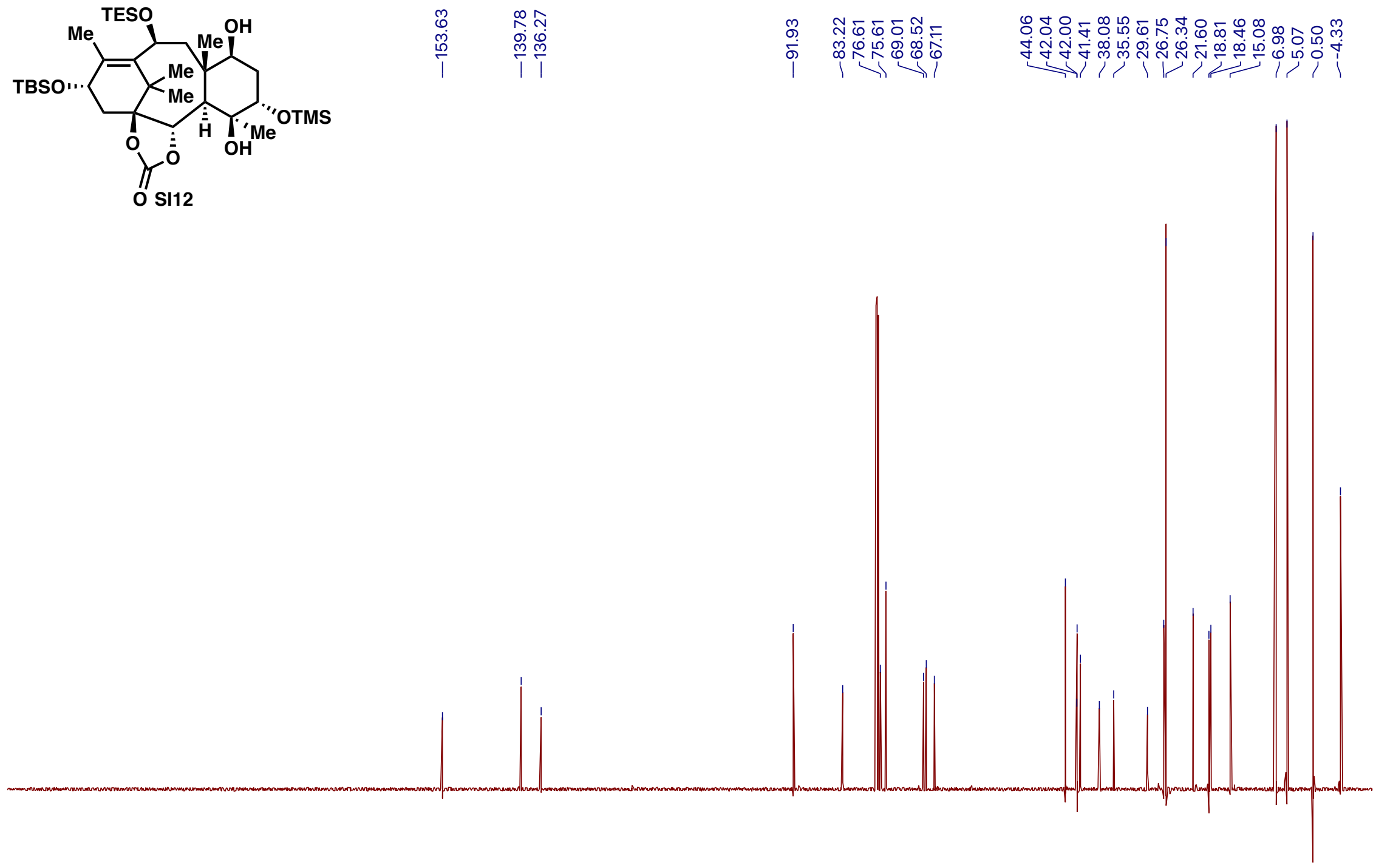

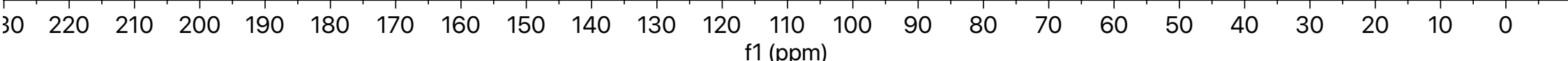




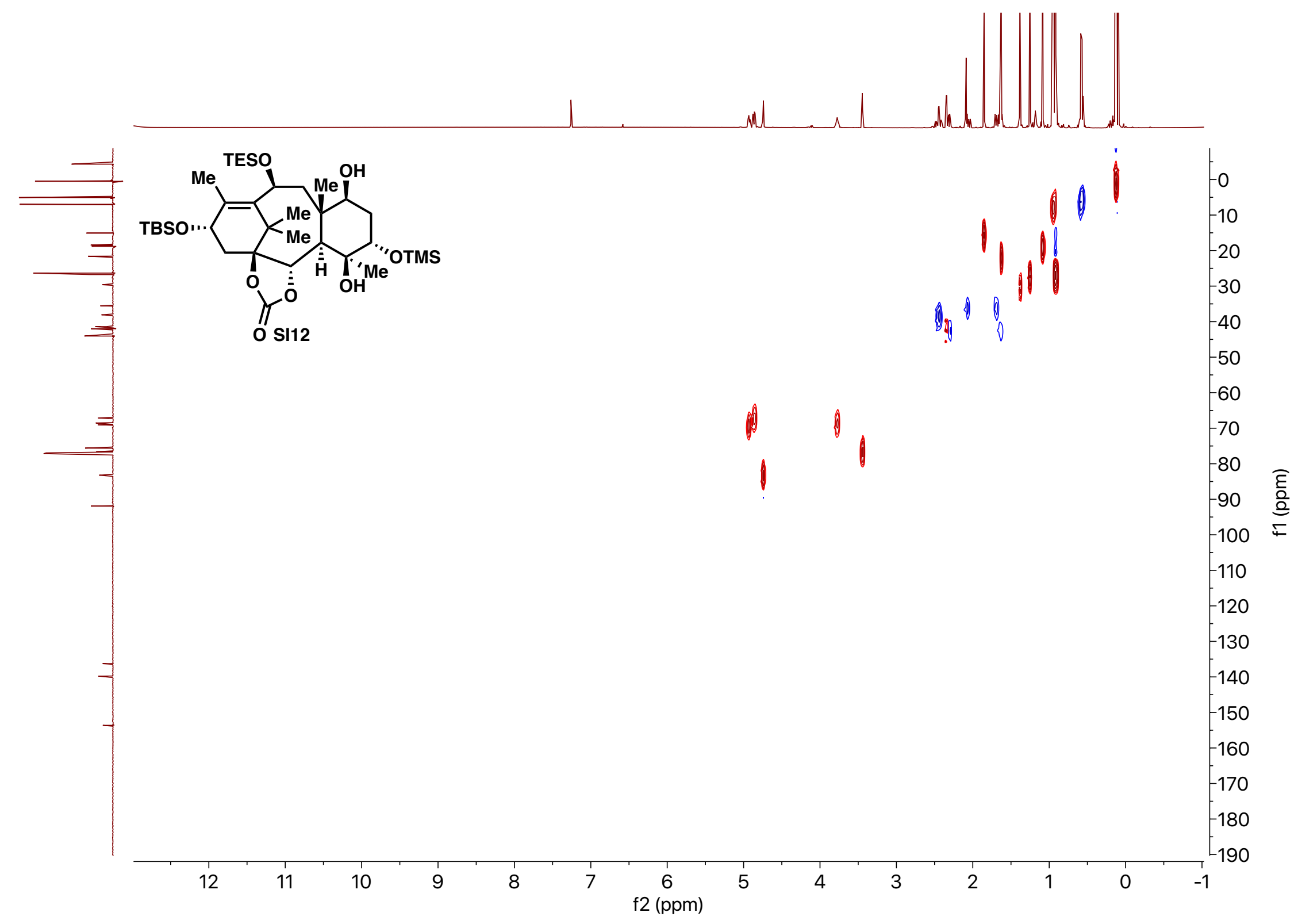

SI-150 


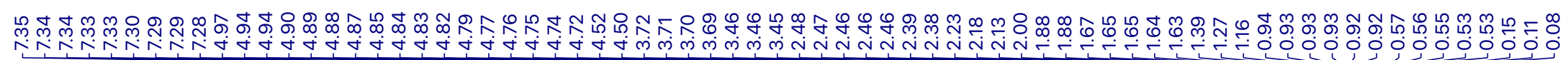

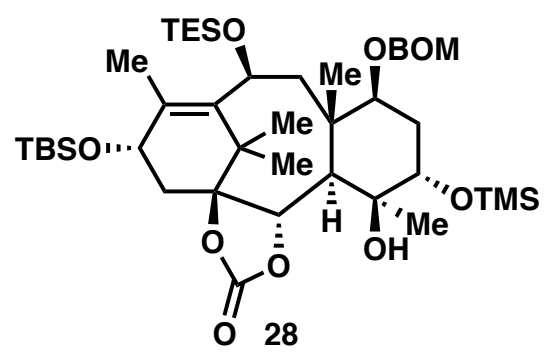

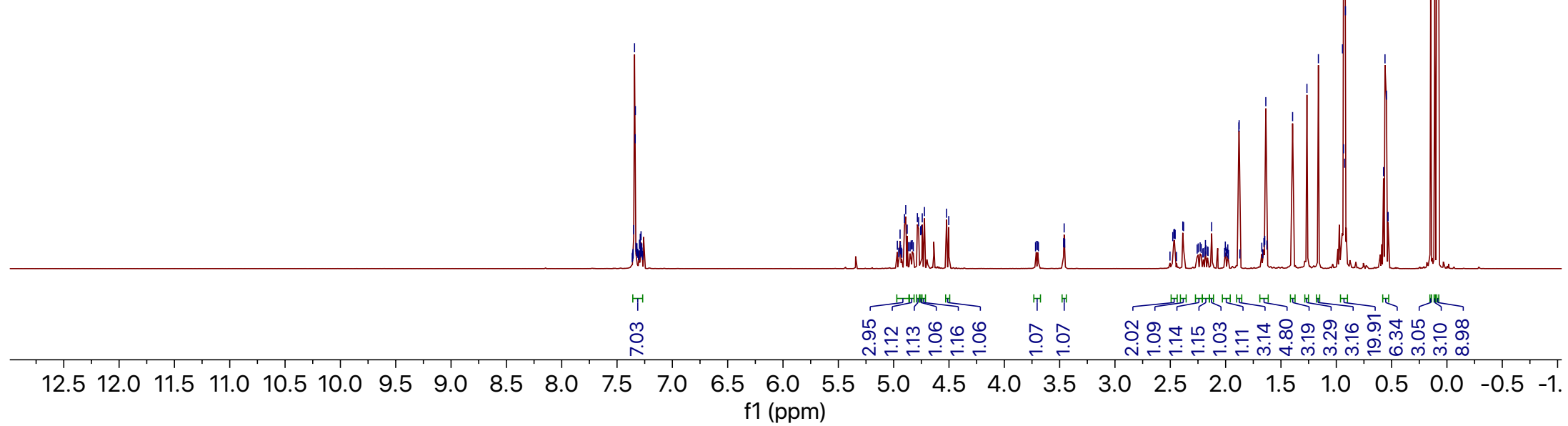



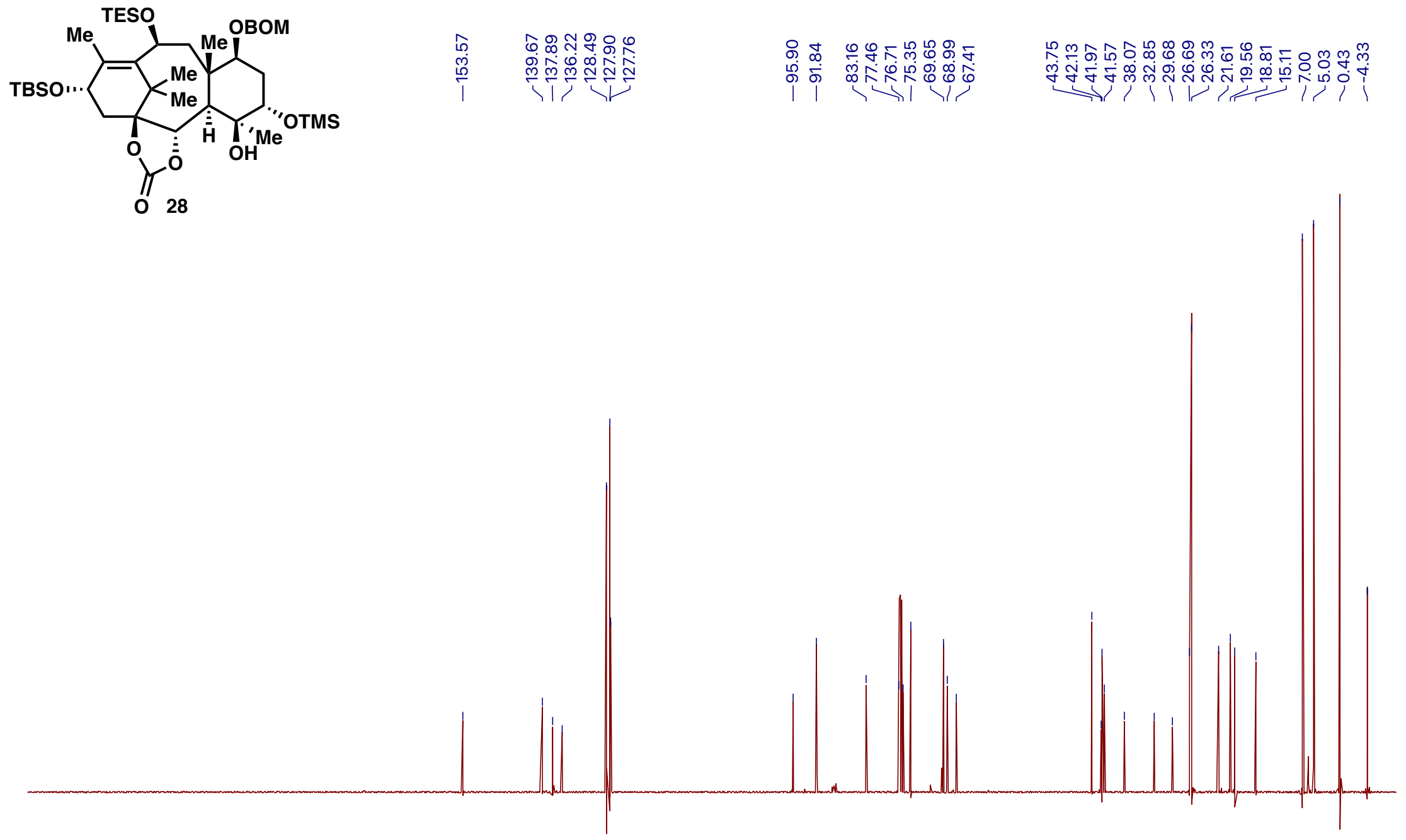

$20 \quad 110$

8070

$60 \quad 50$

$40 \quad 30$

20

100

SI-152 


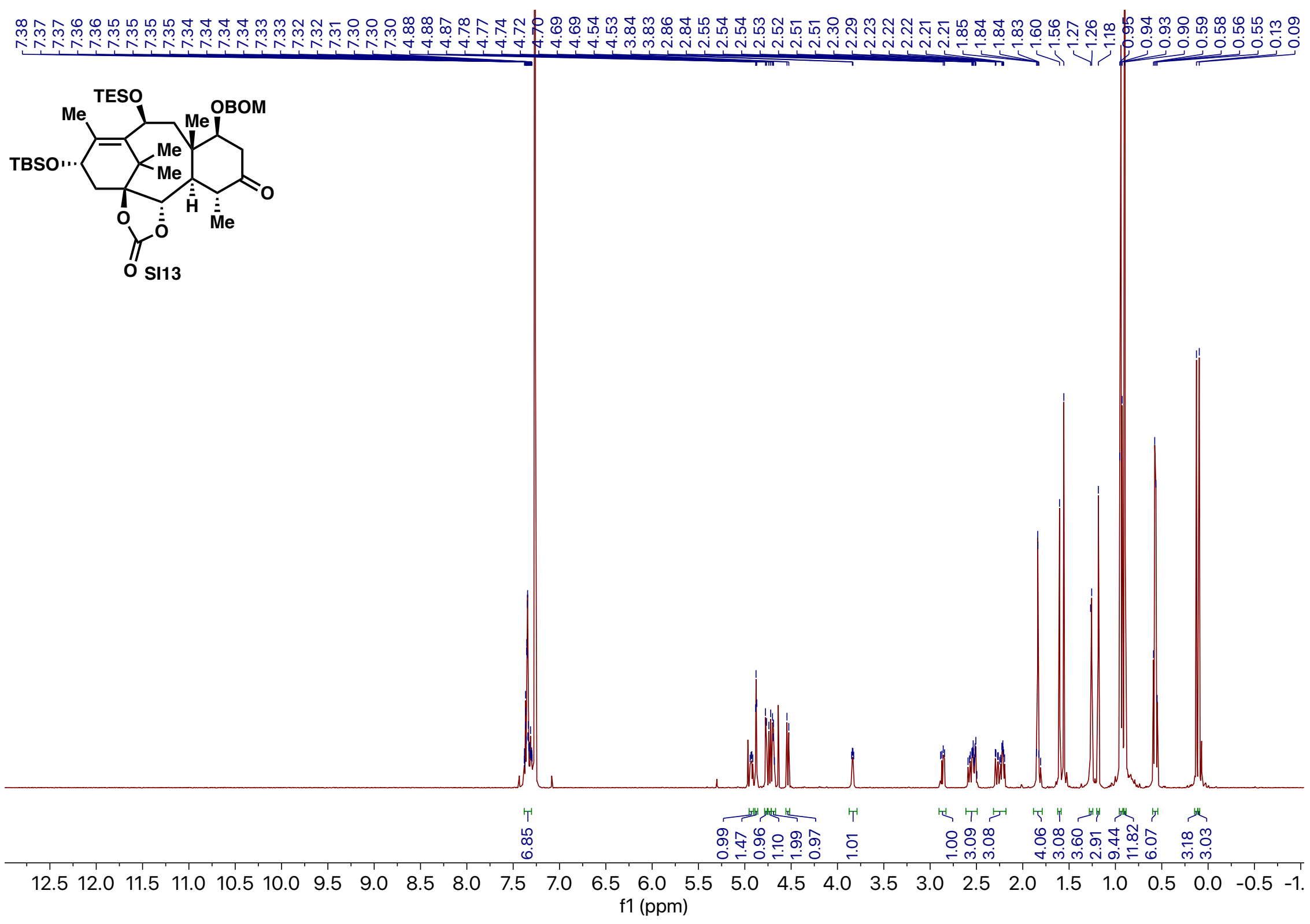

SI-153 


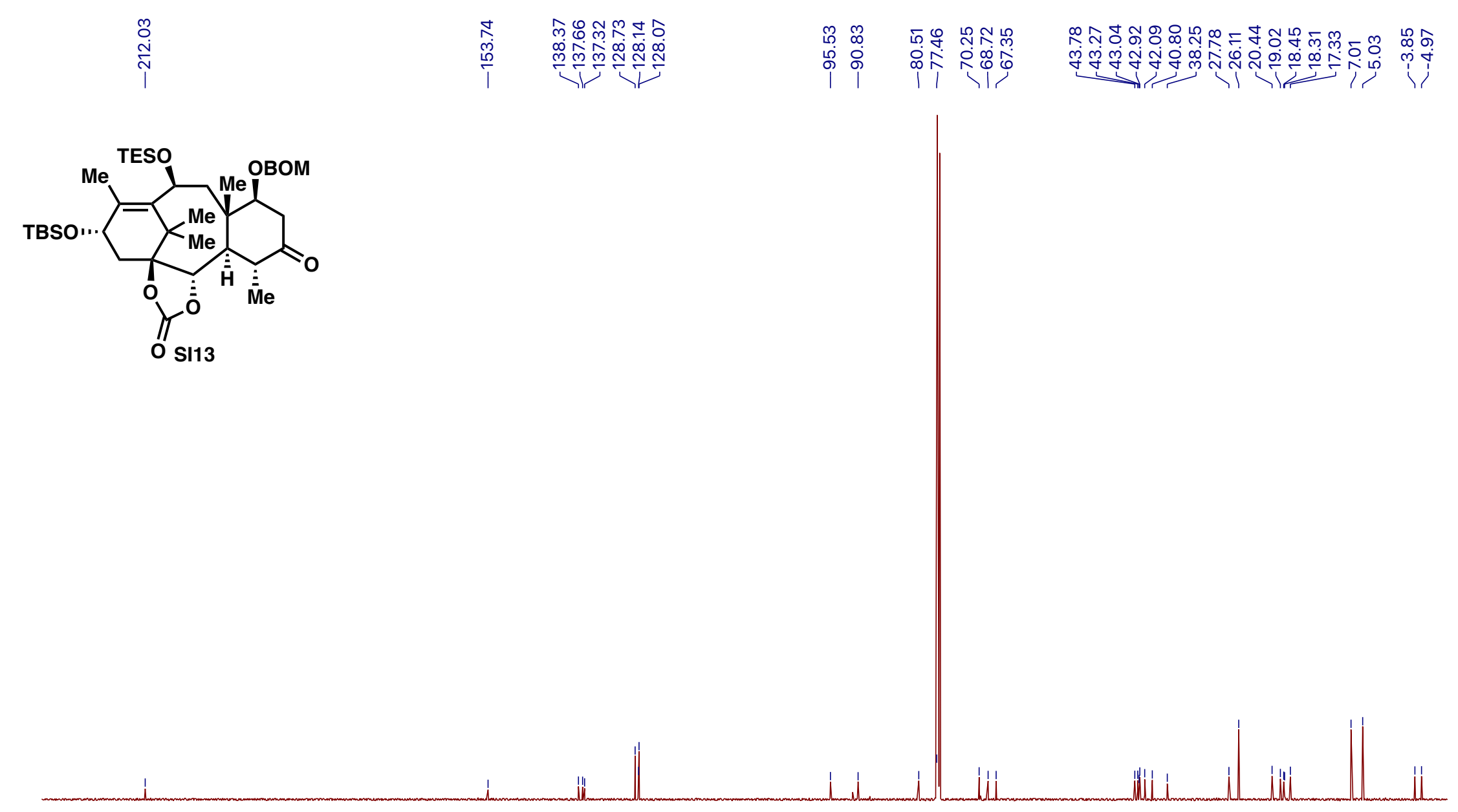

$\begin{array}{llllllllllllllllllllllllllll}220 & 210 & 200 & 190 & 180 & 170 & 160 & 150 & 140 & 130 & 120 & 110 & 100 & 90 & 80 & 70 & 60 & 50 & 40 & 30 & 20 & 10 & 0 & f(p p m)\end{array}$ 


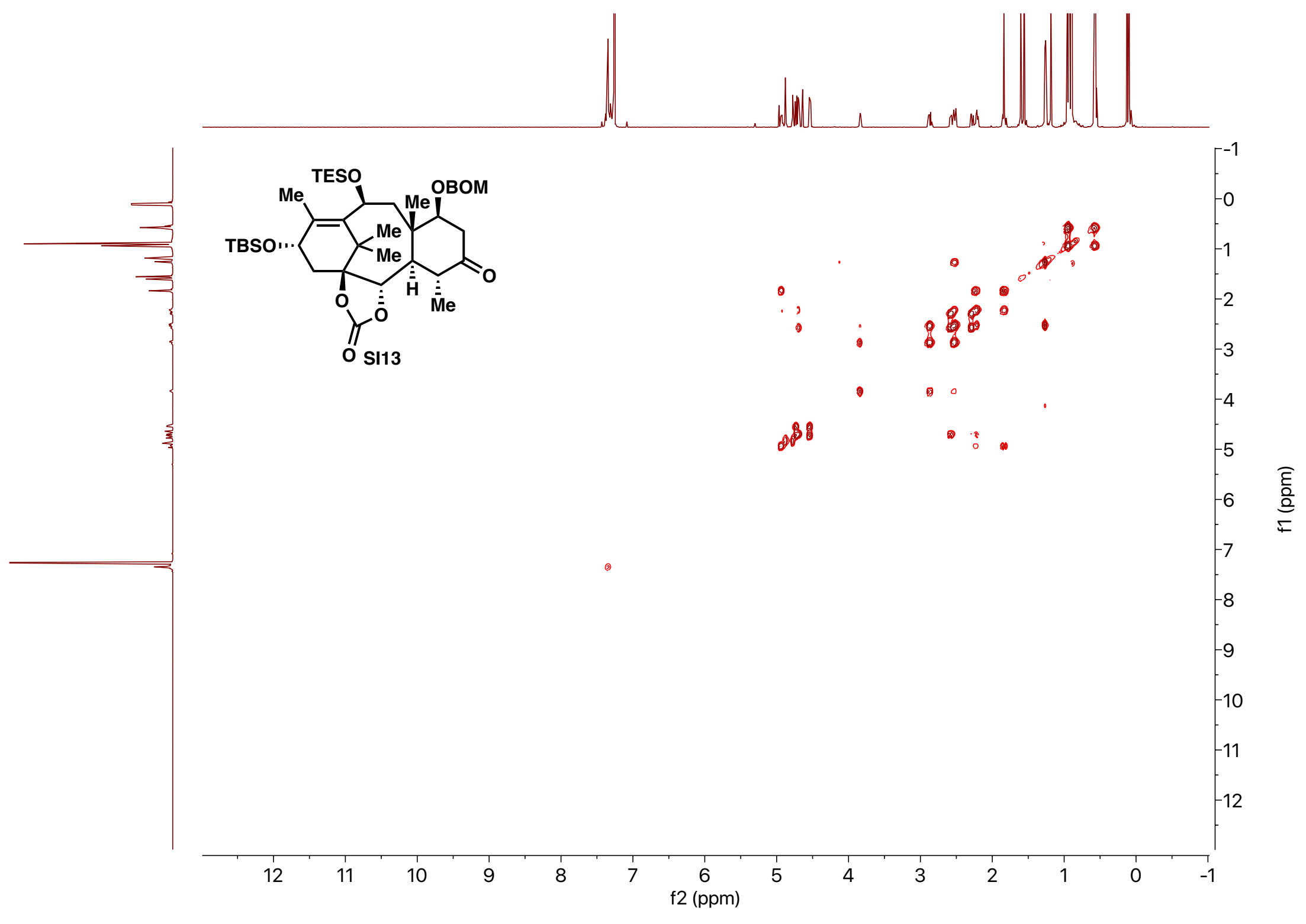

SI-155 


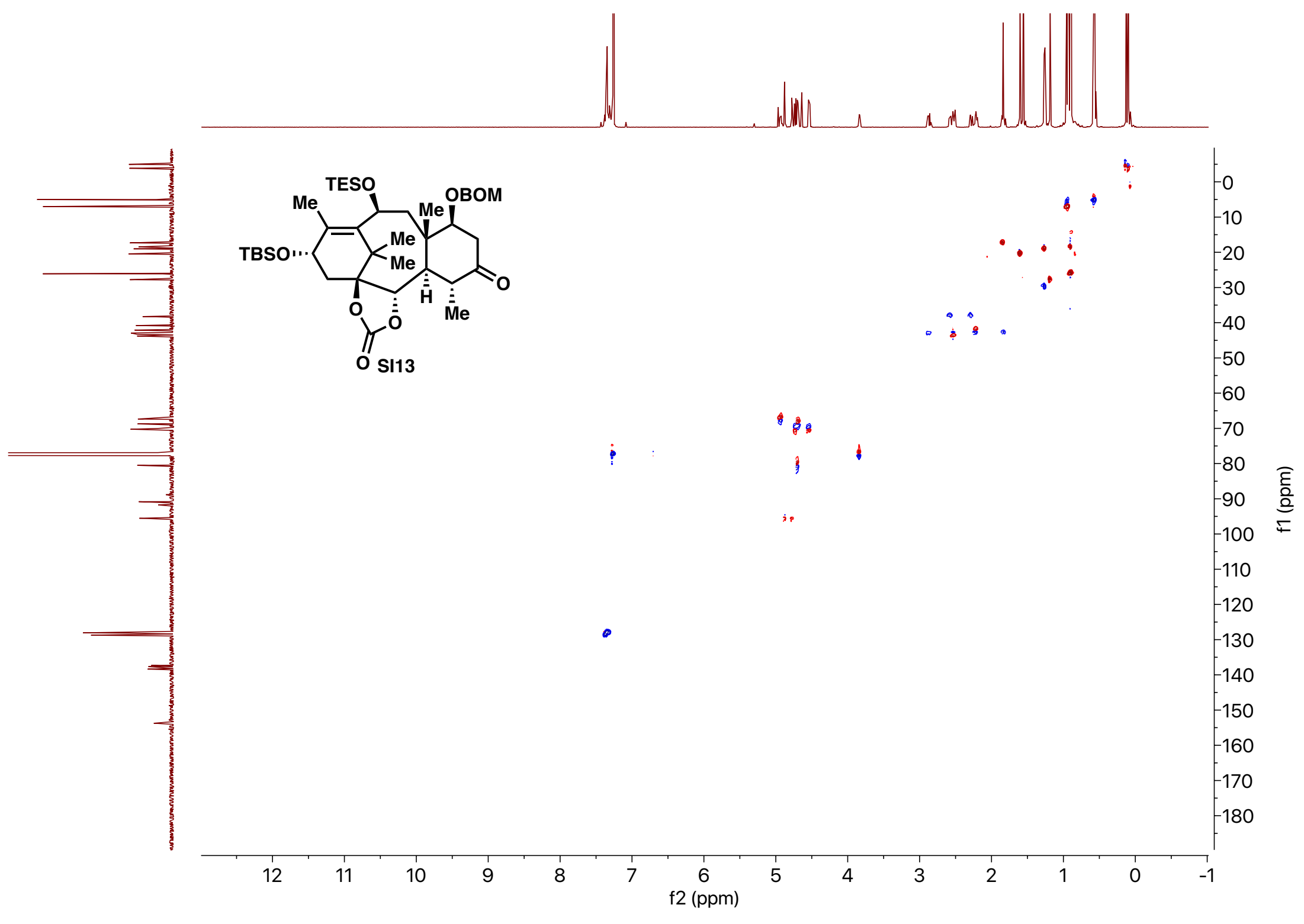

SI-156 


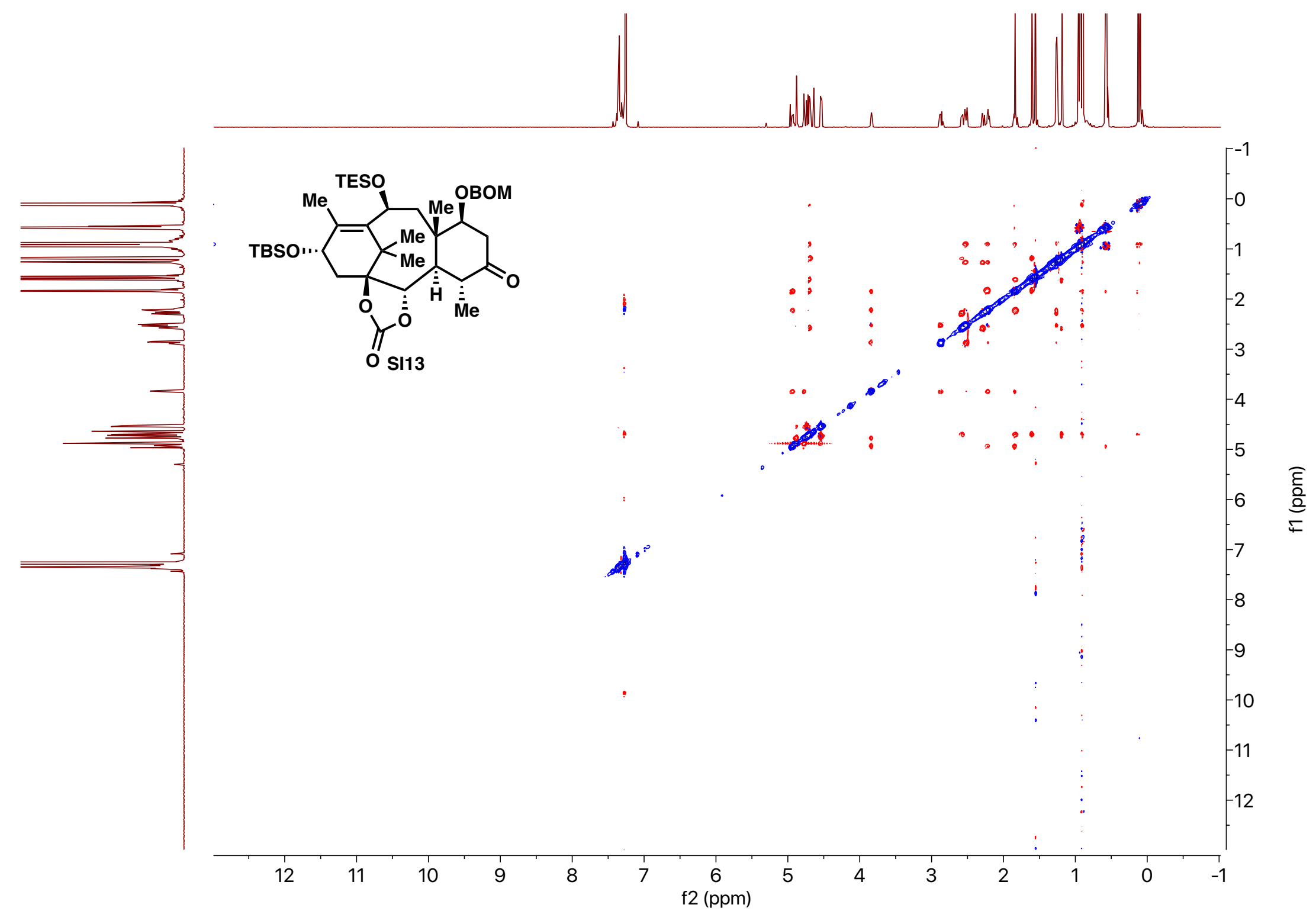

SI-157 

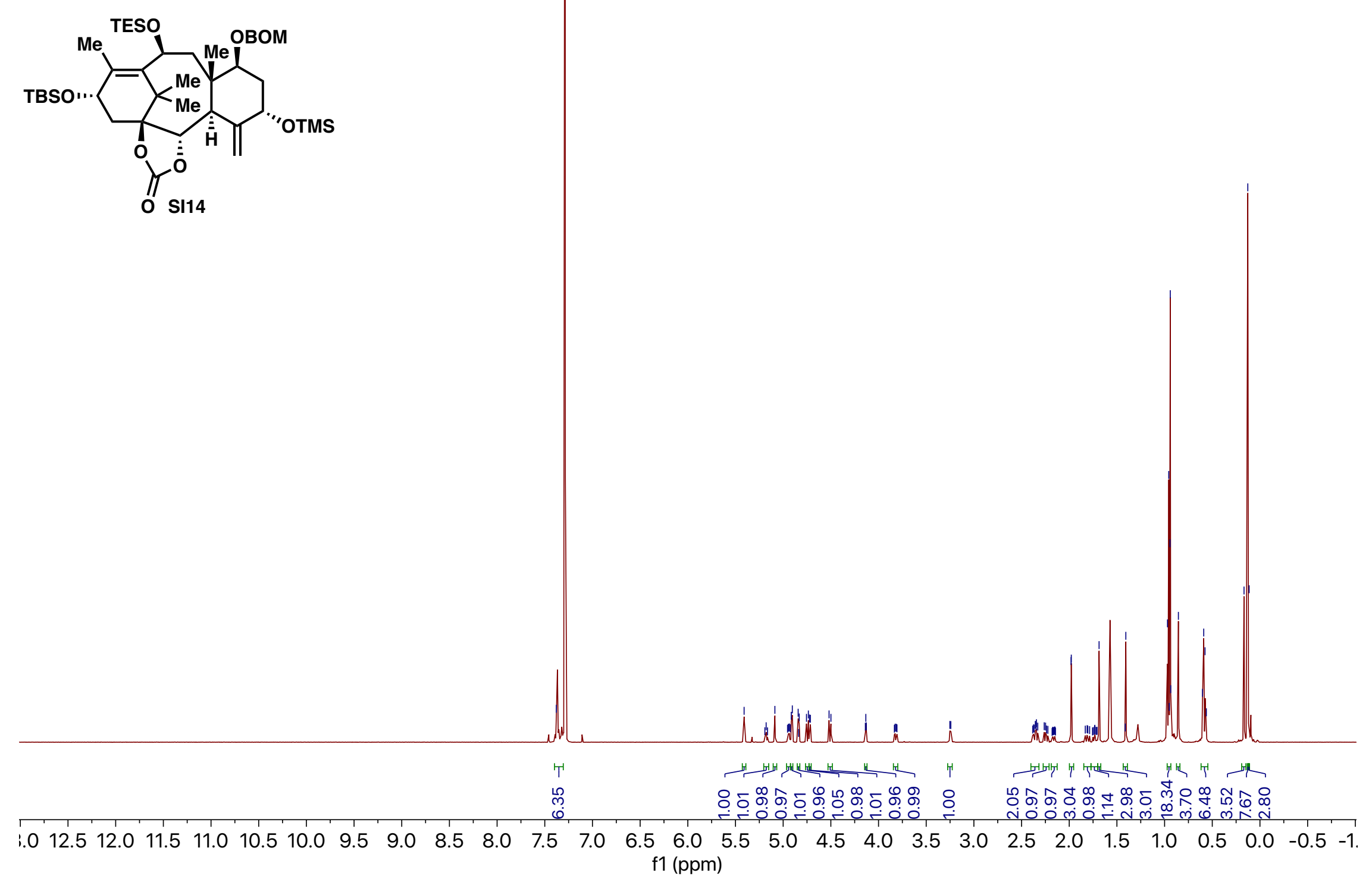

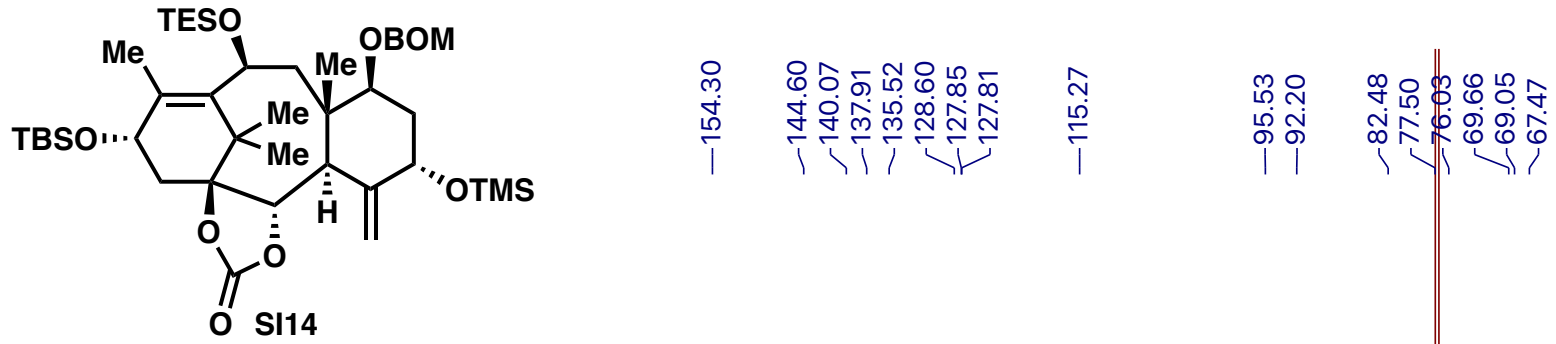

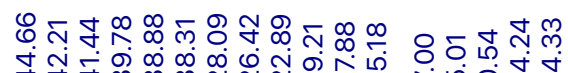

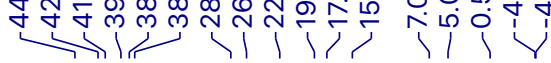

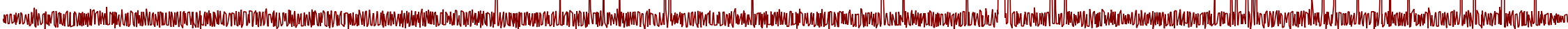




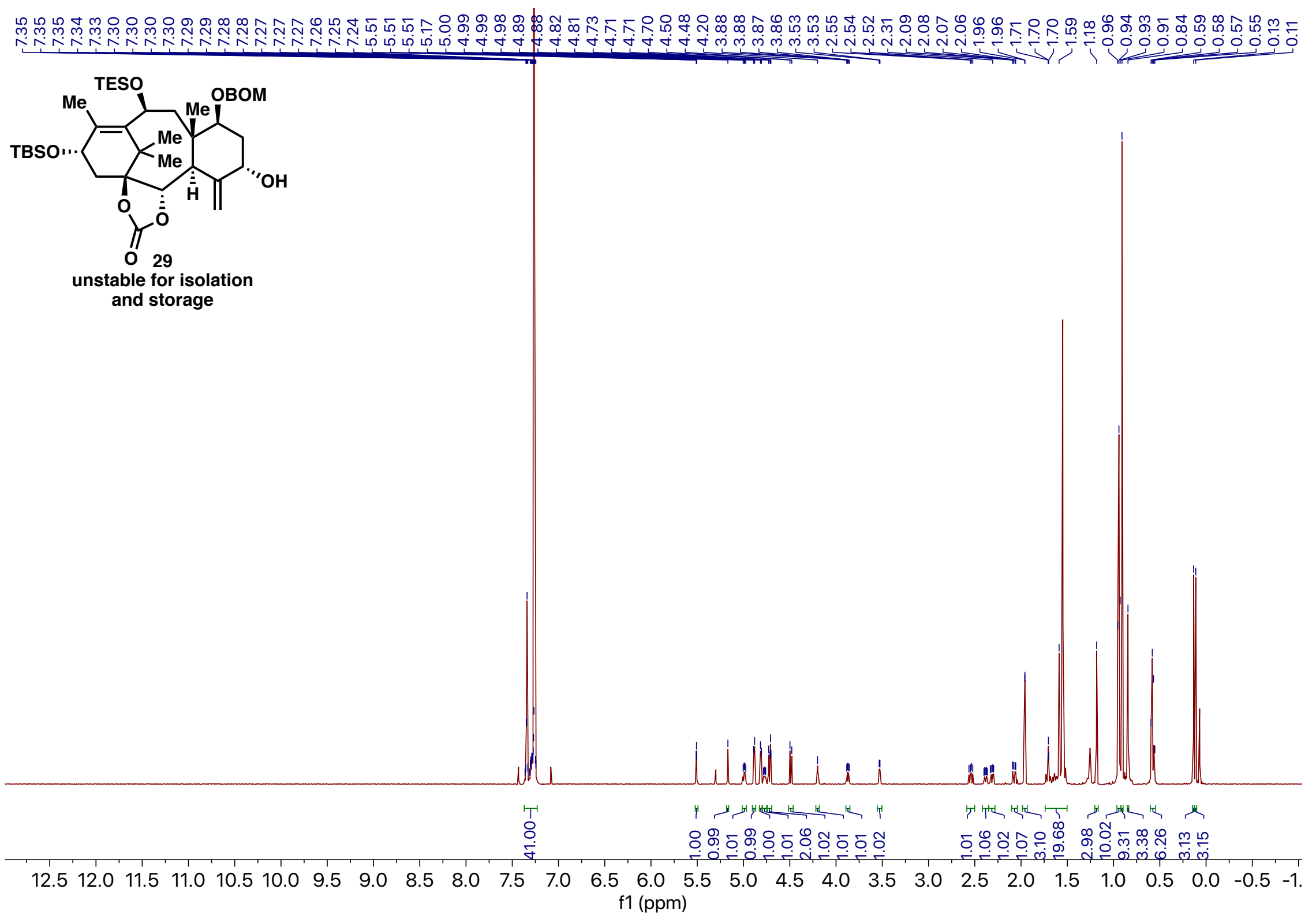

SI-160 


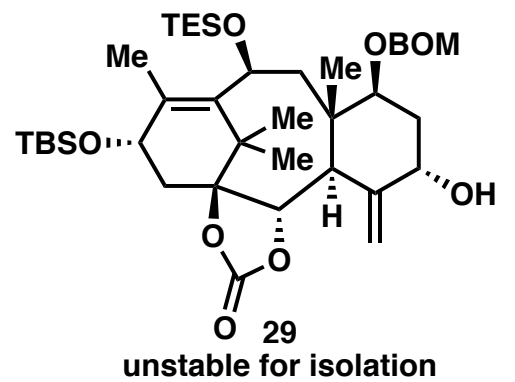
and storage

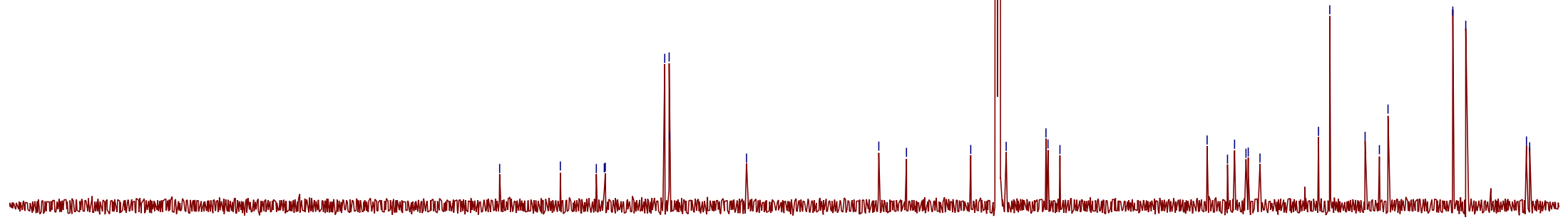

$20 \quad 110 \quad 100$

90

$80 \quad 70$

$60 \quad 50$

40

$30 \quad 20$

$10 \quad 0$

SI-161 


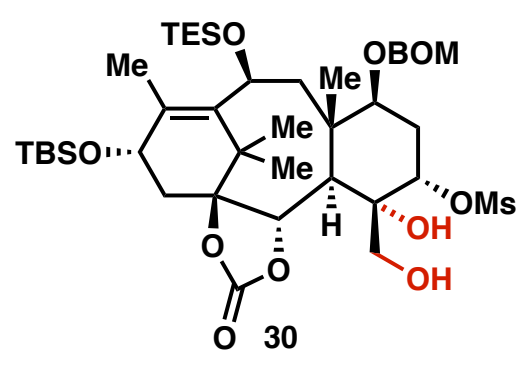



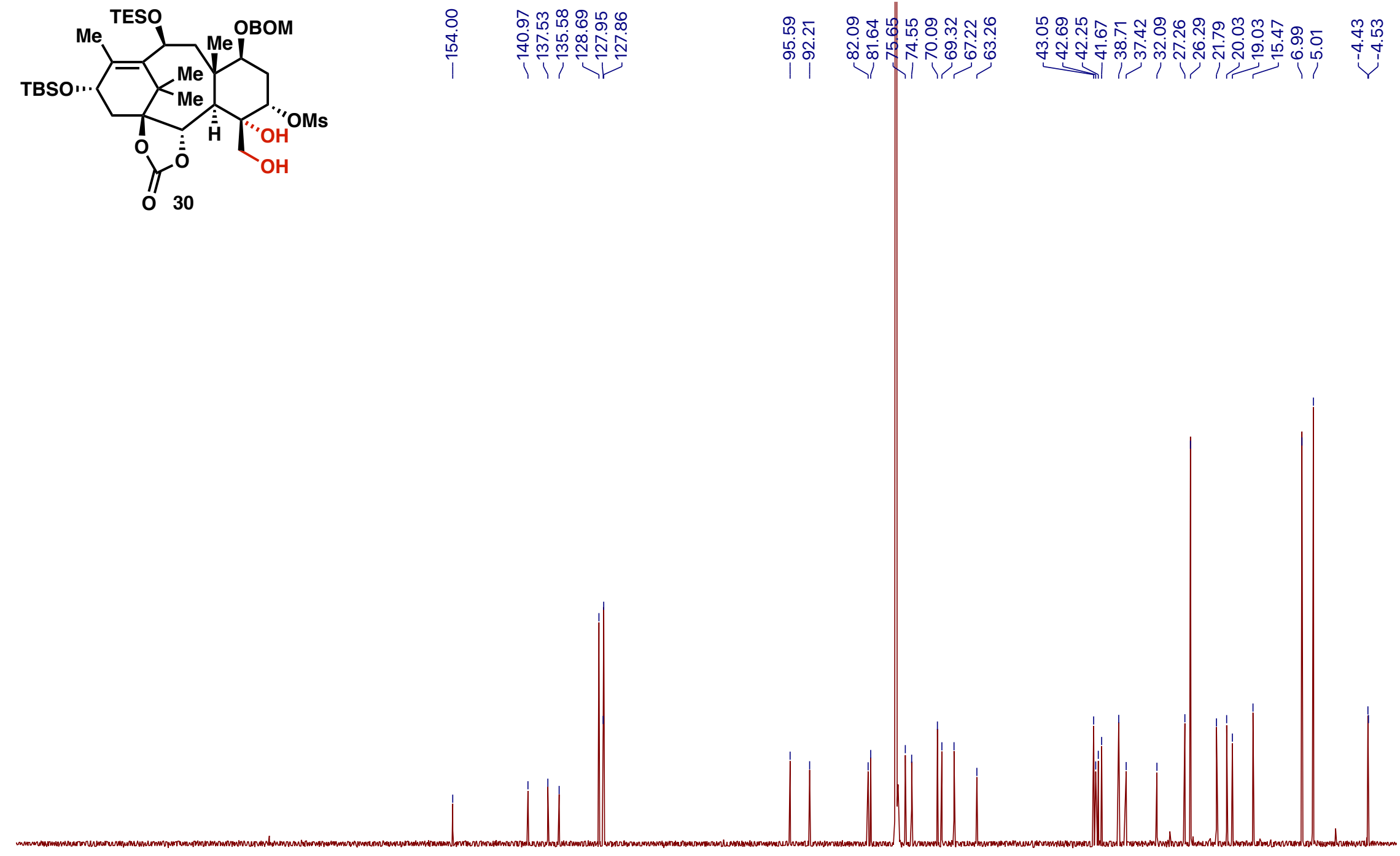


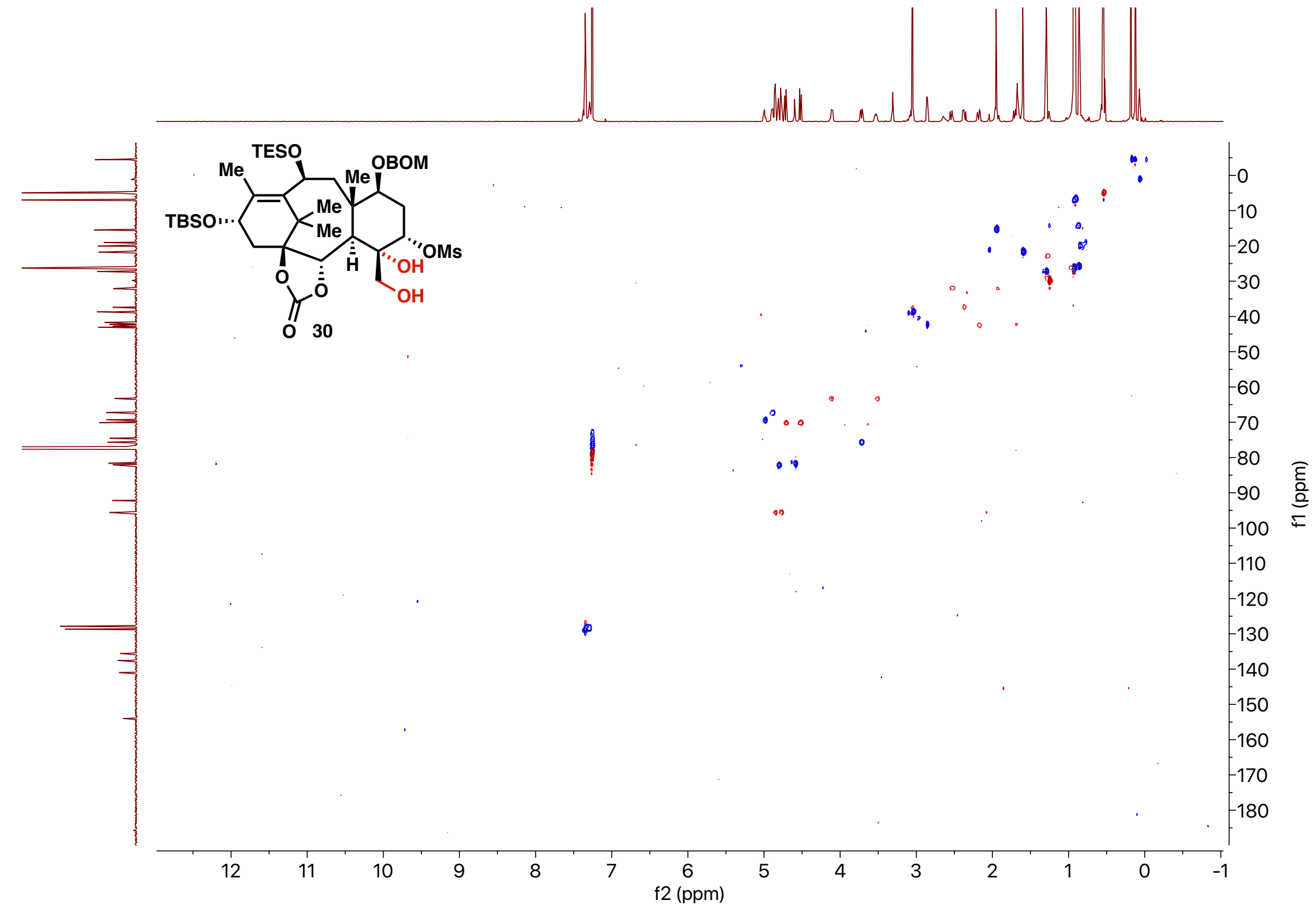

SI-164 


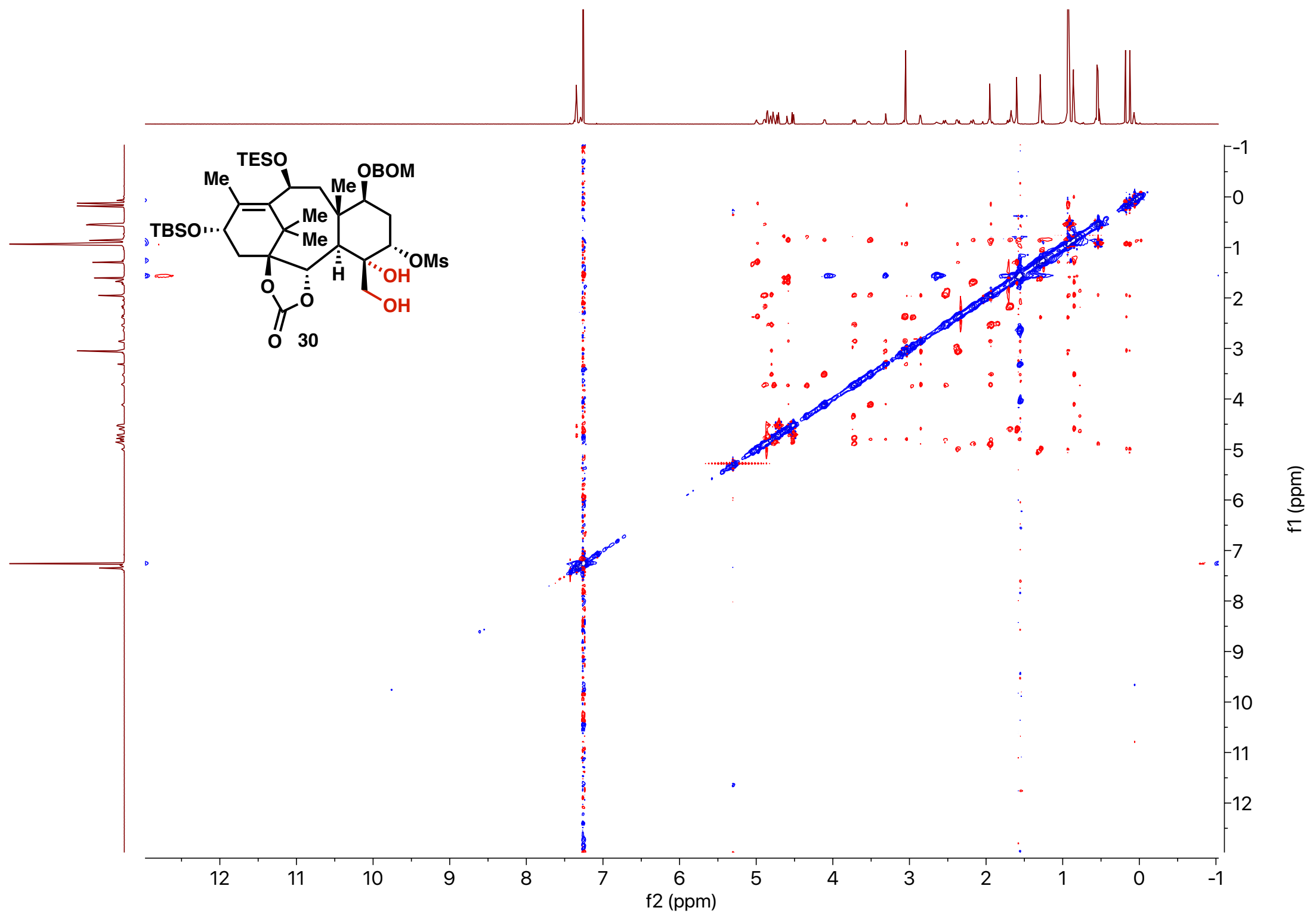

SI-165 


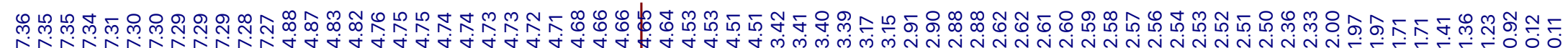

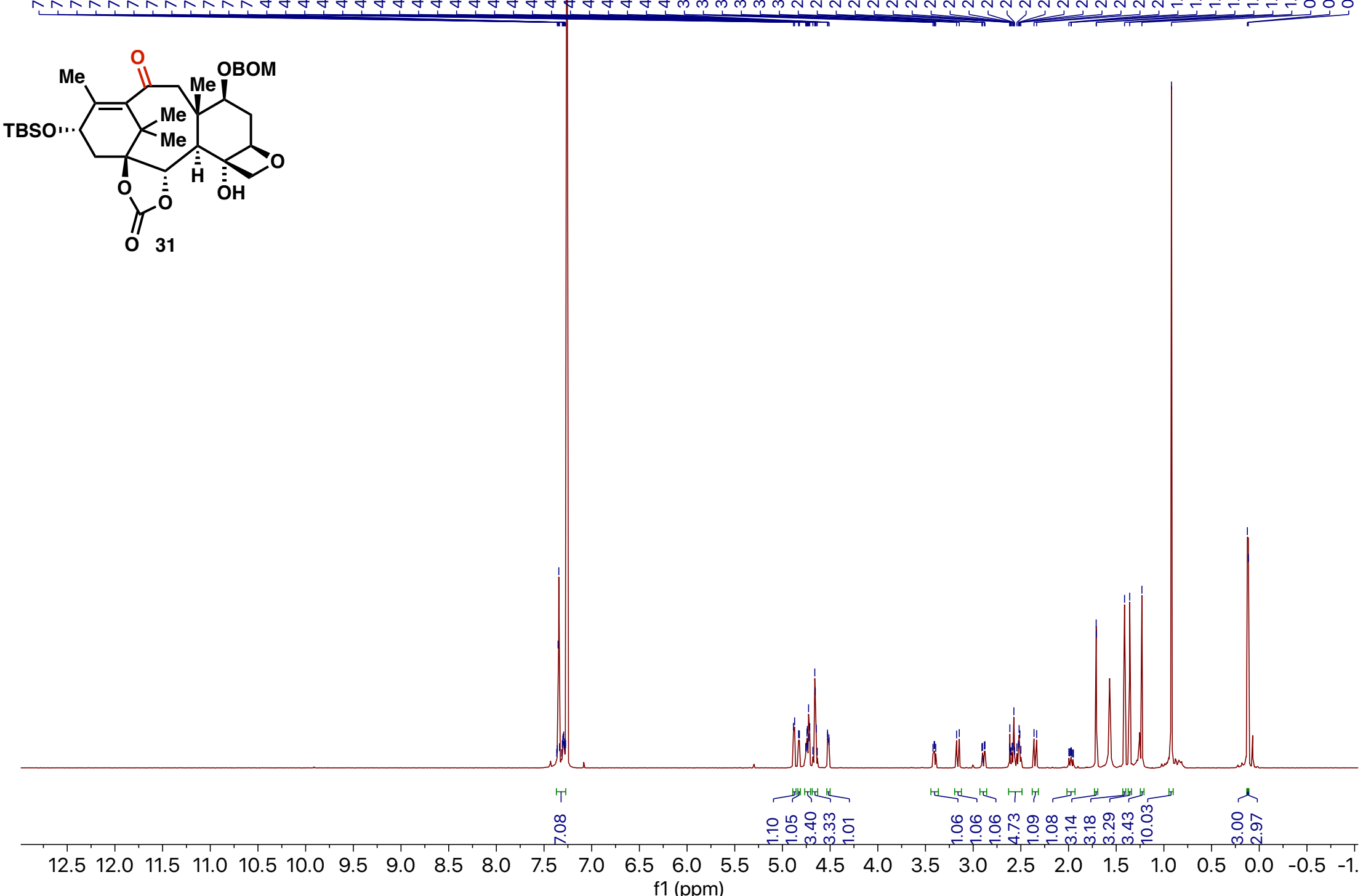




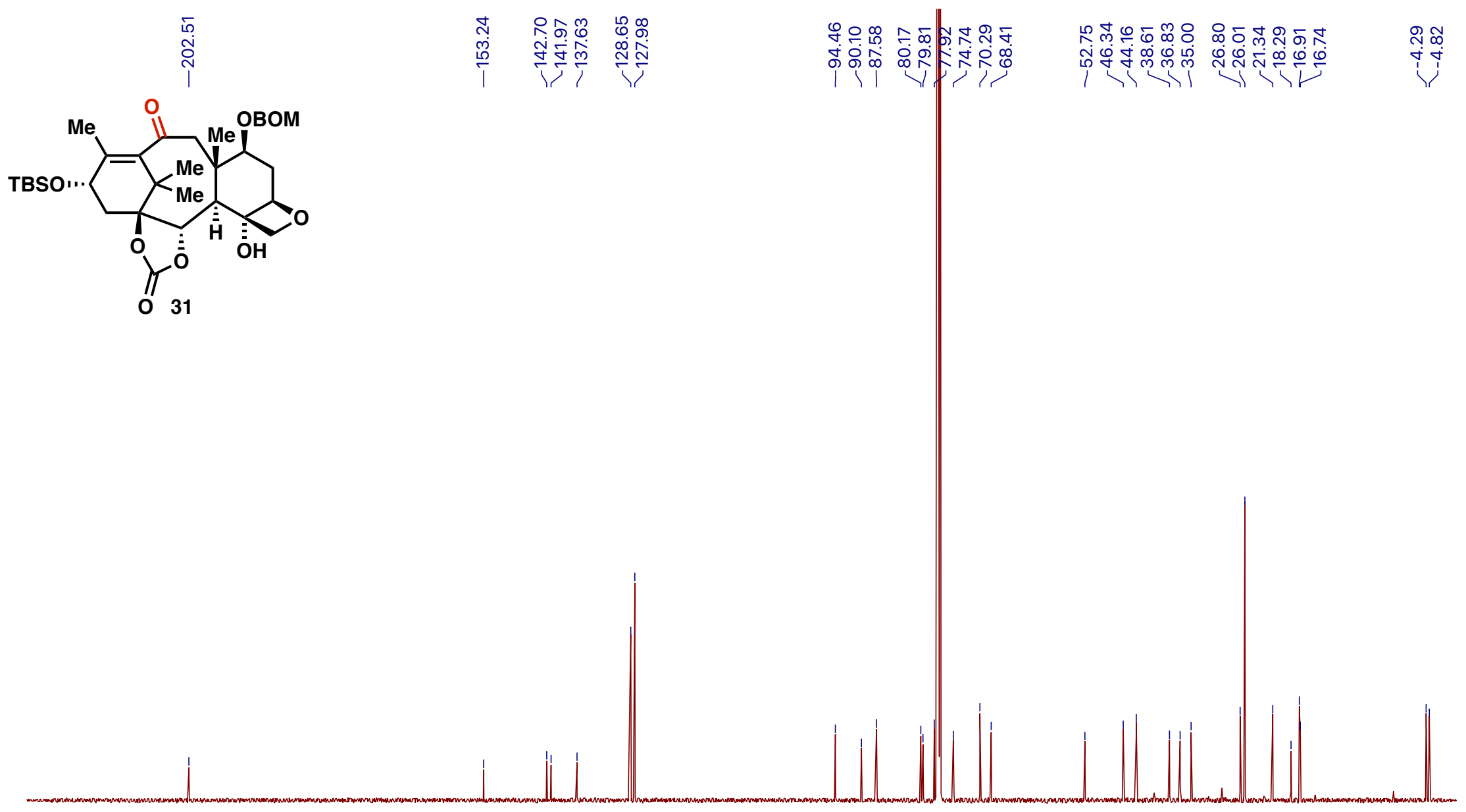

$\begin{array}{lllllllllllllllllllllllllllll}220 & 210 & 200 & 190 & 180 & 170 & 160 & 150 & 140 & 130 & 120 & 110 & 100 & 90 & 80 & 70 & 60 & 50 & 40 & 30 & 20 & 10 & 0 & f 0\end{array}$ 


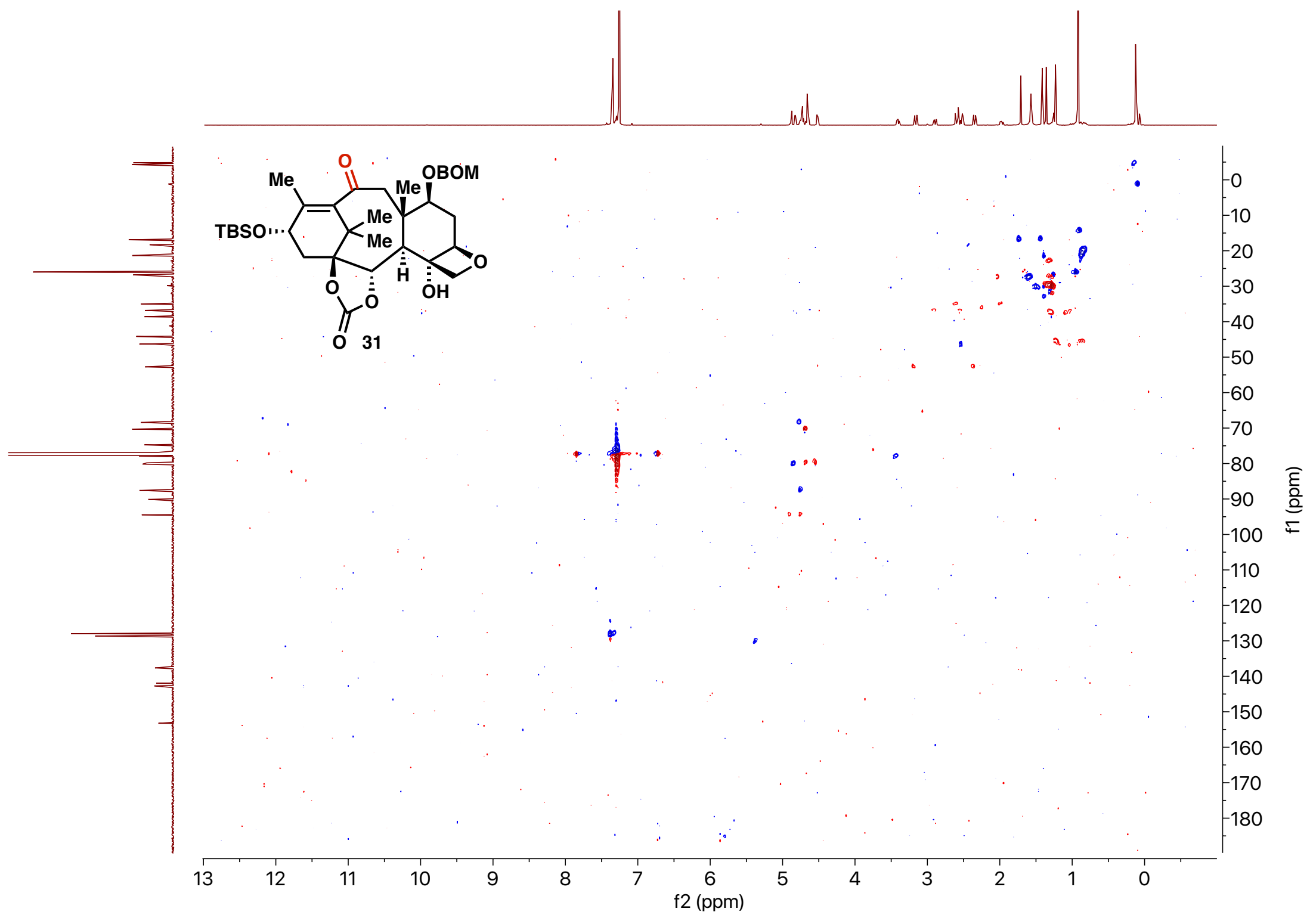

SI-168 


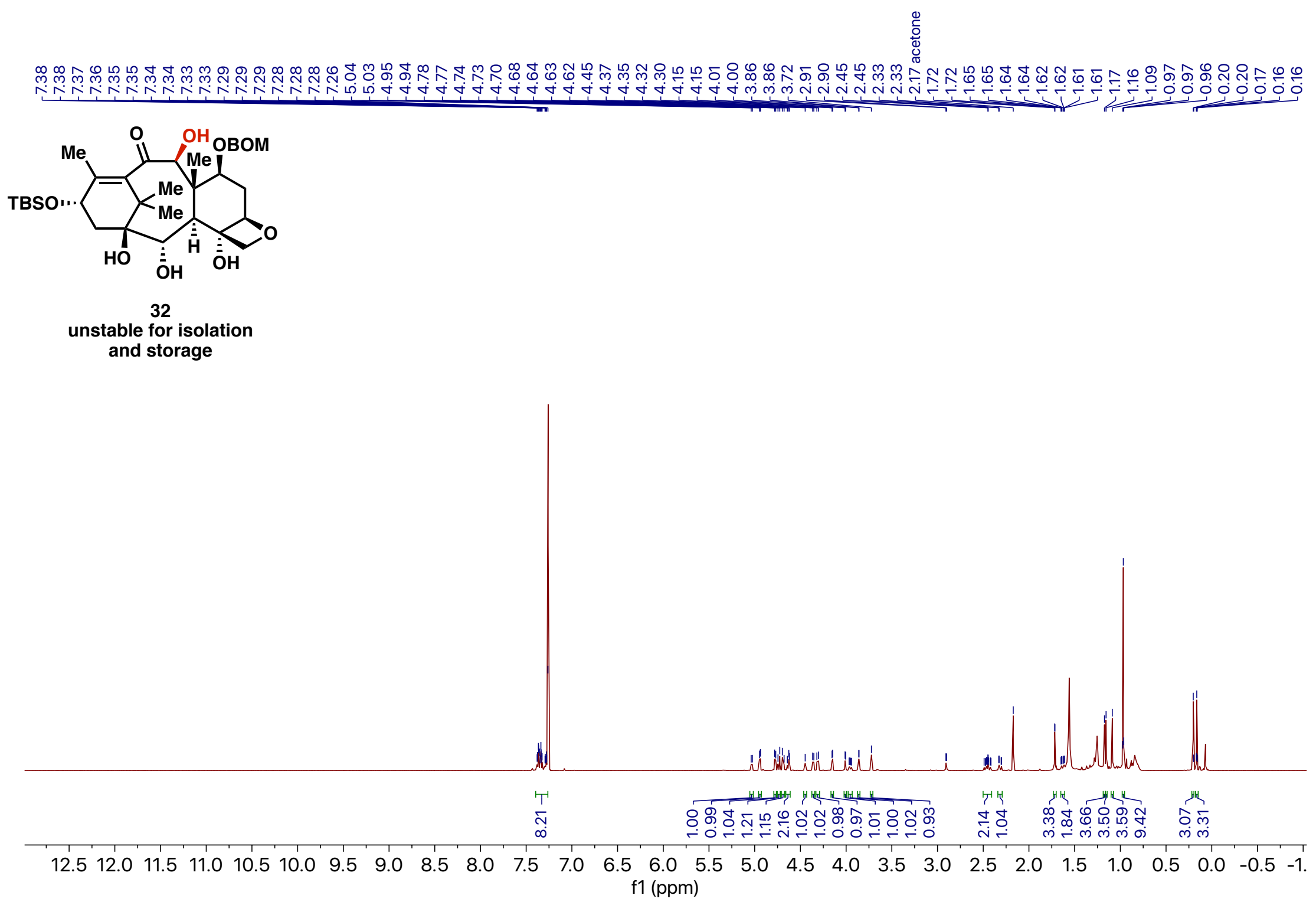




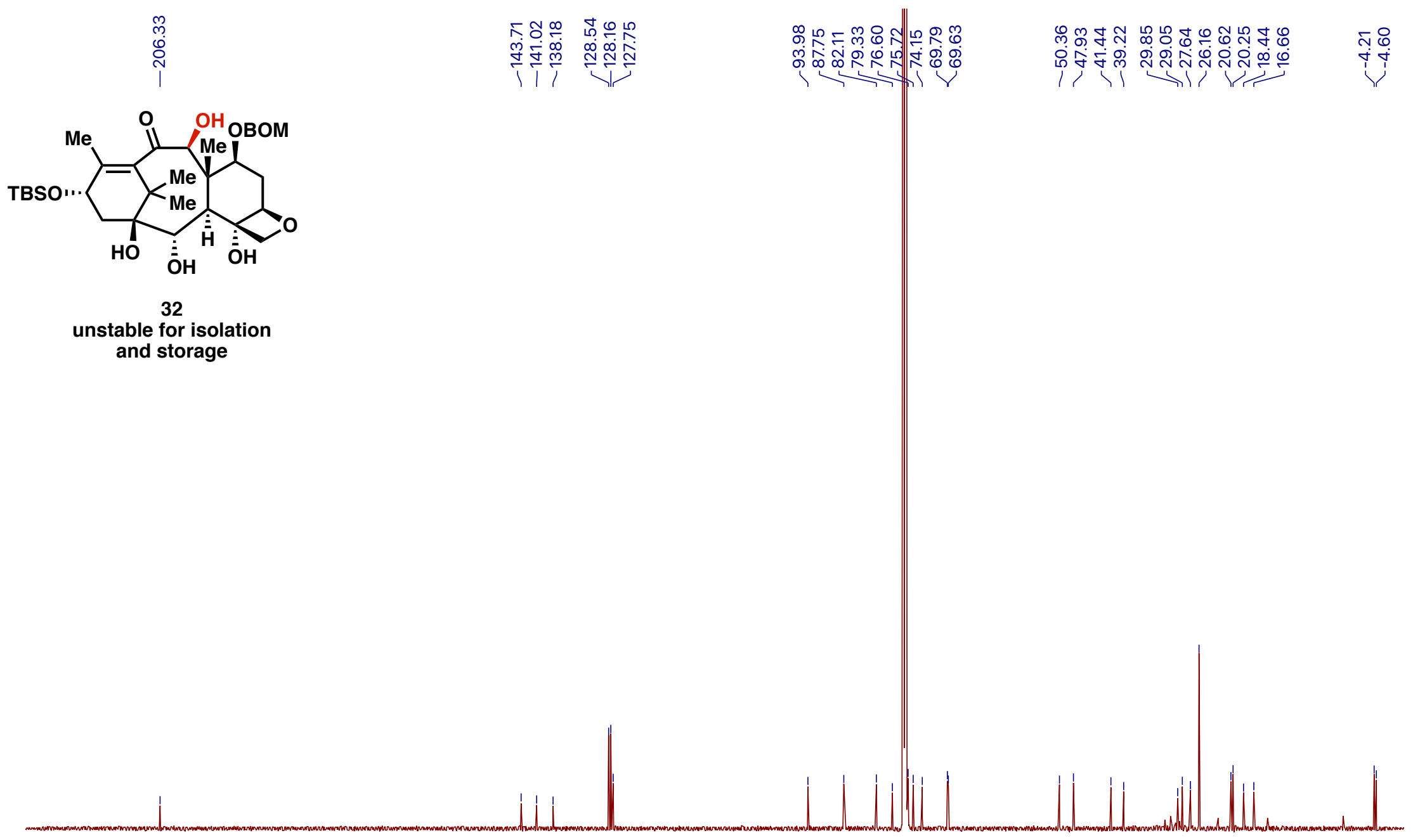

$\begin{array}{lllllllllllllllllllllllllllllll}220 & 210 & 200 & 190 & 180 & 170 & 160 & 150 & 140 & 130 & 120 & 110 & 100 & 90 & 80 & 70 & 60 & 50 & 40 & 30 & 20 & 10 & 0 & \mathrm{f} 1(\mathrm{ppm})\end{array}$ 


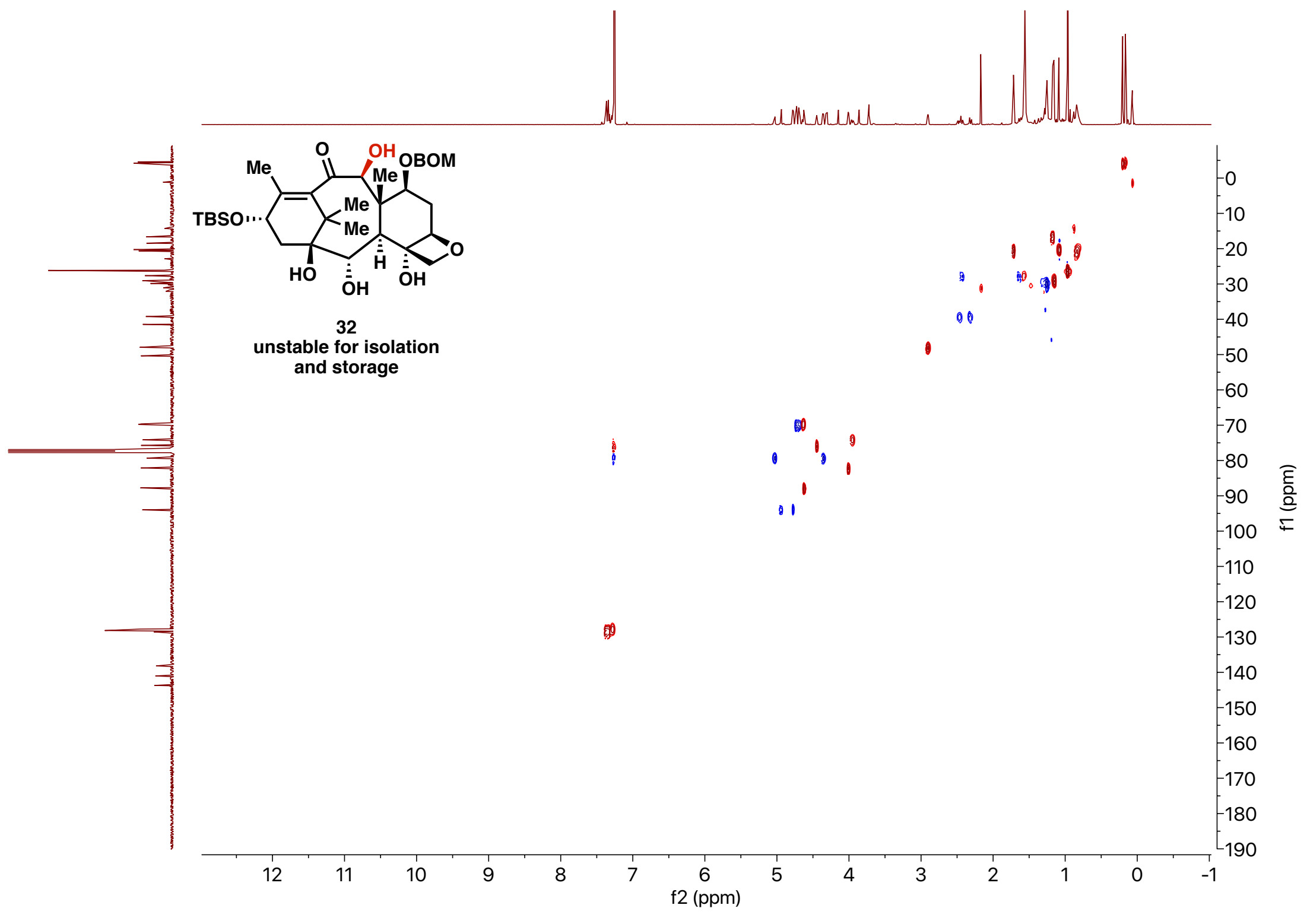




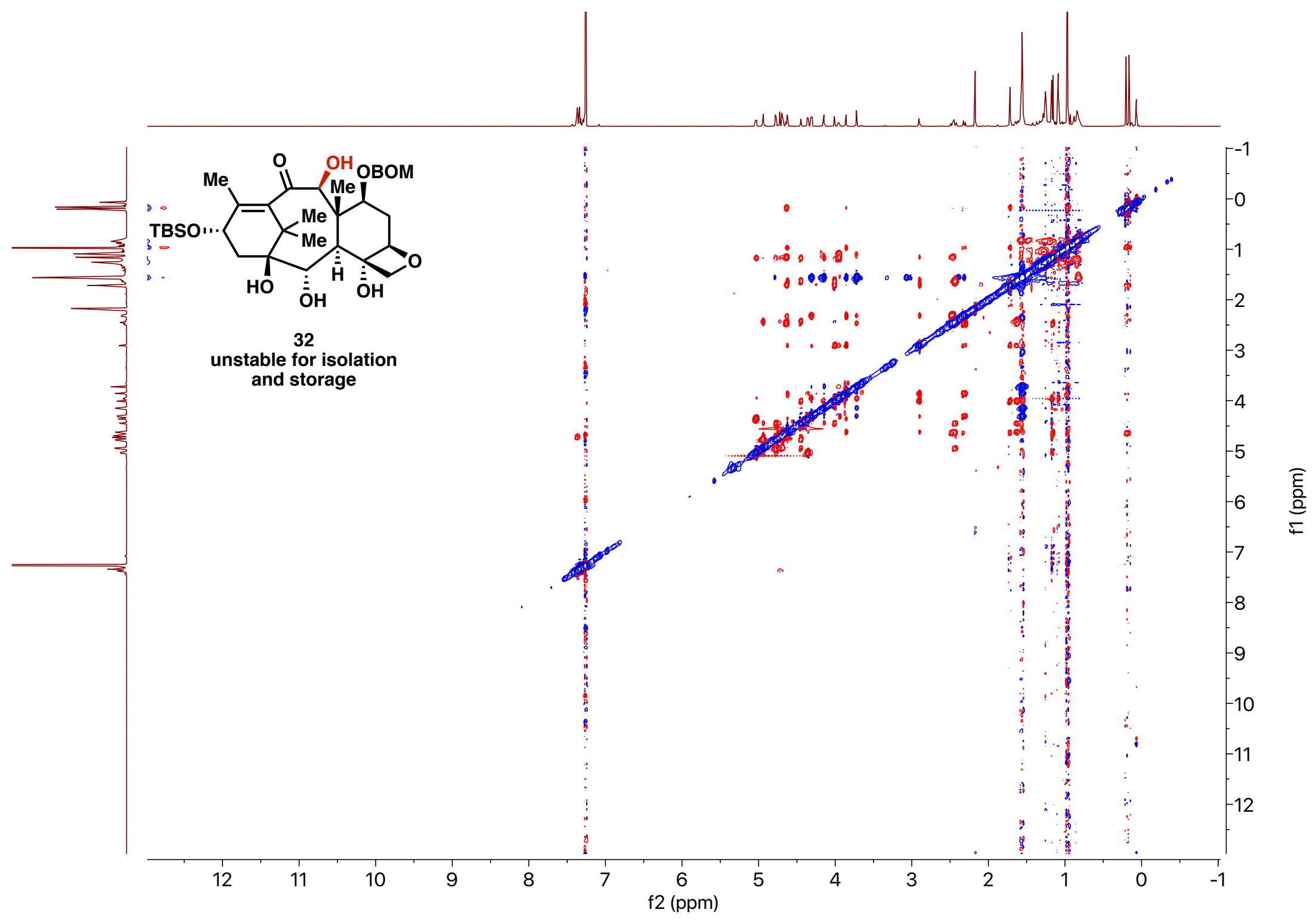


m m m

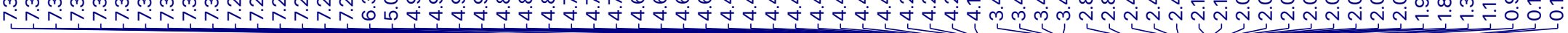
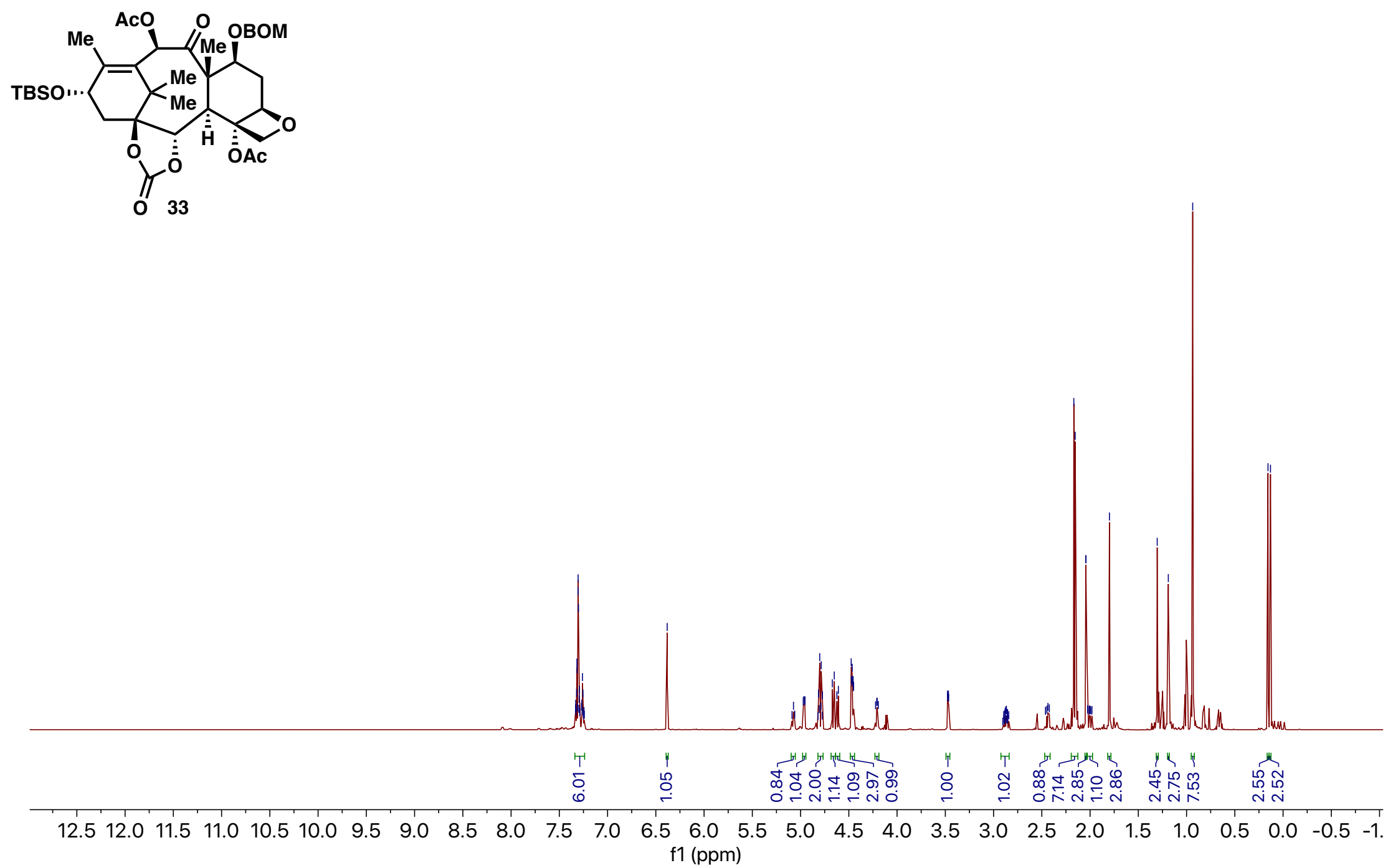


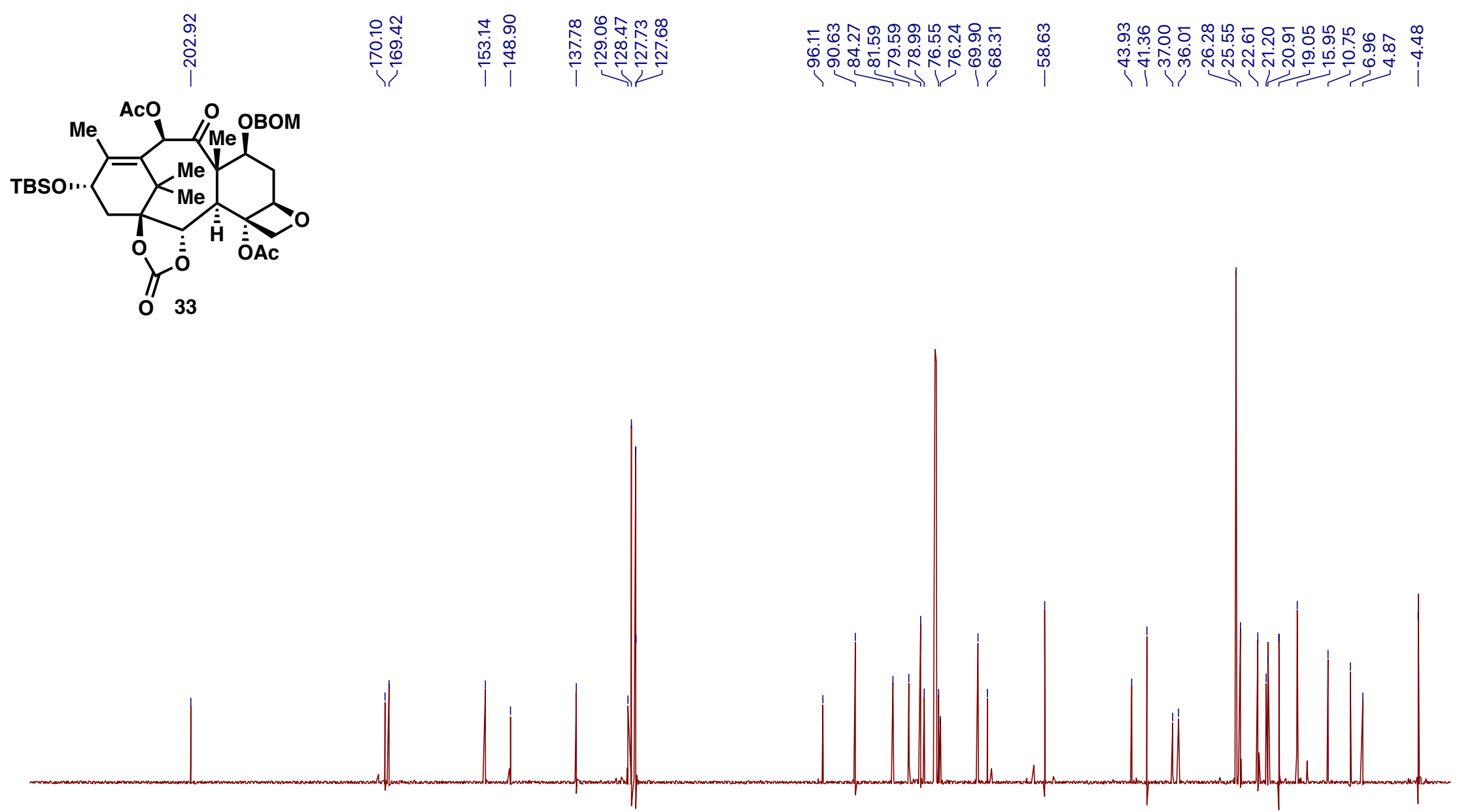

$\begin{array}{lllllllllllllllllllllllll}30 & 220 & 210 & 200 & 190 & 180 & 170 & 160 & 150 & 140 & 130 & 120 & 110 & 100 & 90 & 80 & 70 & 60 & 50 & 40 & 30 & 20 & 10 & 0 & f 0\end{array}$ 


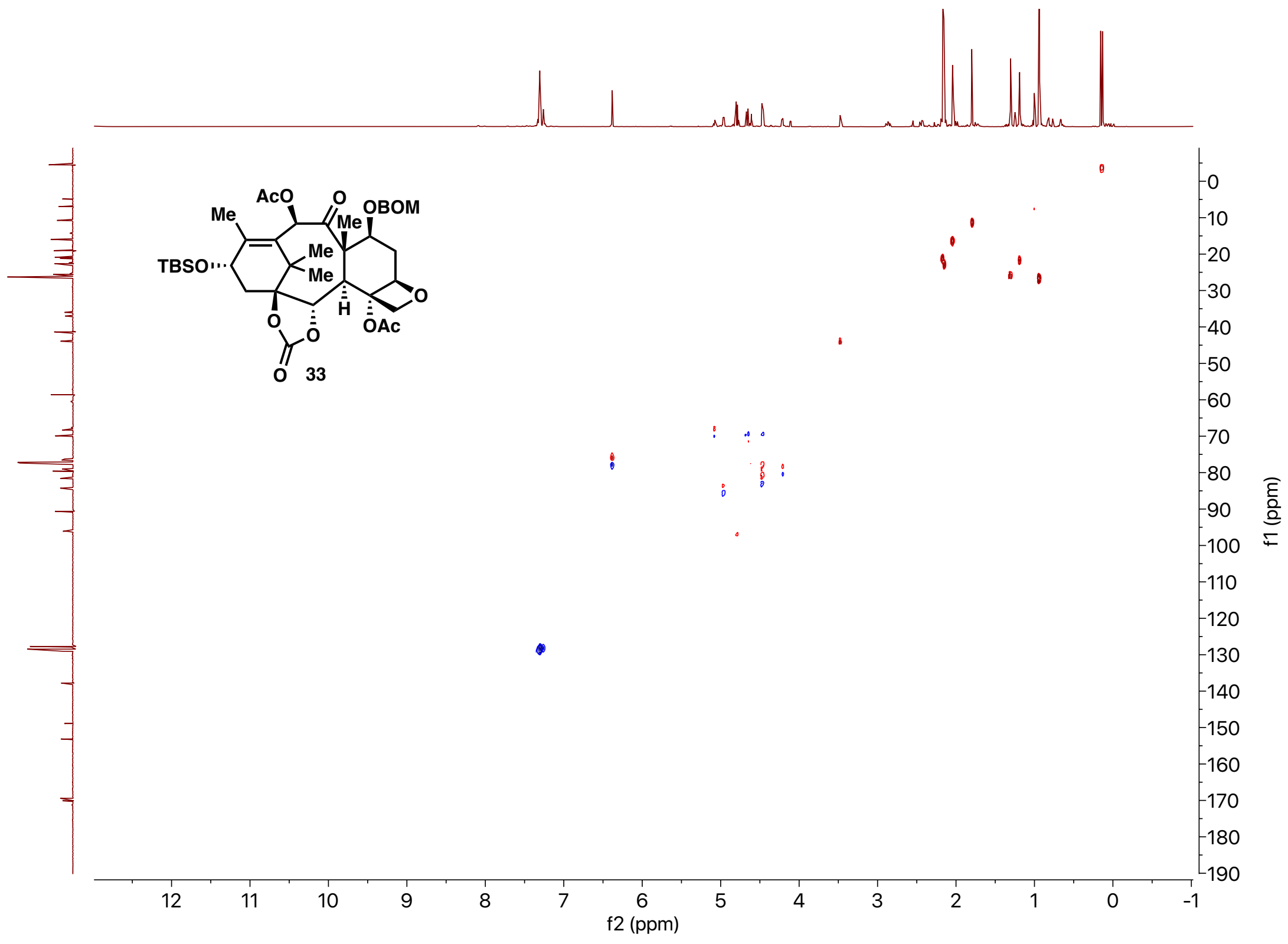

SI-175 


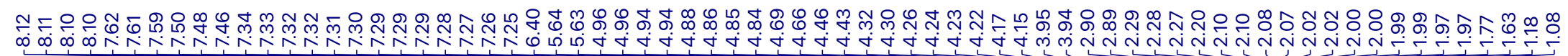

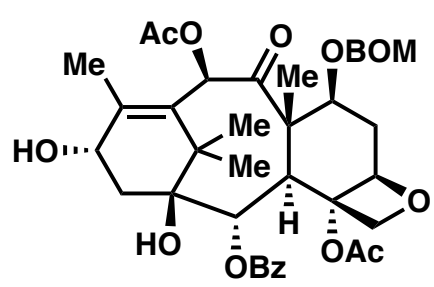

34

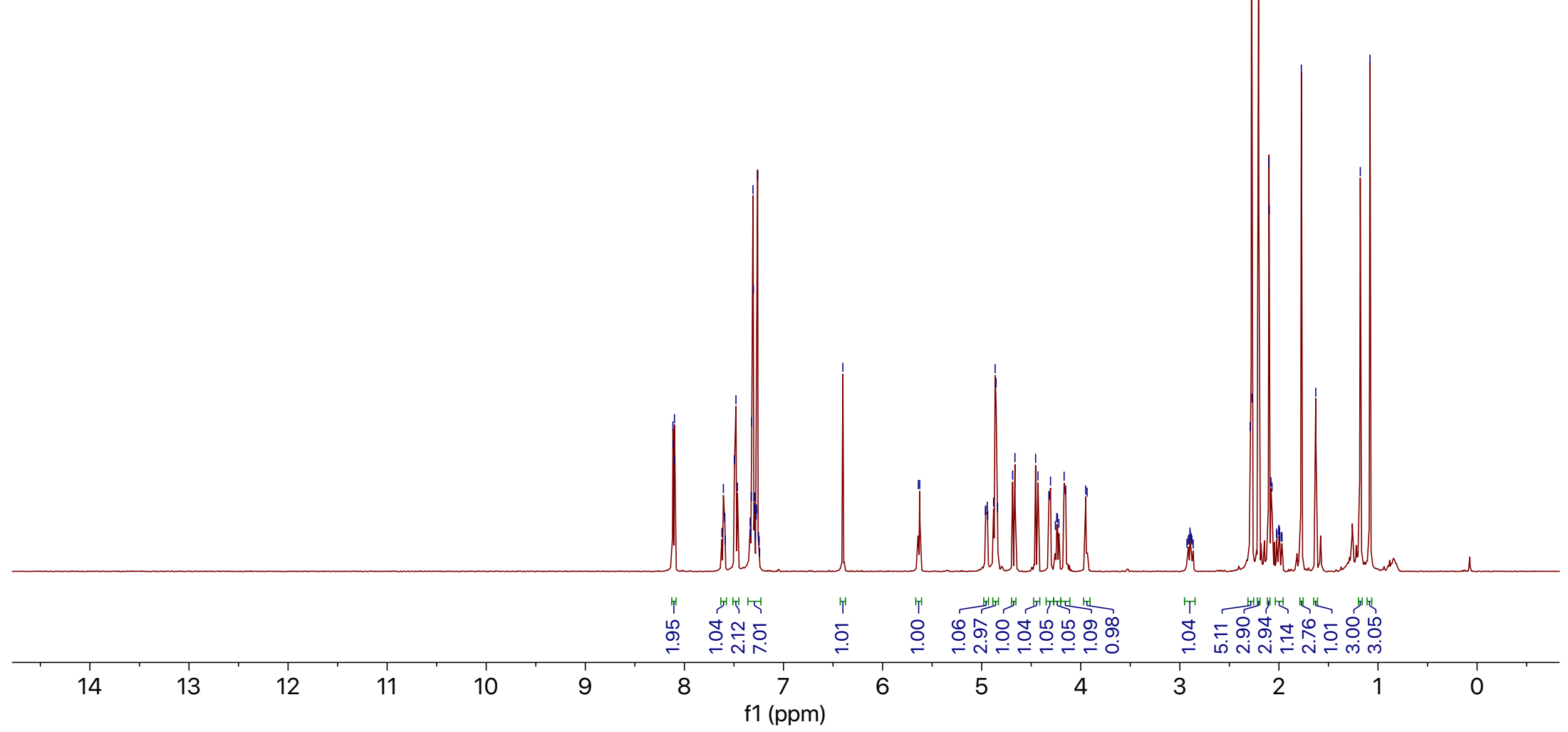



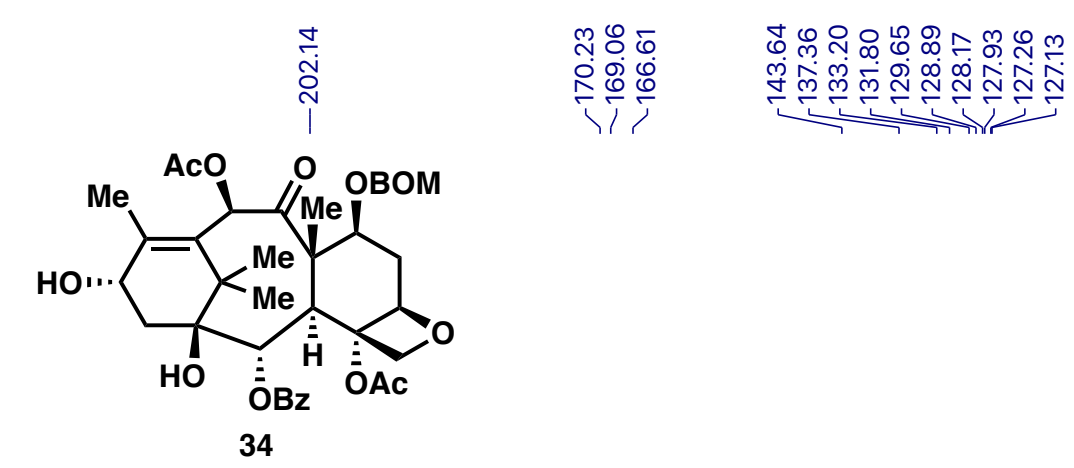

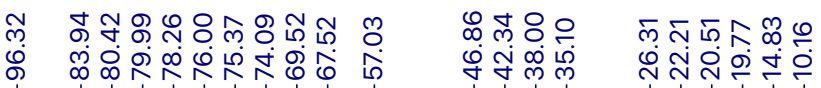

।

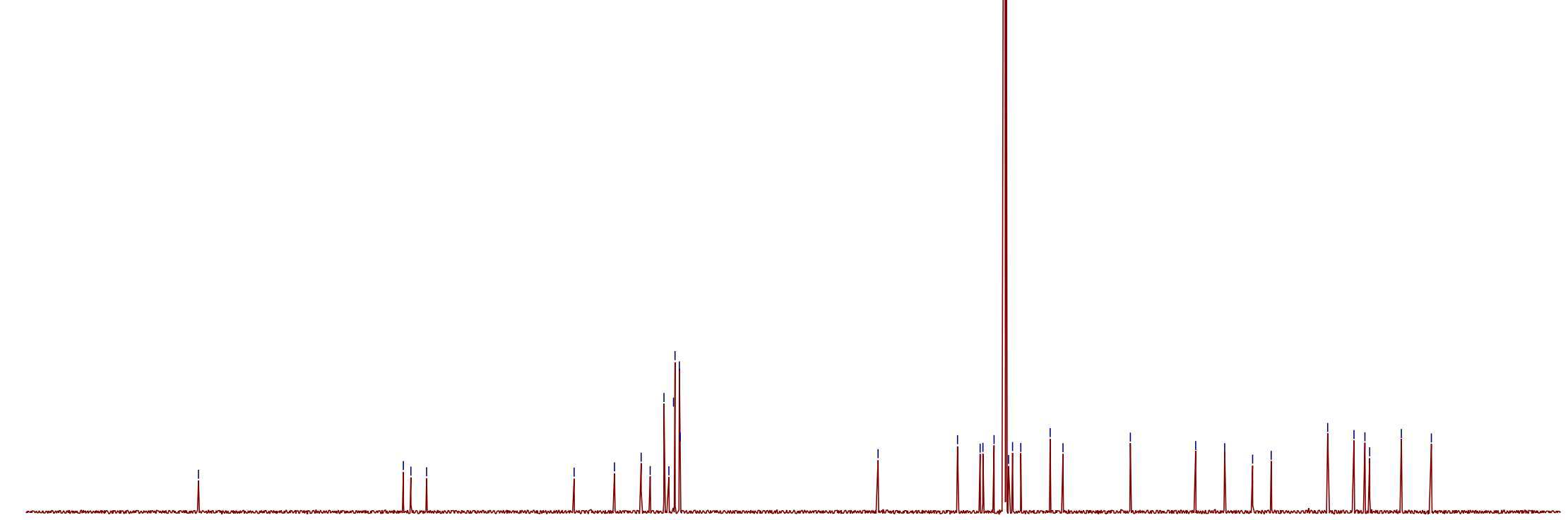

$\begin{array}{lllllll}220 & 210 & 200 & 190 & 180 & 170 & 160\end{array}$

$150140 \quad 130$

120110

f1 (ppm)

$\begin{array}{lllllll}60 & 50 & 40 & 30 & 20 & 10 & 0\end{array}$ 


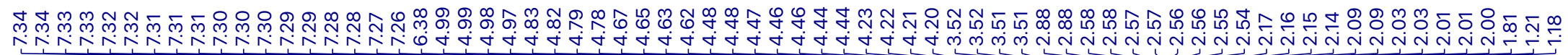
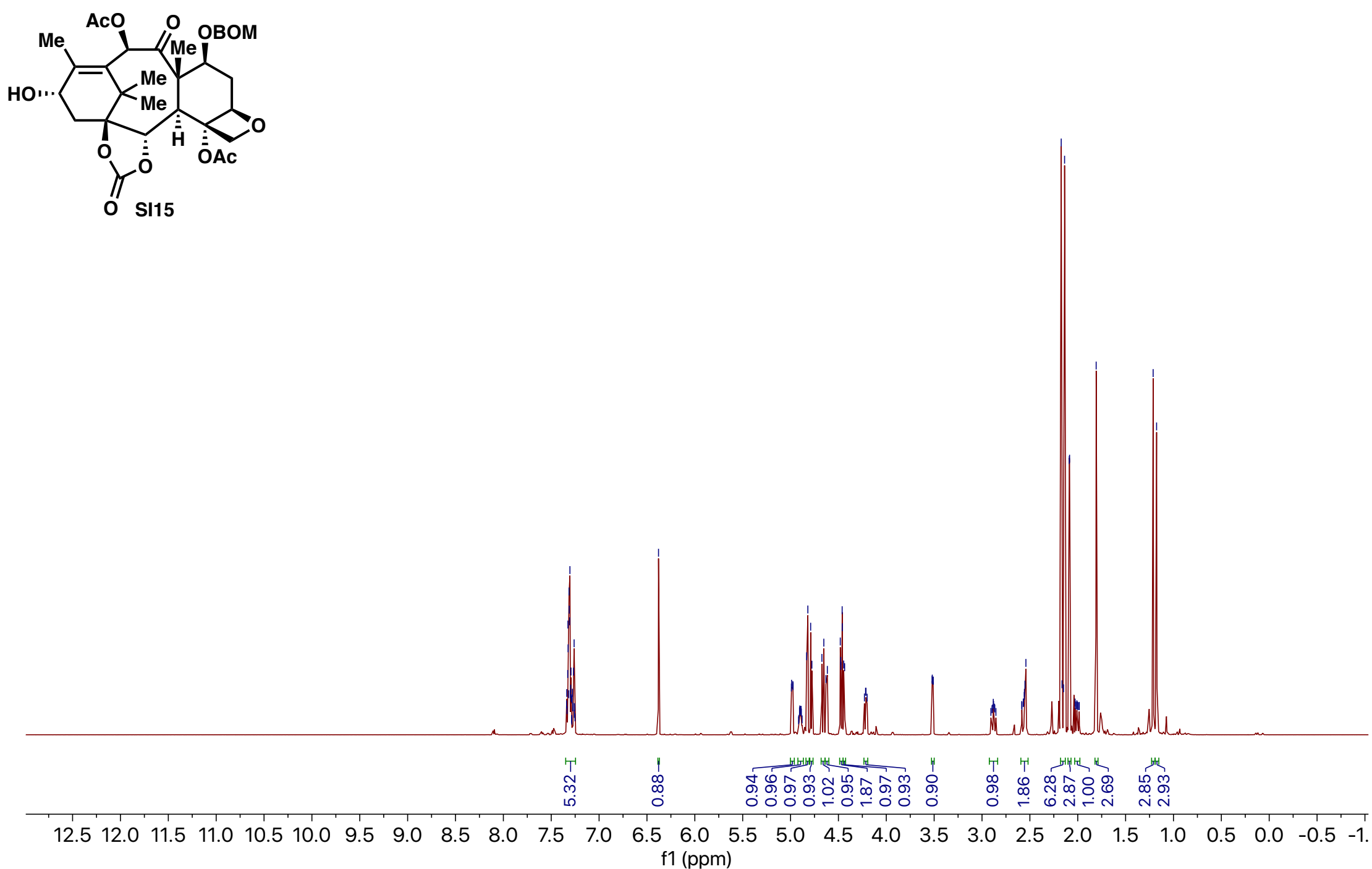


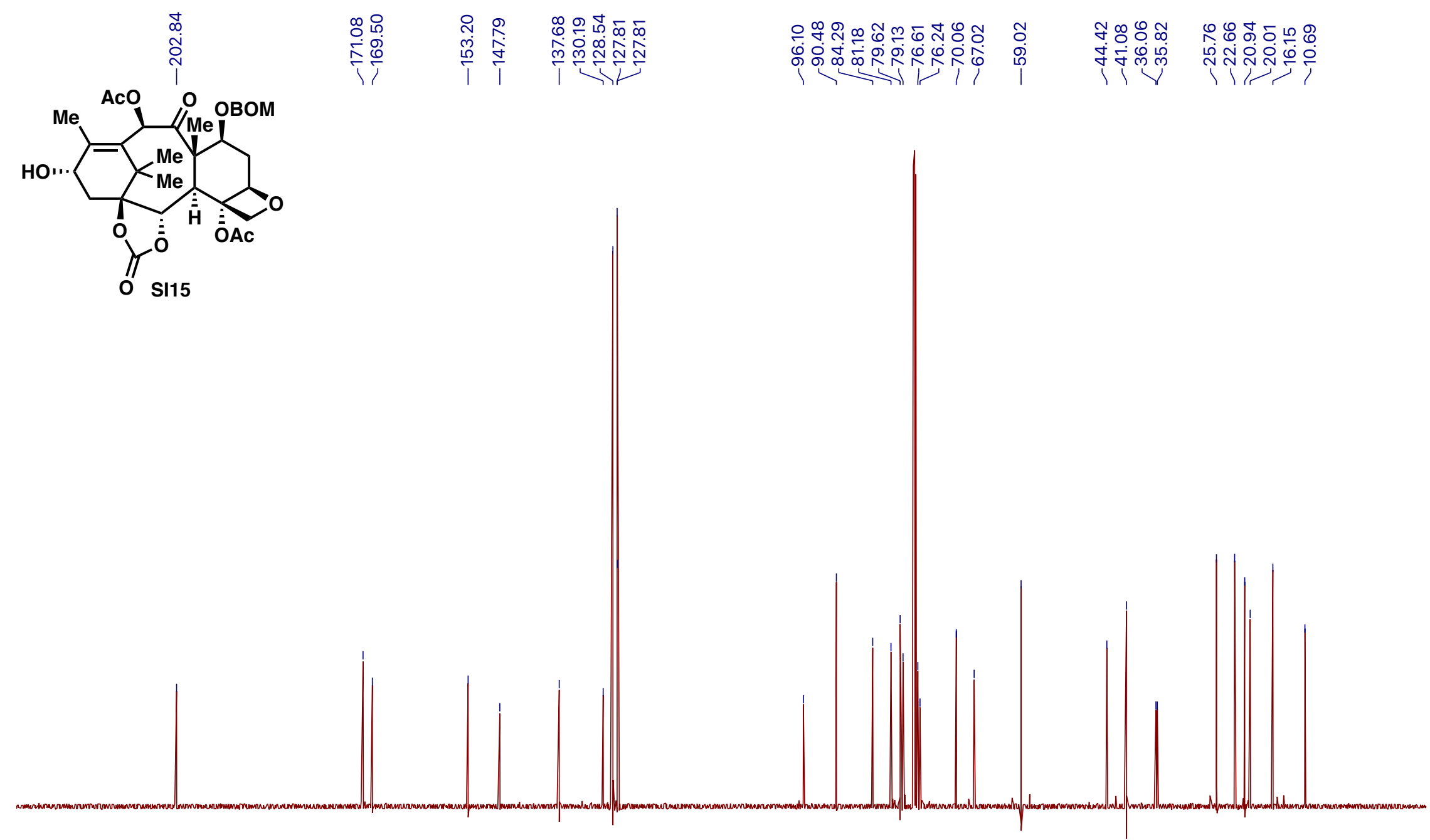

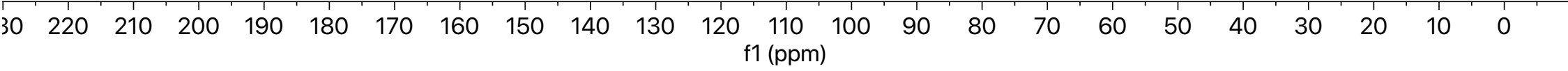




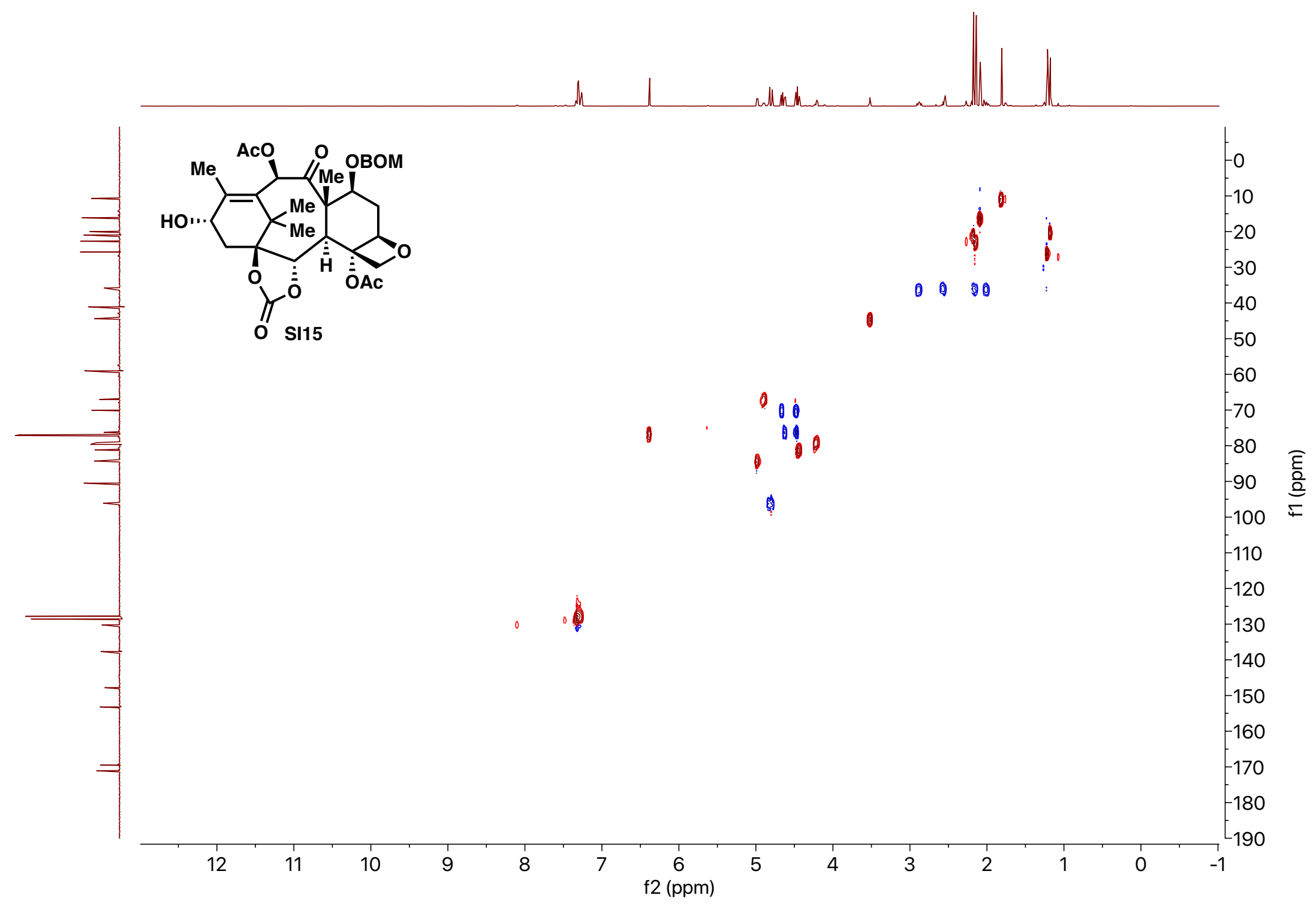

SI-180 


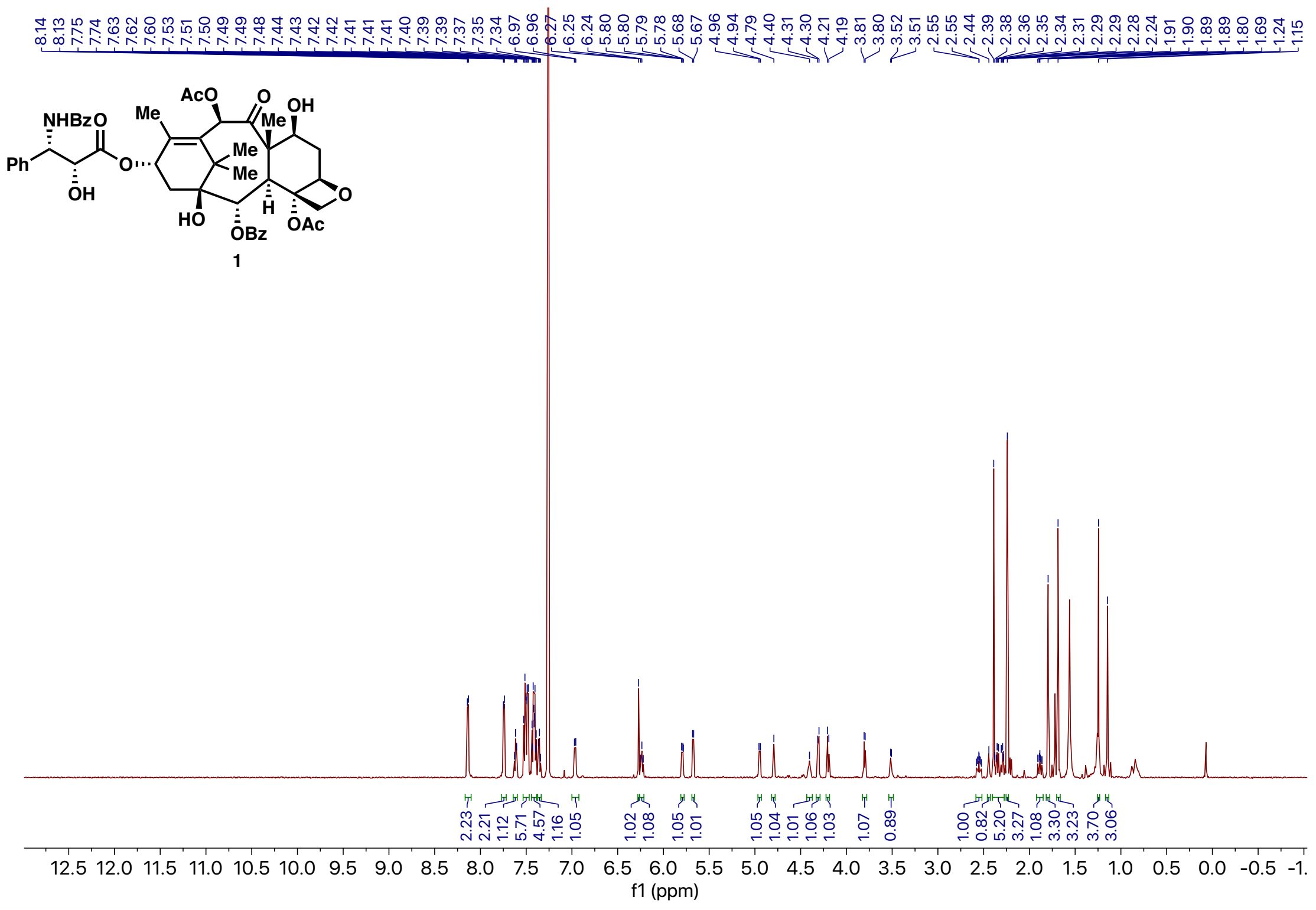




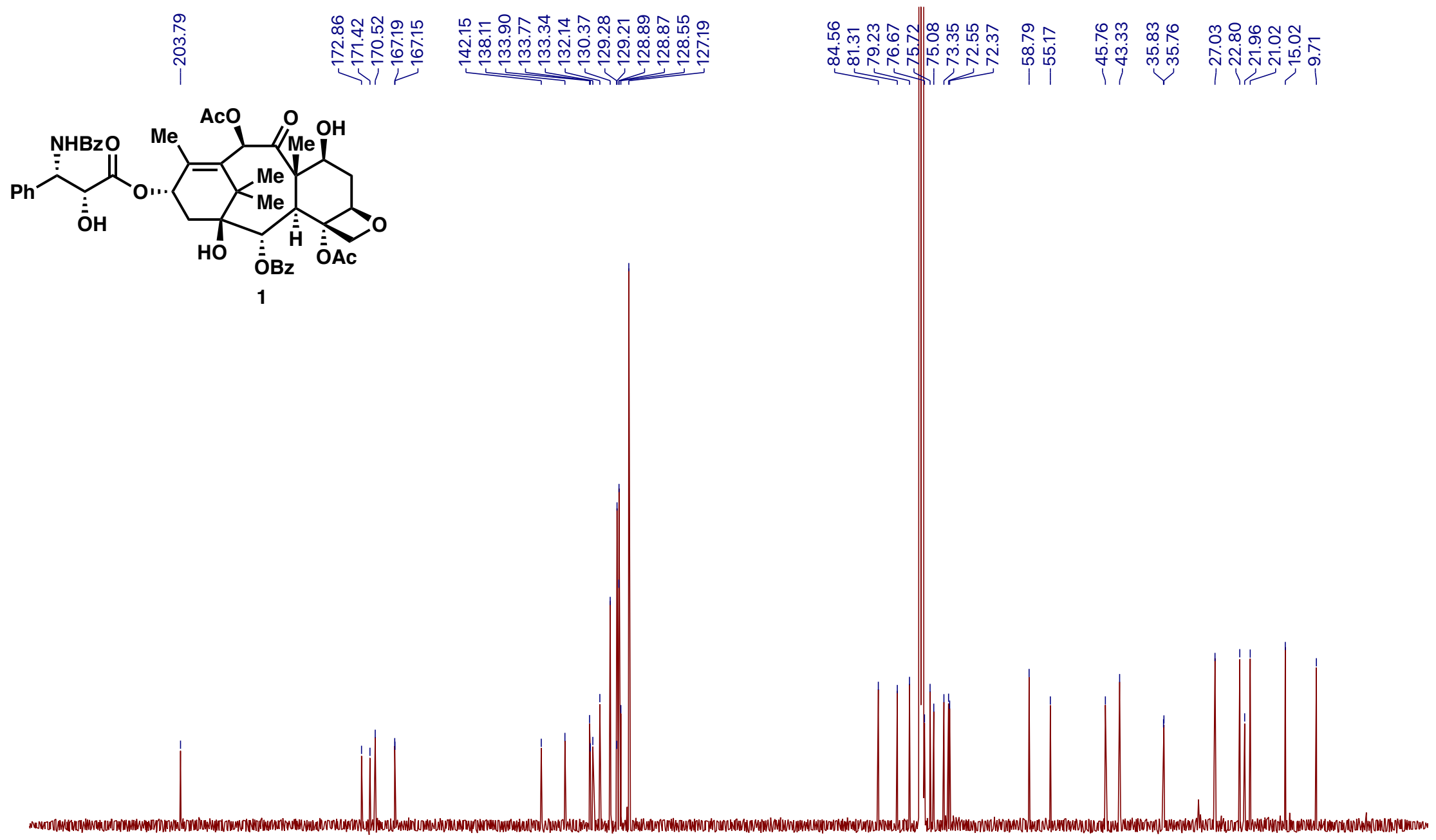

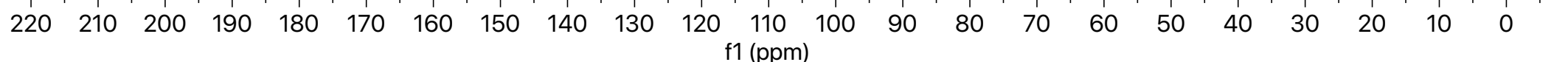




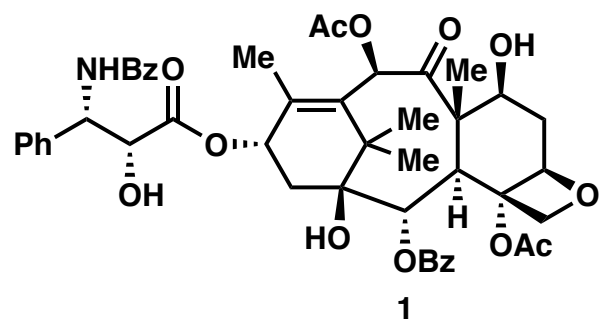

Natural

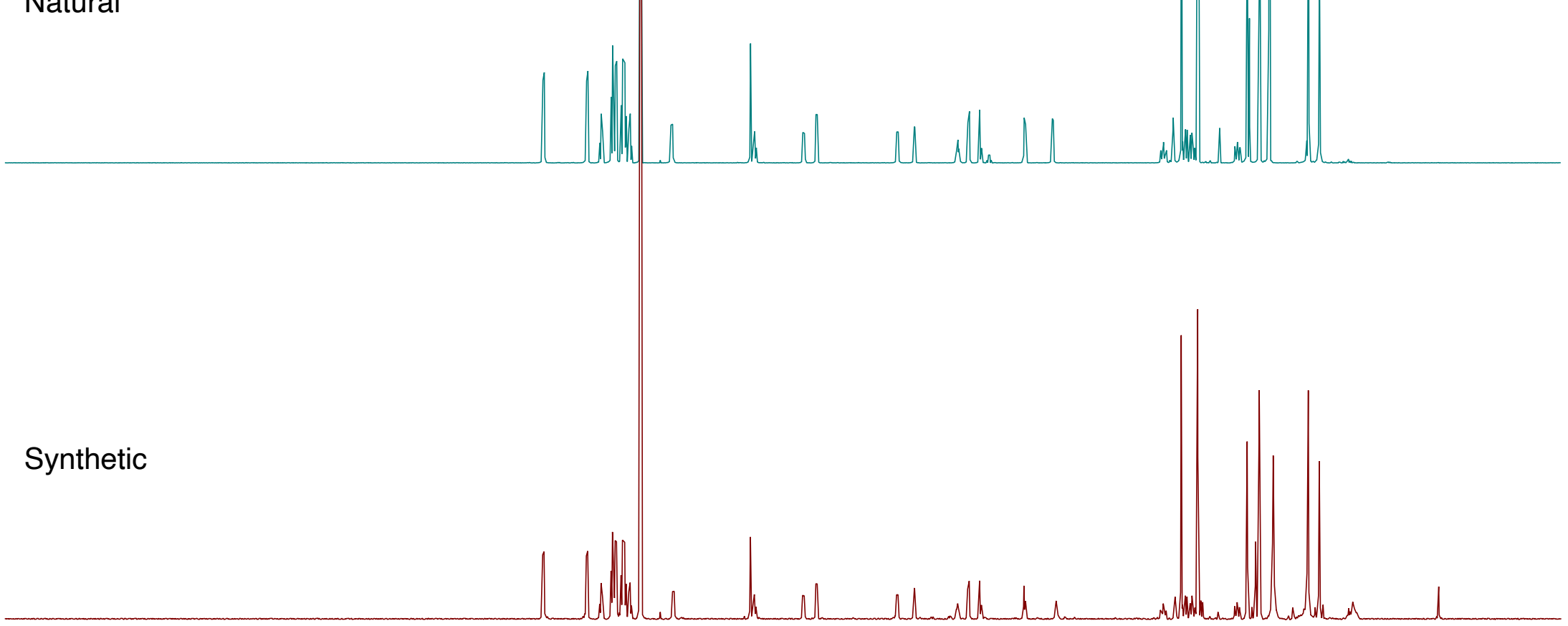

$\begin{array}{llllllllllllllllllllllllllllllllllll}12.5 & 12.0 & 11.5 & 11.0 & 10.5 & 10.0 & 9.5 & 9.0 & 8.5 & 8.0 & 7.5 & 7.0 & 6.5 & 6.0 & 5.5 & 5.0 & 4.5 & 4.0 & 3.5 & 3.0 & 2.5 & 2.0 & 1.5 & 1.0 & 0.5 & 0.0 & -0.5 & -1 .\end{array}$ f1 (ppm) 


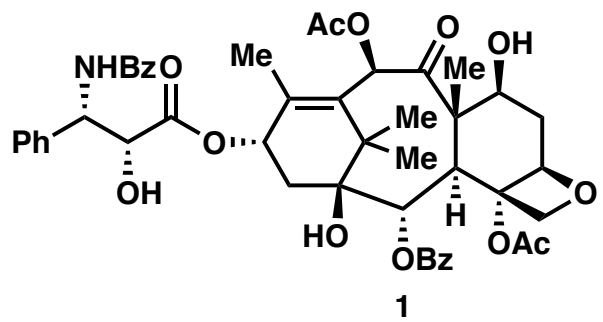

Natural

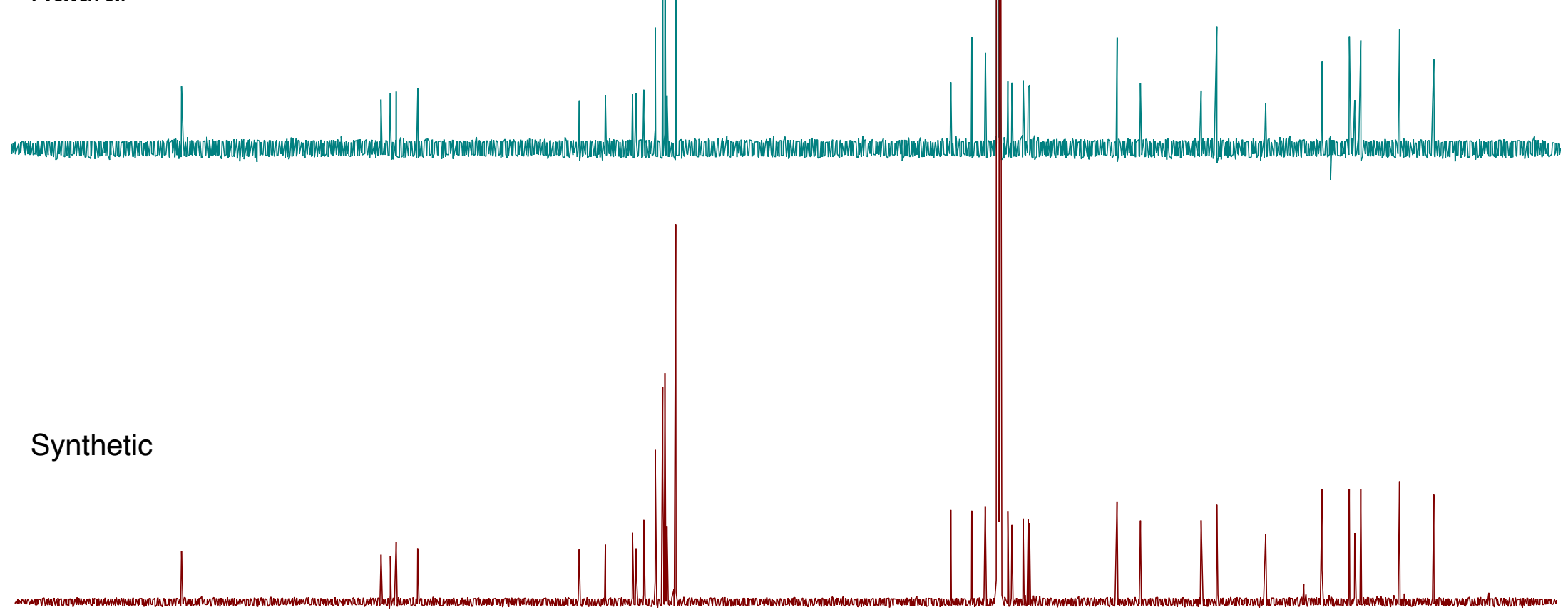

30220
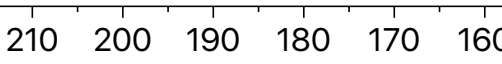

$\begin{array}{llll}150 & 140 & 130\end{array}$

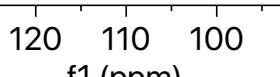

f1 (ppm)

8070

$60 \quad 50$

$40 \quad 30$

20

$10 \quad 0$

SI-184 


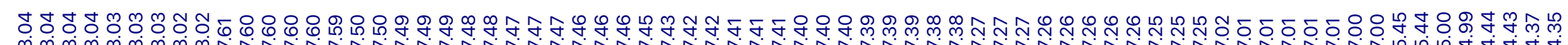

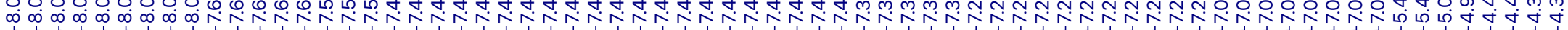

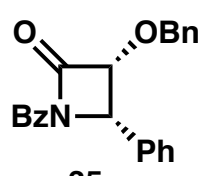

35

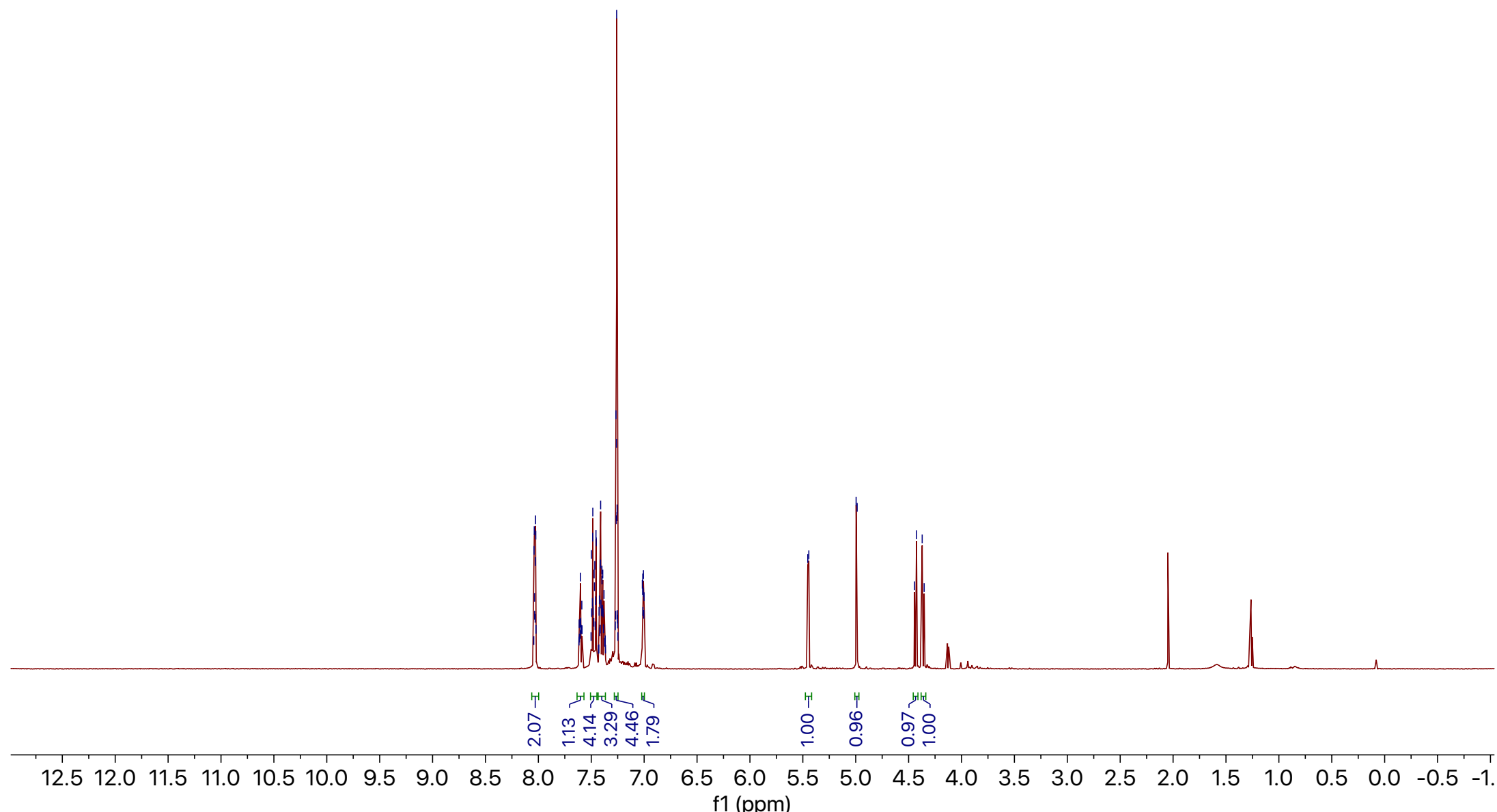

SI-185 


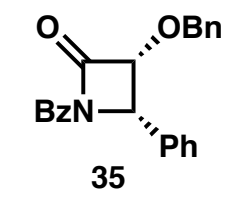

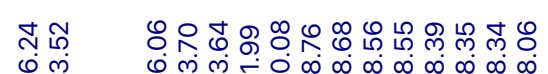

Q

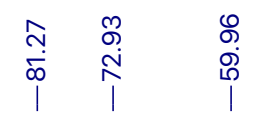

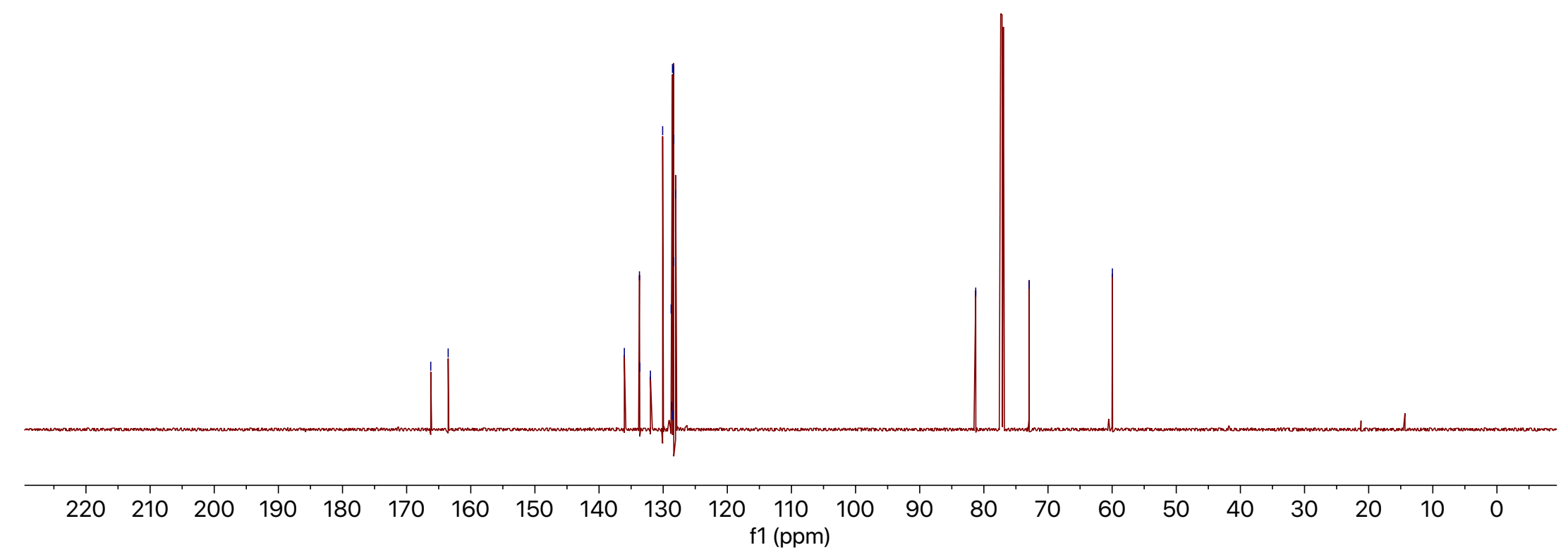

SI-186 


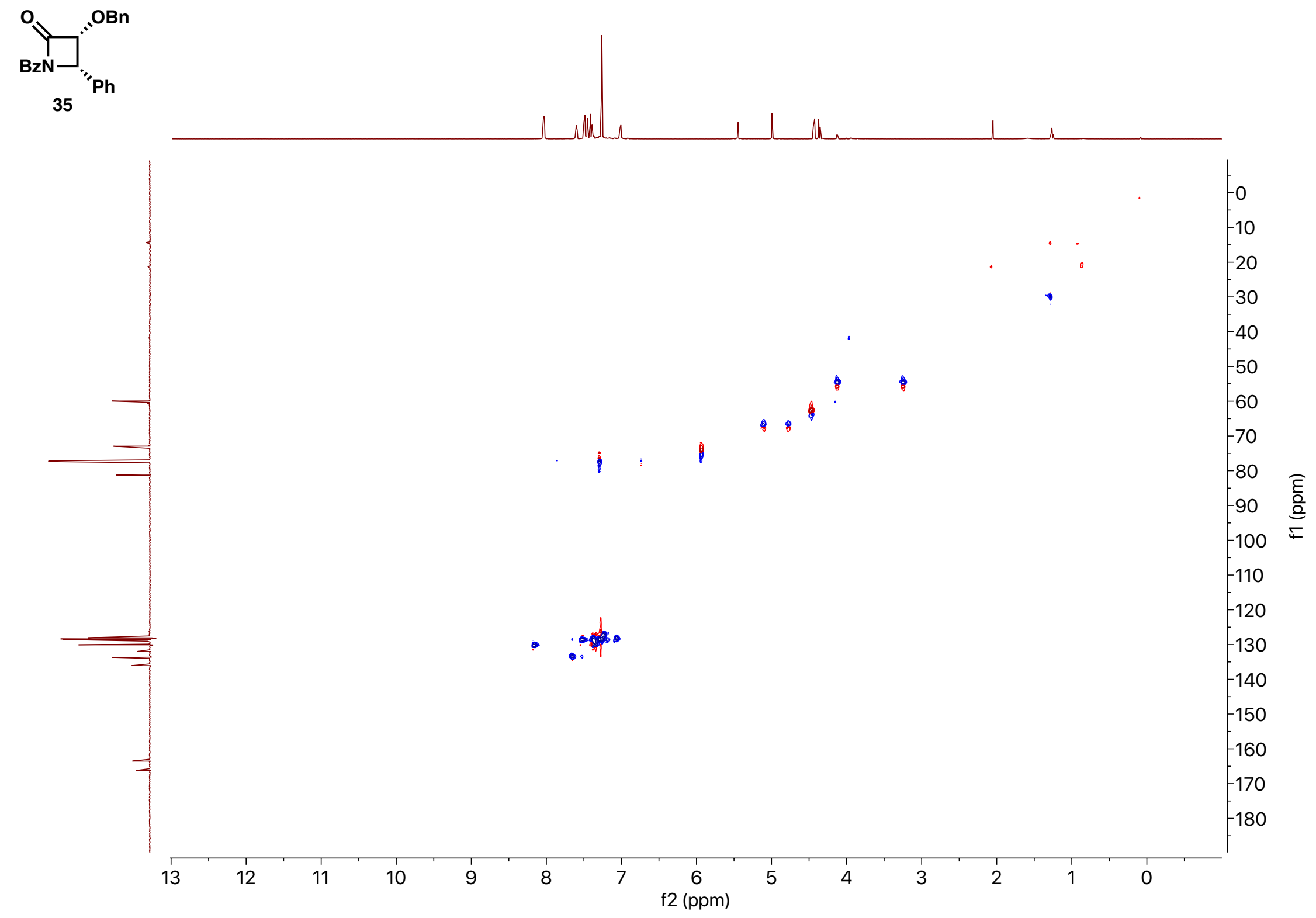

SI-187 


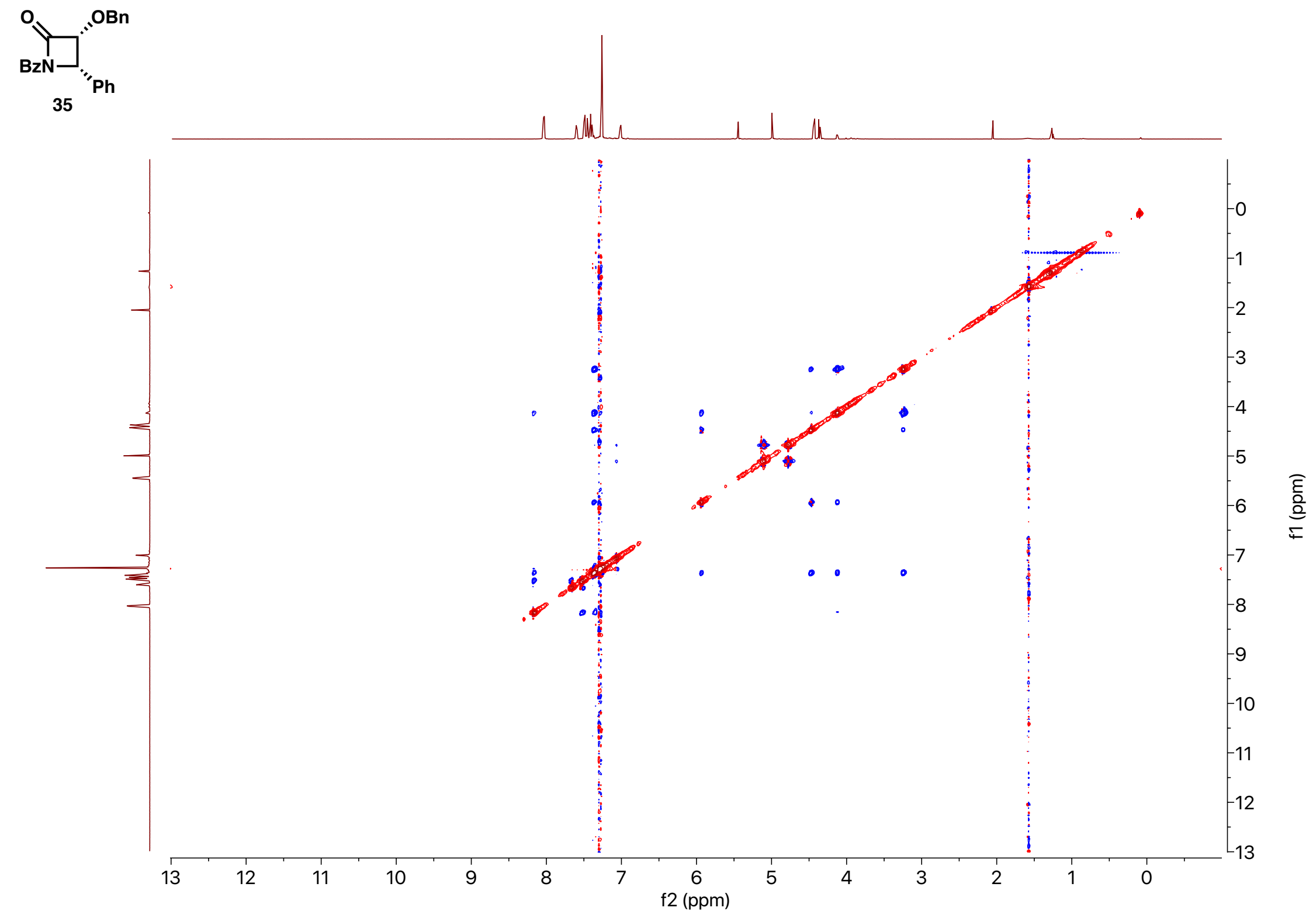

SI-188 